\title{
Ems-Dollard ecosystem model to study changing turbidity and higher trophic level response.
}

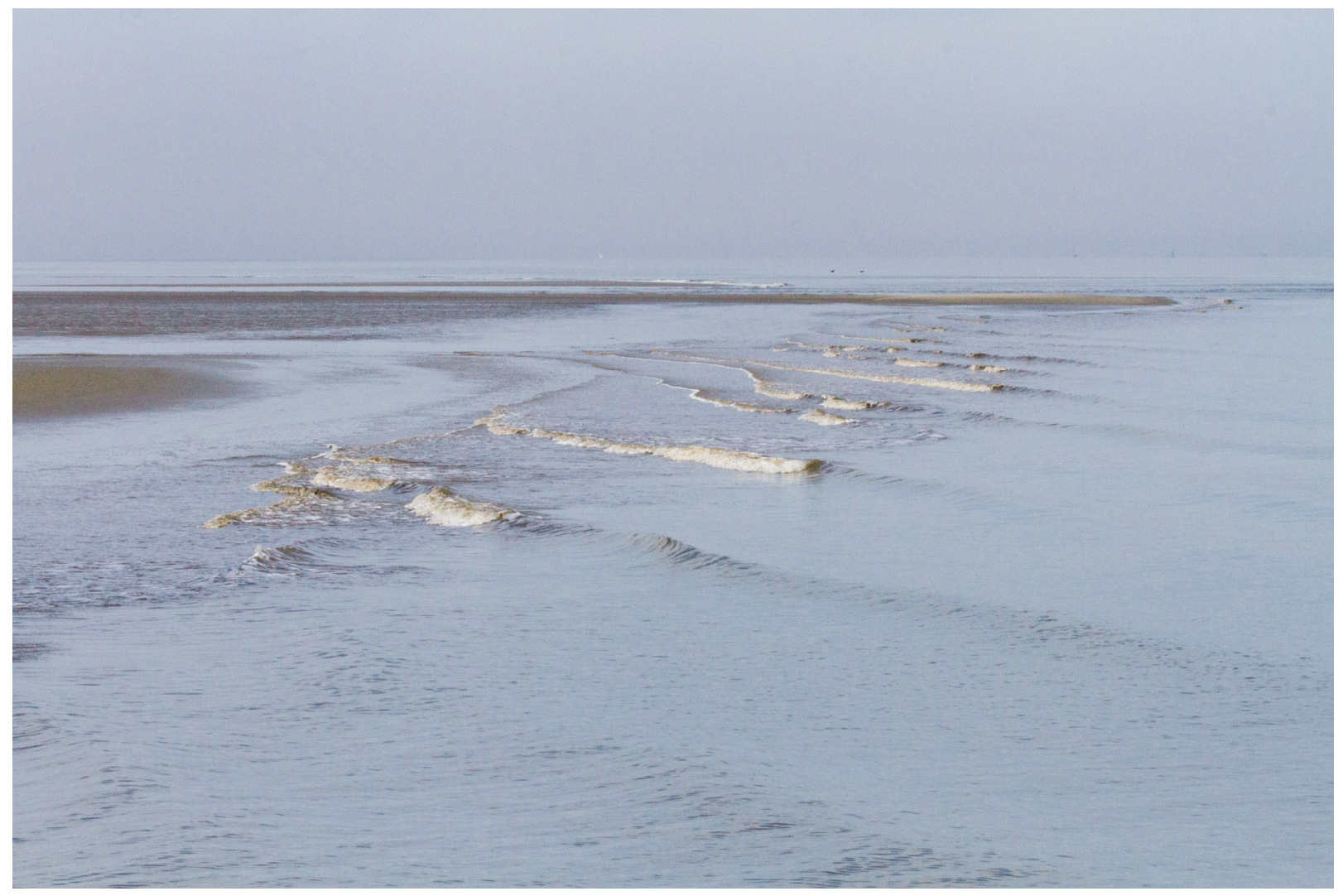

Authors:

AG Brinkman

JE Tamis

Publication date: 2018 Apr 20

This research project was carried out by Wageningen Marine Research at the request of and with funding from the Ministry of Economic Affairs for the purposes of Policy Support Research Theme 'Natuurambitie Grote Wateren' (project no. BO-11-018.01-005).

Wageningen Marine Research, report C058/17 
Brinkman AG \& Tamis JE. 2017. Ems-Dollard ecosystem model to study changing turbidity and higher trophic level response. Wageningen Marine Research (Wageningen University \& Research), Wageningen Marine Research report C058/17, 284pp (62 pp main, 222 pp appendices)

Keywords: Ecosystem model, Ems-Dollard estuary, turbidity, higher trophic level, silt problem

Client: $\quad$ Ministry of Economic Affairs

Drs M. Datema

BAS code: BO-11-018.01-005

This report can be downloaded, paper copies will not be forwarded: https://doi.org/10.18174/421199

Wageningen Marine Research is ISO 9001:2008 certified.

(C) 2016 Wageningen Marine Research Wageningen UR

Wageningen Marine Research The Management of Wageningen Marine Research is not institute of Stichting Wageningen responsible for resulting damage, as well as for damage Research is registered in the Dutchresulting from the application of results or research obtained by traderecord nr. 09098104, Wageningen Marine Research, its clients or any claims related BTW nr. NL 806511618 to the application of information found within its research. This report has been made on the request of the client and is wholly the client's property. This report may not be reproduced and/or published partially or in its entirety without the express written consent of the client. 


\section{Colophon}

With data contributions of:

Niedersächsischer Landesbetrieb für Wasserwirtschaft, Küsten- und Naturschutz (NLWKN)

(DE, Norden)

Royal Institute for Sea Research (Royal NIOZ)

(NL, Texel)

Waterschap Hunze en Aa's

(NL, Veendam)

Waterschap Noorderzijlvest

(NL, Groningen)

Helpdesk Rijkswaterstaat

(NL, Lelystad)

Deltares

(NL, Delft)

Wageningen Marine Research

(NL, Yerseke)

(Details in section 1.4)

Cover photo : : AG Brinkman

Report prepared by : AG Brinkman, Brinkman Adviesbureau/BAB and JE Tamis, Wageningen Marine Research

Postal address : Wageningen Marine Research

Ankerpark 27

1781 AG Den Helder

E-mail

: bert.brinkman@wur.nl

bert.brinkman@brinkman-bab.nl

jacqueline.tamis@wur.nl 
4 of 286 | Wageningen Marine Research report C058/17 


\section{Contents}

\section{Contents}

Introduction

1.1 General 15

1.2 Why this model? 15

1.3 Structure of the report 16

1.4 Thanks 16

$\begin{array}{lll}1.5 & \text { Geographical data sources } & 17\end{array}$

$\begin{array}{lll}1.6 & \text { Software used } & 17\end{array}$

$\begin{array}{lll}1.7 & \text { Responsibilities } & 17\end{array}$

$2 \quad$ Model set-up and area 19

$\begin{array}{lll}2.1 & \text { Compartments } & 19\end{array}$

2.2 The 'real' estuary 21

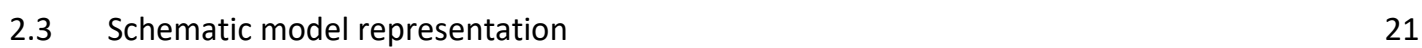

$3 \quad$ Input data 23

3.1 General 23

3.2 Water quantity data 23

3.2.1 Available data $\quad 23$

3.2.2 Missing data $\quad 24$

3.3 Water quality data 24

3.3.1 Water quality data Netherlands inside the system 24

3.3.2 Water quality data outside the system: boundary sites 24

$\begin{array}{lll}\text { 3.3.3 General overview } & 25\end{array}$

$\begin{array}{ll}\text { 3.3.4 Exceptional situations } & 27\end{array}$

$\begin{array}{lll}3.3 .5 & \text { Old data } & 28\end{array}$

$\begin{array}{lll}\text { 3.3.6 Missing data } & 28\end{array}$

$\begin{array}{ll}3.4 & \text { Morphology } \\ \end{array}$

3.4.1 Depth data $\quad 29$

3.4.2 Sediment data Netherlands, Wadden Sea -A 29

3.4.3 Meteorological data 30

$\begin{array}{lll}3.5 & \text { Habitat data } & 31\end{array}$

$4 \quad$ Data for comparison 33

4.1 General 33

$\begin{array}{lll}4.2 & \text { Benthic fauna data } & 33\end{array}$

4.2.1 Netherlands 33

4.2.2 Germany $\quad 34$

$\begin{array}{lll}4.3 & \text { Phytobenthos and benthic primary production } & 34\end{array}$

4.4 Phytoplankton, chlorophyll-data and pelagic primary production 36

$\begin{array}{lll}4.5 & \text { Water levels } & 36\end{array}$

$\begin{array}{lll}4.6 & \text { Older data } & 37\end{array}$ 
$\begin{array}{lll}5.1 & \text { The water column } & 39\end{array}$

$\begin{array}{ll}5.2 & \text { Exchange between compartments }\end{array}$

5.3 Exchange between sub-compartments 39

$\begin{array}{lll}5.4 & \text { Temperature } & 39\end{array}$

5.5 The sediment part, pore water profiles, oxygen and nitrogen consumption in the sediment $\begin{array}{ll}\text { and adsorbing ions } & 40\end{array}$

5.6 Choices for model components 41

5.7 lon/ elements 41

5.8 Phytoplankton 41

5.8.1 Groups 41

$\begin{array}{lll}\text { 5.8.2 Parameters } & 41\end{array}$

5.9 Detritus 42

5.9.1 Groups 42

5.9.2 Parameters 42

5.10 Fauna 42

5.10.1 Overall description of the model 42

5.10.2 Parameters $\quad 42$

$\begin{array}{lll}5.10 .3 & \text { The literature review. } & 43\end{array}$

5.10.4 Model parts describing the effect of silt on zoobenthos growth 43

$\begin{array}{ll}5.11 \text { Zoobenthos } & 44\end{array}$

$\begin{array}{lll}5.11 .1 & \text { Groups } & 44\end{array}$

$\begin{array}{ll}5.12 \text { Zooplankton } & 45\end{array}$

$\begin{array}{lll}5.12 .1 & \text { Groups } & 45\end{array}$

$\begin{array}{lll}5.12 .2 & \text { Parameters } & 45\end{array}$

$\begin{array}{lll}6 & \text { Tuning the model physics } & 47\end{array}$

$\begin{array}{lll}6.1 & \text { General } & 47\end{array}$

$\begin{array}{lll}6.2 & \text { Exchange between compartments } & 47\end{array}$

$\begin{array}{llr}6.3 & \text { Resuspension in each compartment } & 49\end{array}$

$\begin{array}{lll}\text { 6.3.1 Introduction } & 49\end{array}$

$\begin{array}{lll}6.3 .2 & \text { Results } & 49\end{array}$

7 Model results and effect of changing turbidity $\quad 53$

$\begin{array}{lll}7.1 & \text { Introduction } & 53\end{array}$

$\begin{array}{lll}7.2 & \text { Changing turbidity simulated } & 53\end{array}$

$\begin{array}{lll}7.3 & \text { Results } & 53\end{array}$

7.3.1 Extinction coefficient 53

$\begin{array}{lll}\text { 7.3.2 Primary production and dissolved components } & 55\end{array}$

8 Conclusions $\quad 61$

$\begin{array}{lll}8.1 & \text { Overall } & 61\end{array}$

$\begin{array}{lll}8.2 & \text { Most relevant improvements } & 61\end{array}$

8.3 All suggestions for model improvements 62

$\begin{array}{lr}\text { References } & 65\end{array}$

$\begin{array}{lr}\text { Justification } & 71\end{array}$

$\begin{array}{ll}\text { APPENDICES } & 73\end{array}$

A1 Compartment setup and sub-compartments $\quad 75$ 
A2 Overview table main benthic animal groups (next to shellfish) 77

A3 Water quality monitoring sites tributaries $\quad 79$

A4 Completing water quantity data series $\quad 83$

A4.1 Introduction 83

A4.2 Computation method $\quad 85$

A4.2.1 Precipitation data $\quad 85$

A4.2.2 Drainage areas $\quad 86$

A4.2.3 Averaging of data $\quad 88$

A4.2.4 Maximum discharge values $\quad 90$

$\begin{array}{ll}\text { A4.2.5 Completed data sets } & 91\end{array}$

A5 Water quality variables, elaboration of Dutch and German water quality data 95

A5.1 Introduction 95

$\begin{array}{ll}\text { A5.2 Available water quality data } & 96\end{array}$

A5.3 Data consistency check and completion 97

$\begin{array}{ll}\text { A5.3.1 introduction } & 97\end{array}$

$\begin{array}{ll}\text { A5.3.2 Phosphorus compounds } & 98\end{array}$

$\begin{array}{ll}\text { A5.3.3 Nitrogen compounds } & 98\end{array}$

A5.3.4 Carbon compounds $\quad 99$

A5.3.5 Conductivity, salinity and chloride content $\quad 99$

A6 Estimating time series for the whole period 1976-now for all variables 102

A7 Completing temperature data series 103

$\begin{array}{lll}\text { A8 } & \text { Manual adaptation of input time series } & 107\end{array}$

A9 Trajectories sailed in 2012-2013 113

A10 Phytoplankton (and fauna) related to salinity in the model $\quad 114$

A11 Some details on available benthos data $\quad 115$

$\begin{array}{ll}\text { A11.1 General } & 115\end{array}$

$\begin{array}{ll}\text { A11.2 Sampling intensity } & 116\end{array}$

$\begin{array}{ll}\text { A11.3 Benthos classification } & 116\end{array}$

$\begin{array}{ll}\text { A11.4 Benthos density } & 116\end{array}$

$\begin{array}{lll}\text { A11.4.1 Introduction } & 116\end{array}$

$\begin{array}{ll}\text { A11.5 Data conversion } & 116\end{array}$

$\begin{array}{lll}\text { A11.5.1 Mussels } & 117\end{array}$

$\begin{array}{lll}\text { A11.5.2 Mudsnails } & 117\end{array}$

$\begin{array}{lll}\text { A11.5.3 All biomass together } & 117\end{array}$

$\begin{array}{lll}\text { A11.5.4 Classification table } & 120\end{array}$

A12 Depth data 126

$\begin{array}{lll}\text { A13 Water level data } & 127\end{array}$

$\begin{array}{lll}\text { A14 Sediment data } & 128\end{array}$

A14.1 Sediment data Netherlands, North Sea 128

A14.2 Sediment data Dutch Wadden Sea 128

A14.3 Sediment data Germany 128

$\begin{array}{ll}\text { A14.4 Combining these data } & 128\end{array}$

$\begin{array}{ll}\text { A14.5 Missing data } & 128\end{array}$ 
A15.1 GeoSea-data (RWS, 1998) 132

$\begin{array}{ll}\text { A15.2 Estimation of other sediment compounds } & 133\end{array}$

A15.3 Relationship German and Dutch Wadden Sea data 135

A16 Sediment data used $\quad 136$

$\begin{array}{lll}\text { A17 Benthic diatoms } & 137\end{array}$

A17.1 Position of benthic diatoms in the model 137

A17.2 Maximal densities of benthic diatoms 137

$\begin{array}{ll}\text { A17.3 Suspension of benthic diatoms } & 138\end{array}$

A18 Description of growth of a fauna individual and of population mortality 139

A18.1 General 139

$\begin{array}{ll}\text { A18.2 Population mass budgets } & 139\end{array}$

$\begin{array}{ll}\text { A18.3 Individual growth } & 140\end{array}$

$\begin{array}{lll}\text { A18.3.1 Filtration and assimilation rate } & 140\end{array}$

$\begin{array}{lll}\text { A18.3.2 Respiration and excretion } & 142\end{array}$

$\begin{array}{lll}\text { A18.3.3 Digestion } & 143\end{array}$

$\begin{array}{lll}\text { A18.3.4 Faeces } & 143\end{array}$

$\begin{array}{lll}\text { A18.3.5 Pseudofaeces } & 143\end{array}$

$\begin{array}{lll}\text { A18.3.6 Mortality } & 144\end{array}$

A18.4 Steady state food concentration, an important fauna characteristic $\quad 144$

$\begin{array}{ll}\text { A18.5 Maximum mass } & 146\end{array}$

$\begin{array}{ll}\text { A18.6 Growth rate and implications for the respiration parameter } & 147\end{array}$

A18.7 Conclusions 153

$\begin{array}{lll}\text { A18.7.1 Growth and respiration parameters } & 153\end{array}$

A18.7.2 Corrections: suspended solids hindrance, pseudofaeces formation and activity respiration 154

$\begin{array}{lll}\text { A18.7.3 } & \text { Maximum filtration rate coefficient } & 154\end{array}$

A19 Coordinate transformations $\quad 155$

A20 Suspended solid concentrations: resuspension and sedimentation 156

$\begin{array}{ll}\text { A20.1 Introduction } & 156\end{array}$

A20.2 Description of sedimentation, suspension and the concentration profiles in the water column

$\begin{array}{ll}\text { A20.3 The resuspension parameter k1 } & 157\end{array}$

A20.4 Effect of currents 157

$\begin{array}{ll}\text { A20.5 Effect of wind } & 158\end{array}$

$\begin{array}{ll}\text { A20.6 Effect of wind direction } & 158\end{array}$

$\begin{array}{ll}\text { A20.7 Sedimentation: the settling velocity vz } & 159\end{array}$

A20.8 Adaptations related to time constants 160

A21 Habitat suitability characteristics as possible selection criterion for fauna 161

A22 Different ratios chlorophyll-a and cell dry matter 163

A23 Sediment layers, and fauna feeding from the sediment, some details 164

$\begin{array}{ll}\text { A23.1 Sediment layers: solids } & 164\end{array}$

$\begin{array}{ll}\text { A23.2 Sediment layers: dissolved compounds. } & 164\end{array}$

$\begin{array}{ll}\text { A23.3 Diffusive exchange between water and sediment } & 165\end{array}$ 
A23.4 Adaptation of the model: compensation of lowest sediment layer leakage of first order ions

A23.5 Fauna

A23.6 Fauna induced exchange between water and sediment

A24 Literature review on effect of silt on bivalves occurring in the Ems-Dollard estuary

$\begin{array}{lll}\text { A24.1.1 } & \text { Background and aim } & 167\end{array}$

$\begin{array}{lll}\text { A24.1.2 } & \text { Species description } & 167\end{array}$

$\begin{array}{lll}\text { A24.1.2.1 } & \text { Blue mussel } & 167\end{array}$

$\begin{array}{lll}\text { A24.1.2.2 Cockle } & 168\end{array}$

$\begin{array}{lll}\text { A24.1.2.3 Peppery furrow shell } & 168\end{array}$

$\begin{array}{lll}\text { A24.1.2.4 Japanese oyster } & 169\end{array}$

$\begin{array}{lll}\text { A24.1.2.5 } & \text { Razor clam } & 170\end{array}$

$\begin{array}{ll}\text { A24.2 Food collection methods } & 170\end{array}$

$\begin{array}{lll}\text { A24.2.1 Introduction } & 170\end{array}$

$\begin{array}{lll}\text { A24.2.2 } & \text { Filter feeding } & 172\end{array}$

$\begin{array}{lll}\text { A24.2.3 Effects of suspended sediment } & 174\end{array}$

$\begin{array}{lll}\text { A24.2.4 } & \text { Sedimentation Biotic Index } & 176\end{array}$

A24.3 Quantitative effects of suspended sediment on filter feeding 176

A24.3.1 Growth and metabolic costs 176

$\begin{array}{lll}\text { A24.3.1.1 Introduction } & 176\end{array}$

$\begin{array}{lll}\text { A24.3.1.2 } & \text { Blue mussel } & 177\end{array}$

$\begin{array}{lll}\text { A24.3.1.3 Cockle } & 178\end{array}$

$\begin{array}{lll}\text { A24.3.1.4 Peppery furrow shell } & 179\end{array}$

$\begin{array}{lll}\text { A24.3.1.5 Japanese oyster } & 179\end{array}$

$\begin{array}{lll}\text { A24.3.1.6 Razor clam } & 179\end{array}$

$\begin{array}{lll}\text { A24.3.2 } & \text { Pseudofaeces production } & 180\end{array}$

$\begin{array}{lll}\text { A24.3.2.1 } & \text { Blue mussel } & 181\end{array}$

$\begin{array}{lll}\text { A24.3.2.2 Cockle } & 183\end{array}$

$\begin{array}{lll}\text { A24.3.2.3 Peppery furrow shell } & 184\end{array}$

$\begin{array}{lll}\text { A24.3.2.4 Japanese oyster } & 184\end{array}$

$\begin{array}{lll}\text { A24.3.2.5 } & \text { Razor clam } & 185\end{array}$

$\begin{array}{lll}\text { A24.3.3 } & 185\end{array}$

$\begin{array}{lll}\text { A24.3.4 Oxygen demand and extraction efficiency } & 187\end{array}$

$\begin{array}{lll}\text { A24.3.5 } & \text { Filtration-, clearance- and pumping rate } & 188\end{array}$

$\begin{array}{lll}\text { A24.3.5.1 Definitions } & 188\end{array}$

$\begin{array}{lll}\text { A24.3.5.2 } & \text { Normal clearance and pumping rates } & 188\end{array}$

A24.3.5.3 General aspects of effects of suspended sediments 190

$\begin{array}{lll}\text { A24.3.5.4 Blue mussel } & 194\end{array}$

$\begin{array}{llr}\text { A24.3.5.5 Cockle } & 196\end{array}$

$\begin{array}{lll}\text { A24.3.5.6 } & \text { Peppery furrow shell } & 198\end{array}$

$\begin{array}{lll}\text { A24.3.5.7 Japanese oyster } & 198\end{array}$

$\begin{array}{llr}\text { A24.3.5.8 } & \text { Razor clam } & 200\end{array}$ 
A24.4 Synthesis

A24.4.1 Effect of silt concentrations

$\begin{array}{lll}\text { A24.4.2 } & \text { Pseudofaeces production } & 200\end{array}$

A24.4.3 Filtration rates 201

A24.5 Summary and conclusions 202

A24.5.1 Mechanism of filter feeding of bivalves 202

A24.5.2 Effect of suspended sediment 202

A25 Tuning the model $\quad 203$

A25.1 Chloride distribution, dispersion settings and model results 203

$\begin{array}{lll}\text { A25.1.1 Time series results } & 203\end{array}$

$\begin{array}{lll}\text { A25.1.2 Spatial distribution } & 206\end{array}$

$\begin{array}{lll}\text { A25.1.3 Possible improvements } & 207\end{array}$

$\begin{array}{ll}\text { A25.2 Resuspension parameters and model results } & 208\end{array}$

$\begin{array}{lll}\text { A25.2.1 Introduction } & 208\end{array}$

$\begin{array}{lll}\text { A25.2.2 } & 209\end{array}$

A25.2.3 Spatial distribution 211

$\begin{array}{lll}\text { A25.2.4 Conclusions } & 212\end{array}$

A25.2.5 Possible improvements 213

A25.3 Light attenuation and model results 214

$\begin{array}{lll}\text { A25.3.1 Introduction } & 214\end{array}$

$\begin{array}{lll}\text { A25.3.2 Timeseries } & 214\end{array}$

$\begin{array}{lll}\text { A25.3.3 Spatial distribution } & 214\end{array}$

$\begin{array}{lll}\text { A25.3.4 Suggestions and possible improvements } & 214\end{array}$

$\begin{array}{lll}\text { A25.3.5 Light at the sediment surface } & 218\end{array}$

$\begin{array}{ll}\text { A25.4 Biologically active variables } & 219\end{array}$

$\begin{array}{lll}\text { A25.4.1 Introduction } & 219\end{array}$

$\begin{array}{lll}\text { A25.4.2 Introduction (2) } & 219\end{array}$

A25.4.3 Phytobenthos parameter tuning, Mudsnail and Copepod 220

A25.5 Dissolved inorganic matter and model results 221

$\begin{array}{lll}\text { A25.5.1 Dissolved oxygen }\left(\mathrm{O}_{2}\right) \text {, time series } & 221\end{array}$

$\begin{array}{lll}\text { A25.5.2 Spatial distribution } & 221\end{array}$

$\begin{array}{lll}\text { A25.5.3 Suggestions and possible improvements } & 221\end{array}$

A25.6 Dissolved inorganic phosphorus ortho-P 225

$\begin{array}{lll}\text { A25.6.1 Introduction } & 225\end{array}$

$\begin{array}{lll}\text { A25.6.2 Time series } & 227\end{array}$

$\begin{array}{lll}\text { A25.6.3 Spatial distribution } & 228\end{array}$

$\begin{array}{lll}\text { A25.6.4 } & \text { P in pore water and adsorption in sediment top layer } & 229\end{array}$

A25.6.5 Suggestions and possible improvements 229

$\begin{array}{ll}\text { A25.7 Silicate } & 232\end{array}$

$\begin{array}{lll}\text { A25.7.1 Introduction } & 232\end{array}$

$\begin{array}{lll}\text { A25.7.2 Time series } & 232\end{array}$

$\begin{array}{lll}\text { A25.7.3 Spatial distribution } & 233\end{array}$

A25.7.4 Simulated silicate pore water concentrations and adsorbed silicate $\quad 234$ 
$\begin{array}{lll}\text { A25.7.5 Suggestions and possible improvements } & 234\end{array}$

$\begin{array}{ll}\text { A25.8 Nitrate NO3 } & 236\end{array}$

$\begin{array}{lll}\text { A25.8.1 Introduction } & 236\end{array}$

$\begin{array}{lll}\text { A25.8.2 } & \text { Time series } & 237\end{array}$

$\begin{array}{lll}\text { A25.8.3 } & \text { Spatial distribution } & 237\end{array}$

$\begin{array}{lll}\text { A25.8.4 Suggestions and possible improvements } & 237\end{array}$

A25.9 Ammonium NH4 240

$\begin{array}{lll}\text { A25.9.1 Introduction } & 240\end{array}$

$\begin{array}{lll}\text { A25.9.2 Time series } & 240\end{array}$

$\begin{array}{lll}\text { A25.9.3 Suggestions and possible improvements } & 240\end{array}$

A25.10 Pelagic chlorophyll-a 242

$\begin{array}{lll}\text { A25.10.1 Introduction } & 242\end{array}$

$\begin{array}{lll}\text { A25.10.2 Time series } & 242\end{array}$

$\begin{array}{lll}\text { A25.10.3 Spatial distribution } & 245\end{array}$

$\begin{array}{lll}\text { A25.10.4 Suggestions and possible improvements } & 247\end{array}$

$\begin{array}{lll}\text { A25.11 Benthic chlorophyll-a } & 248\end{array}$

$\begin{array}{lll}\text { A25.11.1 Introduction } & 248\end{array}$

$\begin{array}{lll}\text { A25.11.2 Time series } & 248\end{array}$

$\begin{array}{lll}\text { A25.11.3 Spatial distribution } & 250\end{array}$

$\begin{array}{lll}\text { A25.11.4 Suggestions and possible improvements } & 250\end{array}$

A25.12 Zoobenthos and zooplankton 252

$\begin{array}{llr}\text { A25.12.1 Mudnails } & 252\end{array}$

$\begin{array}{lll}\text { A25.12.2 Filter feeders } & 253\end{array}$

$\begin{array}{lll}\text { A25.12.3 Microzooplankton } & 257\end{array}$

$\begin{array}{lll}\text { A25.12.4 Copepods } & 258\end{array}$

$\begin{array}{lll}\text { A25.12.5 Suggestions and possible improvements } & 259\end{array}$

A25.13 Gross pelagic primary production 259

$\begin{array}{lll}\text { A25.13.1 Introduction } & 259\end{array}$

$\begin{array}{llr}\text { A25.13.2 Time series } & 259\end{array}$

$\begin{array}{lll}\text { A25.13.3 Spatial distribution } & 261\end{array}$

A25.13.4 Comparison with the "Riegman et al" 2012-2013 research results 261

A25.13.5 Suggestions and possible improvements 263

A25.14 Net pelagic primary production 263

A25.15 Benthic primary production 264

$\begin{array}{lll}\text { A25.16 Sum net primary production } & 264\end{array}$

A25.17 Oxygen and nitrate sediment penetration depths 266

$\begin{array}{lll}\text { A25.17.1 General } & 266\end{array}$

$\begin{array}{lll}\text { A25.17.2 Spatial distribution } & 267\end{array}$

$\begin{array}{lll}\text { A25.17.3 Time series } & 267\end{array}$

$\begin{array}{lll}\text { A25.17.4 Suggestions and possible improvements } & 267\end{array}$

$\begin{array}{lll}\text { A25.18 Nitrification and denitrification } & 269\end{array}$

$\begin{array}{lll}\text { A25.18.1 Suggestion and possible improvements } & 270\end{array}$

$\begin{array}{lll}\text { A25.19 Conclusions } & 271\end{array}$ 
$\begin{array}{lll}\text { A25.19.1 Data comparison } & 271\end{array}$

A26 One simulation with changed turbidity 272

A26.1 Strategy 272

$\begin{array}{ll}\text { A26.2 Results } & 272\end{array}$

A27 Improvements needed/suggested $\quad 275$

$\begin{array}{ll}\text { A27.1 All suggestions listed } & 275\end{array}$

$\begin{array}{ll}\text { A27.2 Most relevant improvements } & 277\end{array}$

$\begin{array}{llr}\text { A28 Overview of used parameters } & 278\end{array}$

$\begin{array}{ll}\text { A28.1 lons/elements } & 278\end{array}$

$\begin{array}{ll}\text { A28.2 Algae } & 280\end{array}$

$\begin{array}{ll}\text { A28.3 Detritus } & 281\end{array}$

A28.4 Fauna 282

$\begin{array}{ll}\text { A28.5 Spawning } & 285\end{array}$

$\begin{array}{lr}\text { A28.6 Solids } & 286\end{array}$ 


\section{Summary}

In 2015/2016, we constructed an ecosystem model for the Ems-Dollard estuary, based on the existing EcoWasp model for the western Dutch Wadden Sea. It is a box-model with 28 compartments, each divided into three tidal zones, and two subtidal zones, depending on emersion time and depth. Hydrodynamics are simple, containing only flow-through of water and dispersal exchange between the compartments and the outer boundaries. Input comes from fresh water inlets (Ems and Westerwoldse Aa river, all canals) and from the North Sea on an exchange basis.

The model includes, next to several inorganic substances, pelagic and benthic primary production, breakdown of dead organic matter in water and sediment, an extended description of pore water processes, and several higher trophic level fauna groups: shellfish, a sediment browser (snail) microzooplankton and detritus feeding zooplankton. The model thus sets a step forward since the previous model of Deltares in 2014/2015 that concerned primary production alone as biological process.

The report contains model characteristics: model set-up, assumptions, collection and elaboration of quantity and quality of inflow from tributaries, simulation results and a single scenario on reduced suspended matter content in a part of the system. An extensive literature review on the effect of (high) silt concentrations in the water column on filter feeders gives a lot of information on how to model this relationship. Far from all results could be implemented now, but these are worth to process in a next step of the model construction

It appeared that many time series for inlet water, quantity and quality, needed completion.

After tuning, simulations show that the model satisfactorily reproduces values for salinity, suspended matter, light attenuation, oxygen, sediment dwellers ("mudsnails"), pelagic primary production, and to a somewhat lesser extend silicate, chlorophyll and filter feeders ("mussels"). Problems arose for phosphate and nitrate. Based on literature data, results for benthic primary production and microzooplankton in the water column, nitrification and denitrification seem to be in the order of magnitude, although local data are too incomplete or even absent for a good comparison.

A striking conclusion, and probably this is also a reason for the increasing lack of fit for orthophosphate and nitrate the closer one comes to the Dollard area, is that the system has two faces. The part close to the North Sea (the 'outer part') is governed by production: phytoplankton and its processes (including grazing upon algae) are most important, and ortho-phosphate has its maximum near the winter period and its minimum at the end of the algae bloom season. The part close to the river Ems (the 'inner part'), is mineralization driven: degradation of organic matter with highest rates when temperatures are highest, thus shortly after summer and peak values for ortho-phosphate in early autumn. Nitrate consumption must be considerably larger than simulated now. The question then is: where does this organic matter come from? This is not known, although it might very well be transported inwards together with silt, a phenomenon characteristic for most estuaries. Also, this must be relatively fast degrading matter, since more humus-like substances (but slowly degrading) probably are sufficiently available since the hinterland of the area in The Netherlands and Germany is very peaty. It was, and partly still is. 
The exercise with a 30-50\% lowered suspended solid content in a part of the estuary reveals a significant increase in primary production, although one should be very careful with these results since, as said, improvements are possible and needed.

Finally, it appeared that the simulations serve as a kind of system analysis, revealing some special system characteristics and leaving some of these unexplained, for the time being. As one of the results we come up with a couple of topics that deserve extra study or better and more detailed process descriptions. 


\section{Introduction}

\subsection{General}

The Water Framework Directive (WFD) requires EU member states to achieve good ecological and chemical status of all designated water bodies (rivers, lakes, transitional and coastal waters) by 2015. The ecological condition of the Ems-Dollard estuary is subject to many discussions, mostly related to an increased turbidity and its ecological implications. These discussions take place in both The Netherlands and Germany. To identify the problem and to quantify the effect of proposed solutions, the research project 'Research mud dynamics Ems Estuary' (Onderzoek slibhuishouding Eems-Dollard) has been carried out, consisting of a detailed analysis of available data, the collection of new data as well as the improvement and application of numerical models. Results of that project have been published in several reports and are summarized in Taal et al (2015).

Since in the ecological model study as performed in that research phytoplankton and phytobenthos were included, but no higher trophic levels, it was suggested to perform another ecosystem model study that does include higher trophic levels.

In previous studies (i.e. Brinkman 2105) it was already concluded that changes in nutrient levels mostly affect the highest trophic level, and to a much lower extend phytoplankton contents change. Primary production changes are intermediate. These effects are typical for such systems, where bottom-up effects (from nutrients $>>$ filter feeders) are active, and also top-down effects (filter feeders -> phytoplankton) play an important role.

A short overview of existing (model-)knowledge has been presented in Brinkman (2015 ${ }^{\mathrm{b}}$ ), including strengths and shortcomings. As a result, the Ministry of Economic Affairs asked IMARES (presently Wageningen Marine Research) to construct an ecological model based on the existing EcoWaspmodel.

In this study, the ecosystem model has been set up. Next to primary production and related processes, it includes several higher trophic level fauna groups: shellfish, a sediment browser (snail), microzooplankton and detritus feeding zooplankton. Some tests have been performed to tune the model, but nevertheless, it also appeared that not all existing data for the area could be collected and/or used completely. Thus, future improvements can be made. Also, it appeared that the simulations serve as a kind of system analysis, revealing some special system characteristics and leaving some of these unexplained, for the time being. As one of the results we come up with a couple of topics that deserve extra study or better and more detailed process descriptions.

\subsection{Why this model?}

The mentioned report by Taal et al (2015) contains a summary of research project 'Research mud dynamics Ems Estuary'; this project included an ecosystem model study with phytoplankton and phytobenthos as biological variables. Effects of changing turbidity on primary production have been result of the study. A main conclusion was that primary production largely depends on turbidity, in all parts of the Ems-Dollard area. 
However, higher trophic levels (zoobenthos or zooplankton) were absent in that model study. Since these are considered as important food source for fish and birds, it is relevant to include these in a model study as well and so presenting an opportunity to estimate the effects of changing turbity on the growth and abundance of shellfish and some zooplankton groups. This is what is realised in the present report. Scenario studies are not part of the present study, except for an example showing the results of just one scenario: decreasing turbidity in the central part of the system.

\subsection{Structure of the report}

The report covers the main topics of the project and is as short as possible, most details are in the appendices. Both, the main report and the appendices, have the same order: area description, a brief model set-up, description of input data and of data used for comparison, some details on model characteristics and/or tuning and finally results that also include changes that occur after changing the turbidity in a part of the system. The last section (8.2) contains several suggestions for further work and possible improvements.

\subsection{Thanks}

Modelling a system like the Ems-Dollard includes collection of many data. Some of these have been used extensively, some just partly and being useful for further model tuning. We asked several authorities and institutions for their cooperation, which was awarded by all. We thank:

The Helpdesk of Rijkswaterstaat / Behandelgroep Water data for sending us many chemical and biological water quality monitoring data for the Dutch national surface waters, and Bert Bellert for his contribution. Arie Naber provided us with benthos data.

NLWKN (Niedersächsischer Landesbetrieb für Wasserwirtschaft, Küsten- und Naturschutz, Norden, Niedersachsen, Germany) for chemical and biological water quality monitoring data for German waters (Ems-Dollard, Ems river and many canals and tributaries), and for benthos data, with special thanks to Andreas Engels, Hermann Hebbelmann, Michael Reetz, Michael Grotjahn and Gabriele Petri.

Waterschap Hunze en Aa's (Veendam, Netherlands) and Waterschap Noorderzijlvest (Groningen, Netherlands) for chemical and biological water quality monitoring data for Dutch canals and rivers, with special thanks to Floris Knot, Edwin van der Pouw Kraan, Peter Paul Schollema and Anton Bartelds.

NIOZ (Royal Netherlands Institute for Sea Research, Texel, Netherlands) for SIBES 2008-2015 benthos data, with special thanks to Henk van de Veer and Marten Tacoma, and other members of the SIBES-team: Tanya Compton, Anne Dekinga, Sander Holthuijsen, Job ten Horn, Dennis Mosk and Theunis Piersma, the NV Navicula crew, and including many temporary workers that made the project possible. SIBES was funded by the NAM, NWO-ALW/ZKO and Royal NIOZ. Rob Dekker provided us with macro benthos data for an earlier study, used again in his project.

Deltares (Delft, Netherlands) for helping us with depth data for the whole area, with special thanks to Bas van Maren. 
Wageningen Marine Research (previously IMARES) (Yerseke, Netherlands) for the data on shellfish monitoring, with special thanks to Karin Troost and Margriet van Asch, and for structuring the benthos data lists, with special thanks to Babeth van der Weide.

Although not actively involved in the project, the previous work of Roel Riegman (IMARES) must be mentioned since it has been of great value for this project.

Christine Röckmann (Wageningen Marine Research, Den Helder, Netherlands) for helping to organize the data collection and doing most of the correspondence with the institutions mentioned above.

And last, but not least, Martin Baptist (Wageningen Marine Research, Den Helder, Netherlands) for project management.

\subsection{Geographical data sources}

For the maps presented we used several data sources. Source for The Netherlands map is https://www.statsilk.com/maps/download-free-shapefile-maps; Dutch waterways and most Germany data originate from OpenStreetMap data and are licensed under the Open Database 1.0 License (see www.openstreetmap.org for details about the project). The files used contain OpenStreetMap data as of 2014-09-03T20:22:02Z courtesy of http://download.geofabrik.de. Some overview figures originate from Google Earth (https://www.google.com/earth).

\subsection{Software used}

The EcoWasp-model used is written in C (Kernigan et al, 1978), and runs on a Linux workstation. The software structure is explained in two basic technical documents (Brinkman, 1993; Brinkman \& Smit, 1993); and later, many improvements have been implemented. Pre- and after elaboration of data was done with many separate scripts written in $\mathrm{R}$, a language and environment for statistical computing and graphics $(R, 2016)$ and Microsoft Excel and using Microsoft Word as reporting environment.

\subsection{Responsibilities}

The first author is responsible for most of the report, the second for the literature review on relevant benthos characteristics (Appendix A2) and on silt affecting benthos activities (Appendix A24). 
18 of 286 | Wageningen Marine Research report C058/17 


\section{Model set-up and area}

\subsection{Compartments}

The Ems-Dollard area is divided into 28 compartments, starting at Leer in the Ems-river and ending off-shore above the islands, and on the tidal flats between Schiermonnikoog and the mainland in the west, and Juist and the mainland in the east. Inner boundaries of all these compartments are related to sub-basins, but also were drawn "by eye". In Figure 1 this set-up is shown. Outer boundary areas (the river and the offshore compartments) merely serve as a buffer in the model, and results should not be taken too seriously.

Next to the division into main compartments, each of these compartments contains subcompartments, based on their emersion times. Three tidal areas are considered (1-0.5, 0.5-0.2 and 0.2-0.0 fraction of the time above water, and two subtidal areas ('subtidal' down to $-5 \mathrm{~m} \mathrm{NAP}$, and 'gullies' deeper them -5 m NAP). This kind of sub-division implies that sub-compartments are not necessarily all connected; this is explained in Figure 3 , and the overall picture of subcompartments distinguished is shown in Figure 2.

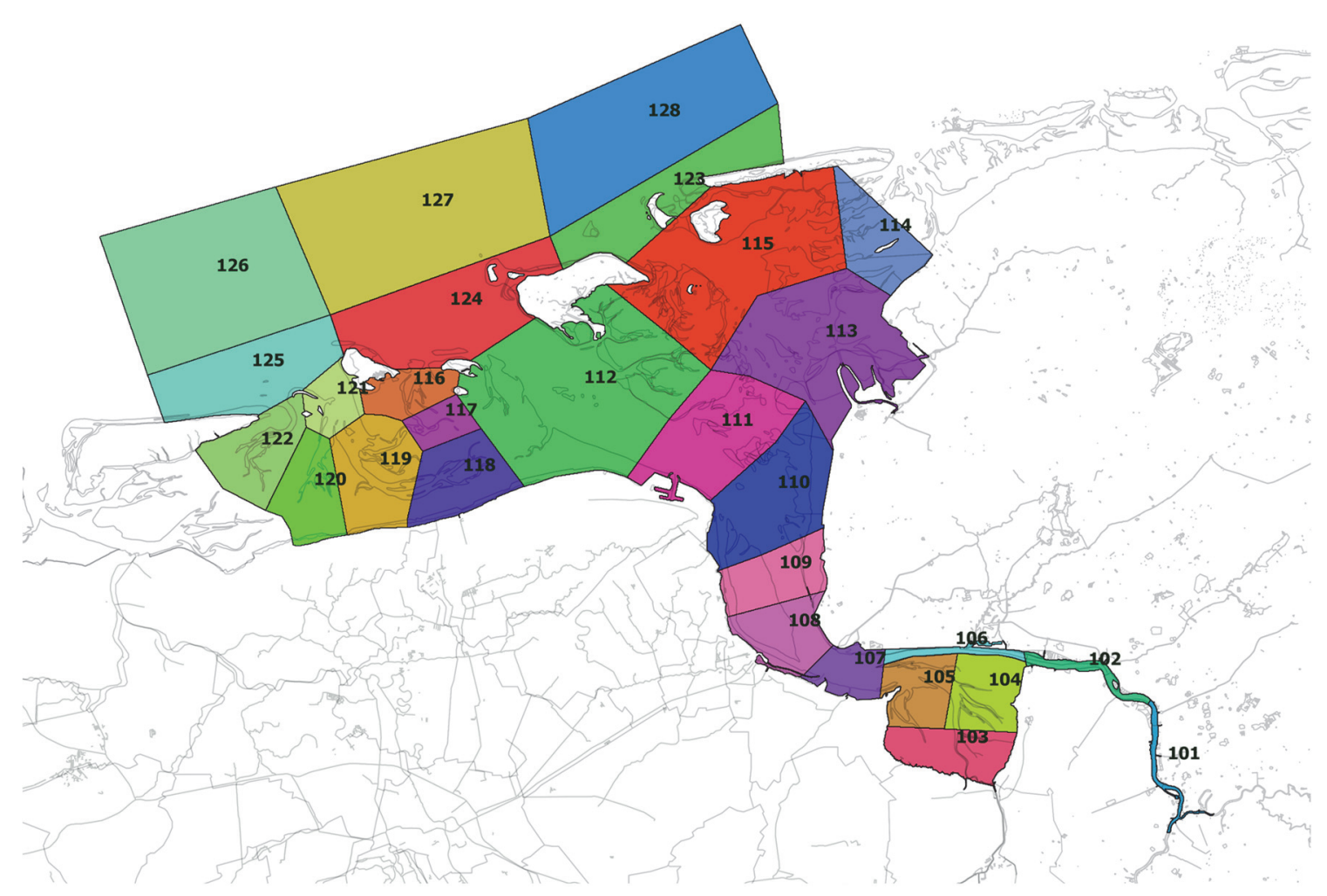

Figure 1 Set-up of main compartments distinguished in the model. Based on their emersion times, three tidal, and two sub-tidal areas are distinguished within each main compartment (see Figure 3). 
By this, the occurrence of phytobenthos and benthic animals having a distribution depending on the emersion period may better be computed. The length of the emersion period is computed/estimated based on the sediment level and the tidal range. Numbering of all these compartments is given in Appendix A1 ("Compartment setup and sub-compartments").

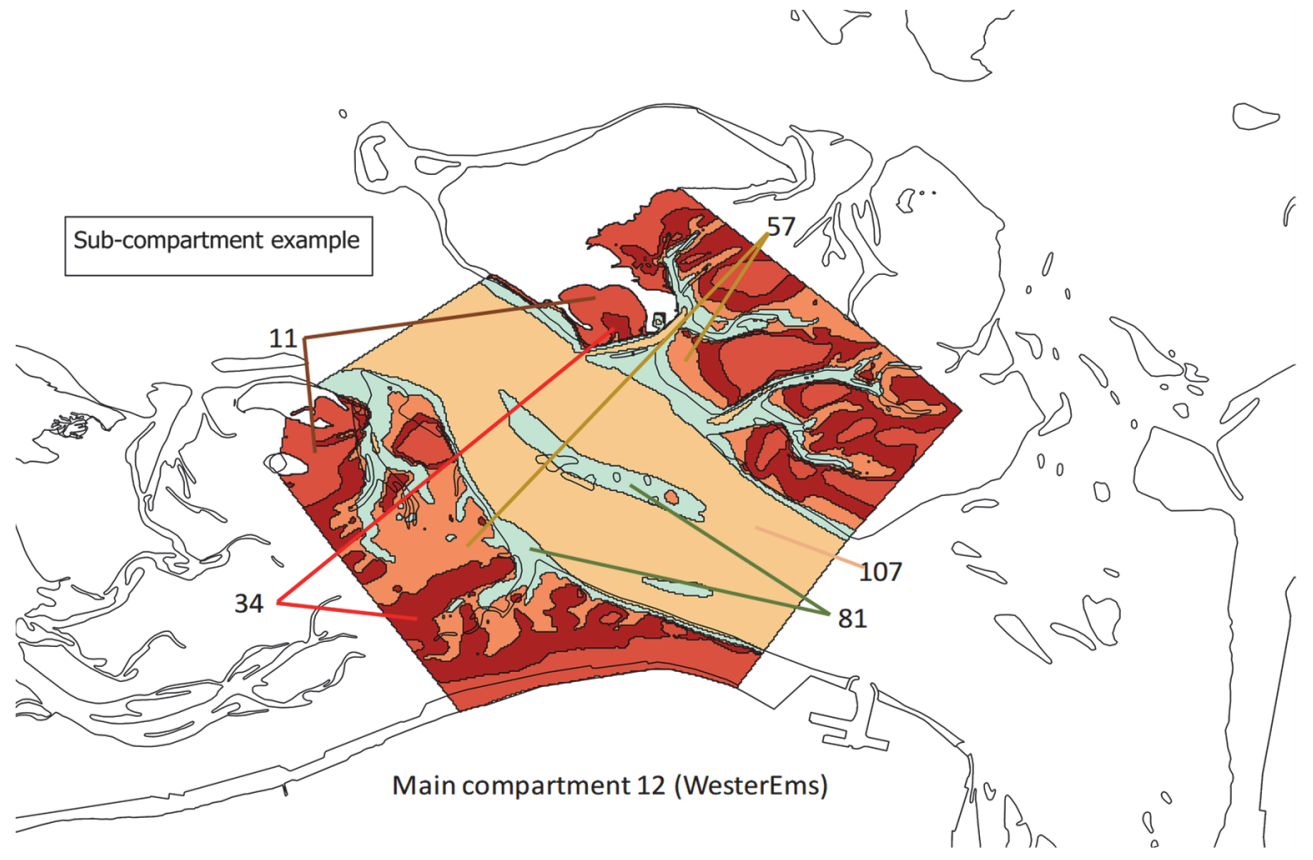

Figure 3 Set-up of sub-compartments. Based on their emersion times, trhee tidal, and two sub-tidal areas are distinguished within each main compartment (Figure 1). That implies that sub-compartments are spread all over the main compartment. Values (both observations and model results) are assigned to all relevant subcompartments in the same main compartment. Here, main compartment 12 (numbered 112 in Figure 1) is shown.

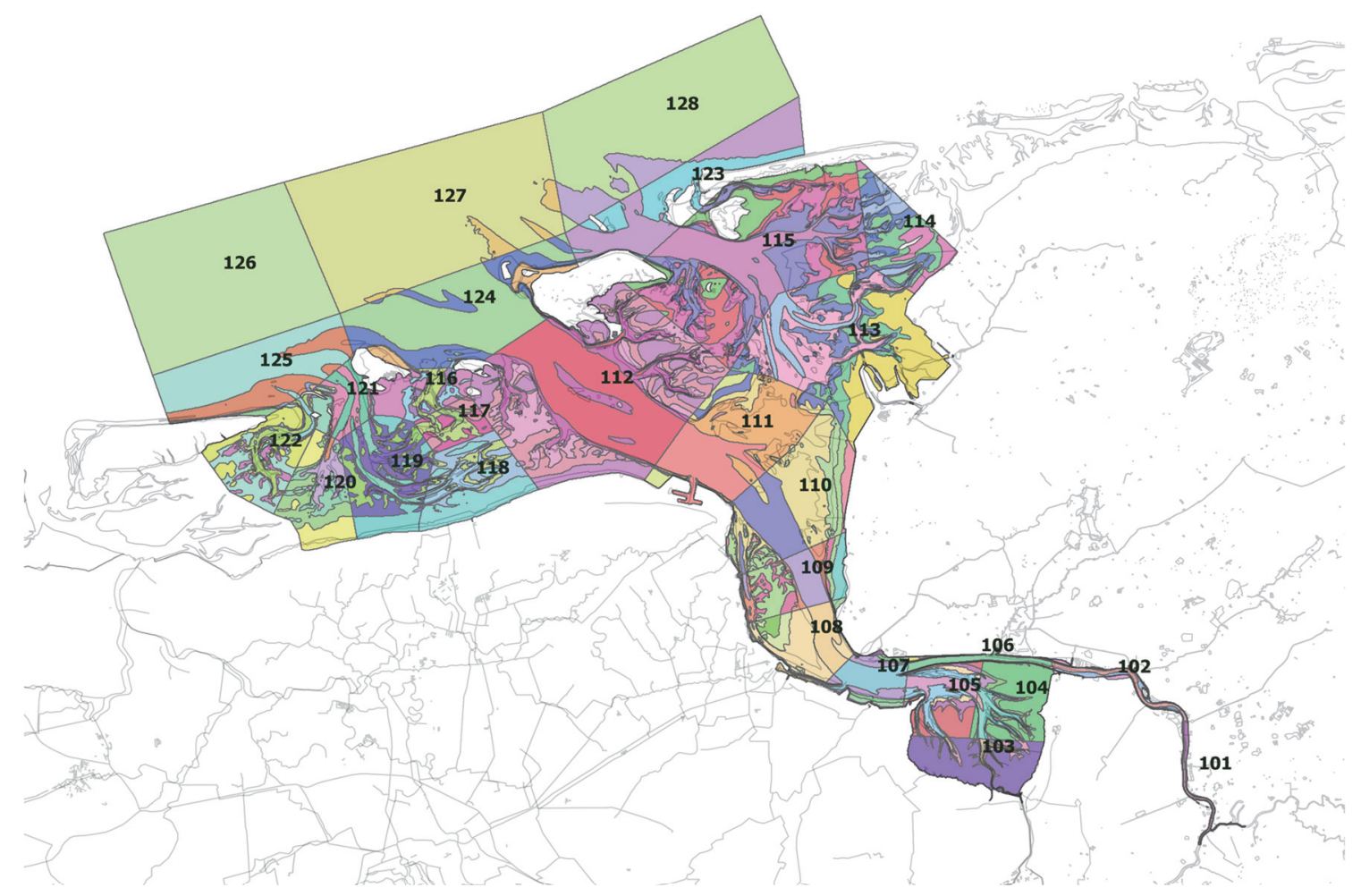

Figure 2 Overview of all sub-compartments. Explanation see Figure 1 and Figure 3. Numbers of main compartments included. 


\subsection{The 'real' estuary}

Some of the data that are available do not cover the whole area (Figure 1), but a smaller part of it. This is shown in Figure 4.

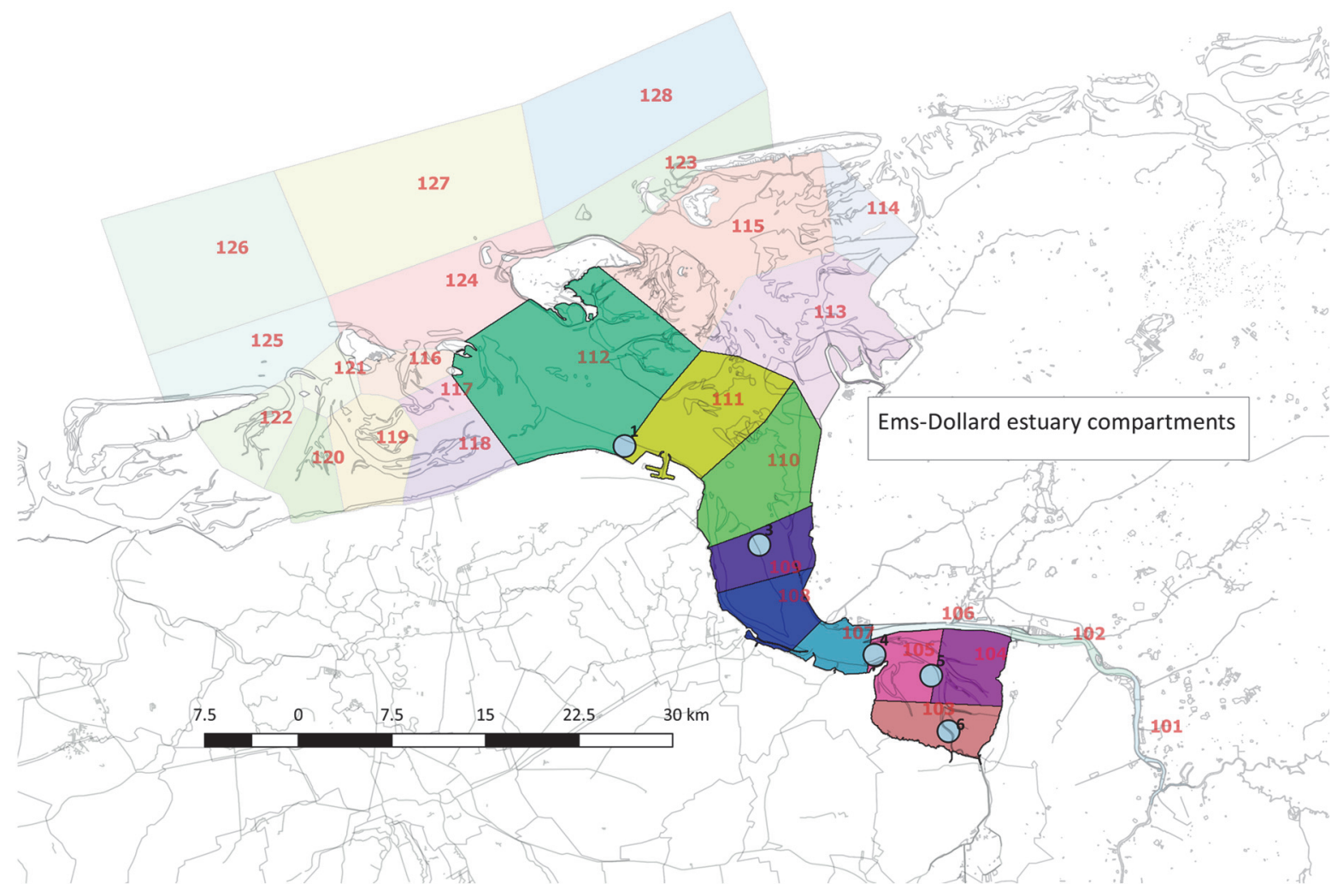

Figure 4 The main part (with the compartments) in the system that have been distinguished in earlier studies (e.g. Colijn, 1983).

\subsection{Schematic model representation}

The model set-up to be used in this Ems-Dollard project (Figure 5) is based on previous applications for the western Dutch Wadden Sea (Brinkman, 2015). Several functional groups are implemented, some additional to the previous model version(s). The most important reason for the implementation of extra groups is that i) areas with (very) low salinity are part of the system and ii) tidal flats are important in the system. Also, a considerable part of the system is very silty, but this can be accounted for by the process descriptions for each fauna group. Phytoplankton groups are extended with fresh water groups, to account for the regions with low(er) salinity.

Fauna groups are extended with a sediment browser and copepods (although finally not tuning satisfactorily), feeding on detritus associated bacteria. Not implemented yet are benthic detritus feeding animals (worms, nematodes). 
See chapter 5 for some details on model characteristics, and more in appendices A1 - A23.

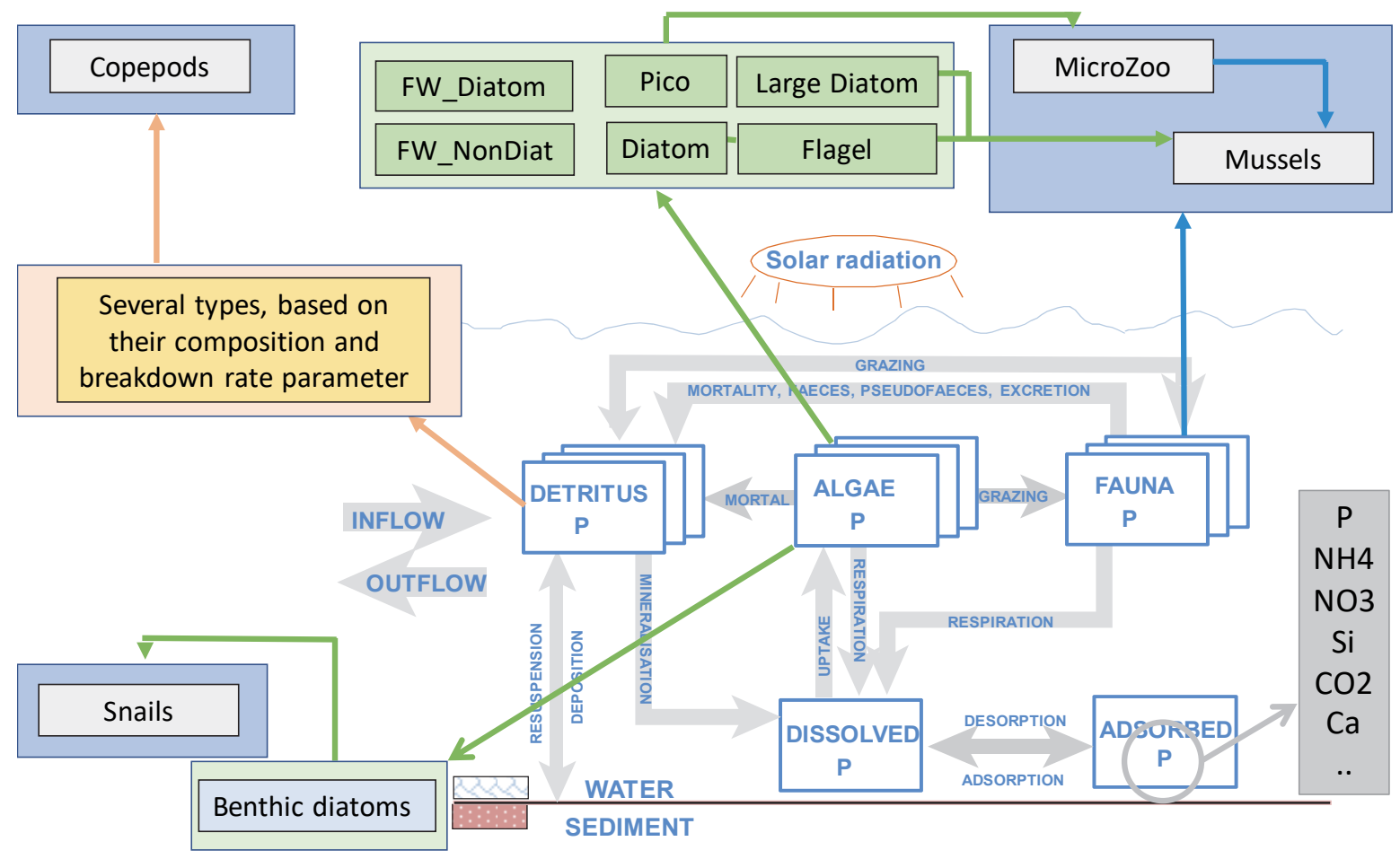

Figure 5 Schematic representation of the Ems-Dollard version of the EcoWasp ecosystem model. ' $P$ ' represents all dissolved components, as mentioned in the grey box. Fauna consists of shellfish ('mussels'), microzooplankton, copepods and snails (sediment browsers). "Mussels" comprised all filter feeding shellfish in the system. Copepods, feeding on detritus, and snails, feeding on benthic algae are the other fauna groups so far. Fresh-water diatoms and non-diatoms are introduced since the input of fresh water is considerable in the area. Picophytoplankton is eaten by microzooplankton; mussels consume the larger phytoplankton species plus microzooplankton. 


\section{Input data}

\subsection{General}

Each ecosystem model needs data describing system characteristics (depth distribution, sediment composition), meteorological data (wind, solar radiation) and the inflow of water from rivers and canals and exchange with external water bodies (quantity and quality: concentrations of relevant substances).

\subsection{Water quantity data}

\subsubsection{Available data}

All relevant inflow sites into the system are pictured in Figure 6. For most of these, (some) inflow data are available. But, and that's valid for all these inflows, none of the datasets were complete. Also, there have been changes during the 1976-2016 simulation period. Pumping stations have been built, or changed, and some outflows changed from free outflow during low tides to -partlyoutflow via a pumping station.

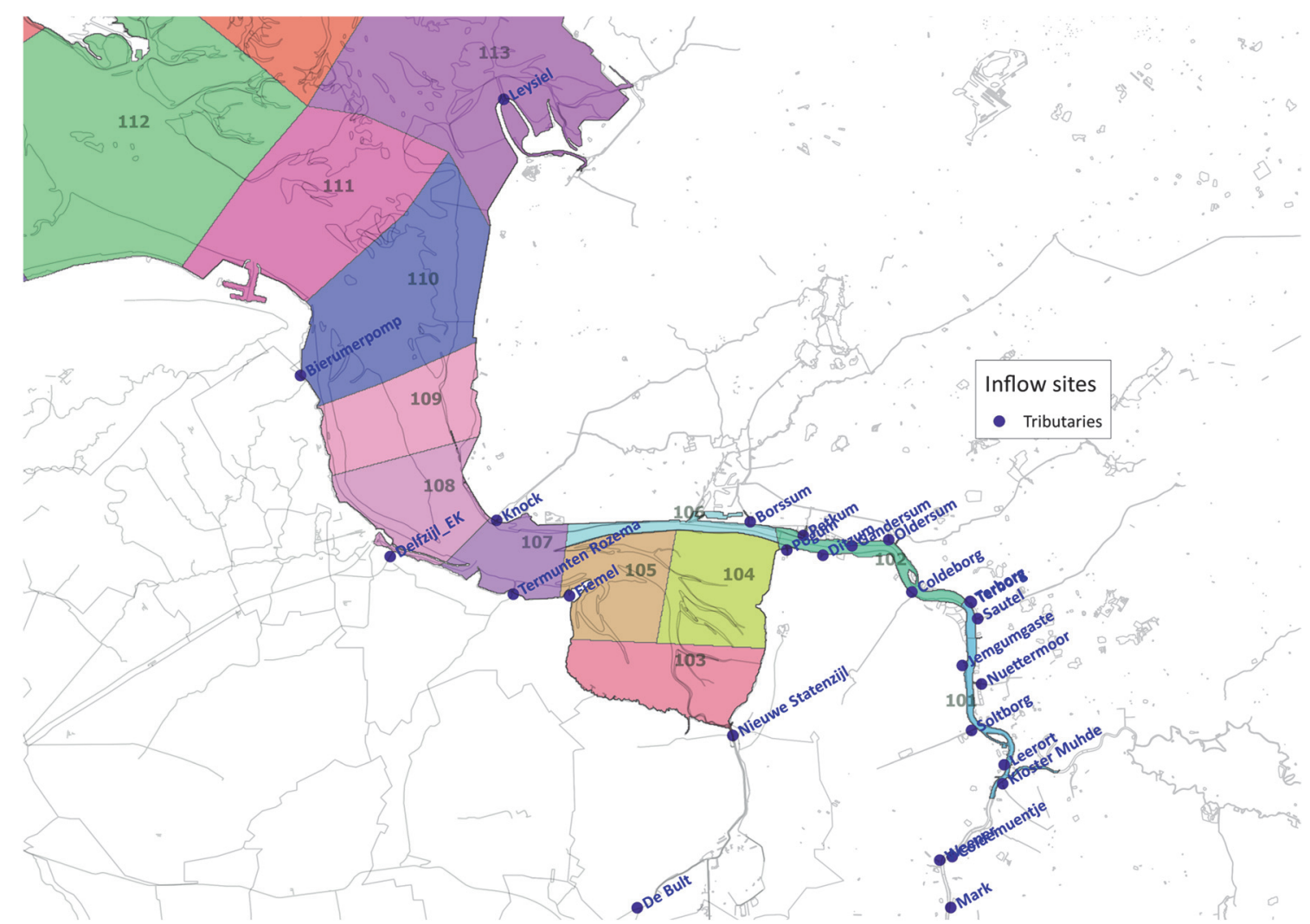

Figure 6 Tributaries in the area. Their importance depends on their annual inflow and nutrient contents. See below (Figure 7) what data inside the system have been used for comparison, and outside the system in the North Sea as boundary values. 


\subsubsection{Missing data}

To make the sets complete, the available data were linked to rainfall and evaporation data (KNMI,2016), and thus, discharge estimates for the remaining periods have been made. Basin areas were needed, and these are (partly estimated) listed in Table 9. The method applied is explained in appendix A4.

For the river Ems, this procedure was not applicable, merely since the discharges are less correlated with the KNMI-precipitation surplus data. Missing values (only a few) are added by hand, simply based on similar situations in other years.

\subsection{Water quality data}

Water quality data are needed to supply the computations with boundary and discharge data. Next, data from inside the system are used to compare model results with observations.

\subsubsection{Water quality data Netherlands inside the system}

Water quality inside the system are not used as state variable, except for temperature. All other values serve as comparison data. Available sites are shown in Figure 7.

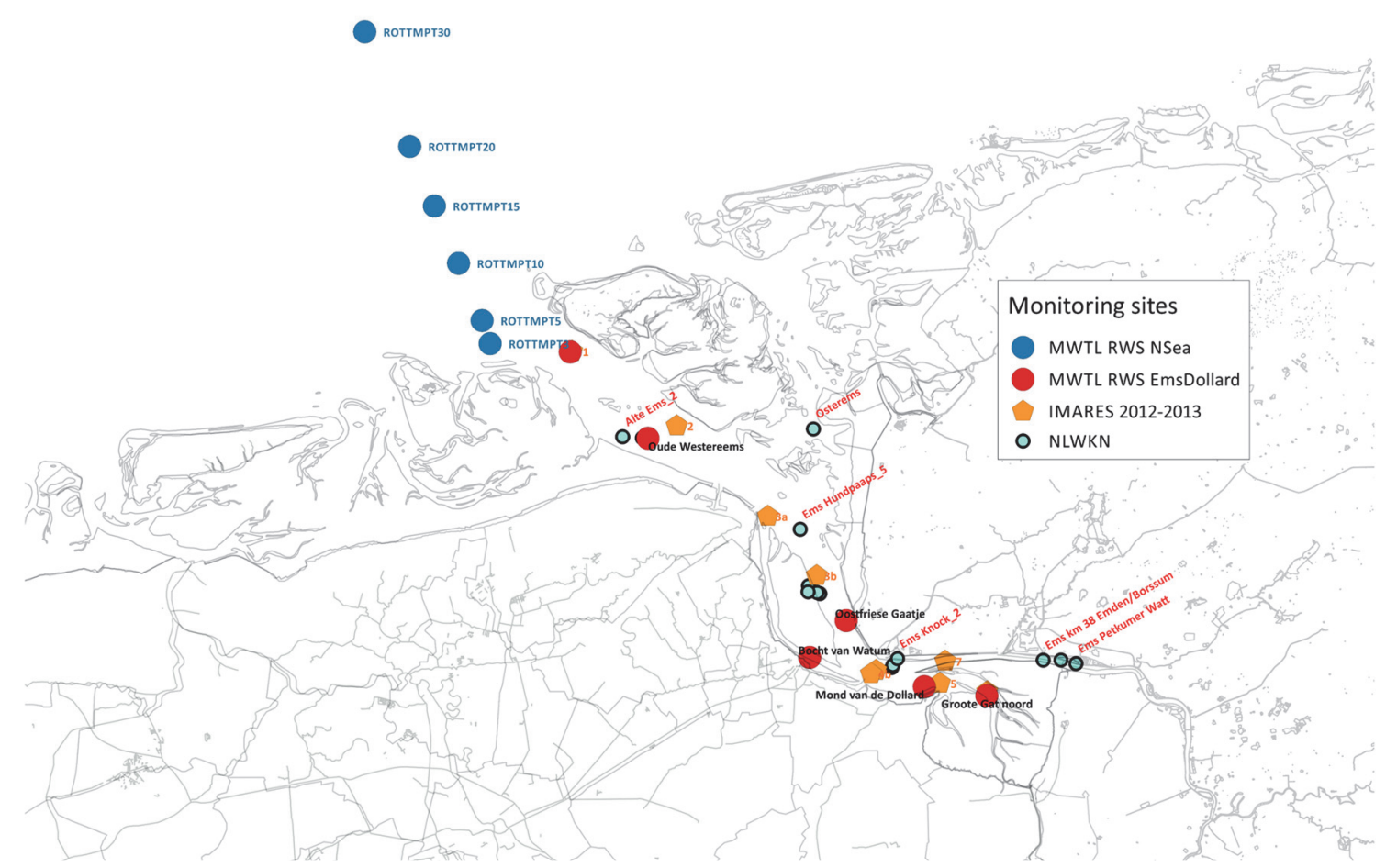

Figure 7 Water quality monitoring sites in the Ems-Dollard (Dutch RWS and German NLWKN) and in the North Sea coastal area.

\subsubsection{Water quality data outside the system: boundary sites}

Data for discharge sites are available close to the discharge site (see Figure 8 for relevant sites). The period for which data are available is listed in A3, Table 6. 
The river Ems is part of the modelled system, but the first available more or less complete data set was at Gandersum and Leer, sites that are part of the modelled system. As a trick, flow data for Gandersum were used, and quality data for Papenburg. Consequently, flow data for the Leda river

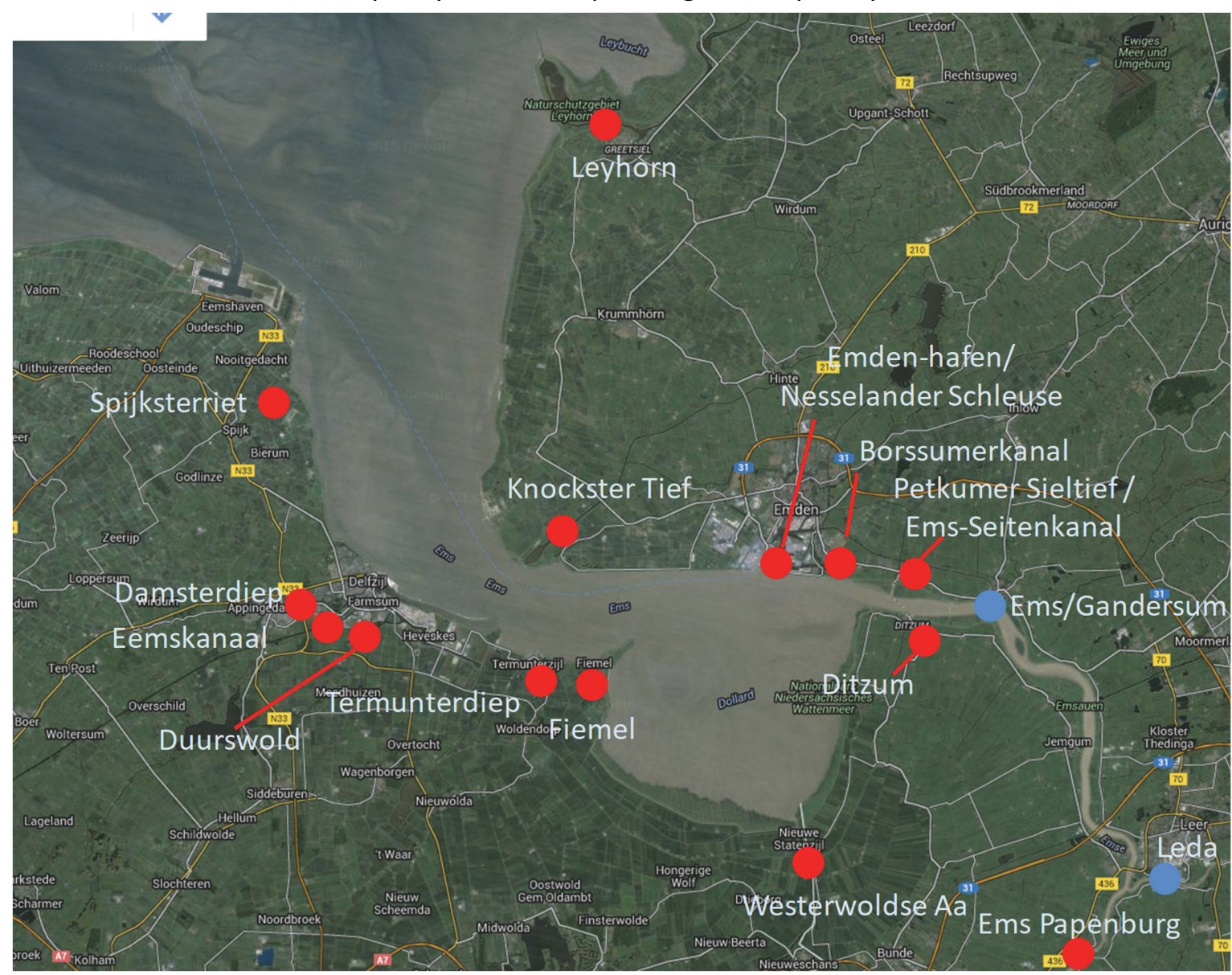

Figure 8 Relevant monitoring sites in Netherlands (authorities Hunze en Aa's and Noorderzijlvest) and Germany (authorities NLWKN), pictured on a Google map.

are omitted.

\subsubsection{General overview}

As an example, two set are briefly presented: the Ems (at Terborg) (Figure 9) and the Eemskanaal (at Delfzijl) (Figure 10). Characteristics are quite different for both sites: the Ems-data reflect the increasing suspended solid content, coupled to an increasing total-P and total- $\mathrm{N}$ content and decreasing oxygen concentrations. The Eemskanaal shows very high phosphorus concentrations

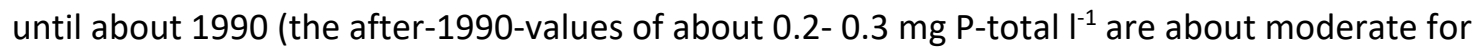
canals, and twice the values needed for a 'good' classification (see Evers \& Knoben 2007; Molen \& Pot 2007a,b). On average there are no serious oxygen problems in the Eemskanaal (most values above 6-7 $\mathrm{mg} \mathrm{O}_{2} \mathrm{l}^{-1}$ ). Until about 2000, ammonium concentrations are high (with $1 \mathrm{mg} \mathrm{NH}_{4}-\mathrm{N} \mathrm{I}^{-1}$, values are high, since target total- $\mathrm{N}$ values of about $1.4 \mathrm{mg} \mathrm{l}^{-1}$ (Evers \& Knoben 2007; Molen \& Pot 2007a,b; Van Liere \& Jonkers, 2002) imply that $\mathrm{NH}_{4}-\mathrm{N}$ values (usually lower to much lower than nitrate- $\mathrm{N}$ and organic- $\mathrm{N}$ ) must be even far below the present values. 

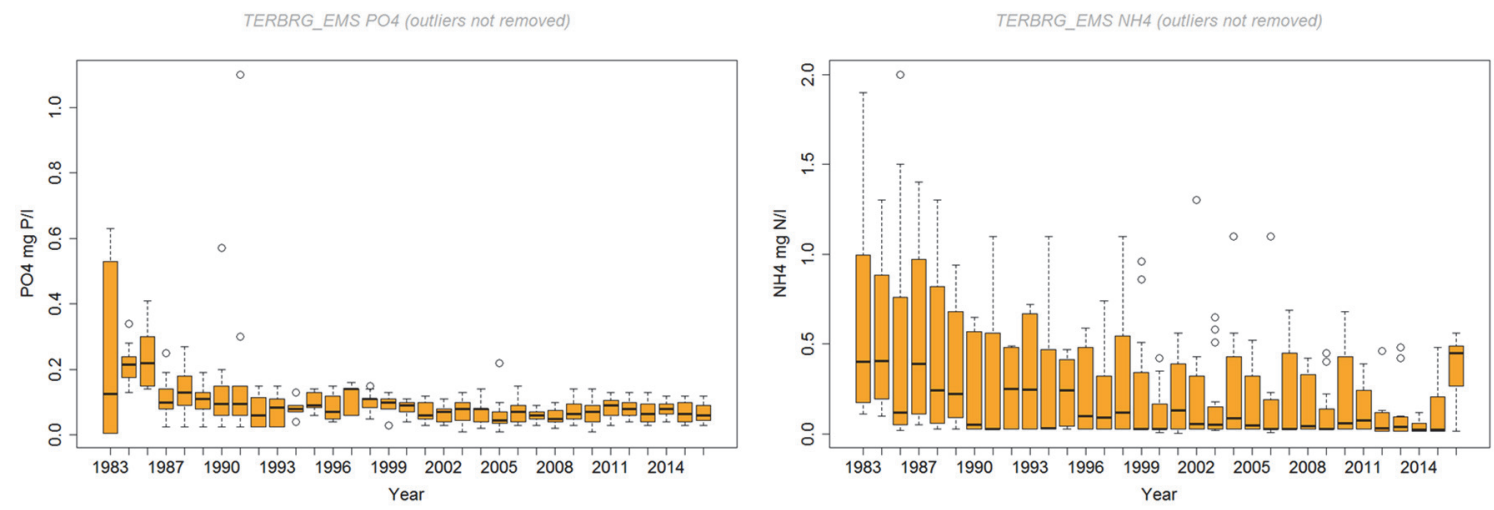

TERBRG_EMS Ptot (outliers not removed)
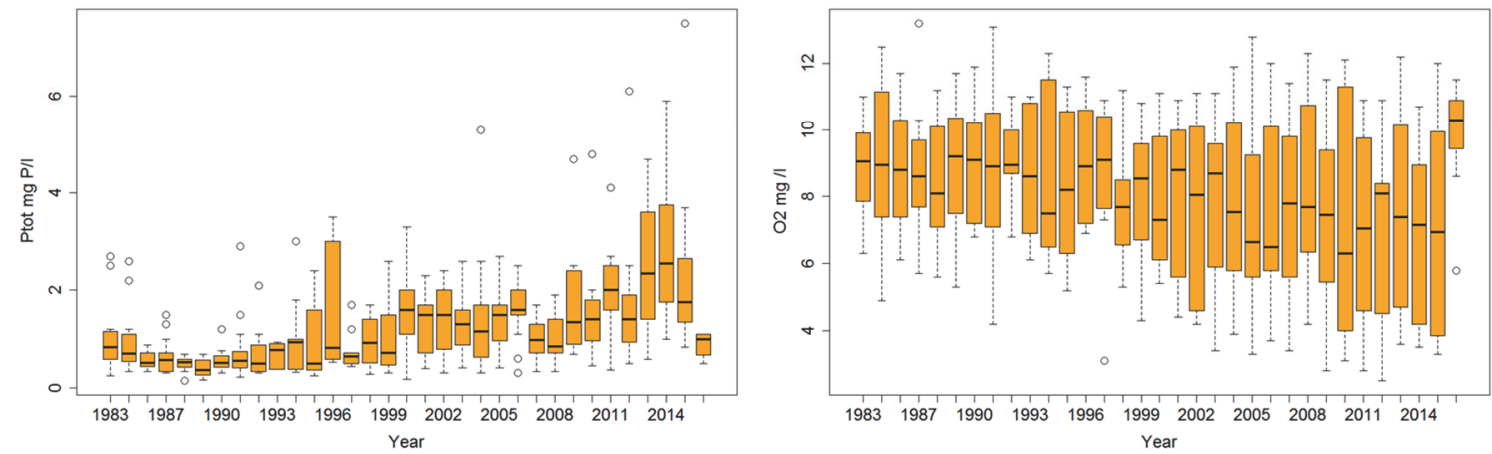

Figure 9 Water quality variables in the Ems river at Terborg (site 85), showing that total phosphorus concentration is quite high for all years and is increasing with the years, whilst oxygen content tends to decrease with the years. These characteristics are related to the increasing suspended solid content of the water. Data before 1983 are to be estimated.
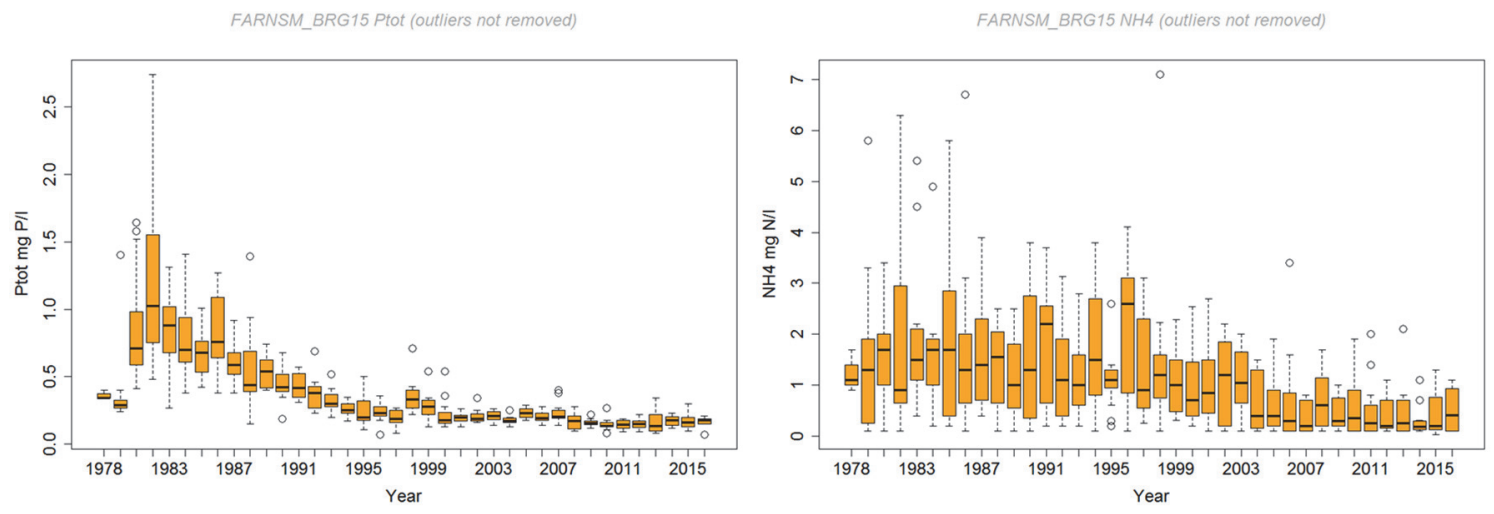

FARNSM_BRG15 PO4 (outilers not removed)
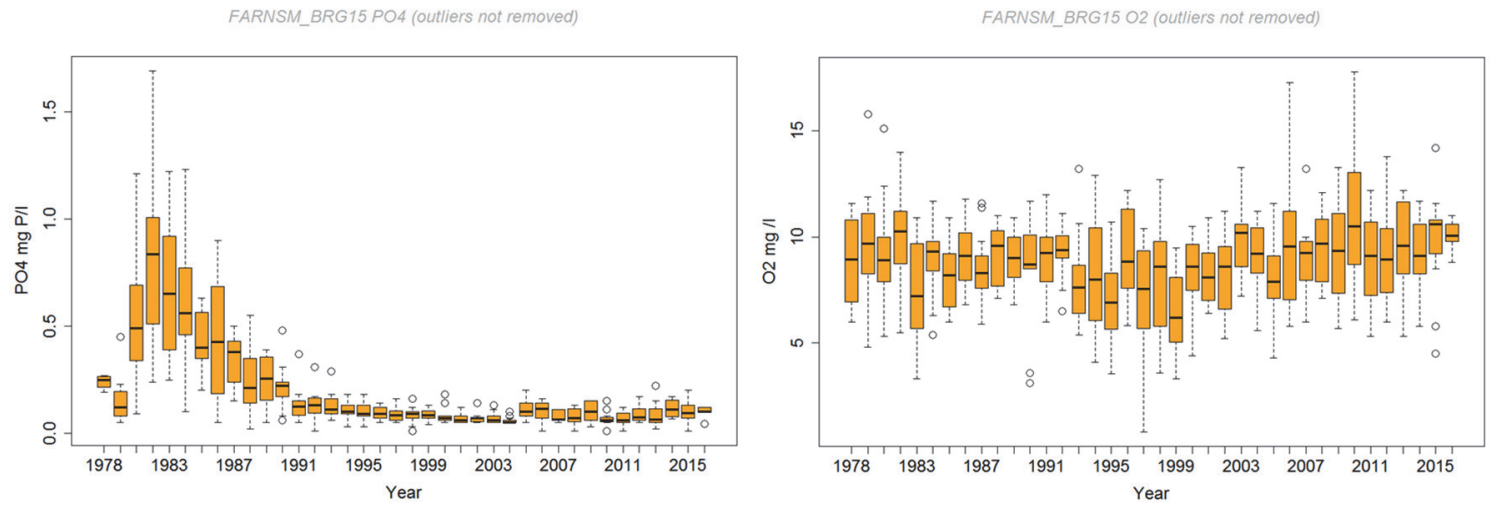

Figure 10 Water quality variables in the Eemskanaal at Delfzijl (Farnsum bridge, site 4102), showing that oxygen problems were not very severe (anymore) in these years, but nutrient concentrations stayed high until about 1990. 


\subsubsection{Exceptional situations}

When looking back in time, the exceptional situation in the beginning of the period we started collecting data for (1976) is striking. The area suffered from a heavy organic potato-starch industry waste water loading. The activities of these factories were concentrated in the autumn months (September-December), and then extremely high values of organic discharges, ammonium and phosphorus were observed, whilst oxygen concentrations dropped to zero. The Groninger canals were then covered with foam. Due to improving waste water treatment, closing of factories, and applying more sophisticated techniques, these discharges disappeared gradually. Around 1980,
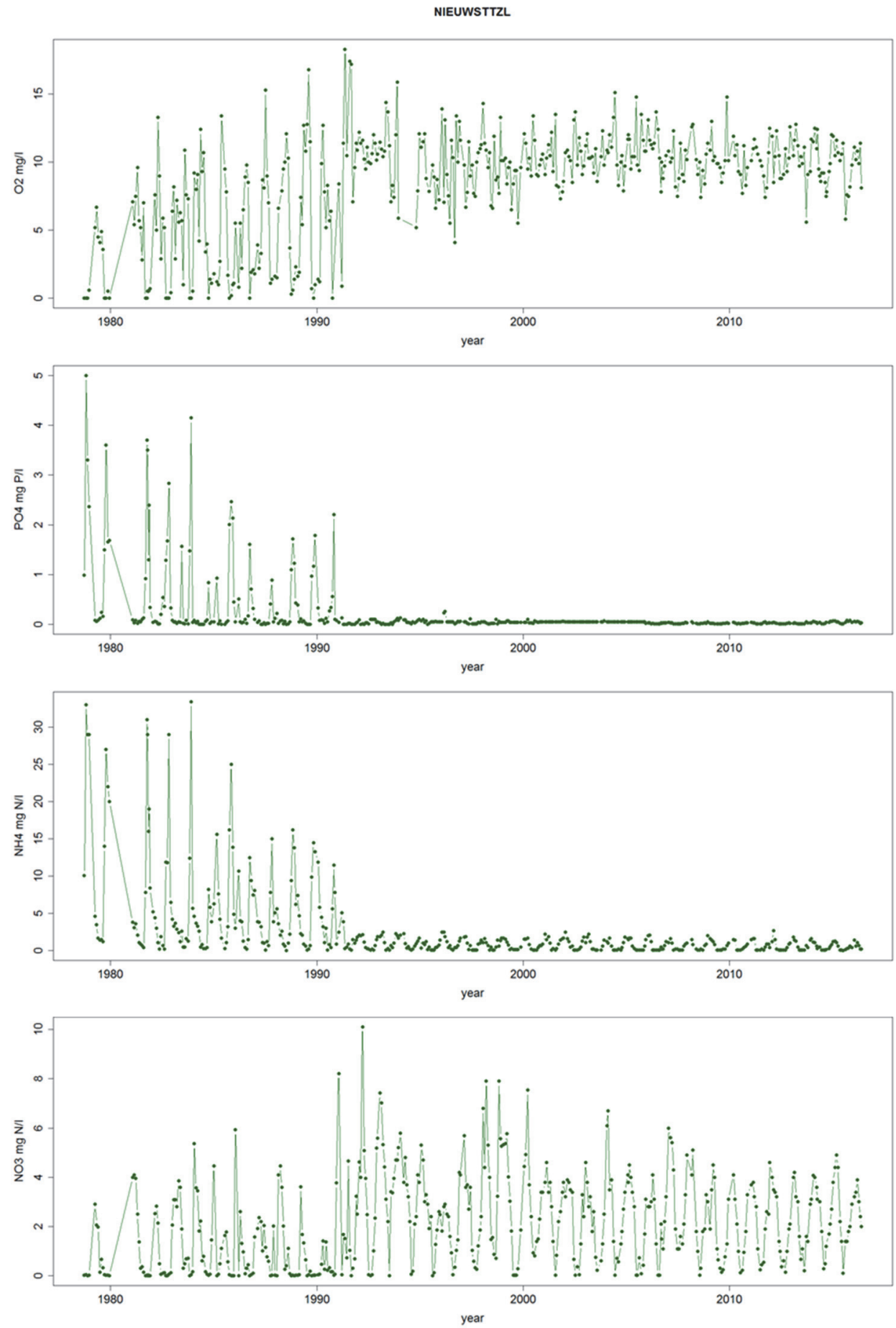

Figure 11 Water quality variables Westerwoldse Aa (Figure 8). Until 1991, discharges of N-compounds (Kjd$N, N H 4)$ and $P$-compounds (particular $P$, ortho-P) were extremely high, and oxygen concentrations were low. 
when most of the data series start, most of the canals already were 'clean'. An exception was the Westerwoldse $\mathrm{Aa}$, where organic matter and nutrient concentrations stayed high until about 1990 (Figure 11, Figure 12).
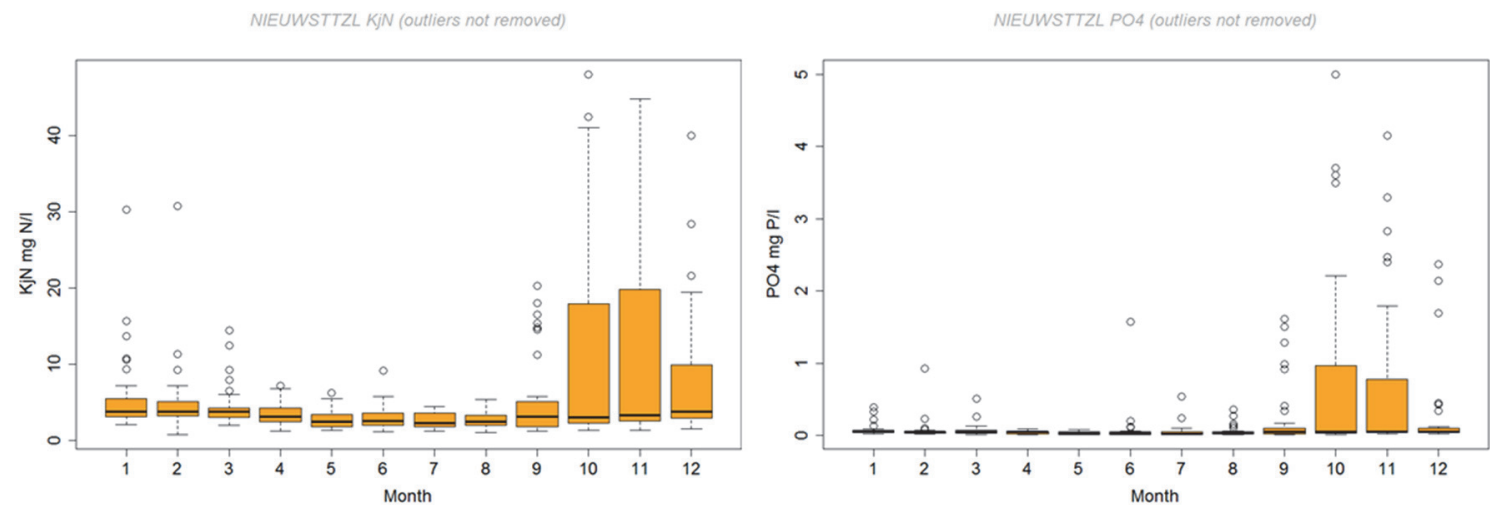

NIEUWSTIZL O2 (outliers not removed)
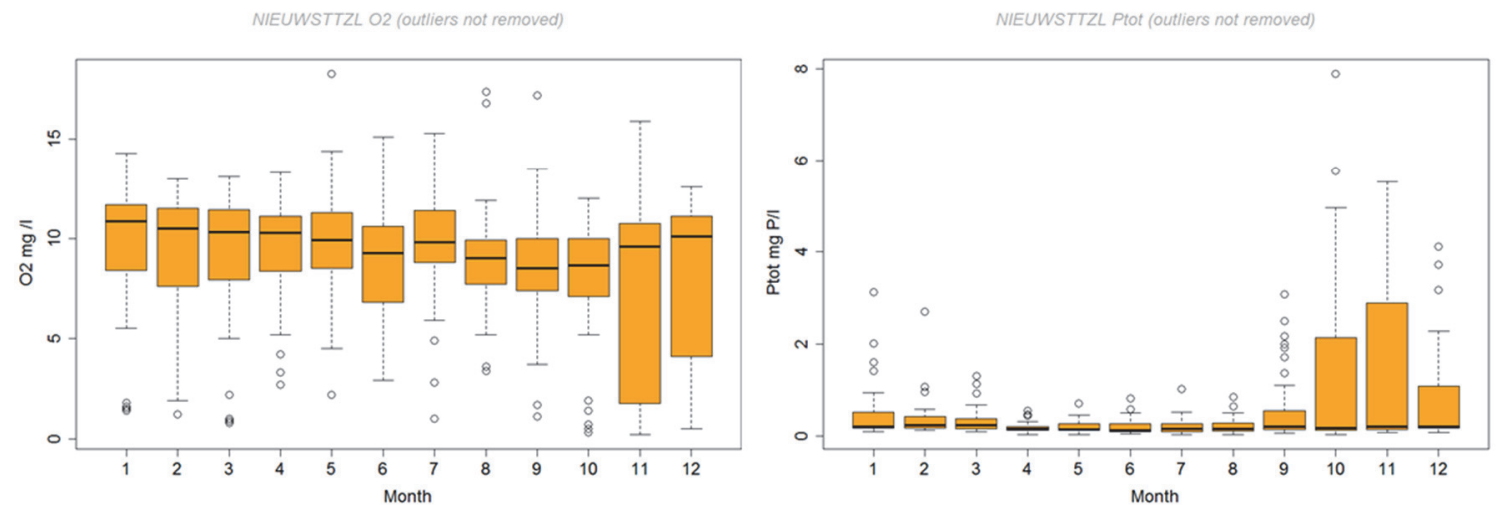

Figure 12 Averages of some water quality variables, showing that extreme values typically occurred in autumn (Sep-Dec)

\subsubsection{Old data}

Some data from literature were available, especially the ones from the BOEDE-research, performed in the period 1973-1980 (Baretta \& Ruardij, 1988). Some monthly water quality (and discharge) data were listed for the period 1977-1982 for the rivers Ems and Westerwoldse Aa. The water quality data were restricted to total and dissolved organic carbon. A few data for oxygen, ortho-P and silicate were mentioned, related to the day in the year. These data have been used here, but -as for the missing data handled as explained in the next section- these values must be considered as approximate, and may well deviate (a lot) from the real values those days.

\subsubsection{Missing data}

For most tributaries data are available, but also large gaps occur. These gaps have been filled in, mostly after a regression analysis comparing existing data for the site with more complete series for another site and sometimes after an analysis of the own data. This is explained in more detail in appendix A5 - A8. 


\subsection{Morphology}

\subsubsection{Depth data}

Thanks to Deltares, that provided depth data for the whole area (and beyond), for all system parts depth data are available. The data had to be elaborated to the $50 \mathrm{~m}$ grid cells we used for all data, this is done using a standard kriging procedure (Nabil, 2015). Result is shown in Figure 13.

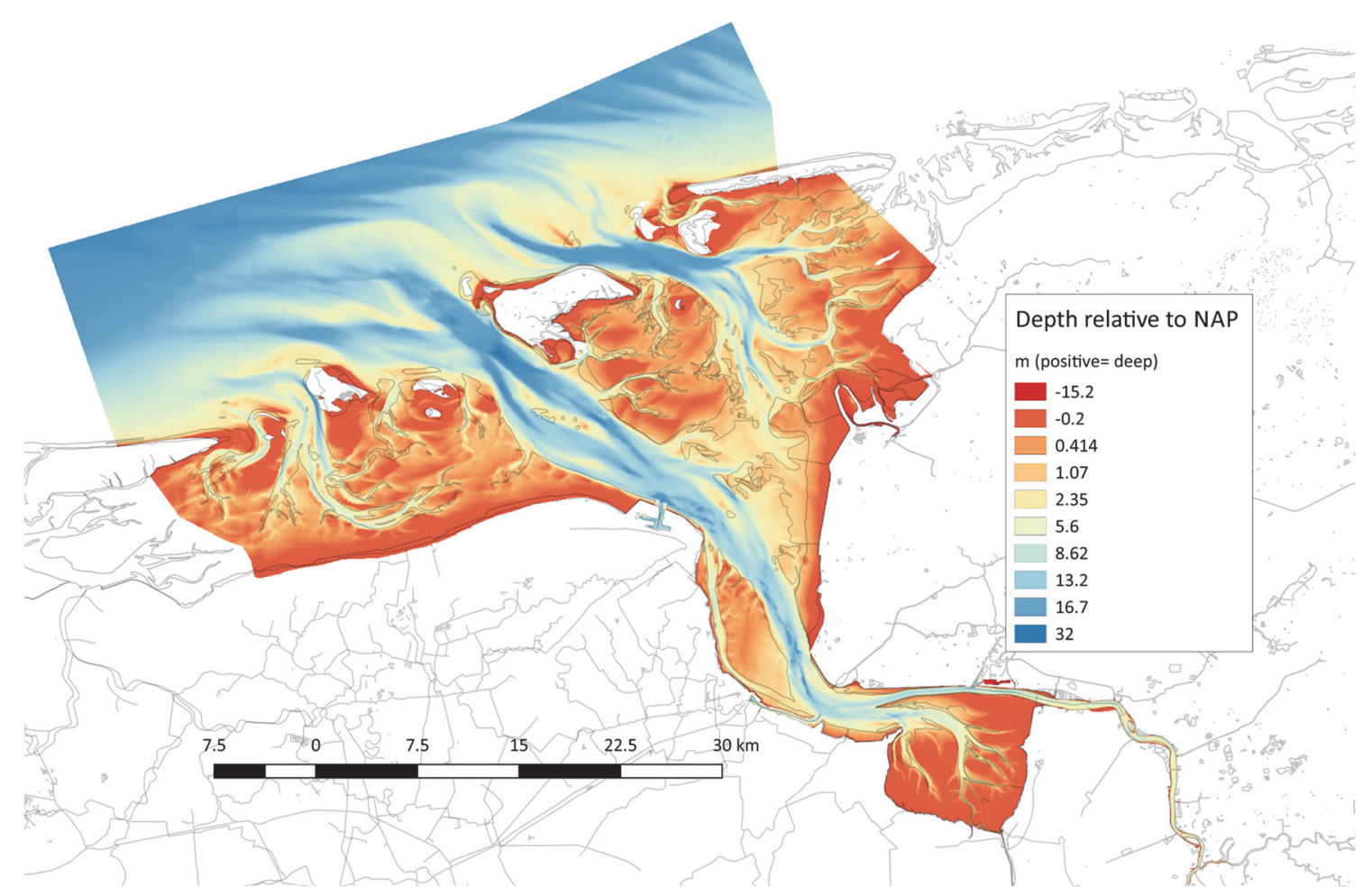

Figure 13 Depth in $m$ relative to NAP (Dutch Ordinary Level)

\subsubsection{Sediment data Netherlands, Wadden Sea -A}

Data were available from the Sedimentatlas, Rijkswaterstaat (RIKZ, 1998). Sampled in the period 1989-1995, mostly one sample per $1 \mathrm{~km}^{2}$. After kriging (by Rijkswaterstaat), they became available on a $50 * 50 \mathrm{~m}$ grid (Brinkman \& Bult, 2003). See Figure 74. Data consist of median grain sizes (M16) for all particles $>16 \mu \mathrm{m}$, and silt content (as mass-\%). Silt is considered as all particles smaller than $63 \mu \mathrm{m}$. Analyses by GeoSea (UK) have been performed with a Malvern Laser Particle Sizer. Missing data (specially at the German side) are estimated. As an example, results for the finest silt fraction (lutum, size 0-2 $\mu \mathrm{m}$ ) are shown in Figure 15. 


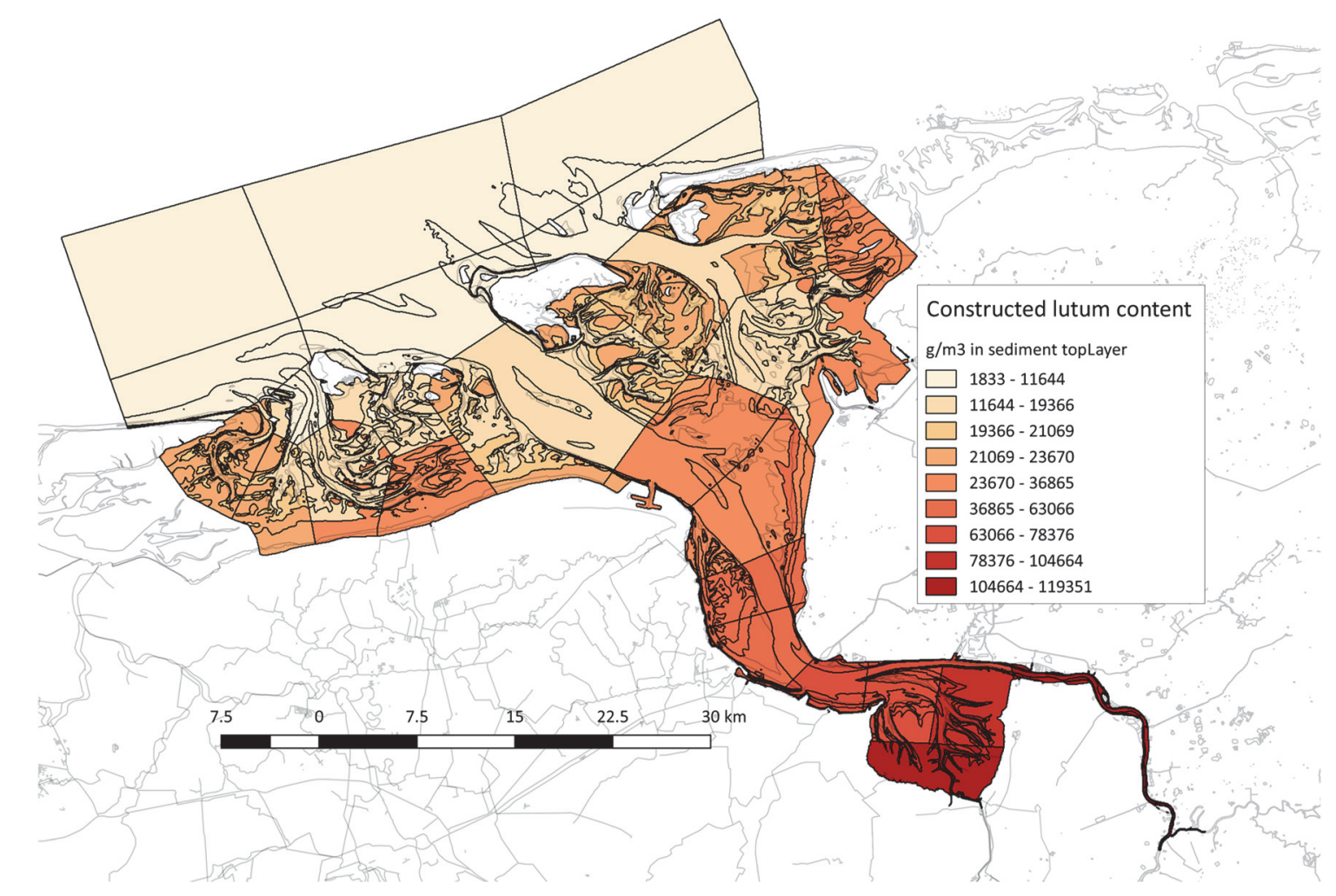

Figure 15 Lutum content (particles $<2 \mu \mathrm{m}$ ) in sediment toplayer. Constructed from Dutch sediment data and extrapolated to German areas. Details in appendix A15

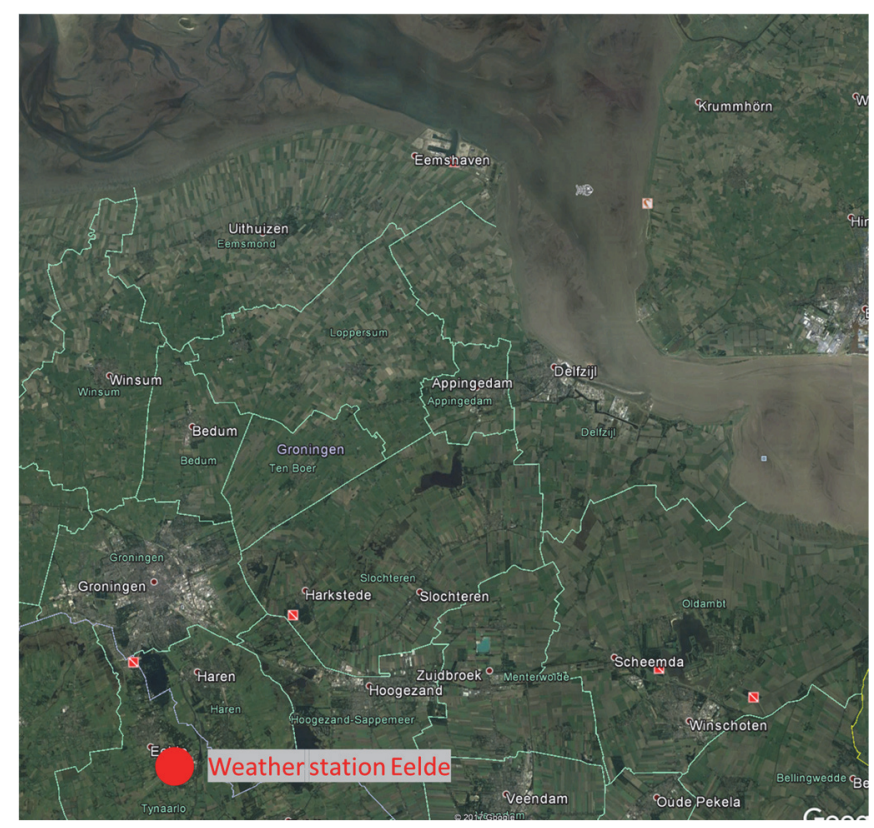

Figure 14 Meteo-station Eelde of the Royal Netherlands Meteorological Institute (KNMI), source of all meteodata on a Google map.

\subsubsection{Meteorological data}

Meteorological data available for the station Eelde from the Royal Netherlands Meteorological Institute (KNMI) were used (Figure 14). Data concern all relevant meteo-data, of which wind velocity and direction, global radiation and air temperature are the relevant ones. Data are available as daily values. Hourly values are available as well, but not needed for this research. 


\subsection{Habitat data}

Based on the occurrence of mussel beds and sublittoral mussel biomass, habitat maps for mussels (Mytilus edulis) have been constructed (see e.g. Brinkman et al, 2002). These maps (and may be similar ones for other animals) can be used to better model the growth/ occurrence of species/groups in parts of the system. Especially since there are large differences in sediment composition in the system (e.g. Figure 74) such knowledge can be applied to distribute the computed animal biomass (per functional group) more correctly: shell fish in the sandier areas, nematodes in the siltier areas, for example. In fact, habitat knowledge is to be used when functional relationships (e.g. why do nematodes prefer very silty areas? or why do lugworms prefer sandier areas?) are lacking or are not modelled explicitly. See Appendix A21. 
32 of 286 | Wageningen Marine Research report C058/17 


\section{Data for comparison}

\subsection{General}

Results from the model computations must be compared to available observations inside the system. These can be (and are) highly variable: concentrations of substances in the water column at different sites (sometimes as time series, sometimes observations during a short period), production data (ditto), contents in the sediment, and more.

\subsection{Benthic fauna data}

We have received benthic fauna data from several sources. For details see appendix A11.

\subsubsection{Netherlands}

- Survey data from IMARES/Wageningen Marine Research: it includes merely (but not only) data from the regular yearly shell fish biomass inventory. Data include tidal and subtidal areas. Sampling was done along transectories and was stratified (meaning that sampling took place there where shellfish was expected or occurred; zeroes were avoided this way). Samples are different in size, the largest one being 2-meter-long and $20 \mathrm{~cm}$ wide, and 10 $\mathrm{cm}$ deep each. For details see e.g. Van den Ende, 2012. According to Van den Ende et al

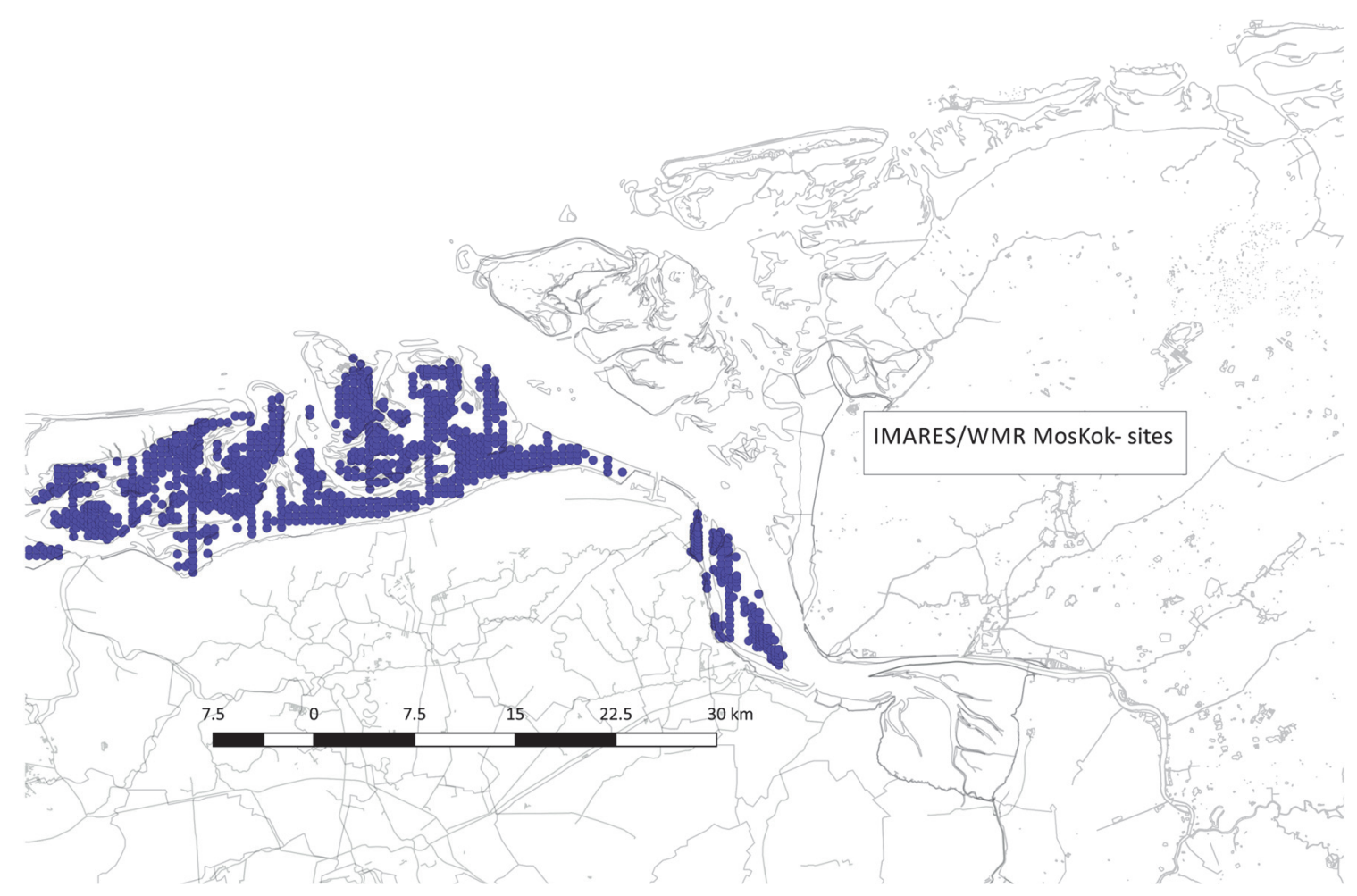

Figure 16 Sampling sites of WMR / IMARES, summary of all years that sampling took place. 
(2012), each sample represents $12.84 \mathrm{ha}$. This value is needed when combing data with other observations, and calculating average densities.

- NIOZ/SIBES data collected in the framework of a large Wadden Sea-wide benthic survey research. Data concern tidal areas only. Some samples have been taken in one year, some cover several years (Figure 17).

- Rijkswaterstaat collected benthos data as well, partly with NIOZ as the sub-contractor, and partly by Altenburg \& Wymenga as subcontractor. Data were collected on a year-basis on a few fixed sites in the Wadden Sea (not shown).

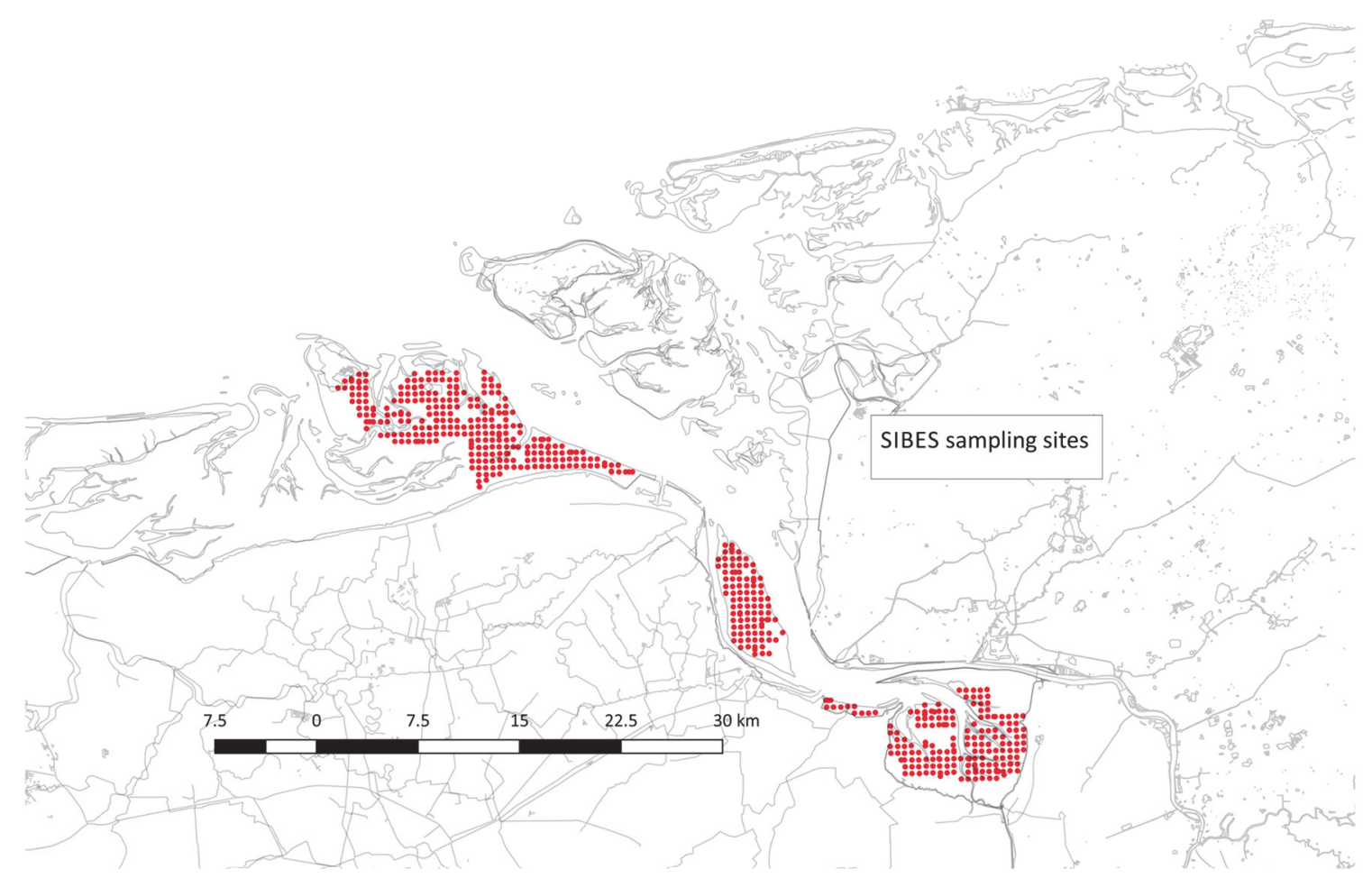

Figure 17 Sampling sites for the SIBES-project (NIOZ, 2016), summary of all years that sampling took place.

\subsubsection{Germany}

Survey data provided by the Niedersächsische Landesbetrieb für Wasserwirtschaft, Küsten- und Naturschutz (NLWKN). Data have been collected between 2008 and now (Figure 18).

\subsection{Phytobenthos and benthic primary production}

Riegman and co-workers from IMARES performed research on benthic primary production (although results were uncertain) including analysis of benthic chlorophyll-a content. See Brinkman et al (2015). In Figure 19, sampling sites are shown. Older results (Colijn \& De Jonge, 1984; De Jonge, 1992; De Jonge \& Van Beusekom, 1992; De Jonge et al, 2012) do not cover the finally chosen simulation period, but still do serve as important comparison values. 


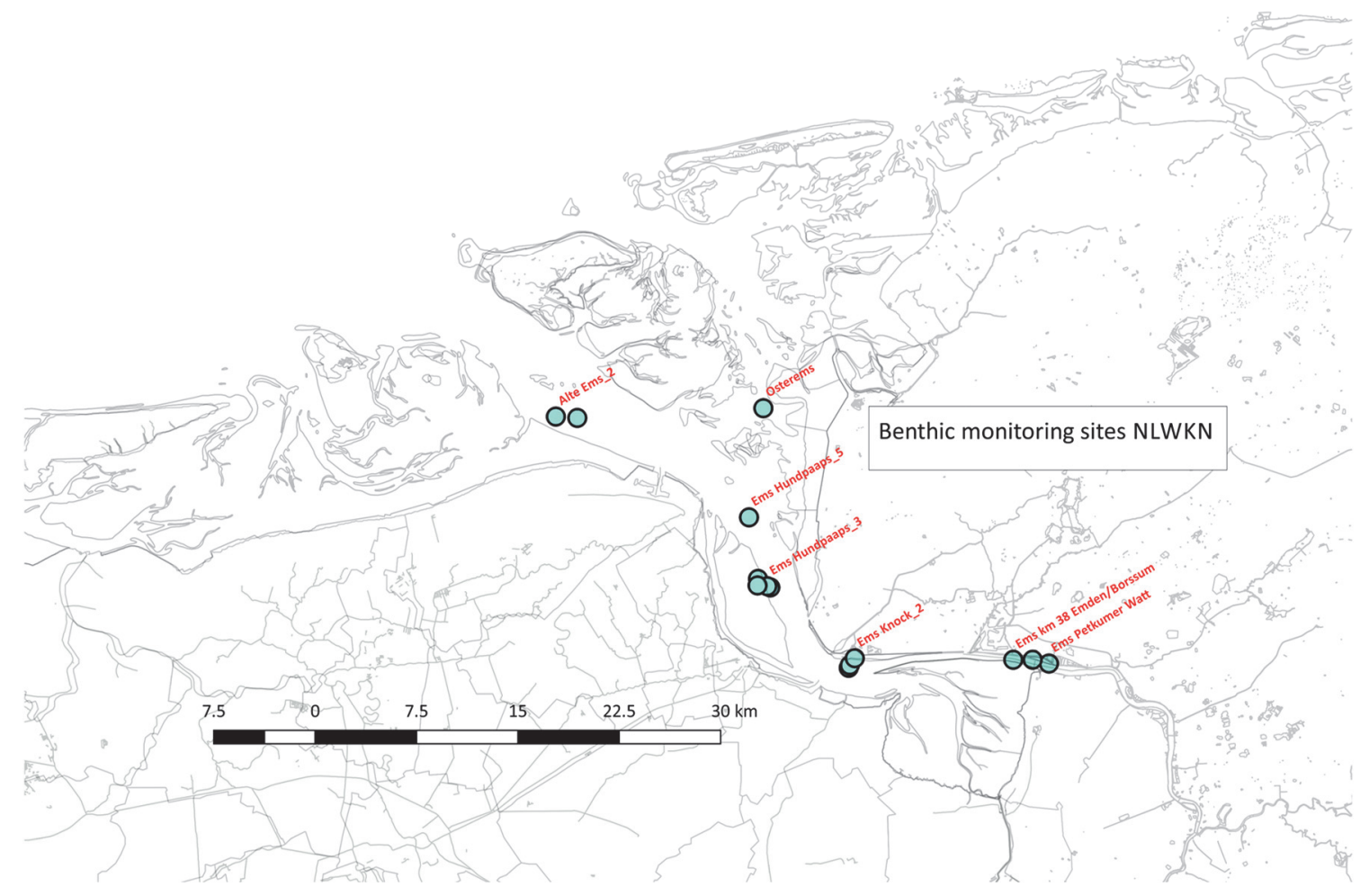

Figure 18 NLWKN benthos monitoring sites

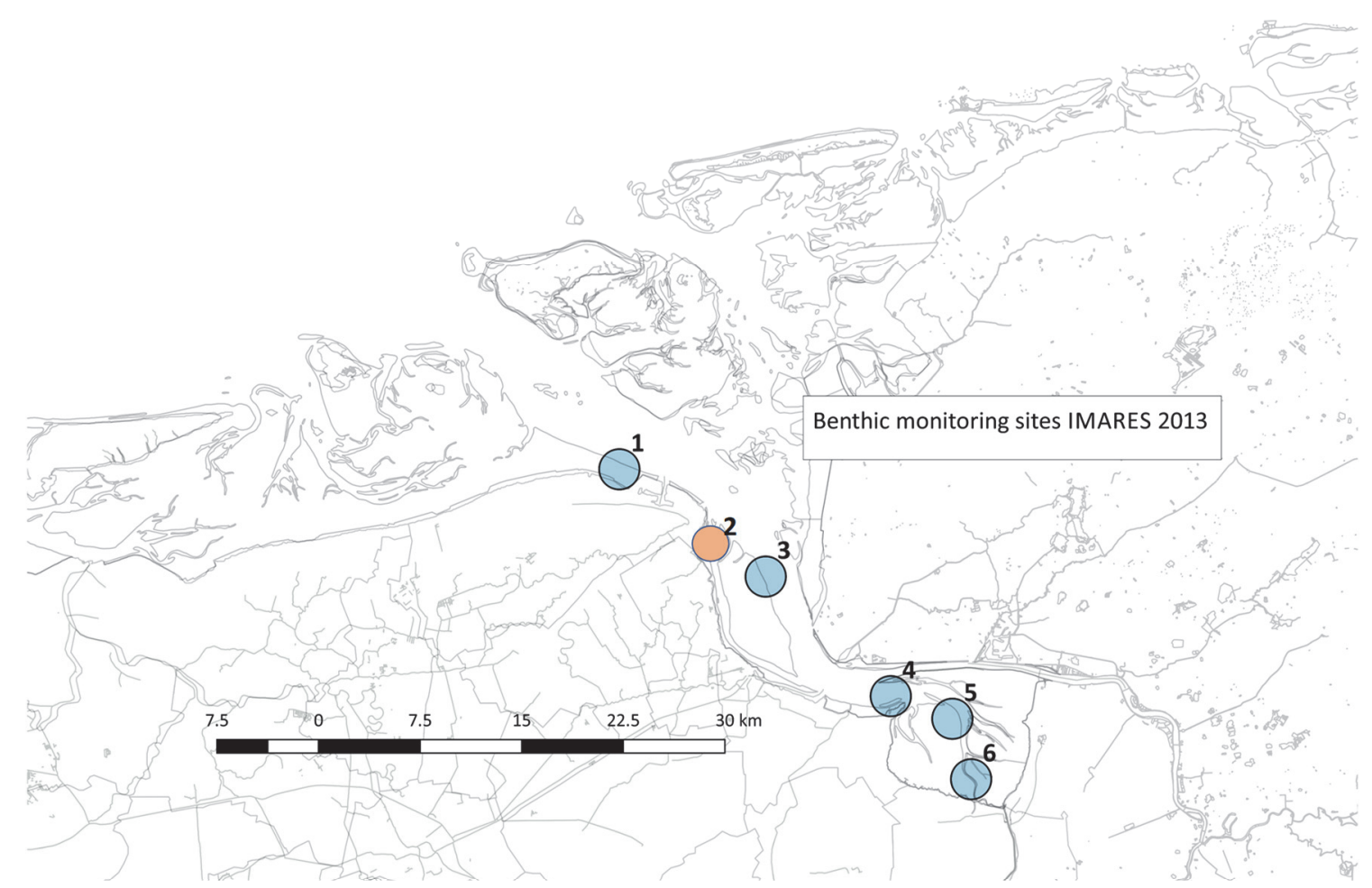

Figure 19 IMARES Benthic primary production measurement sites during the 2013 primary production study. Site 2 (orange) visited only once. 


\subsection{Phytoplankton, chlorophyll-data and pelagic primary production}

Phytoplankton data are mostly available as chlorophyll-a proxy. The latter is part of the monthly Rijkswaterstaat monitoring program and the NLWKN-monitoring program (sites are shown in Figure 7). Next, in some occasions also phytoplankton composition has been measured. Koeman \& Bijkerk performed several phytoplankton analyses for Rijkswaterstaat, and in 2012 \& 2013 for IMARES (Wanink et al, 2014). Rijkswaterstaat data were obtained from the Rijkswaterstaat Helpdesk.

Primary production data are available during some years. At the end of the '70's, Colijn and De Jonge performed a lot of measurements (Colijn, 1983; Colijn \& De Jonge, 1984; De Jonge \& Colijn 1994;). For the present simulations, these are less relevant since most of the available input data are not available before 1980. Recently, in 2012 and 2013, IMARES (Riegman et al) performed about 40 measurements (reported by Brinkman et al, 2015; Brinkman, 2015); sites are mapped in Figure 20. From sampling sites 1 to 6, trajectories have been sailed (Figure 65, appendix A9). Results were then used to estimate whole system pelagic primary production.

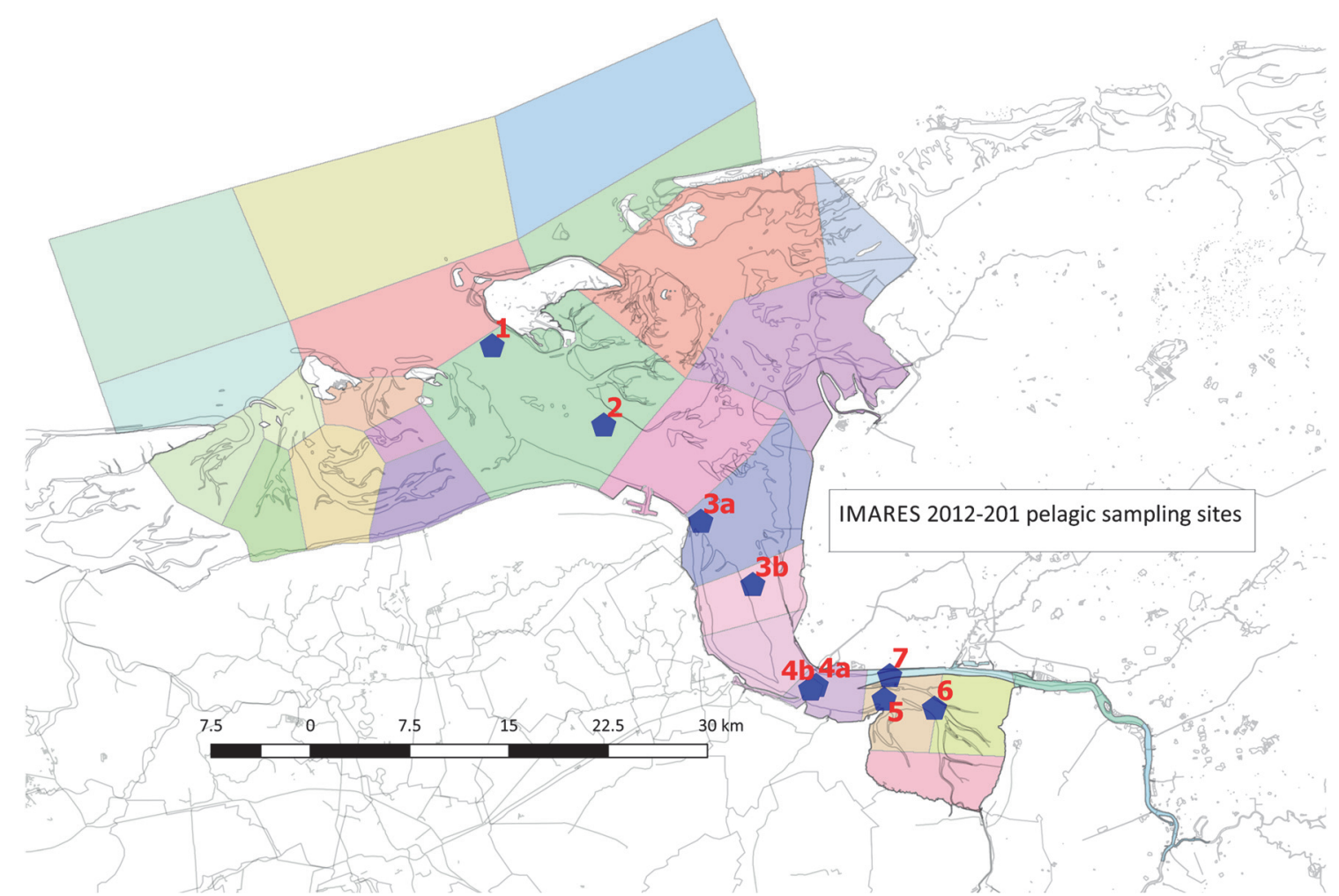

Figure 20 Pelagic sampling points during the 2012-2013 IMARES study on primary production. Site 3a was moved to $3 b$ since it appeared that the site was badly chosen. Also site $4 b$ was moved to $4 b$. Site 7 (Emden channel) has only been sampled once.

\subsection{Water levels}

Water level data are not directly needed for the model, but are useful when comparing model results with water quality data since they make clear in what tidal phase the monitoring activities 
have been performed. Figure 72 (Appendix A13) shows the monitoring sites where data are available. All data concern 10-minute data, and sometimes hourly data.

\subsection{Older data}

Next to the 2012-2013 data, and the already mentioned monitoring data there are only a few data available. Most result from the BOEDE-research in the end of the '70's (Baretta \& Ruardij, 1988), but the system characteristics were different in those days as explained in section 3.3.5.

Next to those data, De Jonge and co-workers assessed data on phytobenthos (chlorophyll densities, production).

Data on higher trophic levels (shrimps, fish) are scarce, but now also less relevant since these fauna groups are not included in the model. 
38 of 286 | Wageningen Marine Research report C058/17 


\section{$5 \quad$ Model characteristics}

\subsection{The water column}

The water column in the present model-setup is assumed to be well mixed. An option to compute profiles of solid components (algae, detritus and inorganic solids) is available in the model, but not activated for the present application.

Computed water column primary production and phytobenthos primary production both take the emersion period and the tidal range (changing light conditions during submersion) into account. See Brinkman $\left(1993^{b}\right)$ for all details.

\subsection{Exchange between compartments}

The hydrodynamical part of the model is very simple (and probably the weakest parts of it). There is a flow-through of incoming fresh water; the pathways of the water flowing through the compartments (e.g. which fraction flows from compartment 111 to 113 and which fraction to 112) are set a priori. There also may be a flow-through of North Sea water (entrance through one tidal inlet, and outflow through another), but that is not relevant in this case, although one may argue that there are two main tidal inlets (Huibert Gat and Memmert) and thus water may flow partly from one inlet to the other, like it happens in the western Dutch Wadden Sea. But this possibility was omitted here. Also, a flow from the Groninger tidal basins to the Ems and next, a flow crossing the Juist tidal flat, is neglected. Especially the input from the Groninger tidal basin needs improvement since it introduces fresh water and thus nutrients into the North-western part of the system.

\subsection{Exchange between sub-compartments}

Sub-compartments share their water column bodies regarding dissolved substances, phytoplankton and detritus (although this can be changed by the parameter choices). Thus, after each time-step (with computations done separately for each sub-compartment), resulting values for these components are averaged and assigned to all (the five) pelagic sub-compartments. This is not the case for suspended sediment: the suspended inorganic sediment concentrations in the water column is done for each sub-compartment separately (this inorganic matter is not transported at all in the model, also not between main compartments).

\subsection{Temperature}

Temperature appeared to be lacking in too many cases, and values were computed based on a simple method handed by Wolf Mooij (Netherlands Institute for Ecology of the Royal Netherlands Academy of Arts \& Sciences). See appendix A7. 


\subsection{The sediment part, pore water profiles, oxygen and nitrogen consumption in the sediment and adsorbing ions}

Sediments of all the sub-compartments are treated separately, i.e., there is no other contact between sediment compartments than via the water column.

The model contains the computation of processes in the sediment and exchange between sediment and overlying water. Exchange is diffusion driven, with an extra term accounting for acceleration of the diffusive (or dispersive) process by benthic animals. Transport to the sediment is a result of sedimentation and biological driven deposition (e.g. by shellfish).

In the sediment and the sediment pore water, several processes take place. Primary production by benthic algae takes place in the sediment top layer; in all layers mineralization occurs. The first produces oxygen and demands consumption of carbon dioxide and nutrients. Mineralization uses oxygen or nitrate, depending on the availability of each, or, in absence of both, anoxic degradation takes place. All these reactions are assumed to be zero order regarding the ions, and first order regarding the substrates. See appendix A12 for some extra information on phytobenthos.

Dissolved ions $\mathrm{P}$ and $\mathrm{Si}$ (ortho-phosphate $\mathrm{H}_{\mathrm{x}} \mathrm{PO}_{4}{ }^{(3-\mathrm{x})}$ - and silicate $\mathrm{H}_{\mathrm{x}} \mathrm{SiO}_{4}{ }^{(4-\mathrm{x})}$ ) ) may adsorb onto solids (see also Brinkman, 1993'); these solids are represented in the model by FeOOH. This implies that in the sediment first order adsorption-desorption reactions take place, next to the already mentioned zero-order reactions. In the present model set-up, other ions do not adsorb, but the model does not limit the number of adsorbing ions; adsorption onto detritus (humus-like matter) can be included as well.

For all ions, pore water profiles are computed every time step by solving the steady state reactiondiffusion equation. For an extensive explanation of the solution of the equation the reader is referred to Brinkman \& Smit (1993).

The use of steady-state profiles implies sudden changes from one profile to another (only slightly different, in most cases), and need a correction to preserve the mass budget. To avoid too large sudden pore water profile changes, a kind of relaxation term is introduced, causing more gradually changing pore water concentrations. The mass budget correction needed is computed and applied; deviations from the mass budget are, weighted based on the actual concentration, divided over all layers.

The first-order situation (with adsorbing ions) contains exponential terms that include the first order reaction rate coefficient. Since these adsorption reactions are very fast, the arguments of the exponential term may become very large or very small. Therefore, the thickness of the sediment layers is restricted; in the present model set-up, a maximum of $25 \mathrm{~cm}$ sediment depth is implemented in the model. This is the lower system boundary.

Each time-step it is first computed what the reaction rates are in the sediment (which depends on the present situation), then what the penetration depth is of oxygen and next the one of nitrate, then the other profiles of ions with zero order reactions terms only, and finally the profiles of those ions that show adsorption behaviour (and thus have first order reactions terms in the diffusion-reaction equation).

Based on these profiles, release to or uptake from the water column is computed using Fick's first law. 


\subsection{Choices for model components}

The EcoWasp ecosystem software has a general structure, allowing users to choose the ions and elements, phytoplankton groups, etc, according to their own choice. Of course, necessary parameters must be provided, and this is the time-consuming part of any new model construction. Note that a distinction is made between the software (is the same for any application) and the model (the software plus the choices for the components and their parameter setting).

\subsection{Ion/ elements}

Ions that are included in the model are listed in appendix A28.1. For all components, data for tributary input are needed. Phosphorus and silicate also adsorb onto sediment particles. All elements occur in the water column and in the sediment, and are subject to sediment/water exchange. Oxygen, carbon dioxide and ammonia are exchanged with the atmosphere.

\subsection{Phytoplankton}

\subsubsection{Groups}

In the existing western Wadden Sea model (Brinkman, 2015) three pelagic algae types have been implemented. Fresh water algae were assumed to die immediately after entering the marine environment.

In the Ems-Dollard area, a larger part of almost fresh and more brackish water is present. Previous research (e.g. Wanink et al, 2014) showed that blue-greens and other fresh water algae stay present in the inner part of the area. Therefore, three new phytoplankton groups were added to the model: freshwater diatoms, fresh water non-diatoms and fresh water blue-greens. Their mortality is related to the water column salinity. And an inverse salinity relationship is implemented for the marine phytoplankton. This is explained in A10. Groups are specified in listed in appendix A28.1. A28.6

\subsubsection{Parameters}

Phytoplankton and phytobenthos parameters are first copied from previous research (Brinkman, 2015), and adapted based on the results from the work of Riegman and co-workers in 2012 and 2013 (reported by Brinkman et al, 2015).

Salinity related parameters are simply chosen by hand, such that fresh water phytoplankton cannot survive in a marine environment, but still exist (a bit) around 10 ppt. marine phytoplankton is assumed to have opposite characteristics, and still can survive around about $10 \mathrm{ppt}$ salinity, but not at lower values.

No salinity relationship is applied to phytobenthos. Phytobenthos is not imported into the system, and therefore such a distinction is less relevant. 


\subsection{Detritus}

\subsubsection{Groups}

The choice for detritus components is the same as it was in previous applications (e.g. Brinkman, 2015). Seven groups are distinguished: fast degrading dead organic matter (with and without silica as component: DEADSUB and DEADDIA), slowly degrading dead organic matter (with and without silica as component: FRAC2 and FRAC2DIA), frustules (FRUSTULES), labile organic matter (LOM) and refractory organic matter (ROM). All types are defined through their composition ( $N$, $P$, etc) and degradation rate constant. All living matter that dies goes 1:1 into dead organic matter. Next, a fraction goes into the next types, and the rest is released as nutrient and $\mathrm{CO}_{2}$. $\mathrm{ROM}$ and FRUSTULES are the last types, most slowly degrading. In fact, degradation of FRUSTULES is more dissolution of the silica than a biochemical process. But for the model, this difference is not relevant. Groups are specified in appendix A28.3.

\subsubsection{Parameters}

Parameters have been copied from previous research (Brinkman et al, 2015). Only the settlement and resuspension parameters were adapted to better account for the detritus distribution between sediment and water column

\subsection{Fauna}

\subsubsection{Overall description of the model}

Here, details of the model are not explained, but the reader is referred to the basic report on EcoWasp (Brinkman, 1993 ${ }^{b}$ ). This report is distributed with this present report as background information.

\subsubsection{Parameters}

All fauna components are defined through their parameter setting: where they live, feed, reproduce, defecate, etc, in the water column or sediment. Also, all food preferences and utilisations are defined by appropriate parameters; and similarly, maximum growth, respiration, mortality rates, etc, are defined through relevant parameters. This implies that only one routine is needed to compute all fauna dynamics.

Most hard to find are mortality parameters. For the western Dutch Wadden Sea, NIOZ data on animal numbers and ages (thanks to Rob Dekker, NIOZ) on the Balgzand area have been used by Brinkman (2015) to find best values. These were copied, and slightly adapted if needed, to the present model area.

Also, the parameter that describes the costs of large silt content in the water column is found after testing the effects. This is based on the presence or absence of animals in silty areas. At first instance, it appeared that the parameter describing the maximum amount of pseudofaeces already accounted for a negative response in high turbid situations. See above. In very turbid cases, the result is that this amount is reached before the amount of 'real' food is caught that the animal needs for its growth. Thus, the animal does not grow sufficiently. 


\subsubsection{The literature review.}

Since we are dealing with a situation where silt concentrations in the water column can reach extremely high values (up to and even above $1 \mathrm{~g} \mathrm{DW}^{-1}$ ) it is very likely that foraging of filter feeding animals is negatively affected.

Therefore, two literature researches were performed: "Characteristics of several species typical for the system" (appendix A2) and "Effects of silt concentration on filtration and growth characteristics of several shellfish species" (appendix A24). Relationships of filter feeding activities with silt content was checked for Blue Mussel, Edible Cockle, Japanese Oyster, Razor Clam and Peppery Furrow Shell (see appendix A24 for a complete overview). Of these animals, the Peppery Furrow Shell (Scrobicularia plana) is a typical inhabitant of muddy areas, while the other species avoid too silty environments.

General, the literature review reveals that Blue mussel (Mytilus edulis) growth probably is impaired when silt concentrations get above $250 \mathrm{mg} \mathrm{l}^{-1}$. Critical limits are related to digestive constraints rather than to metabolic costs of feeding and digestion. For details see appendix A24.3.1.2.

Cockles (Cerasteroderma edule) seem to be well-adapted to turbid environments due to an effective pre-ingestive selection of food. Increasing non-edible suspended solids results in energetic losses due to mucus formation coupled to pseudofaeces production.

Peppery furrow shells (Scrobicularia plana) shows a lower 'scope for growth' when suspended matter is above $100-150 \mathrm{mg} \mathrm{l}^{-1}$.

Japanese oysters (Crassostrea gigas) shows a decreasing scope-for-growth above about $90 \mathrm{mg}$ suspended matter $\mathrm{l}^{-1}$.

Razor clams (Ensis directus) seem to show a high tolerance to high silt concentrations, up to 300 $\mathrm{mg} \mathrm{l}^{-1}$.

On general, the idea is that not all results for the shell fish mentioned above are concistent. For example, the Peppery furrow shell is a typical inhabitant of very silty sediments, but, according to the results mentioned, it has a low tolerance to high silt concentrations. This makes it difficult to find proper parameters for the equations that describe the effect of silt on food uptake and energetic costs of separating edible and unedible suspended matter.

\subsubsection{Model parts describing the effect of silt on zoobenthos growth}

The equations that describe fauna dynamics contain two terms that may account for a (negative) effect of silt concentration.

One is that some animals (like the Blue Mussel Mytilus edulis) produce pseudofaeces; the amount increasing with increasing silt levels. The costs of pseudofaeces formation is a cost term in the mass budget of the animals. It is described as an energetic loss that increases linearly with the amount of pseudofaeces (see below in section 5.11.1.1) produced (as mg DW loss per mg pseudofaeces).

Second, a separate energetic loss term is introduced, called ISS_costs, that increases with increasing presence of inorganic suspended solids. This relationship is not linear, but quadratic: 
costs are ISS_param* (inorganic solids)2. Thus, low suspended solid contents are not relevant here, but higher ones (may) become increasingly important (in a negative way). The idea is that solids hinder the filtration process. ISS_costs has units (mass loss $\left(\mathrm{g} \mathrm{ind}^{-1} \mathrm{~d}^{-1}\right)$ ), and the parameter ISS_param (mass loss $\left(\mathrm{g} \mathrm{ind}^{-1} \mathrm{~d}^{-1}\right)$ (g DM filtered ind $\left.{ }^{-1}\right)^{-2}$ ). Thus, it is different from the pseudofaeces costs (that is only relevant for pseudofaeces producing animals), and it can be used for all animals, including those that do not produce pseudofaeces.

\subsection{Zoobenthos}

\subsubsection{Groups}

Conclusions on which species (or better: functional groups) are most important for the system were based on available species data from NIOZ (the SIBES-project), NLWKN and Wageningen Marine Research / IMARES.

Benthic species and groups considered in the present model are shellfish (feeding on suspended algae by filtering the water column) and sediment browsers (called 'Hydrobia' or 'mudsnails' here, but comprises all animals feeding from microphytobenthos). Other animals and groups, such as lugworms and meiobenthos, are omitted now, but can be included if desired.

\subsubsection{Filter feeders}

One group is included at this moment for which blue mussels are the representing group, although data for other species have been searched for (Tamis, 2017). Parameters as used for the western Wadden Sea were used first (Brinkman, 2015); later, it appeared necessary to change the parameter setting a bit.

Mussels have four stages, three benthic ones (seed, half-grown and adult), and one pelagic stage (larvae). Reproduction takes place in early May, takes two weeks (in the model), and settlement of larvae occurs as soon as they reached a critical size. Since this will differ for each compartment, settlement may occur at different dates. Transfer from one stage to another includes a (large) loss of numbers: spawning is only for a low \% successful, ditto for settlement to the sediment.

Mussels filter the water column and respire in the water column. Benthic stages deposit their faeces on the sediment, larvae do this in the water column. Benthic stages produce pseudofaeces, with a certain maximum (total filtration rate relative to the maximum edible food uptake rate), set to twice the amount of edible algae. Thus, in case of a high turbidity, the maximum edible food uptake cannot be reached because of the excessive pseufaeces production. The factor two is a bit arbitrary. This pseudofaeces is deposited onto the sediment surface. Groups are specified in appendix A28.4 and A28.5 (specifically on spawning characteristics).

\subsubsection{Sediment browsers}

One group is included, as Hydrobia. The estimation procedure was like the shell fish one. But since growth and number data are scarcer, these were roughly estimated. The parameters finally have been tuned to come to acceptable numbers of the snails. Also, it was decided to use one stage, so no reproduction, etc, is of interest now. Improvements are still possible/needed. In a next phase, Littorina littorea may be included as well; both snails then must be distinguished based on their habitat preferences. 


\subsection{Zooplankton}

\subsubsection{Groups}

Pelagic species/groups considered in the present model set-up are microzooplankton, feeding on small picophytoplankton, and copepods, manly feeding on bacteria associated with detritus. The first is a special group in the model, since in turn it can be eaten by shellfish, making shellfish not only a secondary producer but also a secondary consumer. This mechanism is important for the occurrence or disappearance of picophytoplankton.

In previous model versions, little attention was paid to these animals. A major cause was the dominating presence of macrozoobenthos (shell fish). Effects of fresh water is implemented; equations like eqs 1 and 2 are applied accounting for the relationship between mortality and [CI]. If wanted (not done yet) fresh water zooplankton can be included. Also, it appeared at the end that tuning of copepod dynamics was not successful so far. Groups are specified in appendix A28.4 and A28.5 (specifically on spawning characteristics).

\subsubsection{Parameters}

As said above, fauna characteristics are defined by setting the appropriate parameters (5.10.2). Thus, pelagic animals are defined by the parameter that says that their living habitat is the water column, where they also feed and deficate (also defined through the appropriate parameter).

Microzooplankton growth and respiration parameters have first been copied from the western Wadden Sea parameter set (see Brinkman, 2015) and later adapted somewhat to come to animal numbers that seem realistic. This will sound rather tricky (and it is), but since there are no real data available, values for the western Wadden Sea were taken as guiding.

Finding growth and respiration parameters for detritus feeding zooplankton was first done after the outline in appendix A18. Secondly, old BOEDE-data (Baretta \& Ruardij, 1988) have been used to tune growth parameters and thus reach reasonable numbers per unit of volume (although the BOEDE-data are for an earlier period not covered by the present model). Difficulties remained, however, see chapters 6 and appendix A25.12.

For both zooplankton groups, no spawning events and thus no larval stages are distinguished. 
46 of 286 | Wageningen Marine Research report C058/17 


\section{Tuning the model physics}

\subsection{General}

Two parts of the model tuning are done first: inflow into and exchange of water between compartments and resuspension/sedimentation. These both do affect the other simulation results, but themselves, they change with time (daily), but stay (almost) unchanged for all simulations. So, they are not or hardly (sedimentation/resuspension) affected by the other components. The other components may show a high level of interaction and have feedback characteristics (one component affects another, but vice versa it also occurs).

\subsection{Exchange between compartments}

A basic condition for an ecosystem model is that the transport to, from and inside the modelled system is satisfactorily modelled. Since in the present Ems-Dollard system salinity differences are large, ranging from almost fresh water to complete saline conditions, the $\left[\mathrm{Cl}^{-}\right]$-data are very well suited to use when tuning this part of the model. Three aspects are relevant:

- In- and outflow of sea water through tidal inlets or from and to adjacent tidal areas.

- Inflow of water from rivers and canals. The water flows through the system to the sea, and the route that is followed by the water must be described.

- Dispersion between compartments. In the present case, this is almost completely a result of the tidal movements inside the modelled basin.

Of these three processes, the first is neglected. There is one main entrance, and the net input of marine water will be zero, although there may be net flow from the Huibertgat tidal inlet towards the Memmert tidal inlet, or vice versa. Also, there will be a flow across the tidal flat between Schiermonnikoog and the main land, as there will be a flow across the tidal flat between Juist and the main land. As said, these are neglected, but the effect will be discussed later, since it is a shortcoming in the model.
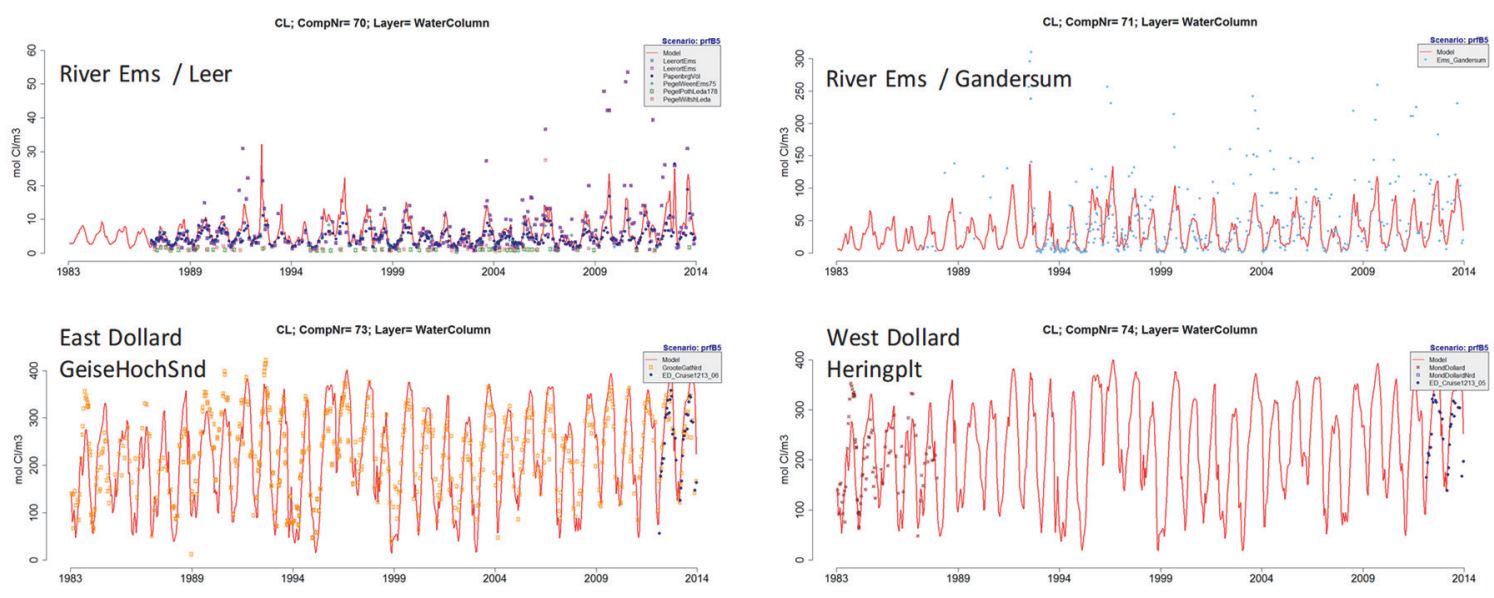

Figure 21 Modelled chloride concentrations in compartments 1, 2, 3 and 4 (subcomp 70,71,73,74), with data. The lower Ems River near Gandersum is not very well modelled, instead of the other three compartments. 

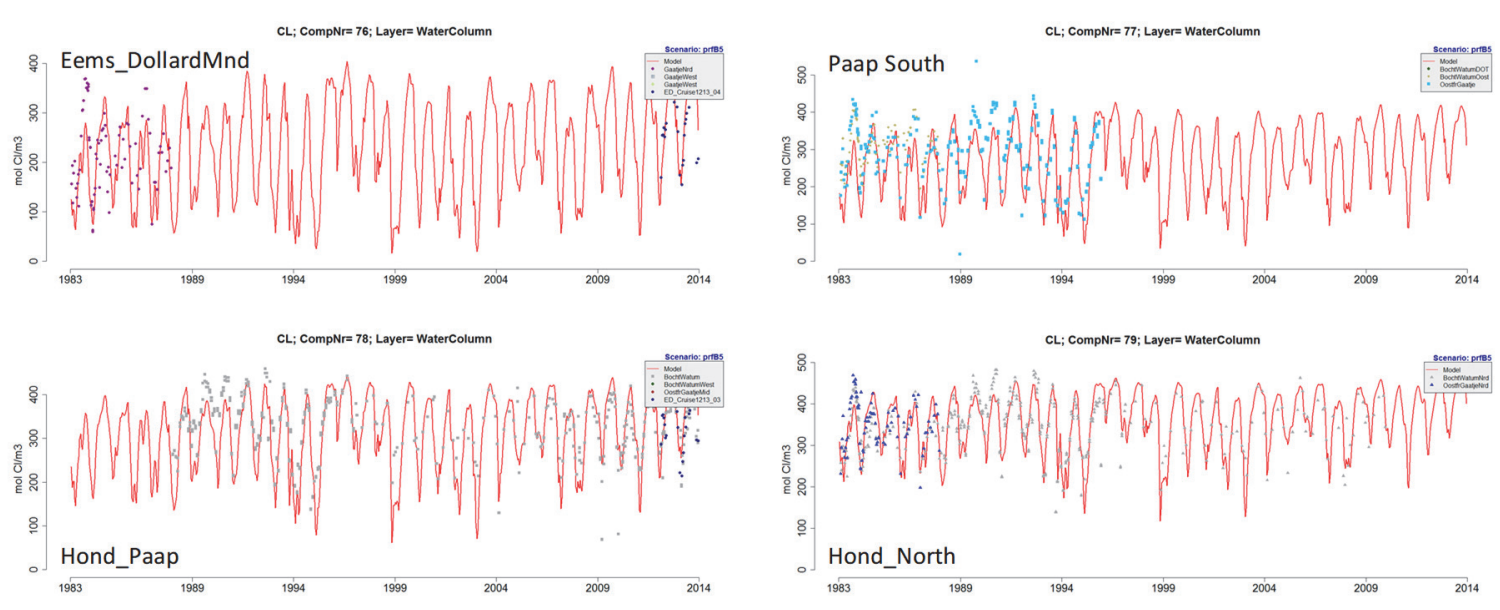

Figure 23 Modelled chloride concentrations in compartments 7, 8, 9 and 10 (subcomp 76,77,78, 79), with data. Except from some extremes, most of the results fit quite well.
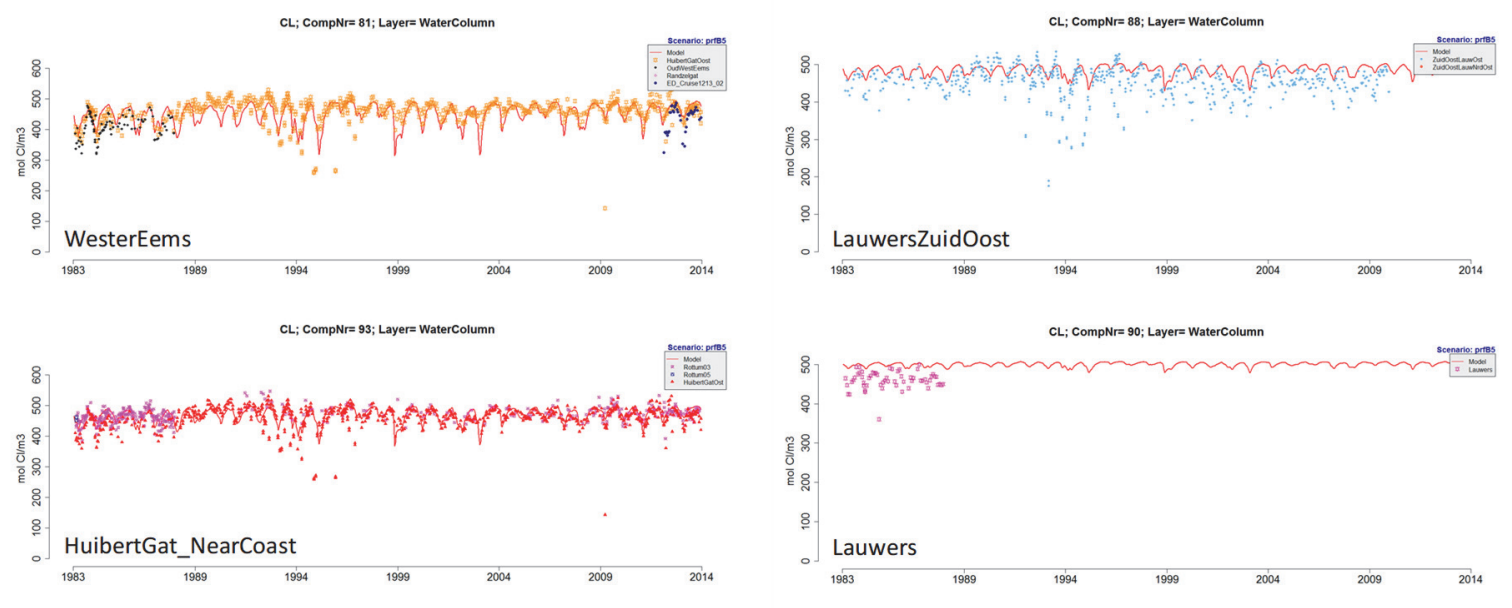

Figure 23 Modelled chloride concentrations in compartments 12, 19, 24 and 21 (subcomp 81, 88, 93 and 90), with data. Both left figures (81 \& 88) show reasonable similarity between data and model. Both right figures show failures. This is because inflow from and / or exchange with the Zoutkamperlaag (where the fresh water from Lauwersmee enters) is neglected.

The inflow of water from rivers and canals is described as well as possible (appendix A4). The route the water follows is a matter of this tuning section, although in most cases it is a relatively simple problem because of the linear shape of the area: all the water flowing in in compartments 1-10 will flow from compartment (i) to compartment (i+1). For compartment 11, some water (estimated at 20\%) will flow to compartment 13 (Easter Ems) (and on to 15, 23), the rest (estimated at 80\%) will flow to compartment 12 (Wester Ems), and on to 24.

Dispersion (tidally driven) is overall governing the distribution of water in the system. Dispersion parameters are expressed in $\mathrm{m}^{3} \mathrm{~s}^{-1}$ : the effective amount of water that is exchanged between two compartments per unit of time. By trial and error, most fitting values are found. In Figure 21 Figure 23 some results are shown; more relevant figures are in appendix A25.

The figures show that despite the very simple transport model that is underlying this EcoWaspmodel description, $\left[\mathrm{Cl}^{-}\right]$-values are described rather accurate. Thus, it is decided to use this part of the model representation. An overview of salinities in the system is in Figure 24. 


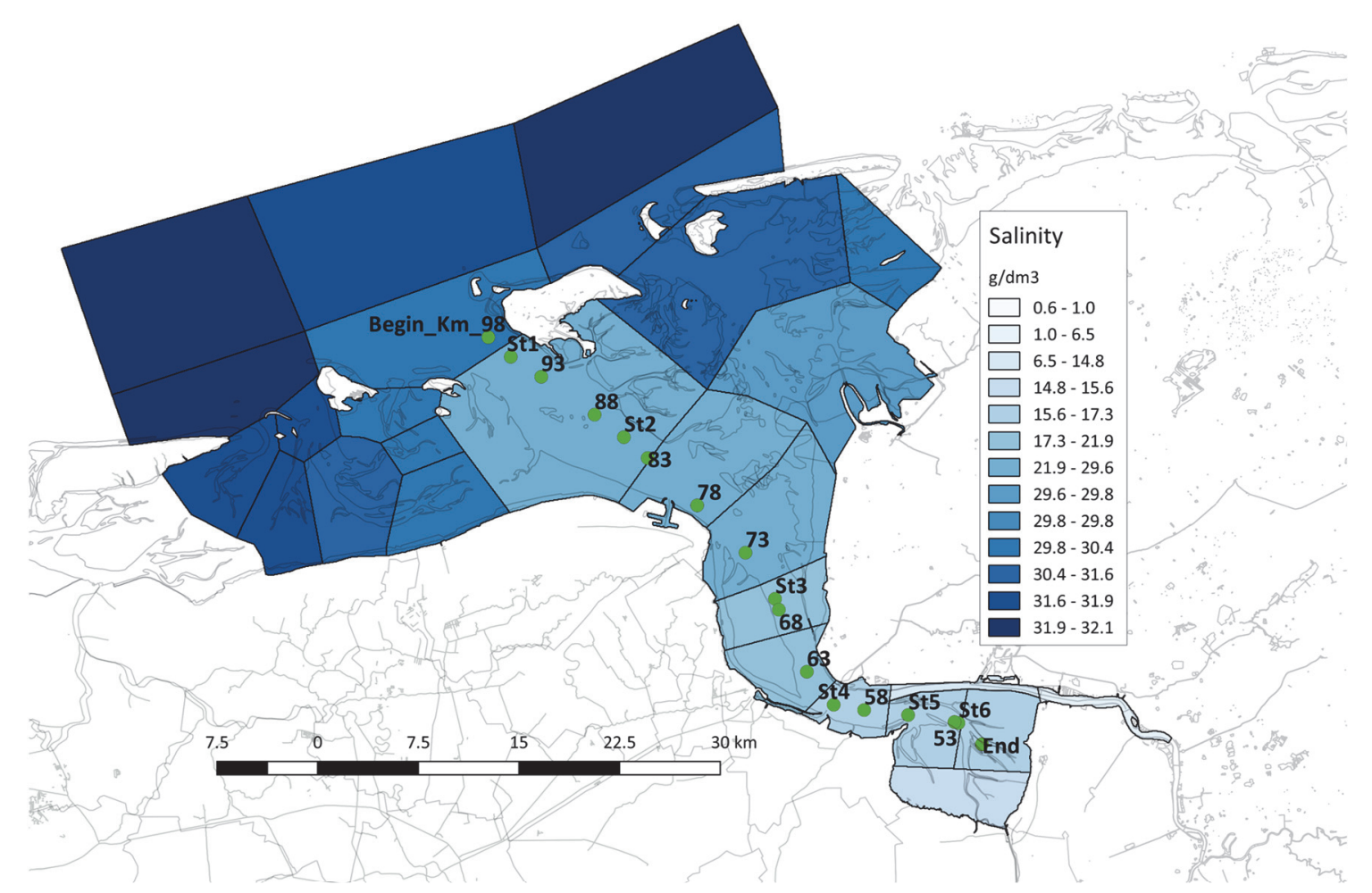

Figure 24 Spatial pattern of all-averaged (years 2010-2013) salinity in the system. Compare with Figure 117. The km-marks are mentioned in Figure 117 as well (starting at Herbrum as km 0)..

\subsection{Resuspension in each compartment}

\subsubsection{Introduction}

Next to the horizontal exchange of water, the vertical exchange between water column and sediment because of wind and water flow is described. Since transport of inorganics solids does not occur in the model (bound to each sub-compartment), resuspension is a process that solely depends on local sediment composition (which is an input data set for the model) plus wind and flow characteristics. The only part that is variable is the content of organic components (detritus, phytobenthos). Since this is a minor part of the suspended matter, it is neglected when tuning the resuspension parameters. Each sub-compartment (see section 2.1) parameter set (see appendix A20.2 for the description) is specifically valid for that sub-compartment.

\subsubsection{Results}

An overview is presented in appendix A20. As an example, seasonal pattern results for compartments 73, 78 and 93 are shown in Figure 25, a 2010-2013 plot in Figure 27, and the computed spatial pattern (average values for the period 2010-2013) are shown in Figure 26. 


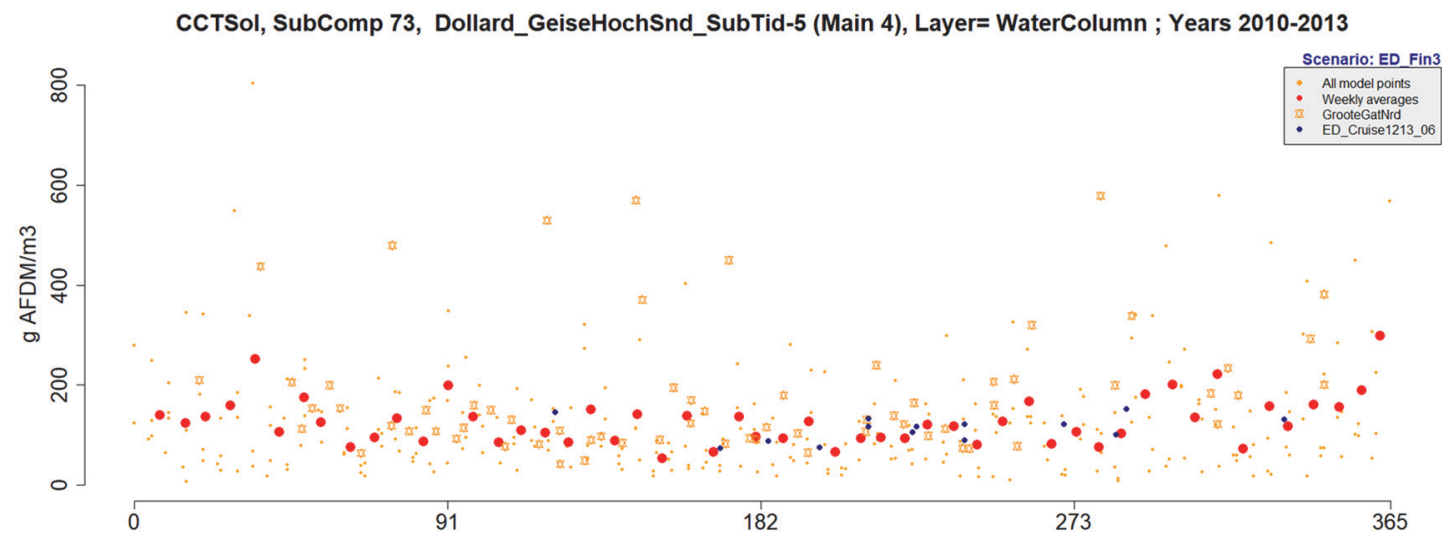

CCTSol, SubComp 78, Hond_Paap_SubTid-5 (Main 9), Layer= WaterColumn ; Years 2010-2013

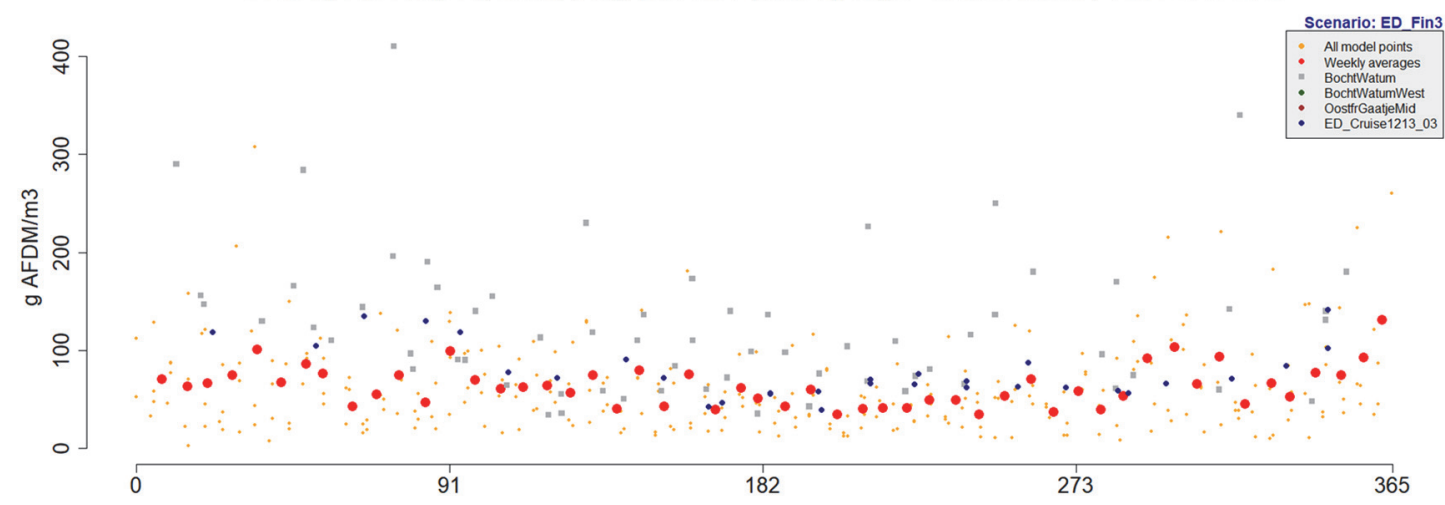

CCTSol, SubComp 93, HuibertGat_NearCoast_SubTid-5 (Main 24), Layer= WaterColumn ; Years 2010-2013

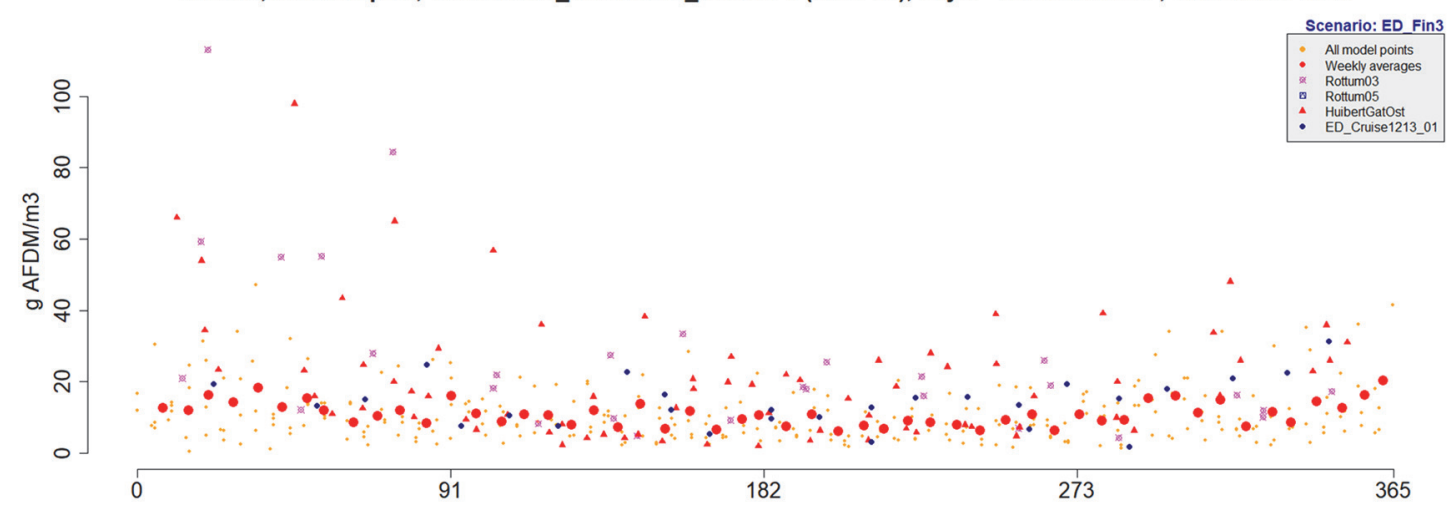

Figure 25 Light attenuation coefficients in the Ems-Dollard, period 2010-2013, seasonal pattern. For compartment numbers, see Table 3. Parameter values for this simulation ("ED_Fin3") are all listed in app. A24 


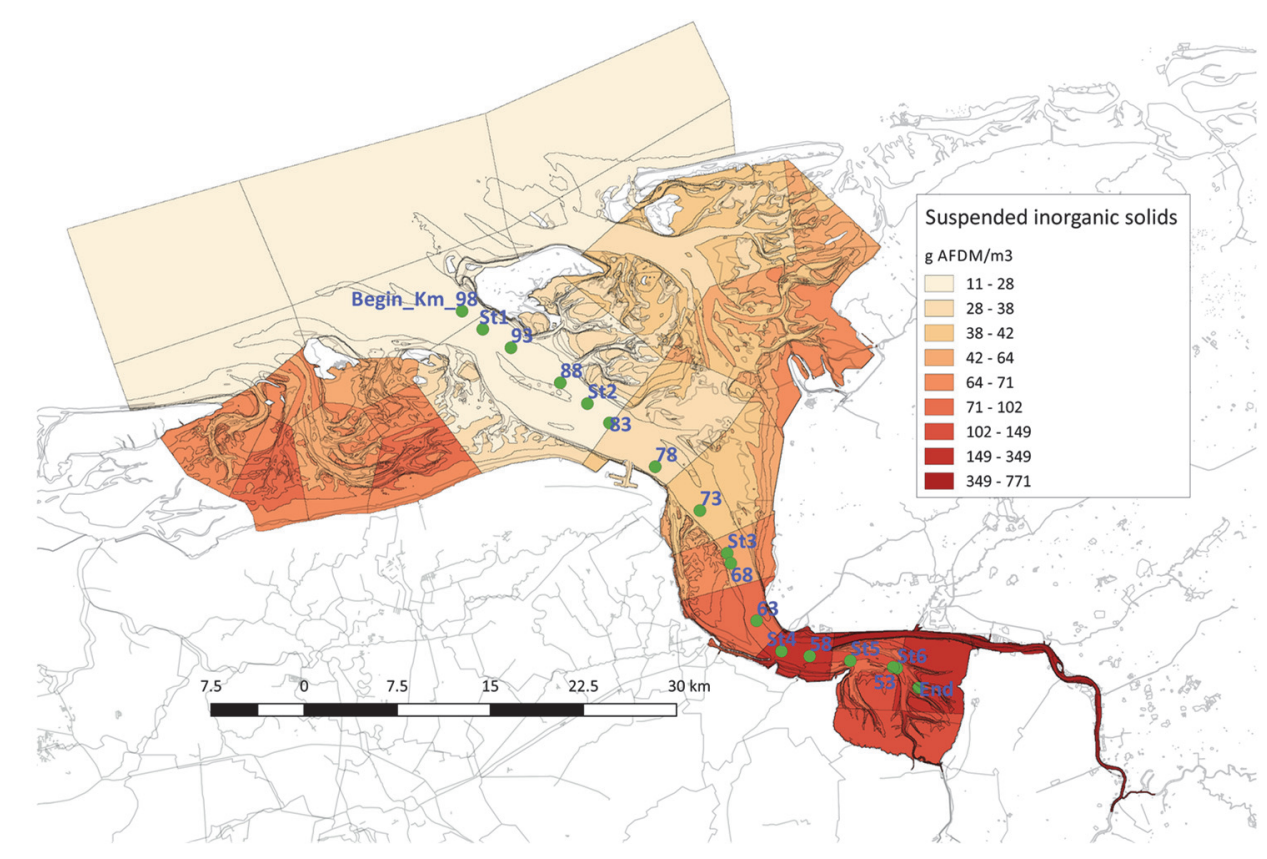

Figure 26 Computed distribution of suspended inorganic solids in the system (averages for the whole 20102013 periode. IMARES 2012-2013 sailing sites are included.

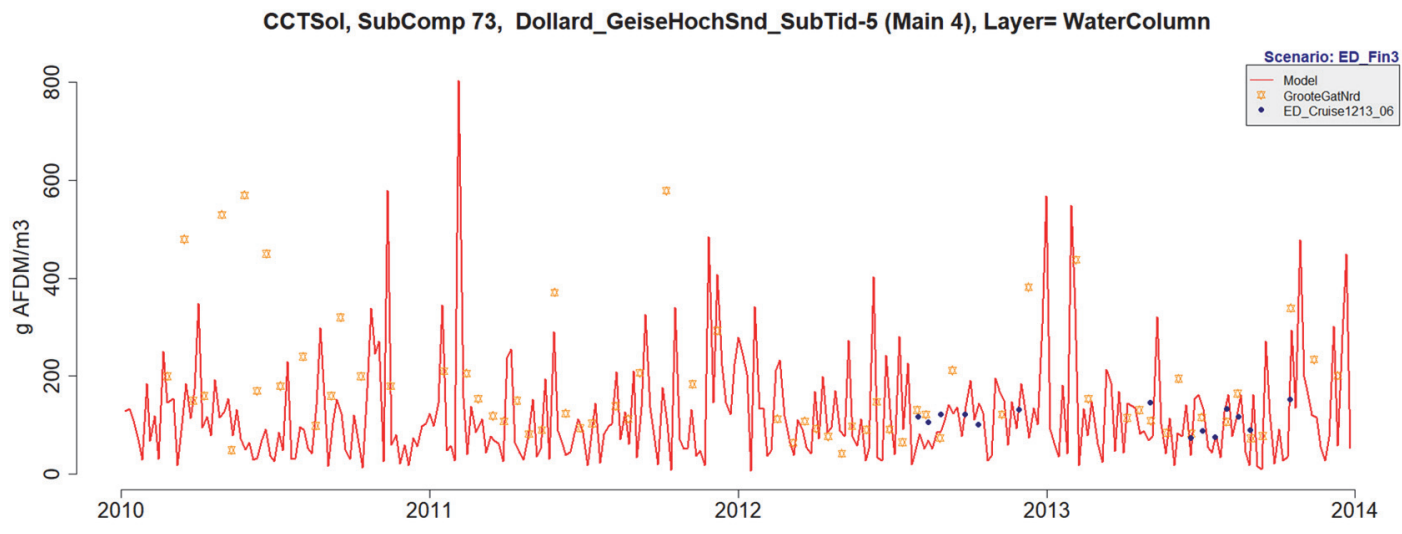

Figure 27 Computed (line) and observations (dots) suspended solids in sub-compartment 73 (main compartment 4, Geise-Hochsand) for the period 2010-2013. 


\section{Model results and effect of changing turbidity}

\subsection{Introduction}

Next to the water transport characteristics and resuspension parameter settings, all biological processes need to be tuned. Most of the tuning already has been done in previous work (see e.g. Brinkman, 2015), but it appeared to be necessary to check several parts of the descriptions. Also, new parameter settings for those groups introduced in the model now are needed. Most of the results are presented in appendix A25. Here just an excerpt is presented. This is done together with the results of a first simulation of effects of changing turbidity. This avoids double presentation of figures, since changes sometimes are small and must be compared next to each other.

\subsection{Changing turbidity simulated}

As a first exercise, one simulation was performed with a changed turbidity. The strategy was that the resuspension parameters for solids and detritus were changed with a factor; this is listed in Table 28. These reductions particularly concern the compartments closest to the site where the Ems river enters the estuary; see Figure 1.

Table 1 Resuspension reduction factor used for the silt-reduction scenario. For compartment numbering see Figure 1. All sub-compartments in each main compartment have the same reduction factor.

\begin{tabular}{|c|c|}
\hline Main compartment & Factor \\
\hline 2 & 0.7 \\
\hline 3 & 0.5 \\
\hline 4 & 0.5 \\
\hline 5 & 0.5 \\
\hline 7 & 0.5 \\
\hline 8 & 0.5 \\
\hline 9 & 0.5 \\
\hline 6 & 0.6 \\
\hline 10 & 0.6 \\
\hline
\end{tabular}

\subsection{Results}

\subsubsection{Extinction coefficient}

The ecological target of changing the turbidity is to improve the light climate in the water column. This is mostly, although not completely since also algae play a role, dependent on the silt and detritus content of the water column. Thus, up a $50 \%$ change is to be expected. This indeed happens, see Figure 28; Figure 29 gives an overall picture for the whole system. Light climates in 
those areas not mentioned in table Table 1 , does (hardly) not change. If so, then it is the effect of changing phytoplankton and/or detritus is the compartment.

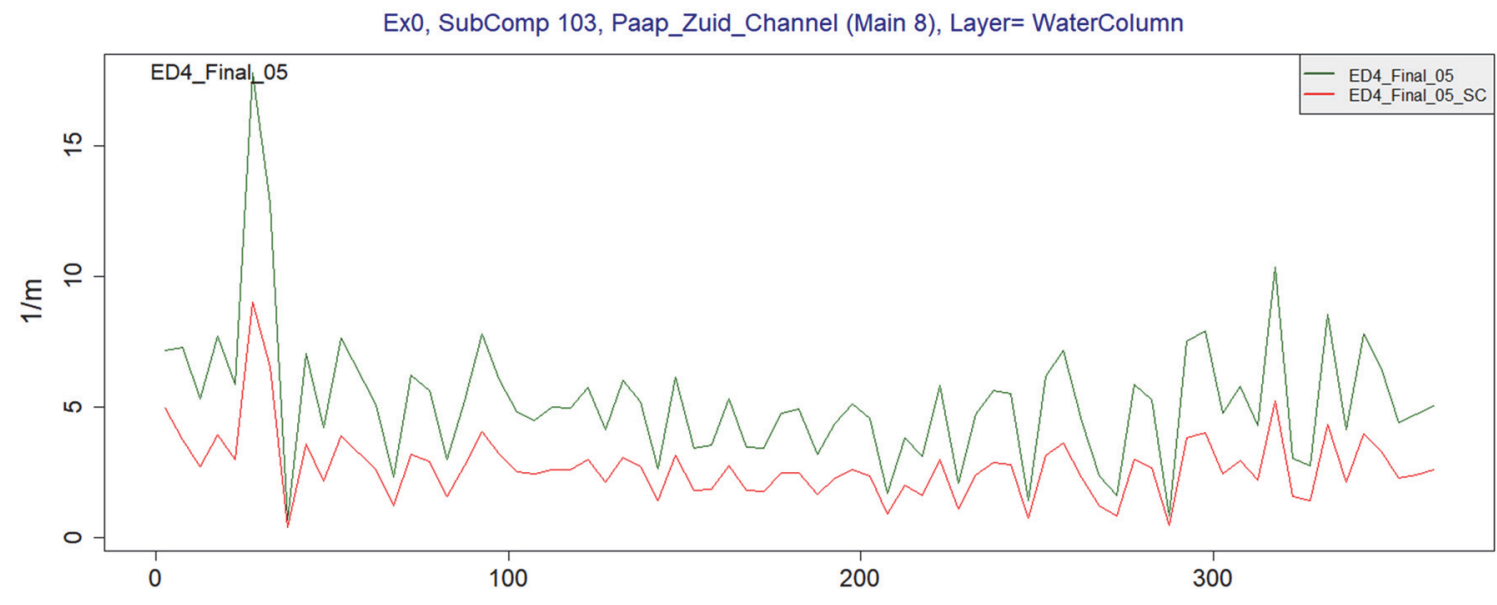

Ex0, SubComp 103, Paap_Zuid_Channel (Main 8), Layer= WaterColumn ; relative to first scen

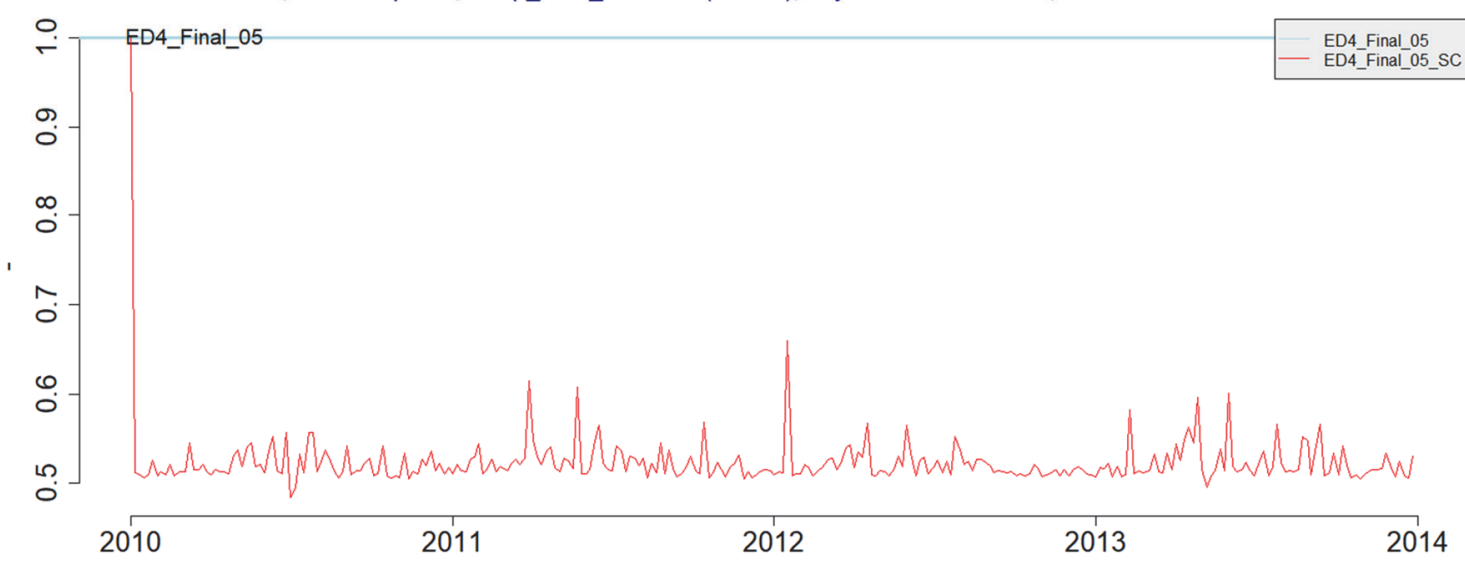

Figure 28 Light attenuation coefficient in sub-compartment 103 (Paap-Zuid, main compartment 8). ED4_Final_05 is the standard, ,ED4_Final_05_SC is the situation with reductios according to Table 1. Upper: absolute values, lower relative value of the reduction (red line) result compared to the standard (blue line, has always value=1). The value of 1 at the very first beginning is because of the initial value are unchanged.
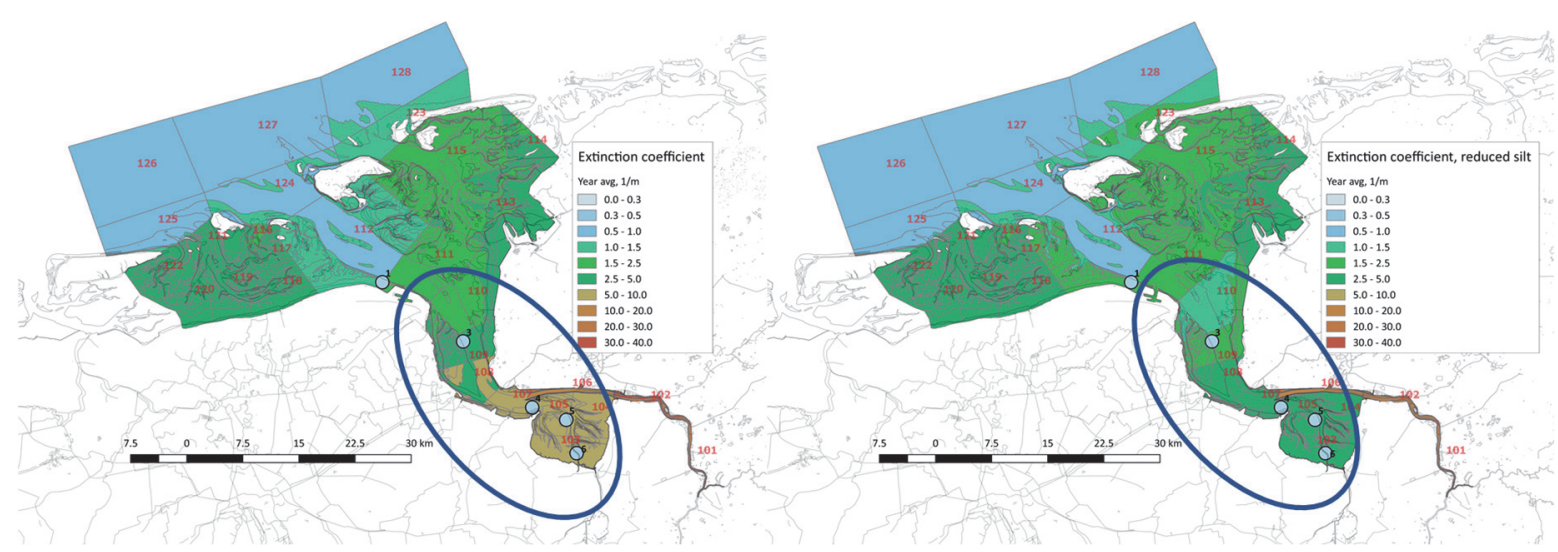

Figure 29 Light attenuation coefficient, year averaged, in the system. The areas where largest changes occur are marked. 


\subsubsection{Primary production and dissolved components}

With a changed light climate, one may also expect changes in primary production and consequently, in dissolved components such as oxygen, phosphorus, nitrate, silicate. However, this only slightly the case. An example of the gross primary production, with the data from the 2012-2013 IMARES research by Riegman et al (Brinkman et al, 2014) is shown in Figure 31, in Figure 30 a spatial overview of the gross and net primary production is presented, including the changes after suspended matter reduction. Changes are visible, but not very clear.

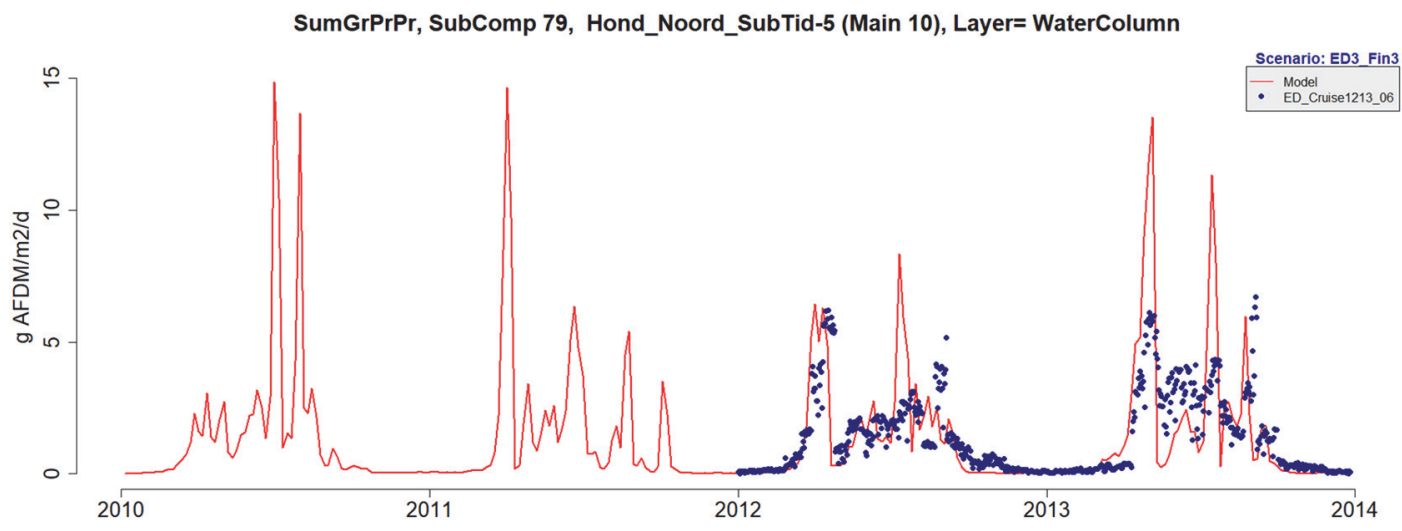

Figure 31 Gross primary production in compartment 79 (main compartment 10), sub-tidal area. Computed (lines) and results from the 2012-2013 IMARES-research (dots, see Brinkman et al 2014). Compartment numbers in Figure 1. Values in $g$ AFDM m ${ }^{-2} d^{-1}$. Other results in appendix A25.13.

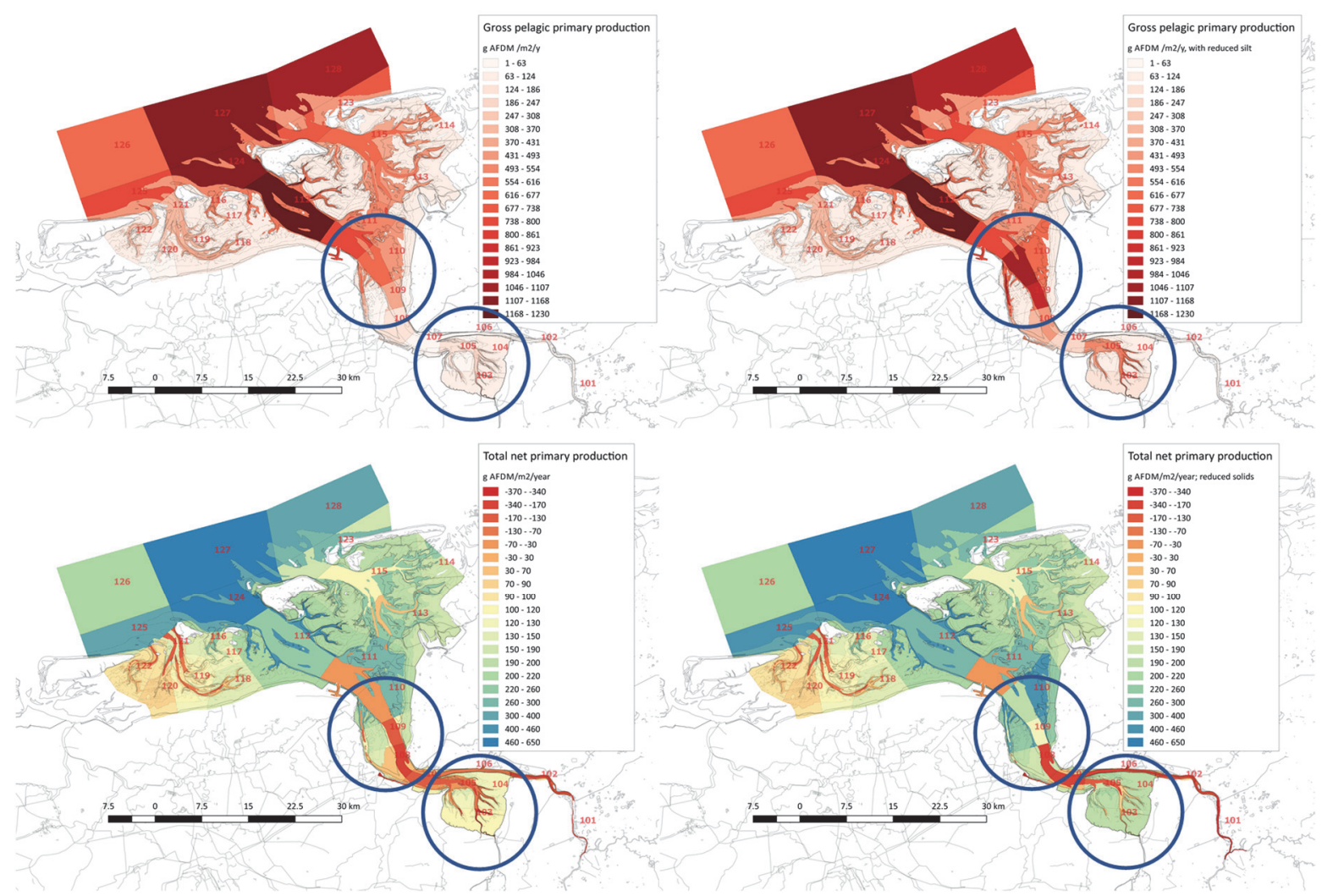

Figure 30 Spatial picture of gross (upper) and net (lower) primary production. Values in $g$ AFDM $m^{-2} y^{-1}$. Left: standard result, right: after the suspended matter reduction as described in Table 1. With circles, the areas with most changes are marked. 


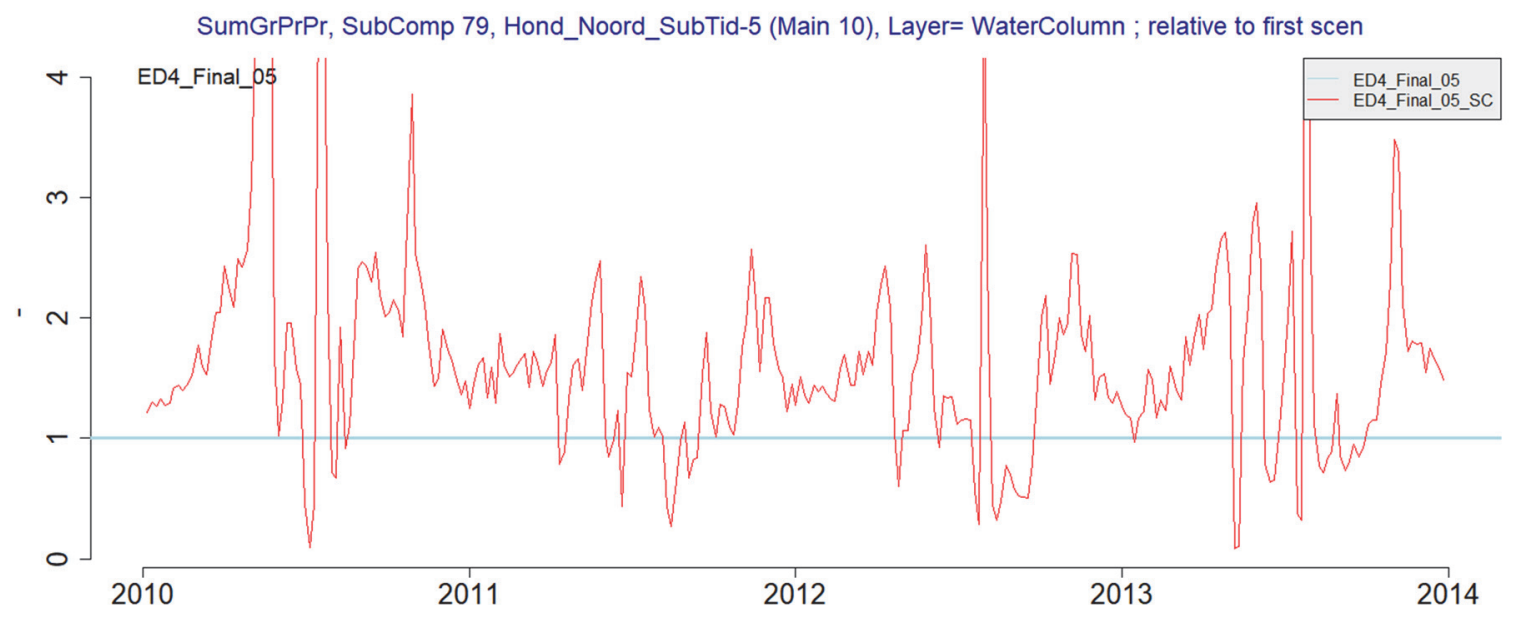

SumGrPrPr, SubComp 79, Hond_Noord_SubTid-5 (Main 10), Layer= WaterColumn ; relative to first scen

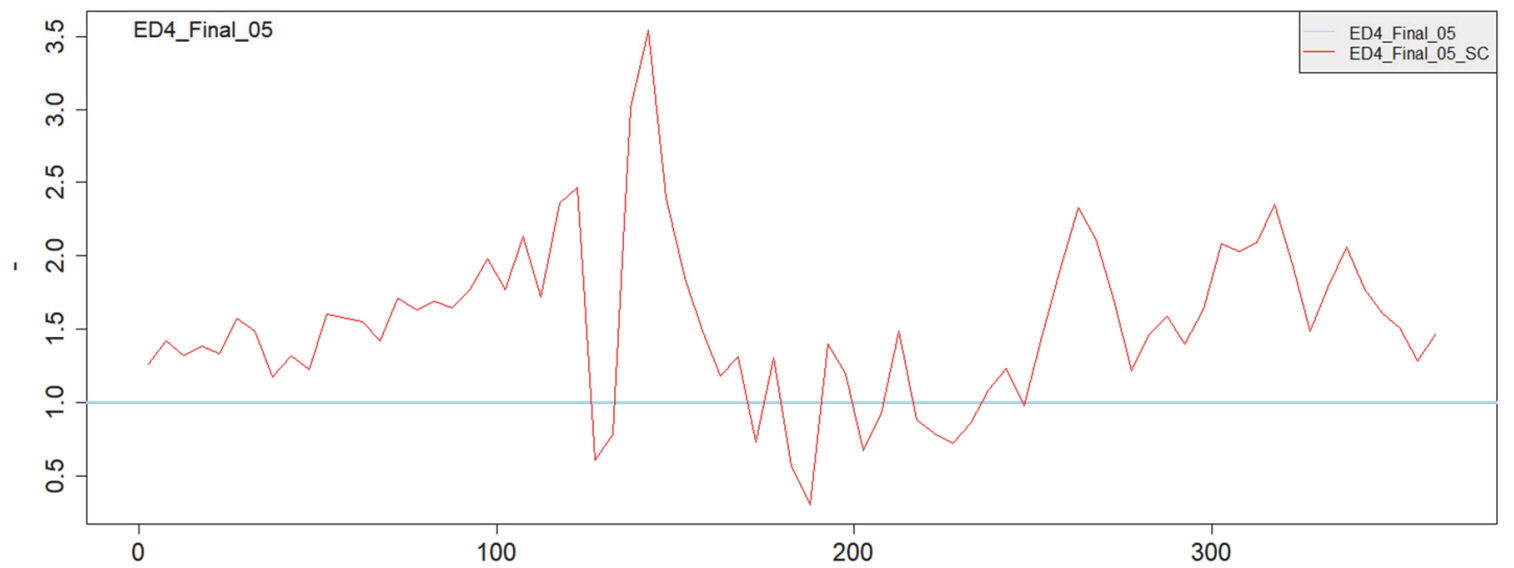

Figure 33 Relative changes in gross primary production in sub-compartment 79 (main compartment 10, area Hond-Noord). Blue line ( $=1.0)$ represents the standard situation (ED4 Final_05), the red line the relative changes. (ED4 Final_05_SC). In some parts of the year, the computed gross primary production gets lower a bit, but overall, the increase is obvious.

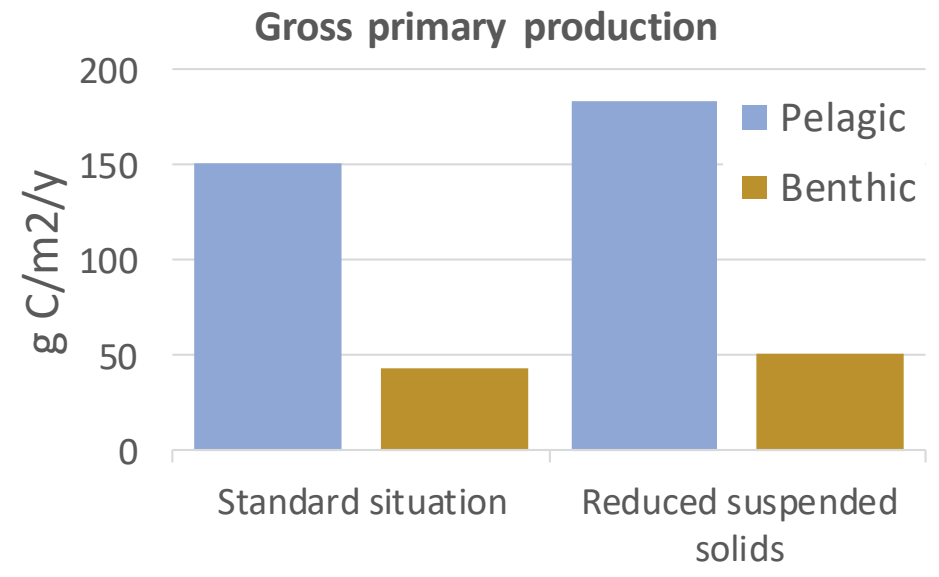

Figure 32 Gross primary production changes in the 'pure' Ems-Dollard estuary (Figure 4) after turbidity reduction (see Table 28)

However, when these changes are made explicit (Figure 33), one can see that locally really changes occurred. This is caused by the strong non-linear relationship between depth and light intensity. Since at lower light intensities gross primary production is almost linearly related with light, also gross primary production changes non-linearly with depth. So far, lower turbidity at least locally affects primary production substantially. 
The question is, what does this mean for the system as a whole? In Figure 32, this is pictured. Here, only the 'real' Ems-Dollard compartments have been considered (Figure 4), so results can be compared with other research results as well. The model computes an increase of the overall gross primary production. Also, total benthic primary production increases a bit; it most likely (not checked) to be caused by a small increase in benthic diatom presence (in the model, these also grow in the water column and thus benefit from the decreased turbidity).

Changes in dissolved components are less visible (Figure 34).

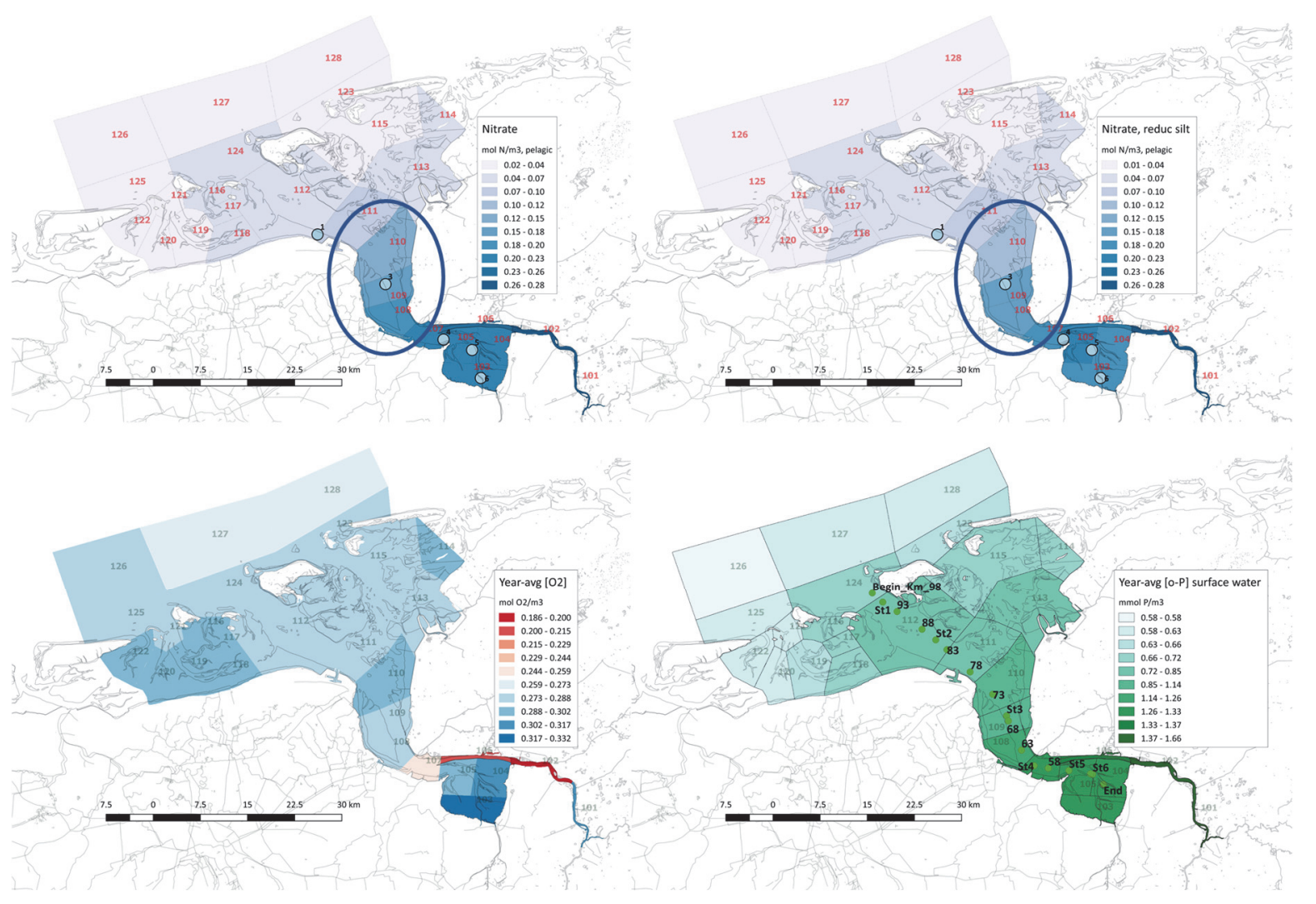

Figure 34 Spatial picture of nitrate (upper), oxygen (lower left) and orth-phosphate (lower right) average concentrations. Values in $\mathrm{mol} \mathrm{m}^{-3}$. For nitrate: left: standard result, right: after the suspended matter reduction as described in Table 1. With circles, the areas with most changes are marked. For both lower maps, differences were not visible.

Also, these differences can be made visible (Figure 35), showing that in the model computations indeed only nitrate changes in a negative way (down to about $80 \%$ ). The other three components hardly change.

The question here is, at the same tie, how reliable these results are? To answer that, one must have a look at the fit between monitoring data and model results. In appendix A25, many more figures are presented. Here, it is only shown in (Figure 36) that

I) For phosphorus, there was a problem finding a good fit between data and model, and this problem has not been solved yet.

II) For silicon, the fit between data and model is not very good, but still acceptable

III) For nitrate, too high values in summer are computed

Results for oxygen fit well with the observations. 

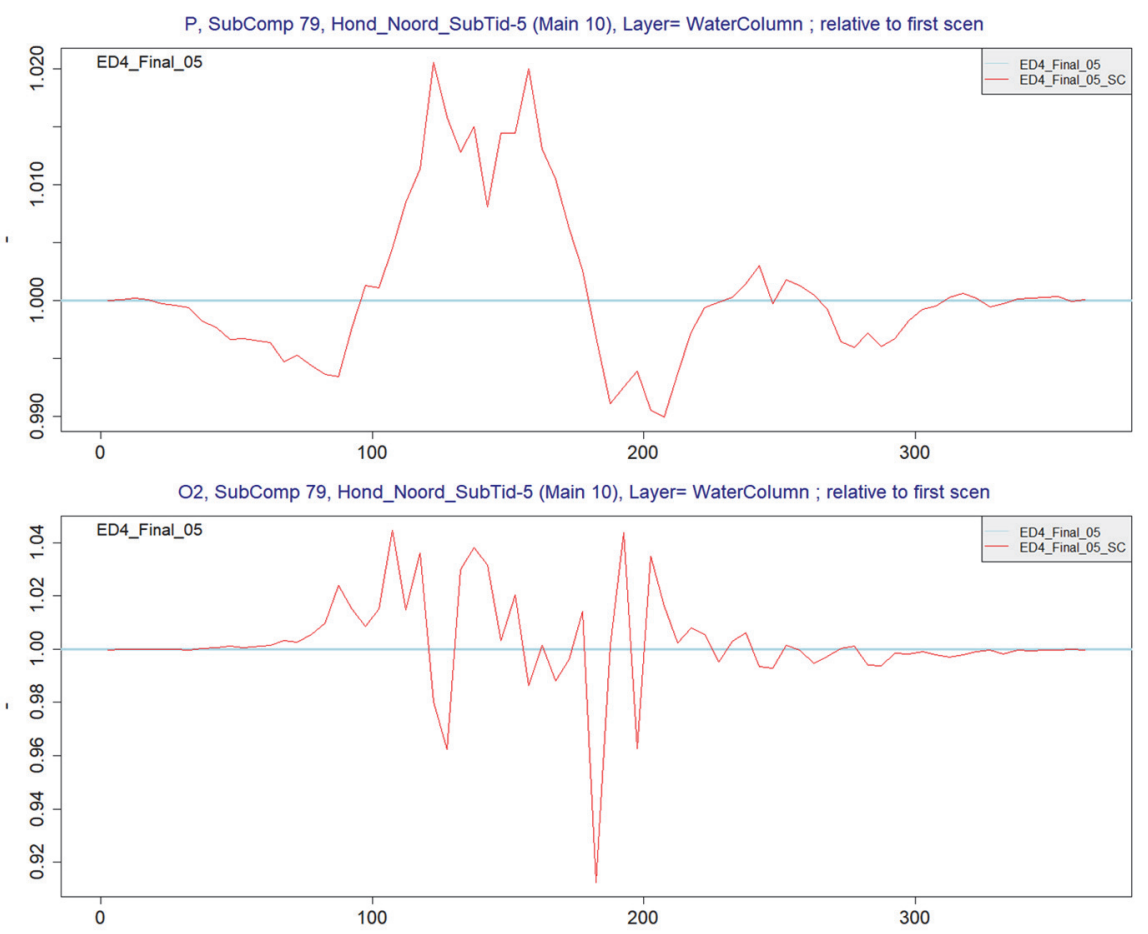

NO3, SubComp 79, Hond_Noord_SubTid-5 (Main 10), Layer= WaterColumn ; relative to first scen
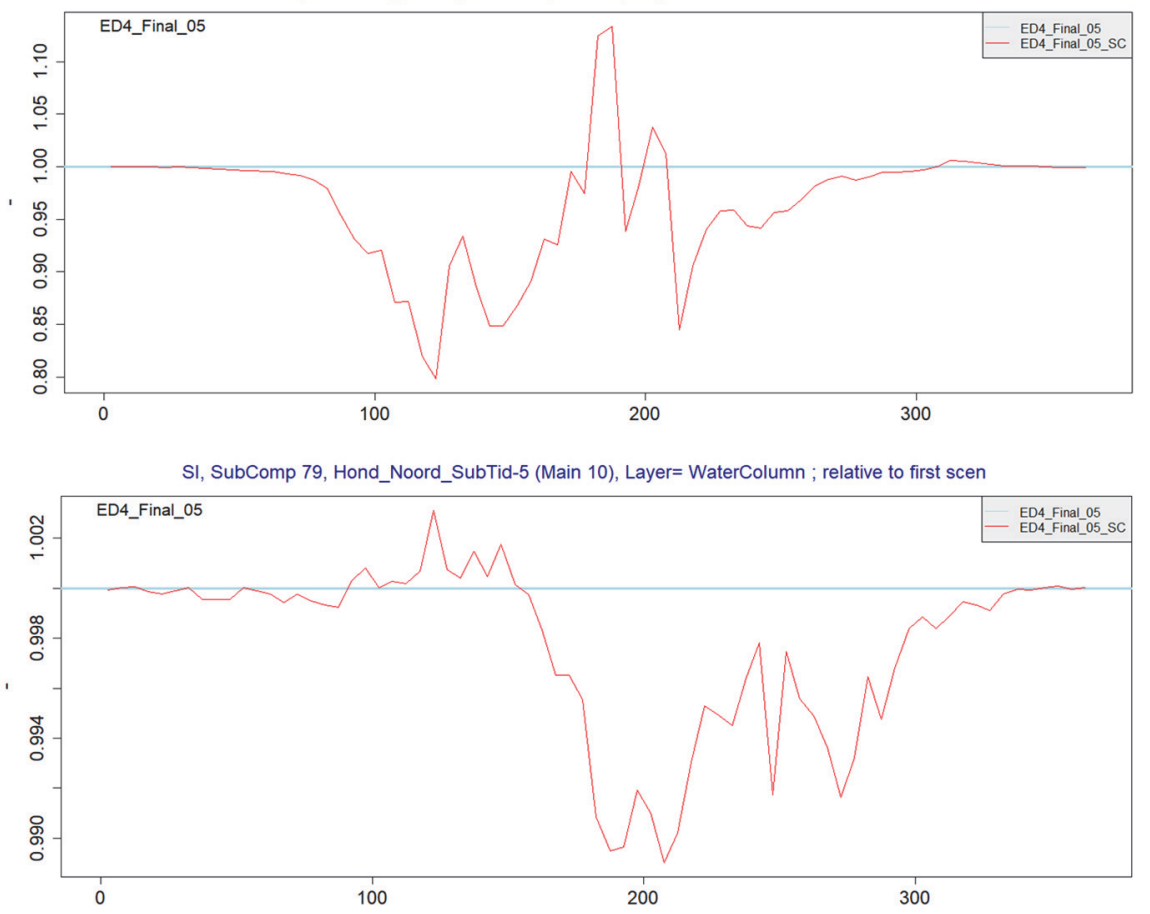

Figure 35 Relative changes in dissolved components in sub-compartment 79 (main compartment 10, area Hond-Noord), seasonal variations averaged for the period 2010-2013. Blue line $(=1.0)$ represents the standard situation (ED4 Final_05), the red line the relative changes. (ED4 Final_05_SC). Upper: orthophosphate, second: oxygen, third: nitrate, lower: silicate. All in $\mathrm{mol} \mathrm{m}^{-2}$.

This lack of fit between ortho-phosphate and nitrate model results and field data implies that the model needs improvement. 

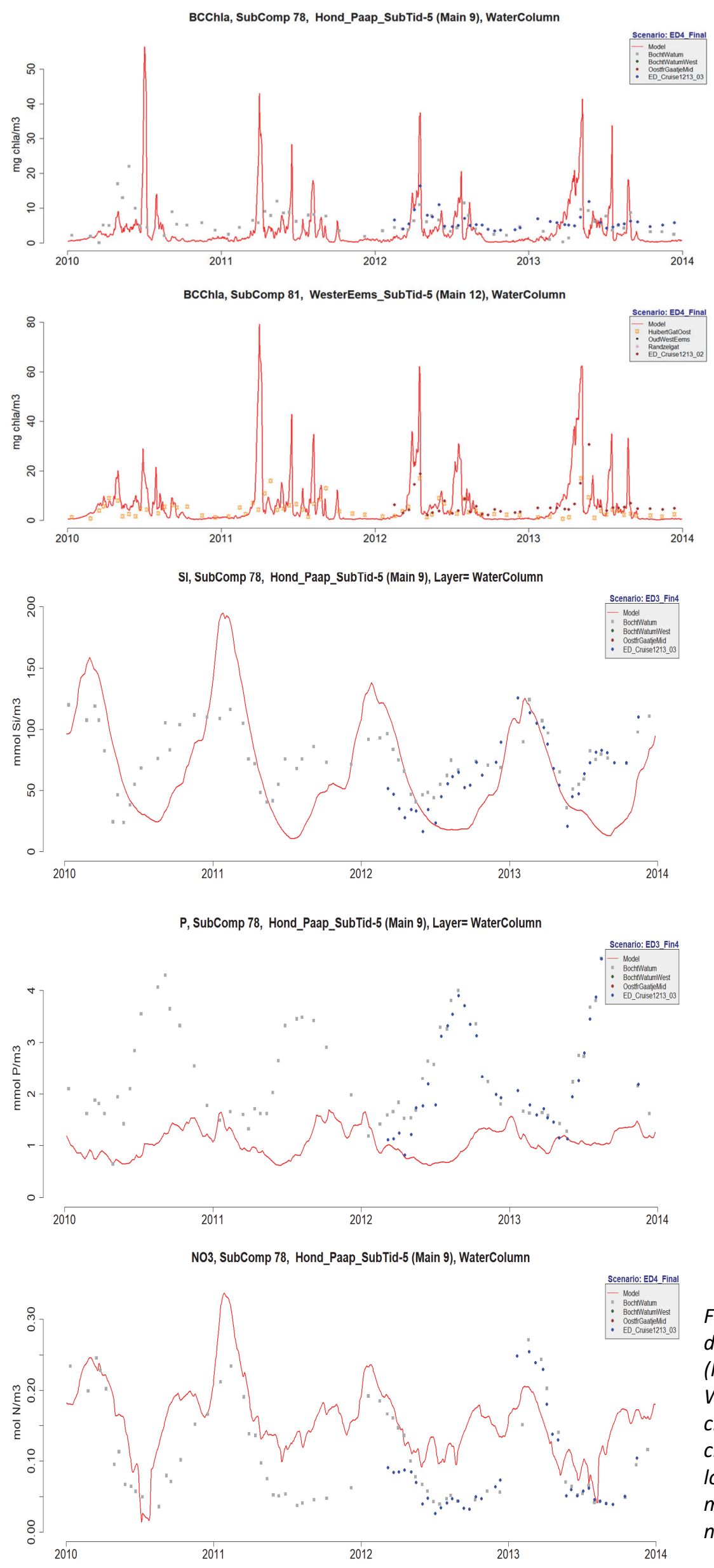

Figure 36 Model results and data for main compartment 9 (Hond \& Paap), and also WesterEms (second) for clorophyll. Upper two: chlorphyll, mid: Si, foruth: $P$, lower: nitrate. Chlorophyll in $\mathrm{mg} \mathrm{m}^{-3}$, Si and $\mathrm{P}$ in $\mathrm{mmol} \mathrm{m}^{-3}$, nitrate in $\mathrm{mol} \mathrm{m}^{-3}$. 
Finally, the question is what it all means for fauna in the system. Here, it is restricted to filter feeders ("Mussels") and sediment browsers ("Hydrobia"). In Figure 37, this is illustrated. Also, data are presented, showing that the model results are rather close to the observations, especially for sediment browsers. Filter feeder densities are somewhat different, but far from extreme. The negative aspect for filter feeders is that the model computes largest densities in gullies, while observation say this is on the tidal flats. This is a subject of concern, see the last chapter on 'possible improvements'.

Nevertheless, the model computes an increase of filter feeders ánd sediment browsers in those areas where reduction of suspended solids is assumed. Also, this extends to neighbour compartments, such as the Dollard.

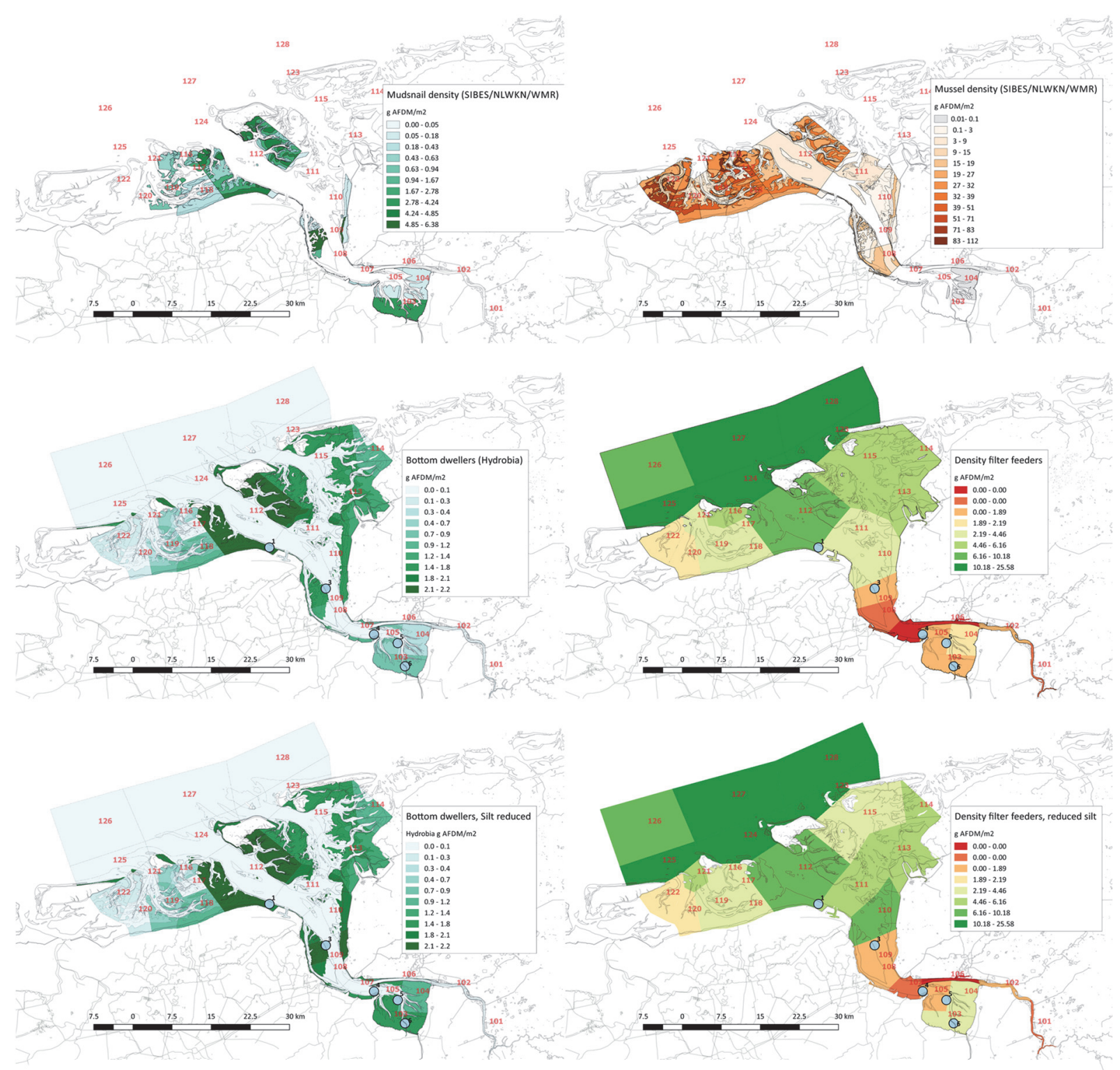

Figure 37 Spatial picture of filter feeders (right) abd sediment browsers (left). Upper: observations (SIBES/NLWKN/WMR; mid: standard situation; lower: after reduction of suspended solids according to Table 1. 


\section{Conclusions}

\subsection{Overall}

We presented a working ecosystem model with a relative simple physical background, but still capable of simulating important fauna characteristics, although several improvements are possible (and needed). It is capable of being used for studying effects of changed turbidity (and other possible changes affecting light or nutrient inputs). Major reason for implementing improvements is that the model appeared to have difficulties computing correct values for ortho-phosphate and nitrate, ánd places filter feeders in gullies where tidal flats are the spots where these animals are found. The reason for the latter deviations is clear: the model does not account for predation upon fauna blow low water line, nor accounts for the effect of suspended solids concentrations being much higher close to the sediment; thus, affecting filter feeding negatively.

Results for several system components however come close to observations; this is valid for sediment browsers, more or less for filter feeders, for gross primary production, oxygen, salinity, light attenuation and, to a somewhat lesser extend silicate, chlorophyll and filter feeders ("mussels"). Some problems arose for phosphate and nitrate. Results for benthic primary production and nitrification and denitrification seem to be in the order of magnitude, although data are too incomplete or even absent for a good comparison. Results for microzooplankton results were -although data were lacking- within the range previously found for the western Dutch Wadden Sea. For copepods, however, we failed to find a suitable parameter set.

A striking conclusion (see appendix A25.6), and probably this is also a reason for the increasing lack of fit for ortho-phosphate and nitrate the closer one comes to the Dollard area, is that the system has two faces. The outer part is governed by production: phytoplankton and its processes (including grazing upon algae) are most important, and ortho-phosphate has its maximum near the winter period and its minimum at the end of the algae bloom season. The inner part is mineralization driven: degradation of organic matter with highest rates when temperatures are highest, thus shortly after summer and top values for ortho-phosphate in early autumn. Nitrate consumption must be considerably larger than simulated now. The question then is: when does this organic matter come from? This is not known, although it might very well be transported inwards together with silt, a phenomenon characteristic for most estuaries. Also, this must be relatively fast degrading matter, since more humus-like substances (but slowly degrading) probably are sufficiently available since the hinterland of the area in The Netherlands and Germany is very peaty. It was, and partly still is.

Thus, what is really happening here is one of the topics to be incorporated into the model description.

\subsection{Most relevant improvements}

In appendix A27, several suggestions and possible improvements is listed. Most topics to be solved first are, according to the authors' opinion: 
i) to include the Lauwersmeer-input (that implies an extension of the model westward),

ii) to implement (==switch on and check) the computation of vertical suspended matter profiles,

iii) to improve the implementation of the effect of suspended solids on fauna,

iv) check the resuspension and distribution of benthic algae, and their activity when suspended (is their productivity similar then?)

v) check the reason of the discrepancy between computed and observed [P]

vi) check the reason of the discrepancy between computed and observed $\left[\mathrm{NO}_{3}\right]$

\subsection{All suggestions for model improvements}

Most of the sections in appendix A25 (and some mentioned in A26) have one or more suggestions for improvements. These are collected here, just as a list, without further explanation.

On boundaries:

- Most important improvement is to include the Lauwersmeer-outflow area (called the Zoutkamperlaag), and (thus) the outflow of fresh water from the Lauwersmeer.

On silt in the system

- Activate the computation of vertical profiles. This option is already present in the model, but not applied yet. Next, the distribution on the tidal flats between the Groninger shore and the islands of Schiermonnikoog and Rottumeroog may be improved. Finally, the German tidal flats now have lower suspended solids simulated than the Dutch 'counter'tidal flats. This does not seem plausible, thus must be improved also.

On extinction:

- The spatial distribution of lower and higher light attenuation coefficients must be improved (although differences certainly are not major): close to the Groningen north shore, and on the German tidal flats.

- Also, the attenuation coefficient, and the concentration of suspended solids, can be adjusted around monitoring site 3 in Figure 126.

On atmosphere/water exchange:

- Check the computed air/water exchange parameters with literature values

- Check the computed sediment/water exchange (not mentioned here) with literature values.

On adsorption of $\mathrm{P}$ and $\mathrm{Si}$ onto sediments

- $\quad$ Find better adsorption parameters

- Compare computed water sediment fluxes with available observations (from other parts of the Wadden Sea or even the North Sea coastal zone)

On phosphorus characteristics: 
- Find out what the cause is of the low [P]-values computed (especially in the inner compartments)

For silicate dynamics:

- Find better parameter values for frustule dissolution

- Find better time series for [Si] in tributaries

For nitrification and denitrification:

- Find better nitrification rate parameters (and probably also: descriptions of the process)

- Check why these results differ from the mentioned previous ones where the fit between data and model results were better.

- Check the computed sediment/water exchange (not mentioned here) with literature values.

For ammonium characteristics:

- Study the effect of ammonium adsorption

- Check further what might cause the overestimation of ammonium.

- Check sediment/water exchange rates with possibly available data

On pelagic chlorophyll:

- Chlorophyll in the Ems river needs better tuning

- Better tune the effect of salinity on freshwater and marine phytoplankton

Tuning both parts better probably will also improve the chlorophyll simulation results for main compartments 107-109 (numbers mentioned in Figure 157), now results are far below observations. These improvements are really needed since these are target compartments for all attempts to improve water transparency.

One other possible improvement is

- Model the chlorophyll content of the phytoplankton. Thus, production rates per unit of biomass change during the season and depend on external conditions. This asks for new descriptions in the model.

On benthic chlorophyll

- Check the reason of the very small differences between the three tidal parts in each main compartment

- Make early spring and late autumn values more like summer values

- Include other fauna that also grazes upon phytobenthos (e.g. worms and/or shellfish).

- check the resuspension and distribution of benthic algae.

On primary production:

- Better check primary productivity data from the Riegman et al research (Brinkman et al, 2015) with the model results here.

- check the activity of suspended benthic algae: is their productivity similar then?

On macrofauna: 
- Make better overviews of the observations of the benthic fauna (then now has been done by the author): take care of the non-normal distributions of animal numbers in the samples. Possibly a Poisson-like distribution analysis is suited better. Also, the method of stratified sampling by IMARES/WMR should better be taken into consideration, this is to use the several areas that are supposed to be representative for the samples taken instead of one uniform size.

- Do compute and check better the relationship between suspended matter and filtration characteristics. The material necessary is present: see appendix A24.

On oxygen and nitrate penetration depths:

- Check computed values with possibly available data (not searched for yet)

- Check results for mineralization rates with possibly available data

On nitrification and denitrification:

- Check the parameters chosen for nitrification and denitrification. Better tuning must be possible. 


\section{References}

Aalderink RH, Lijklema L, Breukelman J, Van Raaphorst W \& Brinkman AG. 1984. Quantification of wind induced resuspension in a shallow lake. Wat. Sci. Tech. 17: 903-914.

Baretta J \& Ruardij P. 1988. Tidal flat estuaries. Springer Verlag. Berlin.353 pp.

Barillé L, Prou J, Héral M, Bourgrier S (1993) No influence of food quality, but ration-dependent retention efficiencies in the Japanese oyster Crassostrea gigas. J Exp Mar Bio Ecol 171:91-106

Barillé L, Prou J, Héral M, Razet D (1997) Effects of high natural seston concentrations on the feeding, selection, and absorption of the oyster Crassostrea gigas (Thunberg). J Exp Mar Bio Ecol 212:149172

Bayne BL, Hawkins AJS, Navarro E (1987) Feeding and digestion by the mussel Mytilus edulis L . ( Bivalvia : Mollusca ) in mixtures of silt and algal cells at low concentrations. J Exp Mar Biol Ecol Ill:1-22

Bayne BL, Hawkins AJS, Navarro E, Iglesias IP (1989) Effects of seston concentration on feeding, digestion and growth in the mussel Mytilus edulis. Mar Ecol Prog Ser 55:47-54

Bird RB, Stewart WE \& Lightfoot EN. 1960 . Transport Phenomena. John Wiley \& Sons, Inc. NY. 780 pp.

Breeuwsma A. 1973. Adsorption of ions on hematite $\left(a-\mathrm{Fe}_{2} \mathrm{O}_{3}\right)$-a colloid chemical study-. PhD thesis. Agricult. Univ. Wageningen

Brinkman AG \& Van Raaphorst W. 1986. De fosfaathuishouding in het Veluwemeer. PhD-thesis. Twente University of Technology, $700 \mathrm{pp}$.

Brinkman AG, $1993^{\mathrm{a}}$. A double-layer model for ion adsorption onto metal oxides, applied to experimental data and to natural sediments of Lake Veluwe, The Netherlands. Hydrobiologia 253: 31-45.

Brinkman AG. 1993 ${ }^{\text {b }}$. Biological processes in the EcoWasp ecosystem model. IBN Research Report 93/6. 111 pp.

Brinkman AG \& Smit JPC. 1993. Porewater profiles in the EcoWasp ecosystem model. IBN Research Report 93/2. $62 \mathrm{pp}$.

Brinkman AG, Dankers NMJ \& Van Stralen MR. 2002 An analysis of mussel bed habitats in the Dutch Wadden Sea. Helgol. Marine Research

Brinkman AG, Bult T. 2002 Geschikte eulitorale gebieden in de Nederlandse Waddenzee voor het ontstaan van stabiele natuurlijke mosselbanken. Alterra rapport 456

Brinkman AG, Smaal AC. 2003 Onttrekking en natuurlijke productie van schelpdieren in de Nederlandse Waddenzee in de periode 1976-1999 Alterra/RIVO. Alterra-rapport 888, 247 pp

Brinkman AG. 2008. Nutriënt- en chlorofylgehaltes in het westelijke en oostelijke deel van de Nederlandse Waddenzee; waarden en trends tussen 1980 en 2005 en mogelijke oorzaken daarvan. Wageningen IMARES/Texel Rapport C112/08

Brinkman AG, 2013. Modelling the effects of mussel seed collectors on the western Dutch Wadden Sea ecosystem. IMARES Wageningen UR Report number C061/13

Brinkman AG, Riegman R, Jacobs P, Kuhn S \& Meijboom A. 2014. Ems-Dollard primary production research. Full data report. Report IMARES/WUR C160/14. 297 pp. 
Brinkman AG, Jacobs P, Jak RG \& Riegman R. 2015. Ems-Dollard primary production research. Concise summary. Report IMARES/WUR C163/14. 78 pp.

Brinkman AG. 2015. Ecosysteemeffecten van lokaal veranderde slibgehaltes in de westelijke Waddenzee. IMARES Rapport C157/15

Brinkman AG. 2015. Blueprint for an Ems-Dollard ecosystem model study. IMARES Report C158/15

Bruns R. 1994 . Benthische Primärproduction, Remineralisation und Nitrifikation und der Austausch anorganischer Stickstoffverbindungen zwischen Sediment und Wasser im Nordsylter Wattenmeer. PhD-Thesis CAU-Universität Kiel. 133 p.

Brussaard CPD, Riegman R, Noordeloos AAM, Cadee GC, Witte H, Kop AJ, Nieuwland G, Van Duyl FC \& Bak RPM. 1995. Effects of grazing, sedimentation and phytoplankton cell lysis on the structure of a coastal pelagic food web. Mar Ecol Prog Ser. 123:259-271

Brussaard CPD, Gast GJ, Van Duyl FC \& Riegman R. 1996. Impact of phytoplankton bloom magnitude on a pelagic microbial food web. Mar Ecol Prog Ser. 144: 211-221

Buschbaum C. 2000. Direct and indirect effects of Littorina littorea (L.) on barnacles growing on mussel beds in the Wadden Sea. Hydrobiologia 440: 119-128

Buschbaum C. 2001. Siedlungsmuster und Wechselbeziehungen von Seepocken (Cirripedia) auf Muschelbänken (Mytilus edulis L.) im Wattenmeer. PhD Thesis Universität Hamburg

Camponelli K (2001) “Ensis directus” (On-line), Animal Diversity Web.

Colijn F. 1983 Primary production in the Ems-Dollard estuary. Ph. D. Thesis, Groningen, The Netherlands, 123 $\mathrm{pp}$

Colijn F \& De Jonge VN. 1984. Primary production of microphytobenthos in the Ems-Dollard Estuary. Marine Ecology Progress Series, 14, 185-196. doi: 10.3354/meps014185.

Compton TJ, Holthuijsen S, Koolhaas A, Dekinga A, Ten Horn J, Smith J, Galama Y, Brugge M, Van der Wal D Van der Meer JVan der Veer HW \& Piersma Th. 2013. Distinctly variable mudscapes: Distribution gradients of intertidal macrofauna across the Dutch Wadden Sea. J. Sea Res. 82: 103-116

Cranford PJ, Ward JE, Shumway SE (2011) Filter Feeding: Variability and Limits of the Aquaculture Biofilter. In: Shumway SE (ed) Shellfish Aquaculture and the Environment. Wiley-Blackwell, p 81-124

De Boer WF. 2000. Ontwikkeling van de biomassa van het microfytobenthos in het Eems-Dollard estuarium in de periode 1992-1999. Koeman en bijkerk, rapport 2000-31

De Jonge VN. 1992. Physical processes and dynamics of microphytobenthos in the Ems estuary (The Netherlands). PhD. Thesis, University of Groningen, 176 pp.

De Jonge VN and Van Beusekom JEE. 1992. Contribution of resuspended microphytobenthos to total phytoplankton in the Ems estuary and its possible role for grazers, Netherlands Journal of Sea Research, 30, 91-105

De Jonge VN \& Colijn F. 1994. Dynamics of microphytobenthos biomass in the Ems estuary. Mar.Ecol. Prog. Ser. 104: 185-196

De Jonge VN, De Boer WF, De Jong DJ \& Brauer VS. 2012. Long-term mean annual microphytobenthos chlorophyll a variation correlates with air temperature. Mar Ecol Progr Ser 468: 43-56 
De Kluijver A, Brinkman AG, Van Duren LA \& Fey F. 2014. Effecten van slib op de ecologische doelen van de KRW Primaire productie, zeegras en bodemdieren in de Waddenzee. Rapport Deltares 1209473-000.

EON-I. 1988. Ecosysteemmodel van de westelijke Waddenzee. Nederlands Instituut voor Onderzoek der Zee, NIOZ-rapport 1988-1

EON-II. 1988. The ecosystem of the western Wadden Sea: field research and mathematical modelling. Nederlands Instituut voor Onderzoek der Zee, EMOWAD-II. NIOZ-rapport 1988-11

Essink K, Bos A. (1985) Growth of three bivalve molluscs transplanted along the axis of the Ems estuary. Netherlands J Sea Res 19:45-51

Essink K, Steyaert FHIM, Mulder HPJ, Jonge VN De, Heuvel T Van, Bergs J Van den (1992) Effects of dredging activities in the Ems estuary and Dutch Wadden sea. Netherlands Institute Sea Res 20

Essink K, Eppinga J \& Dekker R. 1998. Long-term changes (1977-1994) in intertidal macrozoobenthos of the Dollard (Ems estuary) and effects of introduction of the North American Spionid polychaete Marenzellaria cf. wireni. Senckenbergiana maritima 28-211-223

Essink K (1999) Ecological effects of dumping of dredged sediments; options for management. J Coast Conserv 5:69-80

Essink K. 2009. Stomvloed 1509; Geschiedenis van de Dollard. Lezing voor Ubbo Emmius Gymnasium te Leer (Ostfriesland). http://www.verdronkengeschiedenis.nl/nl/stormvloed/docs/12-EssinkDe\%20Dollard-NL-1-opmaak-def.pdf

Evers CHM \& Knoben RAE (2007), Omschrijving MEP en maatlatten voor sloten en kanalen voor de Kaderrichtlijn Water, Utrecht: STOWA.

Foster-Smith RL (1975) The effect of concentration of suspension on the filtration rates and pseudofaecal production for Mytilus edulis L., Cerastoderma edule (L.) and Venerupis pullastra (Montagu). J Exp Mar Bio Ecol 17:1-22

Gittenberger A, Loon W Van (2013) Sensitivities of marine macrozoobenthos to environmental pressures in The Netherlands. Ned Faun Meded 43

Hawkins A, Smith R, Bayne B, Héral M (1996) Novel observations underlying the fast growth of suspensionfeeding shellfish in turbid environments:Mytilus edulis. Mar Ecol Prog Ser 131:179-190

Hawkins AJS, Bayne BL, Bougrier S, Héral M, Iglesias JIP, Navarro E, Smith RFM, Urrutia MB (1998) Some general relationships in comparing the feeding physiology of suspension-feeding bivalve molluscs. J Exp Mar Bio Ecol 219:87-103

Helder W. 1983. Aspects of the nitrogen cycle in Wadden Sea and Ems-Dollard Estuary with emphasis on nitrification. PhD-Thesis RUU Groningen.

Herman PMJ, Middelburg JJ, Van de Koppel J, Heip CHR. 1999. Ecology of etuarine macrobenthos. Advances in Ecological Research 29: 195-240

Hunze en Aa's \& Gemeente Slochteren. 2008. Watersysteemplan Duurswold. Eindconcept. Veendam

Hughes JR (2008) Crassostrea gigas Pacific oyster. In Tyler-Walters H. and Hiscock K. (eds) Marine Life Information Network: Biology and Sensitivity Key Information Reviews, [on-line].

Iglesias JIP, Navarro E, Alvarez Jorna P, Armentina I (1992) Feeding, particle selection and absorption in cockles Cerastoderma edule (L.) exposed to variable conditions of food concentration and quality. J Exp Mar Bio Ecol 162:177-198

Iglesias JIP, Urrutia MB, Navarro E, Alvarez-Jorna P, Larretxea X, Bougrier S, Heral M (1996) Variability of feeding processes in the cockle Cerastoderma edule (L.) in response to changes in seston concentration and composition. J Exp Mar Bio Ecol 197:121-143 
Jak RG, Kaag NHBM, Schobben HPM, Scholten MCT, Karman CC, Schobben JHM (2000) RAM Kwantitatieve dosis-effect relaties.

Jørgensen CB (1996) Bivalve filter feeding revisited. Mar Ecol Prog Ser 142:287-302

Kamermans P, Brummelhuis E, Wijsman J (2011) First pioneering laboratory experiments on filtration, respiration and growth of the razor clam (Ensis directus, Conrad).

Kamermans P, Brummelhuis E, Dedert M (2013) Effect of algae- and silt concentration on clearance- and growth rate of the razor clam Ensis directus, Conrad. J Exp Mar Bio Ecol 446:102-109

Karlsson Ö, Jonsson PR, Larsson AI (2003) Do large seston particles contribute to the diet of the bivalve Cerastoderma edule? Mar Ecol Prog Ser 261:161-173

Kernighan, Brian W.; Ritchie, Dennis M. (February 1978). The C Programming Language (1st ed.). Englewood Cliffs, NJ: Prentice Hall. ISBN 0-13-110163-3.

Kieskamp WM (1994) On nitrous oxide and denitrication in marine sediments with emphasis on intertidal sediments. PhD Thesis Rijskuniversiteit Groningen 169 pp.

Kieskamp WM, Lohse L, Epping E \& Helder W. 1991. Seasonal variation in denitrification rates and nitrous oxide fluxes in intertidal sediments of the western Wadden Sea. Mar. Ecol. Progr. Ser. 72: 145-151

Kiorboe T, Mohlenberg F, Nohr O (1980) Feeding, particle selection and carbon absorption in Mytilus edulis in different mixtures of algae and resuspended bottom material. Ophelia 19:193-205

Kiørboe T, Møhlenberg F, Nøhr O (1981) Effect of suspended bottom material on growth and energetics in Mytilus edulis. Mar Biol 61:283-288

Kohlmeier C. 2004. Modellierung des Spiekerooger Rückseitenwatts mit einem gekoppelten Euler-LagrangeModell auf der Basis von ERSEM. PhD_DIss Carl v Ossietzky Universität Oldenburg.

Kuiper K \& Brinkman AG. 2015. Antropogene bodemberoering, Verkenning Slibhuishouding Waddenzee. IMARES/Deltares. Deltares-nr 1220102-000.

Lannou A, Geairon P, Bougrier S, Raillard O, Heral M, Ra I, Vilar J, Lec I (1992) Effects of the feeding behaviour of Crassostrea gigas (Bivalve Molluscs) on biosedimentation of natural particulate matter. Hydrobiologia 231:85-91

Lick W. 1982. Entrainment, Deposition and Transport of Fine Grained Sediments in Lakes. Hydrobiologia (91), $31-40$

Lohse L. 1996. Sediment-water exchange of nitrogen compounds and oxygen in the North Sea. PhD-diss Rijksuniversiteit Groningen, 86pp

Mainwaring K, Tillin H, Tyler-Walters $\mathrm{H}$ (2014) Assessing the sensitivity of blue mussel beds to pressures associated with human activities. Jt Nat Conserv Committe

Marine Institute (2013) Tools for Appropriate Assessment of Fishing and Aquaculture Activities in Marine and Coastal Natura 2000 Sites Report I : Intertidal and subtidal muds.

Møhlenberg F, Riisgård HU (1978) Efficiency of particle retention in 13 species of suspension feeding bivalves. Ophelia 17:239-246

Navarro E, Iglesias JIP, Ortega MM (1992) Natural sediment as a food source for the cockle Cerastoderma edule (L.): effect of variable particle concentration on feeding, digestion and the scope for growth. J Exp Mar Bio Ecol 156:69-87

Navarro E, Iglesias JIP, Ortega MM, Larretxea X (1994) The Basis for a Functional Response to Variable Food Quantity and Quality in Cockles Cerastoderma edule (Bivalvia, Cardiidae). Physiol Zool 67:468-496

Navarro JM, Widdows J (1997) Feeding physiology of Cerastoderma edule in response to a wide range of seston concentrations. Mar Ecol Prog Ser 152:175-186

Navarro E, Méndez S, Urrutia MB, Arambalza U, Ibarrola I (2016) Digestive selection underlies differential utilization of phytoplankton and sedimentary organics by infaunal bivalves: Experiments with cockles (Cerastoderma edule) using cross-labelled mixed diets. Mar Environ Res 120:111-121 
Newell CR, Wildish DJ, MacDonald BA (2001) The effects of velocity and seston concentration on the exhalant siphon area, valve gape and filtration rate of the mussel Mytilus edulis. J Exp Mar Bio Ecol 262:91-111

Pizzolla PF (2002) Scrobicularia plana Peppery furrow shell. In Tyler-Walters H. and Hiscock K. (eds) Marine Life Information Network: Biology and Sensitivity Key Information Reviews, [on-line].

Postma H. 1961. Transport and accumulation of suspended matter in the Dutch Wadden Sea. Neth. J. Sea Res. 1:148-190

Prins TC, Dankers N, Smaal AC (1994) Seasonal variation in the filtration rates of a semi-natural mussel bed in relation to seston composition 1. J Exp Mar Bio Ecol 176:69-86

R Core Team, 2016. R: A language and environment for statistical computing. R Foundation for Statistical Computing, Vienna, Austria. ISBN 3-900051-07-0, URL http://www.R-project.org/.

R (2016): rdgal package. https://cran.r-project.org/web/packages/rgdal/rgdal.pdf

Ren JS, Ross AH, Schiel DR (2000) Functional descriptions of feeding and energetics of the Pacific oyster Crassostrea gigas in New Zealand. 208:119-130

Rena JS, Ross a H (2001) A dynamic energy budget model of the Pacific oyster Crassostrea gigas. Ecol Modell 142:105-120

Riisgård HU, Funch P, Larsen PS (2015) The mussel filter-pump - present understanding, with a reexamination of gill preparations. Acta Zool 96:273-282

RIKZ (1998) Sedimentatlas Waddenzee. Ministerie van Verkeer en Waterstaat/ Rijksinstituut voor Kust en Zee (RIKZ) Haren, CD ROM.

RWS, 2015. Rijkswaterstaat, http://live.waterbase.nl

Saier B. 2001. Direct and indirect effects of seastars Asterias rubens on mussel beds (Mytilus edulis) in the Wadden Sea. J. Sea. Res 46: 29-42

Saier B. 2001. Ecological comparison of intertidal and subtidal mussel beds (Mytilus edulis L.) in the Wadden Sea. PhD thesis University of Hamburg 164 pp.

Saier B \& Buschbaum C. 2001. Growth of the mussel Mytilus edulis L. in the Wadden Sea affected by tidal emergence and barnacle epibionts. J. Sea Res. 45: 27-36

Saier, B, C. Buschbaum \& K. Reise. 2002 Subtidal mussel beds in the Wadden Sea: threatened oases of biodiversity. . Wadden Sea News Letter 25 (1)

Saraiva S, Meer J van der, Kooijman SALM, Sousa T (2011) Modelling feeding processes in bivalves: A mechanistic approach. Ecol Modell 222:514-523

Sigg L. 1979 Die Wechselwirkung von Anionen und schwachen Saeuren mit a-FeOOH (Goethit) in wässriger Lösung. PhD thesis Eidgenoessischen Technischen Hochschule Zuerich

Smaal AC, Zurburg W (1997) The uptake and release of suspended and dissolved material by oysters and mussels in Marennes-Oléron Bay. Aquut Living Resour 10:23-30

Smaal AC, Brinkman AG, Schellekens T, Jansen J, Agüera A \& Van Stralen MR. 2014. Ontwikkeling en stabiliteit van sublitorale mosselbanken, Samenvattend eindrapport. IMARES WUR Rapport c066.14

Sornin JM, Deslous-Paoli JM, Hesse O (1988) Experimental study of the filtration of clays by the oyster Crassostrea gigas (Thunberg): Adjustment of particle size for best retention. Aquaculture 69:355366 
Taal MD, Schmidt CA, Brinkman AG, Stolte W \& Van Maren DS. 2015. Slib en primaire productie in het Eemsestuarium Een samenvatting van vier jaar meten, modelleren, kennis bundelen en verwerven. Deltares, IMARES \& Rijkswaterstaat.

Talke SA, Swart HE de, Schuttelaars HM (2009) Feedback between residual circulations and sediment distribution in highly turbid estuaries: An analytical model. Cont Shelf Res 29:119-135

Tyler-Walters H (2007) Cerastoderma edule Common cockle. In Tyler-Walters H. and Hiscock K. (eds) Marine Life Information Network: Biology and Sensitivity Key Information Reviews, [on-line].

Tyler-Walters H (2008) Mytilus edulis Common mussel. In Tyler-Walters H. and Hiscock K. (eds) Marine Life Information Network: Biology and Sensitivity Key Information Reviews, [on-line].

Urrutia MB, Iglesias JIP, Navarro E (1997) Feeding behaviour of Cerastoderma edule in a turbid environment: Physiological adaptations and derived benefit. Hydrobiologia 355:173-180

Van den Ende D, Troost K, Van Stralen M, Van Zweeden C, Van Asch, M. 2012. Het mosselbestand en het areaal aan mosselbanken op de droogvallende platen van de Waddenzee in het voorjaar van 2012. IMARES Wageningen UR, rapport C149/12

Van der Laan DJ. 2017. Rijksdriehoek.R. https://github.com/djvanderlaan/rijksdriehoek/blob/master/R/rijksdriehoek.R

Van der Molen DT \& Pot R (2007a), Referenties en maatlatten voor natuurlijke wateren voor de Kaderrichtlijn Water, Amersfoort: STOWA.

Van der Molen DT \& Pot R (2007b) Referenties en maatlatten voor natuurlijke watertypen voor de Kaderrichtlijn Water. Aanvullingen kleine typen, Amersfoort: STOWA.

Van Liere, E \& Jonkers DA. 2002. Watertypegerichte normstelling voor nutriënten in oppervlaktewater. RIVM-rapport 03715005/2002.

Wanink JH, Bijker R \& Brochard CJE. 2014. Phytoplankton of the Ems-Dollard estuary 2013. Koeman en Bijkerk BV. Report 2014-015.

Ward JE, Macdonald LBA, Thompson RJ, Beninger PG (1993) Mechanisms of suspension feeding in bivalves: Resolution of current controversies by means of endoscopy. Limnol Ocean 38:265-272

Ward JE, Shumway SE (2004) Separating the grain from the chaff: Particle selection in suspension- and deposit-feeding bivalves. J Exp Mar Bio Ecol 300:83-130

Widdows J, Fieth P, Worrall CM (1979) Relationships between seston, available food and feeding activity in the common mussel Mytilus edulis. Mar Biol 50:195-207

Wijsman J, Dedert M, Schellekens T, Teal L, Kruchten Y van (2012) Adaptive Monitoring Strategies in dredging ; Case Study Mussels - Modeling the effect of dredging on filter-feeding bivalves.

Wilber DH, Clarke DG (2001) Biological Effects of Suspended Sediments: A Review of Suspended Sediment Impacts on Fish and Shellfish with Relation to Dredging Activities in Estuaries. North Am J Fish Manag 21:855-875

Zemlys P, Daunys D, Razinkovas A (2003) Revision pre-ingestive selection efficiency definition for suspension feeding bivalves: Facilitating the material fluxes modelling. Ecol Modell 166:67-74

Zwarts L. 2004. Bodemgesteldheid en mechanische kokkelvisserij in de Waddenzee. Rapport RIZA/2004.028. ISBN 9036956862. Rijkswaterstaat/RIZA. 


\section{Justification}

Report $\quad: C 058 / 17$

WMR project nr: WMR 4318100124

The scientific quality of this report has been peer reviewed by a colleague scientist and a member of the Management Team of Wageningen Marine Research

Approved $\quad$ : Dr. D Gerla Scientist modelling Wageningen Marine Research

Signature

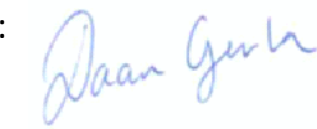

Date $\quad: 2018$ April 20

Approved : Drs. J. Asjes

Manegement Team Wageningen Marine Research

Signature

Date

: 2018 April 20 
Wageningen Marine Research

$T+31(0) 317480900$

E: marine-research@wur.nl

www.wur.eu/marine-research

Visitors' address

- $\quad$ Ankerpark 271781 AG Den Helder

- $\quad$ Korringaweg 5, 4401 NT Yerseke

- $\quad$ Haringkade 1, 1976 CP IJmuiden
Wageningen Marine Research is the Netherlands research institute established to provide the scientific support that is essential for developing policies and innovation in respect of the marine environment, fishery activities, aquaculture and the maritime sector.

\section{Wageningen University \& Research:}

is specialised in the domain of healthy food and living environment.

The Wageningen Marine Research vision

'To explore the potential of marine nature to improve the quality of life'

The Wageningen Marine Research mission

- $\quad$ To conduct research with the aim of acquiring knowledge and offering advice on the sustainable management and use of marine and coastal areas.

- Wageningen Marine Research is an independent, leading scientific research institute

Wageningen Marine Research is part of the international knowledge organisation Wageningen UR (University \& Research centre). Within Wageningen UR, nine specialised research institutes of the Stichting Wageningen Research Foundation have joined forces with Wageningen University to help answer the most important questions in the domain of healthy food and living environment. 


\section{APPENDICES}


74 of 286 | Wageningen Marine Research report C058/17 


\section{A1 Compartment setup and sub-compartments}

All compartments have a 'Main'-number (Figure 38), and a sub-compartments number (Table 2).

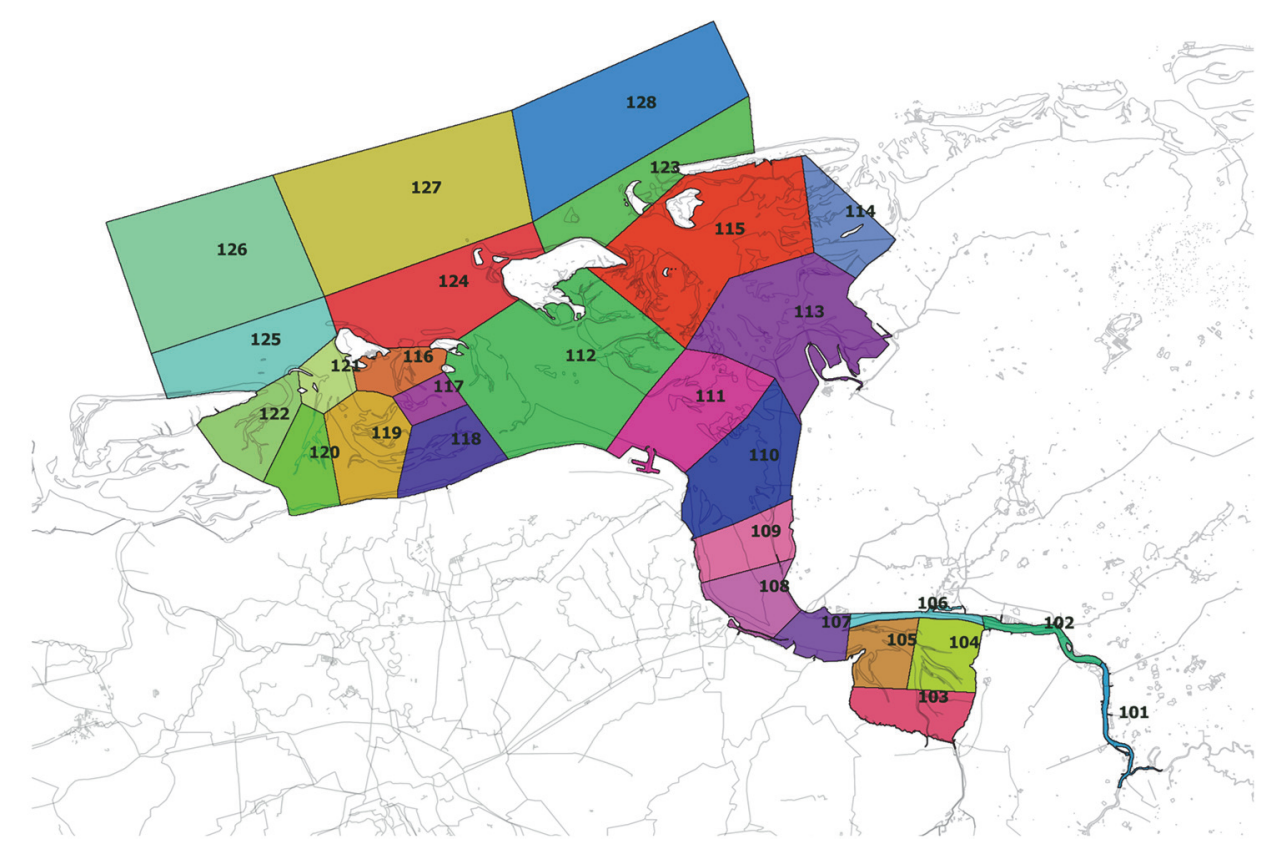

Figure 38 Numbering of main compartments in the model area. Note that in most cases, numbering is without the 100's (so: 1-23, instead of 101-123) 
Table 2 Numbering of all sub-compartments (tidal 1..3, subtidal and channels) of the main compartments (MainComp) (see Figure 38). The name of the main compartment is mentioned, and the sub-compartments are named CompName_tidal_1,..., CompName_channel.

\begin{tabular}{|c|c|c|c|c|c|c|}
\hline MainComp & Tidal_1 & Tidal_2 & Tidal_3 & Subtidal & Channel & CompName \\
\hline 1 & & & & 70 & 97 & Ems_Leer \\
\hline 2 & 1 & 24 & 47 & 71 & 98 & Ems_Gardersum \\
\hline 3 & 2 & 25 & 48 & 72 & & Dollard_NwStatzijl \\
\hline 4 & 3 & 26 & 49 & 73 & 99 & Dollard_GeiseHochSnd \\
\hline 5 & 4 & 27 & 50 & 74 & 100 & Dollard_Heringplt \\
\hline 6 & 5 & 28 & 51 & 75 & 101 & Eems_Vaarwater \\
\hline 7 & 6 & 29 & 52 & 76 & 102 & Eems_DollardMnd \\
\hline 8 & 7 & 30 & 53 & 77 & 103 & Paap_Zuid \\
\hline 9 & 8 & 31 & 54 & 78 & 104 & Hond_Paap \\
\hline 10 & 9 & 32 & 55 & 79 & 105 & Hond_Noord \\
\hline 11 & 10 & 33 & 56 & 80 & 106 & Doekegat \\
\hline 12 & 11 & 34 & 57 & 81 & 107 & WesterEems \\
\hline 13 & 12 & 35 & 58 & 82 & 108 & OsterEms \\
\hline 14 & 13 & 36 & 59 & 83 & 109 & Juist_Flat \\
\hline 15 & 14 & 37 & 60 & 84 & 110 & Memmert \\
\hline 16 & 15 & 38 & 61 & 85 & 111 & RottumOog \\
\hline 17 & 16 & 39 & 62 & 86 & 112 & Horsborn \\
\hline 18 & 17 & 40 & 63 & 87 & 113 & GroningerWad \\
\hline 19 & 18 & 41 & 64 & 88 & 114 & LauwersZuidOost \\
\hline 20 & 19 & 42 & 65 & 89 & 115 & Spruit \\
\hline 21 & 20 & 43 & 66 & 90 & 116 & Lauwers \\
\hline 22 & 21 & 44 & 67 & 91 & 117 & Eilandergat \\
\hline 23 & & & & 92 & 118 & Memmert_NearCoast \\
\hline 24 & 22 & 45 & 68 & 93 & 119 & HuibertGat_NearCoast \\
\hline 25 & 23 & 46 & 69 & 94 & 120 & Lauwers_NearCoast \\
\hline 26 & & & & & 121 & Lauwers_OffCoast \\
\hline 27 & & & & 95 & 122 & Huibertgat_OffCoast \\
\hline 28 & & & & 96 & 123 & Memmert_OffCoast \\
\hline
\end{tabular}




\section{A2 Overview table main benthic animal groups (next to shellfish)}

Table 3 Overview table main benthic animal groups (next to shellfish)

\begin{tabular}{|c|c|c|c|c|}
\hline & $\begin{array}{l}\text { Arenicola marina } \\
\text { (Blow lug, wadpier) }\end{array}$ & $\begin{array}{l}\text { Hydrobia ulvae = Peringia ulvae } \\
\text { (mudsnail) }\end{array}$ & Littorina littorina (Alikruik) & $\begin{array}{l}\text { Meiofauna in } \\
\text { general }\end{array}$ \\
\hline $\begin{array}{l}\text { Max size } \\
(\mathrm{f} / \mathrm{m})\end{array}$ & $\begin{array}{l}\mathrm{f}: 11 \mathrm{~cm} / \mathrm{m}: 12 \mathrm{~cm}- \\
20 \mathrm{~cm}\end{array}$ & $\begin{array}{l}\text { Body: } 05 .-3 \mathrm{~mm} \\
\text { Shell: }<1 \mathrm{~cm} \text { : } \\
\text { up to } 4(<6) \mathrm{mm} \text { or } 2.5-7 \mathrm{~mm} \\
\text { Length/width ratio: } 2: 1\end{array}$ & $\begin{array}{l}\mathrm{f}+\mathrm{m}: 10-12 \mathrm{~mm} \text { at maturity; } \\
\mathrm{m} \text { size range }<30 \mathrm{~mm} \\
\text { Max height: } 52 \mathrm{~mm}\end{array}$ & $>30 \mu \mathrm{m}$ and $<1 \mathrm{~mm}$ \\
\hline $\begin{array}{l}\text { Age of max } \\
\text { size/ } \\
\text { sexual } \\
\text { maturity }\end{array}$ & $1-2$ years & $\begin{array}{l}<1 \text { year; Life span } 1-2.5 \text { years (but: } \\
\text { up to } 5 \text { years in aquaria; >4 years in } \\
\text { the arctic. } \\
\text { Growth rate variable (time of year; } \\
\text { degree parasite infestation) }\end{array}$ & $\begin{array}{l}\text { 2-3 years } \\
\text { Life span 5-10 years } \\
\text { Length + timing of breeding = } \\
\text { f(climate conditions) }\end{array}$ & $\begin{array}{l}\text { short life cylces, } \\
\text { without plankton } \\
\text { stages }\end{array}$ \\
\hline $\begin{array}{l}\text { Reproductio } \\
\text { n: estimate } \\
\text { \# eggs }\end{array}$ & $100,000-1,000,000$ & $\begin{array}{l}50 \text { in one egg mass; Fecundity: } \\
26.5\end{array}$ & $\begin{array}{l}10,000-100,000(100,000 \text { for } \\
\text { large } 27 \mathrm{~mm} \text { shell height) per } \\
\text { year, released on several } \\
\text { separate occasions. } \\
\text { Fecundity (f) increases with size }\end{array}$ & $\begin{array}{l}\text { High abundance and } \\
\text { productivity; } \\
\text { reproduction in many } \\
\text { sedimentary habitats }\end{array}$ \\
\hline $\begin{array}{l}\text { Reproductio } \\
\text { n: where? } \\
\text { Seafloor, } \\
\text { pelagic, ... }\end{array}$ & $\begin{array}{l}\text { Spawning: within the } \\
\text { burrow; } \\
\text { Spawned sperm on the } \\
\text { sediment surface; Eggs } \\
\text { in female burrows }\end{array}$ & $\begin{array}{l}\text { Fertilization: internal } \\
\text { Egg hatching time: 5-8 days } \\
\text { Larval development: for the } \\
\text { planktonic stage up to } 4 \text { weeks, for } \\
\text { a nonfeeding benthic larva } \\
\text { metamorphoses probably lasts just } \\
\text { two days. } \\
\text { Eggs laid preferentially on shells of } \\
\text { live individuals, also on empty } \\
\text { shells, grains of sand. Egg mass } \\
\text { acquires a protective layer of sand } \\
\text { grains. }\end{array}$ & \begin{tabular}{|l} 
Pelagic, directly in the sea \\
|
\end{tabular} & \\
\hline $\begin{array}{l}\text { Reproductio } \\
\text { n: when }\end{array}$ & $\begin{array}{l}\text { at } \mathrm{T}<15 / 13^{\circ} \mathrm{C} \text { (late } \\
\text { autumn, early winter) }\end{array}$ & $\begin{array}{l}\text { varies with latitude } \\
\text { March to October }\end{array}$ & $\begin{array}{l}\text { In estuaries: maximum } \\
\text { spawning in January (early) } \\
\text { Egg release synchronized with } \\
\text { spring tides. }\end{array}$ & \\
\hline Larvae & & $\begin{array}{l}\text { Larval stage: } 11-30 \text { days } \\
\text { Larval/Juvenile dispersal potential } \\
>10 \mathrm{~km}\end{array}$ & $\begin{array}{l}\text { Larval settling time or pelagic } \\
\text { phase can be up to six weeks. }\end{array}$ & \\
\hline
\end{tabular}




\begin{tabular}{|c|c|c|c|c|}
\hline $\begin{array}{l}\text { Food - } \\
\text { Feeds on: }\end{array}$ & $\begin{array}{l}\text { (sub)surface deposits: } \\
\text { micro-organisms } \\
\text { (bacteria), meiofauna }+ \\
\text { benthic diatoms in the } \\
\text { ingested sediment, } \\
\text { detritus, absorbs DOM }\end{array}$ & $\begin{array}{l}\text { (sub)surface deposit feeder } \\
\text { "microbrowser" } \\
\text { Detritus, periphytic microalgae }\end{array}$ & $\begin{array}{l}\text { fine green, brown and red } \\
\text { algae (incl. Ulva lactuca, Ulva } \\
\text { spp., Cladophora spp., } \\
\text { Ectocarpus spp.). } \\
\text { Wide variety of algae, esp. } \\
\text { small, ephemeral species } \\
\text { (diatoms, Enteromorpha, Ulva, } \\
\text { Porphyra), also small newly } \\
\text { settled barnacles + other } \\
\text { invertebrates }\end{array}$ & $\begin{array}{l}\text { benthic microalgae, } \\
\text { other microbes, and } \\
\text { detrital food sources }\end{array}$ \\
\hline $\begin{array}{l}\text { Food size } \\
\text { preference }\end{array}$ & $<2 \mathrm{~mm}$ & $\begin{array}{l}\text { Diatoms } \\
\text { Larger particles }\end{array}$ & Small & detritus \\
\hline $\begin{array}{l}\text { Food } \\
\text { location }\end{array}$ & Sediment (sub)surface & Surface, sub-surface & & In sediment \\
\hline $\begin{array}{l}\text { Preferred } \\
\text { habitat (e.g. } \\
\text { sand, mud, } \\
\text { Surface, } \\
\text { bottom... }\end{array}$ & $\begin{array}{l}\text { Sands of } 100 \mu \mathrm{m} \text { mean } \\
\text { particle size ( } 80 \text { - } \\
200 \mu \mathrm{m}) \text { with organic } \\
\text { content; intertidal } \\
\text { (mid-tidal). } \\
\text { Juveniles: ca } 250 \mu \mathrm{m}\end{array}$ & $\begin{array}{l}\text { sandy mud, muddy sand, mud } \\
\text { all North Sea coasts: Upper-lower-, } \\
\text { eu- and sub- littoral fringe } \\
\text { dispersal up to } 10 \mathrm{~km}\end{array}$ & $\begin{array}{l}\text { Rocky, upper shore to } \\
\text { sublittoral, to } 60 \mathrm{~m} ; \\
\text { if sheltered, also sand, mud } \\
\text { (estuaries, mud-flats) } \\
\text { densities of up to } 200-800 \\
\text { individuals per square meter }\end{array}$ & $\begin{array}{l}\text { Benthic habitat; } \\
\text { shallow water }\end{array}$ \\
\hline $\begin{array}{l}\text { Main } \\
\text { predators }\end{array}$ & $\begin{array}{l}\text { Flatfish, wading birds } \\
\text { (e.g. Numenius } \\
\text { arquata, Limosa } \\
\text { lapponica, Haematopus } \\
\text { ostralegus), ragworm } \\
\text { (Nereis virens, Hediste } \\
\text { diversicolor) }\end{array}$ & Retusa obtusa (Montagu) & $\begin{array}{l}\text { shore birds, fish, crabs } \\
\text { (Carcinus maenas (L.)), lobsters }\end{array}$ & $\begin{array}{l}\text { Larger animals, e.g. } \\
\text { grass shrimp and a } \\
\text { variety of juvenile } \\
\text { fish that utilize } \\
\text { shallow water } \\
\text { nursery habitats }\end{array}$ \\
\hline
\end{tabular}




\section{A3 Water quality monitoring sites tributaries}

Table 4 List of inlets to be chosen, and which one is active.

\begin{tabular}{|c|c|c|c|c|c|}
\hline Inlet_nr & InLetName & Active & Invoercompartiment & Nr_in_CompartNames & Remarks \\
\hline 2 & Leda & 1 & 101 & b28 & River Leda \\
\hline 3 & Ems-Leerort & 0 & 101 & b29 & Ems bij Leer \\
\hline 4 & Ems_Gandersum & 0 & 102 & b30 & Ems bij Gandersum \\
\hline 6 & Borssum_EmKan & 1 & 102 & b42 & Borssum_EmdenKanaal \\
\hline 7 & Knock_Buntweg & 1 & 108 & b44 & Knock_Buntweg \\
\hline 8 & WWA_Statenzijl & 1 & 103 & b46 & Westerwoldse $\mathrm{Aa}$ \\
\hline 12 & EemsKanaal_Farnsum & 1 & 108 & b50 & Eemskanaal \\
\hline 13 & DamsterDiep_BrugDelfzijl & 1 & 108 & b51 & Damsterdiep \\
\hline 14 & Tjariet_Spijk & 1 & 110 & b54 & Tjariet_Spijksterpompen \\
\hline 15 & Leysiel & 1 & 113 & b55 & Leysiel \\
\hline
\end{tabular}

Table 5 Full list of possible inlets, what data are available for what period, and where do the data come from. Note that for most tributaries and rivers, some periods had to be estimated, also when data are available

\begin{tabular}{|c|c|c|c|}
\hline Site / tributary & Data type & From & $\begin{array}{l}\text { Available } \\
\text { period }\end{array}$ \\
\hline Ems-river & Water quality & NLWKN & \\
\hline Gandersum & & & $1992-2016$ \\
\hline Leer-Ort & & & $1987-2016$ \\
\hline Papenburg & & & $1987-2016$ \\
\hline Terborg & & & 1983-2016 \\
\hline Gandersum & Flow & & \\
\hline \multicolumn{4}{|l|}{ Leer-Ort } \\
\hline \multicolumn{4}{|l|}{ Papenburg } \\
\hline \multicolumn{4}{|l|}{ Terborg } \\
\hline Leda & Water quality & NLWKN & \\
\hline Leer & Flow & & $1982-2016$ \\
\hline Amdorf & & & $1998-2016$ \\
\hline \multirow[t]{2}{*}{ Leyhorn } & Water quality & NLWKN & \\
\hline & Flow & & \\
\hline Knockster Tief & Water quality & NLWKN & \\
\hline Buntelsweg & Flow & & $1982-2016$ \\
\hline Twixlum-Doodsh"rn & & & 2015-2016 \\
\hline
\end{tabular}




\begin{tabular}{|c|c|c|c|}
\hline \multirow[t]{2}{*}{ Emden Hafen / Nesselander Schleuse } & Water quality & NLWKN & \\
\hline & Flow & & \\
\hline \multirow[t]{2}{*}{ Borssumerkanal } & Water quality & NLWKN & \\
\hline & Flow & & \\
\hline Petkumer Sieltief & Water quality & NLWKN & 2013 \\
\hline Petkum & Flow & Estimated & \\
\hline \multirow[t]{2}{*}{ Ems Seitenkanal } & Water quality & NLWKN & \\
\hline & Flow & Estimated & \\
\hline Fehntjer Tief & Water quality & NLWKN & $1982-2016$ \\
\hline Oldersum & Flow & Estimated & \\
\hline LeySiel Speicherbecken & Water quality & NLWKN & 2001-2016 \\
\hline Leysiel & Flow & Estimated & \\
\hline Ems-Jade-kanal & Water quality & NLWKN & \\
\hline Westerende-Kirchloog & Flow & Estimated & \\
\hline Sauteler Kanal & Water quality & NLWKN & \\
\hline Neermoor & Flow & Estimated & $1982-2016$ \\
\hline Mittegroáefehn(Nord) & & & 1998-2016 \\
\hline ColdemSchöpfwerk-Tief & Water quality & NLWKN & $1982-2016$ \\
\hline Coldemüntje & Flow & Estimated & \\
\hline \multirow[t]{2}{*}{ Westerwoldse Aa } & Water quality & Hunze \& Aa & $1978-2016$ \\
\hline & Flow & Hunze \& Aa & \\
\hline \multirow[t]{2}{*}{ Termunterdiep } & Water quality & Hunze \& Aa & 2003-nu \\
\hline & Flow & Hunze \& Aa & \\
\hline \multirow[t]{2}{*}{ Eemskanaal } & Water quality & Hunze \& Aa & 1978-nu \\
\hline & Flow & Hunze \& Aa & \\
\hline \multirow[t]{2}{*}{ Fiemel } & Water quality & Hunze \& Aa & 1991-nu \\
\hline & Flow & & \\
\hline \multirow[t]{2}{*}{ Spijksterriet } & Waterkwaliteit & Noorderzijlvest & \\
\hline & Flow & Noorderzijlvest and partly estimated & \\
\hline \multirow[t]{2}{*}{ ED-estuarium DE } & Water quality & & \\
\hline & Silt & & \\
\hline
\end{tabular}




\begin{tabular}{|l|l|l|l|}
\hline & Sediment composition & Geert Aarts & $2006-2015$ \\
\hline & Benthos shellfish & & $2006-2015$ \\
\hline & Benthos rest & & \\
\hline ED-estuarium NL & & & $1975-2012$ \\
\hline & Water quality & RWS & $1975-2012$ \\
\hline & Slib & RWS & 1990 \\
\hline & Bodemsamenstelling & RWS & \\
\hline & & NIOZ & $1990-2015$ \\
\hline & Benthos shellfish & IMARES & $2008-2012$ \\
\hline & Benthos rest & NIOZ & \\
\hline & Phytobenthos & Zelf + plus die van Viktor & $1976-1980$ \\
\hline & $\begin{array}{l}\text { Phytoplankon species/ } \\
\text { groups }\end{array}$ & 2012/2013, ouder 1980, 19?? & $2012 / 2013$ \\
\hline & Primary production & $\begin{array}{l}\text { Old data Viktor de Jonge / Franciscus } \\
\text { Colijn }\end{array}$ & $1976-1980$ \\
\hline & Primary production & IMARES & $2012-2013$ \\
\hline
\end{tabular}

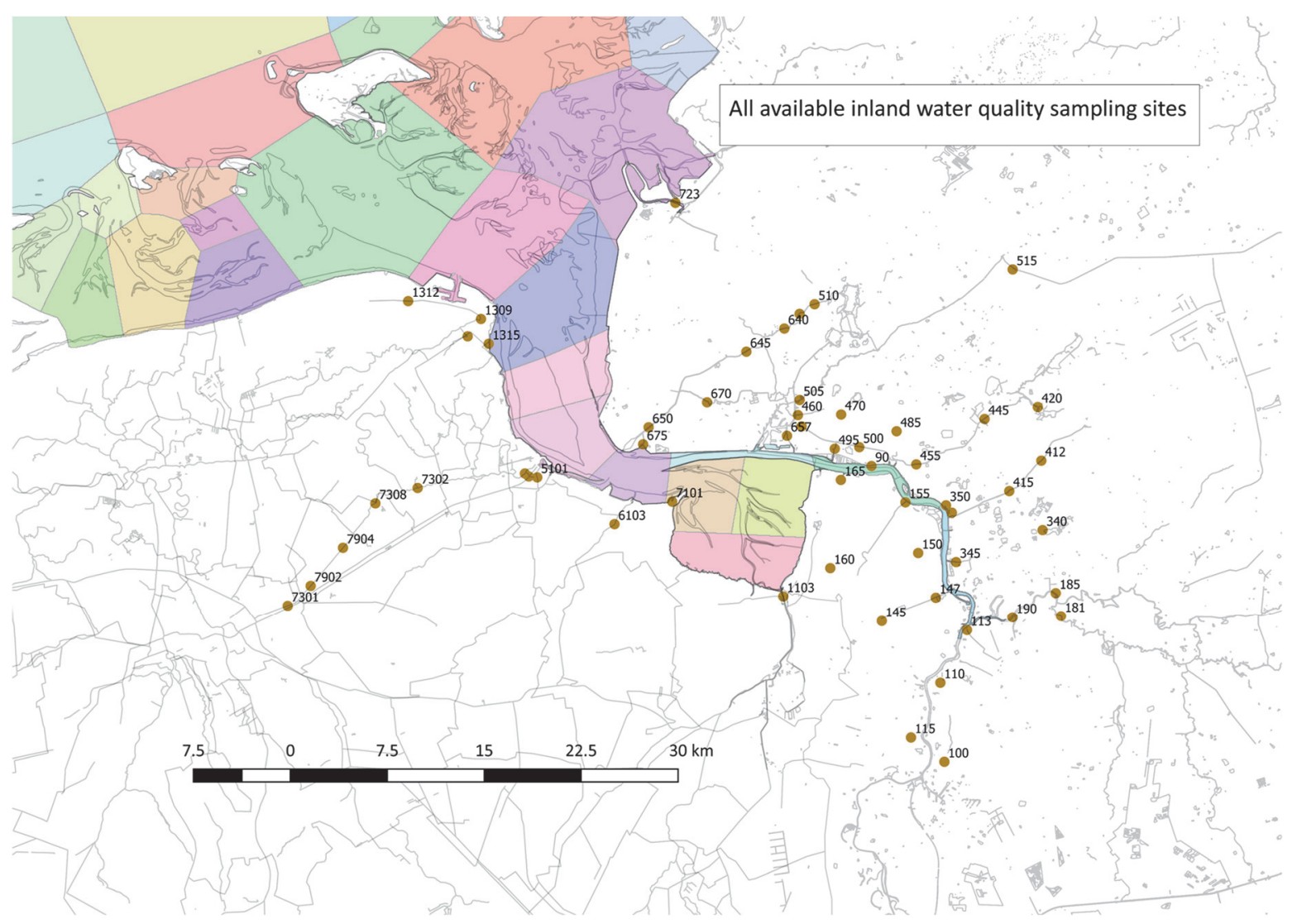

Figure 39 Water quality sampling sites for tributaries and rivers. 
Table 6 Water quality monitoring sites; tributaries to the estuary. Site, ID, first and last date of observations, number of observations and additional information. Data from NLWKN, Hunze en Aa's and Noorderzijlvest.

\begin{tabular}{|c|c|c|c|c|c|c|}
\hline Site & Abbrev & Site_ID & First date & Last date & NrObs & System \\
\hline Papenburg/Voellen & $\begin{array}{r}\text { PAPBRGVOELL } \\
\text { _EMS }\end{array}$ & 70 & 1987-02-04 & 2016-05-11 & 702 & Ems \\
\hline Leerort & $\begin{array}{r}\text { LEERORT_EM } \\
\mathrm{S}\end{array}$ & 80 & 1987-02-04 & $2007-10-10$ & 689 & Ems \\
\hline Terborg & $\begin{array}{r}\text { TERBORG_EM } \\
\mathrm{S}\end{array}$ & 85 & 1983-01-05 & 2016-05-11 & 753 & Ems \\
\hline Gandersum & $\begin{array}{r}\text { GANDERSM_E } \\
\text { MS }\end{array}$ & 90 & 1987-04-02 & 2016-05-11 & 771 & Ems \\
\hline Coldemuentje & COLDM & 110 & $1982-01-18$ & 2001-02-14 & 422 & Coldem.Schoepfw-Tief \\
\hline Amdorf & & 181 & $1987-05-14$ & 2016-02-10 & 264 & Leda \\
\hline Leer & & 190 & $1982-01-18$ & 2003-11-05 & 469 & Leda \\
\hline Mittegrossef.(Nord) & MITTGR_NRD & 360 & $1985-07-17$ & $2015-12-01$ & 177 & Sauteler Kanal \\
\hline Neermoor & & 415 & $1982-01-18$ & $2016-02-02$ & 421 & Sauteler Kanal \\
\hline Oldersum & & 485 & $1982-01-18$ & $2016-02-02$ & 432 & Fehntjer Tief \\
\hline Westerende-Kirchloog & & 510 & $1982-01-18$ & 2016-02-02 & 423 & Ems-Jade-Kanal \\
\hline Buntelsweg & & 650 & $1982-01-18$ & 2016-02-09 & 545 & Knockster Tief \\
\hline Leysiel & & 723 & 2001-02-21 & 2016-02-09 & 190 & Speicherbecken \\
\hline $\begin{array}{l}\text { Losplaats Nieuw } \\
\text { Statenzijl }\end{array}$ & & 1103 & $1978-09-25$ & $2016-05-23$ & 452 & Westerwoldse $\mathrm{Aa}$ \\
\hline Oostpolderbermkanaal & & 1309 & 1978-09-11 & $2016-06-21$ & 448 & Brug Uithuizermeeden \\
\hline Kleine Tjariet & & 1311 & 1978-09-11 & 2015-12-09 & 139 & Duiker Z.O. van Oude Schip \\
\hline Brug 15 Farmsum & & 4102 & $1978-09-18$ & $2016-06-21$ & 475 & Eemskanaal \\
\hline Brug Farmsum & & 5101 & 1978-09-11 & $2016-06-21$ & 449 & Afw.kanaal Van Duurswold \\
\hline Termunterzijldiep & & 6103 & 2003-01-07 & $2016-06-21$ & 140 & Warvenweg thv Baamsum \\
\hline Gemaal Fiemel & & 7101 & 1991-01-15 & $2016-06-21$ & 229 & Uitwateringskanaal \\
\hline Damsterdiep & & 7301 & 1978-09-11 & $2015-12-10$ & 677 & Brug Ruischerbrug \\
\hline Damsterdiep & & 7302 & 1978-09-11 & 2016-06-02 & 241 & Brug Wirdumerdraai \\
\hline Damsterdiep & & 7318 & 1978-09-11 & $2016-06-15$ & 451 & Brug Delfzijl \\
\hline
\end{tabular}




\section{A4 Completing water quantity data series}

\section{A4.1 Introduction}

One of the topics to solve was that most of the quantitative inflow data were incomplete, see e.g. Figure 41. It appeared to be impossible to complete these series by comparing values for several comparable rivers/canals since most of the lacking data concerned the same period(s). Thus, it was decided to estimate discharges using precipitation and evaporation data. These are available for KNMI-station Eelde (KNMI, 2017) for the whole simulation period. Next, the size of the several sub-basins must be known. For the Dutch areas (see Figure 40 and Figure 42).

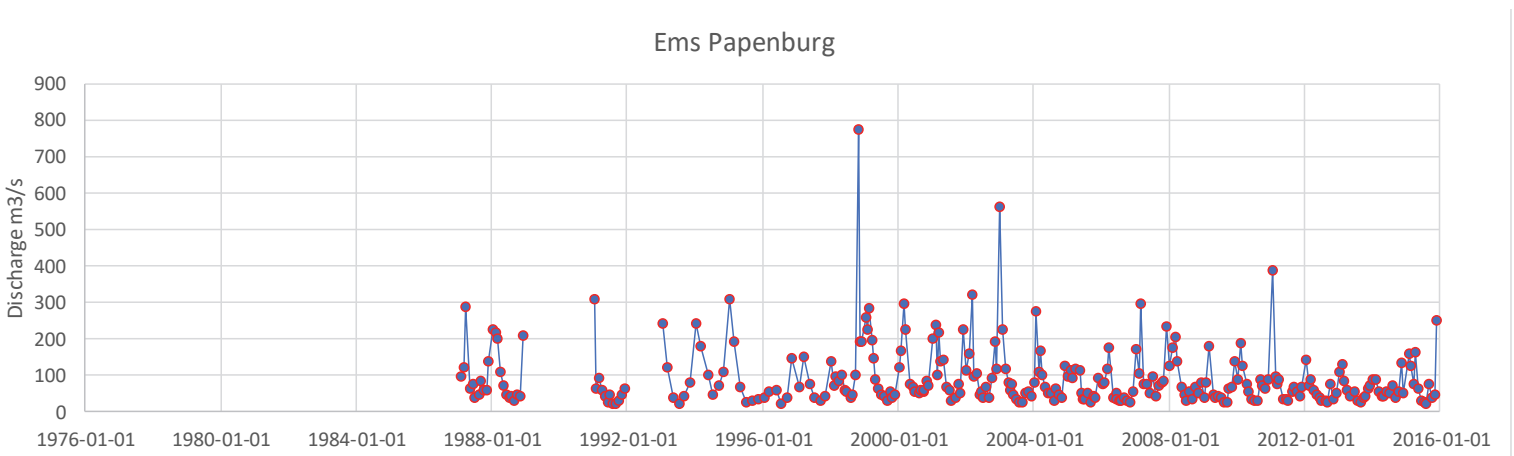

Figure 41 Discharge data for the Ems river at Papenburg (source: NLWKN). Similar sets were available for Leer, Gandersum and Terborg. Old BOEDE-data ((see Baretta \& Ruardij, 1988) made the first few years (until 1981) complete, leaving holes in the 80's and beginning of the 90's.

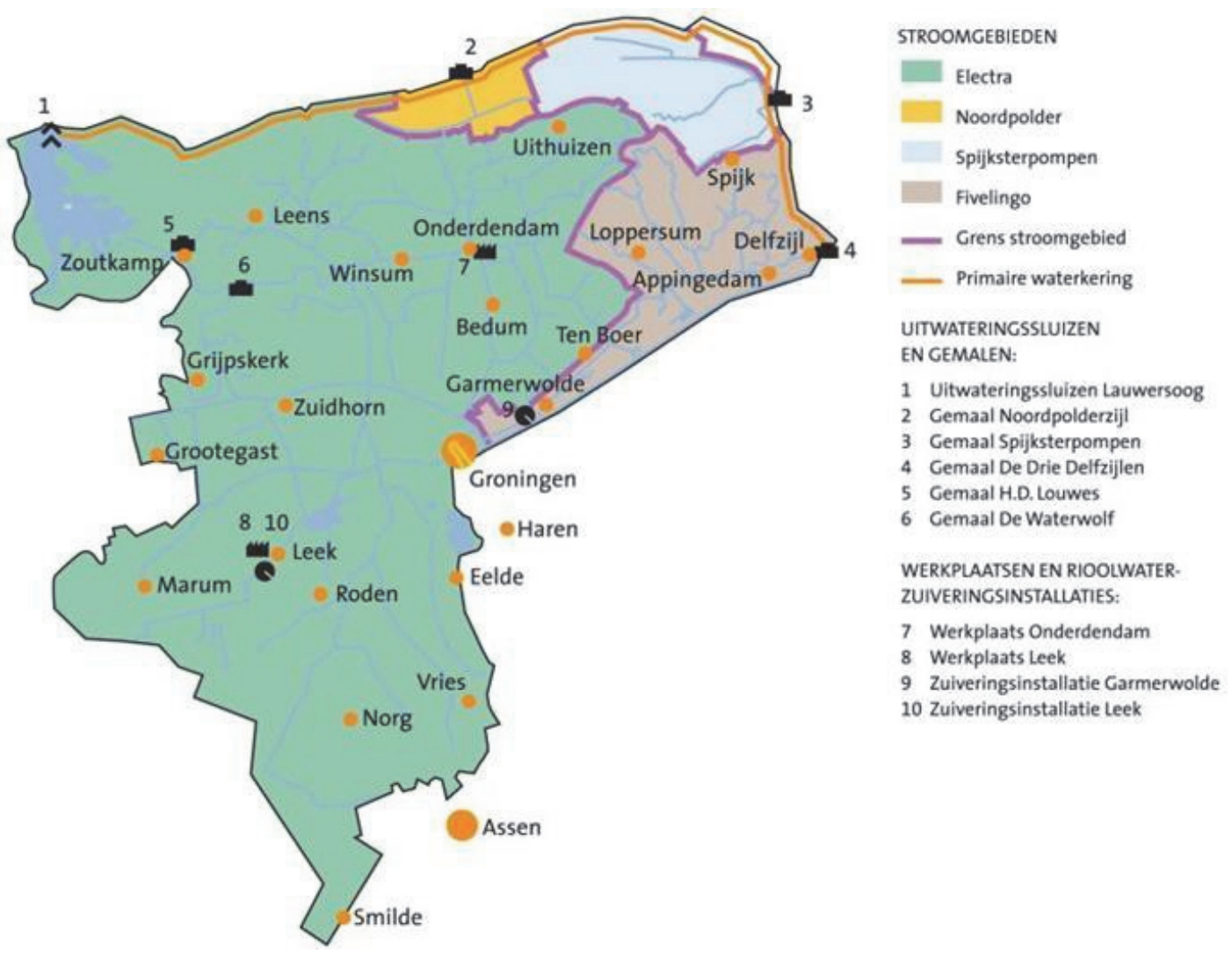

Figure 40 Areas Fivelingo and Spijksterpompen are relevant. From Vos (). 


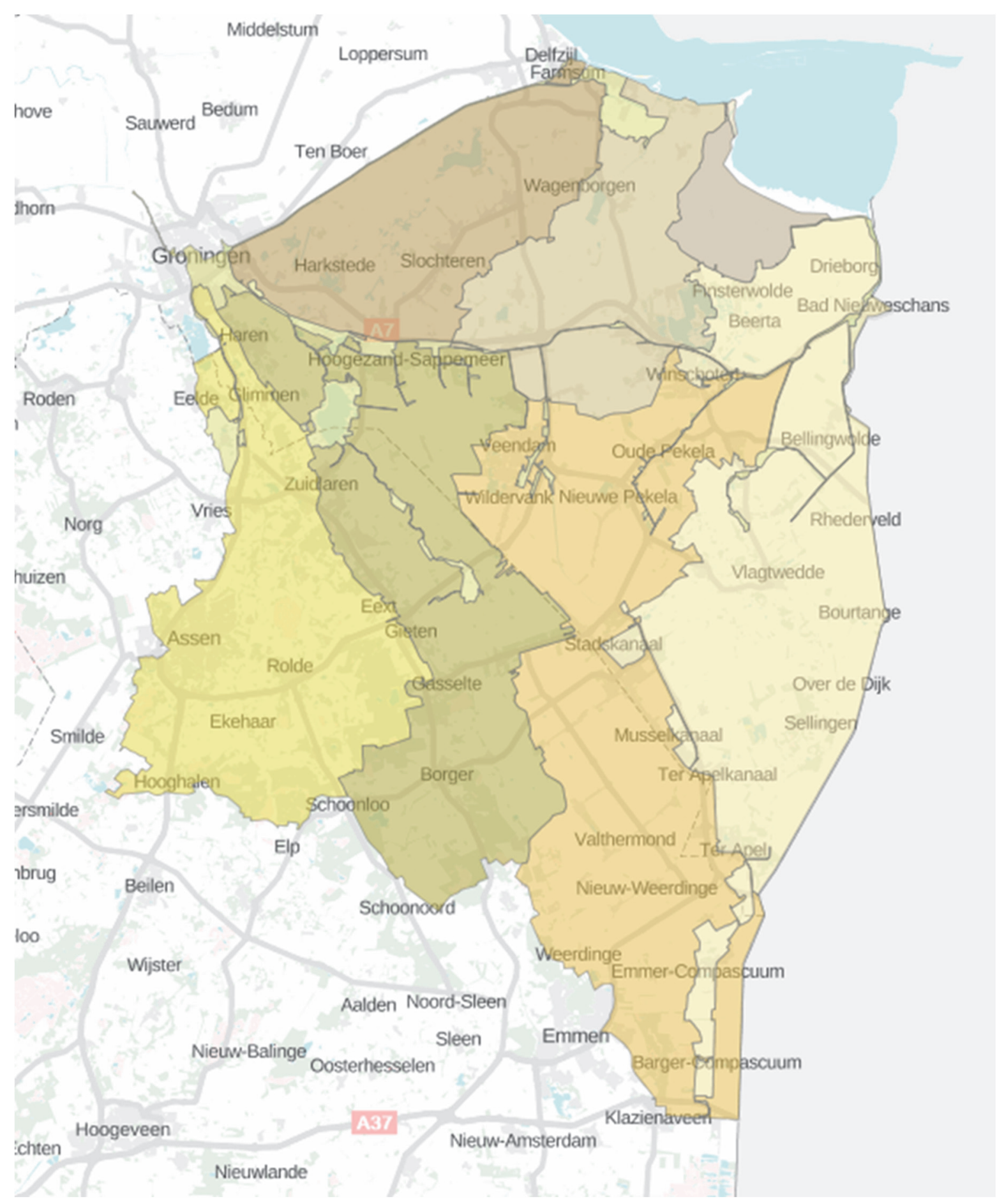

Figure 42 Hunze en Aa's discharge areas. Sizes are partly estimated based on this map. Source: Hunze en Aa.

German discharge areas are not available as map, but the data received from NLWKN contained a discharge area table. The discharge data that were available were related to the precipitation surplus and the discharge area data, and these were rather consistent except for one area where it was needed to adapt the discharge area value. 


\section{A4.2 Computation method}

\section{A4.2.1 Precipitation data}

KNMI (2017) has daily precipitation and evaporation data available. Precipitation is measured, evaporation is a computed value for a standard situation. To start with, simply this computed evaporation value was used. An overview of the net precipitation values is shown in Figure 43. Later, it appeared that there was no need to change this.
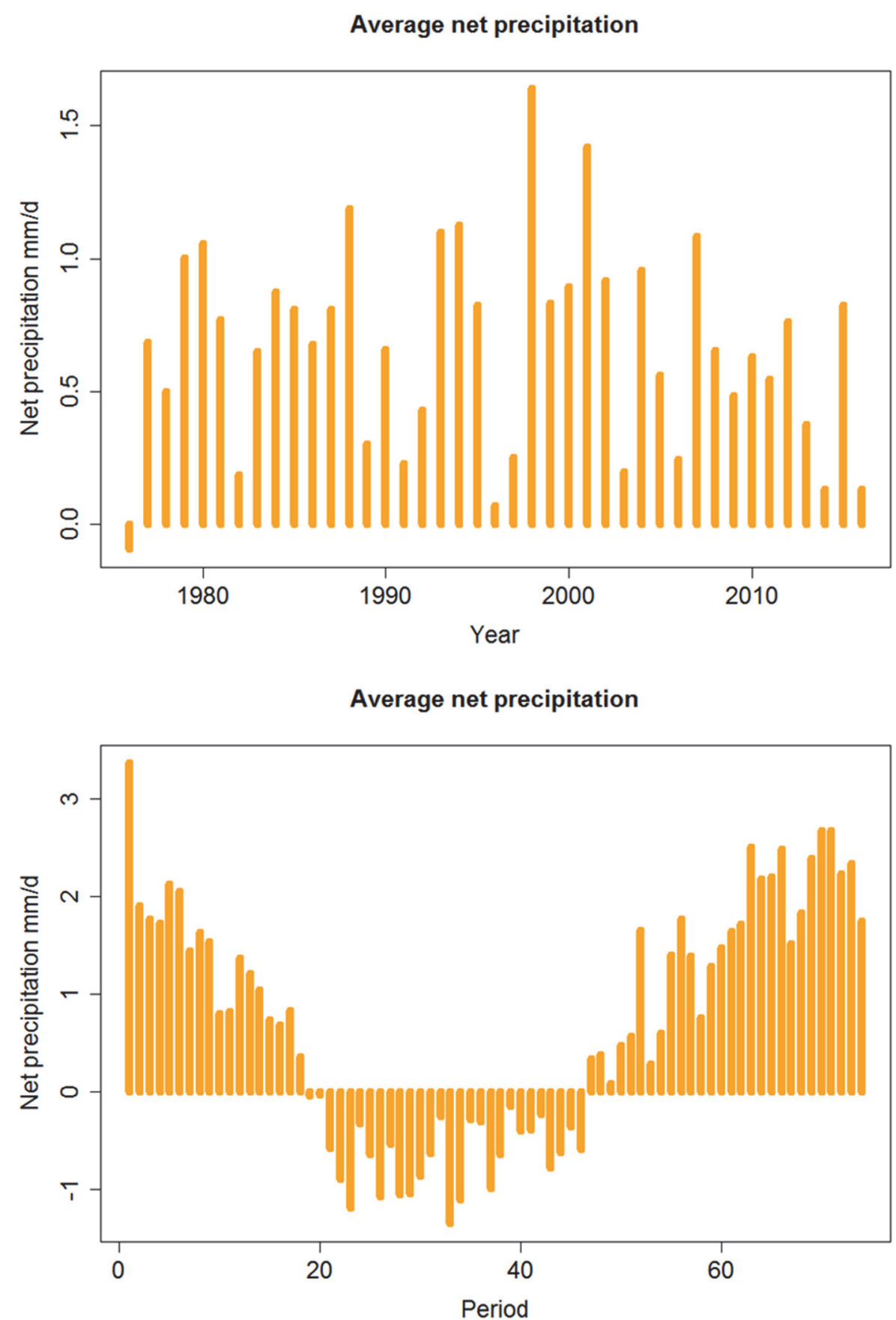

Figure 43 Average net precipitation at KNMI station Eelde (KNMI, 2016). Upper: per year, lower for each 5-days period. 
Discharges have been checked against precipitation values and against difference between precipitation and evaporation, as listed by the KNMI. The latter is not a real evaporation, but a computed value, based on 'standard crop'-characteristics. Thus, it should be treated as an estimate.

\section{A4.2.2 Drainage areas}

The size of the drainage areas was needed. Almost all German data contained these sizes. Size of the Dutch areas were found on the several sites of both the 'Waterschappen' and some on pumping station sites. Data are listed in Table 7 and Table 8, and the drainage area overview is presented in Table 9 (section 3.2.2).

Table 7 German discharge sites and drainage areas

\begin{tabular}{|c|c|c|c|c|}
\hline Site & Data available & Type & Remark & Range \\
\hline Ditzum & $\begin{array}{l}\text { 1987-2015, monthly } \\
\text { data, with gaps. } \\
\text { Omissions: } \\
\text { 1976-1986, } \\
\text { 1989-1990, } \\
1992\end{array}$ & Flow $\mathrm{m}^{3} / \mathrm{s}$ & $5.000 \mathrm{ha}$ & \\
\hline Retkum & $\begin{array}{l}\text { 1987 - 2015. Monthly } \\
\text { data, with gaps. } \\
\text { Omissions: } \\
1976-1986, \\
1988-1990\end{array}$ & Flow $\mathrm{m}^{3} / \mathrm{s}$ & $\begin{array}{l}\text { Discharge area: } \\
\text { Discharge downstream from } \\
\text { Gandersum }\end{array}$ & \\
\hline Oldersum & $\begin{array}{l}\text { Discharge from Ems- } \\
\text { Seitenkanal }\end{array}$ & Flow $\mathrm{m}^{3} / \mathrm{s}$ & $\begin{array}{l}\text { Discharge area: } 20.000 \text { ha } \\
\text { Discharge upstream from } \\
\text { Gandersum }\end{array}$ & \\
\hline Petkum & $\begin{array}{l}\text { 1984-2016. } \\
\text { Monthly data, with } \\
\text { gas. } \\
\text { Omissions: } \\
1976-1984, \\
1986-1987 \\
1989-1991\end{array}$ & Flow $\mathrm{m} 3 / \mathrm{s}$ & Discharge area: 5.000 ha & \\
\hline Borssum & & & $\begin{array}{l}\text { Discharge area: } 12.800 \text { ha } \\
\text { Flows into Ems near Emden }\end{array}$ & \\
\hline Pogum & & & $\begin{array}{l}\text { Discharge area: } 5.800 \text { ha } \\
\text { Flows into Ems, south side, near } \\
\text { inflow into Dollard }\end{array}$ & \\
\hline Knock & $\begin{array}{l}\text { 1992-2015, monthly } \\
\text { data } \\
\text { Omissions: } \\
\text { 1976-1991 }\end{array}$ & Flow $\mathrm{m}^{3} / \mathrm{s}$ & $\begin{array}{l}\text { Discharge area: } 34.000 \text { ha } \\
\text { Discharge into Ems-Dollard }\end{array}$ & \\
\hline Leysiel & $\begin{array}{l}1992-2015 . \\
\text { Monthly data } \\
\text { No gaps }\end{array}$ & Flow $\mathrm{m}^{3} / \mathrm{s}$ & $\begin{array}{l}\text { Discharge area: } 12.000 \text { ha } \\
\text { Discharge into Oster-Ems }\end{array}$ & \\
\hline & & & & \\
\hline & & & & \\
\hline
\end{tabular}


Table 8 Dutch discharge sites and drainage areas

\begin{tabular}{|c|c|c|c|c|}
\hline Site & Data available & Type & Remark & Range \\
\hline Spijksterpompen & $\begin{array}{l}1994-1999 / 10 / 05 \\
\text { 2000/07/08-2016 } \\
\text { Omissions: } \\
\text { 1976-1993 } \\
\text { 1999/10-15 - } \\
2000 / 07 /-8\end{array}$ & Daily data $\left(\mathrm{m}^{3} / \mathrm{d}\right)$ & $\begin{array}{l}\text { Spijksterpompen: consist of a } \\
\text { pumpingstation and a weir. Data } \\
\text { for the latter are less ccurate. } \\
\text { Exists since } 1978 \text {. } \\
\text { Pumpinstation capacity: } 2 \text { electro } \\
\text { engines, } 335 \mathrm{~m}^{3} / \mathrm{min}^{3} \text { each, total } \\
670 \mathrm{~m}^{3} / \mathrm{min}=12 \mathrm{~m}^{3} / \mathrm{s} \text {. }\end{array}$ & $\begin{array}{l}\text { Avg } 1.1 \mathrm{~m}^{3} / \mathrm{s} \\
\operatorname{Max} 27 \mathrm{~m}^{3} / \mathrm{s}\end{array}$ \\
\hline De Drie Delfzijlen & $\begin{array}{l}\text { 1980-1990 } \\
1991-1993 \\
1994-1999-10-02, \\
2005-02-15-2016 \\
\text { Omission: } \\
1976-1979 \\
1991-1993 \text { (daily) } \\
1999 / 10 / 2- \\
2005 / 02 / 15 \\
\text { Site exists since } 1972 .\end{array}$ & $\begin{array}{l}\text { Daily data }\left(\mathrm{m}^{3} / \mathrm{d}\right) \\
\text { Year sums } \\
\text { Daily data }\left(\mathrm{m}^{3} / \mathrm{d}\right)\end{array}$ & $\begin{array}{l}\text { Three pumps, two diesel (650 } \\
\mathrm{m}^{3} / \mathrm{min} \text { each), one electric }(250 \\
\left.\mathrm{m}^{3} / \mathrm{min}\right) \text {, total } 1500 \mathrm{~m}^{3} / \mathrm{min}=25 \\
\mathrm{~m}^{3} / \mathrm{s} \text {. Discharge area Fivelingo- } \\
\text { boezem (from Damsterdiep): } \\
16.000 \text { ha. }\end{array}$ & $\begin{array}{l}\text { Avg } \approx 2.3 \mathrm{~m}^{3} / \mathrm{s} \\
\operatorname{Max} 28 \mathrm{~m}^{3} / \mathrm{s}\end{array}$ \\
\hline Eemskanaalsluizen & $\begin{array}{l}\text { 1980-1990 } \\
1991-1994 \\
1995-1996 \\
1997-2015 \\
\text { Omission: } \\
\text { 1976-1979 } \\
1991-1994 \text { (daily) }\end{array}$ & $\begin{array}{l}\text { Daily data }\left(\mathrm{m}^{3} / \mathrm{d}\right) \\
\text { Year sums } \\
\text { Monthly data } \\
\text { Daily data }\end{array}$ & $\begin{array}{l}\text { Sluices for connecting the } \\
\text { Eemskanaal with the Delfzijl } \\
\text { harbour and thus, the Esm-Dollard }\end{array}$ & Avg $\approx 5 \mathrm{~m}^{3} / \mathrm{s}$ \\
\hline Duurswold & $\begin{array}{l}1980-1990 \\
1991-1993 \\
1995-1998 \\
2000-2015 \\
\text { Exists since } 1933 . \\
\\
\text { Omission: } \\
1976-1979 \\
1991-1993 \text { (daily) } \\
1994 \\
1999\end{array}$ & $\begin{array}{l}\text { Daily data } \mathrm{m}^{3} / \mathrm{d} \\
\text { Year sums } \\
\text { Flows in } \mathrm{m}^{3} / \mathrm{s} \text { and } \\
\mathrm{m}^{3} / \text { day for pumping } \\
\text { station and for } \\
\text { sluices (and both) }\end{array}$ & $\begin{array}{l}\text { Discharge to Delfzijl harbour. } \\
\text { Three pumps, } 2 \text { electric, one } \\
\text { diesel, } 650 \mathrm{~m}^{3} / \mathrm{min} \text { each, total } \\
1950 \mathrm{~m}^{3} / \mathrm{min}^{3}\left(32.5 \mathrm{~m}^{3} / \mathrm{s}\right) \\
\text { Discharge area Duurswoldboezem, } \\
21.500 \text { ha. }\end{array}$ & $\begin{array}{l}\text { Avg } 2.6 \mathrm{~m}^{3} / \mathrm{s} \\
\operatorname{Max} 28 \mathrm{~m}^{3} / \mathrm{s}\end{array}$ \\
\hline Rozema & $\begin{array}{l}1990-1990 \\
1991-1993 \\
1995-1998 \\
2001-2015 \\
\text { Omission: } \\
1976-1979 \\
1991-1994 \\
1999-2000 \\
\end{array}$ & \begin{tabular}{|l|} 
Daily data $\mathrm{m}^{3} / \mathrm{d}$ \\
Year sums \\
Flows in $\mathrm{m}^{3} / \mathrm{s}$ and \\
$\mathrm{m}^{3} /$ day for pumping \\
station
\end{tabular} & $\begin{array}{l}\text { Discharge to Eems-Dollard } \\
\text { (Termunterzijl). Four gas driven } \\
\text { pumps, } 3^{*} 650 \mathrm{~m}^{3} / \mathrm{s}, 1^{*} 750 \mathrm{~m}^{3} / \mathrm{s}, \\
\max 2700 \mathrm{~m}^{3} / \mathrm{min}^{2}\left(45 \mathrm{~m}^{3} / \mathrm{s}\right) \\
\text { Discharge area (Oldambtboezem,): } \\
30.000 \text { ha. }\end{array}$ & $\begin{array}{l}\text { Avg } 2.4 \mathrm{~m}^{3} / \mathrm{s} \\
\max 58.3 \mathrm{~m}^{3} / \mathrm{s}\end{array}$ \\
\hline Fiemel & $\begin{array}{l}1999-2015 \\
\text { Exists since } 1997 .\end{array}$ & $\begin{array}{l}\text { Flows in } \mathrm{m} 3 / \text { day \& } \\
\mathrm{m} 3 / \mathrm{s}\end{array}$ & $\begin{array}{l}\text { Discharge to Dollard mouth. Three } \\
\text { electric pumps, } 130 \mathrm{~m}^{3} / \mathrm{min} \text { each, } \\
\text { total } 390 \mathrm{~m}^{3} / \mathrm{min} \text {. } \\
\text { Includes a fish passage } \\
\text { construction. }\end{array}$ & $\begin{array}{l}\operatorname{Avg} 0.37 \mathrm{~m}^{3} / \mathrm{s} \\
\max 6.3 \mathrm{~m}^{3} / \mathrm{s}\end{array}$ \\
\hline $\begin{array}{l}\text { Nieuw-Statenzijl / } \\
\text { Westerwoldse Aa }\end{array}$ & $\begin{array}{l}\text { 1980-1989 } \\
1992-1994 \\
\text { 1995/10/13- } \\
\text { 2009/1/1 } \\
\text { 2004/1/1-2015/12/31 } \\
\text { Omissions: }\end{array}$ & $\begin{array}{l}\text { Flow in } \mathrm{m}^{3} / \mathrm{d} \\
\text { Year sums } \\
\text { Flow in } \mathrm{m}^{3} / \mathrm{d}\end{array}$ & $\begin{array}{l}\text { Discharge of river (no pumping } \\
\text { station). } \\
\text { Discharge Westerwoldse Aa } \\
\text { depends on (1) Veelerveen-weir } \\
\text { (discharge area } 19.000 \text { ha). } \\
\text { Westerwolde Aa discharge area = }\end{array}$ & $\begin{array}{l}\text { Avg } 6-10 \mathrm{~m}^{3} / \mathrm{s} \\
\operatorname{Max}=94 \mathrm{~m}^{3} / \mathrm{s}\end{array}$ \\
\hline
\end{tabular}




\begin{tabular}{|l|l|l|l|l|}
\hline $\begin{array}{l}1976-1979 \\
1990-1995 / 10 / 13 \\
2000-2003\end{array}$ & & $\begin{array}{l}56.000 \text { ha), ratio= 2.9, plus (2) flow } \\
\text { at 'De Bult' }\end{array}$ & \\
\hline $1992-1994$ : daily data & & & \\
\hline & & & & \\
\hline
\end{tabular}

A summary is given in Table 9.

Table 9 Summary of drainage areas for the several sites mentioned in Figure 6. German data from NLWKN; Dutch areas partly estimated.

\begin{tabular}{|c|c|c|c|c|c|}
\hline Site & Area_Basin_km2 & Site & Area_Basin_km2 & Site & Area_Basin_km2 \\
\hline Borssum & 128 & Fiemel & 100 & Oldersum & 200 \\
\hline Buschfelder & 25 & Jemgumer & 17 & Petkum & 50 \\
\hline Coldeborg & 39.8 & Knock & 340 & Pogum & 58 \\
\hline Coldemüntje & 30 & Leysiel & 120 & Rozema & 300 \\
\hline Ditzum & 50 & Mark & 36 & Sautel & 189 \\
\hline DrieDelfzijlen & 16 & Muhde & 18 & Soltborger & 46 \\
\hline Duurswold & 215 & NieuwStatenzijl & 560 & Spijksterpompen & 100 \\
\hline EemsKanaal & 500 & Nüttermoor & 50 & Stapelmoor & 21 \\
\hline & & & & Terborg & 23 \\
\hline
\end{tabular}

\section{A4.2.3 Averaging of data}

Discharges never react immediately to rainfall or evaporation. Therefore, weekly or monthly net precipitation values were tested.

Two checks have been performed. In the first, all data (discharges and meteo-data) were grouped in weekly data, in the second it concerned 30-day groups. Conclusions were:

- weekly data showed a weak correlation between discharge and meteo data

- precipitation data alone showed a much worse correlation with discharges than the difference between precipitation and evaporation did.

Thus, only monthly discharge and meteo data and net precipitation values are used.

Any lag-period due to soil saturation, etc, has bene omitted. This is a serious simplification, but accepted for the time being. 

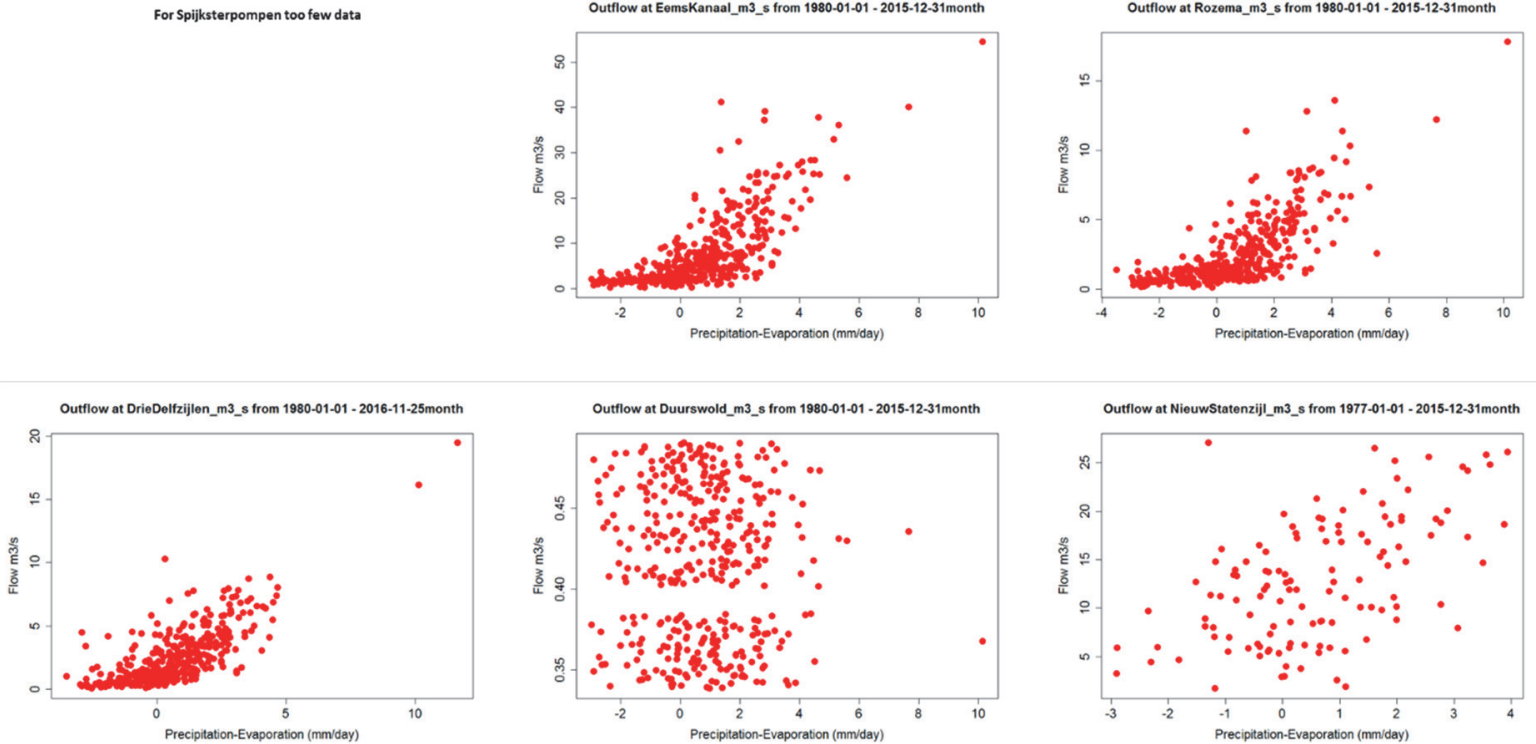

Figure 44 Discharges at the Dutch side of the system, related to the difference between precipitation and a standard crop-evaporation. Measurements. 

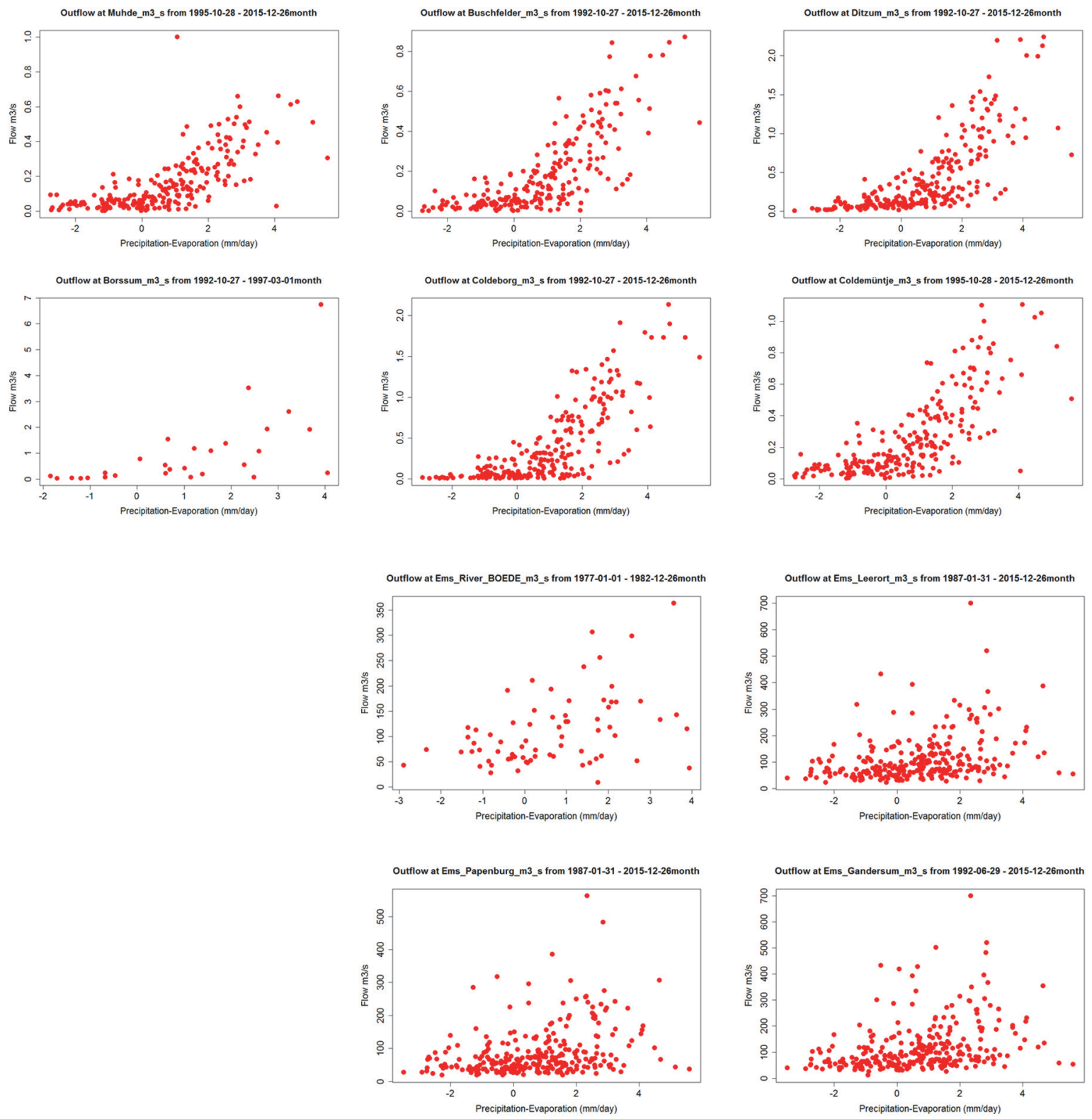

Figure 45 Discharges at the German side of the system (1), related to the difference between precipitation and a standard crop-evaporation. Measurements.

\section{A4.2.4 Maximum discharge values}

The net precipitation values sometimes may lead to high discharge values. Based on the available data, a maximum value is used, and a surplus is 'store' and added to the next averaging period. This maximum value also was varied a bit, sometimes taken as $0.7 *$ that maximum value. 

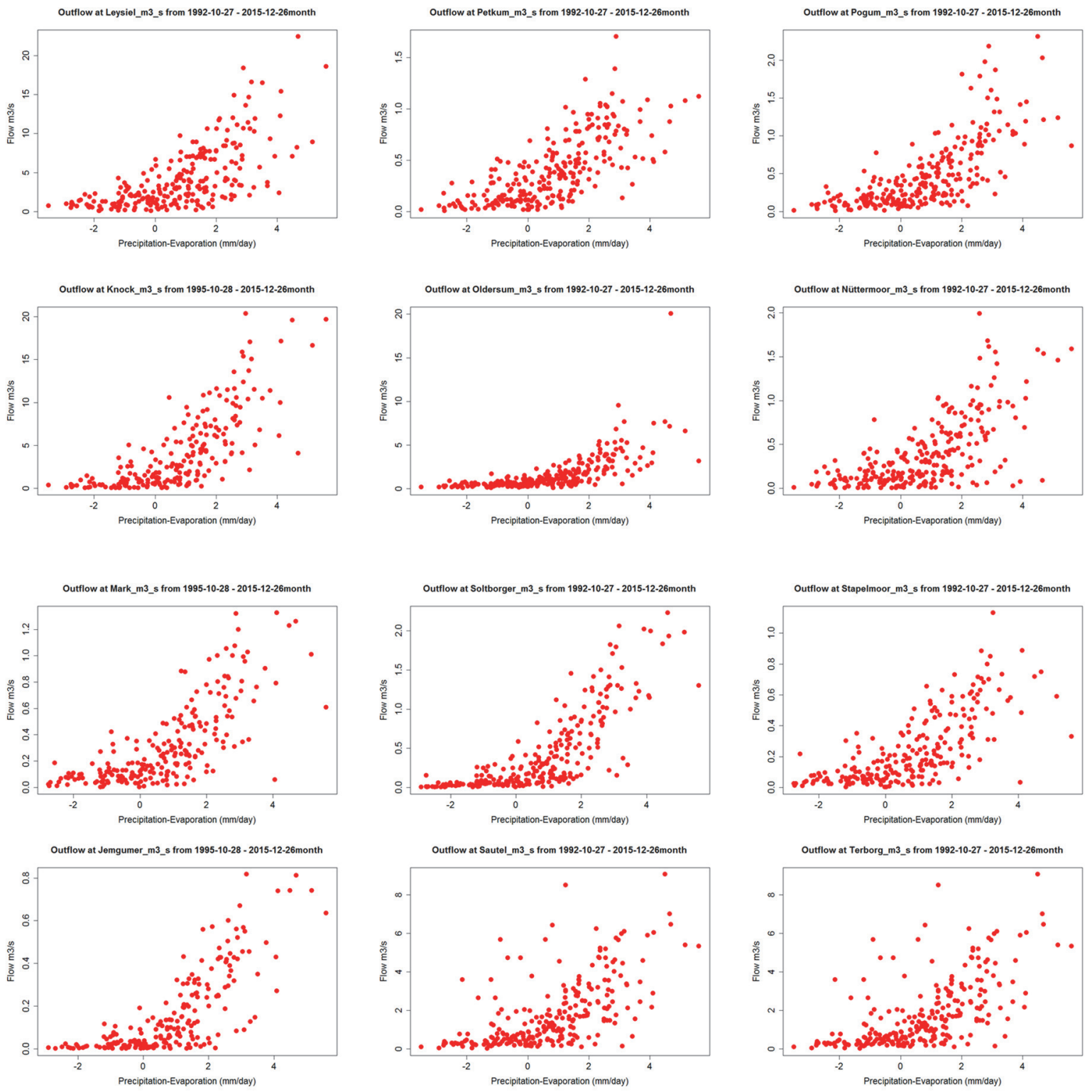

Figure 46 Discharges at the German side of the system (2), related to the difference between precipitation and a standard crop-evaporation. Measurements.

Discharges are not zero when net precipitation gets close to zero or even is negative Figure 44 Figure 46. This will be related to lag periods. Also, discharges can be rather low at a high precipitation surplus. This occurs after dry periods, and rainfall then first saturates soils.

\section{A4.2.5 Completed data sets}

Completed discharge values are presented in Figure 47 - Figure 50. These have been used for the EcoWasp-model computations. 

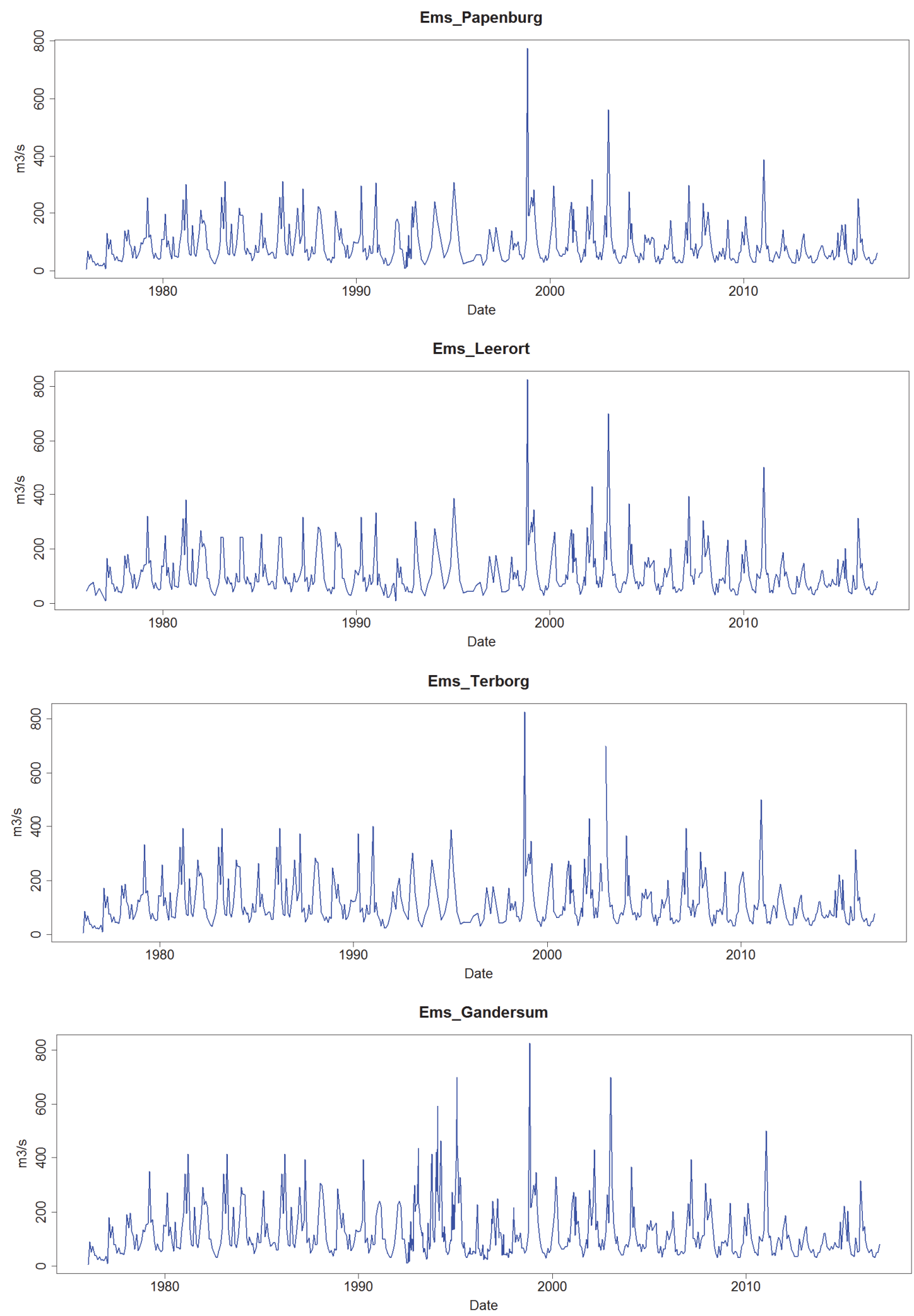

Figure 47 Discharge values for the river Ems. Data plus empty periods completed. 

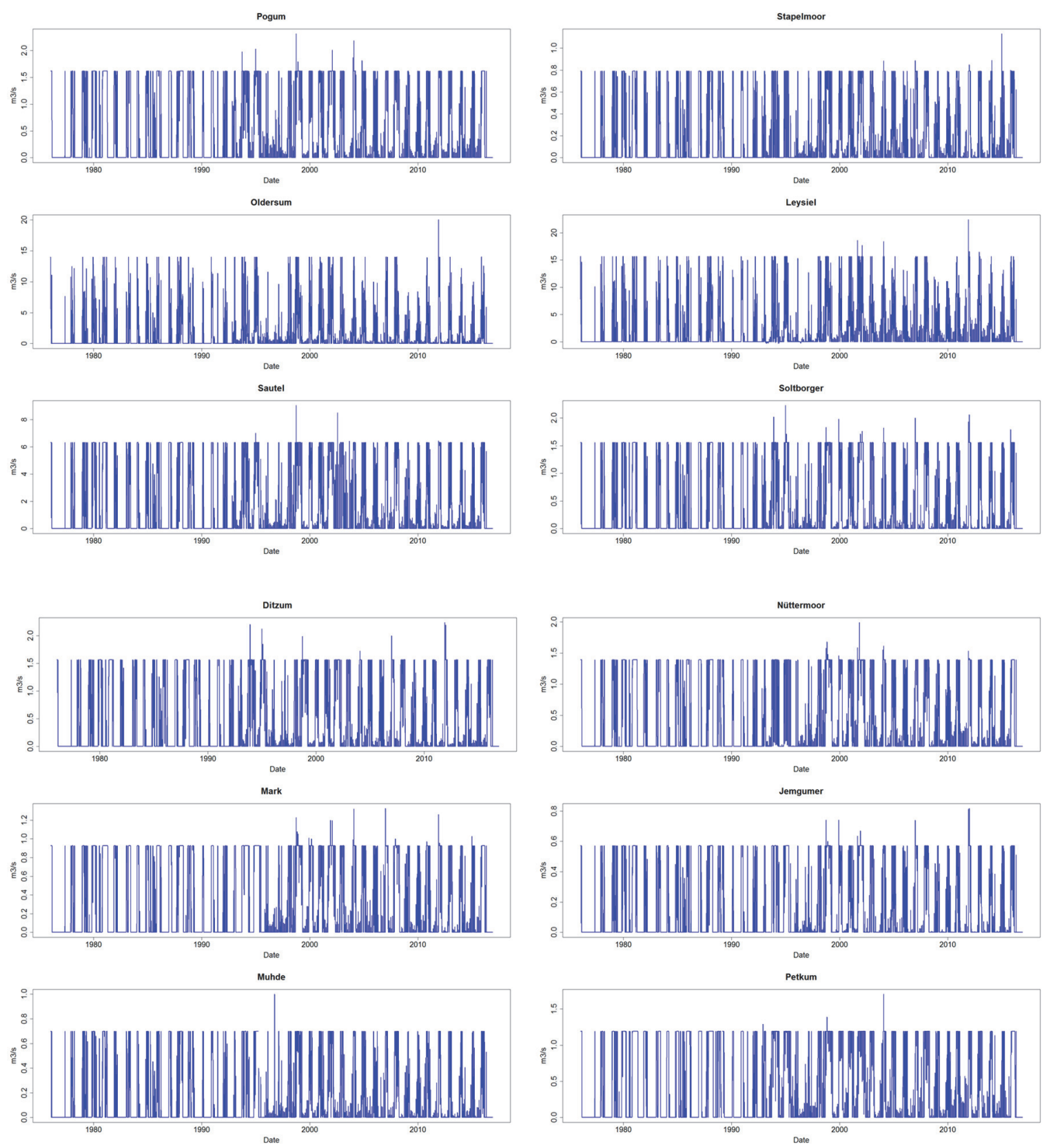

Figure 48 Discharge values for a number of German tributaries. Data plus empty periods completed; empty periods are most common. 

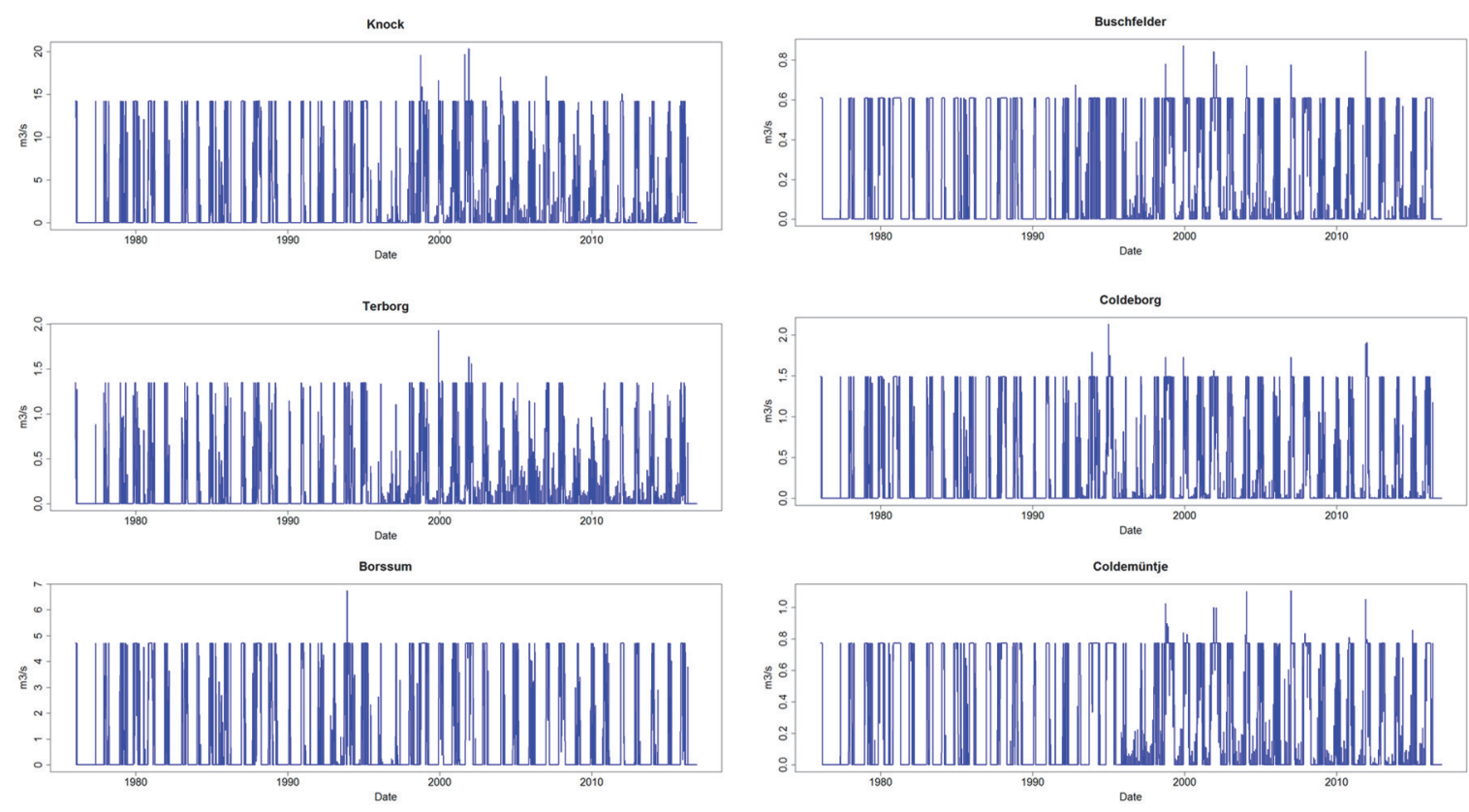

Figure 49 Discharge values for a number of German tributaries. Data plus empty periods completed; empty periods are most common.
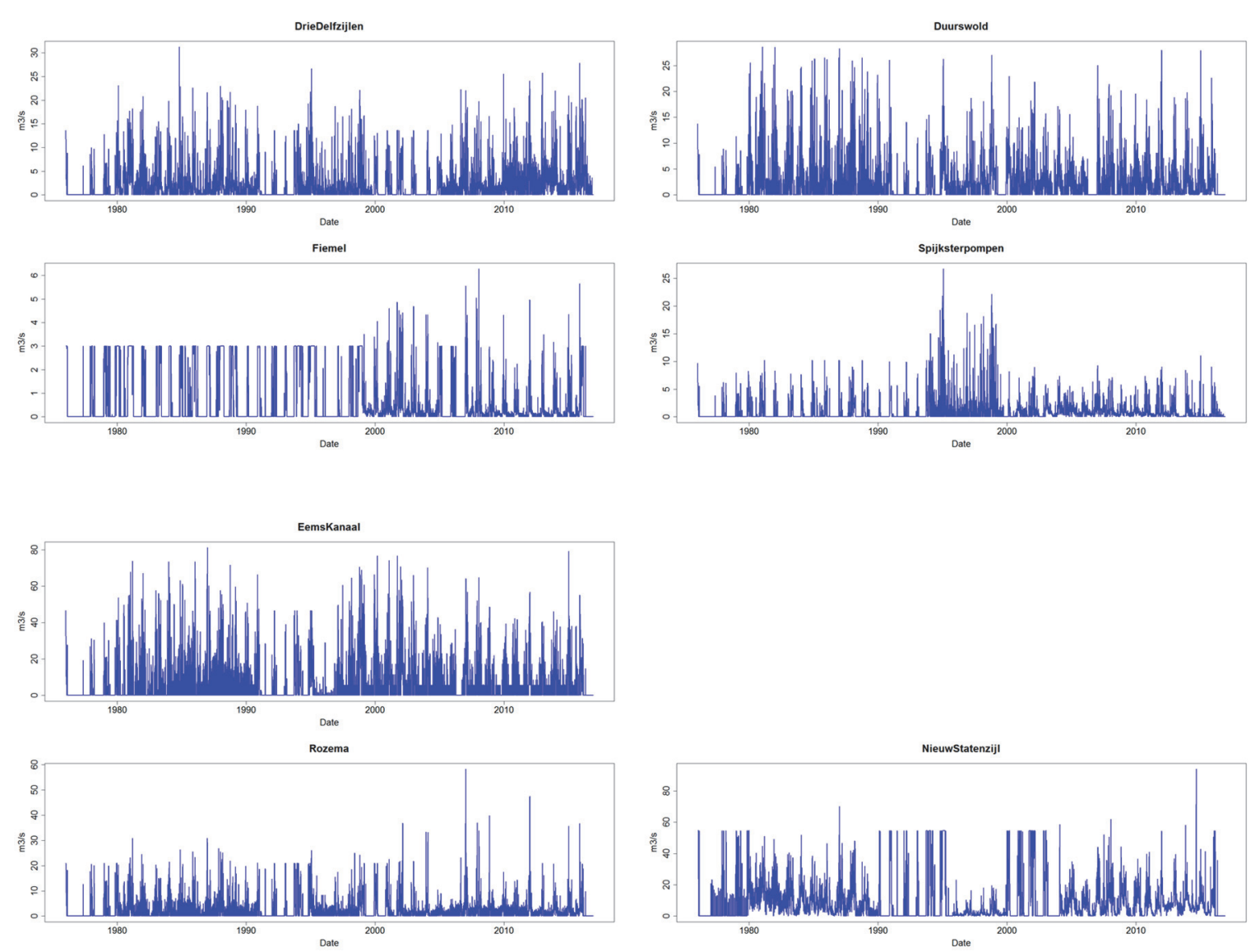

Figure 50 Discharge values for Dutch tributaries. Data plus empty periods completed; data cover most of the years 


\section{A5 Water quality variables, elaboration of Dutch and German water quality data}

\section{A5.1 Introduction}

The Netherlands Rijkswaterstaat (Netherlands Water Board) collects water quality data for socalled Rijkswateren in The Netherlands, from marine water bodies as well as from fresh water systems. These data are primarily collected because of the 'Wet Verontreiniging Oppervlaktewateren' (WVO) from 1969 and, from December 2009 the 'Waterwet' (Water Act), which replaces the WVO. The data serve as indicators whether the water quality targets are achieved or not.

These data also are an important source of information for ecosystem modelling activities; the data give information for boundary conditions, and are very useful as comparison to model results.

The idea is that from the available data some other quantities may be derived that are not directly measured. Especially an estimate for the amounts of algae, diatoms/non-diatoms, humus (refractory organic matter, ROM) and labile organic matter (LOM) may be computed. A first computation concerns the estimation of the phytoplankton content from chlorophyll-a data, the composition of algae (N \& P) and the composition of the remaining fractions: detritus (N \& P) and inorganic matter $(\mathrm{P})$.

A second computation concerns the composition of the dissolved organic fraction (DOM = dissolved organic matter). It appeared from a first check that probably two fractions may be distinguished: one type of DOM that is always present, and one type that appears in the summer period.

A third computation may concern an estimation of the presence of phytoplankton, detritus and the one or two dissolved organic compounds for the whole period relevant for ecosystem simulations. Both the above mentioned two computations concern existing data, this third one implies that for periods where no data exist so far, estimates for DOM, phytoplankton and detritus are produced. 


\section{A5.2 Available water quality data}

From Table 10 is it clear that i) not the whole simulation period (1976-now) is covered by the necessary data sets and ii) the resolution is not always very high. In some cases, only a few data per year were available.

Therefore, values for the empty dates are estimated by comparing the existing data to other systems that do have a complete data set, finding a regression model, and using that model to compute expected data. See below, section A5.3 and on. See also appendices A6 - A8.

Table 10 Overview of available water quality data

\begin{tabular}{|c|c|c|c|c|c|c|}
\hline Site & Abbrev & Site_ID & First date & Last date & NrObs & System \\
\hline Papenburg/Voellen & $\begin{array}{r}\text { PAPBRGVOELL } \\
\text { EMS }\end{array}$ & 70 & $1987-02-04$ & 2016-05-11 & 702 & Ems \\
\hline Leerort & $\begin{array}{r}\text { LEERORT_EM }^{\mathrm{S}} \\
\end{array}$ & 80 & 1987-02-04 & 2007-10-10 & 689 & Ems \\
\hline Terborg & $\begin{array}{r}\text { TERBORG_EM } \\
\mathrm{S}\end{array}$ & 85 & 1983-01-05 & 2016-05-11 & 753 & Ems \\
\hline Gandersum & $\begin{array}{r}\text { GANDERSM_E } \\
M S\end{array}$ & 90 & 1987-04-02 & 2016-05-11 & 771 & Ems \\
\hline Coldemuentje & COLDM & 110 & $1982-01-18$ & 2001-02-14 & 422 & Coldem.Schoepfw-Tief \\
\hline Amdorf & & 181 & $1987-05-14$ & 2016-02-10 & 264 & Leda \\
\hline Leer & & 190 & $1982-01-18$ & 2003-11-05 & 469 & Leda \\
\hline Mittegrossef.(Nord) & MITTGR_NRD & 360 & $1985-07-17$ & $2015-12-01$ & 177 & Sauteler Kanal \\
\hline Neermoor & & 415 & $1982-01-18$ & 2016-02-02 & 421 & Sauteler Kanal \\
\hline Oldersum & & 485 & 1982-01-18 & 2016-02-02 & 432 & Fehntjer Tief \\
\hline Westerende-Kirchloog & & 510 & $1982-01-18$ & 2016-02-02 & 423 & Ems-Jade-Kanal \\
\hline Buntelsweg & & 650 & $1982-01-18$ & 2016-02-09 & 545 & Knockster Tief \\
\hline Leysiel & & 723 & 2001-02-21 & 2016-02-09 & 190 & Speicherbecken \\
\hline $\begin{array}{l}\text { Losplaats Nieuw } \\
\text { Statenzijl }\end{array}$ & & 1103 & $1978-09-25$ & 2016-05-23 & 452 & Westerwoldse $\mathrm{Aa}$ \\
\hline Oostpolderbermkanaal & & 1309 & 1978-09-11 & 2016-06-21 & 448 & Brug Uithuizermeeden \\
\hline Kleine Tjariet & & 1311 & 1978-09-11 & 2015-12-09 & 139 & Duiker Z.O. van Oude Schip \\
\hline Brug 15 Farmsum & & 4102 & $1978-09-18$ & 2016-06-21 & 475 & Eemskanaal \\
\hline Brug Farmsum & & 5101 & 1978-09-11 & 2016-06-21 & 449 & Afw.kanaal Van Duurswold \\
\hline Termunterzijldiep & & 6103 & 2003-01-07 & 2016-06-21 & 140 & Warvenweg thv Baamsum \\
\hline Gemaal Fiemel & & 7101 & 1991-01-15 & 2016-06-21 & 229 & Uitwateringskanaal \\
\hline Damsterdiep & & 7301 & 1978-09-11 & 2015-12-10 & 677 & Brug Ruischerbrug \\
\hline Damsterdiep & & 7302 & 1978-09-11 & 2016-06-02 & 241 & Brug Wirdumerdraai \\
\hline Damsterdiep & & 7318 & 1978-09-11 & 2016-06-15 & 451 & Brug Delfzijl \\
\hline
\end{tabular}

In none of the canals or rivers, silicon-data were available; only for the North Sea boundary were Rijkswaterstaat performed the monitoring. In a first attempt, simply IJsselmeer-data were copied. These turned out to be much too low, and next, river IJssel data from monitoring station Kampen were used. For some reasons, these turned out to be probably too low, and were raised artificially to values that finally gave best simulation results.

Also, German phytoplankton data started only in 2003. Some data could be completed based on a regression analysis. This was the case for the Ems/Gandersum -station. These values were copied 
to Papenburg and Leer (the latter is hardly used as input for the present model set-up). Similar, data for the Ems-Seitenkanal at Borssum could be completed following a regression analysis, and used for the other canals and tributaries that lacked these data.

Particulate organic matter data also are missing for most of the fresh water discharges. These were estimated, see appendix A8.

It is realised that this approach is far from ideal, but it is the best possibility now.

Table 11 Water quality monitoring sites. Additional data produced. Columns: target site, new dates, and site that was used as comparison site (basic data site).

\begin{tabular}{|c|c|c|c|c|}
\hline Site /target & Site_ID & First date & Last date & Basic site, used for comparison \\
\hline Papenburg/Voellen & 70 & 1983-01-05 & 2016-05-11 & Terborg / Ems \\
\hline Leerort & 80 & 1983-01-05 & 2016-05-11 & Terborg / Ems \\
\hline Gandersum & 90 & 1983-01-05 & 2016-05-11 & Terborg / Ems \\
\hline Coldemuentje & 110 & 1978-09-11 & 2016-06-21 & Brug Farmsum \\
\hline Amdorf & 181 & 1983-01-05 & 2016-05-11 & Terborg / Ems \\
\hline Leer & 190 & 1983-01-05 & 2016-05-11 & Terborg / Ems \\
\hline Mittegrossef.(Nord) & 360 & 1978-09-11 & 2016-06-21 & Brug Farmsum \\
\hline Neermoor & 415 & 1978-09-11 & 2016-06-21 & Brug Farmsum \\
\hline Oldersum & 485 & 1978-09-11 & 2016-06-21 & Brug Farmsum \\
\hline Westerende-Kirchloog & 510 & $1978-09-11$ & 2016-06-21 & Brug Farmsum \\
\hline Buntelsweg & 650 & $1978-09-11$ & 2016-06-21 & Brug Farmsum \\
\hline Leysiel & 723 & 1978-09-11 & 2016-06-21 & Brug Farmsum \\
\hline $\begin{array}{l}\text { Losplaats Nieuw } \\
\text { Statenzijl }\end{array}$ & 1103 & $1978-09-25$ & $2016-05-23$ & Westerwoldse Aa \\
\hline Oostpolderbermkanaal & 1309 & 1978-09-11 & 2016-06-21 & Brug Uithuizermeeden \\
\hline Kleine Tjariet & 1311 & 1978-09-11 & 2015-12-09 & Duiker Z.O. van Oude Schip \\
\hline Brug 15 Farmsum & 4102 & $1978-09-18$ & 2016-06-21 & Oostpolderbermkanaal \\
\hline Brug Farmsum & 5101 & $1978-09-11$ & 2016-06-21 & Oostpolderbermkanaal \\
\hline Termunterzijldiep & 6103 & 2003-01-07 & 2016-06-21 & Warvenweg thv Baamsum \\
\hline Gemaal Fiemel & 7101 & 1991-01-15 & 2016-06-21 & Oostpolderbermkanaal \\
\hline Damsterdiep & 7301 & 1978-09-11 & 2015-12-10 & Oostpolderbermkanaal \\
\hline Damsterdiep & 7302 & 1978-09-11 & 2016-06-02 & Oostpolderbermkanaal \\
\hline Damsterdiep & 7318 & 1978-09-11 & 2016-06-15 & Oostpolderbermkanaal \\
\hline
\end{tabular}

\section{A5.3 Data consistency check and completion}

\section{A5.3.1 introduction}

The data as they are present in the files from Rijkswaterstaat, NLWKN, Hunze en Aa's and Noorderzijlvest are not applicable without a check on errors and inconsistencies. Also, data completion is needed.

Data completion may be necessary because of the way the data have been stored. As an example, of the four phosphorus components (ortho-phosphate, dissolved organic phosphate, particulate phosphate and total phosphate) usually not all four are mentioned. If three of these four components have been measured, the fourth can be computed; the practice however is that not always the same three are stored (and, not always three of the four have been analysed at all), so for each monitoring point it must be checked which ones have been stored, and thus, which one 
can be computed. Especially for the nitrogen compounds this concerns a couple of possible combinations.

Thus, if possible, lacking values have computed and added to the tables. This has been done for the combinations:

TOC - POC - DOC : total, particulate and dissolved organic carbon

TP, partP, Pnf, PO4Pnf $\quad:$ total, particulate, total dissolved after filtration and ortho-phosphate

TN, partN, KjN, Nnf, $\mathrm{NH}_{4}, \mathrm{NO}_{2}, \mathrm{NO}_{3}, \mathrm{NO}_{2} \mathrm{NO}_{3}$

: total, particulate, Kjeldahl- $\mathrm{N}$, total dissolved $\mathrm{N}$ after filtration Ammonium, nitrite, nitrate and the sum of $\mathrm{NO}_{2}$ and $\mathrm{NO}_{3}$

Sal, geleid, $\mathrm{Cl}$ : Salinity, Conductivity and chloride-concentration.

Details, and the way it was done is described below. Not mentioned in the list is the content of organic dissolved $\mathrm{N}$ and -P; computed data are added to the table. Also, from suspended solids and data on glowing rests provide a possibility to compute POC as well. These data have been added as well.

Also, data consistency is checked. It appeared that sometimes typos occur. For example, total phosphorus may be lower than ortho-phosphorus in the monitoring records. Sometimes it is possible to repair such an error (when it is obvious what typo was made), and sometimes it is a reason to replace the value by a NA.

\section{A5.3.2 Phosphorus compounds}

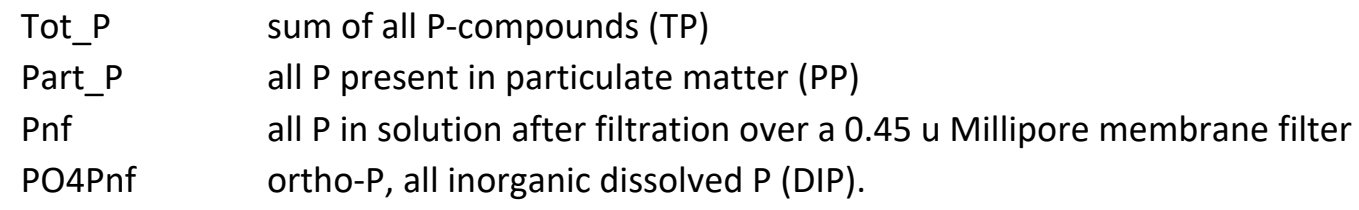

Not mentioned, but added to the data is

DOP all dissolved organic P: the difference between P_nf and PO4Pnf

Pnf is analysed after destruction. Since ortho-P is analysed after adding acid to the solution, the distinction between DOP and DIP is not very precise, and probably a part of DOP is measured as DIP.

In all cases Tot- $P=P a r t-P+P n f$ is used to complete one of the three variables if two of the others are present in the table, and, DOP=Pnf-PO4Pnf is used to find values for DOP.

However, in most case, Pnf is not present, and thus, part_P and/or DOP are not computed. A cheap trick is applied then, and it is assumed that $D O P==0$. After that, part-P and Pnf both are computed.

\section{A5.3.3 Nitrogen compounds}

$\begin{array}{ll}\text { Tot_N } & \text { sum of all } \mathrm{N} \text {-compounds (TN) } \\ \text { Part_N } & \text { all } \mathrm{N} \text { present in particulate matter (PN) } \\ \mathrm{Nnf} & \text { all } \mathrm{N} \text { in solution after filtration over a } 0.45 \text { u Millipore membrane filter } \\ \mathrm{KjN} & \text { Kjeldahl-N, this is all the oxidizable } \mathrm{N} \text {, thus the sum of part-N + NH4+DON } \\ & \begin{array}{l}\text { (dissolved organic } \mathrm{N}) \\ \text { dissolved ammonium }\left(\mathrm{NH}_{4}^{+}\right)\end{array} \\ \mathrm{NH} 4 \mathrm{~N} & \begin{array}{l}\text { nitrite } \\ \mathrm{NO2}\end{array} \\ \mathrm{NO3} & \text { nitrate }\end{array}$


NO2+NO3 sum of nitrate en nitrite.

Not measured, but present is

DON dissolved organic N (also the difference Nnf-NH4-NO2NO3)

Completed is:

$\mathrm{NO} 2+\mathrm{NO} 3=\mathrm{NO} 2 \mathrm{NO} 3$

PartN = Tot-N $-\mathrm{Nnf}$

Added is

DON $\quad$ Nnf $-\mathrm{NH} 4-\mathrm{NO} 2 \mathrm{NO3}$

However, in most case, Nnf is not present, and thus, part_N and/or DON are not computed. A cheap trick is applied then, and it is assumed that DON==0. After that, part-N and Nnf both are computed. Similarly, this is also done for those records where NO2-values are absent. In that case NO2NO3 is set equal to NO3.

\section{A5.3.4 Carbon compounds}

TOC total organic carbon

POC particulate organic carbon

DOC dissolved organic carbon

Completed is

TOC $\quad=P O C+D O C$

Added is

POM_1 $=$ TOC $/ \alpha_{\mathrm{C}, \mathrm{POM}} . \alpha_{\mathrm{C}, \mathrm{POM}}$ is the mass ratio of $\mathrm{C}$ to total organic matter. For Organic matter as $\mathrm{CH}_{2} \mathrm{O}, \alpha_{C, P O M}$ is strictly 0.4 , but for natural matter, $\alpha_{C, P O M}$ has a bit lower value.

POM_2 = ZS* $100-$ perGR)/100. ZS = the content of suspended solids, and perGr the percentage glowing rest. If POM_1 is absent, POM_2 is added.

\section{A5.3.5 Conductivity, salinity and chloride content}

These values are related, and can be used to convert the one into the other.

Chlorinity concerns, based on the method of analysis, the sum of chloride, bromide and iodide in water-expressed as gram per kilogram. But, the two latter forms are of minor importance, and thus is the measured value like the chloride content.

Salinity is the total of dissolved matter in water $\left(\mathrm{g} \mathrm{kg}^{-1}\right)$; the relationship with chlorinity is (Wooster, Lee, and Dietrich, 1969):

$S=1.80655\left[\mathrm{Cl}^{-}\right]$

$$
\left(\mathrm{g} \mathrm{kg}^{-1}\right)
$$

[Cl-] is expressed in $\mathrm{g} \mathrm{kg}^{-1}$.

Conductivity strongly depends on the content of conducting anions and cations, and thus on salinity. 
There are precise conversions (UNESCO-standard; Fofonoff \& Millard, 1983), but for the present situation a simpler conversion as the one by Williams (1986) is satisfactory:

Salinity $=0.4665$ cond $(25)^{1.0878}$

with

$$
\operatorname{cond}(25)=\frac{\operatorname{cond}(T)}{1+f t \cdot(T-25)}
$$

Salinity $\quad \mathrm{g} \mathrm{kg}^{-1}$,

Cond (=conductivity) in $\mathrm{mS} \mathrm{cm}^{-1}$

T

${ }^{\circ} \mathrm{C}$.

In many cases, conductivity is expressed in $\mu \mathrm{S} \mathrm{cm}^{-1}$, thus a factor 1000 higher.

Williams gives $\mathrm{ft}=0.02$, but he used salinity as $\mathrm{g}^{-1}$ (not per unit of mass). At $\mathrm{T}=30^{\circ} \mathrm{C}$ the values are about the same, but deviate for lower temperatures (Figure 51). By choosing $\mathrm{ft}=0.0185$ these deviations disappear (Figure 53).

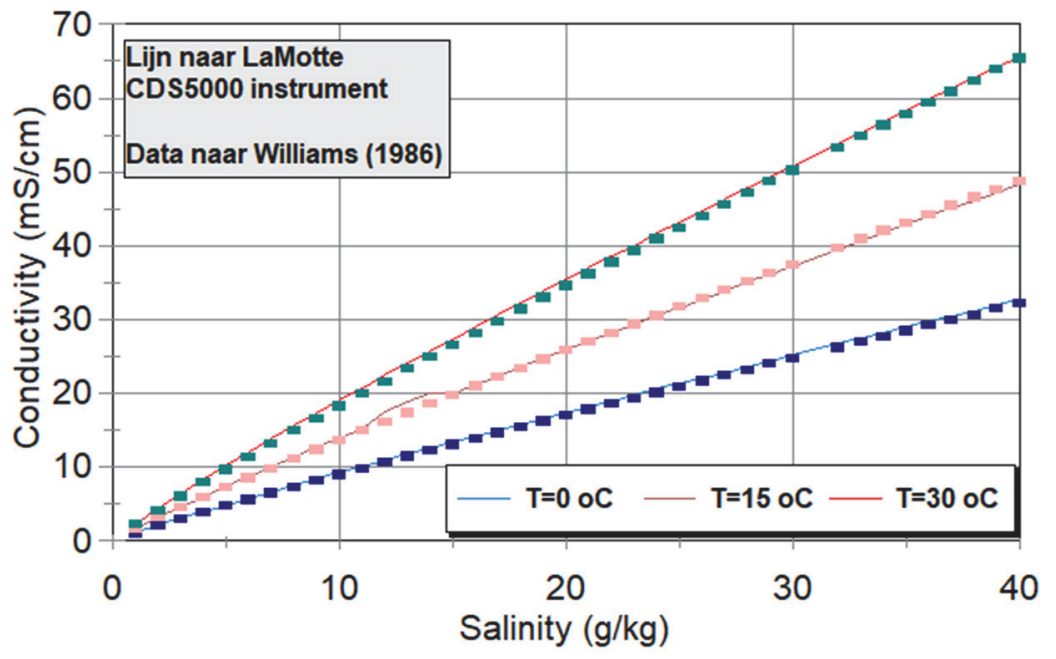

Figure 51 Conductivity and salinity after Williams (1986)

In The Netherlands, $20^{\circ} \mathrm{C}$ is used as standard temperature for the conductivity measurements. The ratio between cond $(20)$ en cond $(T)$ is:

$\operatorname{cond}(T)=\operatorname{cond}(20) \cdot \frac{1+f t \cdot(T-25)}{1+f t \cdot(20-25)}$

Rijkswaterstaat uses in its tables conductivity at $20^{\circ} \mathrm{C}$, and uses the UNESCO-method (Fofonoff \& Millard, 1983) as temperature relationship. The relationships are given in Figure 53. Additions 
based on salinity and chlorinity are shown in showing that these additions are consistent with the other data (Figure 52).

The German data (NLWKN) are based on conductivity values at $25^{\circ} \mathrm{C}$. These have been recomputed to values at $20^{\circ} \mathrm{C}$.

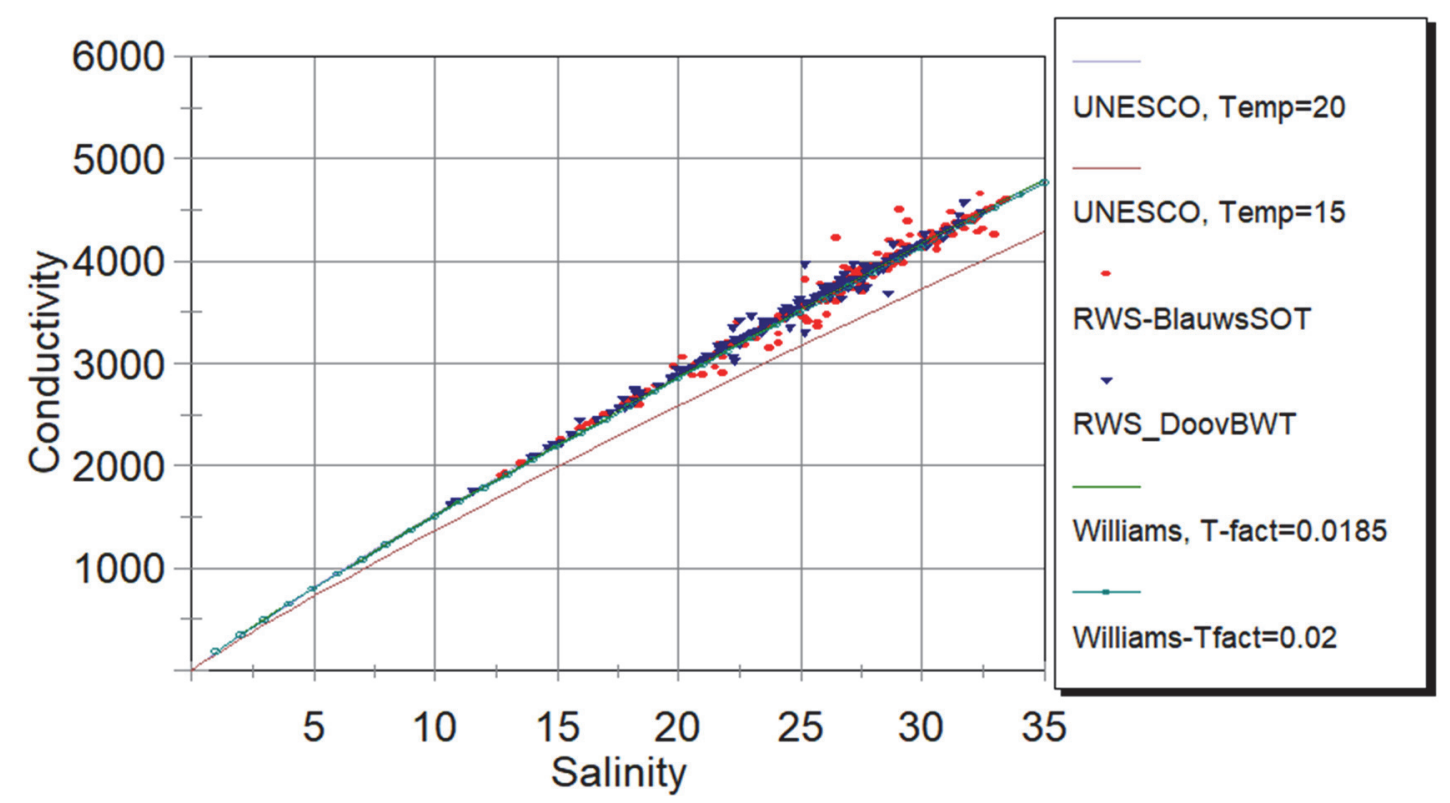

Figure 53 Salinity and conductivity according to UNESCO (Fofonoff \& Millard1983), Williams (1986) and RWS-measurements in the western Dutch Wadden Sea (Blauwe Slenk-Oost and Doove Balg-West)

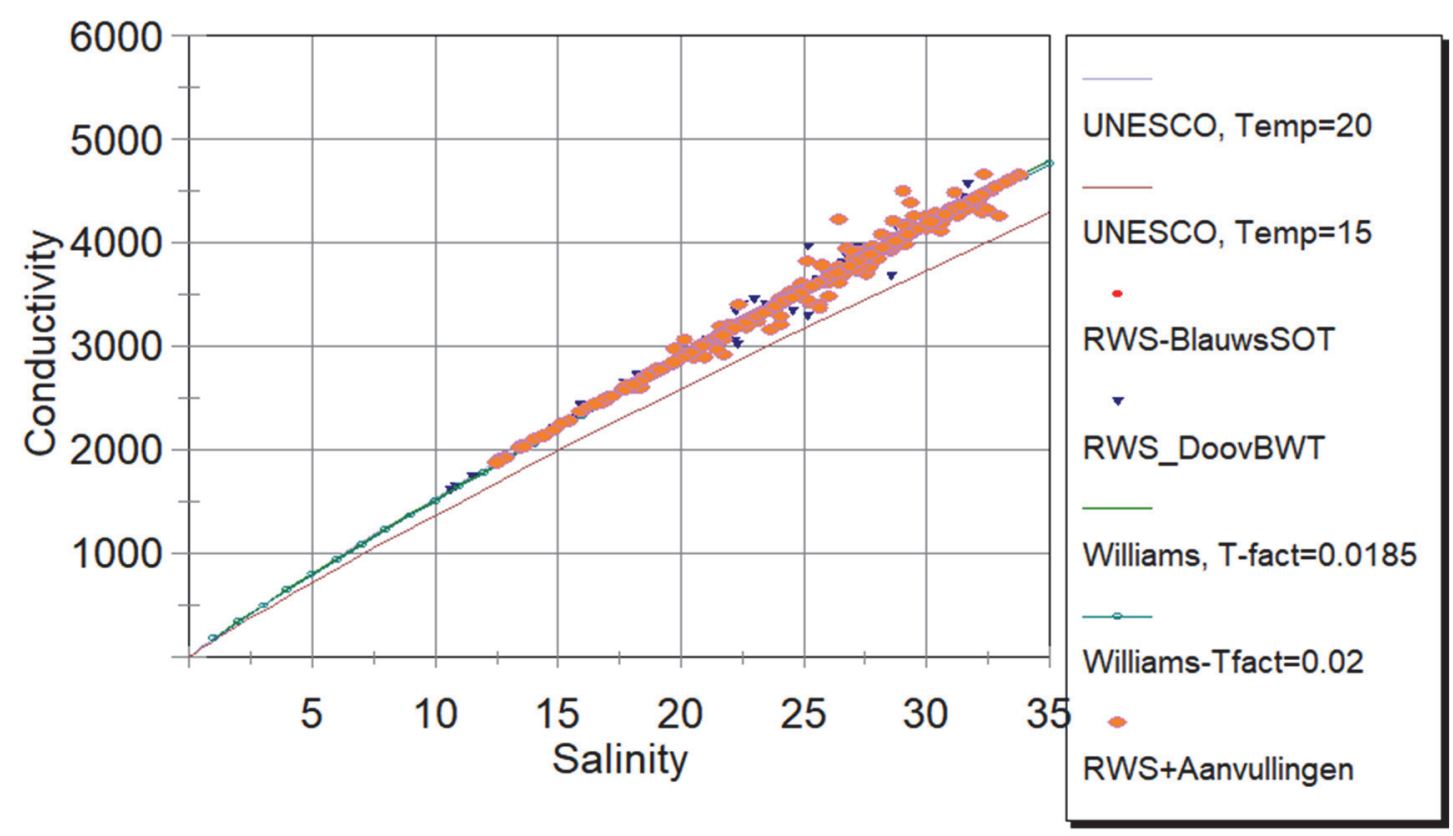

Figure 52 As Figure 53, plus the addition to the Rijkswaterstaat dataset 


\section{A6 Estimating time series for the whole period 1976-now for all variables}

When simulating the ecological behaviour of an ecosystem, boundary values should be as good as possible. In several cases, some elaboration of existing data is necessary to obtain best possible time series. Some of the canal data exist a limited period, and can be compared with another more or less similar- dataset. Using these relationships, best estimates for the outflow characteristics may be computed.

The used R-model could be

$\operatorname{Val}_{\text {SiteB }}=a+b \cdot$ Val $_{\text {SiteA }}+$ factor $($ Month $)$

here, a linear relationship is assumed, with some variation allowed per month. This variation implies that per month another offset (the value for parameter a) is allowed. In a previous exercise, it has proved to be rather successful (Brinkman, 2008), but the fixed value for the offset appeared to be a disadvantage, especially when state variables were considered that showed a large change in concentration with time. A second model

$\operatorname{Val}_{\text {SiteA }}=a+b \cdot V_{\text {Sal }} l_{\text {SiteB }} *$ factor $($ Month $)$

has proven to suit better. 


\section{A7 Completing temperature data series}

Water quality data for the monitoring sites inside the system are needed for comparison, and then incomplete data sets are not limiting the computations. This is not valid for temperature, since it affects all biological processes. Thus, incomplete temperature data sets must be completed.

Two methods have been tested, both resulting in three estimated temperature data sets; one for deep, middle deep and shallow systems each.

The first method is based on a statistical method: measured values and running-average data series for air temperature and wind velocity (both KNMI-data for weather station Eelde, KNMI 2016) are evaluated using a GAM. The resulting model is used to predict temperature values for the whole 1976-2016 period.

The second method is based on an idea by Wolf Mooij (NIOO, Wageningen), saying that water temperature can readily be estimated from air temperature using a three-parameter model (in fact: four parameters, but one is not active). The model reads

$T w a t 1=T w a t 0+\frac{d T}{d t} \Delta t$

With Twat0 and Twat1 temperatures on each day and its previous day, dT/dt the increase rate of T and $\Delta \mathrm{t}$ the time step (which is 1 day in this case).

$\mathrm{dT} / \mathrm{dt}$ follows from

Tdiff $=$ Tair - Twat

$x x 1=$ Tdiff $* b b$

$d d a y=$ YearDay $-d d 0$

$s s 1=\sin \left(2 \frac{\pi \cdot d d a y}{365.25}\right) \cdot a a$

$\frac{d T}{d t}=x x 1+s s 1+f f$

And thus:

$T w a t=T w a t+\frac{d T}{d t} \Delta t$

Here, aa, bb, dd 0 and ff are parameter. dd 0 is set to 81 as standard. At that day in the year, ss 1 is zero. aa and bb both account for the sensitivity of $\mathrm{dT} / \mathrm{dt}$ for changing conditions. aa regulates the effect of the day-in-the-year, and bb the influence of air temperature on the changing water temperature. It will be clear that for deep waters aa and bb will be smaller than for shallow waters.

Table 12 Temperature parameters for three different water types.

\begin{tabular}{|c|c|c|c|}
\hline Parameter $\backslash$ Water & Shallow & Middle & Deep \\
\hline aa & 0.40 & 0.10 & 0.03 \\
\hline bb & 0.40 & 0.15 & 0.05 \\
\hline dd0 & 81 & 81 & 81 \\
\hline $\mathrm{ff}$ & 0 & 0 & 0 \\
\hline
\end{tabular}



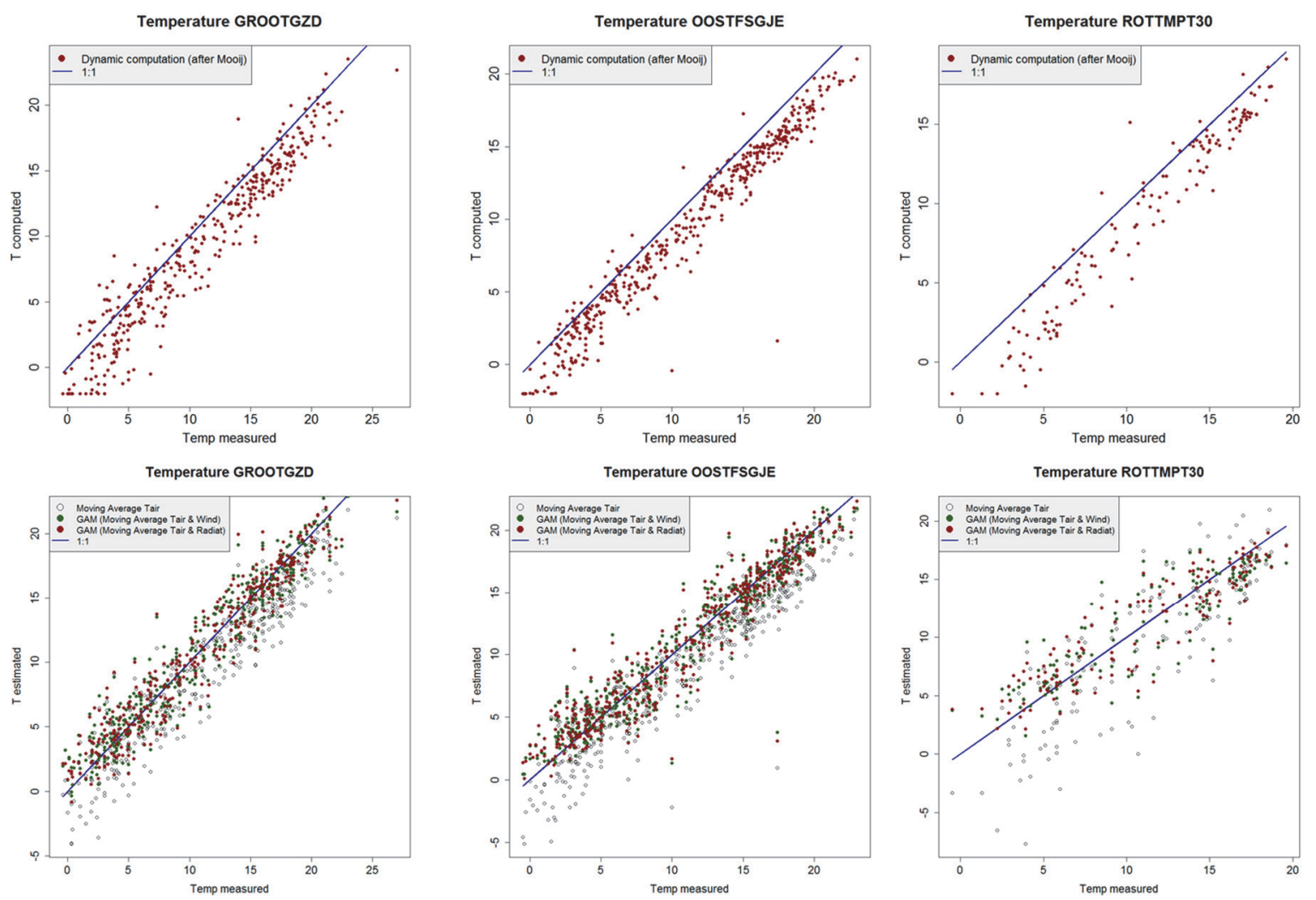

Figure 54 Measured (X-axis) and estimated (Y-axis) temperatures at thee sites. Groote Gat Zuid (left, shallow), OostFriese 6

Temperature GROOTGZD
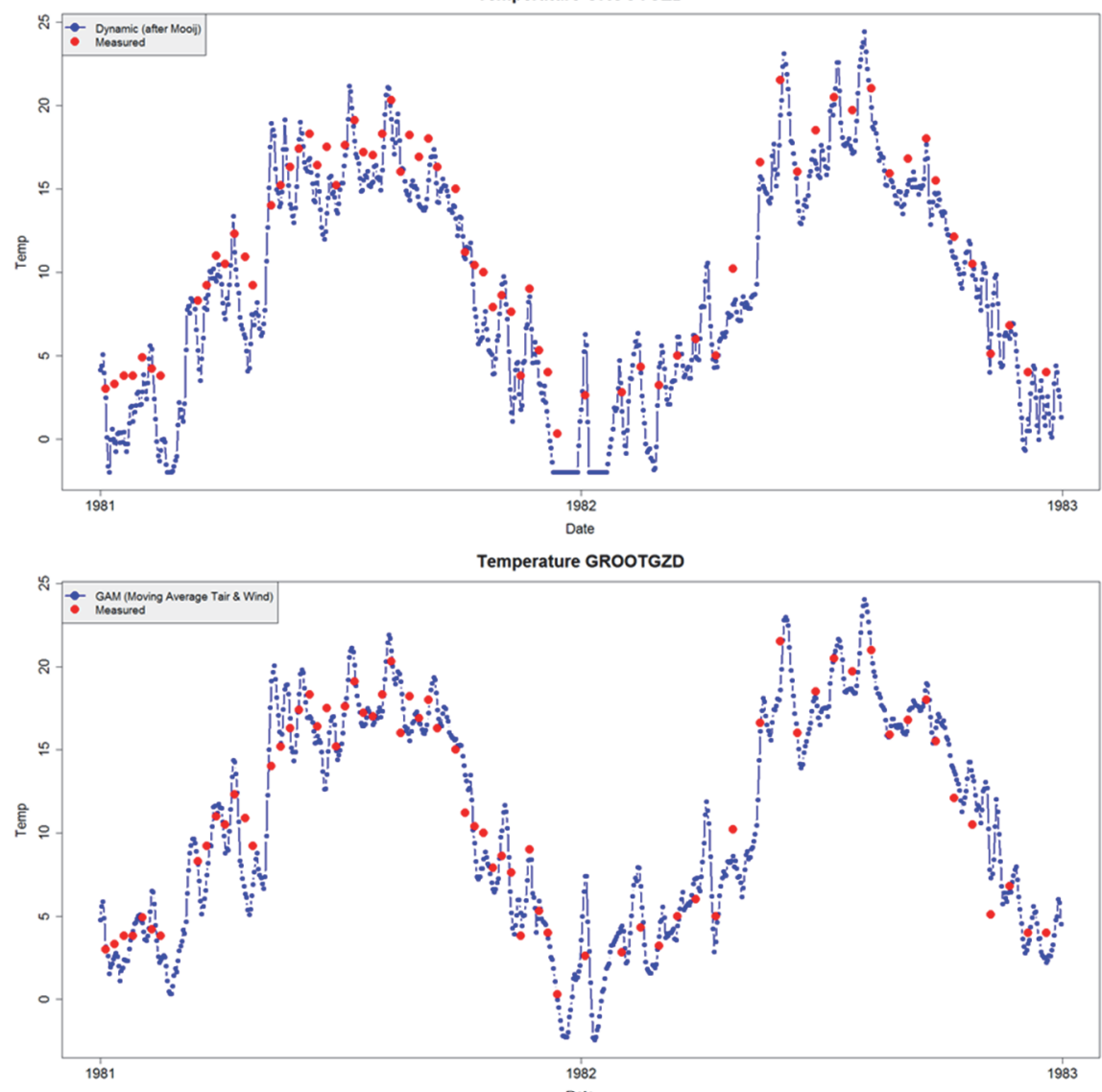

Figure 55 Computed (blue dots) and measured (red dots) temperature at Groote Gat Zuid (shallow area, Dollard, years 1981 - 1982. Upper: dynamic method (with -2 ${ }^{\circ} \mathrm{C}$ as arbitrarily chosen minimum value), lower: statistical method. 

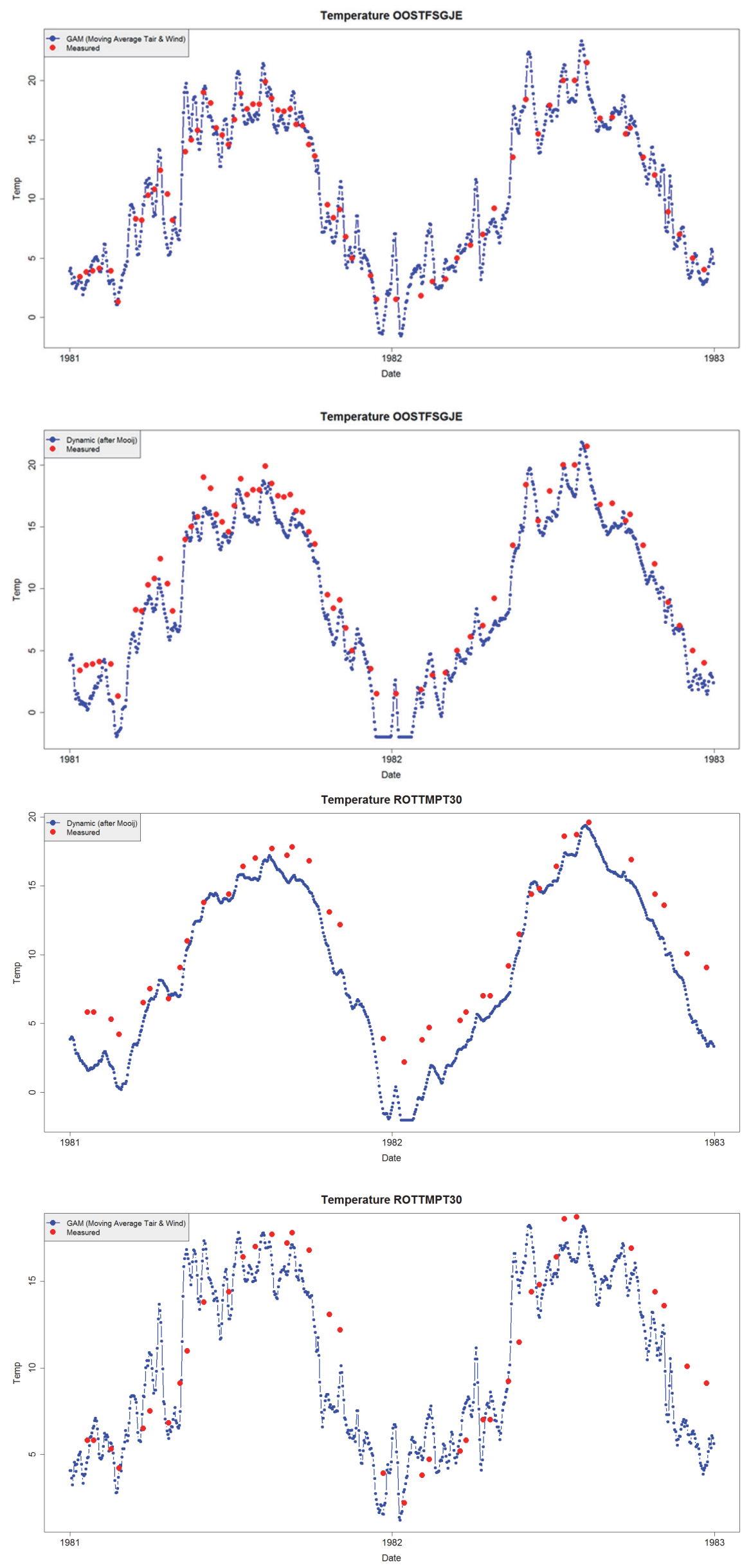

Figure 56 Computed (blue dots) and measured (red dots) temperature at Oostfriese Gaatje (middle deep area, upper) and Rottum 30 (deep area, lower). Years 1981-1982. Each: dynamic method first (with $-2^{\circ} \mathrm{C}$ as arbitrarily chosen minimum value), statistical method second. 
The results of these exercises are applied to water all similar bodies, and serve as template for all compartments. 


\section{A8 Manual adaptation of input time series}

Despite all the attempts to correct and complete data series, some gaps remain. Then, manual completion or correction was the final possibility to create complete time series for an input.

This was done for several situations, listed here.

i) For almost all German inflows chlorophyll-a data are available from 2003. These are 'corrected' by taking the Westerwoldse Aa- data for fresh water diatoms and nondiatoms. Hopefully, the symmetry is good enough since de WWA originates from a peaty area, as do most of the German inflows.

ii) Detritus-data. It appeared that none of the fresh water discharges contained data for detritus, called ROM (Refractory organic matter) in the model. This was added by hand, but it appeared to be rather difficult to find data that might be trusted.

First studied was a relationship between $\mathrm{BZV}_{5}$ (biological oxygen demand after 5 days of incubation at $20^{\circ} \mathrm{C}$ ) and CZV (the chemical oxygen demand, after total oxidation of the sample) (both expressed in $\mathrm{mg} \mathrm{O}_{2} \mathrm{dm}^{-3}$; the latter is supposed to be 32/30 times the content of organic matter in the sample). This is dissolved + particulate matter.

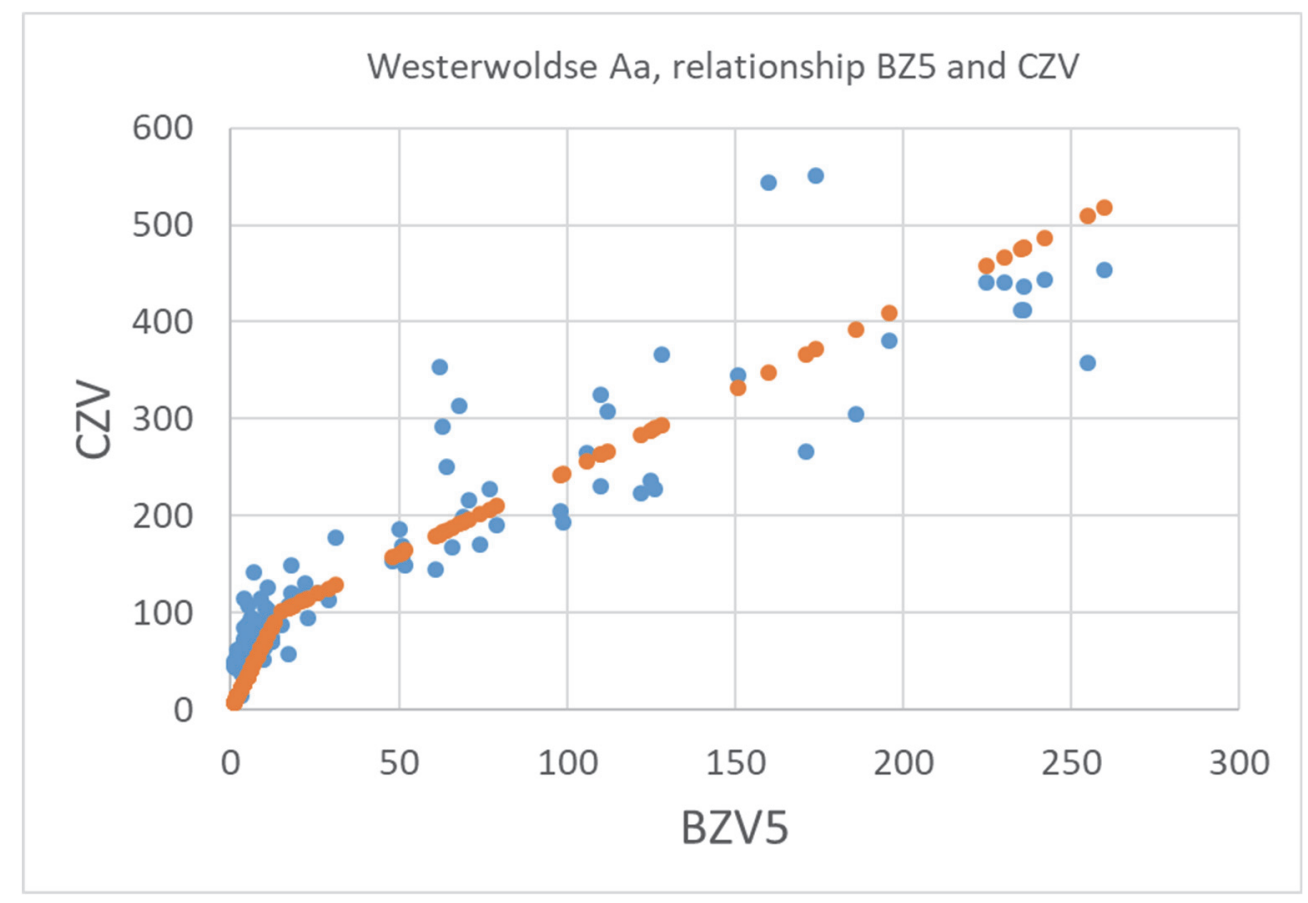

Figure 57 Relationship between BZV5 and CZV for the Westerwoldse Aa. This was used to construct data series for detritus for all fresh water discharges, based on existing BZV ${ }_{5}$ data, or simply copied from more or less similar discharges. The latter choice was rather ad hoc.

Westerwoldse Aa.

Figure 57 shows the relationship for the Westerwoldse Aa where no POC or TOC data were available (except from some scarce values). 

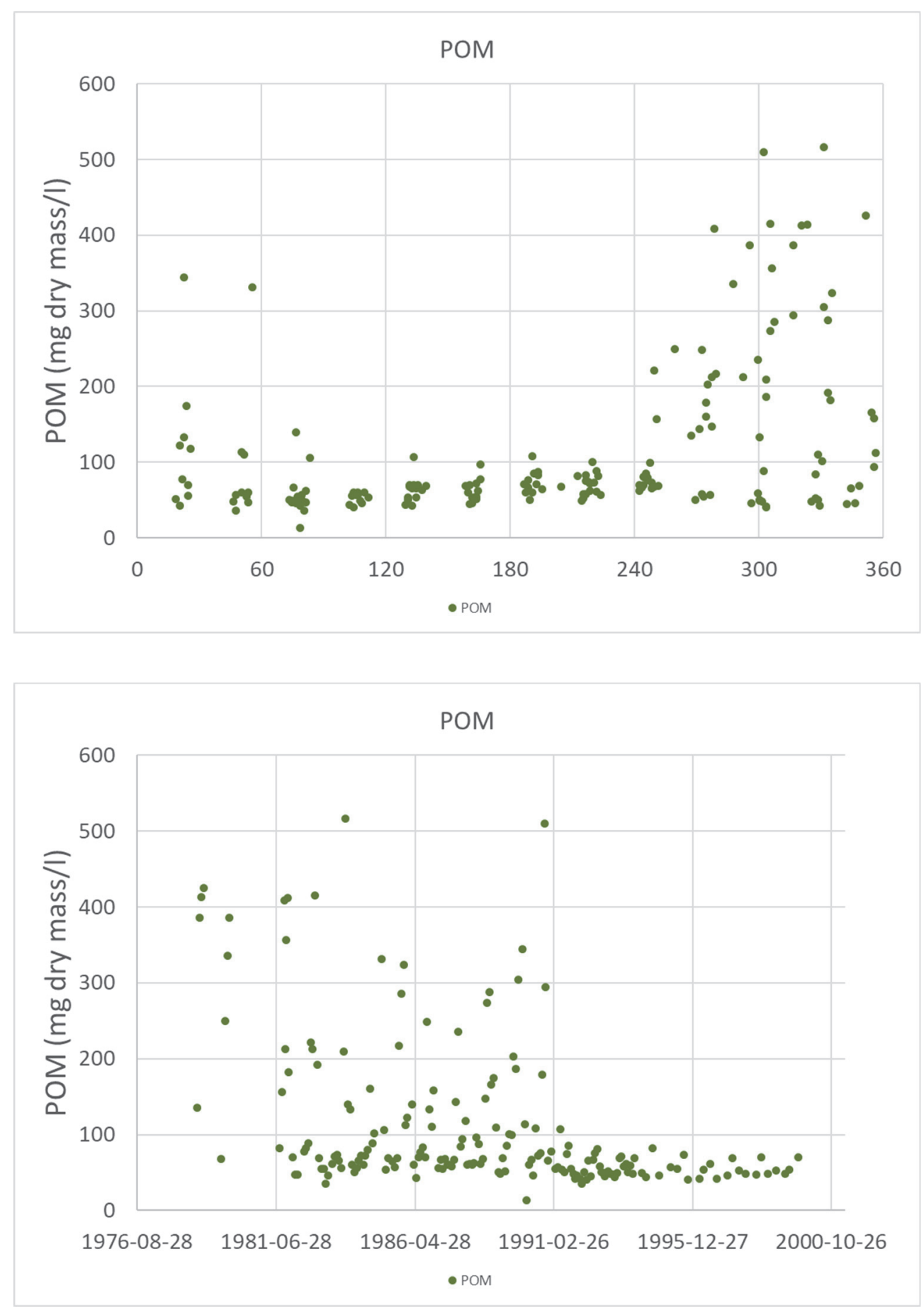

Figure 58 POM-values as 30/12 * POC values, Westerwoldse Aa. Upper: as function of day in the year, lower: 1978-2000 values.

From Figure 58 it becomes clear that values are high before 1991 and, it concerns solely the autumn and winter period; the time of the potato-starch and sugar season. These series are used to produce data series for the period after 1991: these stay the same for all days. The data were analysed per month and per year (Figure 60); and a final estimate was constructed as shown in Figure 59. 


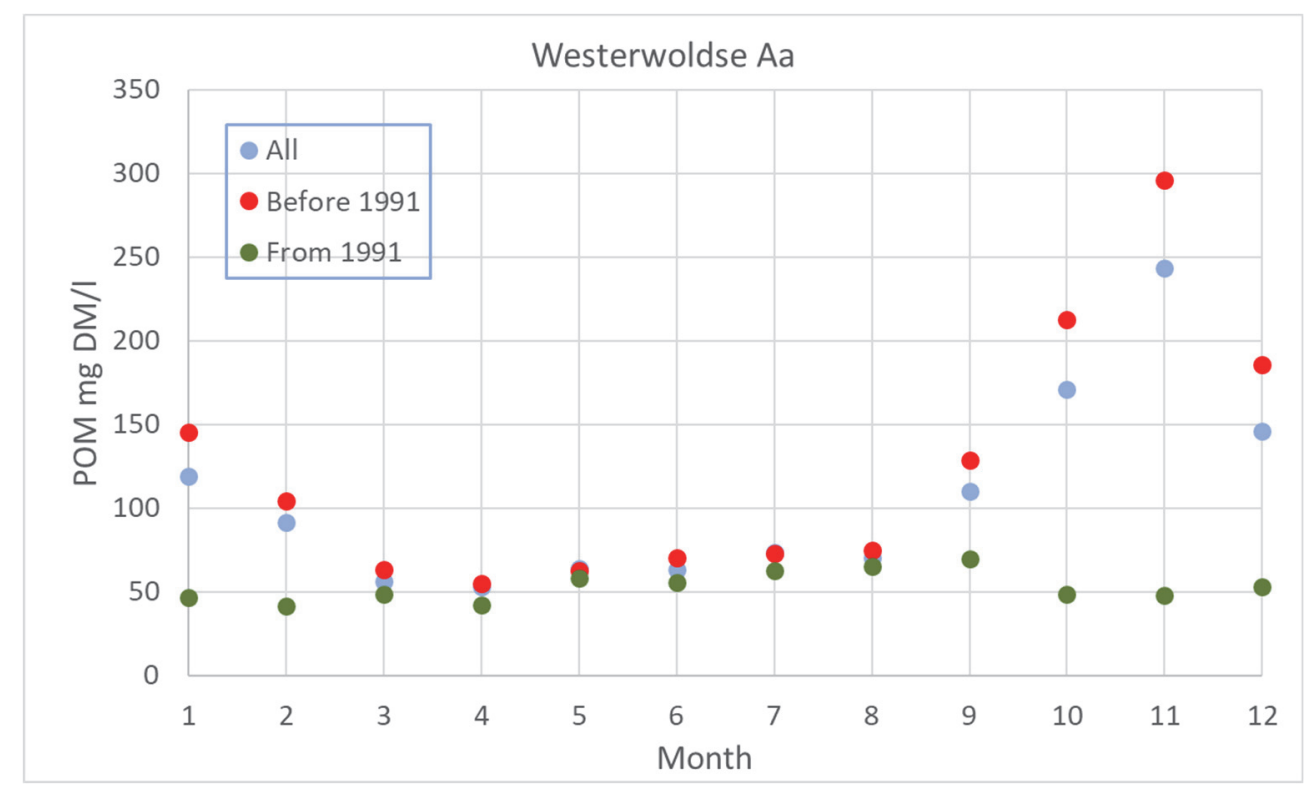

Figure 60 Monthly average for particulate organic mater in the Westerwoldse Aa, derived from chemical oxygen demand values $(* 30 / 32)$.

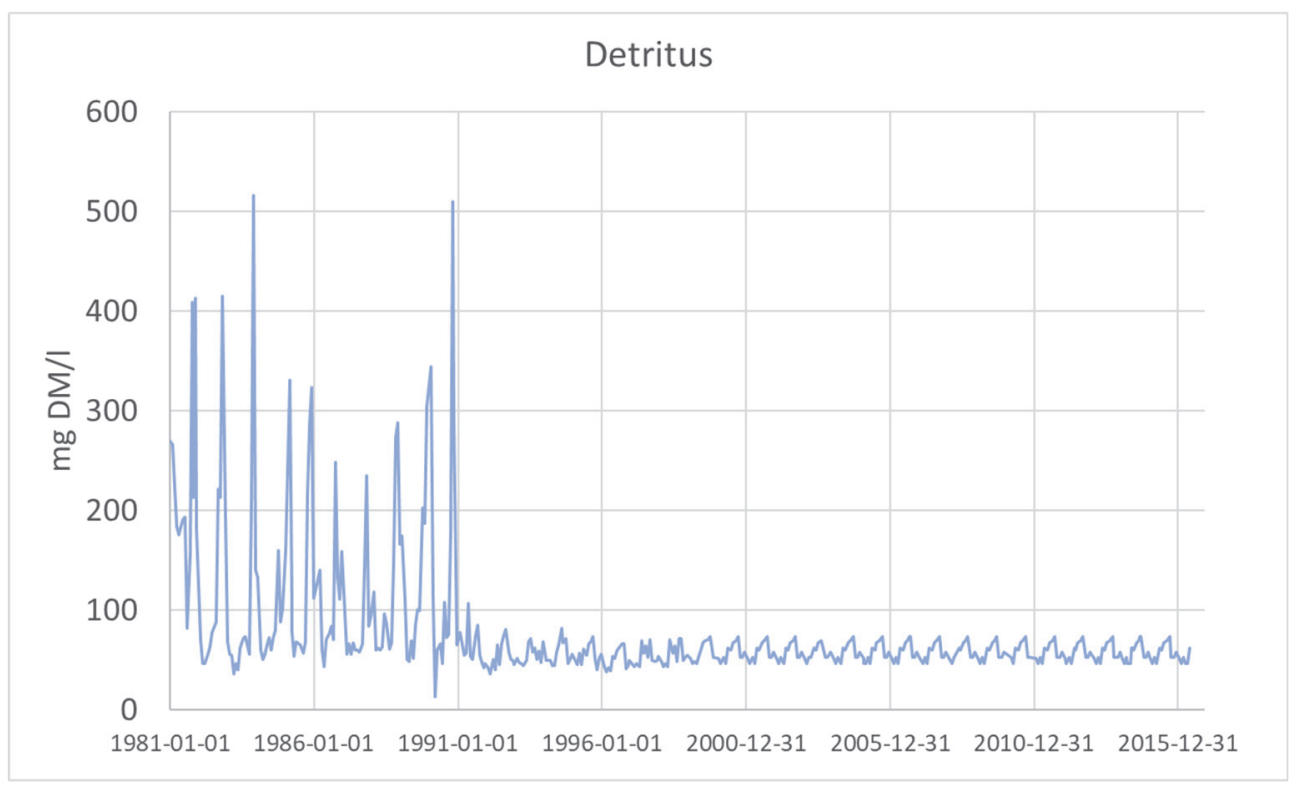

Figure 59 Detritus values estimated from year and month averages. The latter are very different for the periodes before and from 1991.

\section{Leda at Amdorf}

Figure 61 shows available data for particulate organic matter (expressed as dry matter, detritus) and biological oxygen demand $B Z V_{5}$. Here it is clear that all those years, $B Z V_{5}$ roughly stays the same, while the detritus content increases drastically. The explanation seems that the relatively easily degradable matter does not change a lot, but the refractory organic matter (humus-like substances) increase a lot. However, earlier chemical oxygen demand data seem to point at a rather constant level of refractory matter. Striking is the difference between the Leda CZV-values and those for the Westerwoldse Aa (Figure 57); in the WWA it goes up to $500 \mathrm{mg} / \mathrm{l}$, more than 
three times the maximum Leda-values. This is especially the case in the 80's, when still organic waste water discharges were reality in de WWA.

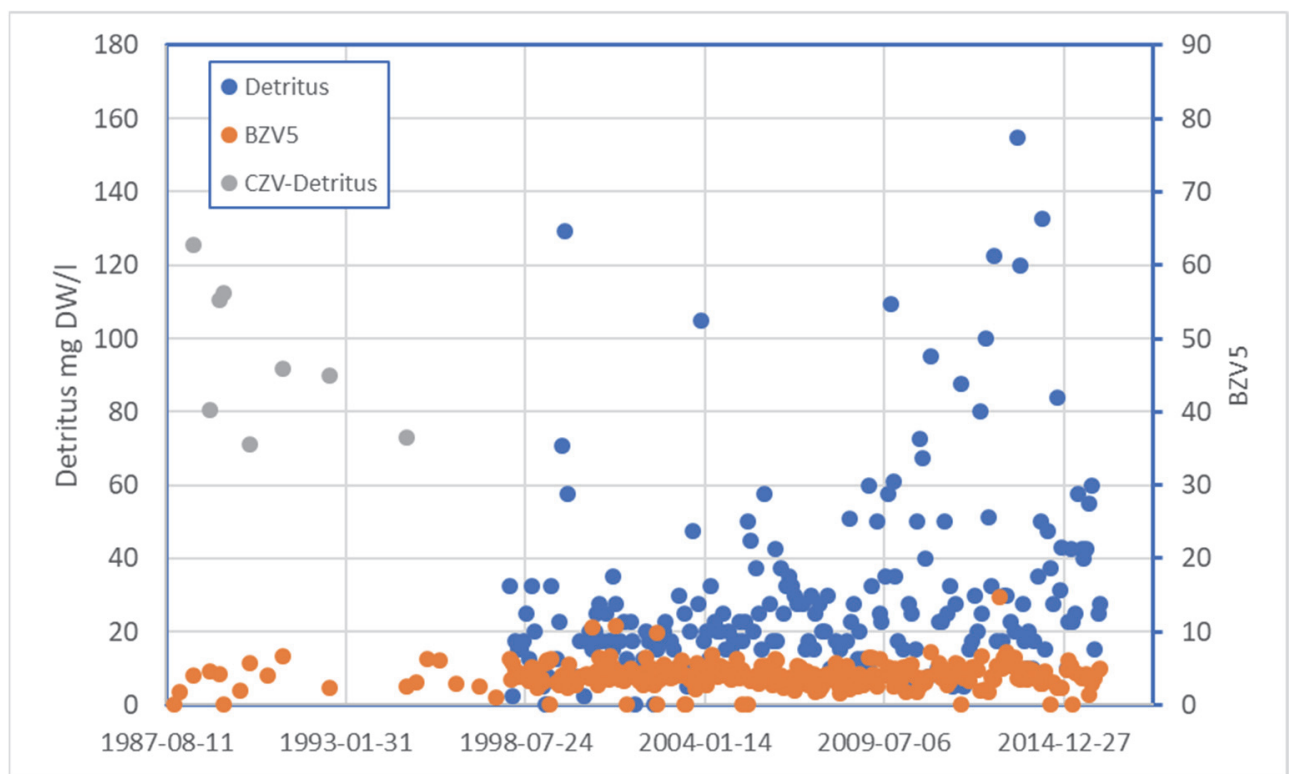

Figure 61 Data for Amdorf (Leda) for POC, expressed of detritus-dry mass (mg dry mass/I), $\mathrm{BZV}_{5}$ (biological oxygen demand, $\mathrm{mg} \mathrm{O}_{2} / \mathrm{l}$ ) and CZV (chemical oxygen demand, $\mathrm{mg}_{\mathrm{O}} \mathrm{O} / \mathrm{l}$ ). Amdorf is roughly $10 \mathrm{~km}$ upstream from Leer.

\section{Ems at Papenburg}

Next, data for the Ems at Papenburg are relevant (Figure 62 and Figure 63). The latter shows a clear seasonal pattern the first a slight increase from the beginning of the 90 's.

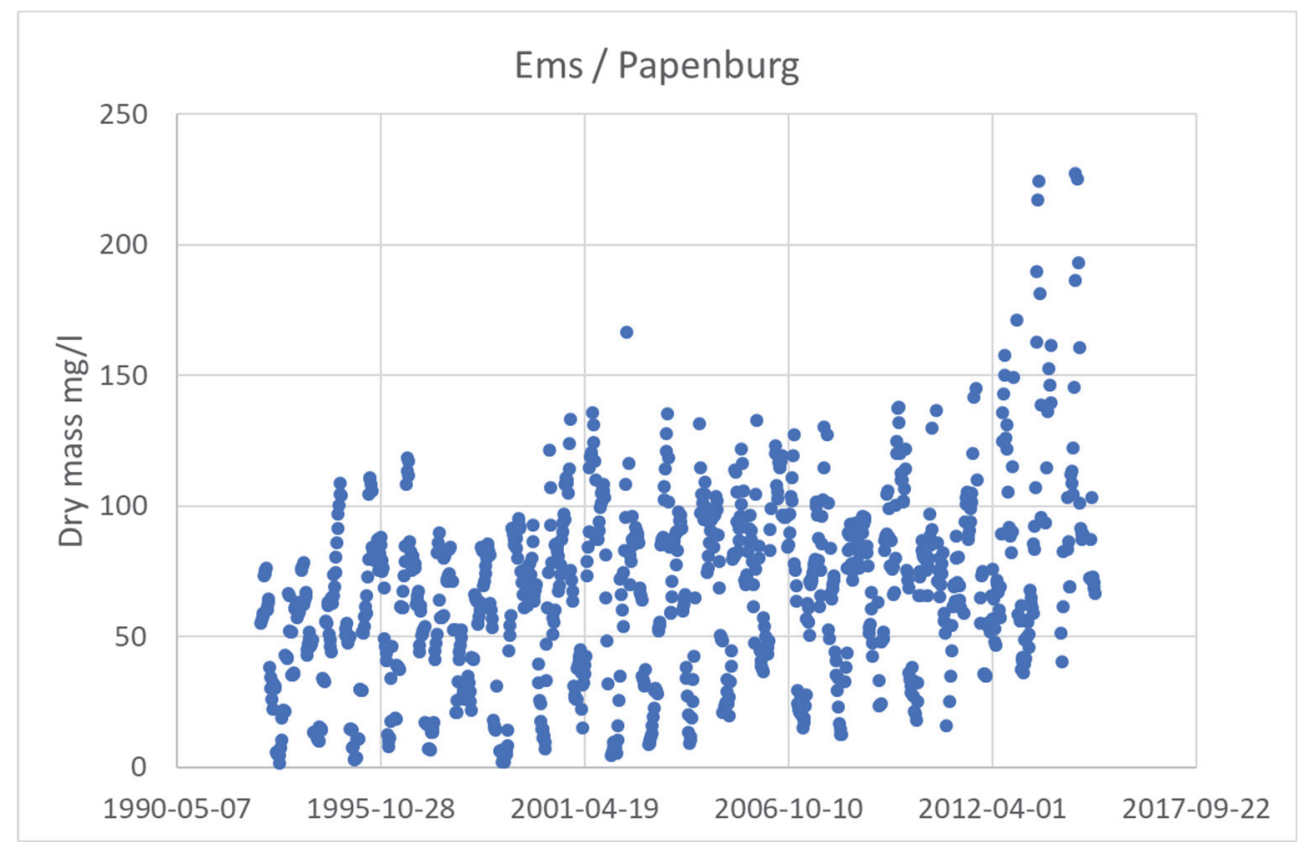

Figure 62 Detritus data (as 30/12*POC)-data for the river Ems at Papenburg Voellen. Timeseries. 


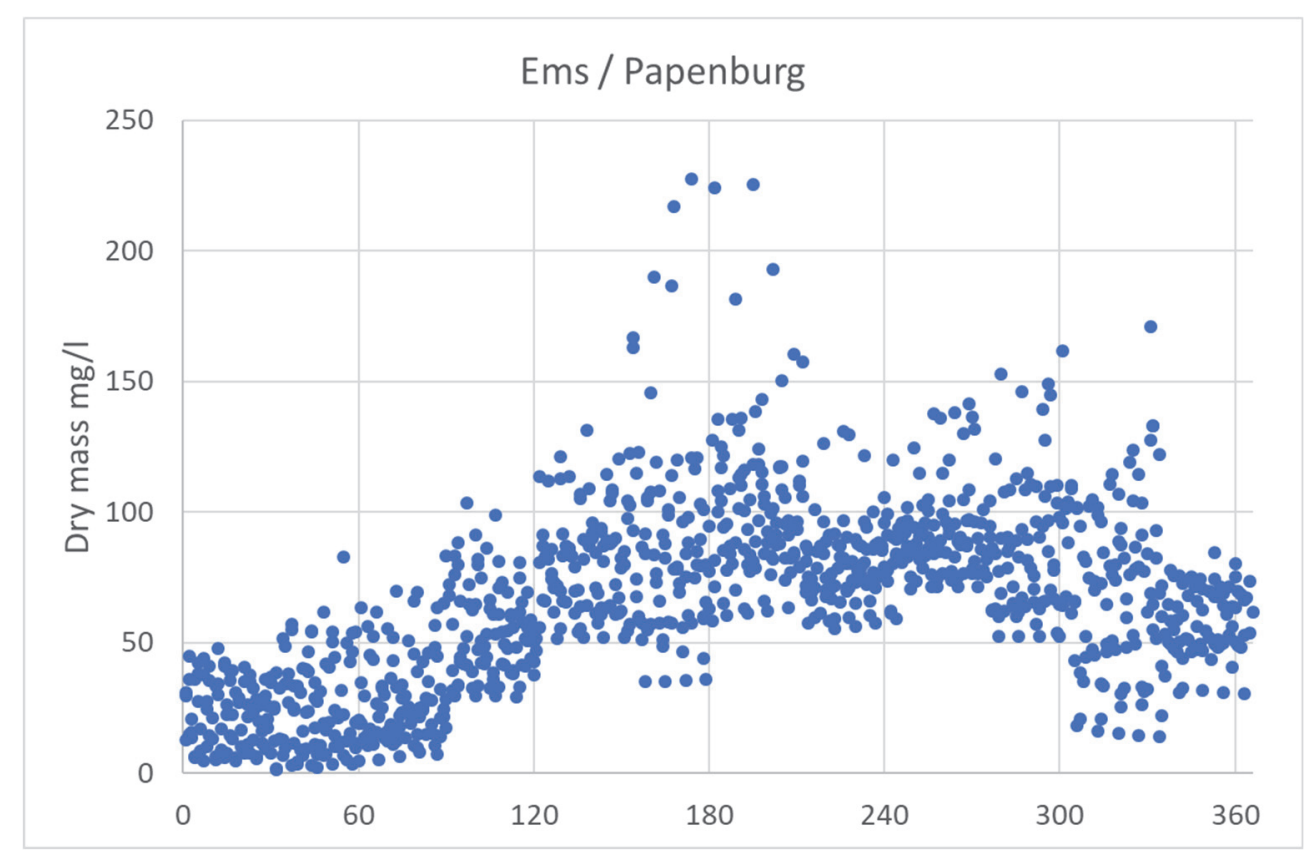

Figure 63 Detritus data (as 30/12*POC)-data for the river Ems at Papenburg Voellen as a function of the day in the year.

These data were analysed (year and seasonal variations), and used to construct data series for the period before 1992. Declining a bit for earlies years, and with the seasonal pattern found.

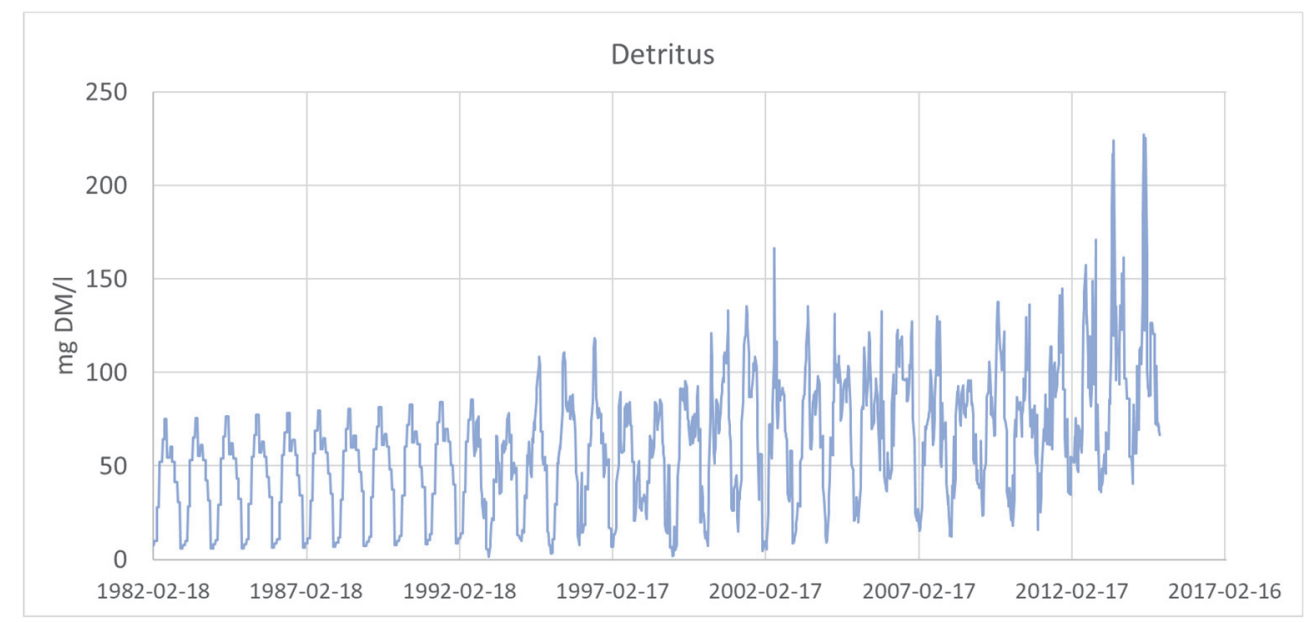

Figure 64 Constructed time series for detritus in the Ems at Papenburg

iii) Silicon data were lacking for all the fresh water discharges. After Lake IJssel-data (at Kornwerderzand) appeared to be too low, it was decided to take river IJssel-data from monitoring station Kampen.

iv) Phosphorus data for the Westerwoldse Aa contain observations that, for some reason, do not fit into the simulation results for the Dollard-area. This is especially the case for ortho-phosphate (dissolved inorganic P). It must be investigated further what the matter is, in this case; but for the time being, a new time-series was constructed based on the total-P and ortho-P data: the differences between both are made smaller. This 
is nothing more than a trick at this moment, but is was needed to make the data (simulations and observations) in the system match. 


\section{A9 Trajectories sailed in 2012-2013}

During the 2012-2013 research by Riegman et al (reported Brinkman et al, 2015), 40 times a trajectory was sailed from the outer Ems to the Dollard area (Figure 65). Data from this research are used here.

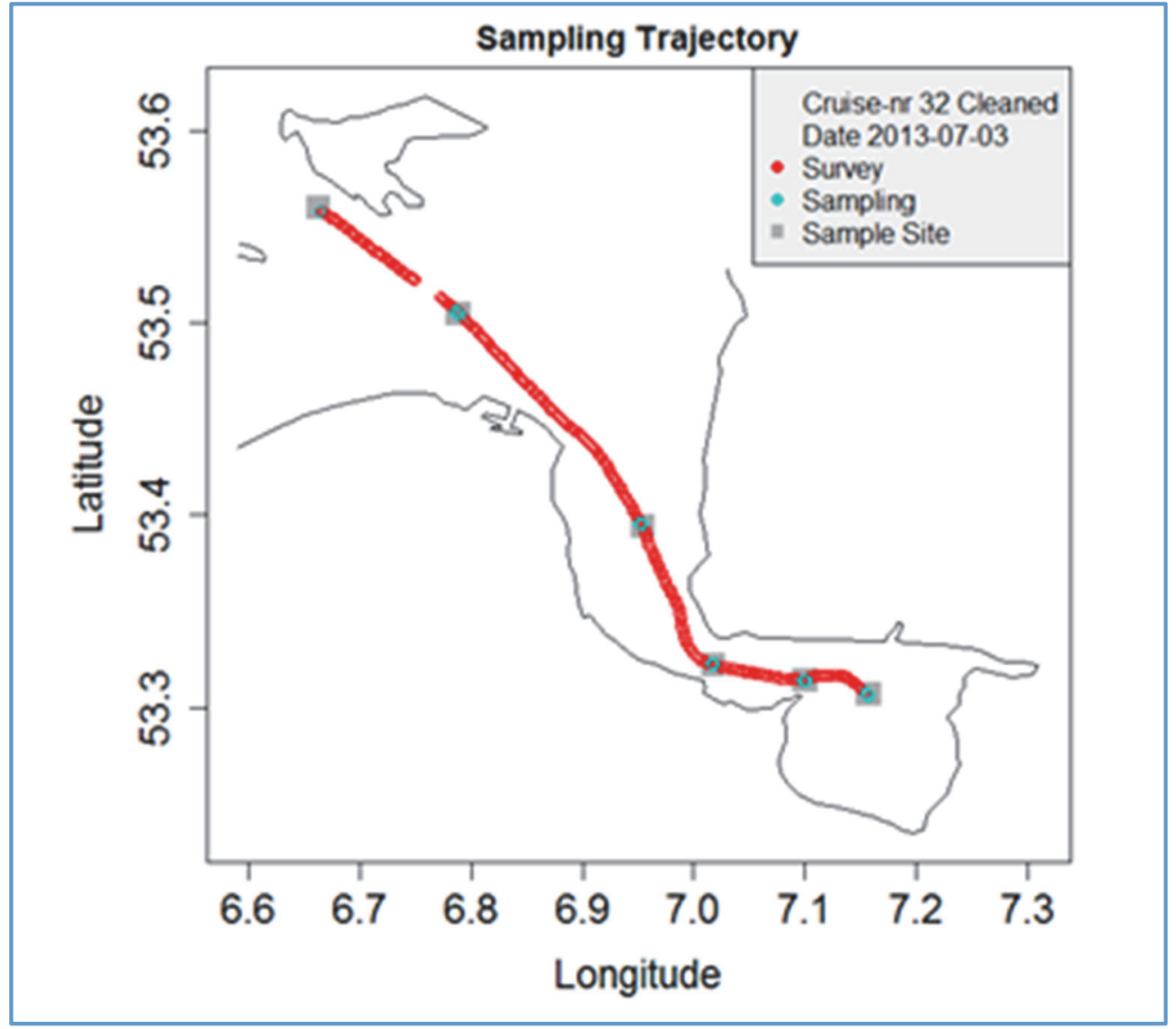

Figure 65 Sampling trajectory during the 2012-2013 IMARES study on primary production 


\section{A10 Phytoplankton (and fauna) related to salinity in the model}

The effect of salinity on phytoplankton occurrence is modelled by an equation that includes an (extra) mortality with salinity:

$$
\left.\operatorname{Mortality}_{(\text {fresh }}\right)=\text { Mortality }_{f 0} \cdot\left\{1+\frac{K_{1}[C l]}{K_{2}+[C l]}\right\}
$$

With $[\mathrm{Cl}]=0$, there is no mortality change; while mortality increases with a factor $\left(1+\mathrm{K}_{1}\right)$ if $[\mathrm{Cl}]=$ maximal.

Vice versa, marine phytoplankton mortality has a higher mortality if salinity declines:

$$
\begin{aligned}
& \text { Mortality(marine) } \\
& \qquad \text { Mortality }_{m 0} \cdot\left\{1+K_{3}\left(1-\frac{[C l]}{K_{4}+[C l]}\right)\right\}
\end{aligned}
$$

Standard value for all four parameters is 0.0 (no effects).
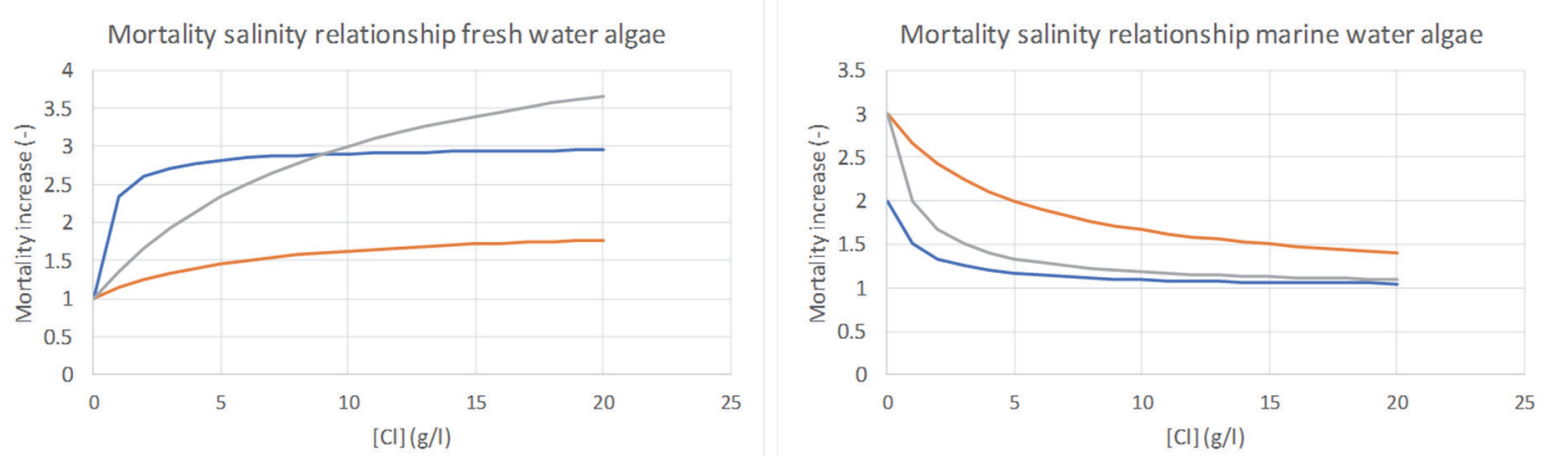

Figure 66 Examples of mortality depending on [Cl-] in water column. Left: fresh water phytoplankton extra mortality, according to eq.1, right: marine phytoplankton extra mortality, according to eq.2. Several values of K1..K4 used, showing that several shapes can be applied.

This equation can also be applied to fauna mortality and thus affecting fauna occurrence in area with a different salinity. 


\section{A11 Some details on available benthos data}

\section{A11.1 General}

As written in section 4.2, data were available from several sources. Unfortunately, the formats were different. In all cases, data files contained a species list (sometimes not-specified, just the name of the Genus). Species names just as scientific names and not always consistent, e.g. for mudsnails Hydrobia and Peringia both occurred.

Coordinates finally had to be as (multiple of 50$)+25$. This was to ensure that coordinate comparison could be successful. These are referred to as ' $50 \mathrm{~m}$ cell coordinates'.

The NLWKN-set contained names of the sites, with coordinates in a separate table, that had to be transformed into Dutch RD-coordinates, and then to the 50-m cell-coordinates as used by the GISpresentation of most data.

The SIBES-dataset contained coordinates of the sites, that have been transformed to the $50-\mathrm{m}$

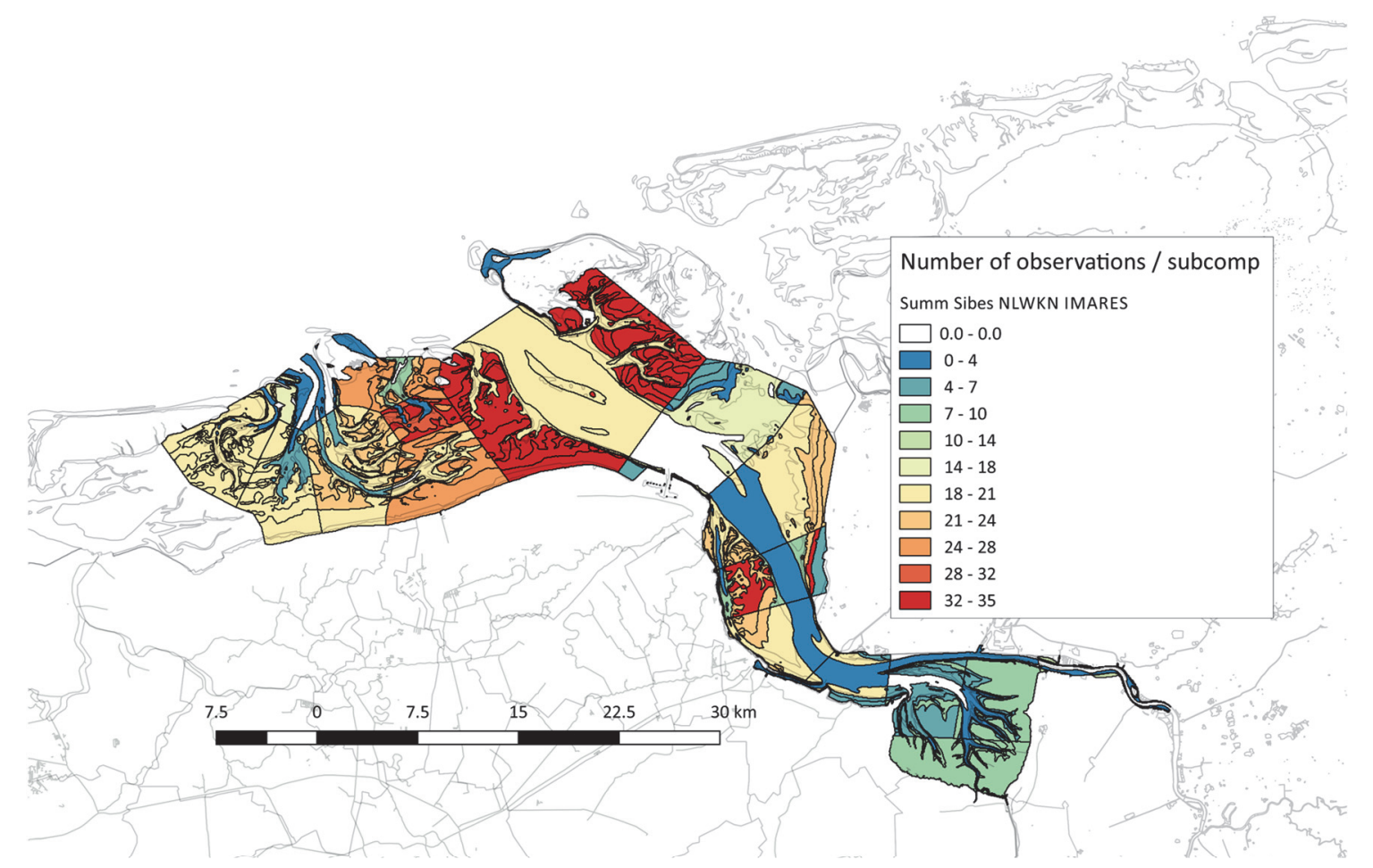

Figure 67 Number of observations (in all available years) by all three institutions, summed per subcompartment. Note that some parts of the sub-compartments may not have been visited at all, but are coloured according to visits in other parts of that same sub-compartment. See also the remarks under Figure 3 and Figure 2 (section 2.1). Also note that the samping sites are situated according to their coordinates, and these may have been called subtidal here, but have been sampled as tidal flats. Thus, subtidal sampling does not per se mean that samples in deeper water have been taken (and vice versa).

cell-coordinates as used by the GIS-presentation of most data. The table contains the scientific species name, plus a 'running name' (in Dutch). Data are as ash-free dry mass/m2 (AFDM m ${ }^{-2}$ ) and number $\mathrm{m}^{-2}$.

The IMARES/WMR-dataset contains coordinates of the sites, partly in WGS-84, and partly as ED50. These have been transformed to the desired $50 \mathrm{~m}$ cell coordinates. Unfortunately, the set contains, next to numbers $/ \mathrm{m} 2$, fresh masses/m2. These have been recomputed to AFDM/m2, using a factor 
20 for mussels, Mya's and the small molluscs (supposing these have a relatively thin shell). For cockles, a factor 30 has been used, and for Japanese oysters a factor 35 . This is just an estimate, and it would have been very convenient if also ash-free dry masses would have bene available.

The RWS-data we received were in a very special format, and at the moment not really understandable. For the time being, we skipped this data set; a part of the data are also included in the NIOZ / SIBES-dataset.

\section{A11.2 Sampling intensity}

Some sites or areas have been visited more than once, some other areas have never been visited for monitoring (or data simply were not available). An overview is given in Figure 67.It shows that the Dutch Wadden Sea North of Groningen, and the tidal flats of Hond and Paap have been visited relatively often. The Dollard area has less intensively been sampled.

\section{A11.3 Benthos classification}

Based on a classification provided by Babeth vd Weide (WMR), the available benthos data were grouped into a limited number of functional groups or even phylae. The whole classification is listed in Table 16, at the end of this appendix.

\section{A11.4 Benthos density}

\section{A11.4.1 Introduction}

For a complete overview and data analysis, see Compton et al (2013). Here, a few results are presented, just to serve as comparison with the model results. Here it should be kept in mind that the model sometimes still does not properly distinguish between tidal flats and the subtidal/gully areas. This is especially the case for benthic filter feeders, that find a much better foraging site in the subtidal. However, predation by fish and epibenthos is not incorporated well, which will lower the environmental suitability for shell fish below the low water level. Also, vertical suspended matter profiles are not computed yet, and thus, worse filtration conditions (higher or even much higher levels of suspended matter) are not modelled well. Thus, filter feeder values are assigned to main compartments in the model result, rather than to separate sub-compartments. This is a topic that needs improvement (see also appendix A27).

\section{A11.5 Data conversion}

Data in the IMARES-database were in the form of fresh mass $/ \mathrm{m} 2$ and numbers $/ \mathrm{m} 2$; the SIBES-data and NLWKN-data concerned ash-free dry masses. The fresh masses (including shells and whatsoever) were transformed to ash-free dry mass, using a conversion factor. This factor differed per group/species, and ranged from 20-30 for shell fish (mostly 20, for cockles and oysters 30 has been used), starfish and crabs to 5 for worms although these are hardly relevant in the WMR/IMARES-database. 


\section{A11.5.1 Mussels}

We considered mussels as group containing benthic filter feeding Mollusca, see Table 13. Inside the Dollard area, hardly any filter feeders occur.

Table 13 Species gathered together as 'mussels'. Selection on column 'Select' from Table 16. Small and Large denote boundaries of small, medium and large individuals (this needs a correction for Ensis in a next phase). .

\begin{tabular}{|l|l|r|r|l|r|r}
\hline Species & Phylum & Phyl_Small & Phyl_Large & Select & Group_Small & Group_Large \\
\hline Cerastoderma edule & Mollusca & $1.00 \mathrm{E}-01$ & $1.00 \mathrm{E}+00$ & Mussels & $1.00 \mathrm{E}-01$ & $1.00 \mathrm{E}+00$ \\
\hline Ensis directus & Mollusca & $1.00 \mathrm{E}-01$ & $1.00 \mathrm{E}+00$ & Mussels & $1.00 \mathrm{E}-01$ & $1.00 \mathrm{E}+00$ \\
\hline Mytilus edulis & Mollusca & $1.00 \mathrm{E}-01$ & $1.00 \mathrm{E}+00$ & Mussels & $1.00 \mathrm{E}-01$ & $1.00 \mathrm{E}+00$ \\
\hline Scrobicularia plana & Mollusca & $1.00 \mathrm{E}-01$ & $1.00 \mathrm{E}+00$ & Mussels & $1.00 \mathrm{E}-01$ & $1.00 \mathrm{E}+00$ \\
\hline Ensis sp. & Mollusca & $1.00 \mathrm{E}-01$ & $1.00 \mathrm{E}+00$ & Mussels & $1.00 \mathrm{E}-01$ & $1.00 \mathrm{E}+00$ \\
\hline
\end{tabular}

\section{A11.5.2 Mudsnails}

Mudsnails are mainly Hydrobia ulvae (== Peringia ulvae), a small snail mainly feeding on benthic algae. Distribution over the whole area, not specifically in a certain part (Figure 69). Also, Periwinkles occur, but in low densities, and are not mentioned further.

Table 14 Species gathered together as mudsnails. Note that Peringia and Hydrobia concerns the same species, but the name changed recently. Both names occur in the lists. Retusa is of minor importance. Selection on column 'Select' from Table 16. Small and Large denote boundaries of small, medium and large individuals

\begin{tabular}{|l|l|r|r|l|r|r|}
\hline Species & Phylum & Phyl_Small & \multicolumn{1}{|c|}{ Phyl_Large } & Select & Group_Small & Group_Large \\
\hline Peringia ulvae & Mollusca & $1.00 \mathrm{E}-01$ & $1.00 \mathrm{E}+00$ & Mudsnail & $1.00 \mathrm{E}-03$ & $1.00 \mathrm{E}-02$ \\
\hline Retusa obtusa & Mollusca & $1.00 \mathrm{E}-01$ & $1.00 \mathrm{E}+00$ & Mudsnail & $1.00 \mathrm{E}-03$ & $1.00 \mathrm{E}-02$ \\
\hline Hydrobia ulvae & Mollusca & $1.00 \mathrm{E}-01$ & $1.00 \mathrm{E}+00$ & Mudsnail & $1.00 \mathrm{E}-03$ & $1.00 \mathrm{E}-02$ \\
\hline
\end{tabular}

\section{A11.5.3 All biomass together}

Observed densities of all fauna together (as listed in Table 16) is shown in Figure 70. The main difference between this figure and the previous Figure 68 and Figure 69 together concerns worms (Annelida), benthic carnivores (starfish, crabs, etc) and Arthropoda (like Corophium). In some subcompartments very high biomass densities have been observed. Number one by far concerns the larger molluscs (sum of Mytilus, Ensis, Crassostrea, Scrobicularia, Cerasteroderma; see also Table 16) since these may occur in dense beds.

Most occurring species (table is from the SIBES-database) are listed in Table 15. 


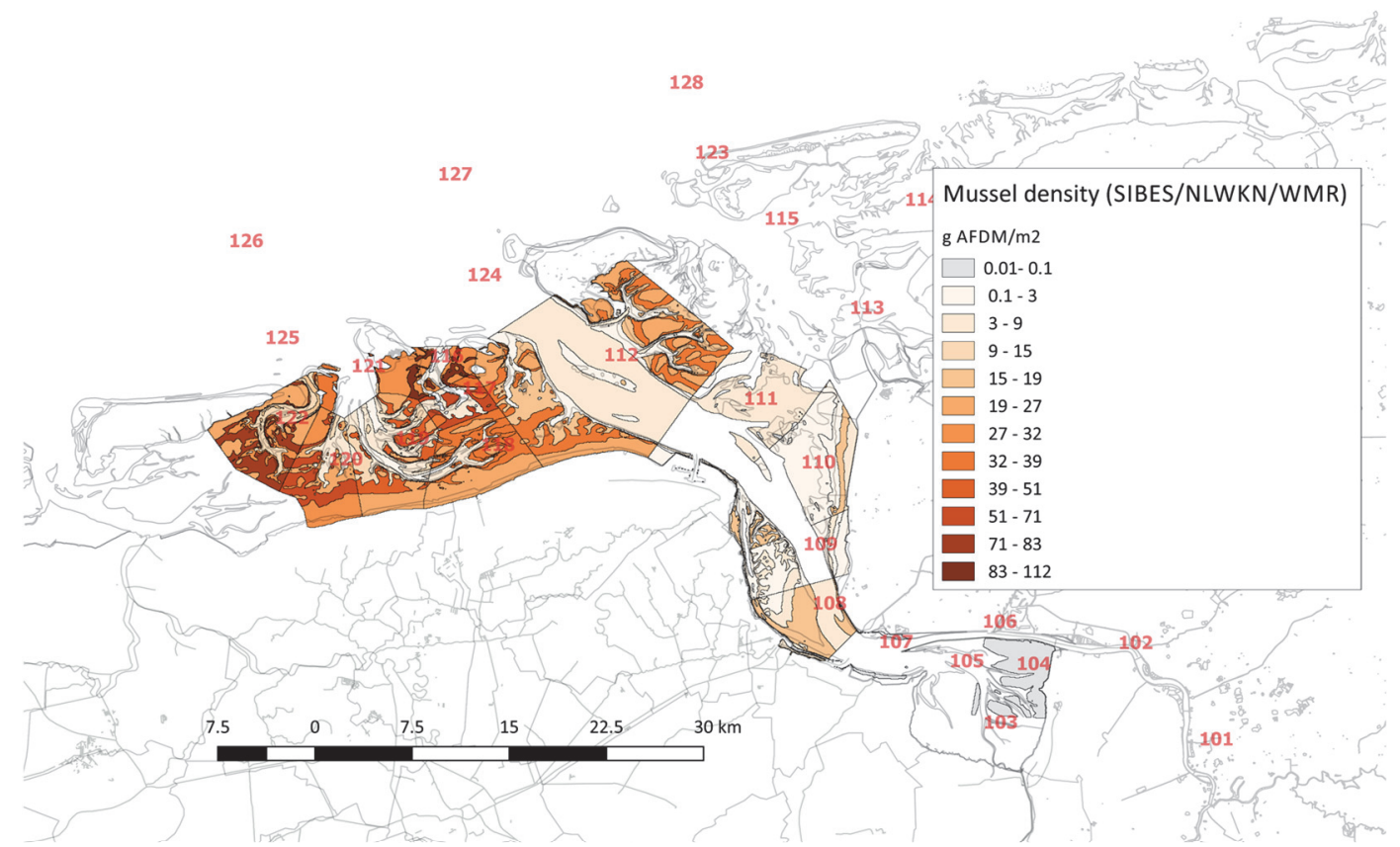

Figure 68 Benthic filter feeder biomass density (average of all observations); it is called 'mussels' here, but also concerns Cockle, Japanese Oyster, Ensis and Scrobularia. See also Table 13

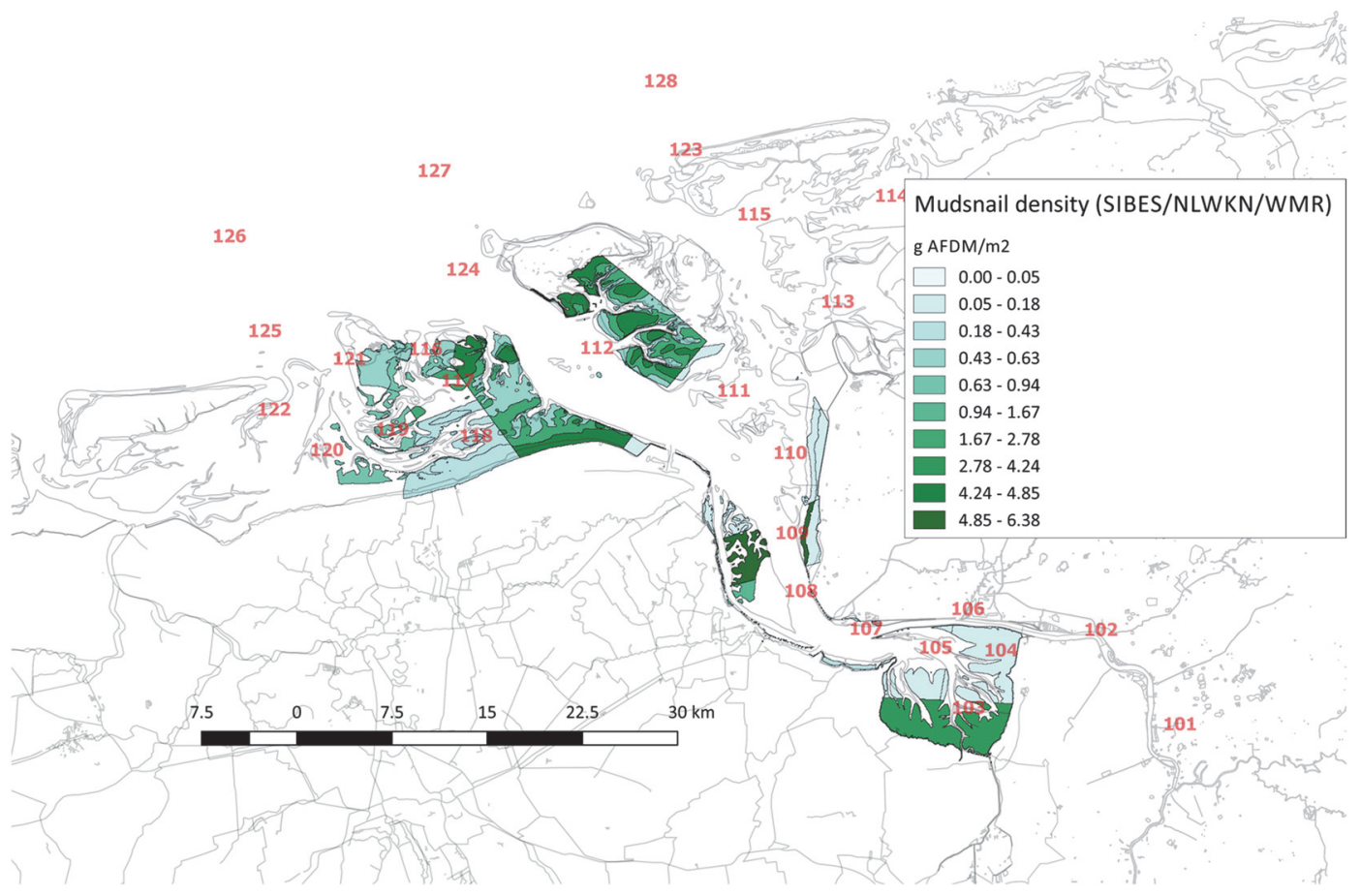

Figure 69 Density of mudsnails in the system ( $\mathrm{AFDM} / \mathrm{m} 2)$, as average for all observations. 


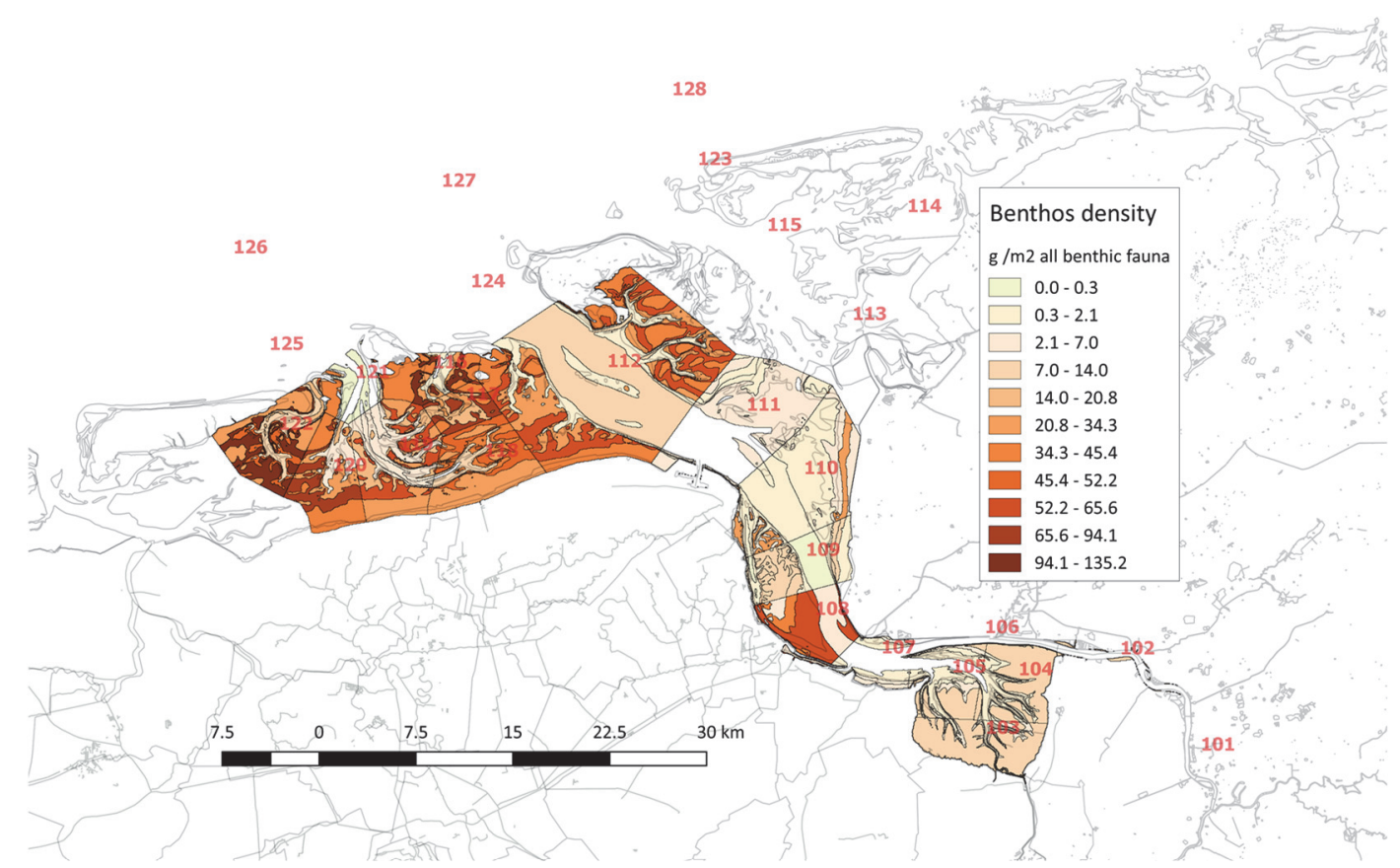

Figure 70 Density of all benthic fauna together in the system (g AFDM/m2), as average for all observations (thus, also including years outside the final simulation period). Areas with high densities mostly contain Blue mussel beds.

Table 15 Species in the SIBES-database with highest biomass densities (tidal flats, investigated area in Figure 17, section 4.2.1).

\begin{tabular}{|l|r|l|l|r|l|}
\hline Species & $\begin{array}{r}\text { g AFDM } \\
\mathrm{m}^{-2}\end{array}$ & Phylum & Species & g AFDM/m2 & Phylum \\
\hline Crassostrea gigas & 16.83 & Mollusca & Ensis directus & 1.64 & Mollusca \\
\hline Petricolaria pholadiformis & 10.69 & Mollusca & Hediste diversicolor & 1.40 & Annelida \\
\hline Mytilus edulis & 10.26 & Mollusca & Alitta succinea & 1.36 & Annelida \\
\hline Arenicola marina & 7.94 & Annelida & Scolelepis bonnieri & 1.24 & Annelida \\
\hline Lepidochitona cinerea & 7.84 & Mollusca & Scoloplos armiger & 1.14 & Annelida \\
\hline Cerastoderma edule & 6.23 & Mollusca & Macoma balthica & 1.01 & Mollusca \\
\hline Praunus inermis & 5.63 & Arthropoda & Magelona mirabilis & 0.94 & Annelida \\
\hline Lanice conchilega & 5.37 & Annelida & Carcinus maenas & 0.91 & Arthropoda \\
\hline Scrobicularia plana & 4.63 & Mollusca & Marenzelleria viridis & 0.76 & Annelida \\
\hline Alitta virens & 2.59 & Annelida & Scolelepis spec. & 0.74 & Annelida \\
\hline Mya arenaria & 2.49 & Mollusca & Scolelepis foliosa & 0.74 & Annelida \\
\hline Peringia ulvae & 2.37 & Mollusca & Abra alba & 0.70 & Mollusca \\
\hline Tellina tenuis & 2.31 & Mollusca & Bylgides sarsi & 0.61 & Annelida \\
\hline Nephtys hombergii & 2.30 & Annelida & Crangon crangon & 0.60 & Arthropoda \\
\hline Littorina littorea & 1.98 & Mollusca & Crepidula fornicata & 0.56 & Mollusca \\
\hline Bathyporeia pilosa & 1.83 & Arthropoda & Nephtys spec. & 0.54 & Annelida \\
\hline Ensis directus & 1.64 & Mollusca & Heteromastus & 0.51 & Annelida \\
\hline
\end{tabular}




\section{A11.5.4 Classification table}

In the next table it is listed how the species have been grouped, generally to phylum (column 2) and with a bit more detail to more groups (column 'select').

Table 16 Classification of all animals observed in the system by IMARES/WMR, SIBES and NLWKN. Two classifications are possible: after Phylum, and after some extra classes ('Select'), where most important species are grouped, or species are distinguished separately (like the Blue Mussel, Mytilus edulis). The sizes give what we considered as small ( $<$ the given value), large ( $>$ the given value), and medium (between small and large). This may be different for both cases.

\begin{tabular}{|c|c|c|c|c|c|c|}
\hline Species & Phylum & Phyl_Small & Phyl_Large & Select & Group_Small & Group_Large \\
\hline Alitta succinea & Annelida & $1.00 \mathrm{E}-03$ & $1.00 \mathrm{E}-02$ & Annelida & $1.00 \mathrm{E}-03$ & $1.00 \mathrm{E}-02$ \\
\hline Alitta virens & Annelida & $1.00 \mathrm{E}-03$ & $1.00 \mathrm{E}-02$ & Annelida & $1.00 \mathrm{E}-03$ & $1.00 \mathrm{E}-02$ \\
\hline Aphelochaeta marioni & Annelida & $1.00 \mathrm{E}-03$ & $1.00 \mathrm{E}-02$ & Annelida & $1.00 \mathrm{E}-03$ & $1.00 \mathrm{E}-02$ \\
\hline Arenicola marina & Annelida & $1.00 \mathrm{E}-03$ & $1.00 \mathrm{E}-02$ & Annelida & $1.00 \mathrm{E}-03$ & $1.00 \mathrm{E}-02$ \\
\hline Aricidea minuta & Annelida & $1.00 \mathrm{E}-03$ & $1.00 \mathrm{E}-02$ & Annelida & $1.00 \mathrm{E}-03$ & $1.00 \mathrm{E}-02$ \\
\hline Bylgides sarsi & Annelida & $1.00 \mathrm{E}-03$ & $1.00 \mathrm{E}-02$ & Annelida & $1.00 \mathrm{E}-03$ & $1.00 \mathrm{E}-02$ \\
\hline Capitella capitata & Annelida & $1.00 \mathrm{E}-03$ & $1.00 \mathrm{E}-02$ & Annelida & $1.00 \mathrm{E}-03$ & $1.00 \mathrm{E}-02$ \\
\hline Eteone longa & Annelida & $1.00 \mathrm{E}-03$ & $1.00 \mathrm{E}-02$ & Annelida & $1.00 \mathrm{E}-03$ & $1.00 \mathrm{E}-02$ \\
\hline Eteone sp. & Annelida & $1.00 \mathrm{E}-03$ & $1.00 \mathrm{E}-02$ & Annelida & $1.00 \mathrm{E}-03$ & $1.00 \mathrm{E}-02$ \\
\hline Eumida sanguinea & Annelida & $1.00 \mathrm{E}-03$ & $1.00 \mathrm{E}-02$ & Annelida & $1.00 \mathrm{E}-03$ & $1.00 \mathrm{E}-02$ \\
\hline Eunereis longissima & Annelida & $1.00 \mathrm{E}-03$ & $1.00 \mathrm{E}-02$ & Annelida & $1.00 \mathrm{E}-03$ & $1.00 \mathrm{E}-02$ \\
\hline Hediste diversicolor & Annelida & $1.00 \mathrm{E}-03$ & $1.00 \mathrm{E}-02$ & BenthCarn & $1.00 \mathrm{E}-03$ & $1.00 \mathrm{E}-02$ \\
\hline Heteromastus filiformis & Annelida & $1.00 \mathrm{E}-03$ & $1.00 \mathrm{E}-02$ & Annelida & $1.00 \mathrm{E}-03$ & $1.00 \mathrm{E}-02$ \\
\hline Lanice conchilega & Annelida & $1.00 \mathrm{E}-03$ & $1.00 \mathrm{E}-02$ & AnnelidaPel & $1.00 \mathrm{E}-03$ & $1.00 \mathrm{E}-02$ \\
\hline Magelona johnstoni & Annelida & $1.00 \mathrm{E}-03$ & $1.00 \mathrm{E}-02$ & Annelida & $1.00 \mathrm{E}-03$ & $1.00 \mathrm{E}-02$ \\
\hline Magelona mirabilis & Annelida & $1.00 \mathrm{E}-03$ & $1.00 \mathrm{E}-02$ & Annelida & $1.00 \mathrm{E}-03$ & $1.00 \mathrm{E}-02$ \\
\hline Magelona spec. & Annelida & $1.00 \mathrm{E}-03$ & $1.00 \mathrm{E}-02$ & Annelida & $1.00 \mathrm{E}-03$ & $1.00 \mathrm{E}-02$ \\
\hline Malmgreniella darbouxi & Annelida & $1.00 \mathrm{E}-03$ & $1.00 \mathrm{E}-02$ & Annelida & $1.00 \mathrm{E}-03$ & $1.00 \mathrm{E}-02$ \\
\hline Marenzelleria viridis & Annelida & $1.00 \mathrm{E}-03$ & $1.00 \mathrm{E}-02$ & Annelida & $1.00 \mathrm{E}-03$ & $1.00 \mathrm{E}-02$ \\
\hline Microphthalmus sczelkowii & Annelida & $1.00 \mathrm{E}-03$ & $1.00 \mathrm{E}-02$ & Annelida & $1.00 \mathrm{E}-03$ & $1.00 \mathrm{E}-02$ \\
\hline Myrianida prolifera & Annelida & $1.00 \mathrm{E}-03$ & $1.00 \mathrm{E}-02$ & Annelida & $1.00 \mathrm{E}-03$ & $1.00 \mathrm{E}-02$ \\
\hline Nephtys caeca & Annelida & $1.00 \mathrm{E}-03$ & $1.00 \mathrm{E}-02$ & BenthCarn & $1.00 \mathrm{E}-03$ & $1.00 \mathrm{E}-02$ \\
\hline Nephtys cirrosa & Annelida & $1.00 \mathrm{E}-03$ & $1.00 \mathrm{E}-02$ & BenthCarn & $1.00 \mathrm{E}-03$ & $1.00 \mathrm{E}-02$ \\
\hline Nephtys hombergii & Annelida & $1.00 \mathrm{E}-03$ & $1.00 \mathrm{E}-02$ & BenthCarn & $1.00 \mathrm{E}-03$ & $1.00 \mathrm{E}-02$ \\
\hline Nephtys longosetosa & Annelida & $1.00 \mathrm{E}-03$ & $1.00 \mathrm{E}-02$ & BenthCarn & $1.00 \mathrm{E}-03$ & $1.00 \mathrm{E}-02$ \\
\hline Nephtys spec. & Annelida & $1.00 \mathrm{E}-03$ & $1.00 \mathrm{E}-02$ & BenthCarn & $1.00 \mathrm{E}-03$ & $1.00 \mathrm{E}-02$ \\
\hline Nereide sp. & Annelida & $1.00 \mathrm{E}-03$ & $1.00 \mathrm{E}-02$ & Annelida & $1.00 \mathrm{E}-03$ & $1.00 \mathrm{E}-02$ \\
\hline Oligochaeta sp. & Annelida & $1.00 \mathrm{E}-03$ & $1.00 \mathrm{E}-02$ & Annelida & $1.00 \mathrm{E}-03$ & $1.00 \mathrm{E}-02$ \\
\hline Ophelia rathkei & Annelida & $1.00 \mathrm{E}-03$ & $1.00 \mathrm{E}-02$ & Annelida & $1.00 \mathrm{E}-03$ & $1.00 \mathrm{E}-02$ \\
\hline Phyllodoce maculata & Annelida & $1.00 \mathrm{E}-03$ & $1.00 \mathrm{E}-02$ & Annelida & $1.00 \mathrm{E}-03$ & $1.00 \mathrm{E}-02$ \\
\hline Phyllodoce mucosa & Annelida & $1.00 \mathrm{E}-03$ & $1.00 \mathrm{E}-02$ & Annelida & $1.00 \mathrm{E}-03$ & $1.00 \mathrm{E}-02$ \\
\hline Phyllodoce spec. & Annelida & $1.00 \mathrm{E}-03$ & $1.00 \mathrm{E}-02$ & Annelida & $1.00 \mathrm{E}-03$ & $1.00 \mathrm{E}-02$ \\
\hline Polydora cornuta & Annelida & $1.00 \mathrm{E}-03$ & $1.00 \mathrm{E}-02$ & Annelida & $1.00 \mathrm{E}-03$ & $1.00 \mathrm{E}-02$ \\
\hline Pygospio elegans & Annelida & $1.00 \mathrm{E}-03$ & $1.00 \mathrm{E}-02$ & Annelida & $1.00 \mathrm{E}-03$ & $1.00 \mathrm{E}-02$ \\
\hline Scolelepis bonnieri & Annelida & $1.00 \mathrm{E}-03$ & $1.00 \mathrm{E}-02$ & Annelida & $1.00 \mathrm{E}-03$ & $1.00 \mathrm{E}-02$ \\
\hline Scolelepis foliosa & Annelida & $1.00 \mathrm{E}-03$ & $1.00 \mathrm{E}-02$ & Annelida & $1.00 \mathrm{E}-03$ & $1.00 \mathrm{E}-02$ \\
\hline Scolelepis spec. & Annelida & $1.00 \mathrm{E}-03$ & $1.00 \mathrm{E}-02$ & Annelida & $1.00 \mathrm{E}-03$ & $1.00 \mathrm{E}-02$ \\
\hline
\end{tabular}




\begin{tabular}{|c|c|c|c|c|c|c|}
\hline Scolelepis squamata & Annelida & $1.00 \mathrm{E}-03$ & $1.00 \mathrm{E}-02$ & Annelida & $1.00 \mathrm{E}-03$ & $1.00 \mathrm{E}-02$ \\
\hline Scoloplos armiger & Annelida & $1.00 \mathrm{E}-03$ & $1.00 \mathrm{E}-02$ & Annelida & $1.00 \mathrm{E}-03$ & $1.00 \mathrm{E}-02$ \\
\hline Spio martinensis & Annelida & $1.00 \mathrm{E}-03$ & $1.00 \mathrm{E}-02$ & Annelida & $1.00 \mathrm{E}-03$ & $1.00 \mathrm{E}-02$ \\
\hline Spiophanes bombyx & Annelida & $1.00 \mathrm{E}-03$ & $1.00 \mathrm{E}-02$ & Annelida & $1.00 \mathrm{E}-03$ & $1.00 \mathrm{E}-02$ \\
\hline Streblospio benedicti & Annelida & $1.00 \mathrm{E}-03$ & $1.00 \mathrm{E}-02$ & Annelida & $1.00 \mathrm{E}-03$ & $1.00 \mathrm{E}-02$ \\
\hline Travisia forbesii & Annelida & $1.00 \mathrm{E}-03$ & $1.00 \mathrm{E}-02$ & Annelida & $1.00 \mathrm{E}-03$ & $1.00 \mathrm{E}-02$ \\
\hline Ampharete acutifrons & Annelida & $1.00 \mathrm{E}-03$ & $1.00 \mathrm{E}-02$ & Annelida & $1.00 \mathrm{E}-03$ & $1.00 \mathrm{E}-02$ \\
\hline Ampharete finmarchica & Annelida & $1.00 \mathrm{E}-03$ & $1.00 \mathrm{E}-02$ & Annelida & $1.00 \mathrm{E}-03$ & $1.00 \mathrm{E}-02$ \\
\hline Ampharete $\mathrm{sp}$. & Annelida & $1.00 \mathrm{E}-03$ & $1.00 \mathrm{E}-02$ & Annelida & $1.00 \mathrm{E}-03$ & $1.00 \mathrm{E}-02$ \\
\hline Ampharetinae & Annelida & $1.00 \mathrm{E}-03$ & $1.00 \mathrm{E}-02$ & Annelida & $1.00 \mathrm{E}-03$ & $1.00 \mathrm{E}-02$ \\
\hline Amphichaeta sannio & Annelida & $1.00 \mathrm{E}-03$ & $1.00 \mathrm{E}-02$ & Annelida & $1.00 \mathrm{E}-03$ & $1.00 \mathrm{E}-02$ \\
\hline Amphicteis gunneri & Annelida & $1.00 \mathrm{E}-03$ & $1.00 \mathrm{E}-02$ & Annelida & $1.00 \mathrm{E}-03$ & $1.00 \mathrm{E}-02$ \\
\hline Anaitides mucosa & Annelida & $1.00 \mathrm{E}-03$ & $1.00 \mathrm{E}-02$ & Annelida & $1.00 \mathrm{E}-03$ & $1.00 \mathrm{E}-02$ \\
\hline Aricidea (Aricidea) minuta & Annelida & $1.00 \mathrm{E}-03$ & $1.00 \mathrm{E}-02$ & Annelida & $1.00 \mathrm{E}-03$ & $1.00 \mathrm{E}-02$ \\
\hline Aricidea cerrutii & Annelida & $1.00 \mathrm{E}-03$ & $1.00 \mathrm{E}-02$ & Annelida & $1.00 \mathrm{E}-03$ & $1.00 \mathrm{E}-02$ \\
\hline Autolytus prolifera & Annelida & $1.00 \mathrm{E}-03$ & $1.00 \mathrm{E}-02$ & Annelida & $1.00 \mathrm{E}-03$ & $1.00 \mathrm{E}-02$ \\
\hline Autolytus sp. & Annelida & $1.00 \mathrm{E}-03$ & $1.00 \mathrm{E}-02$ & Annelida & $1.00 \mathrm{E}-03$ & $1.00 \mathrm{E}-02$ \\
\hline Boccardiella ligerica & Annelida & $1.00 \mathrm{E}-03$ & $1.00 \mathrm{E}-02$ & Annelida & $1.00 \mathrm{E}-03$ & $1.00 \mathrm{E}-02$ \\
\hline Capitella minima & Annelida & $1.00 \mathrm{E}-03$ & $1.00 \mathrm{E}-02$ & Annelida & $1.00 \mathrm{E}-03$ & $1.00 \mathrm{E}-02$ \\
\hline Capitellidae & Annelida & $1.00 \mathrm{E}-03$ & $1.00 \mathrm{E}-02$ & Annelida & $1.00 \mathrm{E}-03$ & $1.00 \mathrm{E}-02$ \\
\hline Caulleriella killariensis & Annelida & $1.00 \mathrm{E}-03$ & $1.00 \mathrm{E}-02$ & Annelida & $1.00 \mathrm{E}-03$ & $1.00 \mathrm{E}-02$ \\
\hline Cirratulidae & Annelida & $1.00 \mathrm{E}-03$ & $1.00 \mathrm{E}-02$ & Annelida & $1.00 \mathrm{E}-03$ & $1.00 \mathrm{E}-02$ \\
\hline Enchytraeus albidus & Annelida & $1.00 \mathrm{E}-03$ & $1.00 \mathrm{E}-02$ & Annelida & $1.00 \mathrm{E}-03$ & $1.00 \mathrm{E}-02$ \\
\hline Enchytraeus capitatus & Annelida & $1.00 \mathrm{E}-03$ & $1.00 \mathrm{E}-02$ & Annelida & $1.00 \mathrm{E}-03$ & $1.00 \mathrm{E}-02$ \\
\hline Eteoninae & Annelida & $1.00 \mathrm{E}-03$ & $1.00 \mathrm{E}-02$ & Annelida & $1.00 \mathrm{E}-03$ & $1.00 \mathrm{E}-02$ \\
\hline Eumida bahusiensis & Annelida & $1.00 \mathrm{E}-03$ & $1.00 \mathrm{E}-02$ & Annelida & $1.00 \mathrm{E}-03$ & $1.00 \mathrm{E}-02$ \\
\hline Fabricia stellaris & Annelida & $1.00 \mathrm{E}-03$ & $1.00 \mathrm{E}-02$ & Annelida & $1.00 \mathrm{E}-03$ & $1.00 \mathrm{E}-02$ \\
\hline Gattyana cirrosa & Annelida & $1.00 \mathrm{E}-03$ & $1.00 \mathrm{E}-02$ & Annelida & $1.00 \mathrm{E}-03$ & $1.00 \mathrm{E}-02$ \\
\hline Harmothoe imbricata & Annelida & $1.00 \mathrm{E}-03$ & $1.00 \mathrm{E}-02$ & Annelida & $1.00 \mathrm{E}-03$ & $1.00 \mathrm{E}-02$ \\
\hline Harmothoe impar & Annelida & $1.00 \mathrm{E}-03$ & $1.00 \mathrm{E}-02$ & Annelida & $1.00 \mathrm{E}-03$ & $1.00 \mathrm{E}-02$ \\
\hline Harmothoe nodosa & Annelida & $1.00 \mathrm{E}-03$ & $1.00 \mathrm{E}-02$ & Annelida & $1.00 \mathrm{E}-03$ & $1.00 \mathrm{E}-02$ \\
\hline Harmothoe sp. & Annelida & $1.00 \mathrm{E}-03$ & $1.00 \mathrm{E}-02$ & Annelida & $1.00 \mathrm{E}-03$ & $1.00 \mathrm{E}-02$ \\
\hline Heterochaeta costata & Annelida & $1.00 \mathrm{E}-03$ & $1.00 \mathrm{E}-02$ & Annelida & $1.00 \mathrm{E}-03$ & $1.00 \mathrm{E}-02$ \\
\hline Lagis koreni & Annelida & $1.00 \mathrm{E}-03$ & $1.00 \mathrm{E}-02$ & Annelida & $1.00 \mathrm{E}-03$ & $1.00 \mathrm{E}-02$ \\
\hline Limnodrilus hoffmeisteri & Annelida & $1.00 \mathrm{E}-03$ & $1.00 \mathrm{E}-02$ & Annelida & $1.00 \mathrm{E}-03$ & $1.00 \mathrm{E}-02$ \\
\hline Lumbricillus lineatus & Annelida & $1.00 \mathrm{E}-03$ & $1.00 \mathrm{E}-02$ & Annelida & $1.00 \mathrm{E}-03$ & $1.00 \mathrm{E}-02$ \\
\hline Magelona sp. & Annelida & $1.00 \mathrm{E}-03$ & $1.00 \mathrm{E}-02$ & Annelida & $1.00 \mathrm{E}-03$ & $1.00 \mathrm{E}-02$ \\
\hline Malacoceros fuliginosus & Annelida & $1.00 \mathrm{E}-03$ & $1.00 \mathrm{E}-02$ & Annelida & $1.00 \mathrm{E}-03$ & $1.00 \mathrm{E}-02$ \\
\hline Malacoceros tetracerus & Annelida & $1.00 \mathrm{E}-03$ & $1.00 \mathrm{E}-02$ & Annelida & $1.00 \mathrm{E}-03$ & $1.00 \mathrm{E}-02$ \\
\hline Malmgreniella castanea & Annelida & $1.00 \mathrm{E}-03$ & $1.00 \mathrm{E}-02$ & Annelida & $1.00 \mathrm{E}-03$ & $1.00 \mathrm{E}-02$ \\
\hline Manayunkia aestuarina & Annelida & $1.00 \mathrm{E}-03$ & $1.00 \mathrm{E}-02$ & Annelida & $1.00 \mathrm{E}-03$ & $1.00 \mathrm{E}-02$ \\
\hline Marenzelleria sp. & Annelida & $1.00 \mathrm{E}-03$ & $1.00 \mathrm{E}-02$ & Annelida & $1.00 \mathrm{E}-03$ & $1.00 \mathrm{E}-02$ \\
\hline Marenzelleria wireni & Annelida & $1.00 \mathrm{E}-03$ & $1.00 \mathrm{E}-02$ & Annelida & $1.00 \mathrm{E}-03$ & $1.00 \mathrm{E}-02$ \\
\hline Marionina argentea & Annelida & $1.00 \mathrm{E}-03$ & $1.00 \mathrm{E}-02$ & Annelida & $1.00 \mathrm{E}-03$ & $1.00 \mathrm{E}-02$ \\
\hline Neanthes succinea & Annelida & $1.00 \mathrm{E}-03$ & $1.00 \mathrm{E}-02$ & Annelida & $1.00 \mathrm{E}-03$ & $1.00 \mathrm{E}-02$ \\
\hline Neanthes virens & Annelida & $1.00 \mathrm{E}-03$ & $1.00 \mathrm{E}-02$ & Annelida & $1.00 \mathrm{E}-03$ & $1.00 \mathrm{E}-02$ \\
\hline Nephtys sp. & Annelida & $1.00 \mathrm{E}-03$ & $1.00 \mathrm{E}-02$ & BenthCarn & $1.00 \mathrm{E}-03$ & $1.00 \mathrm{E}-02$ \\
\hline Nereididae & Annelida & $1.00 \mathrm{E}-03$ & $1.00 \mathrm{E}-02$ & BenthCarn & $1.00 \mathrm{E}-03$ & $1.00 \mathrm{E}-02$ \\
\hline
\end{tabular}




\begin{tabular}{|c|c|c|c|c|c|c|}
\hline Nereis sp. & Annelida & $1.00 \mathrm{E}-03$ & $1.00 \mathrm{E}-02$ & BenthCarn & 1.00E-03 & $1.00 \mathrm{E}-02$ \\
\hline Oligochaeta & Annelida & $1.00 \mathrm{E}-03$ & $1.00 \mathrm{E}-02$ & Annelida & $1.00 \mathrm{E}-03$ & $1.00 \mathrm{E}-02$ \\
\hline Orbiniidae & Annelida & $1.00 \mathrm{E}-03$ & $1.00 \mathrm{E}-02$ & Annelida & $1.00 \mathrm{E}-03$ & $1.00 \mathrm{E}-02$ \\
\hline Paranais litoralis & Annelida & $1.00 \mathrm{E}-03$ & $1.00 \mathrm{E}-02$ & Annelida & $1.00 \mathrm{E}-03$ & $1.00 \mathrm{E}-02$ \\
\hline Polychaeta & Annelida & $1.00 \mathrm{E}-03$ & $1.00 \mathrm{E}-02$ & Annelida & $1.00 \mathrm{E}-03$ & $1.00 \mathrm{E}-02$ \\
\hline Polydora ciliata & Annelida & $1.00 \mathrm{E}-03$ & $1.00 \mathrm{E}-02$ & Annelida & $1.00 \mathrm{E}-03$ & $1.00 \mathrm{E}-02$ \\
\hline Polydora sp. & Annelida & $1.00 \mathrm{E}-03$ & $1.00 \mathrm{E}-02$ & Annelida & $1.00 \mathrm{E}-03$ & $1.00 \mathrm{E}-02$ \\
\hline Polynoidae & Annelida & $1.00 \mathrm{E}-03$ & $1.00 \mathrm{E}-02$ & Annelida & $1.00 \mathrm{E}-03$ & $1.00 \mathrm{E}-02$ \\
\hline Scoloplos (Scoloplos) armiger & Annelida & $1.00 \mathrm{E}-03$ & $1.00 \mathrm{E}-02$ & Annelida & $1.00 \mathrm{E}-03$ & $1.00 \mathrm{E}-02$ \\
\hline Spio filicornis & Annelida & $1.00 \mathrm{E}-03$ & $1.00 \mathrm{E}-02$ & Annelida & $1.00 \mathrm{E}-03$ & $1.00 \mathrm{E}-02$ \\
\hline Spio goniocephala & Annelida & $1.00 \mathrm{E}-03$ & $1.00 \mathrm{E}-02$ & Annelida & $1.00 \mathrm{E}-03$ & $1.00 \mathrm{E}-02$ \\
\hline Spionidae & Annelida & $1.00 \mathrm{E}-03$ & $1.00 \mathrm{E}-02$ & Annelida & $1.00 \mathrm{E}-03$ & $1.00 \mathrm{E}-02$ \\
\hline Streblospio shrubsolii & Annelida & $1.00 \mathrm{E}-03$ & $1.00 \mathrm{E}-02$ & Annelida & $1.00 \mathrm{E}-03$ & $1.00 \mathrm{E}-02$ \\
\hline Tharyx killariensis & Annelida & $1.00 \mathrm{E}-03$ & $1.00 \mathrm{E}-02$ & Annelida & $1.00 \mathrm{E}-03$ & $1.00 \mathrm{E}-02$ \\
\hline Tubificidae & Annelida & $1.00 \mathrm{E}-03$ & $1.00 \mathrm{E}-02$ & Annelida & $1.00 \mathrm{E}-03$ & $1.00 \mathrm{E}-02$ \\
\hline Tubificoides benedii & Annelida & $1.00 \mathrm{E}-03$ & $1.00 \mathrm{E}-02$ & Annelida & $1.00 \mathrm{E}-03$ & $1.00 \mathrm{E}-02$ \\
\hline Tubificoides heterochaetus & Annelida & $1.00 \mathrm{E}-03$ & $1.00 \mathrm{E}-02$ & Annelida & $1.00 \mathrm{E}-03$ & $1.00 \mathrm{E}-02$ \\
\hline Tubificoides pseudogaster & Annelida & $1.00 \mathrm{E}-03$ & $1.00 \mathrm{E}-02$ & Annelida & $1.00 \mathrm{E}-03$ & $1.00 \mathrm{E}-02$ \\
\hline Bathyporeia pilosa & Arthropoda & $5.00 \mathrm{E}-03$ & $5.00 \mathrm{E}-02$ & Arthropoda & $5.00 \mathrm{E}-03$ & $5.00 \mathrm{E}-02$ \\
\hline Bathyporeia sarsi & Arthropoda & $5.00 \mathrm{E}-03$ & $5.00 \mathrm{E}-02$ & Arthropoda & $5.00 \mathrm{E}-03$ & $5.00 \mathrm{E}-02$ \\
\hline Bathyporeia spec. & Arthropoda & $5.00 \mathrm{E}-03$ & $5.00 \mathrm{E}-02$ & Arthropoda & $5.00 \mathrm{E}-03$ & $5.00 \mathrm{E}-02$ \\
\hline Bodotria scorpioides & Arthropoda & $5.00 \mathrm{E}-03$ & $5.00 \mathrm{E}-02$ & Arthropoda & $5.00 \mathrm{E}-03$ & $5.00 \mathrm{E}-02$ \\
\hline Cancer pagurus & Arthropoda & $5.00 \mathrm{E}-03$ & $5.00 \mathrm{E}-02$ & Crabs & $5.00 \mathrm{E}-02$ & $5.00 \mathrm{E}-01$ \\
\hline Carcinus maenas & Arthropoda & $5.00 \mathrm{E}-03$ & $5.00 \mathrm{E}-02$ & Crabs & $5.00 \mathrm{E}-02$ & $5.00 \mathrm{E}-01$ \\
\hline Carcinus maenas megalopa & Arthropoda & $5.00 \mathrm{E}-03$ & $5.00 \mathrm{E}-02$ & Crabs & $5.00 \mathrm{E}-02$ & $5.00 \mathrm{E}-01$ \\
\hline Corophium sp. & Arthropoda & $5.00 \mathrm{E}-03$ & $5.00 \mathrm{E}-02$ & Arthropoda & $5.00 \mathrm{E}-03$ & $5.00 \mathrm{E}-02$ \\
\hline Crangon crangon & Arthropoda & $5.00 \mathrm{E}-03$ & $5.00 \mathrm{E}-02$ & Arthropoda & $5.00 \mathrm{E}-03$ & $5.00 \mathrm{E}-02$ \\
\hline Eurydice pulchra & Arthropoda & $5.00 \mathrm{E}-03$ & $5.00 \mathrm{E}-02$ & Arthropoda & $5.00 \mathrm{E}-03$ & $5.00 \mathrm{E}-02$ \\
\hline Gammarus locusta & Arthropoda & $5.00 \mathrm{E}-03$ & $5.00 \mathrm{E}-02$ & Arthropoda & $5.00 \mathrm{E}-03$ & 5.00E-02 \\
\hline Gammarus spec. & Arthropoda & $5.00 \mathrm{E}-03$ & $5.00 \mathrm{E}-02$ & Arthropoda & $5.00 \mathrm{E}-03$ & $5.00 \mathrm{E}-02$ \\
\hline Hemigrapsus takanoi & Arthropoda & $5.00 \mathrm{E}-03$ & $5.00 \mathrm{E}-02$ & Arthropoda & $5.00 \mathrm{E}-03$ & $5.00 \mathrm{E}-02$ \\
\hline Idotea linearis & Arthropoda & $5.00 \mathrm{E}-03$ & $5.00 \mathrm{E}-02$ & Arthropoda & $5.00 \mathrm{E}-03$ & $5.00 \mathrm{E}-02$ \\
\hline Melita palmata & Arthropoda & $5.00 \mathrm{E}-03$ & $5.00 \mathrm{E}-02$ & Arthropoda & $5.00 \mathrm{E}-03$ & $5.00 \mathrm{E}-02$ \\
\hline Mesopodopsis slabberi & Arthropoda & $5.00 \mathrm{E}-03$ & $5.00 \mathrm{E}-02$ & Arthropoda & $5.00 \mathrm{E}-03$ & $5.00 \mathrm{E}-02$ \\
\hline Praunus inermis & Arthropoda & $5.00 \mathrm{E}-03$ & $5.00 \mathrm{E}-02$ & Arthropoda & $5.00 \mathrm{E}-03$ & $5.00 \mathrm{E}-02$ \\
\hline Urothoe poseidonis & Arthropoda & $5.00 \mathrm{E}-03$ & $5.00 \mathrm{E}-02$ & Arthropoda & $5.00 \mathrm{E}-03$ & $5.00 \mathrm{E}-02$ \\
\hline Amphibalanus improvisus & Arthropoda & $5.00 \mathrm{E}-03$ & $5.00 \mathrm{E}-02$ & Arthropoda & $5.00 \mathrm{E}-03$ & $5.00 \mathrm{E}-02$ \\
\hline Atylus swammerdamei & Arthropoda & $5.00 \mathrm{E}-03$ & $5.00 \mathrm{E}-02$ & Arthropoda & $5.00 \mathrm{E}-03$ & $5.00 \mathrm{E}-02$ \\
\hline Austrominius modestus & Arthropoda & $5.00 \mathrm{E}-03$ & $5.00 \mathrm{E}-02$ & Arthropoda & $5.00 \mathrm{E}-03$ & $5.00 \mathrm{E}-02$ \\
\hline Balanidae & Arthropoda & $5.00 \mathrm{E}-03$ & $5.00 \mathrm{E}-02$ & Arthropoda & $5.00 \mathrm{E}-03$ & 5.00E-02 \\
\hline Balanus crenatus & Arthropoda & $5.00 \mathrm{E}-03$ & $5.00 \mathrm{E}-02$ & Arthropoda & 5.00E-03 & $5.00 \mathrm{E}-02$ \\
\hline Balanus improvisus & Arthropoda & $5.00 \mathrm{E}-03$ & $5.00 \mathrm{E}-02$ & Arthropoda & $5.00 \mathrm{E}-03$ & $5.00 \mathrm{E}-02$ \\
\hline Bathyporeia elegans & Arthropoda & $5.00 \mathrm{E}-03$ & $5.00 \mathrm{E}-02$ & Arthropoda & $5.00 \mathrm{E}-03$ & $5.00 \mathrm{E}-02$ \\
\hline Bathyporeia guilliamsoniana & Arthropoda & $5.00 \mathrm{E}-03$ & $5.00 \mathrm{E}-02$ & Arthropoda & $5.00 \mathrm{E}-03$ & $5.00 \mathrm{E}-02$ \\
\hline Bathyporeia pelagica & Arthropoda & $5.00 \mathrm{E}-03$ & $5.00 \mathrm{E}-02$ & Arthropoda & $5.00 \mathrm{E}-03$ & $5.00 \mathrm{E}-02$ \\
\hline Bathyporeia sp. & Arthropoda & $5.00 \mathrm{E}-03$ & $5.00 \mathrm{E}-02$ & Arthropoda & $5.00 \mathrm{E}-03$ & $5.00 \mathrm{E}-02$ \\
\hline Bathyporeia tenuipes & Arthropoda & $5.00 \mathrm{E}-03$ & $5.00 \mathrm{E}-02$ & Arthropoda & $5.00 \mathrm{E}-03$ & $5.00 \mathrm{E}-02$ \\
\hline Brachyura & Arthropoda & $5.00 \mathrm{E}-03$ & $5.00 \mathrm{E}-02$ & Arthropoda & $5.00 \mathrm{E}-03$ & $5.00 \mathrm{E}-02$ \\
\hline
\end{tabular}




\begin{tabular}{|c|c|c|c|c|c|c|}
\hline Corophium acherusicum & Arthropoda & $5.00 \mathrm{E}-03$ & $5.00 \mathrm{E}-02$ & Arthropoda & $5.00 \mathrm{E}-03$ & $5.00 \mathrm{E}-02$ \\
\hline Corophium arenarium & Arthropoda & $5.00 \mathrm{E}-03$ & $5.00 \mathrm{E}-02$ & Arthropoda & $5.00 \mathrm{E}-03$ & $5.00 \mathrm{E}-02$ \\
\hline Corophium lacustre & Arthropoda & $5.00 \mathrm{E}-03$ & $5.00 \mathrm{E}-02$ & Arthropoda & $5.00 \mathrm{E}-03$ & $5.00 \mathrm{E}-02$ \\
\hline Corophium volutator & Arthropoda & $5.00 \mathrm{E}-03$ & $5.00 \mathrm{E}-02$ & Arthropoda & $5.00 \mathrm{E}-03$ & $5.00 \mathrm{E}-02$ \\
\hline Corystes cassivelaunus & Arthropoda & $5.00 \mathrm{E}-03$ & $5.00 \mathrm{E}-02$ & Arthropoda & $5.00 \mathrm{E}-03$ & $5.00 \mathrm{E}-02$ \\
\hline Crangonidae & Arthropoda & $5.00 \mathrm{E}-03$ & $5.00 \mathrm{E}-02$ & Arthropoda & $5.00 \mathrm{E}-03$ & $5.00 \mathrm{E}-02$ \\
\hline Crustacea & Arthropoda & $5.00 \mathrm{E}-03$ & $5.00 \mathrm{E}-02$ & Arthropoda & $5.00 \mathrm{E}-03$ & $5.00 \mathrm{E}-02$ \\
\hline Cumacea & Arthropoda & $5.00 \mathrm{E}-03$ & $5.00 \mathrm{E}-02$ & Arthropoda & $5.00 \mathrm{E}-03$ & $5.00 \mathrm{E}-02$ \\
\hline Cumopsis goodsir & Arthropoda & $5.00 \mathrm{E}-03$ & $5.00 \mathrm{E}-02$ & Arthropoda & $5.00 \mathrm{E}-03$ & $5.00 \mathrm{E}-02$ \\
\hline Decapoda & Arthropoda & $5.00 \mathrm{E}-03$ & $5.00 \mathrm{E}-02$ & Arthropoda & $5.00 \mathrm{E}-03$ & 5.00E-02 \\
\hline Diptera & Arthropoda & $5.00 \mathrm{E}-03$ & $5.00 \mathrm{E}-02$ & Arthropoda & $5.00 \mathrm{E}-03$ & $5.00 \mathrm{E}-02$ \\
\hline Gammaridae & Arthropoda & $5.00 \mathrm{E}-03$ & $5.00 \mathrm{E}-02$ & Arthropoda & $5.00 \mathrm{E}-03$ & $5.00 \mathrm{E}-02$ \\
\hline Gammarus crinicornis & Arthropoda & $5.00 \mathrm{E}-03$ & $5.00 \mathrm{E}-02$ & Arthropoda & $5.00 \mathrm{E}-03$ & $5.00 \mathrm{E}-02$ \\
\hline Gammarus salinus & Arthropoda & $5.00 \mathrm{E}-03$ & $5.00 \mathrm{E}-02$ & Arthropoda & $5.00 \mathrm{E}-03$ & $5.00 \mathrm{E}-02$ \\
\hline Gammarus sp. & Arthropoda & $5.00 \mathrm{E}-03$ & $5.00 \mathrm{E}-02$ & Arthropoda & $5.00 \mathrm{E}-03$ & $5.00 \mathrm{E}-02$ \\
\hline Gammarus zaddachi & Arthropoda & $5.00 \mathrm{E}-03$ & $5.00 \mathrm{E}-02$ & Arthropoda & $5.00 \mathrm{E}-03$ & $5.00 \mathrm{E}-02$ \\
\hline Gastrosaccus spinifer & Arthropoda & $5.00 \mathrm{E}-03$ & $5.00 \mathrm{E}-02$ & Arthropoda & $5.00 \mathrm{E}-03$ & $5.00 \mathrm{E}-02$ \\
\hline Haustorius arenarius & Arthropoda & $5.00 \mathrm{E}-03$ & $5.00 \mathrm{E}-02$ & Arthropoda & $5.00 \mathrm{E}-03$ & $5.00 \mathrm{E}-02$ \\
\hline Hyperia galba & Arthropoda & $5.00 \mathrm{E}-03$ & $5.00 \mathrm{E}-02$ & Arthropoda & $5.00 \mathrm{E}-03$ & $5.00 \mathrm{E}-02$ \\
\hline Idotea balthica & Arthropoda & $5.00 \mathrm{E}-03$ & $5.00 \mathrm{E}-02$ & Arthropoda & $5.00 \mathrm{E}-03$ & $5.00 \mathrm{E}-02$ \\
\hline Jaera (Jaera) istri & Arthropoda & 5.00E-03 & $5.00 \mathrm{E}-02$ & Arthropoda & $5.00 \mathrm{E}-03$ & $5.00 \mathrm{E}-02$ \\
\hline Ligia oceanica & Arthropoda & $5.00 \mathrm{E}-03$ & $5.00 \mathrm{E}-02$ & Arthropoda & $5.00 \mathrm{E}-03$ & $5.00 \mathrm{E}-02$ \\
\hline Liocarcinus holsatus & Arthropoda & $5.00 \mathrm{E}-03$ & $5.00 \mathrm{E}-02$ & Arthropoda & $5.00 \mathrm{E}-03$ & 5.00E-02 \\
\hline Lophogaster typicus & Arthropoda & $5.00 \mathrm{E}-03$ & $5.00 \mathrm{E}-02$ & Arthropoda & $5.00 \mathrm{E}-03$ & $5.00 \mathrm{E}-02$ \\
\hline Monoculodes carinatus & Arthropoda & $5.00 \mathrm{E}-03$ & $5.00 \mathrm{E}-02$ & Arthropoda & $5.00 \mathrm{E}-03$ & $5.00 \mathrm{E}-02$ \\
\hline Mysidacea & Arthropoda & $5.00 \mathrm{E}-03$ & $5.00 \mathrm{E}-02$ & Arthropoda & $5.00 \mathrm{E}-03$ & $5.00 \mathrm{E}-02$ \\
\hline Neomysis integer & Arthropoda & $5.00 \mathrm{E}-03$ & $5.00 \mathrm{E}-02$ & Arthropoda & $5.00 \mathrm{E}-03$ & $5.00 \mathrm{E}-02$ \\
\hline Nymphon brevirostre & Arthropoda & $5.00 \mathrm{E}-03$ & $5.00 \mathrm{E}-02$ & Arthropoda & $5.00 \mathrm{E}-03$ & $5.00 \mathrm{E}-02$ \\
\hline Nymphon sp. & Arthropoda & $5.00 \mathrm{E}-03$ & $5.00 \mathrm{E}-02$ & Arthropoda & $5.00 \mathrm{E}-03$ & $5.00 \mathrm{E}-02$ \\
\hline Pagurus bernhardus & Arthropoda & $5.00 \mathrm{E}-03$ & $5.00 \mathrm{E}-02$ & Arthropoda & $5.00 \mathrm{E}-03$ & $5.00 \mathrm{E}-02$ \\
\hline Pandalus montagui & Arthropoda & $5.00 \mathrm{E}-03$ & $5.00 \mathrm{E}-02$ & Arthropoda & $5.00 \mathrm{E}-03$ & $5.00 \mathrm{E}-02$ \\
\hline Pontocrates altamarinus & Arthropoda & $5.00 \mathrm{E}-03$ & $5.00 \mathrm{E}-02$ & Arthropoda & $5.00 \mathrm{E}-03$ & $5.00 \mathrm{E}-02$ \\
\hline Praunus flexuosus & Arthropoda & $5.00 \mathrm{E}-03$ & $5.00 \mathrm{E}-02$ & Arthropoda & $5.00 \mathrm{E}-03$ & $5.00 \mathrm{E}-02$ \\
\hline Sacculina carcini & Arthropoda & $5.00 \mathrm{E}-03$ & $5.00 \mathrm{E}-02$ & Arthropoda & $5.00 \mathrm{E}-03$ & $5.00 \mathrm{E}-02$ \\
\hline Schistomysis kervillei & Arthropoda & $5.00 \mathrm{E}-03$ & $5.00 \mathrm{E}-02$ & Arthropoda & $5.00 \mathrm{E}-03$ & $5.00 \mathrm{E}-02$ \\
\hline Schistomysis ornata & Arthropoda & $5.00 \mathrm{E}-03$ & $5.00 \mathrm{E}-02$ & Arthropoda & $5.00 \mathrm{E}-03$ & $5.00 \mathrm{E}-02$ \\
\hline Schistomysis spiritus & Arthropoda & $5.00 \mathrm{E}-03$ & $5.00 \mathrm{E}-02$ & Arthropoda & $5.00 \mathrm{E}-03$ & $5.00 \mathrm{E}-02$ \\
\hline Stenothoe marina & Arthropoda & $5.00 \mathrm{E}-03$ & $5.00 \mathrm{E}-02$ & Arthropoda & $5.00 \mathrm{E}-03$ & $5.00 \mathrm{E}-02$ \\
\hline Alcyonidioides mytili & Bryozoa & & & Bryozoa & & \\
\hline Bowerbankia sp. & Bryozoa & & & Bryozoa & & \\
\hline Conopeum reticulum & Bryozoa & & & Bryozoa & & \\
\hline Conopeum seurati & Bryozoa & & & Bryozoa & & \\
\hline Einhornia crustulenta & Bryozoa & & & Bryozoa & & \\
\hline Electra crustulenta & Bryozoa & & & Bryozoa & & \\
\hline Electra pilosa & Bryozoa & & & Bryozoa & & \\
\hline Farrella repens & Bryozoa & & & Bryozoa & & \\
\hline Chaetognatha & Chaetognatha & & & Chaetognatha & & \\
\hline Syngnathus rostellatus & Chordata & & & Chordata & & \\
\hline
\end{tabular}




\begin{tabular}{|c|c|c|c|c|c|c|}
\hline Abietinaria abietina & Cnidaria & & & Cnidaria & & \\
\hline Actiniaria & Cnidaria & & & Cnidaria & & \\
\hline Anthozoa & Cnidaria & & & Cnidaria & & \\
\hline Clytia hemisphaerica & Cnidaria & & & Cnidaria & & \\
\hline Cordylophora caspia & Cnidaria & & & Cnidaria & & \\
\hline Eudendrium rameum & Cnidaria & & & Cnidaria & & \\
\hline Eudendrium ramosum & Cnidaria & & & Cnidaria & & \\
\hline Laomedea flexuosa & Cnidaria & & & Cnidaria & & \\
\hline Obelia bidentata & Cnidaria & & & Cnidaria & & \\
\hline Obelia dichotoma & Cnidaria & & & Cnidaria & & \\
\hline Obelia longissima & Cnidaria & & & Cnidaria & & \\
\hline Obelia sp. & Cnidaria & & & Cnidaria & & \\
\hline Opercularella lacerata & Cnidaria & & & Cnidaria & & \\
\hline Sagartia sp. & Cnidaria & & & Cnidaria & & \\
\hline Sagartia troglodytes & Cnidaria & & & Cnidaria & & \\
\hline Sertularia cupressina & Cnidaria & & & Cnidaria & & \\
\hline Tubularia indivisa & Cnidaria & & & Cnidaria & & \\
\hline Tubularia larynx & Cnidaria & & & Cnidaria & & \\
\hline Asterias rubens & Echinodermata & $5.00 \mathrm{E}-02$ & $5.00 \mathrm{E}-01$ & Echinodermata & 5.00E-02 & $5.00 \mathrm{E}-01$ \\
\hline Echinocardium cordatum & Echinodermata & $5.00 \mathrm{E}-02$ & $5.00 \mathrm{E}-01$ & Echinodermata & $5.00 \mathrm{E}-02$ & $5.00 \mathrm{E}-01$ \\
\hline Ophiura ophiura & Echinodermata & $5.00 \mathrm{E}-02$ & $5.00 \mathrm{E}-01$ & Echinodermata & $5.00 \mathrm{E}-02$ & $5.00 \mathrm{E}-01$ \\
\hline Pedicellina cernua & Entoprocta & & & Entoprocta & & \\
\hline Abra alba & Mollusca & $1.00 \mathrm{E}-01$ & $1.00 \mathrm{E}+00$ & Mollusca_Small & $1.00 \mathrm{E}-01$ & $1.00 \mathrm{E}+00$ \\
\hline Abra tenuis & Mollusca & $1.00 \mathrm{E}-01$ & $1.00 \mathrm{E}+00$ & Mollusca_Small & $1.00 \mathrm{E}-01$ & $1.00 \mathrm{E}+00$ \\
\hline Cerastoderma edule & Mollusca & $1.00 \mathrm{E}-01$ & $1.00 \mathrm{E}+00$ & Mussels & $1.00 \mathrm{E}-01$ & $1.00 \mathrm{E}+00$ \\
\hline Crassostrea gigas & Mollusca & $1.00 \mathrm{E}-01$ & $1.00 \mathrm{E}+00$ & Mollusca_large & $1.00 \mathrm{E}-01$ & $1.00 \mathrm{E}+00$ \\
\hline Crepidula fornicata & Mollusca & $1.00 \mathrm{E}-01$ & $1.00 \mathrm{E}+00$ & Periwinkle & $1.00 \mathrm{E}-02$ & $1.00 \mathrm{E}-01$ \\
\hline Ensis directus & Mollusca & $1.00 \mathrm{E}-01$ & $1.00 \mathrm{E}+00$ & Mussels & $1.00 \mathrm{E}-01$ & $1.00 \mathrm{E}+00$ \\
\hline Kurtiella bidentata & Mollusca & $1.00 \mathrm{E}-01$ & $1.00 \mathrm{E}+00$ & Mollusca_Small & $1.00 \mathrm{E}-01$ & $1.00 \mathrm{E}+00$ \\
\hline Lepidochitona cinerea & Mollusca & $1.00 \mathrm{E}-01$ & $1.00 \mathrm{E}+00$ & Periwinkle & $1.00 \mathrm{E}-01$ & $1.00 \mathrm{E}+00$ \\
\hline Littorina littorea & Mollusca & $1.00 \mathrm{E}-01$ & $1.00 \mathrm{E}+00$ & Periwinkle & $1.00 \mathrm{E}-02$ & $1.00 \mathrm{E}-01$ \\
\hline Macoma balthica & Mollusca & $1.00 \mathrm{E}-01$ & $1.00 \mathrm{E}+00$ & Mollusca_Small & $1.00 \mathrm{E}-01$ & $1.00 \mathrm{E}+00$ \\
\hline Mya arenaria & Mollusca & $1.00 \mathrm{E}-01$ & $1.00 \mathrm{E}+00$ & Mollusca_large & $1.00 \mathrm{E}-01$ & $1.00 \mathrm{E}+00$ \\
\hline Mytilus edulis & Mollusca & $1.00 \mathrm{E}-01$ & $1.00 \mathrm{E}+00$ & Mussels & $1.00 \mathrm{E}-01$ & $1.00 \mathrm{E}+00$ \\
\hline Peringia ulvae & Mollusca & $1.00 \mathrm{E}-01$ & $1.00 \mathrm{E}+00$ & Mudsnail & $1.00 \mathrm{E}-03$ & $1.00 \mathrm{E}-02$ \\
\hline Petricolaria pholadiformis & Mollusca & $1.00 \mathrm{E}-01$ & $1.00 \mathrm{E}+00$ & Mollusca_large & $2.00 \mathrm{E}-01$ & $1.00 \mathrm{E}+00$ \\
\hline Retusa obtusa & Mollusca & $1.00 \mathrm{E}-01$ & $1.00 \mathrm{E}+00$ & Mudsnail & $1.00 \mathrm{E}-01$ & $1.00 \mathrm{E}+00$ \\
\hline Scrobicularia plana & Mollusca & $1.00 \mathrm{E}-01$ & $1.00 \mathrm{E}+00$ & Mussels & $1.00 \mathrm{E}-01$ & $1.00 \mathrm{E}+00$ \\
\hline Tellina tenuis & Mollusca & $1.00 \mathrm{E}-01$ & $1.00 \mathrm{E}+00$ & Mollusca_Small & 1.00E-01 & $1.00 \mathrm{E}+00$ \\
\hline Ventrosia ventrosa & Mollusca & $1.00 \mathrm{E}-01$ & $1.00 \mathrm{E}+00$ & Mollusca_Small & $1.00 \mathrm{E}-01$ & $1.00 \mathrm{E}+00$ \\
\hline Ensis sp. & Mollusca & $1.00 \mathrm{E}-01$ & $1.00 \mathrm{E}+00$ & Mussels & 1.00E-01 & $1.00 \mathrm{E}+00$ \\
\hline Fabulina fabula & Mollusca & $1.00 \mathrm{E}-01$ & $1.00 \mathrm{E}+00$ & Mollusca_Small & $1.00 \mathrm{E}-01$ & $1.00 \mathrm{E}+00$ \\
\hline Gastropoda & Mollusca & $1.00 \mathrm{E}-01$ & $1.00 \mathrm{E}+00$ & Periwinkle & $1.00 \mathrm{E}-01$ & $1.00 \mathrm{E}+00$ \\
\hline Hydrobia ulvae & Mollusca & $1.00 \mathrm{E}-01$ & $1.00 \mathrm{E}+00$ & Mudsnail & $1.00 \mathrm{E}-01$ & $1.00 \mathrm{E}+00$ \\
\hline Mysella bidentata & Mollusca & $1.00 \mathrm{E}-01$ & $1.00 \mathrm{E}+00$ & Mollusca_Small & $1.00 \mathrm{E}-01$ & $1.00 \mathrm{E}+00$ \\
\hline Solenidae & Mollusca & $1.00 \mathrm{E}-01$ & $1.00 \mathrm{E}+00$ & Mollusca_Small & $1.00 \mathrm{E}-01$ & $1.00 \mathrm{E}+00$ \\
\hline Tellimya ferruginosa & Mollusca & $1.00 \mathrm{E}-01$ & $1.00 \mathrm{E}+00$ & Mollusca_Small & $1.00 \mathrm{E}-01$ & $1.00 \mathrm{E}+00$ \\
\hline Varicorbula gibba & Mollusca & $1.00 \mathrm{E}-01$ & $1.00 \mathrm{E}+00$ & Mollusca_Small & $1.00 \mathrm{E}-01$ & $1.00 \mathrm{E}+00$ \\
\hline
\end{tabular}




\begin{tabular}{|l|l|l|l|l|l|l|}
\hline Nematoda & Nematoda & & & Nematoda & & \\
\hline Nemertini sp. & Nemertea & & & Nemertea & & \\
\hline Nemertea & Nemertea & & & Nemertea & & \\
\hline Nemertini & Nemertea & & & Nemertea & & \\
\hline Turbellaria & Platyhelminthes & & Platyhelminthes & \\
\hline Halichondria panicea & Porifera & & & Porifera & & \\
\hline
\end{tabular}




\section{A12 Depth data}

Depth data of the Dutch Wadden Sea were obtained from Deltares as ( $x, y$, Depth), and covered a larger part of the area than needed. Data had a non-uniform density distribution, with less data per unit of area on the North Sea than inside the area. Data were reduced to the simulation area and through an R-kriging routine (Nabil, 2015) computed for a 50*50m grid cell representation of the area (Figure 71).

Next, average depths for each sub-compartment was computed; these results are part of the model input.

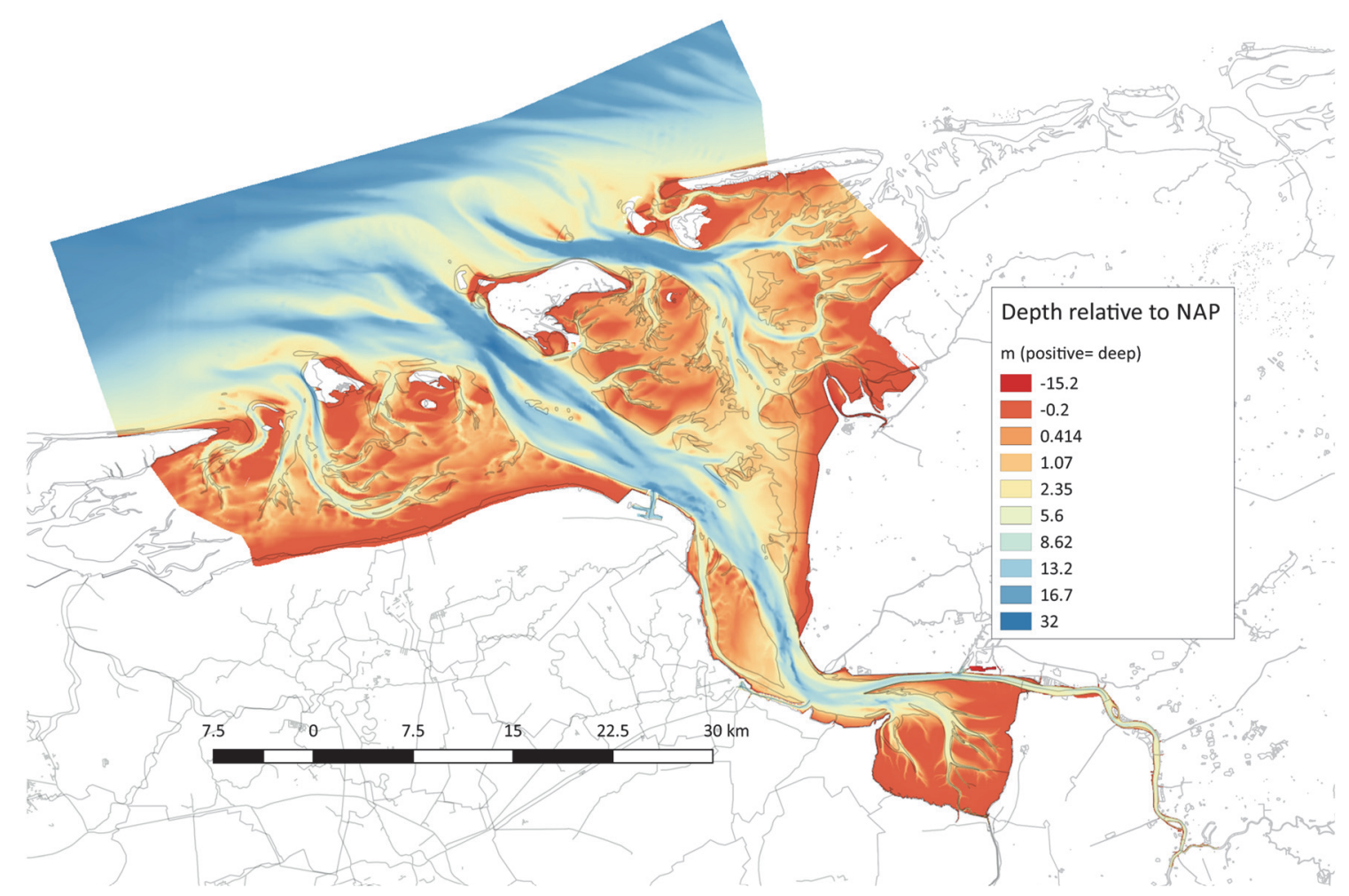

Figure 71 Depth WaddenSea (Source: Deltares) 


\section{A13 Water level data}

Water level data were available for several sites (Figure 72), and thus it can be computed in what tidal phase all time series data have been assessed. However, this has not been used in the present model evaluation.

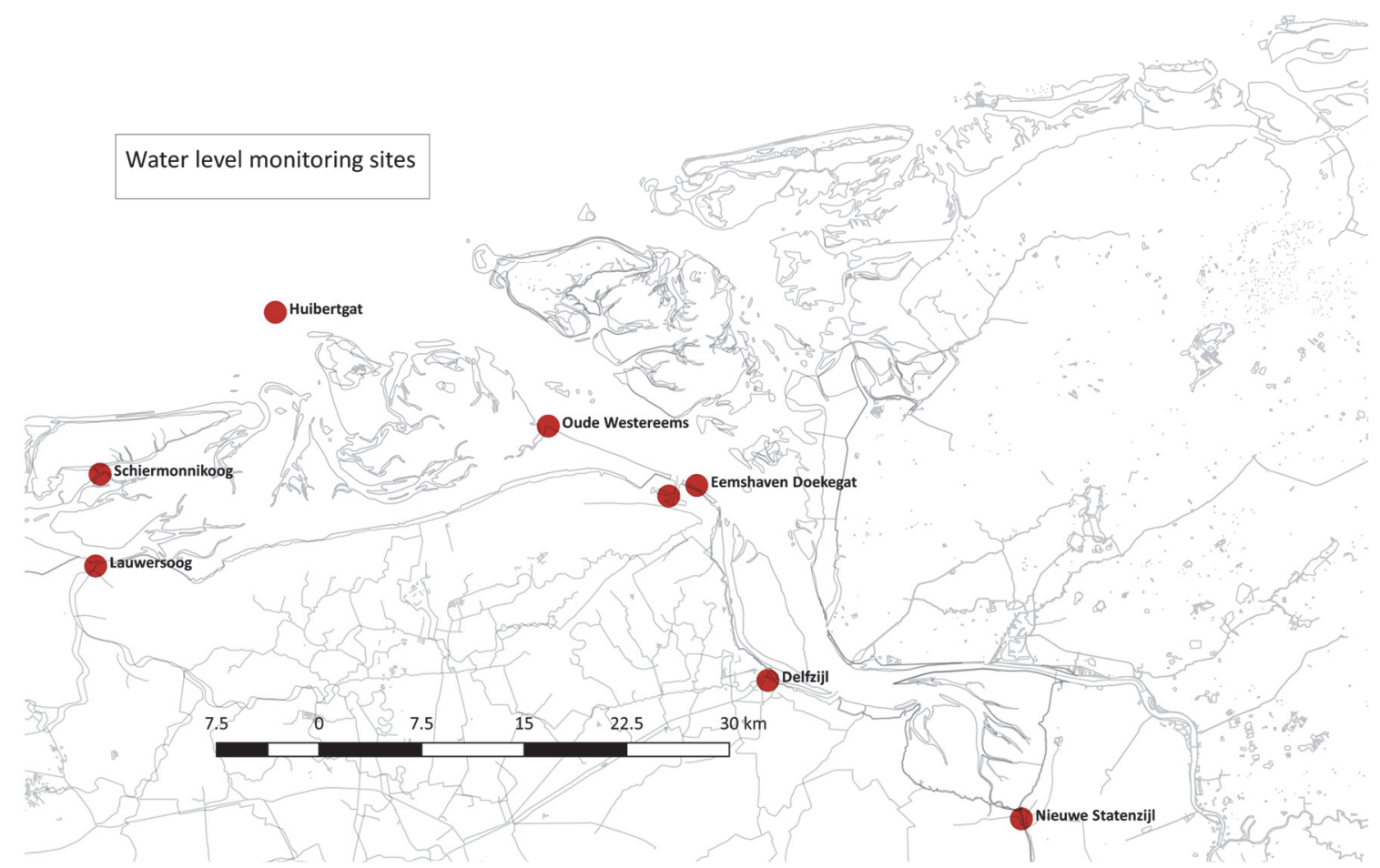

Figure 72 Monitoring stations water level. Available data: Huibertgat, Delfzijl, Schiermonnikoog, Lauwersoog whole period 1976-2016 (the latter two are a bit outside the study area), Nieuw Statenzijl 1979-2016, Eemshaven 1978-2016, and Oude Westereems and Eemshaven Doekegat 1983-1986. 


\section{A14 Sediment data}

\section{A14.1 Sediment data Netherlands, North Sea}

Data were available from the former Rijks-Geologische Dienst, later NITG (Utrecht), and nowadays part of Deltares. Most data are available, after kriging, on a $250 * 250 \mathrm{~m}$ grid basis. Data consist of median grain sizes (M00) for all particles (Figure 73), and silt content (as mass-\%). Silt is considered as all particles smaller than $63 \mu \mathrm{m}$. Analyses by NITG (NL) have been performed with a Particle Sizer (type unknown).

\section{A14.2 Sediment data Dutch Wadden Sea}

Data from the period 1988-1995 were available (RIKZ, 1998) (Figure 74). Some later data do exist, but were not collected yet. There is a discussion on the reliability of the data, see appendix A15.

\section{A14.3 Sediment data Germany}

Data were available for the same sampling sites as depth (Figure 75). Values for 69 grain size classes have been provided, and as summary: mass fractions of silt $(<63 \mu \mathrm{m})$, sand $(63-2000 \mu \mathrm{m})$ and gravel (> $2000 \mu \mathrm{m}$ ). Unfortunately, almost all German data are outside the simulation area; sampling sites inside the simulation area (Figure 75) appeared to have depth data only.

\section{A14.4 Combining these data}

The previous three data sets must be combined. The four sets must be compatible, and thus it is checked whether overlapping data are consistent (Figure 76). This is rather tricky, since there is a discussion on the silt analyses by GeoSea (see e.g. Zwarts et al, 2004). Simply take mean values (if more values are available) is not realistic: the Zwarts-correction implied that the GeoSea-values became much lower than had been measured (see appendix A15).

All GeoDea-silt data were multiplied by a factor 0.48 to get the new data; North Sea silt-data were taken unchanged. Then, al other variables (see appendix A15) were computed from the silt content.

Several data are pair-plotted in Figure 76, and show similarities and relationships between data from the three datasets for the same $50 * 50 \mathrm{~m}$ grid cell. E.g. the plot cell $(1,2)$ tells us the difference between the GeoSea-data and the Zwarts-corrected data. Cell $(2,4)$ shows that the NSsilt data and the Zwarts-data are more or less similar.

\section{A14.5 Missing data}

Since data for the German area (outside the RIKZ-data area, Figure 74) are scarce, it was decided to copy values from similar Dutch sites. Thus, sediment composition data for those German subcompartments outside the area shown in Figure 74 are based on estimations. 


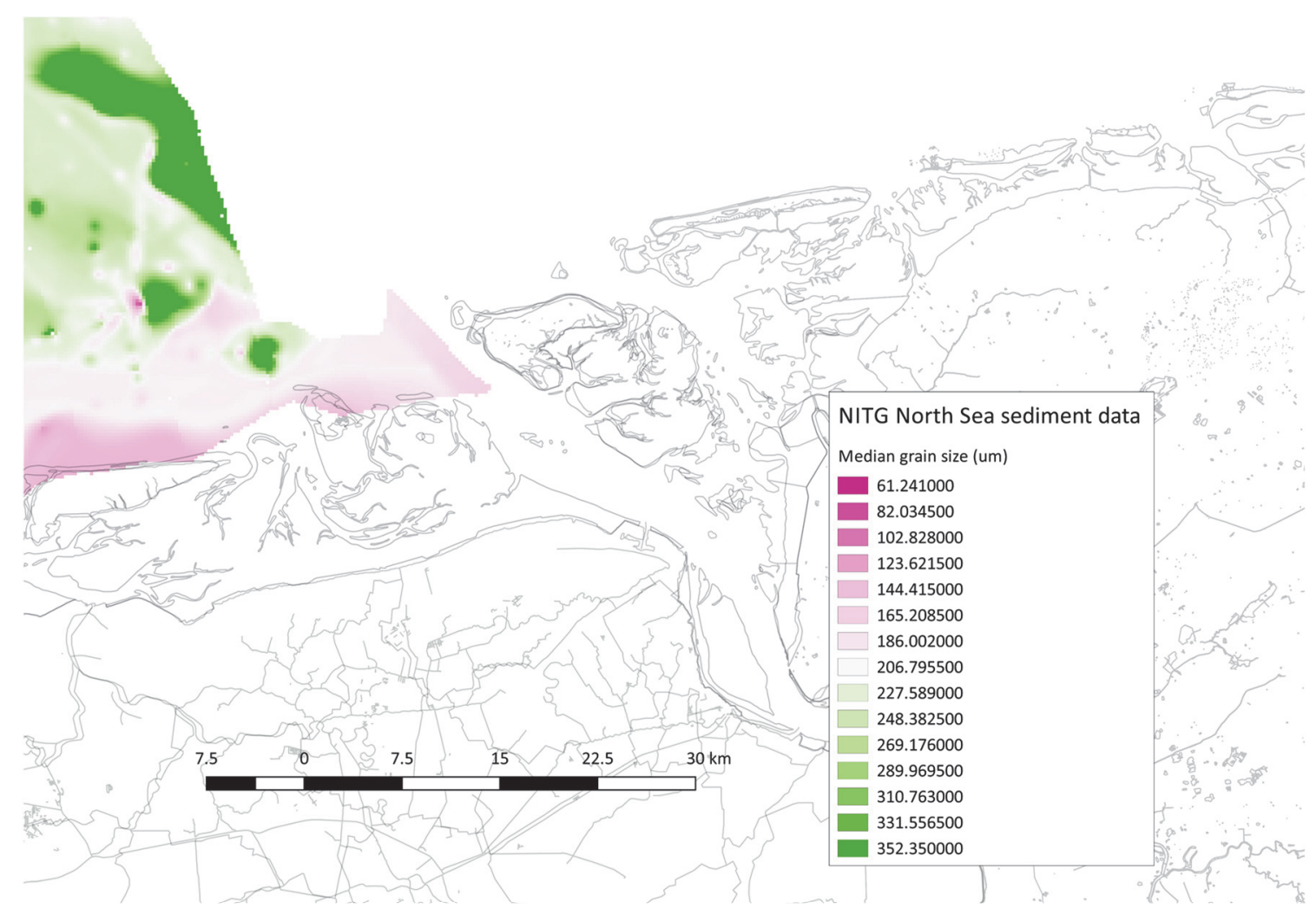

Figure 73 Available sediment data for the North Sea coastal zone; simulation compartments included. Data from NITG.

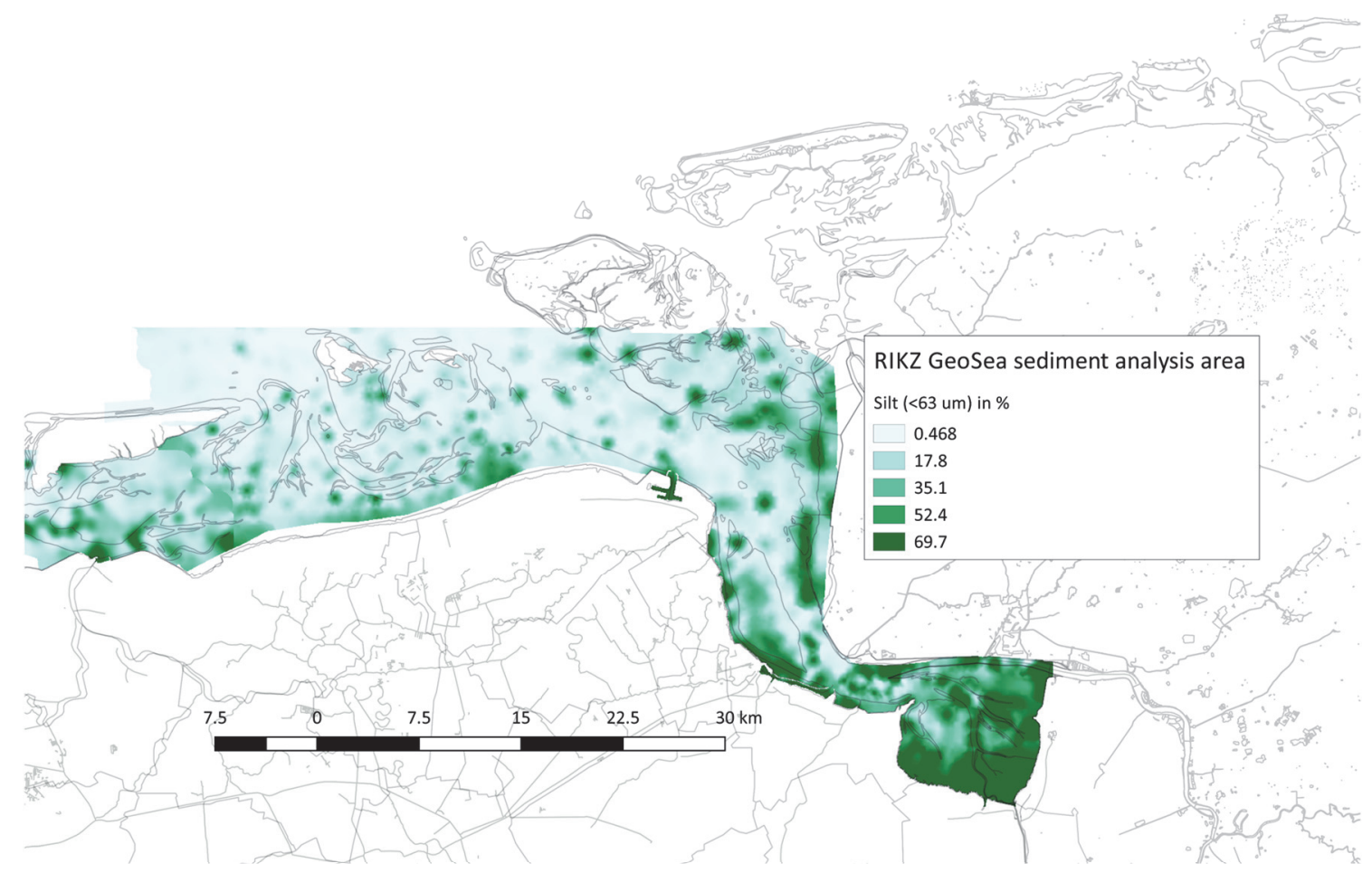

Figure 74 GEOSEA-sediment data (silt content in mass-\%). 


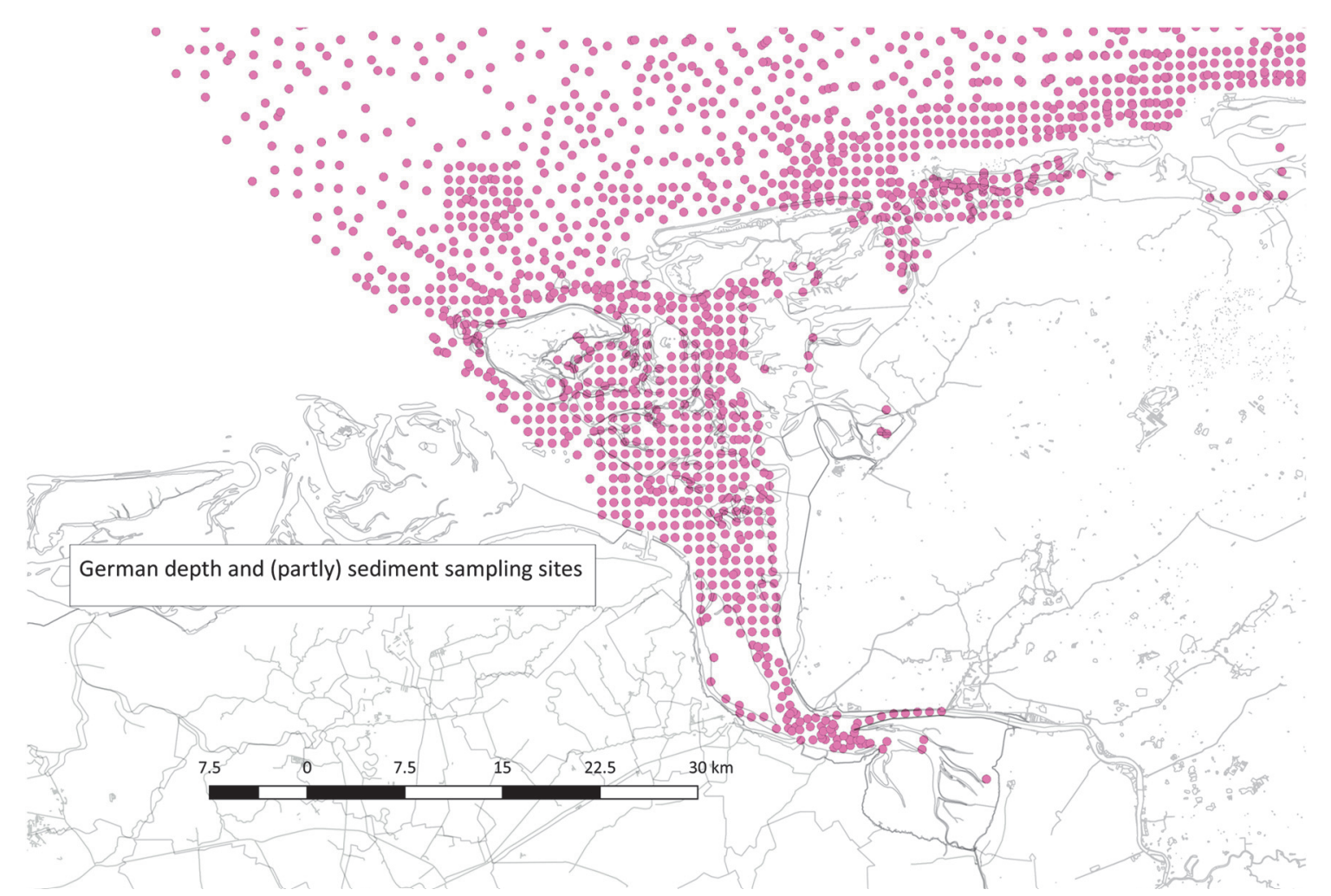

Figure 75 German sampling sites. Allmost all sites appeared to have depth data only. Just a few sites -all outside the modelling area- contained sediment composition data. 


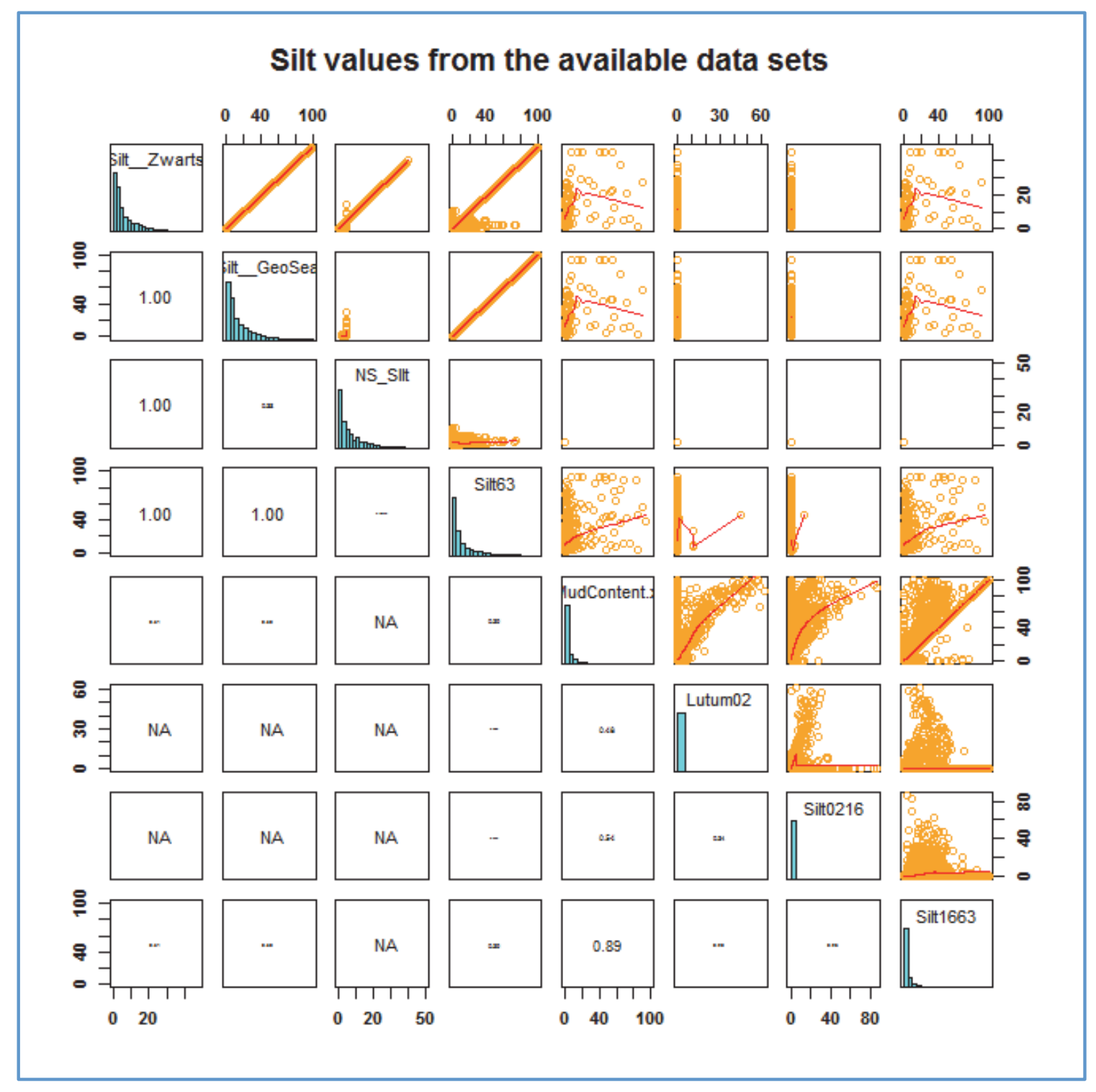

Figure 76 Sediment silt content, all data sets together. Silt_GeoSea concerns the Rijkswaterstaat "Sedimentatlas"-set (RWS, 1998); elaborated (and corrected) by Zwarts 9204): Silt Zwarts. Silt63 is the same GeoSea-dataset, but extended to the German Wadden Sea. NS_Silt is the NITG-dataset. Mudcontent, Lutum02, Silt0216 and Silt1663 concerns the German data set. MudContent being the sum of the other three (separate) fractions. These separate fractions have been measured (are not computed from MudContent) and show some, but not a very clear relationship with MudContent. In those cases where there is an overlap with the Dutch Wadden Sea data, the relationship is weak, although preferably it should be 1:1. Similarly, the relationship between NS_Silt and Silt63 had to be 1:1, but also here it appears that it is weak. Simply take mean values (if more values are available) is not realistic: the Zwarts-correction implied that the GeoSeavalues became much lower than had been measured. In appendix A15 it is explained how this question was handled. 


\section{A15 Elaboration sediment data}

\section{A15.1 GeoSea-data (RWS, 1998)}

Sediment data are available after a systemwide sampling and analyses by GeoSea in the late ' 80 's and early '90's (RIKZ 1998). Analyses were performed with a Malvern particles sizer; see RIKZ 1998 for all details. There is some discussion on the correctness of the data for silt content, see Zwarts (1988). Zwarts (2004) compared GeoSea-analyses results obtained with other analysis methods and-equipment.

As a result, he found some conversion factors enabling an estimate for the content of fine particles (as: mass-\% of total dry matter):

$$
\begin{aligned}
& \text { Slib0063 (all <63 } \mu \mathrm{m})=0.48 * \text { GeoSea-silt (RIKZ 1998) (mass-\%) } \\
& \text { Slib0016 (all <16 } \mu \mathrm{m}) \quad=0.1879 * \text { GeoSea-silt (RIKZ 1998) }(\text { mass-\%) } \\
& =0.391 * \text { Slib0063 }
\end{aligned}
$$

Fine particles may be sub-divided:

$$
\begin{aligned}
\operatorname{Lutum}(<2 \mu \mathrm{m}) & =0.423+0.61 * \text { Slib0016 }(\text { mass }-\%) \text {, or: } \\
& =0.63 * \text { Slib0016 (mass-\%) }
\end{aligned}
$$

Thus, it follows that:

$$
\begin{array}{lll}
\text { Slib0216 }(2 \mu \mathrm{m}<\text { Slib0216 }<16 \mu \mathrm{m}) & =\text { Slib0016 }- \text { Lutum } \quad \text { (mass-\%) } & 3 \\
\text { Slib1663 }(16 \mu \mathrm{m}<\text { Slib1663 }<63 \mu \mathrm{m}) & =\text { Slib0063 - Slib0016 (mass-\%) }
\end{array}
$$

From Lake Veluwe data (Brinkman \& Van Raaphorst, 1986) it is derived that, using a simple linearregression analysis:

Humus

$$
=0.27 * \text { Lutum }(\text { mass }-\%)
$$

For this project, also an estimate is made fro the particle fraction between 63-125 and 125-250 $\mu \mathrm{m}$. this was based on the median grainsize (M16, $\mu \mathrm{m}$; the index 16 implies that only particles $>16$ $\mu \mathrm{m}$ have been used by GeoSea when computing the median grain size value) and an assumed normal size distribution for the sand fraction. The standard deviation used was estimated based on several tests (not mentioned in more detail here), using median values for all particles together (M0). Then the question: at which M0 would the same M16 been found; this gives estimates for the fraction fr63-125 and fr125-250. This method gives different results depending on the M16value.

Of course, this is a simple method, but for the time being it is used for the model exercises. It follows:

$$
\begin{array}{lll}
\text { Sand63+ }(\text { all }>63 \mu \mathrm{m}) \quad=100-\text { Silt0063 } \text { (mass-\%) }- \text { Humus } \quad \text { (mass-\%) } & 6 \\
\text { Sand63125 }(63 \mu \mathrm{m}<\text { Sand63125 }<125 \mu \mathrm{m}) & =\text { fr125*100 }- \text { Silt0063 }- \text { Humus } & \text { (mass-\%) } \\
\text { Sand125250 }(125 \mu \mathrm{m}<\text { Sand125250<250 } \mu \mathrm{m})=\text { fr250*100 }- \text { fr125*100 } & \text { (mass-\%) }
\end{array}
$$




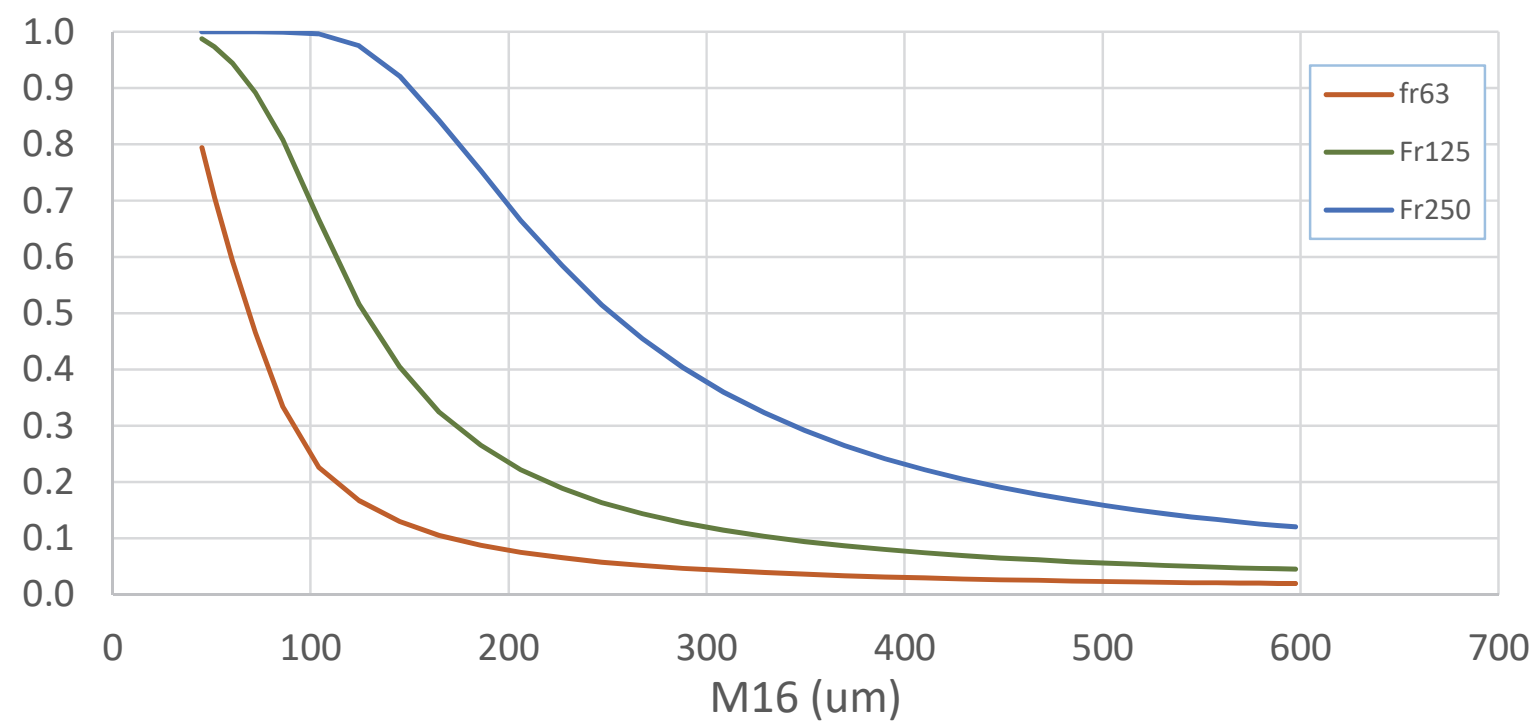

Figure 77 Example of values form fr63, fr125 and fr250 at a certain value for M16. Note that $Y$-value $=0.5$ is reached at $M 16(=X)=72$ for fr63. Only at low M16-values M16 there is a substantial difference between M16 and $\mathrm{MO}$.

\section{A15.2 Estimation of other sediment compounds}

With the already mentioned data for Lake Veluwe (Brinkman \& Van Raaphorst, 1986) another analysis has been performed, giving an estimate for the pore water content, content of organic matter and several inorganic components. These analyses gave:

$\mathrm{Fe}$

$$
\begin{aligned}
& =4.27+0.612 * \text { Silt0016 } \\
& =1.3 * \text { Lutum }
\end{aligned}
$$$$
\text { (mg Fe/g) }
$$$$
\text { (Lutum in mass-\%, Fe in } \mathrm{mg} / \mathrm{g} \mathrm{DM} \text { ) }
$$

$P$$$
=0.192+0.0149 * \text { Silt0016 }
$$$$
=0.0393 * \text { Lutum }
$$$$
\text { (mg } \left.\mathrm{P} \mathrm{g}^{-1} \mathrm{DM}\right) \text {, lutum in mass-\% }
$$

$\mathrm{Ca}$

$$
=10.56+1.13 * \text { Silt0016 }
$$$$
=2.65 * \text { Lutum }
$$$$
\text { (mg Ca g } \left.{ }^{-1} \mathrm{DS}\right)
$$

$\mathrm{CO} 3$

$$
=0.62 * \text { Lutum }
$$

$\left(\mathrm{mg} \mathrm{CO} \mathrm{CO}^{-1} \mathrm{DS}\right)$

10

$\mathrm{Mg}$

$$
\begin{aligned}
& =1.27+0.193 * \text { Silt0016 } \\
& =0.411 * \text { Lutum }
\end{aligned}
$$


Several phosphate adsorption fractions have been determined as well (following Hieltjes, 1980; see Brinkman \& Van Raaphorst, 1986):

$$
\begin{array}{lll}
\text { P_NH4 } & =0.00138 * \text { Lutum } & \left(\mathrm{mg} \mathrm{P} \mathrm{g}^{-1} \mathrm{DS}\right) \\
\mathrm{P}_{-} \mathrm{OH} & =0.00724 * \text { Lutum } & \left(\mathrm{mg} \mathrm{P} \mathrm{g}^{-1} \mathrm{DS}\right) \\
\mathrm{P}_{-} \mathrm{HCl} & =0.0130 * \text { Lutum } & \left(\mathrm{mg} \mathrm{P} \mathrm{g}^{-1} \mathrm{DS}\right) \\
\text { P_Extractable } & =\text { sum of the three fractions }) & \\
& =0.0215 * \text { Lutum } &
\end{array}
$$

All these analyses give the relative content $\mathrm{mg} \mathrm{g}^{-1}$ sediment, while the amount per unit volume is required. Thus, the sediment porosity must be included. Its values follow from the iron content (also from the Lake Veluwe analyses, computed for the silt-values mentioned above):

$\mathrm{H}_{2} \mathrm{O}=18.73+1.212 * \mathrm{Fe} \quad\left(\mathrm{mg} \mathrm{g}^{-1} \mathrm{DS}\right)$

Now the volume fraction of water can be computed. The total volume of $1 \mathrm{~g}$ sediment is (solid mass solid / $\rho_{\text {solids }}+$ water mass). $\rho_{\text {solids }}$ is the specific mass of the solids $\left(\mathrm{g} \mathrm{m}^{-3}\right)$, estimated at $2.610^{3}$ $\left(\mathrm{g} \mathrm{m}^{-3}\right)$, a value often used:

mass- $\% \mathrm{H}_{2} \mathrm{O}=\frac{v o l \% H 2 O \cdot \rho_{\mathrm{H} 2 \mathrm{O}}}{v o l \% H 2 O \cdot \rho_{\mathrm{H} 2 \mathrm{O}}+(100-v o l \% H 2 O) \cdot \rho_{\text {sol }}}$

$\rho_{\mathrm{H} 2 \mathrm{O}}$ is the specific mass $\left(\mathrm{g} \mathrm{m}^{-3}\right)$ van water. After rewriting eq(7) it follows that

vol\% $\mathrm{H} 2 \mathrm{O}=\frac{100 \cdot \mathrm{mass} \% \mathrm{H} 2 \mathrm{O} \cdot \rho_{\text {sol }}}{100 \cdot \rho_{\mathrm{H} 2 \mathrm{O}}+\mathrm{mass}_{\mathrm{S}} \mathrm{H} 2 \mathrm{O} \cdot \rho_{\mathrm{sol}}-\mathrm{mass} \% \mathrm{H} 2 \mathrm{O} \cdot \rho_{\mathrm{H} 2 \mathrm{O}}}$

$\rho_{\mathrm{H} 2 \mathrm{O}}=1$, en $\rho_{\text {sol }}$ is $2.60\left(\mathrm{~kg} \mathrm{dm}^{-3}\right.$, or $\left.2.610^{6} \mathrm{~g} \mathrm{~m}^{-3}\right)$.

These equations give the opportunity to estimate the Wadden Sea sediment composition, since the volume- $\%$ of the solid $=100-\mathrm{vol}-\% \mathrm{H}_{2} \mathrm{O}$, and the mass per $\mathrm{m}^{3}$ follows directly from

massa_m $\mathrm{m}^{-3}=$ volume- $\%$ of the solid $/ 100 * 2.610^{3} *$ fraction dry matter.

Now the absolute mass values of all relevant components in the sediment are known. Of course, the procedure can be improved, but at this moment it is the best-available data et for the EDecosystem modelling. 


\section{A15.3 Relationship German and Dutch Wadden Sea data}

Available data for the Dutch Wadden Sea, North Sea and the German part of the simulation area are shown in Figure 78: there is hardly a correlation. Basically, this is the result of the existing variability of the area; samples that are supposed to originate from the same site may very well concern different sediment composition.

An important question is whether the North Sea and the German data must be recompiled, like the GeoSea data. According to Figure 78 (upper right, lower left) it seems not necessary, although the data are very scattered. For the North Sea data, it seems also to be the case. GeoSea data clearly are higher than the North Sea data, and thus it was decided to keep these data as they are, and just recompile all the GeoSea data (Silt63 in Figure 76) according to eqs 1-6. After that: values are simply averaged in those cases where more than one data was available for a grid cell.

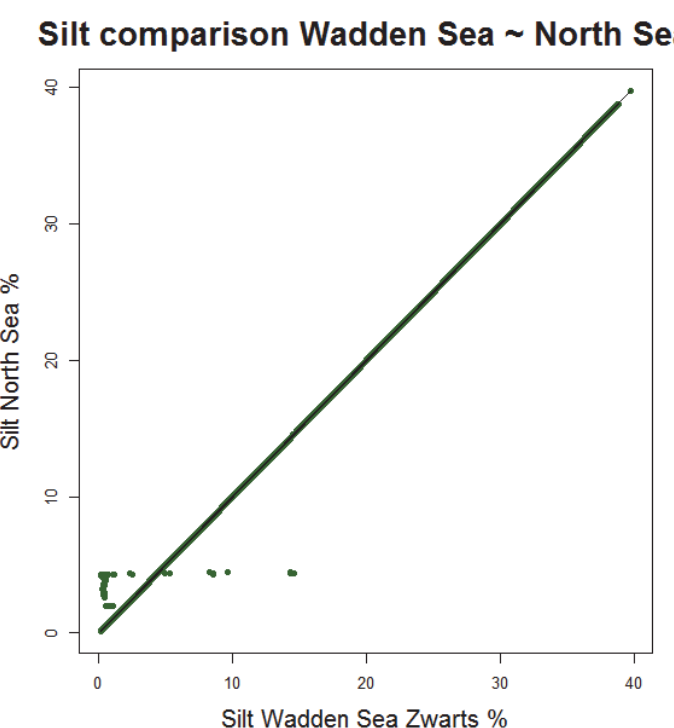

Silt comparison Wadden Sea NL DE

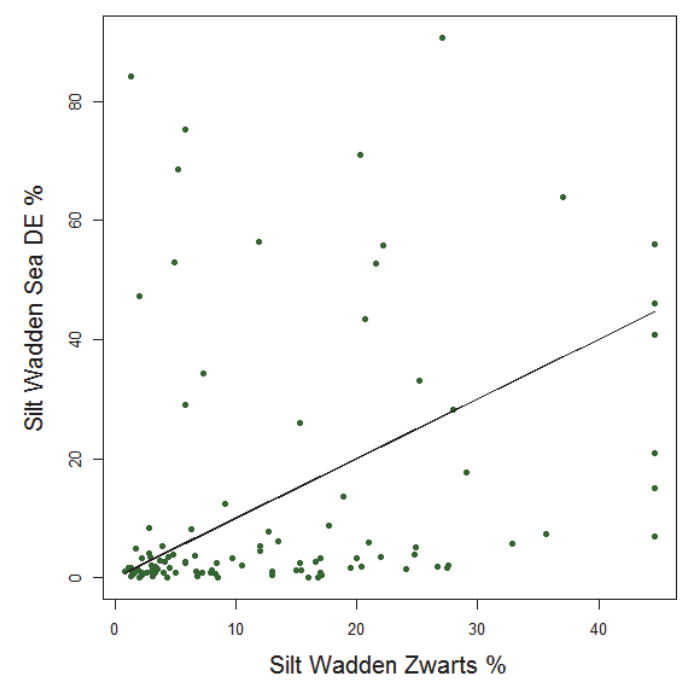

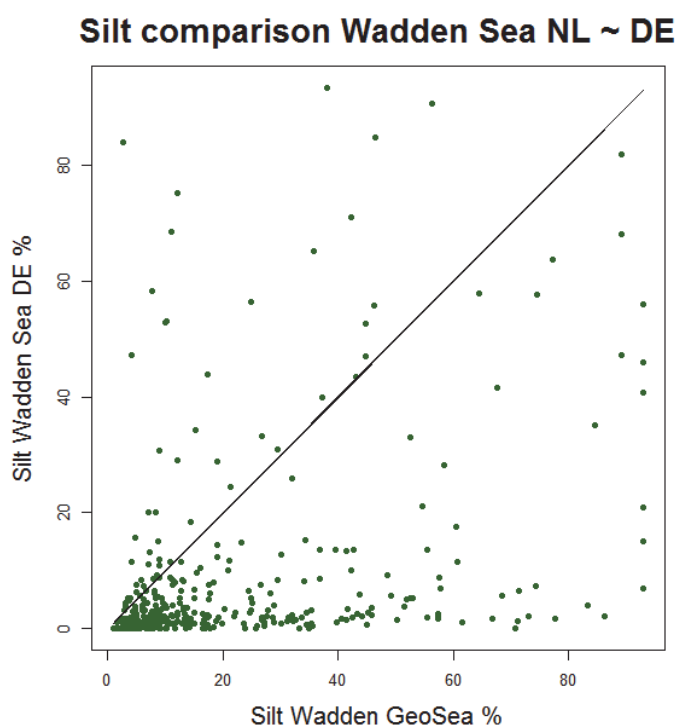

Silt comparison Wadden Sea NL North Sea

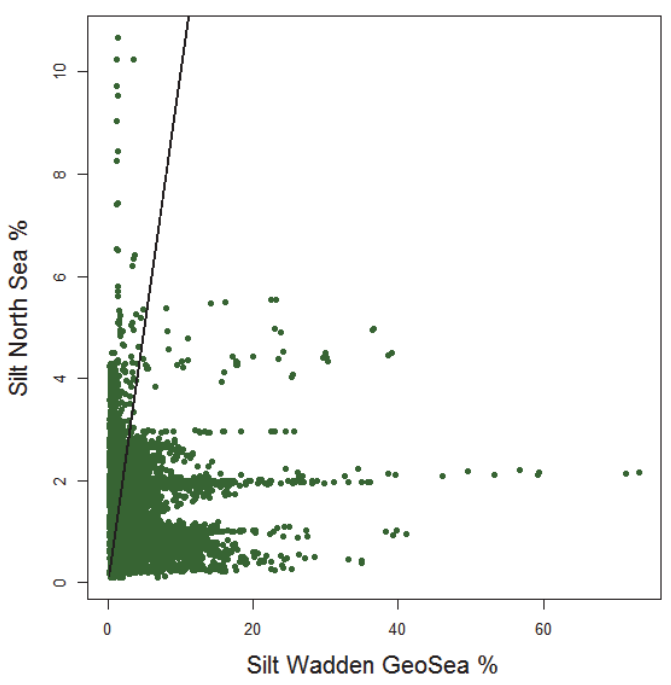

Figure 78 Relationship between the Dutch (Zwart, GeoSea) and German sediment data (silt content) (upper right, lower left), and Dutch Wadden Sea and North Sea-data (upper left, lower right). 


\section{A16 Sediment data used}

With the data presented in appendix A14 and the conversions mentioned in appendix A15, plus an estimate for the composition of the not analysed German parts of the system we came to a list of sediment characteristics in terms of lutum and silt content, and derived values for e.g. phosphorus (Figure 79). Patterns of most other characteristics are similar since these have been derived from the same underlying data.

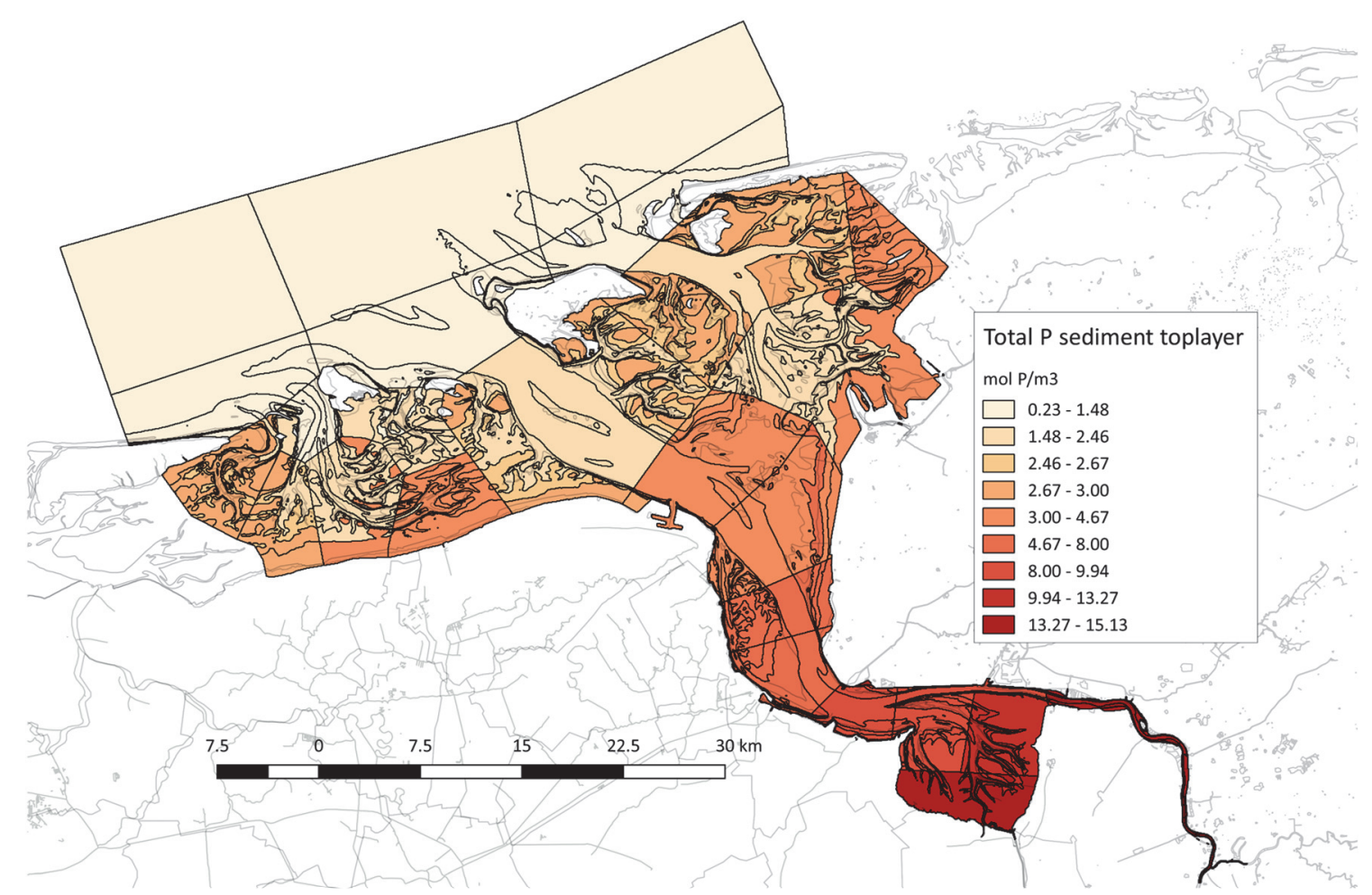

Figure 79 Example of sediment toplayer characteristics in the area: content of total phosphorus $\left(\mathrm{mol}^{\mathrm{P}} \mathrm{m}^{-3}\right)$ Values are different for each sub-compartment. Main compartments and their numbers are included. 


\section{A17 Benthic diatoms}

\section{A17.1 Position of benthic diatoms in the model}

Benthic diatoms in the model are present in the upper sediment layer. This layer has, on the tidal flats, a thickness of $1 \mathrm{~cm}$. It is assumed that light availability is optimal. Nutrient concentrations are the mean values of that upper sediment layer (Figure 80). Concentrations of all dissolved

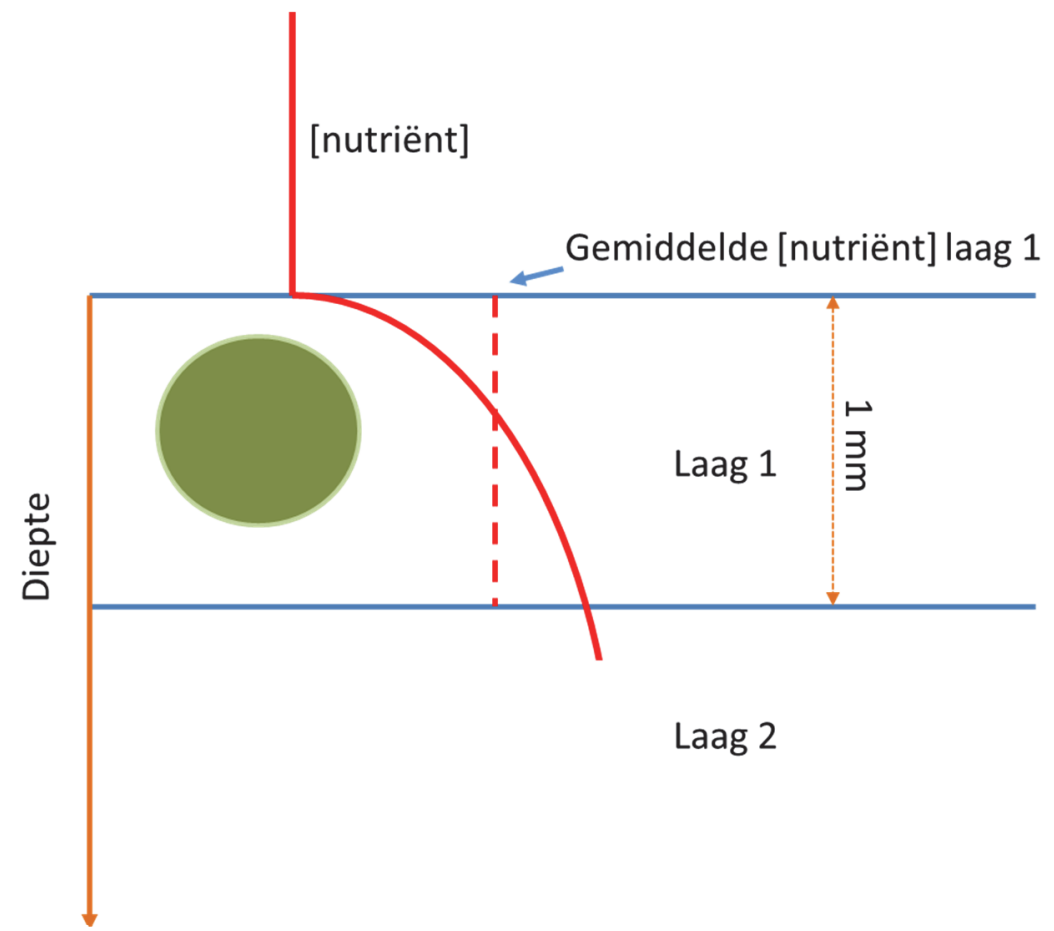

Figure 80 Benthic diatoms have optimal light conditions and 'see' the average nutrient content of the sediment top layer

components (and thus: also nutrients) are computed every time step from the steady-state solution of the reaction-diffusion equation that described the pore water profiles of each component.

This first assumption (optimal light conditions) was first used because of its simplicity. After the IMARES Ems-Dollard field and laboratory research by Riegman and colleagues (reported by Brinkman et al, $2015^{\mathrm{a}, \mathrm{b}}$ ), it appeared that this assumption wasn't that bad; therefore, it is used in the present model setup as well.

\section{A17.2 Maximal densities of benthic diatoms}

If the number of benthic diatoms increases they may occupy more space than available. Since algae do not have a volume in the model (one of the parts to be improved in future), occupying space is not a limiting factor in the simulations. Therefore, an artificial maximum density is introduced, set as an a-priority parameter. Maximum benthic chlorophyll-a values of about 200 $\mathrm{mg}$ chla $\mathrm{m}^{-2}$ are found in the Ems-Dollard (De Jonge et al; 2012). The IMARES 2012-2013 data (Brinkman et al, 2015a,b) show maximum values of 100-150 mg chla $\mathrm{m}^{-2}$. These concern 2-5 mm thick sediment layers, and imply about 20-100 $\mathrm{g}$ chla $\mathrm{m}^{-3}$ and about 2000-10000 g DW benthic diatom $\mathrm{m}^{-3}$. For the simulations, a maximum of $2000 \mathrm{~g} \mathrm{DW}$ benthic diatoms $\mathrm{m}^{-3}$ is used. 
Table 17 Average phytobenthos-chlorophyll-a values for tidal flats from Colijn \& De Jonge, 1983

\begin{tabular}{|c|l|c|c|}
\hline Station & Where & Present compartment numbers & $\begin{array}{l}\text { Chla } \mathrm{mg} / \mathrm{m} 2 \text { on } \\
\text { tidal flat }\end{array}$ \\
\hline 1 & Outer Ems & 112 / near Groningen coast & 121 \\
\hline 2 & Mid Ems & $110 /$ near Groningen coast & 42.1 \\
\hline 3 & Dollard & $105 /$ near Reide & 82.6 \\
\hline 4 & Mid Ems & $110 /$ Paap & 42.1 \\
\hline 5 & Dollard & $105 /$ close to gully & 82.6 \\
\hline 6 & Dollard & $103 /$ close to Nw Statenzijl & 82.6 \\
\hline
\end{tabular}

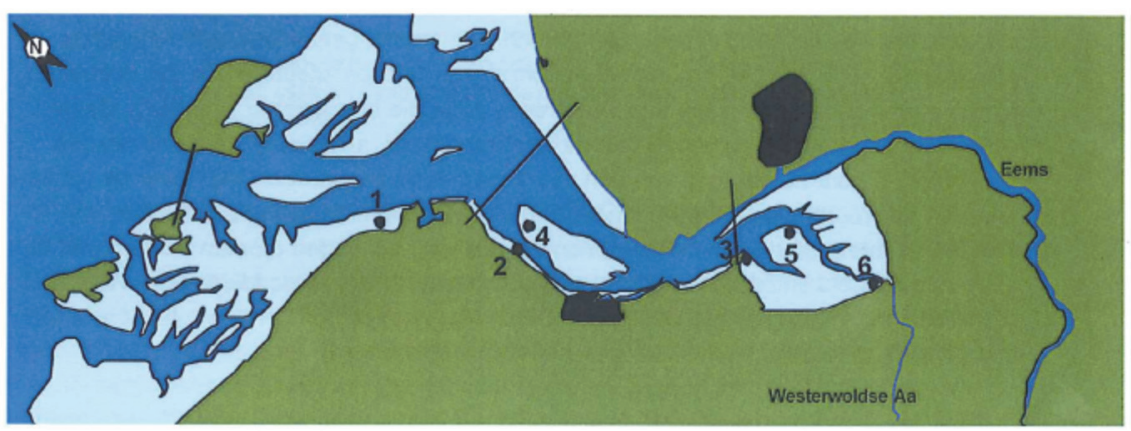

Figure 81 Locations sampled for microphytobenthos in the periods between 1976 and 1978, and between 1992 and 1999 (from de Boer, 2010).

\section{A17.3 Suspension of benthic diatoms}

As silt and other benthic matter, benthic diatoms may be suspended in the water column. This has already been mentioned by De Jonge \& Van Beusekom (1992) and De Jonge \& Colijn (1994). In the water column, benthic diatoms contribute to the computed pelagic primary production, just as the other algae do. The parameter settings (light and nutrient relationships) remain unchanged compared to the benthic situation. 


\section{A18 Description of growth of a fauna individual and of population mortality}

\section{A18.1 General}

This appendix A18 gives a description of the way fauna objects were modelled in the EcoWaspmodel. Also, it is explained how to come to a first estimate of growth and respiration parameters based on the maximum animal length (mass) and the time needed to reach a, say, 2/3 of that maximum length (mass).

\section{A18.2 Population mass budgets}

Generally, a population biomass density is described by

$$
M_{i}=\overline{W_{i}} \cdot N_{i}
$$

where $\mathrm{W}_{\mathrm{i}}$ is the average mass ( $\mathrm{g}$ ) of individual animals in class $\mathrm{i}$, and $\mathrm{N}_{\mathrm{i}}$ the number of animals per unit of volume (number. $\mathrm{m}^{-3}$ ). Changes in population biomass is a result from changes in both mass and numbers.

Changes in animal average mass (W) follow from

$$
\frac{d \overline{W_{i}}}{d t}=\text { uptake+ respiration }+ \text { excretion }+ \text { digestion }+ \text { faeces }+ \text { spawning } W
$$

$$
\left(g \text { DW ind }{ }^{-1} d^{-1}\right)
$$

and changes in numbers $\mathrm{N}$ from

$$
\frac{d \overline{N_{i}}}{d t}=\text { mortality+ grazing + spawningN } \quad \text { (ind } \mathrm{m}^{-3} \mathrm{~s}^{-1} \text { ) }
$$

The conversion from the spawned biomass SpawningW to spawned numbers SpawningN is done through the larval mass. The first-born larvae get a start mass which is a model parameter. Later born larvae get the mass the already existing larvae already have reached because of uptake and losses. This construction avoids some numerical problems. Spawning occurs in certain periods, set by parameter values; presently it is not related to computed environmental conditions.

Since ion budgets are computed following N.dW/dt and W.dN/dt, a dW/dt. DN/dt-correction is implemented. 


\section{A18.3 Individual growth}

Each animal filters or searches a certain system volume per unit of time. This determines the grazing losses in terms of animal numbers (in case they are prey), and this times the prey masses, it determines the amount of food in terms of biomass that can be taken up by a predator.

\section{A18.3.1 Filtration and assimilation rate}

As a short explanation, the uptake term is given in more detail. Fauna food uptake rate depends on a) the maximum filtration rate times the food concentration, and b) the maximum assimilation rate is determined by the animal's growth capability.

The maximum filtration rate is

max_filtration_rate $=F_{1}(T) \cdot F_{2}\left(\cdot \overline{W_{i}}\right) \cdot \overline{W_{i}} \quad\left(\mathrm{~m}^{3}\right.$ ind $\left.^{-1} \mathrm{~d}^{-1}\right)$

or, with $F_{2}(W)$ written explicitly:

$\max _{-}$filtration_rate $=F_{1}(T) \cdot a_{m f} \overline{W_{i}}{ }^{b m f} \cdot \overline{W_{i}} \quad\left(\mathrm{~m}^{3} \mathrm{ind}^{-1} \mathrm{~d}^{-1}\right)$

with $a_{m f}$ and $b_{m f}$ as allometric maximum filtration rate coefficients.

Thus, the maximum amount of food (max_uptake_rate) that can be caught is:

$\max \_$uptake_rate $=[$Food $] \cdot \max _{-}$filtration _rate $\quad\left(\mathrm{g} \mathrm{DW} \mathrm{ind}^{-1} \mathrm{~d}^{-1}\right)$

The maximum assimilation rate is on the one hand determined by the food availability:

assim rate $=\beta_{\text {ass }} \cdot \max \underline{\text { uptake rate }}$

(g AFDW ind $\left.{ }^{-1} d^{-1}\right)\left(4^{c}\right)$

and on the other hand, by the maximum gross growth capability of the animal:

assim_rate $_{2}=F_{3}(T) \cdot F_{4}\left(\cdot \overline{W_{i}}\right) \cdot \overline{W_{i}}$

$\left(\right.$ g AFDW ind $\left.{ }^{-1} d^{-1}\right) \quad\left(4^{d}\right)$

or, with $\mathrm{F}_{4}(\mathrm{~W})$ written explicitly:

assim_rate $_{2}=F_{3}(T) \cdot a_{\text {ass }}{\overline{W_{i}}}^{\text {bass }} \cdot \overline{W_{i}} \quad$ (g AFDW ind $\left.{ }^{-1} \mathrm{~d}^{-1}\right) \quad\left(4^{\mathrm{d} 1}\right)$

[Food] is the total concentration of food particles in the water (g AFDW $\mathrm{m}^{-3}$ ) and $F_{1}(T)$ and $F_{3}(T)$ are temperature dependency functions. $\mathrm{F}_{2}(\mathrm{~W})$ and $\mathrm{F}_{4}(\mathrm{~W})$ are the allometric functions that describe the weight dependencies of max_filtration_rate and assim_rate $2 . \beta_{\text {ass }}$ denotes the assimilation efficiency. Multiplication with W (as done here) gives the individual assim_rate and max_filtration_rate, respectively.

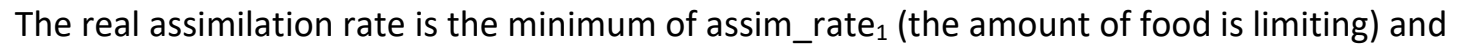
assim_rate 2 (the animal cannot grow faster): 
assim_rate $=\operatorname{MIN}\left(\right.$ assim_rate $_{1}$, assim_rate $\left._{2}\right) \quad\left(\mathrm{g} \mathrm{AFDW} \mathrm{ind}^{-1} \mathrm{~d}^{-1}\right)$

Consequently, the uptake rate (the amount of food taken up) is

uptake_rate $=$ assim_rate $/ \beta_{\text {ass }} \quad \quad\left(\mathrm{g} \mathrm{AFDW}\right.$ ind $\left.^{-1} \mathrm{~d}^{-1}\right)$

There's a complicating factor: certain shellfish species produce pseudofaeces. A part of the filtered matter is laid aside together with some mucus-like matter that 'glues' together these particles. In the field, this pseudofaeces will partly be resuspended after a while, but not all, and these biologically settled particles form hills rich in clay and organic matter. After consolidation, these sediment elevations become very stable, and may form suitable substrates for future larval settlements.

There are two possibilities in a shellfish life regarding the filtration strategy:

- the real filtration simply is the maximum rate, until [Food] ${ }^{*}$ max_filtration_rate* $\beta_{\text {ass }}$ reaches the assim_rate 2 . In case of pseudofaeces formation, EcoWasp uses an extra pseudofaeces parameter PsfFactor that says that between that point and assim_rate2/ $\beta_{\text {ass }}$ * PsfFactor the maximum filtration rate is continued. The extra amount of filtered matter (the amount of pseudofaeces) is max_filtration_rate*[Food] - assim_rate2/ $\beta_{\text {ass }}$ * PsfFactor, and is considered to be the produced pseudofaeces. As soon as [Food]*max_filtration_rate exceeds assim_rate2/ $\beta_{\text {ass }} *$ PsfFactor, filtration slows down proportionally. The second possibility is:

- the real filtration rate is always the maximum rate, and all extra matter is laid aside as pseudofaeces. In this case, and extra pseudofaeces filtration factor is not needed.

Following the first filtration strategy, there are three situations possible:

- there's not enough food available: the filtration rate is maximal: eq. $4^{\mathrm{a}}$

- there's enough food available, but the surplus is not very high: the filtration rate is still the maximum filtration rate, until the max_filtration_rate*[Food] reaches Psffactor* assim_rate $2 / \beta_{\text {ass }}$

- there's a large surplus of food, and the filtration rate slows down proportionally until the real filtration rate*[Food] equals Psffactor*maximum assimilation rate / assimilation efficiency.

Following the second filtration strategy, there's only on possibility:

- the filtration rate is always at its maximum

The pseudofaeces production begins as soon as the assimilation reaches its maximum. All extra food is laid aside. The modellers problem is that in extreme cases (very high food concentration) the filter feeders lay aside large amounts of filtered algae, and thus remove a large part of the algae without really using this resource. 
Therefore, in the model the first approach is implemented. Equations are given below.

\section{A18.3.2 Respiration and excretion}

The description of respiration consists of three terms: a basic metabolism term, an activity related term and a term that describes the negative effect of inorganic particles in the water: these may affect filtration negatively if their concentration is too high. The activity related term is zero when there is no feeding activity, but it increases when the animal searches for food. Thus:

respiratio $n=$ rest respiratio $n+$ activity respiratio $n+$ particle _hindrance _ $\cos t s$

$$
\text { (g AFDW ind }{ }^{-1} d^{-1} \text { ) }
$$

or:

$$
\begin{array}{ll}
\text { restrespiration }=-F_{5}(\text { Temp }) \cdot F_{6}\left(\cdot \overline{W_{i}}\right) \cdot \overline{W_{i}} & \left(\mathrm{~g} \mathrm{AFDW} \mathrm{ind} \mathrm{d}^{-1} \mathrm{~d}^{-1}\right) \\
\text { activityespiratio }=-k_{r a} \cdot \text { real_filtration } \underline{\text { ratt }} & \left(\mathrm{g} \mathrm{AFDW} \mathrm{ind} \mathrm{d}^{-1} \mathrm{~d}^{-1}\right)
\end{array}
$$

\section{particle_hindrance $\cos t s=-k_{p h} \cdot r e a l \_f i l t r a t i o n$ rate $\cdot[$ inorganicprticles]}

$$
\text { (g AFDW ind }{ }^{-1} d^{-1} \text { ) }
$$

The real_filtration rate is given by

$\mathrm{F}_{6}\left(\mathrm{~W}_{\mathrm{i}}\right)$ is the allometric equation for mass dependent rest respiration, and multiplied with the individual mas, it gives the individual respiration rate:

$$
F_{6}(W)=a_{r r} \bar{W}^{b r r} \bar{W} \quad \text { (g AFDW } \text { ind }^{-1} \mathrm{~d}^{-1} \text { ) }
$$

In eq. $6^{\mathrm{a} 1}, \mathrm{a}_{\mathrm{rr}}$ and $\mathrm{b}_{\mathrm{rr}}$ are the rest respiration coefficients. The activity related respiration constant $\mathrm{k}_{\mathrm{ra}}$ has different units and, thus, has a different meaning.

The real_filtration_rate is nothing else than the space that an individual has searched or filtered for food per until of time $\left(\mathrm{m}^{3}\right.$ ind $\left.^{-1} \mathrm{day}^{-1}\right)$; the conversion factor $\mathrm{k}_{\mathrm{ra}}$ thus describes the amount of energy (expressed in $\mathrm{g}$ DW in the model) needed per $\mathrm{m}^{3}$ searched volume. One of the ideas is that this factor is the same for all animals that show the same food collection behaviour; and consequently, it may have much lower values for fauna species that search a volume of water instead of filtering it.

Particle hindrance may be relevant in case the animal e.g. filters water and catches inedible particles, such as sand grains or silt particles. The parameter $k_{p h}$ accounts for the energetic costs of separating the sand or silt. Also, turbidity may negatively affect the prey search possibilities of a hunter.

Excretion is described similarly. Respiration is an oxygen demanding process, and the products are carbon dioxide and nutrients following the stoichiometric composition of the animal. Chemically, respiration is the reverse reaction of assimilation (in the model). The product of excretion is some detrital matter; set through a model parameter. Different chemical composition of the detrital product and the animal is accounted for. 


\section{A18.3.3 Digestion}

The food particles taken up must be digested. This amount of food taken up is [Food]*real_filtration_rate, and thus, the digestion costs are:

digestion_cos $t s=k_{d i g} \cdot[$ Food $] \cdot$ real_filtration_rate

\section{A18.3.4 Faeces}

Faeces is the difference between food uptake and assimilation, or (1- $\left.\beta_{\text {ass }}\right)$ * food uptake:

$$
\text { faeces }=\left(1-\beta_{\text {ass }}\right) \cdot \text { uptake_rate }
$$

\section{A18.3.5 Pseudofaeces}

As mentioned above (filtration and assimilation rate), as soon as the max_filtration rate times [Food] exceeds the assim_rate $2 / \beta_{\text {ass }}{ }^{*}$ PsfFactor the filtration rate may slow down (first case of both filtration strategy possibilities).

Thus, there are three cases:

1- [Food] is low, filtration rate is maximum:

real_filtr_rate $=\max _{-}$filtration_rate

pseudo_faeces $=0$

2- [Food] is more than needed, but

max_filtration_rate $\cdot[$ Food $]<=$ PsfFactor.uptake_rate:

real_filtr_rate $=\max _{-}$filtration_rate

pseudo_faeces $=\max _{-}$filtration_rate $[$Food $]-$uptake_rate

3- [Food] is more than needed, and max_filtration_rate.[Food]>PsfFactor-uptake_rate:

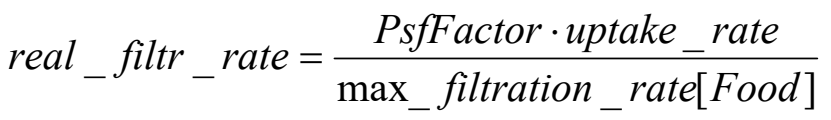

pseudo_faeces $=p s f \cdot$ uptake_rate

Note that in case the animal is always filtering at maximum rate, the pseudofaeces production is pseudo_faeces $=\max \_$filtration_rate $*[$ Food $]-$ uptake_rate 
The costs concern the mucus produced:

pseudo_faeces_costs $=p s f_{c} \cdot p s e u d o_{-}$faeces

(g AFDW ind ${ }^{-1} \mathrm{~d}^{-1}$ )

The constant psf $\mathrm{c}_{\mathrm{c}}$ has units $\left(\mathrm{g} \mathrm{g}^{-1}\right)$; it says that a certain weight fraction of the pseudofaeces produced is needed to produce the mucus. Note that here the inorganic particles are included as well, so pseudofaeces in eq.10 is $>$ the pseudofaeces in eqs.9.

\section{A18.3.6 Mortality}

Change in numbers by mortality is described following:

$$
\text { mortality } \left.=-F_{12}(\text { Temp }) \cdot F_{13}\left(\text { age, condition } \overline{W_{i}}\right) \cdot N_{i} \quad \text { (ind } \mathrm{m}^{-3} \mathrm{~d}^{-1}\right)
$$

where $k_{\text {mor }}$ is a rate constant $\left(d^{-1}\right)$. The terms $k_{\text {mor }}$ and $F($ age, condition, mass) must be reconsidered every time new predators are introduced in or removed from a model setup, or when other feeding parameters are changed.

The function F13 depends, for the time being, on individual mass alone. So, condition dependence is not implemented yet:

$$
F_{13}\left(\operatorname{age}, \text { condition } \overline{W_{i}}\right)=k_{\text {mor }} W^{\text {bmor }}
$$

This equation basically describes all the mortality not modelled in the term grazing; consequently, if grazing upon the animal occurs, the mortality parameter needs to be adjusted. In the model, it is checked whether predation upon the fauna species occurs, the mortality parameter $k_{\text {mor }}$ is decreased

$$
k_{\text {mor_real }}=k_{\text {mor }} * k_{p p}
$$

The factor $k_{p p}$ is the reduction parameter if a predator is present $\left(k_{p p}<1\right)$.

\section{A18.4 Steady state food concentration, an important fauna characteristic}

An important characteristic of the prey-predator system is the food concentration at which assimilation and losses are equal, and thus, growth rate is zero. There is a relationship between this steady state food concentration and the size (individual mass) of an animal, to be derived from eq (2) after setting $d W / d t=0$, and omitting spawningW. Also, excretion is not separated from respiration, and thus it follows:

$0=$ uptake + respiratio $n+$ digestion + particle hindrance

Pseudofaeces production is not relevant since food content is low, and thus, there's no possibility to lay aside any food surplus. The question whether the animal should decrease it's filtering rate also is not relevant either; it always filters at its maximum rate.

When also omitting the temperature effects, it follows that 


$$
\begin{gathered}
0=[\text { Food }] \cdot F_{2}(W) \cdot W \cdot \beta_{\text {ass }}-F_{6}(W) \cdot W-k_{d i f} \cdot[\text { Food }] \cdot F_{2}(W) \cdot W \cdot \beta_{a s s}-k_{r a} \cdot F_{2}(W) \cdot W- \\
-k_{p h} \cdot F_{2}(W) \cdot W \cdot[\text { inorganic particles }]
\end{gathered}
$$

( or: 0 = assimilation - rest respiration - digestion - activity respiration - particle hindrance).

To solve eq.15, the terms containing [Food] are separated from the others, giving:

$[$ Food $] \cdot F_{2}(W) \cdot W \cdot \beta_{a s s}+k_{d i f} \cdot[$ Food $] \cdot F_{2}(W) \cdot W \cdot \beta_{a s s}=F_{6}(W) \cdot W+k_{r a} \cdot F_{2}(W) \cdot W+$

$$
+k_{p h} \cdot F_{2}(W) \cdot W \cdot[\text { inorganic particles }]
$$

Rearranged a bit, and with inorganic_particles shortened to in_part:

$[$ Food $] \cdot\left(F_{2}(W) \cdot W \cdot \beta_{\text {ass }}\left(1-k_{\text {dig }}\right)=F_{6}(W) \cdot W+k_{r a} \cdot F_{2}(W) \cdot W+k_{p h} \cdot F_{2}(W) \cdot W \cdot[\right.$ in_part $]$

and it follows for the steady state food concentration [Food $]_{s t}$ :

$[\text { Food }]_{s s t}=\frac{F_{6}(W) \cdot W+k_{r a} \cdot F_{2}(W) \cdot W+k_{p h} \cdot F_{2}(W) \cdot W \cdot[\text { in_part }]}{\left(F_{2}(W) \cdot W \cdot \beta_{\text {ass }}\left(1-k_{d i g}\right)\right.}$

The activity respiration term and the particle hindrance term usually are smaller or even much smaller than the rest respiration term $\mathrm{F}_{6}(\mathrm{~W}) \cdot \mathrm{W}$. Consequently, [Food] will mainly depend on the quotient rest respiration / maximum filtration rate. Rest respiration is described in eq. $6^{\mathrm{a}}{ }^{1}$, and maximum filtration in eq. $4^{\mathrm{a}}$. Omitting activity respiration and particle hindrance for the moment, the steady state food concentration is given by

$[\text { Food }]_{s s t}=\frac{a_{r r} W^{b r r} \cdot W}{a_{m f} W^{b m f} \cdot W \cdot \beta_{a s s}\left(1-k_{d i g}\right)}=\frac{1}{\beta_{a s s}\left(1-k_{d i g}\right)} \frac{a_{r r}}{a_{m f}} W^{(b r r-b m f)}$

Since the digestion parameter $k_{\text {dig }}$ and the assimilation efficiency $\beta_{\text {ass }}$ are more less constant for most fauna species, as are the exponent parameters $b_{r r}$ and $b_{m f}$, it is the quotient $a_{r r} / a_{m f}$ in the first place that says at which food concentration growth reaches a steady state.

Here, the distinction between hunters and filtering animals becomes clear. The first type of predators needs to search for a prey, depends on visibility, needs to have a large volume search rate. Therefore, $a_{m f}$ is large and the animals can survive at low [Food].

Since $b_{r r}>b_{m f}(0$ and -0.35 or -0.30 , respectively) [Food] increases with $W$. This is an important conclusion, implying that at low [Food] only small individuals of a species can survive.

If the contribution of activity related respiration and of particle hindrance is considered, [Food $]_{s t}$ is adjusted somewhat. 


\section{A18.5 Maximum mass}

The same equations as above can be rearranged, to derive $W_{\max }$ with $d W / d t=0$, as a function of [Food]. Mind that [Food] determines $W_{\max }$ only in case of low [Food]. Thus, for the maximum possible mass, food is not limiting, and growth is determined by the maximum assimilation rate according to eq. $4^{\mathrm{d} 1}$.

Consequently, the digestion term from eq. 2 does not contain the maximum filtration times food concentration, but the maximum assimilation rate divided by the assimilation efficiency.

Thus:

$$
\begin{array}{r}
0=F_{4}(W) \cdot W-F_{6}(W) \cdot W-k_{\text {dig }} \cdot \frac{F_{4}(W) \cdot W}{\beta_{a s s}}-k_{r a} \cdot F_{2}(W) \cdot W- \\
\quad-k_{p h} \cdot F_{2}(W) \cdot W \cdot[\text { inorganic particles] }
\end{array}
$$

In both the particle hindrance and activity related respiration term the real filtration rate is needed $\left[\mathrm{F}_{2}(\mathrm{~W}) \cdot \mathrm{W}\right]$. At high [Food], the real filtration rate is lower or even much lower than the maximum filtration rate, and consequently, both terms become less important. So, if both are omitted for the time being, and with eqs $4^{\mathrm{d} 1}$ and $6^{\mathrm{a} 1}$ substituted, it follows that

$$
0=a_{a s s} \cdot W^{\text {bass }} \cdot W-a_{r r} W^{b r r} \cdot W-k_{d i g} \cdot \frac{a_{a s s} \cdot W^{\text {bass }} \cdot W}{\beta_{a s s}}
$$

Rearranged, and dividing all by ( $\left.\mathrm{W}^{\text {bass }} \mathrm{W}\right)$ it gives

$$
0=a_{a s s}-a_{r r} W^{\text {brr-bass }}-k_{\text {dig }} \cdot \frac{a_{\text {ass }}}{\beta_{\text {ass }}}
$$

Or, after moving the term with $\mathrm{W}$ to the left-hand side:

$$
a_{r r} W^{\text {brr-bass }}=a_{\text {ass }}-k_{\text {dig }} \cdot \frac{a_{\text {ass }}}{\beta_{\text {ass }}}
$$

and thus, it follows for the maximum individual biomass $\mathrm{W}_{\max }$ that

$$
W_{\max }=\left(\frac{a_{a s s}-k_{d i g} \cdot \frac{a_{a s s}}{\beta_{a s s}}}{a_{r r}}\right)^{\frac{1}{b r r-b a s s}}
$$

$W_{\max }=\left(\frac{a_{a s s}\left(1-\frac{k_{d i g}}{\beta_{a s s}}\right)}{a_{r r}}\right)^{\frac{1}{b r r-b a s s}}$ or $\quad$ (g AFDW ind $\left.{ }^{-1}\right)$

Eq. $19 \mathrm{~b}$ says that if $k_{\text {dig }}=\beta_{\text {ass }}, W_{\max }=0$. This is logical because all assimilated food is needed to sustain the digestion, and no food is left for the real growth. 
To give an idea of this relationship, some parameter choices may be applied: since $\mathrm{k}_{\text {dig }} \approx 0.16$, and $\beta_{\text {ass }} \approx 0.75$ is a common value, it follows, with $b_{\text {rr }}=0$ and $b_{\text {ass }}=-0.35$, that

$$
W_{\max }=\left(\frac{0.78 a_{a s s}}{a_{r r}}\right)^{\frac{1}{b r r-b a s s}}=0.504\left(\frac{a_{a s s}}{a_{r r}}\right)^{2.85}
$$

\section{A18.6 Growth rate and implications for the respiration parameter}

A third characteristic of a fauna group is the time needed to reach a certain mass. Since we're dealing with exponential growth, 1-1/e (about $68 \%$ ) of the maximum value might be a useful criterion. Also in this case, food limitation is not the case, and thus, growth is determined by the maximum assimilation rate, and not the maximum filtration rate * [Food]. So, the growth equation reads, with omitting again the activity related respiration and particle hindrance:

$$
\frac{d W}{d t}=a_{a s s} \cdot W^{\text {bass }} \cdot W-a_{r r} W^{b r r} \cdot W-k_{d i g} \cdot \frac{a_{a s s} \cdot W^{\text {bass }} \cdot W}{\beta_{a s s}}
$$

Or, since the first and the last right-hand term can be taken together, and performing the multiplication with $\mathrm{W}$ :

$$
\frac{d W}{d t}=a_{a s s} \cdot\left(1-\frac{k_{d i g}}{\beta_{a s s}}\right) \cdot W^{b a s s+1}-a_{r r} W^{b r r+1}
$$

\section{Substituting}

$$
a_{a}=a_{a s s} \cdot\left(1-\frac{k_{d i g}}{\beta_{a s s}}\right)
$$

it gives:

$$
\frac{d W}{d t}=a_{a} \cdot W^{b a s s+1}-a_{r r} W^{b r r+1}
$$

Thus, the differential equation

$$
\frac{d W}{a_{a} \cdot W^{b a s s+1}-a_{r r} W^{b r r+1}}=d t
$$


must be solved.

The primitive of the left-hand term is a bit easier to obtain when $b_{r r}=0$ is substituted; this is a normal value for $b_{r r}$. In that case

$P\left(\frac{d W}{a_{a} \cdot W^{\text {bass }+1}-a_{r r} W}\right)=P\left(\frac{d W}{\left(a_{a} \cdot W^{\text {bass }}-a_{r r}\right) W}\right)=\frac{-1}{b_{a s s} a_{r r}} \ln \left(\frac{W^{\text {bass }}}{-a_{r r}+a_{a} W^{\text {bass }}}\right)$

Thus, the solution for eq. $20^{\mathrm{d}}$ is

$\frac{-1}{b_{a s s} a_{r r}} \ln \left(\frac{W^{\text {bass }}}{-a_{r r}+a_{a} W^{\text {bass }}}\right)=t$

or:

$\ln \left(\frac{W^{\text {bass }}}{-a_{r r}+a_{a} W^{\text {bass }}}\right)=-b_{\text {ass }} a_{r r} t$

Thus, the solution for eq.20d, with $b_{r r}=0$ as a common choice, follows from

$\left[\ln \left(\frac{W^{\text {bass }}}{-a_{r r}+a_{a} W^{\text {bass }}}\right)\right]_{t=0}^{t=t}=\left[-b_{\text {ass }} a_{r r} t\right]_{t=0}^{t}$

or

$\ln \left(\frac{W_{t}^{\text {bass }}}{-a_{r r}+a_{a} W_{t}^{\text {bass }}}\right)-\ln \left(\frac{W_{0}^{\text {bass }}}{-a_{r r}+a_{a} W_{0}^{\text {bass }}}\right)=-b_{\text {ass }} a_{r r} t$

or

$\ln \left(\frac{\left.\frac{\left(W_{t}^{\text {bass }}\right.}{-a_{r r}+a_{a} W_{t}^{\text {bass }}}\right)}{\left(\frac{W_{0}^{\text {bass }}}{-a_{r r}+a_{a} W_{0}^{\text {bass }}}\right)}\right)=-b_{a s s} a_{r r} t$

Now, with

$\gamma=-b_{a s s} a_{r r}$

$Y Y=\left(\frac{W_{0}^{\text {bass }}}{-a_{r r}+a_{a} W_{0}^{\text {bass }}}\right)$ 
this gives

$$
\ln \left(\frac{\left(\frac{W_{t}^{\text {bass }}}{-a_{r r}+a_{a} W_{t}^{\text {bass }}}\right)}{Y Y}\right)=\gamma t
$$

And thus:

$$
\left(\frac{W_{t}^{\text {bass }}}{-a_{r r}+a_{a} W_{t}^{\text {bass }}}\right)=Y Y \exp (\gamma t)
$$

Rearranging, it follows:

$$
\begin{aligned}
& W_{t}^{\text {bass }}=\left(-a_{r r}+a_{a} W_{t}^{\text {bass }}\right) Y Y \exp (\gamma t) \\
& -> \\
& W_{t}^{\text {bass }}-a_{a} W_{t}^{\text {bass }} Y Y \exp (\gamma t)=-a_{r r} \cdot Y Y \exp (\gamma t) \\
& -> \\
& W_{t}^{\text {bass }}=\frac{-a_{r r} \cdot Y Y \exp (\gamma t)}{1-a_{a} Y Y \exp (\gamma t)} \\
& -> \\
& W_{t}=\left[\frac{-a_{r r} \cdot Y Y \exp (\gamma t)}{1-a_{a} Y Y \exp (\gamma t)}\right]^{\frac{1}{\text { bass }}}
\end{aligned}
$$

YY and $\gamma$ can be substituted again, giving

$$
W_{t}=\left[\frac{-a_{r r} \cdot\left(\frac{W_{0}^{\text {bass }}}{-a_{r r}+a_{a} W_{0}^{\text {bass }}}\right) \exp \left(-b_{a s s} a_{r r} t\right)}{1-a_{a}\left(\frac{W_{0}^{\text {bass }}}{-a_{r r}+a_{a} W_{0}^{\text {bass }}}\right) \exp \left(-b_{\text {ass }} a_{r r} t\right)}\right]^{\frac{1}{\text { bass }}}
$$

The limit case is simple: for $\mathrm{t}->\infty$, both exponents are $\infty$ as well (because $\mathrm{b}_{\text {ass }}<0$ ), consequently, the term " 1 " in the denominator disappears, as does the factor with $W_{0}$ in nominator and denominator, and it follows that

$$
W_{\infty}=\left[\frac{a_{r r}}{a_{a}}\right]^{\frac{1}{\text { bass }}}
$$

which is the same result as given in eqs. $\left(19^{\mathrm{b}}, 19^{\mathrm{b} 1}\right)$ since $\left[\frac{a_{r r}}{a_{a}}\right]^{\frac{1}{b a s s}}==\left[\frac{a_{a}}{a_{r r}}\right]^{\frac{-1}{\text { bass }}}$. 
Now, the question is at what time $\left(\mathrm{t}_{\mathrm{Fr}}\right) \mathrm{W}_{\mathrm{t}} / \mathrm{W}_{\infty}=$ a certain fraction $(\mathrm{Fr})$ of the final (maximum) mass. This is given by

$$
\left.\frac{\left[-a_{r r} \cdot\left(\frac{W_{0}^{\text {bass }}}{-a_{r r}+a_{a} W_{0}^{\text {bass }}}\right) \exp \left(-b_{a s s} a_{r r} t_{F r}\right)\right.}{\left.1-a_{a}\left(\frac{W_{0}^{\text {bass }}}{-a_{r r}+a_{a} W_{0}^{\text {bass }}}\right) \exp \left(-b_{a s s} a_{r r} t_{F r}\right)\right]^{\frac{1}{\text { bass }}}}\right]_{\infty}=F r
$$

Now, $W_{\infty}$ is a value, and eq. $19^{\text {b1 }}$ should not be used. Instead, it gives the relationship between $\mathrm{a}_{\mathrm{rr}}$ and $\mathrm{a}_{\text {ass, }}$, and is used to remove all the $\mathrm{a}_{\text {ass }}$-parameters from eq. $25^{\mathrm{a}}$.

With

$Q=W_{0}^{\text {bass }}$

and

$a_{a}=a_{r r} \cdot W_{\infty}^{\text {-bass }}$

and

$E E=\exp \left(-b_{a s s} a_{r r} t_{F r}\right)$

It gives

$\frac{-a_{r r} \cdot\left(\frac{Q}{-a_{r r}+a_{r r} \cdot W_{\infty}^{-b a s s} \cdot Q}\right) E E}{1-a_{r r} \cdot W_{\infty}^{-b a s s}\left(\frac{Q}{-a_{r r}+a_{r r} \cdot W_{\infty}^{-b a s s} \cdot Q}\right) E E}=\left(F r \cdot W_{\infty}\right)^{\text {bass }} \Rightarrow$

$\frac{-\left(\frac{Q}{-1+W_{\infty}^{- \text {bass }} \cdot Q}\right) E E}{1-W_{\infty}^{- \text {bass }}\left(\frac{Q}{-1+W_{\infty}^{- \text {bass }} \cdot Q}\right) E E}=\left(F r \cdot W_{\infty}\right)^{\text {bass }}=>$

$-\left(\frac{Q}{-1+W_{\infty}^{\text {-bass }} \cdot Q}\right) E E=\left(F r \cdot W_{\infty}\right)^{\text {bass }} \cdot\left(1-W_{\infty}^{\text {-bass }}\left(\frac{Q}{-1+W_{\infty}^{-b a s s} \cdot Q}\right) E E\right)$

With

$Q W=\left(\frac{Q}{-1+W_{\infty}^{- \text {bass }} \cdot Q}\right)$ 
one gets

$-Q W \cdot E E=\left(F r \cdot W_{\infty}\right)^{\text {bass }} \cdot\left(1-W_{\infty}^{-b a s s} \cdot Q W \cdot E E\right)$

or

$\left(F r \cdot W_{\infty}\right)^{\text {bass }} \cdot W_{\infty}^{- \text {bass }} \cdot Q W \cdot E E-Q W \cdot E E=\left(F r \cdot W_{\infty}\right)^{\text {bass }}$

or

$\left(F r^{\text {bass }} \cdot W_{\infty}^{\text {bass }} \cdot W_{\infty}^{- \text {bass }}-1\right) \cdot Q W \cdot E E=\left(F r \cdot W_{\infty}\right)^{\text {bass }}$

or

$$
E E=\frac{\left(F r \cdot W_{\infty}\right)^{\text {bass }}}{\left(F r^{\text {bass }}-1\right) \cdot Q W}=\frac{\left(F r \cdot W_{\infty}\right)^{\text {bass }}}{\left(F r^{\text {bass }}-1\right) \cdot Q W}
$$

The term EE contains $\mathrm{a}_{\mathrm{rr}}\left(\mathrm{eq} \cdot 25^{\mathrm{b}}\right.$ ), and thus

$$
\begin{gathered}
\exp \left(-b_{a s s} a_{r r} t_{F r}\right)=\frac{\left(F r \cdot W_{\infty}\right)^{\text {bass }}}{\left(F r^{\text {bass }}-1\right) \cdot Q W}=> \\
a_{r r}=\frac{\ln \left(\frac{\left(F r \cdot W_{\infty}\right)^{\text {bass }}}{\left(F r^{\text {bass }}-1\right) \cdot Q W}\right)}{-b_{\text {ass }} t_{F r}}
\end{gathered}
$$

Eq.28 can be simplified a bit, since $Q W$ from eq. $27^{\text {a }}$ contains $W_{\infty}$ bass, and can be rewritten to

$$
Q W=\left(\frac{Q \cdot W_{\infty}^{\text {bass }}}{Q-W_{\infty}^{\text {bass }}}\right)
$$

And after substitution into eq. 28 this gives

$$
a_{r r}=\frac{\ln \left(\frac{F r^{\text {bass }}\left(Q-W_{\infty}^{\text {bass }}\right)}{\left(F r^{\text {bass }}-1\right) \cdot Q}\right)}{-b_{\text {ass }} t_{F r}}
$$

Using eq. $25^{\mathrm{b}}$ for $\mathrm{Q}$, it follows that

$$
a_{r r}=\frac{\ln \left(\frac{F r^{\text {bass }} \cdot\left(W_{0}^{\text {bass }}-W_{\infty}^{\text {bass }}\right)}{\left(F r^{\text {bass }}-1\right) \cdot W_{0}^{\text {bass }}}\right)}{-b_{\text {ass }} t_{F r}}
$$


Thus, if $\mathrm{W}_{0}$ and $\mathrm{W}_{\infty}$ are known, and the time $t_{\mathrm{Fr}}$ (days) as the moment that a certain intermediate size is reached, the respiration parameter $\mathrm{a}_{\mathrm{rr}}$ and the uptake parameter $\mathrm{a}_{\text {ass }}$ both can be computed directly.

Also, after substitution of a relevant value for $W_{0}$ and $W_{\infty}$ is becomes clear that $a_{r r}$ is not very sensitive for the choice for $\mathrm{W}_{0}$ : typical values for $\mathrm{W} 0$ are of the order of $10^{-5} \mathrm{gram}$ or lower, and for $\mathrm{W}_{\infty} 1$ gram or more. The term

$$
\frac{\left(W_{0}^{\text {bass }}-W_{\infty}^{\text {bass }}\right)}{W_{0}^{\text {bass }}}
$$

gets very close to 1 in case $W_{0} / W_{\infty} \ll<10^{-3}$ (Figure 82)

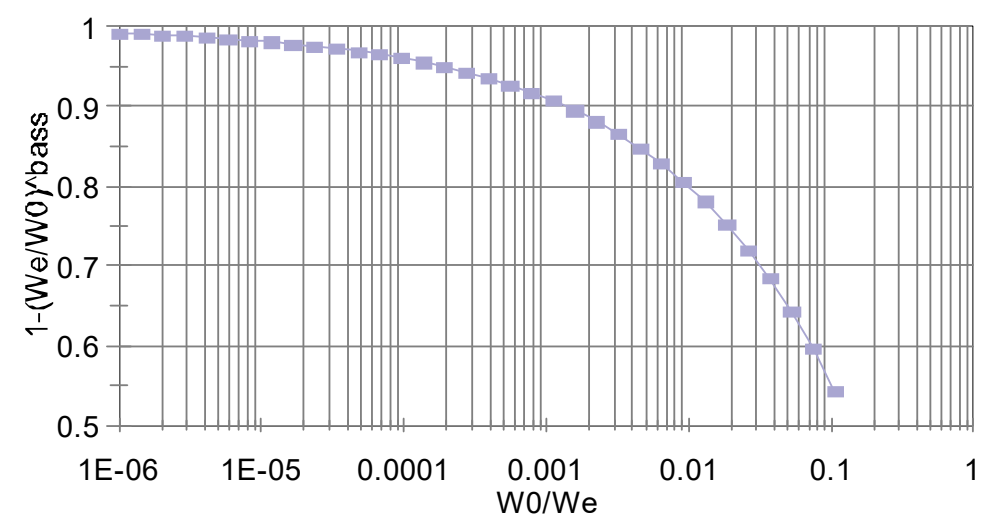

Figure $82 \mathrm{Eq} .28 \mathrm{c}$ as a function of $W_{0} / W_{\infty}$

In fact, this means that $\mathrm{Fr}$ and $\mathrm{t}_{\mathrm{Fr}}$ are the most important characteristics determining $\mathrm{a}_{\mathrm{rr}}$; from Figure 83 -showing the logarithm of $\mathrm{Fr}^{\text {bass }} /\left(\mathrm{Fr}^{\text {bass }}-1\right)$ - it becomes clear that a proper choice is $\mathrm{Fr} \approx$ 0.3 à 0.8 . In case $\mathrm{Fr}$ gets close to 1 , the logarithm increases drastically, and consequently, so does $t_{\mathrm{Fr}}$ in eq. $28^{\mathrm{a}, \mathrm{b}}$.

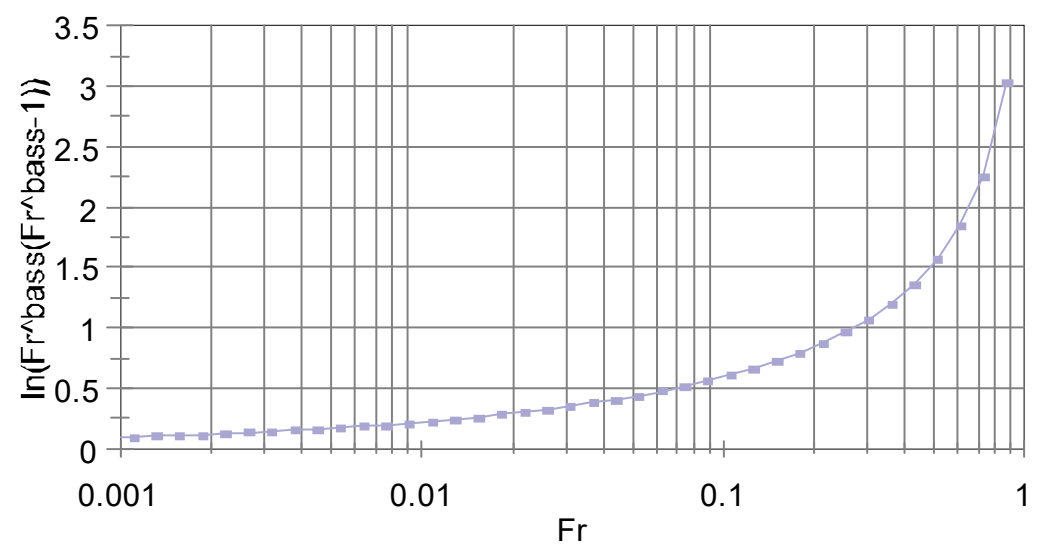

Figure $83 \ln \left(\mathrm{Fr}^{\text {bass }} /\left(\mathrm{Fr}^{\text {bass }}-1\right)\right.$ as a function of $\mathrm{Fr}\left(\mathrm{Fr}=W_{\mathrm{tFr}} / W_{\infty}\right)$ 
In case one must deal with length instead of mass, the mass-length relationship

$$
\begin{aligned}
& W=a L^{b} \\
& \text { or } \\
& L=\left(\frac{W}{a}\right)^{\frac{1}{b}}
\end{aligned}
$$

is relevant.

If $\mathrm{Fr} \approx 0.3$ à 0.8 is a proper choice, the related length ratios are

$$
\frac{L_{F r}}{L_{\infty}}=\frac{\left(\frac{W_{F r}}{a}\right)^{\frac{1}{b}}}{\left(\frac{W_{\infty}}{a}\right)^{\frac{1}{b}}}=\left(\frac{W_{F r}}{W_{\infty}}\right)^{\frac{1}{b}}=0.6-0.9
$$

Thus, the time an animal reaches roughly $60-90 \%$ of its maximum length provides a good possibility to compute the respiration parameter $\mathrm{a}_{\mathrm{rr}}$. See Figure 84 for more details.

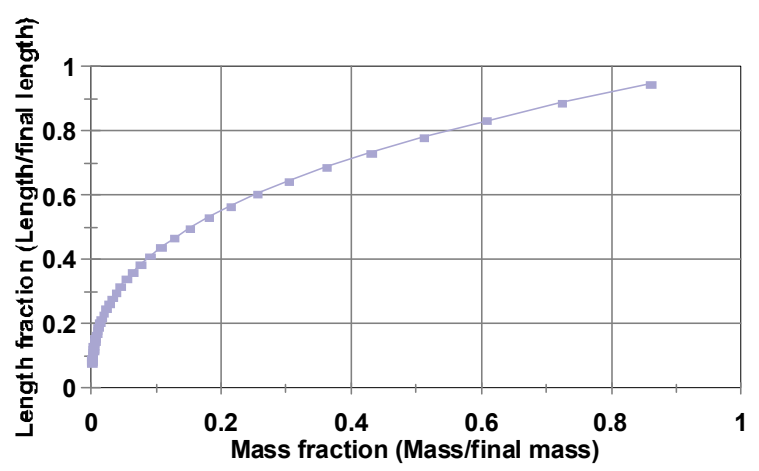

Figure 84 Relationship mass fraction <> length fraction, Mass=a.Length^2.7

\section{A18.7 Conclusions}

\section{A18.7.1 Growth and respiration parameters}

In the above section, relationships between the respiration parameter, the maximum uptake parameter and two individual size data are derived.

Both the respiration parameter and the uptake parameter $\left(\mathrm{a}_{\mathrm{rr}}\right.$ and $\left.\mathrm{a}_{\mathrm{ass}}\right)$ can easily be computed as soon as the maximum size is known, and the moment at which a certain intermediate length or size is reached. 
The assumption is that the growth and respiration rate dependence on mass is the same for the whole life cycle. Also, in the equations above it is assumed that the respiration - mass dependence is linear.

\section{A18.7.2 Corrections: suspended solids hindrance, pseudofaeces formation and activity respiration}

Suspended solids hindrance, pseudofaeces formation costs and activity respiration costs are to be considered as corrections; they lead to a lower maximum biomass and a longer period needed to reach a certain fraction of this this maximum. As soon as data are available on a lower maximum individual biomass in case of particle hindrance or pseudofaeces production, these parameters may be estimated also.

\section{A18.7.3 Maximum filtration rate coefficient}

The maximum filtration rate coefficient $\mathrm{a}_{\mathrm{mf}}$ (eq. $4^{\mathrm{a}}$ ) follows from the relationship between the level of [Food] combined with the maximum individual mass that is reached under food limiting conditions (eq. 17). Note that this maximum mass is different from the theoretically possible maximum, being the mass that can be reached in case food is not limiting (eq. $19^{\text {b) }}$. 


\section{A19 Coordinate transformations}

Two transformations have been applied to get compatible RD-values (the Dutch reference system, "Rijksdriehoek-coordinatenstelsel").

One is from the German Gauss-Krüger reference system. A one-step transformation was not available and thus, the values were first transformed to WGS94, and in a second step to RD.

\section{Gaus-Krüger to WGS94}

A straight-forward method is available as an R-library: routine spTransform from the rgdal-library (https://CRAN.R-project.org/package=rgdal) $(R, 2016)$.

\section{WGS94 to RD}

The routine used was

onhttps://github.com/djvanderlaan/rijksdriehoek/blob/master/R/rijksdriehoek.R (Van der Laan, 2017). 


\section{A20 Suspended solid concentrations: resuspension and sedimentation}

\section{A20.1 Introduction}

The model contains (partly estimated) data on the sediment composition in the area; this includes the content of lutum $(<2 \mu \mathrm{m}$ ), silt (fine 2-16 $\mu \mathrm{m}$ and course 16-63 $\mu \mathrm{m}$ ) and sand (fine 63-125 $\mu \mathrm{m}$ and course 125-250 $\mu \mathrm{m}$ ). An organic fraction, called humus in the model (or: refractory organic matter, ROM) is distinguished as well.

Based of flow velocities, wind speed and water depth, resuspension of matter is computed. Together with best estimates for settling velocities this gives simulated values for suspended matter concentrations in the water column.

\section{A20.2 Description of sedimentation, suspension and the concentration profiles in the water column}

A general equation describing the solid content in a water column contains the flux of resuspended matter to the water column and the settling flux of matter from the water column to the sediment.

With

$\left[\mathrm{C}_{\mathrm{H}}\right]=$ concentration of suspended solids $\left(\mathrm{g} \mathrm{m}^{-3}\right)$ at the sediment/water-interface

$\mathrm{H}=$ depth of water column $(\mathrm{m})$

[CB] = concentration of solids in sediment top layer $\left(\mathrm{g} \mathrm{m}^{3}\right)$

$\mathrm{k}_{1}=$ resuspension parameter $\left(\mathrm{m} \mathrm{d}^{-1}\right)$

$v_{z} \quad=$ settling velocity of suspended matter $\left(m^{-1}\right)$

both fluxes are gives as follows:

suspension flux $=k_{1}[C B] \quad\left(\mathrm{g} \mathrm{m}^{-2} \mathrm{~d}^{-1}\right)$

and

settling flux $=v_{z}\left[C_{H}\right] \quad\left(\mathrm{g} \mathrm{m}^{-2} \mathrm{~d}^{-1}\right)$

And thus, rate of change of solid content in the water column is:

$\frac{d\left[C_{H}\right]}{d t}=\frac{1}{H}\left(k_{1}[C B]-v_{Z}\left[C_{H}\right]\right) \quad\left(\mathrm{g} \mathrm{m}^{-3} \mathrm{~d}^{-1}\right)$

in case there is no vertical profile assumed, the average concentration in the water column $\overline{[C]}=$ $\left[\mathrm{C}_{\mathrm{H}}\right]$.

The equilibrium situation is reached at $d\left[C_{H}\right] / d t=0$, thus:

$C_{H}=\frac{k_{1}}{v_{z}}[C B]$ $\left(\mathrm{g} \mathrm{m}^{-3}\right)$

The parameters $k_{1}$ and $v_{z}$ need further descriptions. 


\section{A20.3 The resuspension parameter $k 1$}

The formulation of the resuspension parameter $k_{1}$ used in EcoWasp is

$\boldsymbol{k}_{1}=\boldsymbol{k}_{\text {flow }}+\boldsymbol{k}_{\text {wind }} \quad\left(\mathrm{m} \mathrm{d}^{-1}\right)$

Both parts of eq. E.5 are explained in the next sections. here, they appear as separate terms, but in fact, they cannot be separated completely. An important reason is that resuspension usually does not occur under quiet flow+wind conditions: there's a threshold value below which no resuspension occurs. Just above a certain threshold, resuspension starts to occur, irrespective whether wind or current is the actual cause. As soon as resuspension occurs, say because of wind alone, any additional effect of currents will simply add to the resuspension flux; and vice versa. So, the threshold should apply to the combined effects (or combined impulse flux) of wind and currents. The threshold value it selves depends on the cohesion of the sediment particles, which specially is a topic in silty sediments, or sediments with a large content of phytobenthos or benthic bacteria. This implies that eq. E.5 can better be replaced by

$\boldsymbol{k}_{1}=\boldsymbol{f}\left(\boldsymbol{k}_{\text {flow }}+\boldsymbol{k}_{\text {wind }}\right) \quad\left(\mathrm{m} \mathrm{d}^{-1}\right)$

The resuspension parameter $k_{1}$ is affected by the hydrodynamical conditions (a result of flow and waves and/or wind) and the conditions of the sediment surface (e.g. roughness and erodibility of the particles that may be affected by the presence of bacteria and or benthic algae).

The settlement velocity $\mathrm{v}_{\mathrm{z}}$ depends on the characteristics of the solid particles, and of the water. Although often several types of particles (or several size classes) are distinguished, the accompanying $\mathrm{v}_{\mathrm{z}}$-values usually are fixed. However, the particles, and thus their settling velocity, may be affected by the formation of aggregates.

Theoretical equations for the $\mathrm{v}_{\mathrm{z}}$-parameter do exist and are given here. In our case, we must rely partly on measured time series of [C]: the final parameter setting will be determined by the fit between computed and measured suspended solid contents in the Wadden Sea. This is also caused by the fact that in the ecosystem model, solids are grouped, and thus a particle type is a group of roughly similar particles, but without a precise density and/or size. In the next pages, the concentration "[]"-notation is omitted.

\section{A20.4 Effect of currents}

Presently, $\mathrm{k}_{\text {flow }}$ is a parameter computed as $\mathrm{k}_{\mathrm{f}}$ * maximum flow induced shear stress. Max flow induced shear stress $\left(\mathrm{N} \mathrm{m}^{-2}\right)$ has previously been computed by Alkyon, and $\mathrm{k}_{\mathrm{f}}$ was roughly estimated at $10^{-11} \mathrm{~m} \mathrm{~s}^{-1}$.

In the model, it is formulated as:

$\mathbf{k}_{\text {flow }}=\mathbf{k}_{\text {flowfact }} \cdot$ FlShstress $\quad\left(\mathrm{m} \mathrm{d}^{-1}\right)$

Here, FIShStress is the bottom shear stress $\left(\mathrm{N} \mathrm{m}^{-2}\right)$ computed as the maximum value (at maximum flow velocity); data delivered by RWS and $\mathrm{k}_{\text {flowfact }}$ a proportionality constant $\left(\mathrm{s} \mathrm{kg}^{-1}\right)$, including a factor $86400\left(\mathrm{~s} \mathrm{~d}^{-1}\right)$ needed to come to $\mathrm{m} \mathrm{d}^{-1}$ for $\mathrm{k}_{\text {flow }}$. 
During the simulations, it appeared that the effect of flow must be set to small values. This doubtlessly has to do with the characteristics of suspending solids: if suspended by flow, the particles mainly stay close to the bottom, while wind driven suspension is mixed through the entire water column. And, all the data have been collected close to the water surface. Also, the main effect on primary production will be in the top layer of the water column. Thus, current effects are reduced to small values in the present model set-up.

\section{A20.5 Effect of wind}

Wind induces waves, and wave energy is transported to the sediment/water-interface where it may cause suspension of solids. One may describe this process in roughly three ways (Aalderink et al, 1984): a deterministic one, based on physical and hydrodynamical descriptions, a statistical one and one based on time-series analysis. The latter usually (but not necessarily) is a combination of an extension of the second method, and a simplification of the first. Lick (1982) followed such an approach, combining computed shear stresses and measured erosion rates of sediment particles. A general description used for wind related resuspension, also applied by Aalderink et al (1984), is

$$
\boldsymbol{k}_{\text {wind }}=\boldsymbol{r}_{\mathbf{1}}\left(\boldsymbol{W}_{10}^{r 3}-\text { opw }^{r 3}\right) \quad\left(\mathrm{m} \mathrm{d}^{-1}\right)
$$

with

$r_{1}=$ effect parameter, depending on sediment characteristics

$\mathrm{W}_{10}=$ wind speed on $10 \mathrm{~m}$ elevation (standard) $\left(\mathrm{m} \mathrm{s}^{-1}\right)$, since wave action is mainly driven by wind

opw = threshold value for wind speed $\left(\mathrm{m} \mathrm{s}^{-1}\right)$. Below opw, no resuspension occurs. Note that $k_{\text {wind }}$ cannot be negative; this is taken care of in the model

$r_{3}=$ parameter that reflects a non-linear relationship between wind speed and resuspension power

The parameters $r_{1}$ and opw may be different for different types of particles. Particles that are small and/or have a low specific mass may erode easily, and thus have a low opw-value. On the other hand, particles that are large and/or have a high specific mass may have large opw-values.

\section{A20.6 Effect of wind direction}

Also, it was decided to implement a dependency of wind direction. The position of many site (compartments) is thus that not only wind speed, but also wind direction probably affects resuspension; sometimes an area is exposed, or just sheltered depending on where the winds come from.

The relationship between the effective $k_{1}\left(k_{1 \text { (effect) }}\right)$ and the $k_{1}$ from eq. E. 5 is:

$k_{1}=k_{1} \cdot\left(1.0+\zeta \cdot\left(\right.\right.$ richt - richt $\left.\left._{0}\right)\right) \quad\left(\mathrm{m} \mathrm{d}^{-1}\right)$

In which $\zeta$ is a proportionality constant, richt the direction of the wind, and richt $t_{0}$ the direction from which the wind affect is minimal. Richt and richt $t_{0}$ in degrees relative to north $(=0)$. Two values 
for richt $t_{0}$ are asked by the model, and thus all the cases where there is a shelter from two directions are covered as well.

For a few sites and years some computed suspended matter concentrations are given in the next section.

\section{A20.7 Sedimentation: the settling velocity vz}

The sedimentation flux is proportional to the solid content of the water and the settling velocity $\mathrm{v}_{\mathbf{z}}$ (see eq.E.2). The settling velocity $v_{z}$ is determined by density differences between the suspended particles and the water, the particle radius and the viscosity of the water. The latter is important because in many cases, the settling particle exhibits a shear stress at the particle's surface. Temperature affects the water viscosity, and is therefore-in theory- a variable too.

The general settlement flux is given by eq.E.2, with $C_{Z}$ instead of $C_{H}$ : $C_{Z}$ is the concentration of solids in the water at a certain depth $\mathrm{z}$ :

$$
\text { settling flux }=v_{z}\left[C_{z}\right] \quad\left(g^{-2} \mathrm{~d}^{-1}\right)
$$

The settling velocity $v_{z}$ is the parameter that depends on the physical properties of water and of the particles mentioned above:

$\boldsymbol{v}_{\mathrm{z}}=\boldsymbol{f}\left(\boldsymbol{\rho}_{\text {solid }}, \boldsymbol{\rho}_{\text {water }}, \boldsymbol{v}_{\text {water }}, \boldsymbol{d}_{\text {solid }}\right) \quad\left(\mathrm{m} \mathrm{d}^{-1}\right)$

with

$\rho \quad=$ specific mass of solid or water $\left(\mathrm{g} \mathrm{dm}^{-3}\right)$

$v \quad=$ kinematic viscosity of water $\left(\mathrm{m}^{2} \mathrm{~d}^{-1}\right)$

$\mathrm{d} \quad=$ diameter of solid particle $(\mathrm{m})$

The quantitative relationship between the settling velocity and the physical properties is given by Stokes' law (Bird et al, 1960), if necessary extended with a factor $f(0<f 1)$ that describes the influence of the particles' shape:

$v_{z}=\frac{1}{18} f \frac{g}{v} \frac{\rho_{s}-\rho_{w}}{\rho_{w}} d^{2} \cdot s p d \quad\left(\mathrm{~m} \mathrm{~d}^{-1}\right)$

$\mathrm{f} \quad=1$ in case of an ideal sphere (-)

g = gravity acceleration $\left(\mathrm{m} \mathrm{s}^{-2}\right)$

$\mathrm{d} \quad=$ particle's diameter $(\mathrm{m})$

$s p d=$ seconds per day, needed for the transformation of seconds- to day-units ( $s p d=86400$ ).

Temperature and salinity are implicit factors in the kinematic viscosity $v$ and the specific mass of the water $\rho_{\mathrm{w}}$. These are not given here, but described in a separate document.

Additionally, suspended particles may flocculate. Especially detritus and fine inorganic matter such as silt may together form larger aggregates, and thus increase their settling velocity by an increased 'diameter' $\mathrm{d}$. Such a flocculation is of importance when highly turbulent waters suddenly reach quiet sites, or when fresh water enters a saline environment. The reason for this is that fine matter (clays, humus matter) partly is stabilised by an electrochemical surface charge; this charge 
stabilisation becomes less effective in a situation with high ionic concentrations. The electrostatic double layer of the particles is affected by the ions (especially the anions). As a result, the electric potential at a certain distance from the particle surface drops to much lower (absolute) values in the presence of high ion concentrations, and thus, mutual repulsion becomes much less effective in a saline environment.

\section{A20.8 Adaptations related to time constants}

The EcoWasp-model runs with timesteps of about 0.1 day; a maximum of 1 day and a minimum of about 0.05 day (to be changed if wanted) is usually set when running the model. Too large values may lead to numerical instabilities or inconsistencies. The latter may concern negative values for suspended solids or other components that show high e.g. reaction or settlement rates.

A 0.1-day timestep for resuspending components still may lead to inconsistencies, and therefore an adaptation is implemented. 


\section{A21 Habitat suitability characteristics as possible selection criterion for fauna}

Based on the occurrence of mussel beds and sublittoral mussel biomass, habitat maps for mussels (Mytilus edulis) have been constructed. These maps (and may be similar ones for other animals) can be used to better model the growth/ occurrence of species/groups in parts of the system. Especially since there are large differences in sediment composition in the system (e.g. Figure 74) such knowledge can be applied to distribute the computed animal biomass (per functional group) more correctly: shell fish in the sandier areas, nematodes in the siltier areas, for example. In fact, habitat knowledge is to be used when functional relationships (e.g. why do nematodes prefer very silty areas? or why do lugworms prefer sandier areas?) are lacking or are not modelled explicitly. An example of the mussel bed habitat suitability map is presented in Figure 85. 

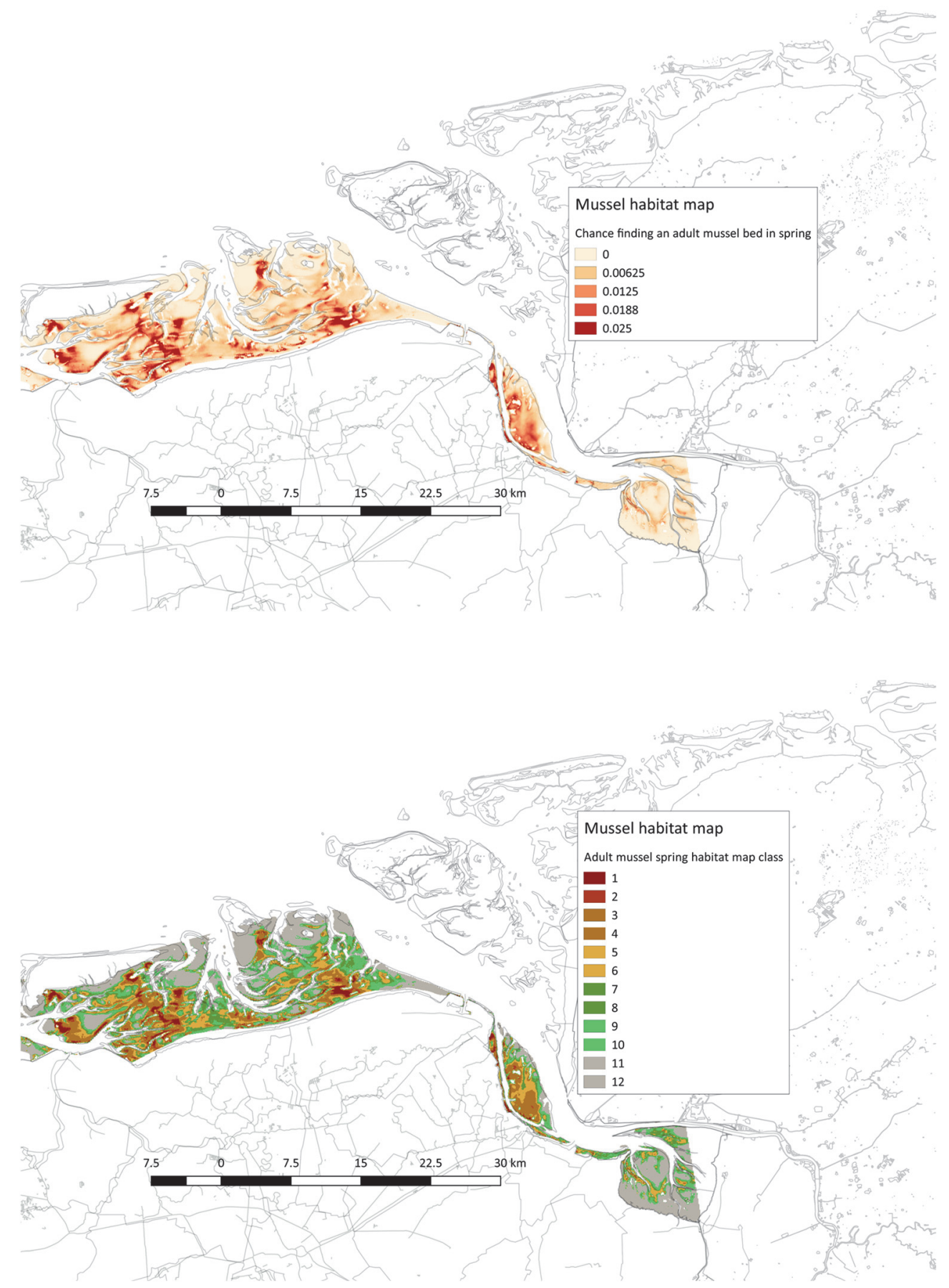

Figure 85 Example of a mussel habitat map for the Ems-Dollard area (Dutch part only). Adult mussel occurrence in spring, Based on 1994-2009 data. Upper map: chance of finding a mussel bed merely consisting of adult mussels. Lower: ranking of the areas (1=best $1 \%$ of all areas, $12=$ less best (30\%) of all areas. See table below for info on which class belongs to which \% of the area. .

\begin{tabular}{|c|c|c|c|c|c|c|c|c|c|c|c|}
\hline Class & $\%$ & Class & $\%$ & Class & $\%$ & Class & $\%$ & Class & $\%$ & Class & $\%$ \\
\hline 1 & $0-1$ & 3 & $2-5$ & 5 & $10-15$ & 7 & $20-25$ & 9 & $30-40$ & 11 & $50-70$ \\
\hline 2 & $1-2$ & 4 & $5-10$ & 6 & $15-20$ & 8 & $25-30$ & 10 & $40-50$ & 12 & $70-100$ \\
\hline
\end{tabular}




\section{A22 Different ratios chlorophyll-a and cell dry matter}

Based on data by Koeman \& Bijkerk (Wanink, 2014) and the results of the IMARES-research in 2013, the ratio cell-carbon/cell-chlorophyll-a could be estimated. Estimated because the cell volumes (and thus masses) were computed from the cell sizes, and this comes with difficulties because of the often-irregular shapes. Sampling sites are in Figure 87, results in Figure 87. Here, this is just for illustrating the possible difficulties when converting algae biomass to chlorophyll.

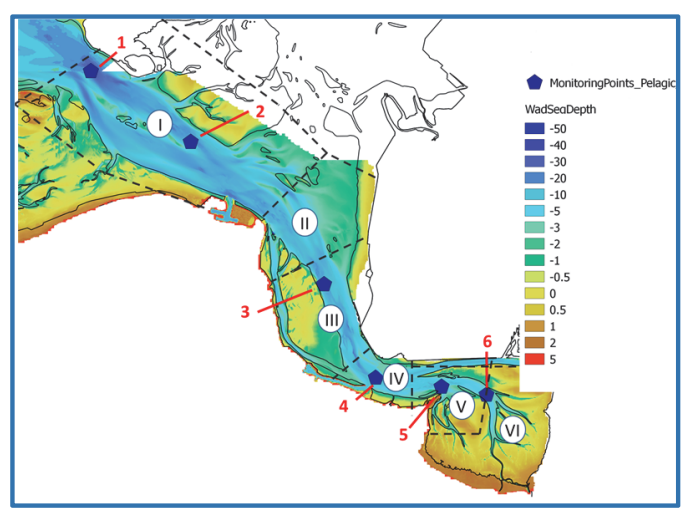

Figure 87 Pelagic sampling points during the 2012-2013 IMARES study on primary production (see Brinkman et al, 2014)
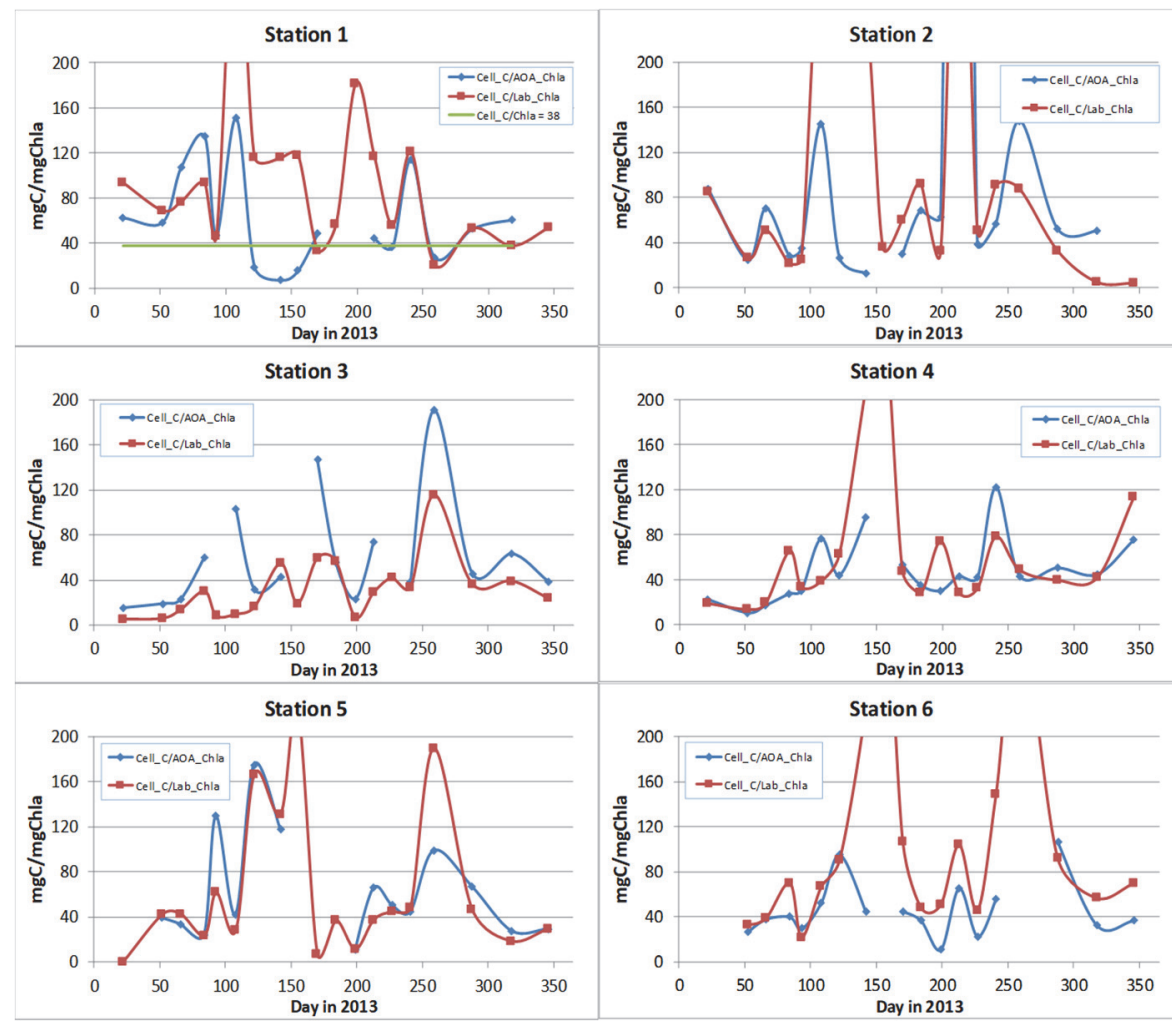

Figure 87 Ratio cell-carbon content and chlorophyll-a in 2013 in the Ems-Dollard. Cel carbon following Koeman \& Bijkerk-data (see Wanink et al, 2014) and chlorophyll-a according to the work of Riegman et al (reported by Brinkman et al, 2014). 


\section{A23 Sediment layers, and fauna feeding from the sediment, some details}

\section{A23.1 Sediment layers: solids}

The sediment compartment in EcoWasp (see for many details Brinkman \& Smit, 1993) consist of several layers with a fixed thickness. These values are part of the initial input files defining the system. All solids (inorganic, detritus, phytobenthos, and fauna living in the sediment) are defined as mass (number) per unit of volume $\left(\mathrm{m}^{3}\right)$ in each of the relevant fixed layers. Between the several layers there exists an exchange, a basic exchange rate coefficient is determined as initial value. The existence of fauna may imply an increasing exchange rate; one of the fauna parameters is regulating that effect.

\section{A23.2 Sediment layers: dissolved compounds.}

Since the model computes pore water profiles, based on the steady state solution of the combined diffusion-reaction equation (with zero order reaction terms for all components that are subject to biochemical transformations, and first order reaction terms for all components that show an adsorption/desorption reaction), the fixed layers are not sufficient. Therefore, variable layers are introduced, of which the thickness is determined by the penetration depths of oxygen and nitrate as electron acceptors (in that order). Thus, there are three types of oxidation states: a top layer where oxygen is used by bacteria (oxidizing organic matter, ammonium and a substance called 'reduced matter') or produced by phytobenthos, a second one where nitrate is used (oxidising organic matter and 'reduced matter') and a third one where both are absent, and organic matter is decomposed into $\mathrm{CO} 2$ and 'reduced matter'. This 'reduced matter' is called $\mathrm{CH}_{4}$ in the model (to give it a composition; it is not really methane). Thus, this 'reduced matter' can be oxidized in the upper layers, ánd the water column.

The pore water profiles thus have a zero-order profile (most components, except phosphorus and silicon) or a first-order profile (phosphorus and silicon). A third group concerns those substances that are not produced or consumed at all: especially chloride $\left(\mathrm{Cl}^{-}\right)$. Also, $\mathrm{Ca}^{2+}, \mathrm{Mg}^{2+}$ may belong to this group if no reactions are defined. The model automatically selects the situation based on the characteristics mentioned in the input definition files.

The concept of penetration depths implies that these depths do not coincide with the fixed layer depths. Thus, we arrive at two more layers in the sediment system: the variable layers. The number of variable layers always is equal to the number of fixed layers + number of electron acceptors (two in this example). Since the variable layers have changing depths, depending on the conditions (oxygen and nitrate levels in the water column, sediment temperature affecting sediment conversion rates), the mass budgets need an extra check and correction every time step. This is not further explained here.

All profiles have the water column concentration as upper boundary condition. Boundary condition of all zero-order profiles is that there is no diffusion through the lowest boundary (first order derivative is zero). Boundary condition for all first-order profiles is that the limit value for very deep in the sediment is not infinite. There's no further explanation here, but that the pore water profile equation contains two exponential terms, one with a negative argument and one with a positive argument. The positive one may lead to infinite values for depth== very large. 
Next condition for each layer is that the fluxes through each plane between two variable layers is the same in both variable layers, and the pore water concentrations at this plane is the same in both layers. These conditions are necessary and sufficient to solve the steady-state reactiondiffusion equation. The set of zero-order equations is solved by subsequently solving the set, starting at layer 1 , and down to the last (deepest) layer, the set of first-order equations is solved following a Gauss-elimination procedure.

\section{A23.3 Diffusive exchange between water and sediment}

The pore water profiles as found after the reaction-diffusion equations are solved are used (according to Ficks first law) to compute the fluxes through each sediment plane, including the sediment-water surface. This gives the amount of dissolved matter that is released into the water, or taken up by the sediment, for each time step.

\section{A23.4 Adaptation of the model: compensation of lowest sediment layer leakage of first order ions}

It appeared to be necessary to adapt the model in those cases where adsorption of ions onto sediment particles occurs.

For most ions that only have zero-order kinetics (or kinetics made zero-order), the boundary condition for the lowest sediment layer (the bottom of the sediment system) is that the flux through the bottom plane is zero. Thus, mass budgets are closed (at least here). This concerns $\mathrm{NO}_{3}{ }^{-}, \mathrm{CO}_{2}, \mathrm{O}_{2}$ and $\mathrm{NH}_{4}{ }^{+}$. Although nitrification is not zero order in ammonium nor oxygen, the reaction is made zero-order each timestep. Those ions that do not react (like $\mathrm{Cl}^{-}$) have a zero flux at the sediment lower layer as well.

For silicate and phosphate, that have an adsorption term, the lowest layer boundary condition was that the [] at infinite depth is a real number. See Brinkman \& Smit (1993) for a complete explanation. However, it appeared that this introduced errors that were not foreseen before. Thus, since now, the rest-flux through the lowest sediment plane is assigned to the water column, and thus, mass budgets are closed.

\section{A23.5 Fauna}

In the EcoWasp-model, input data and results for fauna are expressed as number and biomass per $\mathrm{m}^{2}$, except for animals that live in the water column: then the number and biomass per $\mathrm{m}^{3}$ is mentioned. However, inside the model, as computations are performed using numbers and biomass per $\mathrm{m}^{3}$. Therefore, all initial data are transformed from numbers $\mathrm{m}^{-2}$ to numbers $\mathrm{m}^{-3}$. The layer thickness involved always is the thickness of the fixed layers (see below).

Bottom dwellers (such as snails) search the fixed layers with a certain rate $\left(\mathrm{m}^{3} \mathrm{~s}^{-1}\right)$, and may find the desired food (present as $g$ AFDM m ${ }^{-3}$ ). This is not different from the animals feeding from the water column. Note that in the present model set-up, animals searching the sediment for food do this per fixed layer. So, a certain search depth that may involve more layers is not implemented (yet). 


\section{A23.6 Fauna induced exchange between water and sediment}

The model asks for fauna model parameter telling where fauna finds the food, respires, excretes, defecates. Respiration is a process that uses oxygen, but also transforms body mass into the original components ( $\mathrm{N}$ is always released as $\mathrm{NH}_{4}{ }^{+}$). Thus, also phosphate is released, for example. In case this respiration is into the water column (which is mostly the case), $\mathrm{O}_{2}$ is taken up from and $P$ (etc) is released into the water column. This is called fauna induced exchange, and is separately stored by the model. 


\section{A24 Literature review on effect of silt on bivalves occurring in the Ems-Dollard estuary}

\section{A24.1 Introduction}

\section{A24.1.1 Background and aim}

The Ems-Dollard Estuary is known as a highly turbid area (Talke et al. 2009) which has an influence on the ecosystem functioning. The suspended particulate matter (SPM) concentration increases from $<50 \mathrm{mg} / \mathrm{l}$ at the mouth of the estuary to around $150 \mathrm{mg} / \mathrm{l}$ up the river. Highest values found in 2012/2013 are above 300-400 mg DW/I, up to an occasional $700 \mathrm{mg}$ DW/I.

Suspended particles are considered to have an impact in three ways: decrease in vision of visual predators, decrease of light used by primary producers, and interference with the respiratory and filter apparatus of filter feeders. This document addresses the latter effect with the aim to support the development of an ecological model of the Ems-Dollard Estuary. It describes the available knowledge on the effect of suspended sediments on filter feeding bivalves, i.e. Blue mussel (Mytilus edulis), Cockle (Cerastoderma edule), Peppery furrow shell (Scrobicularia plana), Japanese oyster (Crassostrea gigas) and Razor clam (Ensis directus).

Questions addressed are:

- Qualitative description of effects

$\circ$ What is the mechanism of filter feeding of bivalves?

- How does suspended sediment affect the mechanism?

- What are the effects (e.g. lower/less food intake; higher respiration energy demand causing growth inhibition; mortality)?

- Quantitative effects

- At what concentration do effects occur (i.e. dose/response relationships)?

- What is the amount of pseudofaeces in relation to the suspended sediment concentration?

- What are the costs (energy) to produce pseudofaeces, concerning mainly the required mucus?

- What are the filtration rates in relation to the suspended sediment concentration?

\section{A24.1.2 Species description}

\section{A24.1.2.1 Blue mussel}

The blue mussel (Mytilus edulis) occurs from the high intertidal to the shallow subtidal attached by fibrous byssus threads to suitable substrata. Found on rocks and piers in sheltered harbours and estuaries, often occurring as dense masses. The shell colour varies, usually purple or blue but sometimes brown (Figure 88). Length varies, specimens usually ranging from $5-10 \mathrm{~cm}$ although some populations never attain more than $2-3 \mathrm{~cm}$ (Tyler-Walters 2008). 


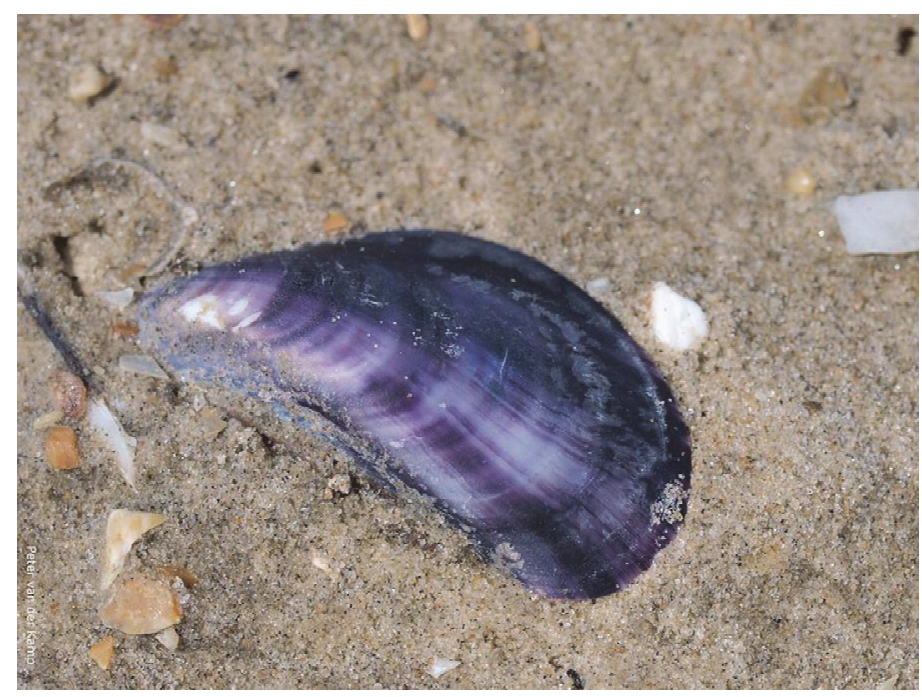

Figure 88 Blue mussel, Mytilus edulis (photo Peter van der Kamp).

\section{A24.1.2.2 Cockle}

The cockle (Cerastoderma edule) is often abundant in estuaries and sheltered bays. It inhabits the surface of sediments, burrowing to a depth of no more than $5 \mathrm{~cm}$. Found on clean sand, muddy sand, mud or muddy gravel from the middle to lower intertidal, sometimes subtidal. The shell is solid, thick, equivalve, globular and broadly oval in outline (Figure 89); up to $5 \mathrm{~cm}$ long but usually less (Tyler-Walters 2007).

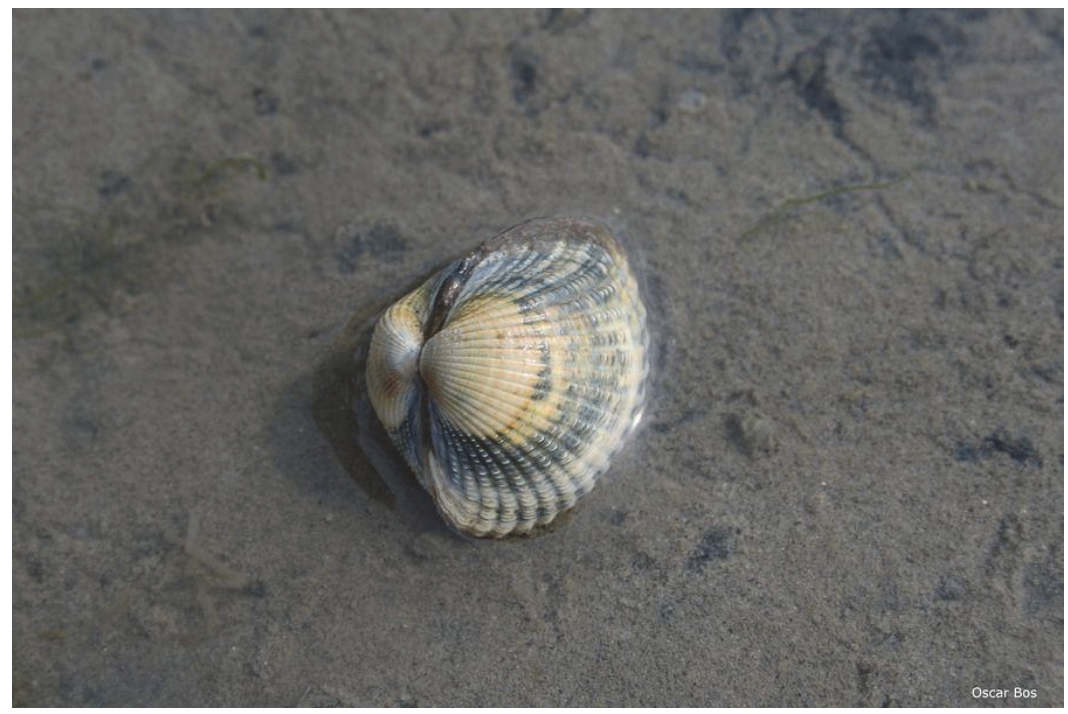

Figure 89 Cockle, Cerastoderma edule (photo Oscar Bos).

\section{A24.1.2.3 Peppery furrow shell}

The peppery furrow shell (Scrobicularia plana) is found in estuarine and intertidal conditions and can tolerate low salinities in thick mud or muddy sand. The shell can be anything from white through yellowish to a pale brownish-grey in colour, up to $6,5 \mathrm{~cm}$ in diameter (Figure 90). It 
burrows up to $20 \mathrm{~cm}$ deep in sediments and it feeds by extending a siphon above the sediment when the tide is in (Pizzolla 2002).

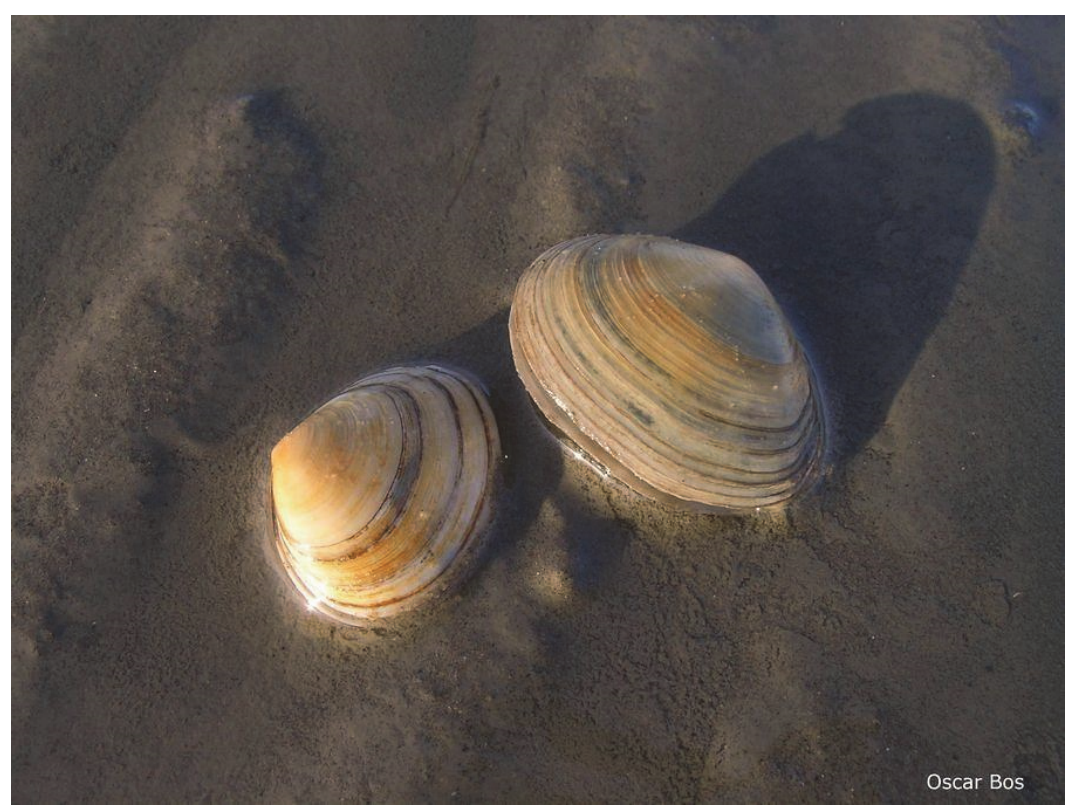

Figure 90 Peppery furrow shell, Scrobicularia plana (photo Oscar Bos).

\section{A24.1.2.4 Japanese oyster}

The Japanese oyster or Pacific oyster (Crassostrea gigas) is an exotic species and found on the lower shore and shallow sublittoral to a depth of around $80 \mathrm{~m}$. The shell can grow up $18 \mathrm{~cm}$ long and has an off-white to yellow or bluish grey colour (Figure 91). The shell often has deep purple patches (Hughes 2008).

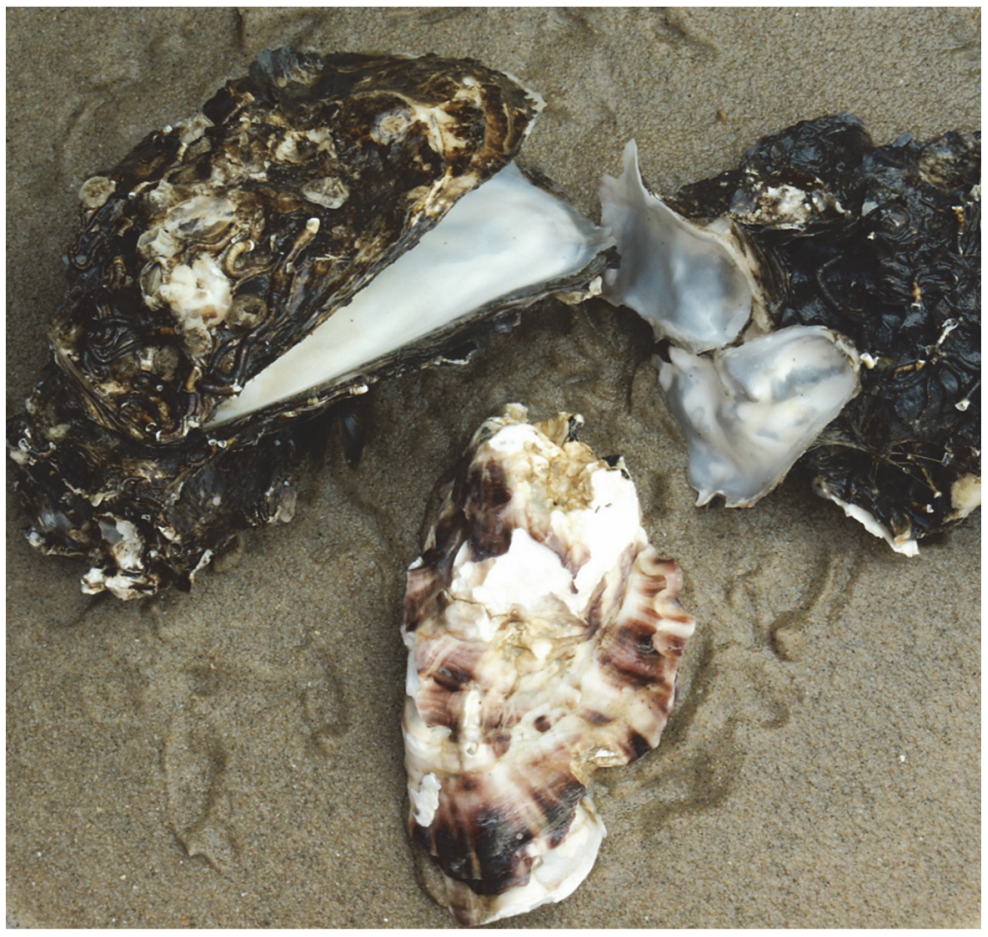

Figure 91 Japanese oyster, Crassostrea gigas

(http://www.strandvondsten.nl/tweekleppigen/japanse_oester/). 


\section{A24.1.2.5 Razor clam}

The razor clam (Ensis directus or Ensis americanus) has been introduced from America and is found in estuaries in the infralittoral and circalittoral, buried in decimetres of sand or muddy sand. It has a whitish-grey colour with brown drawings and can grow at least $20 \mathrm{~cm}$ long with a length six times as wide, slightly curved (Figure 92). When feeding, E. directus stays very close to the surface and its siphons are sticking up through the surface (Camponelli 2001). When disturbed they quickly withdraw into their holes in the sediment.

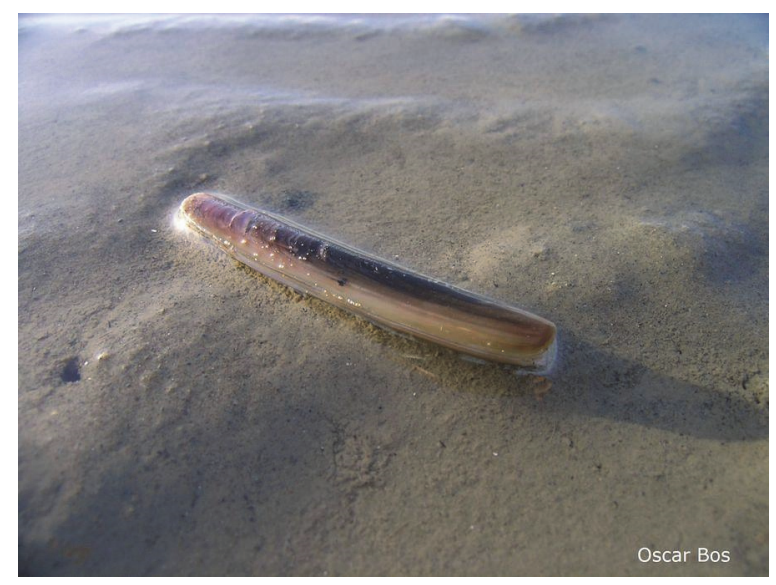

Figure 92 Razor clam, Ensis directus or Ensis americanus (photo Oscar Bos).

\section{A24.2 Food collection methods}

\section{A24.2.1 Introduction}

Uptake of particles from the environment is the first step in a sequence of events that ultimately leads to ingestion of food. In general, particle feeding is characterized by efficient uptake, ingestion and assimilation of particulate matter. Differences in food collection methods of particlefeeding molluscs exist and molluscs can be separated into two broad categories based on the mechanism of particle uptake and processing (Ward \& Shumway 2004):

1. those that utilize mucous nets or strings (external to, or within the mantle cavity) to collect material, predominantly in the class Gastropoda (i.e. snails and slugs) thus outside the scope of this document, and;

2. those that rely on ciliated (hair like) structures (proboscides, ctenidia) for particle collection, transport and processing.

There are also a few molluscs that rely on both mechanisms for particle collection.

Molluscs that rely on ciliated structures for particle collection include the (see Figure 93):

- Lamellibranchiate bivalves which use the ctenidia to capture suspended or deposited material (Figure 93, A and B), and the

- Protobranchiate bivalves, which mostly deposit feed using a pair of palp proboscides (Figure 93C). 
As this study is focussed on the effect of increased suspended sediments on filter feeding bivalves the latter mechanism (Figure 93C) is outside the scope of this study. The species that are relevant for this study are blue mussel, cockle, peppery furrow shell, Japanese oyster and razor clam. Cockle, peppery furrow shell and razor clam inhabit the surface of sediments, burrowed in the sand or mud like most of the bivalves occurring in het Dutch intertidal zone. Food collection in these species is schematically presented by Figure 93B. The blue mussel and the Japanese oyster live above the seafloor, mostly attached to hard substrate and can form banks. The schematically presentation of food collection in these species is shown in Figure 93A.

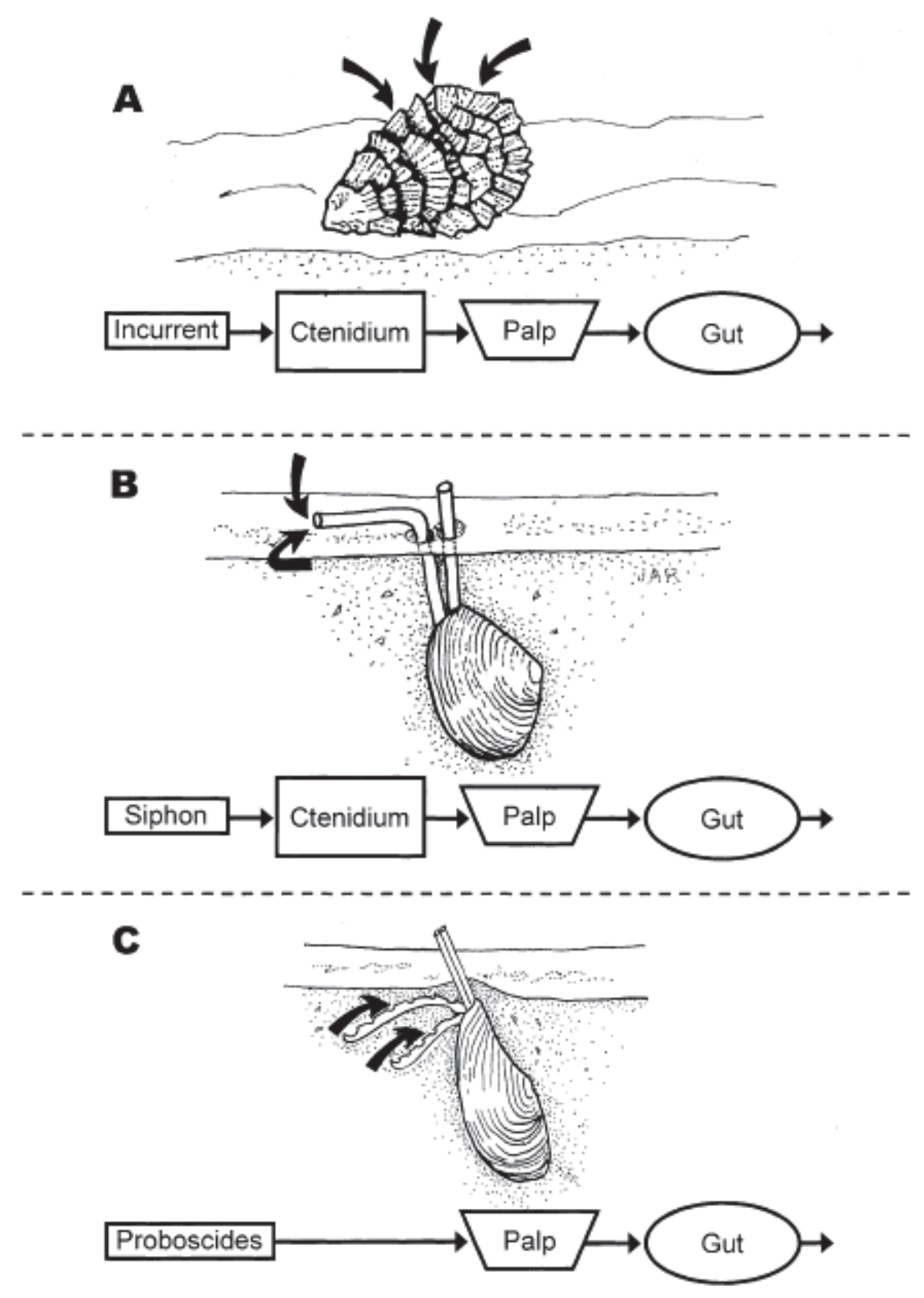

Figure 93 Diagram of the major modes of food collection in the Bivalvia (arrows). Flow charts demonstrate uptake and movement of particles from the environment (left) through the main feeding compartments: $(A)$ suspension feeding via an inhalant aperture or siphon 


\section{A24.2.2 Filter feeding}

Bivalves use their gills and palps for feeding. The particle capture organ of the gill (which also serves as the respiratory organ) is called the ctenidium (Cranford et al. 2011). An individual has two pairs of gills and two pairs of palps (Figure 95). Suspension - feeding lamellibranchiate bivalves rely on ciliated structures to capture and transport suspended particulate matter for selection and ingestion. According to recent understanding, particles are separated from the water current at the entrance to the interfilamentous canals of the gill and transferred onto the frontal surface by the action of the laterofrontal cirri (Ifc) that have a fixed, alternating beat pattern (Figure 94). A water current is produced which displaces water within the shells and replaces it with water from outside the shells (i.e. the pump). Particles from the water are captured by the gills and edible parts are roughly selected. Particle retention efficiency in Mytilus edulis and 12 other species of suspension feeding bivalves from different habitats and with different gill types has been determined by analysing the particle size distribution in the water entering and leaving the

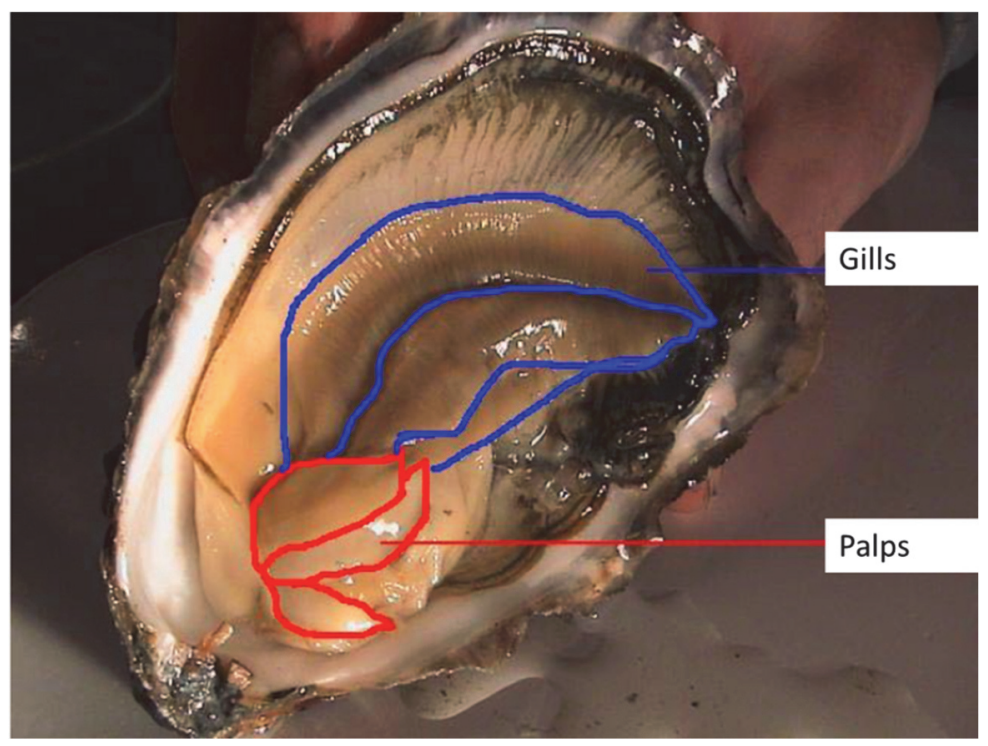

Figure 94 The gills and palps of the Japanese oyster

inhalent and exhalent apertures (Møhlenberg \& Riisgård 1978). Particles $\geq 4 \mu \mathrm{m}$ were completely withheld by $M$. edulis and the retention efficiency decreased only slightly down to $2 \mu \mathrm{m}$, where it was appr. $80 \%$. Particle retention efficiency was below 50\% for particles of $1 \mu \mathrm{m}$ (Møhlenberg \& Riisgård 1978). It is suggested that particles larger than $4 \mu \mathrm{m}$ are stopped and transferred to the frontal side, whereas smaller particles either follow the flow around the Ifc or they are stopped by the Ifc's branching cilia (Riisgård et al. 2015).

Bivalves utilize the digestible part of the SPM, such as phytoplankton, bacteria and decomposable detritus (Essink 1999). The digestible parts are transported to the palps whereas non-edible parts are secreted as pseudofaeces (Zemlys et al. 2003). The complete mixture of particulate matter suspended in the water column is also referred to as seston, which applies to all particulates, including plankton, organic detritus, and inorganic material. The total amount of SPM is a measure of food quantity whereas the organic content of the SPM is a measure of the food quality (Barillé et al. 1997). 


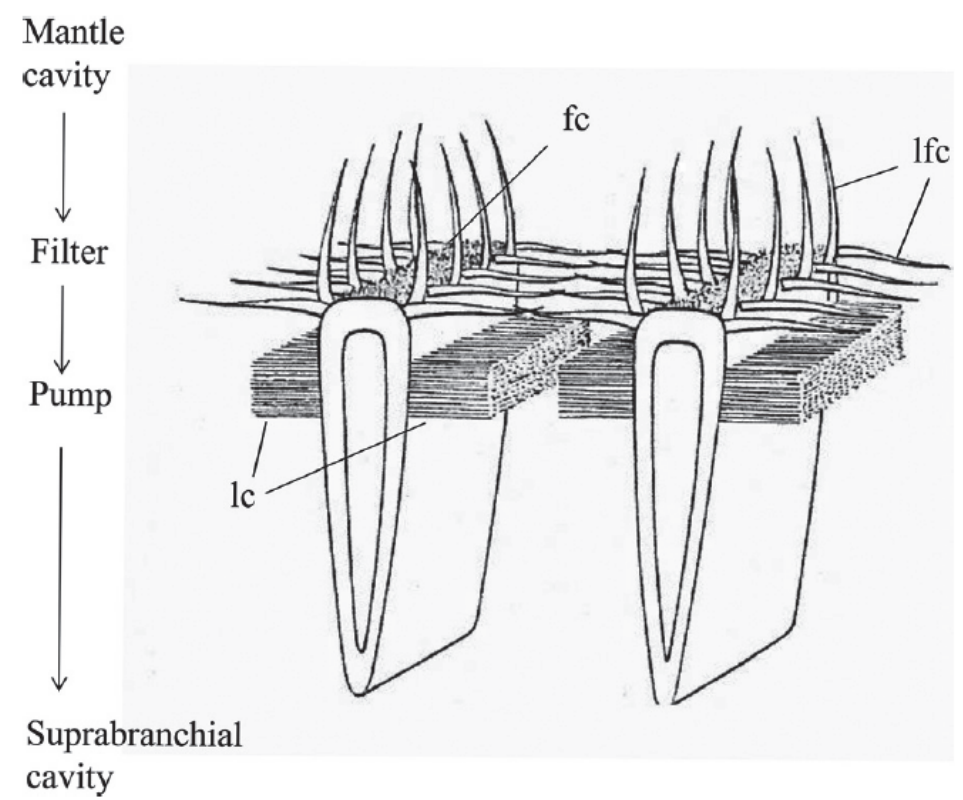

Figure 95 Cross-section of two gill filaments of mussel Mytilus edulis; Ifc = laterofrontal cirri (alternating cirri beat synchronously and differ half a phase with the two adjacent ones; effective stroke upwards); Ic = lateral cilia (effective stroke downwards),

A review of literature by (Ward \& Shumway 2004) indicates that capture efficiency of bivalves increases non-linearly with particle diameter and that some species are more efficient at capturing small particles than others. Capture, however, is not merely a mechanical sieving process. For example, in M. edulis, the distance between branching cilia of the laterofrontal cirri varies from about 1.0 to $1.7 \mu \mathrm{m}$ would mean a near $100 \%$ capture of all particles down to $>2 \mu \mathrm{m}$, which is clearly not the case (Ward \& Shumway 2004). Qualitative factors can also influence particle capture. Mussels cleared phytoplankton particles at a higher rate than silt particles at total concentrations of less than 20,000 particles/ml. The mechanisms involved in the discrimination of particles with equivalent spherical diameter by the bivalve ctenidium are not yet understood. One possibility is that some types of particles are more efficiently retained by the ctenidium, resulting in a higher capture rate (encounter rate $\mathrm{x}$ retention efficiency). Enhanced retention could be a consequence of specific interactions between the extracellular matrix of living cells, and cilia and mucus of the bivalve ctenidia (Ward \& Shumway 2004). For example, sugar-containing compounds on the cell surfaces of marine diatoms have a higher stickiness then silt, leading to enhanced retention. Bivalves can selectively reject certain species of phytoplankton which are disposed as pseudofaeces.

Large particles like plant detritus, benthic diatoms and invertebrate larvae may accumulate in the near-bed layer and form a significant food source for benthic bivalve, as observed for the cockle Cerastoderma edule (Karlsson et al. 2003). C. edule can discriminate between living chlorophyllrich cells and inert organic particles and prefers microalgae vs detritus (Navarro et al. 2016). It is suggested that phytoplankton would be the main contributor to growth of $\mathrm{C}$. edule during the seasons of phytoplankton abundance and detrital matter would decisively contribute to body maintenance along the resting season (autumn/early winter) (Navarro et al. 2016).

Mucus plays an important role in the normal feeding process of undisturbed bivalves (Ward et al. 1993) (Jørgensen 1996). When particles arrive in the dorsal tract they are carried toward the 
mouth in a sludge of low-viscosity mucus. A considerable basal amount of mucus is continuously produced and ingested, regardless of particle concentration in the external medium. Small amounts of mucus may be carried in suspension in the surface currents to the mouth and ingested, whereas larger amounts of mucus are rejected as pseudofaeces (Jørgensen 1996).

\section{A24.2.3 Effects of suspended sediment}

Although adult bivalves are silt-tolerant organisms, their survival in naturally silty areas does not necessarily indicate they are unaffected by high concentrations of suspended sediment (Wilber \& Clarke 2001). As mentioned previously, increased suspended matter may cause interference with the respiratory and filter apparatus of filter feeders. Potential effects of increased suspended matter concentrations in the water column on bivalves are:

- Decreased food intake (clearance rate, filtration rate)

An increase of the seston with inorganic particles (sediment), due to e.g. dredging or dumping, may lead to a decrease in the net food intake of filter feeding bivalves per unit of time (Essink 1999). Clearance rate (the volume of water cleared per unit time, e.g., L $\mathrm{h}^{-1}$ ), also expressed as filtration rate (the mass of particles cleared per unit time, e.g., $\mathrm{mg}$ $\mathrm{h}^{-1}$ ), was found to decline with increasing particle concentration (Widdows et al. 1979), see Figure 96.

- Increase of pseudofaeces Unusable material is separated from the food particles and is excreted as pseudo faces without passing the digestive organs. A higher silt content will lead to a higher production of pseudo faces (Essink, 2005). (Kiorboe et al. 1980). There is however a limit to the pseudofaeces production; above a certain SPM the production will decline, see Figure 96.

- Decreased ventilation rate Increasing particle concentration causes a decline in ventilation rate and therewith a decrease in the amount of oxygen delivered to the gills per unit time (Widdows et al. 1979).

- Higher respiration energy demand

As response to a decrease in the amount of oxygen delivered to the gills (see above), $M$. edulis has been shown to increase extraction efficiency exponentially with increasing particle concentration (Widdows et al. 1979). Therewith the oxygen intake was kept constant, within a range of SPM. This requires additional energy.

- Adjusted morphology

Many bivalve species are able to adjust their gills and palps, i.e. their ctenidial morphology, to cope with high levels of suspended matter (Ward \& Shumway 2004). The ctenidial morphology is known to vary over time and between regions with different turbidity. Such morphological plasticity may result in functional changes in capture efficiency, clearance rate and particle selection in response to ambient seston concentrations (Ward \& Shumway 2004). Using different mixtures of silt and phytoplankton it was shown that $C$. gigas adjusted its capture efficiency in response to seston quantity, but not quality (Barillé et al. 1993). It was suggested that $C$. gigas controls the interfilamentary spaces of the ctenidium as a compensatory response to high seston loads (Barillé et al. 1993). Mussels from the Wadden Sea, with SPM between 40 and 400 $\mathrm{mg} / \mathrm{l}$, have relatively small gills (high SPM) and relatively large palps (to sort out the large 
amount of particles). In the North Sea, where the SPM is often below $20 \mathrm{mg} / \mathrm{l}$, the mussels have larger gills and smaller palps because there is hardly any unusable material that needs to be sorted out. The relative size of gills and palps of mussels from locations with less than $50 \mathrm{mg} / \mathrm{I} \mathrm{SPM}$ seems to be linear correlated with the SPM (Essink, 2005).

- Higher assimilation energy demand

The assimilated energy for the process of food intake and digestion could increase if there are less utilizable components in SPM (Widdows et al. 1979). The cost of maintenance of a large ciliary gill pump is considerable and only relatively little energy can be saved by stopping or reducing the beat frequency of the water-pumping cilia. Therefore, continuous feeding with a 'minimal scaled' pump is cheaper than discontinuous feeding with a correspondingly larger pump (Riisgård et al. 2015).

- Growth inhibition

Several studies indicate that growth is inhibited by low rates of ventilation of the mantle cavity, regardless of the food intake (Jørgensen 1996). Growth of bivalves was found to be strongly correlated with the chlorophyll a- and organic matter content of SPM (Essink \& Bos 1985). Furthermore, there is a maximum of suspended matter concentration that can be filtered by bivalves. Above this maximum there is little or no energy available for growth of the organism (Essink, 2005). A higher silt content could thus inhibit growth.

- Mortality

Prolonged exposure to very high SPM concentrations could lead to mortality (Mainwaring et al. 2014).

These adverse effects of increased SPM mostly follow an optimum curve relationship (e.g. (Widdows et al. 1979, Essink 1999, Mainwaring et al. 2014). Therefore, when the SPM is relatively low, positive effects may initially occur due to an increase in SPM. Only when exceeding optimum values adverse effects may occur. This should be considered when interpreting the quantitative effects, as described in the next section (Appendix A24.3).

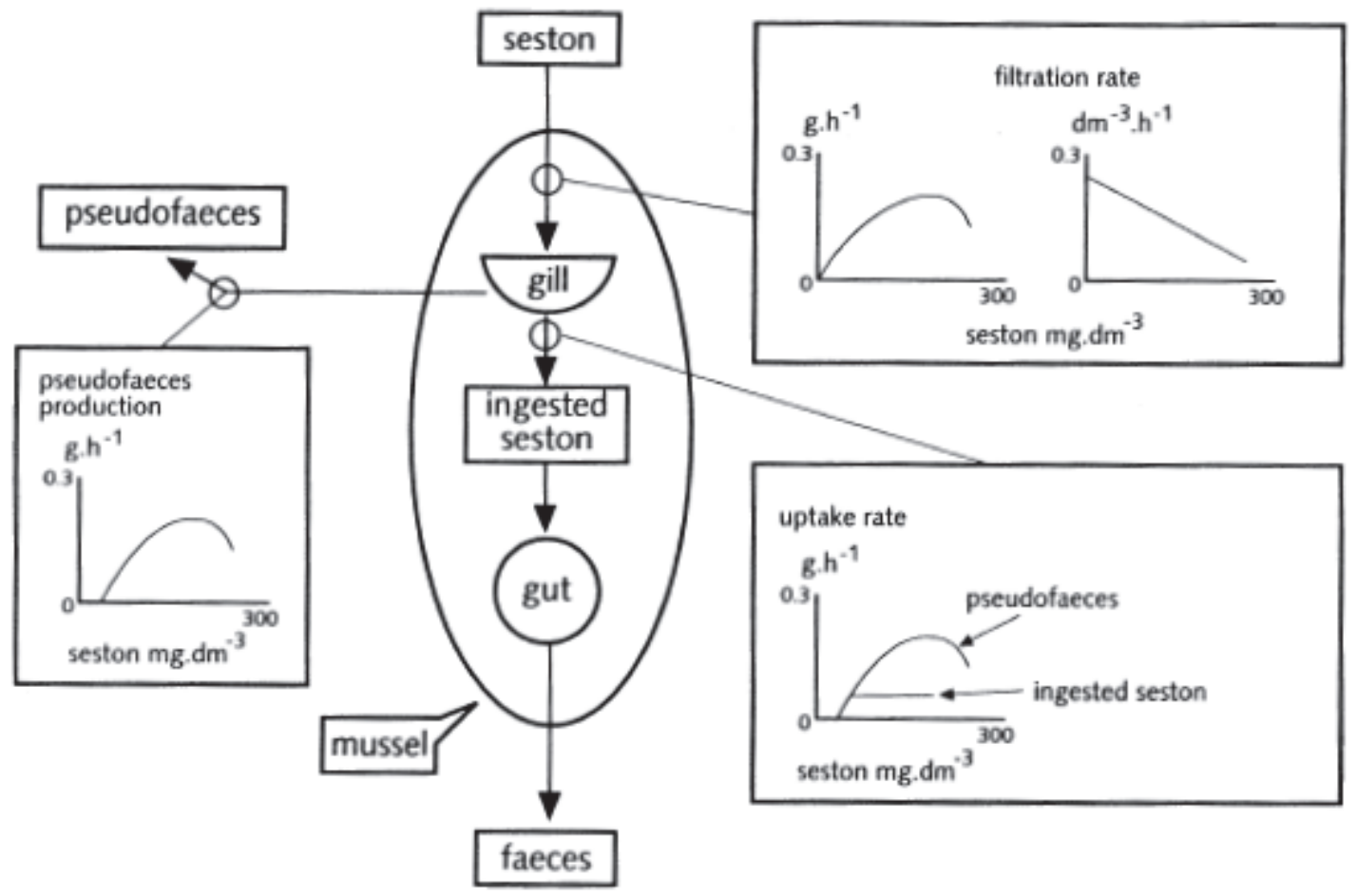

Figure 96 Schematic presentation of filtration and ingestion of suspended matter (seston) and of pseudofaeces production by Mytilus edulis as a function of suspended matter concentration ((Essink 1999), after (Widdows et al. 1979)). 


\section{A24.2.4 Sedimentation Biotic Index}

The relative sensitivities of 309 common invertebrate species in Dutch marine waters were presented for environmental and anthropogenic pressures (among which sedimentation) by Gittenberger \& van Loon (2013). The SEBI (Sedimentation Biotic Index) describes the sensitivity of species to suspended matter (especially sand and mud) that precipitates onto the macrozoobenthos (Gittenberger \& Loon 2013). Benthic species that live in an area where a lot of sediment sinks to the bottom should be able to clean themselves of this sediment to prevent suffocation and blocking of food intake. This ability and therefore the sensitivity of a species to sedimentation is at least partly dependent on the trophic group of a species (Gittenberger \& Loon 2013). The following groups are distinguished (Gittenberger \& Loon 2013):

- Group I. Species very sensitive to sedimentation in general. In clear waters, the species is present in relatively high densities.

- Group II. Species sensitive to high sedimentation. They prefer to live in areas with some sedimentation, but don't easily recover from strong fluctuations in sedimentation.

- Group III. Species insensitive to higher amounts of sedimentation, who however don't recover easily from strong fluctuations in sedimentation.

- Group IV. Second-order opportunistic species insensitive to higher amounts of sedimentation. Although they are sensitive to strong fluctuations in sedimentation, their populations recover relatively quickly and even benefit. This causes their population sizes to increase significantly in areas after a strong fluctuation in sedimentation.

- Group V. First-order opportunistic species. Species which are significantly benefitting from higher amounts of sedimentation and fluctuations in sedimentation.

The relevant bivalve species for the underlying study (C. gigas, E. directus, M. edulis, C. edule and S. plana) were all classified as group II: species sensitive to high sedimentation (Gittenberger \& Loon 2013).

\section{A24.3 Quantitative effects of suspended sediment on filter feeding}

\section{A24.3.1 Growth and metabolic costs}

\section{A24.3.1.1 Introduction}

Research into the naturally occurring diets for suspension-feeding bivalves concerns the role of suspended silt, where in some studies a proportion of silt in the diet has been found to enhance growth and in others to inhibit growth (Bayne et al. 1987). As the feeding rate reaches an optimum at certain SPM concentrations, see e.g. Figure 96, it is likely that observations of increased growth at higher proportions of silt are related to relatively low levels of silt, i.e. below concentrations of maximum filtration.

Short-term increases of particulate inorganic matter has less impact on the growth performance of the shellfish compared to a continuous increase (Wijsman et al. 2012). The timing is also important. In the winter period, when the activity of the shellfish is low, the impact of increased suspended sediment concentration is much lower than during summertime. 


\section{A24.3.1.2 Blue mussel}

As explained previously, increased suspended matter can inhibit growth, but there are also stimulating effects reported. Such positive effects occur when the SPM is below the optimum value, such as known for Mytilus edulis.

There are reports of faster growth of Mytilus edulis in more turbid environments but these refer to SPM-concentrations of ca. $90 \mathrm{mg} / \mathrm{l}$ as compared to $10 \mathrm{mg} / \mathrm{l}$ (Essink 1999). In this range of SPM, the bivalve can make a profitable use of the higher organic matter concentrations at higher SPMconcentrations, agreeing with the first part of the optimum curve relationship presented by Widdows et al. (1979). Concentrations above $250 \mathrm{mg} / \mathrm{l}$ have been shown to impair the growth of filter-feeding organisms, though not significantly (Essink 1999). Field experiments carried out in the Ems estuary indicate that an increase of suspended matter concentrations by $10-20 \%$ is not likely to affect growth and condition of M. edulis (Essink et al. 1990 in (Essink et al. 1992)).

Critical limits to growth for $M$. edulis relate to digestive constraints rather than to metabolic costs of feeding and digestion (Bayne et al. 1989). For mussels an exponential relationship between metabolic costs and net absorption rate has been observed (Bayne et al. 1989). In the summer, the maximum ingestion rate $(2.35 \mathrm{mg} / \mathrm{h})$ for $M$. edulis greatly exceeds the maintenance ration and provides an energy excess for growth and the production of gametes. This summer value for food availability (1.1 $\mathrm{mg}$ of food per litre filtered) corresponds to the optimum ration, that is, the ration at which maximum growth efficiency occurs (Widdows et al. 1979).

The energetic cost of maintaining a large gill pump based on gill respiration is considerable, so that continuous feeding with a 'minimal scaled' pump is cheaper than discontinuous feeding with a correspondingly larger pump (Riisgård et al. 2015).

Young specimens of three bivalve species were transplanted at the start of the growing season from the Wadden Sea to 5 sites in the Ems estuary (Essink \& Bos 1985). Changes in shell lengths and ash-free dry weights (ADW) of the soft parts were determined by monthly sampling. Growth of all bivalves was relatively good in the outer part to decrease towards the inner parts of the estuary, reaching very low values in the innermost part for M. edulis. Growth was found to be significantly correlated to suspended matter (Table 18).

A study on the effect of suspended bottom material on growth of $M$. edulis found growth rate to be increased by 30 to $70 \%$ by the addition of $5 \mathrm{mg}$ silt/l (Kiørboe et al. 1981). It should be noted that SPM concentrations were relatively low (highest exposure was $20 \mathrm{mg} / \mathrm{l}$ ) thus the mussels were probably exposed to concentrations below the optimum SPM. 
Table 18 Regression equations $(y-a x+b)$ and linear correlation coefficients $(r)$ for the relation between shell length and ADW (y) of the 3 bivalves (Mytilus edulis, Mya arenaria, Cerastoderma edule) and the environmental variables $(x)$ salinity, suspended mat

\begin{tabular}{|c|c|c|c|c|c|c|c|c|c|}
\hline \multirow[t]{2}{*}{ Species } & \multirow[t]{2}{*}{ Environmental variable } & \multirow[t]{2}{*}{ Date } & \multirow[t]{2}{*}{$N$} & \multicolumn{3}{|c|}{ Shell length $(\mathrm{mm})$} & \multicolumn{3}{|c|}{$A D W(m g)$} \\
\hline & & & & $r$ & a & $b$ & $r$ & $a$ & $b$ \\
\hline \multirow[t]{4}{*}{ Mytilus } & Salinity & Aug. 15 & 117 & 0.66 & 0.46 & 20.21 & 0.84 & 17.2 & -75.2 \\
\hline & Suspended matter & Aug. 15 & 117 & -0.77 & -0.03 & 28.65 & -0.80 & -0.9 & 218.2 \\
\hline & POM in susp. matter & Aug. 15 & 117 & 0.29 & 0.35 & 20.79 & 0.59 & 20.6 & -159.2 \\
\hline & Chlor. in susp. matter & Aug. 15 & 117 & 0.81 & 0.02 & 21.31 & 0.76 & 0.5 & 6.6 \\
\hline \multirow[t]{6}{*}{ Mya } & Salinity & May 21 & 102 & & & & 0.15 & & \\
\hline & & June 18 & 110 & & & & 0.26 & 2.0 & 33.5 \\
\hline & Susp. matter & May 21 & 102 & & & & -0.14 & & \\
\hline & & June 18 & 110 & & & & -0.11 & & \\
\hline & POM in susp. matter & June 18 & 110 & & & & 0.40 & 4.8 & -6.3 \\
\hline & Chlor. in susp. matter & June 18 & 110 & & & & 0.13 & & \\
\hline \multirow[t]{12}{*}{ Cerastoderma } & Salinity & May 21 & 124 & 0.55 & 0.19 & 9.08 & 0.55 & 1.6 & 2.6 \\
\hline & & June 18 & 110 & 0.80 & 0.49 & 9.90 & 0.89 & 7.1 & -34.2 \\
\hline & & July 15 & 31 & 0.91 & 0.78 & 8.13 & 0.91 & 12.5 & -92.4 \\
\hline & Susp. matter & May 21 & 124 & -0.56 & -0.02 & 12.96 & -0.57 & -0.2 & 34.8 \\
\hline & & June 18 & 110 & -0.64 & -0.02 & 17.61 & -0.74 & -0.3 & 78.0 \\
\hline & & July 15 & 31 & -0.92 & -0.05 & 23.01 & -0.92 & -0.8 & 145.2 \\
\hline & POM in susp. matter & May 21 & 124 & 0.20 & 0.11 & 9.97 & 0.09 & & \\
\hline & & June 18 & 110 & 0.72 & 0.67 & 6.97 & 0.75 & 9.0 & -68.0 \\
\hline & & July 15 & 31 & 0.92 & 1.13 & 2.74 & 0.92 & 17.9 & -175.7 \\
\hline & Chlor. in susp. matter & May 21 & 124 & 0.16 & & & 0.01 & & \\
\hline & & June 18 & 110 & 0.48 & 0.01 & 12.76 & 0.53 & 0.1 & 7.9 \\
\hline & & July 15 & 31 & 0.94 & 0.03 & 9.80 & 0.89 & 0.4 & -56.7 \\
\hline
\end{tabular}

\section{A24.3.1.3 Cockle}

In contrast to mussels, for cockles, metabolic behaviour appears best described by a linear dependence on absorption, meaning that the combined costs of feeding, digestion, and growth calculated as the specific dynamic action (SDA = total metabolism - maintenance metabolism) represent a constant fraction of absorption (10\%) throughout the entire range of food levels (Navarro et al. 1994). Therefore, no extra metabolic costs are incurred with increasing ration, and SFG experiences no restrictions from metabolic expenditure at high food availability. At low food/silt ration, however, maintenance costs become a relevant proportion of absorbed energy (up to $87 \%$ at the maintenance ration), setting a lower limit to food levels capable of sustaining positive growth (Navarro et al. 1994).

Cerastoderma edule is well adapted to living in turbid environments due to an effective preingestive selection of organic particulates, together with increasing filtration and rejection rates. However, increased siltation and suspended sediment concentration results in loss of energy and carbon as mucus due to increased pseudofaeces production (Tyler-Walters 2007).

In the field study of the Wadden Sea, where bivalve species were from the Wadden Sea to the Ems estuary (Essink \& Bos 1985), growth of $C$. edule reached very low values in the innermost part of the estuary. Growth was found to be significantly correlated to suspended matter (Table 18). 


\section{A24.3.1.4 Peppery furrow shell}

The peppery furrow shell (S. plana) was classified by Gittenberger \& Loon (2013) as group II: species sensitive to high sedimentation. The sensitivity class is equal to those of the other relevant bivalve species for the underlying study (C. gigas, E. directus, M. edulis, C. edule).

S. plana was judged by the Marine Institute (2013) to have 'medium-high resistance' to on-going siltation events and population effects are assumed to be limited. Recovery is assessed as 'very high' so that the sensitivity of this species to high sedimentation is judged to be 'low-not sensitive' (Marine Institute 2013).

No specific information on growth effects of increased sedimentation was found for S. plana.

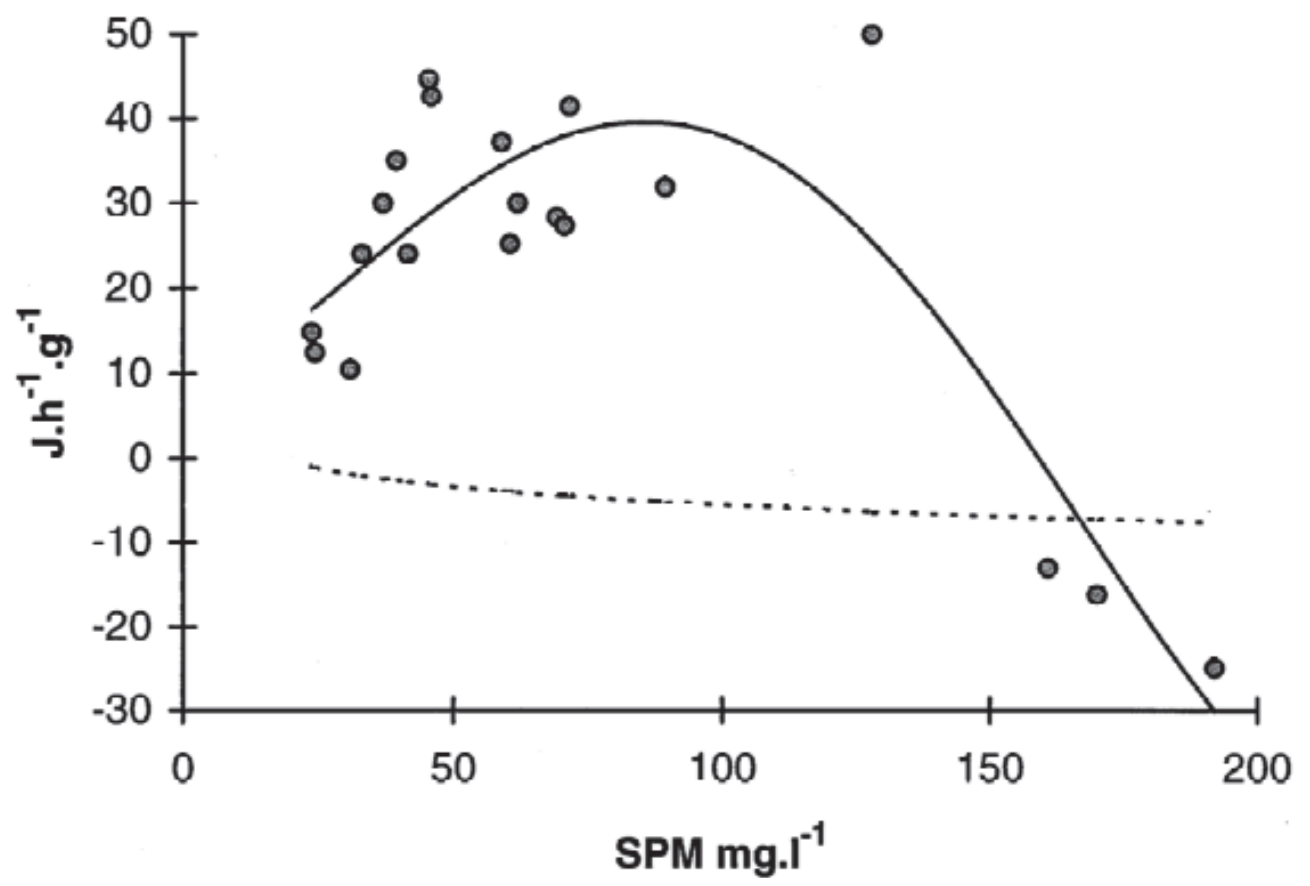

Figure 97 Scope for Growth (SFG; J/h/g ) as a function of suspended particulate matter (SPM; $m g / l$ ). SFG is compared with the expected trend without selection ability (broken line. (Barillé et al. 1997).

\section{A24.3.1.5 Japanese oyster}

The oyster $C$. gigas selectively rejects inorganic from organic particles, enriching the ingested fraction. This selection process is less efficient at high particle concentration, scope for growth (SFG) steadily decreases above $90 \mathrm{mg} / \mathrm{l}$ (Figure 97), suggesting that $C$. gigas cannot fully counteract the food dilution which causes a low food quality occurring at high seston loads (Barillé et al. 1997).

\section{A24.3.1.6 Razor clam}

Laboratory experiments were carried out with Ensis directus to estimate growth rate as a function of the amount of food and presence of silt (Kamermans et al. 2013). Maximum growth rate of $E$. directus was $0.24 \mathrm{~mm}$ increase in shell length and $2 \%$ increase in wet weight per day. Long-term (10 weeks) exposure to silt concentrations of $300 \mathrm{mg} / \mathrm{l}$ showed significantly higher growth than 
the $150 \mathrm{mg} / \mathrm{l}$ treatment. This indicates that exposure to a high silt concentration did not induce a reduction in growth, but stimulated growth (Kamermans et al. 2013). This could mean that the optimum value of SPM for growth has not yet been reached, even at $300 \mathrm{mg} / \mathrm{l}$.

\section{A24.3.2 Pseudofaeces production}

Filter feeding bivalves are able selectively to enrich the organic content of ingested matter relative to filtered matter, preferentially rejecting inorganic matter prior to ingestion as pseudofaeces

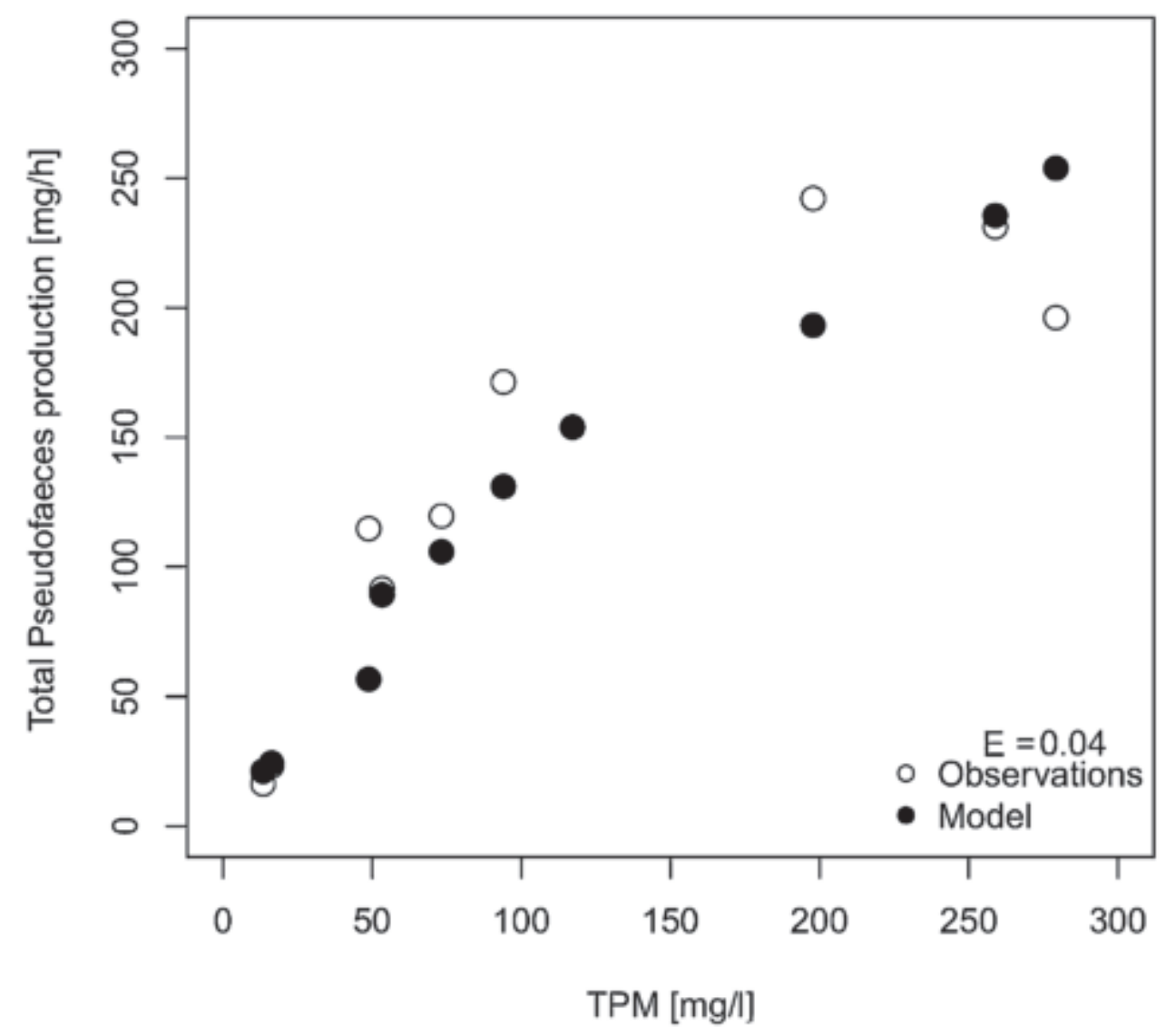

Figure 98 Pseudofaeces production: model (closed symbols) vs. observations for M. edulis by Widdows et al. (1979) (open symbols). E-value reflects the variance of the error obtained for each data point and TPM represents total particulate matter concentration in the water column (Saraiva et al. 2011)

(Hawkins et al. 1996, 1998, Zemlys et al. 2003). The composition of seston has an important effect on the response of animals to high concentration of particles (as also described further in this document) and thus also the production of pseudofaeces. With low-quality diets, resorting to pseudofaeces production would confer advantages over alternative mechanisms (i.e. clearance rate reduction), due to sorting capabilities and subsequent organic enrichment of ingested ration (Navarro et al. 1994).

Pseudofaeces production starts above a certain threshold value for SPM concentration (Widdows et al. 1979, Kiorboe et al. 1980, Navarro et al. 1992, Navarro \& Widdows 1997). Pseudofaeces production of bivalves related to SPM concentration was modelled by Saraiva et al. (2011), see Figure 98. 


\section{A24.3.2.1 Blue mussel}

It has It has been reported that Mytilus edulis is relatively tolerant of turbidity and siltation, thriving in areas that would be harmful to other suspension feeders. Mytilus edulis possesses efficient shell cleaning and pseudofaeces expulsion mechanisms to remove silt (Moore, 1977 in (Tyler-Walters 2008)), although it should be noted that pseudofaeces production involves an energetic burden (Navarro \& Widdows 1997) (Figure 99).

Based on experiments where Mytilus edulis was exposed to enhanced silt concentrations, it was concluded that the species is well adapted to silt concentrations up to $55 \mathrm{mg} / \mathrm{l}$ (Kiorboe et al. 1980), which was the maximum concentration tested. The experimental food suspension was a mixture of Phaeodactylum (10000 cells $/ \mathrm{ml}$ in all experiments) and silt. Pseudofaeces production started above a threshold silt concentration of about $1 \mathrm{mg} / \mathrm{l}$. A slight but statistically significant increase of ingestion rate with increasing concentration of silt was found (Kiorboe et al. 1980). It should be noted that this effect was observed at relatively low concentrations of silt. The amount of suspended material retained increased with increasing concentration in the range of concentrations investigated (Figure 100).

In the experiments where $M$. edulis was fed with pure algae no pseudofaeces were produced, whereas in all experiments with silt added to the suspension some of the retained material was rejected as pseudofaeces (Figure 101). Figure 100 shows the pseudofaeces production as function of material retained. The difference between the regression line and the line $X=Y$ (i.e. the difference between material retained and pseudofaeces produced) corresponds to the amount of material ingested.

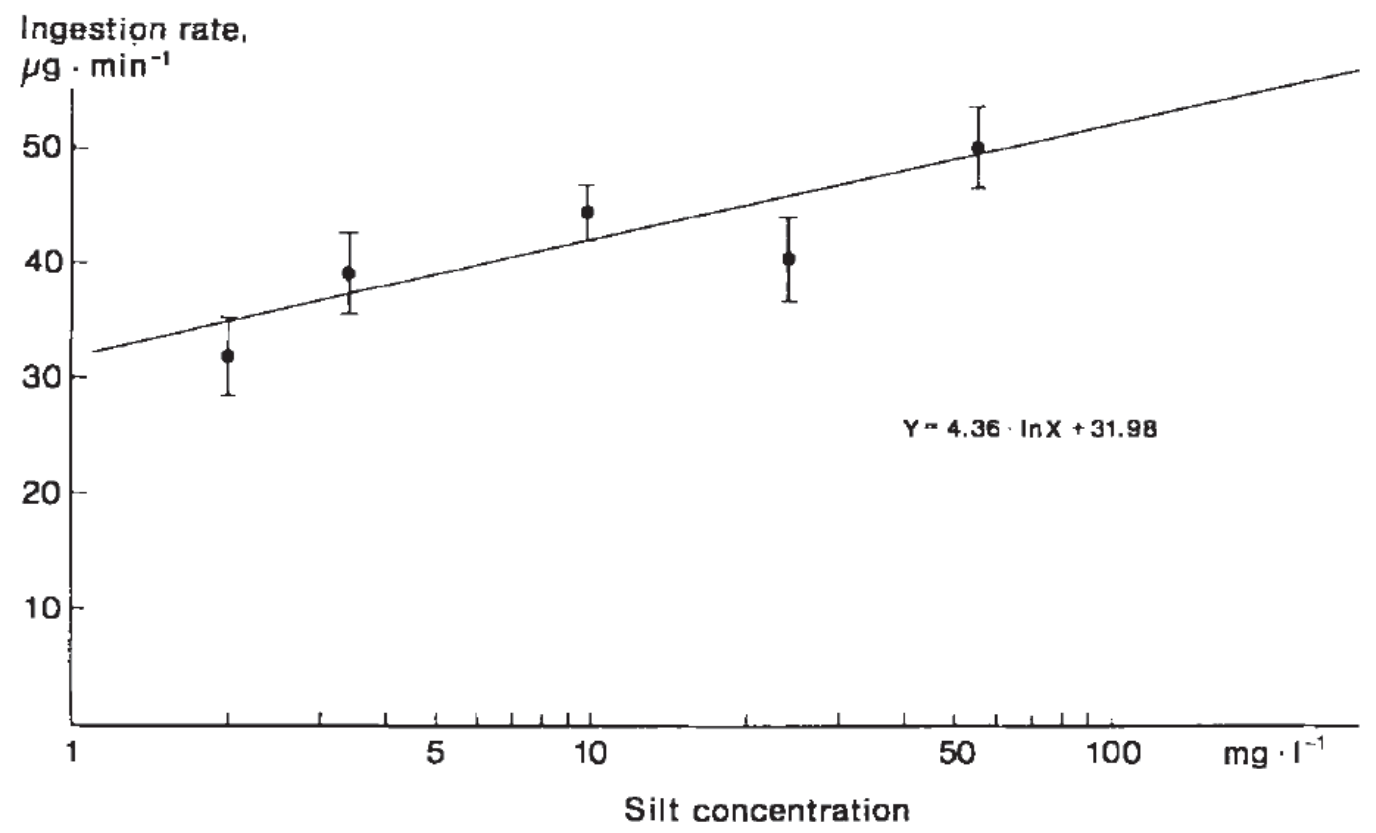

Figure 99 Ingestion rate ( $\pm 95 \%$ confidence limits) of Mytilus edulis as a function of silt concentration. The regression line is based on single measurements (Kiorboe et al. 1980) 


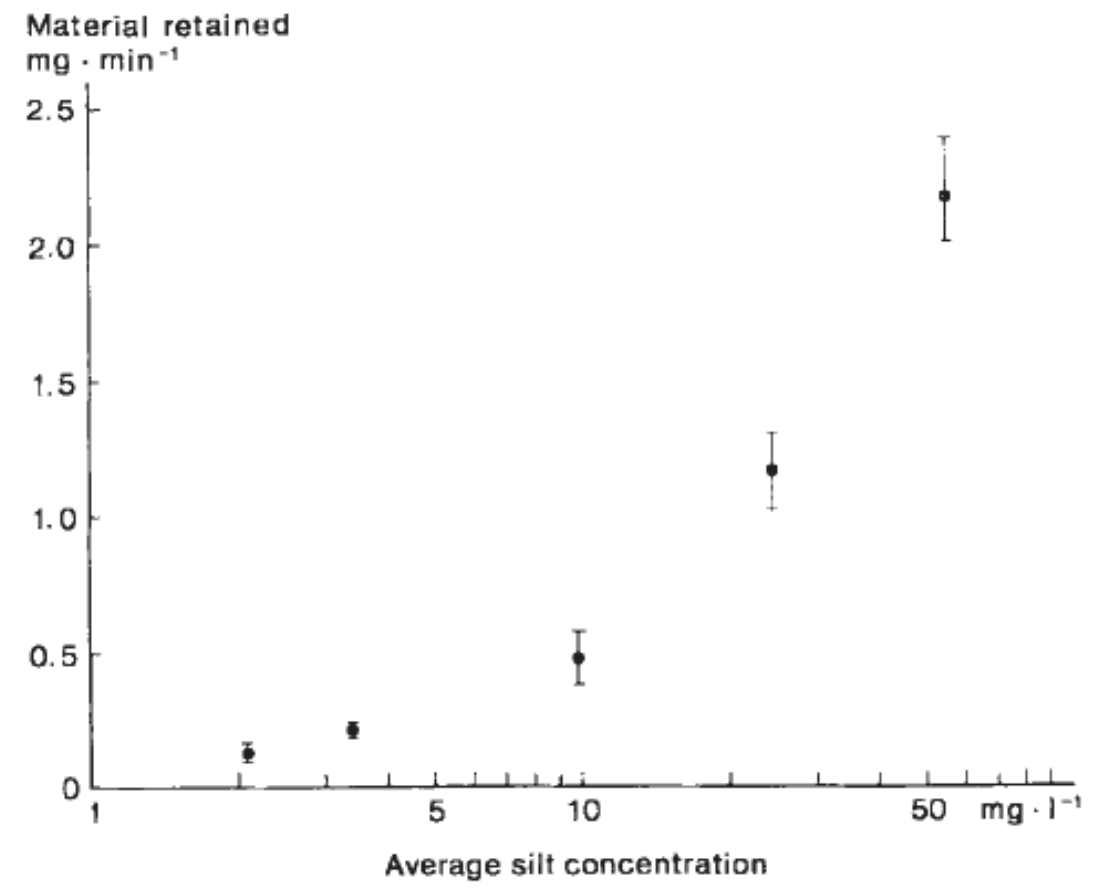

Figure 100 Dry weight of material retained per minute by Mytilus edulis as a function of silt concentration. Mean values $\pm 95 \%$ confidence limits (Kiorboe et al. 1980).

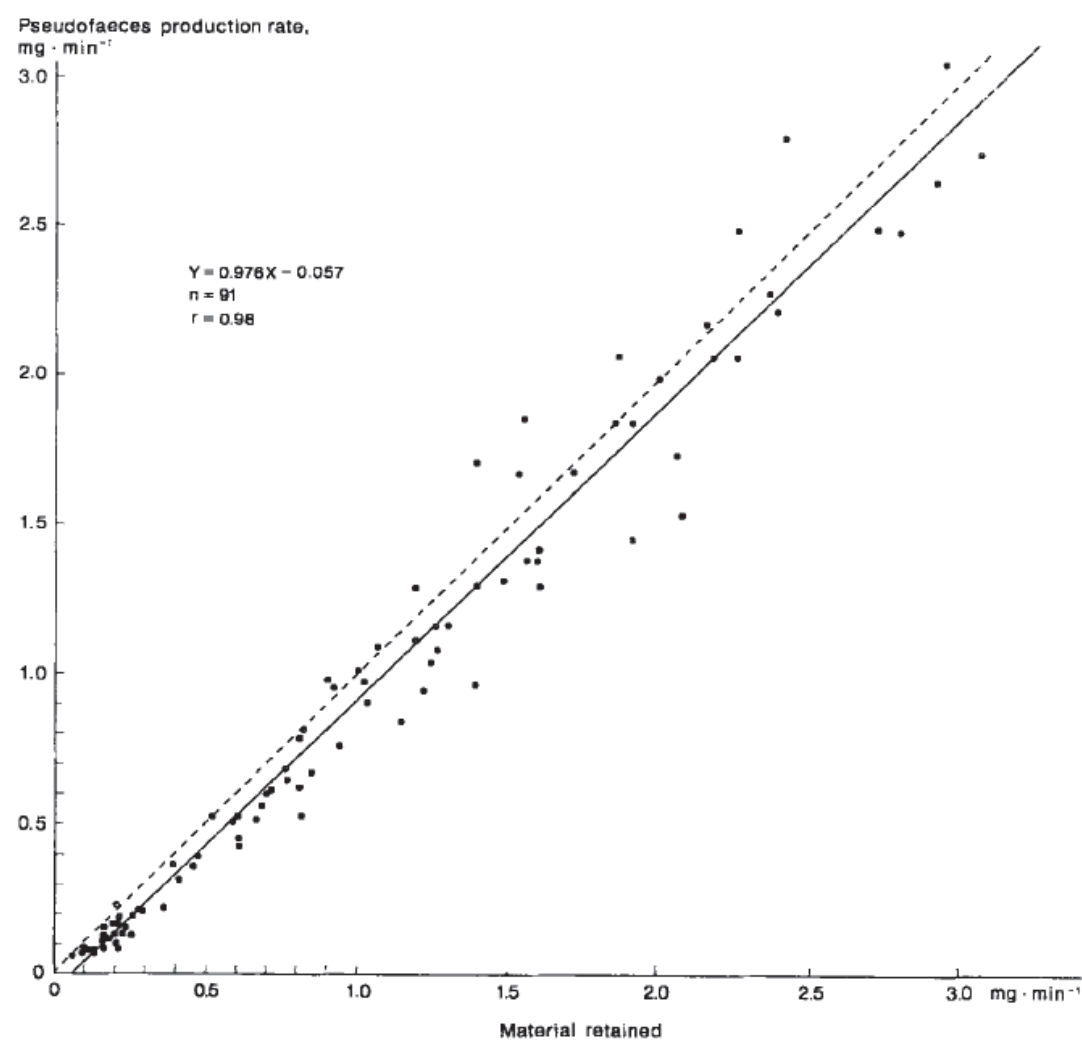

Figure 101 Pseudofaeces production by Mytilus edulis as a function of material retained. The dotted line is the line $X=Y$. The solid line is the regression line (Kiorboe et al. 1980). 
Pseudofaeces production has been determined for increasing concentrations of various types of suspension to arrive at some indication of the efficiency and the methods bivalves show in controlling ingestion (Foster-Smith 1975). For Mytilus edulis this was mainly controlled by increasing the proportions of material rejected as pseudofaeces. The minimum concentrations at which pseudofaeces was formed varied with the type of suspension (Table 19). M. edulis showed a rapid increase in the proportion of suspension rejected between concentrations of 10-100 $\times 10^{3}$ cells $/ \mathrm{ml}$ of Phaeodacty/um: thus at a concentration $100 \times 10^{3}$ cells $/ \mathrm{ml} 67.7 \pm 9.5 \%$ was rejected

Table 19 Minimum concentration causing pseudofaeces to form (Foster-Smith 1975)

\begin{tabular}{lcccc}
$\begin{array}{l}\text { Type of } \\
\text { suspension }\end{array}$ & $\begin{array}{c}\text { Size and } \\
\text { range }(\mu \mathrm{m})\end{array}$ & \multicolumn{3}{c}{$\begin{array}{c}\text { Minimum concentrations }\left(\times 10^{3} \mathrm{p} / \mathrm{ml}\right) \text { causing } \\
\text { pseudofaeces to form in } \\
\text { C. } \text { edule }\end{array}$} \\
\hline Phaeodactylum & $29 \pm 4.5$ & M. edulis & V. pullastra \\
Graphite 1 & $31.5,19-53$ & $10-20$ & $40-50$ & $50-75$ \\
Graphite 2 & $22.5,13-38.5$ & $10-20$ & - & - \\
Alumina 1 & $17.5,7.5-31.5$ & $15-25$ & - & - \\
Platymonas & $13 \pm 2.0$ & $20-25$ & $75-100$ & $100-150$ \\
Alumina 2 & $8.0,2.5-16$ & $10-20$ & - & - \\
Isochrysis & $5.3 \pm 1.5$ & $150-200$ & 250 & - \\
\hline
\end{tabular}

and at the highest concentrations used $\left(850 \times 10^{3}\right.$ cells $\left./ \mathrm{ml}\right) 90.3 \pm 7.2 \%$ was rejected (Foster-Smith 1975).

\section{A24.3.2.2 Cockle}

Cockles (Cerastoderma edule) fed with diets consisting of particles low in food value were found to regulate rates of food ingestion by keeping clearance rates nearly constant and by increasing pseudofaeces production (Iglesias et al. 1992, 1996).

According to experiments with cockles C.edule (Navarro et al. 1992), the proportion of filtered total particulate matter (TPM) which is ingested (IR/FR) $)_{T P M}$, increases as filtration rate (FR: $\mathrm{mg} / \mathrm{h}$ ) decreases, at a rate given by the expression:

$(\mathrm{IR} / \mathrm{FR})_{\mathrm{TPM}}=0.195+1.875 / \mathrm{FR}_{\mathrm{TPM}}\left(\mathrm{r}^{2}=0.935, \mathrm{p}<0.001\right)$

The critical filtration rate above which pseudofaeces production is initiated can be calculated by setting IR/FR = 1 in the equation above, amounting to $2.33 \mathrm{mg} T P M / h$, corresponding to $1.54 \mathrm{mg}$ $\mathrm{TPM} / \mathrm{I}$, that represents the threshold seston concentration for $C$. edule (Navarro et al. 1992). A different study found that pseudofaeces production for $C$. edule started at a concentration of 4.8 $\mathrm{mg} / \mathrm{l}$, showing its highest values at around $300 \mathrm{mg} / \mathrm{l}$ (Navarro \& Widdows 1997).

Pseudofaeces production for $C$. edule has been determined for increasing concentrations of various types of suspension (Foster-Smith 1975). C. edule rejected $39.5 \pm 15.5 \%$ of the Phaeodactylum filtered at the highest concentrations used. The species show a steady increase in the proportion of material rejected with increasing concentration. The minimum concentrations at which they form pseudofaeces vary with the type of suspension (Table 19). 


\section{A24.3.2.3 Peppery furrow shell}

No information was found for this species.

\section{A24.3.2.4 Japanese oyster}

The Japanese oyster Crassostrea gigas cultivated in the Bay of Marennes-Oleron, where resuspension processes have a major influence on food availability and quality, were exposed to turbidity levels from 20 to $350 \mathrm{mg} /$ I (Barillé et al. 1997). Food was diluted by the fine resuspended sediment, and organic content of particulate matter in the water column decreased from $30 \%$ to $10 \%$ with increasing seston loads. Below seston concentrations of $90 \mathrm{mg} / \mathrm{l}$ ingestion rate was regulated by pseudofaeces production. Above $90 \mathrm{mg} / \mathrm{l}$, a sharp reduction of filtration and rejection rates suggests physical constraints limiting food acquisition (Barillé et al. 1997).

The amount of matter retained by the gills of $C$. gigas and rejected as pseudofaeces (PF, $\mathrm{mg} \mathrm{h}^{-1} \mathrm{~g}$ $\left.\mathrm{DW}^{-1}\right)$ was linearly correlated with the total amount of suspended matter (TSM, $\mathrm{mg}^{-1}$ ) in seawater (Figure 102, (Lannou et al. 1992)):

$\mathrm{PF}=1.81 \mathrm{TSM}-8.35(r=0.94, n=92)$

The relationship between TSM and faeces production ( $F, m g h-1 \mathrm{~g} D W-1)$ can be described as (Figure 102, Lannou et al. 1992)):

$\mathrm{F}=-3.09+11.96\left(1-\mathrm{e}^{-0.24 \mathrm{TSM}}\right)(r=0.61, n=92)$
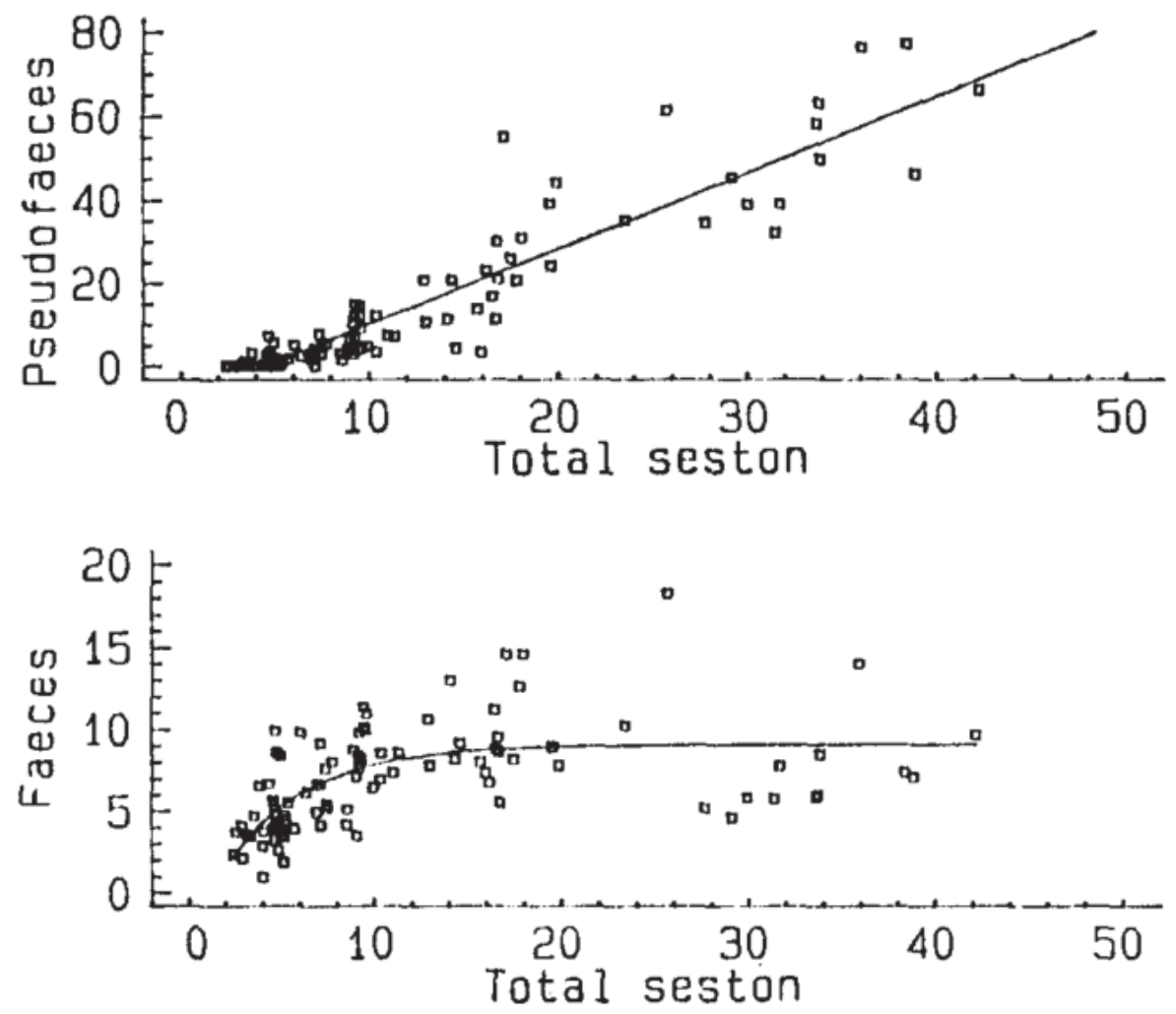

Figure 102 Effect of particle concentration ( $\mathrm{mg} / \mathrm{l})$ on pseudofaeces (above) and faeces (below) production (mg h-1 g DW-1) by Crassostrea gigas (Lannou et al. 1992).

The amount of faeces reaches a constant mean value of $8.9 \mathrm{mg} \mathrm{h}^{-1} \mathrm{~g} \mathrm{DW}^{-1}$ when seston concentration increases (Figure 102). The proportion of faeces versus pseudofaeces is over 1 when 
seston concentrations are below $10 \mathrm{mg} / \mathrm{l}$, and remains less than 1 when seston concentrations are higher than $10 \mathrm{mg} / \mathrm{l}$ (Lannou et al. 1992).

For the oyster Crassostrea gigas a direct significant relation $(P<0.05)$ was determined between the rate of total energy loss $(E, J g D W / h)$ and the rate of ejection of pseudofaeces $(P, m g g D W / h)$ :

$E=0.121 * P^{1.12}$ (Sornin et al. 1988).

\section{A24.3.2.5 Razor clam}

Based on experience with Mytilus edulis, Cerastoderma edule L. and Venerupis pullastra (FosterSmith, 1975), Kamermans et al. (2011) assumed that 2 mg dry weight (DW) $\mathrm{I}^{-1}$ represents the optimal particle concentration for Ensis directus, just below the pseudofaeces threshold (Kamermans et al. 2011). Experiments showed that pseudofaeces were produced by all size classes of $E$. directus and at all silt concentrations, ranging from 50 to $300 \mathrm{mg} / \mathrm{l}$ (Kamermans et al. 2013). Production of pseudofaeces increased with silt concentration and clam size.

\section{A24.3.3 Mortality and reproduction}

The sensitivity of $M$. edulis to increased suspended matter was assessed based on a review of literature (Mainwaring et al. 2014). It was reported that concentrations of particulates as high a $440 \mathrm{mg} / \mathrm{l}$ did not affect $M$. edulis and that mortality was only occurred when mud was added to the experiment bringing the concentrations up to $1220 \mathrm{mg} / \mathrm{l}$ (Purchon, 1937 in Mainwarring et al., 2014). It is suggested that discrepancy between studies may be due to the volume of water used in the experiment. A different study is cited by Mainwarring et al. (2014) (i.e. Loosanoff, 1962) that found that in small quantities of turbid water (due to particulates) the mussel can filter out all the particulates within a few minutes whereas in volumes $>50$ gallons per individual the mussel becomes exhausted before the turbidity has been significantly lowered, causing it to close its shell and die.

The relationship between increased suspended matter and the resulting effects on different North Sea species were described as a function of increased mortality or reduced reproduction by (Jak et al. 2000). The numerical values of the parameters in these functions were, as far as possible, estimated based on data from literature, dealing with the sensitivity of the considered species, or otherwise of related species or biota in general, for the regarded disturbance. In Table 20 estimated lethal effect fractions are presented for some species as result of exceeding a suspended matter concentration of $200 \mathrm{mg} / \mathrm{l}$ in the lower water layer. The threshold value $\mathrm{d}$ represents the maximum number of days with no effect, and value $c$ which represents the effect size (fraction) for each succeeding day. The same kind of data are also given for reproduction effects. The dose-response relationship was described using a negative linear relation with threshold, see also Figure 103 (Jak et al. 2000):

$$
\begin{aligned}
& x \leq d \Rightarrow y=1 \\
& d<x<\left(d+\left(\frac{1}{c}\right)\right) \Rightarrow y=(1-c(x-d)) \\
& x \geq\left(d+\left(\frac{1}{c}\right)\right) \Rightarrow y=0
\end{aligned}
$$

With:

$d=$ threshold, disturbance intensity at which effect will occur;

$c=$ intensity-effect coefficient, which describes the slope of the function; 
$y=$ the effect on survival/ reproduction (fraction between 0 en 1 ) and;

$\mathrm{x}=$ the disturbance intensity of the potential exposure.

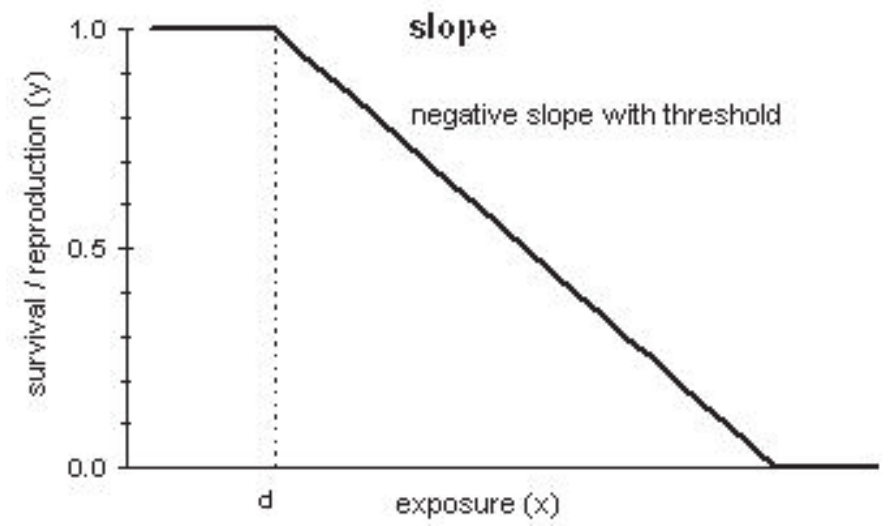

Figure 103 Generic presentation of a negative linear relation with threshold (Jak et al. 2000).

Table 20 Lethal and reproduction effect as result of exceeding a suspended matter concentration in the lower water layer of $200 \mathrm{mg} / \mathrm{l}$. The threshold value d represents the maximum number of days with no effect, and value c represents the effect size (fraction) for each succeeding day (Jak et al. 2000).

\begin{tabular}{|ccccccc|}
\hline Mortality & \multicolumn{3}{c}{ No effect } & \multicolumn{5}{c|}{ Effect size } \\
Species & d & $\min$ & $\max$ & c & $\min$ & $\max$ \\
\hline $\begin{array}{c}\text { Mussel bed } \\
\text { Cockle bed }\end{array}$ & 20 & 9.06 & 44.14 & 0.05 & 0.03 & 0.10 \\
\hline Reproduction & 10 & 3.98 & 25.11 & 0.05 & 0.02 & 0.10 \\
\hline Species & d & No effect & & & Effect size & \\
\hline $\begin{array}{c}\text { Mussel bed } \\
\text { Cockle bed }\end{array}$ & 5 & 2.55 & 9.82 & 0.1 & 0.05 & 0.20 \\
\hline
\end{tabular}

A synthesis of studies that report biological responses to known suspended sediment concentrations provides some mortality values for bivalves (Wilber \& Clarke 2001), see Figure 104. For larvae and eggs the lowest effect value reported was $400 \mathrm{mg} / \mathrm{L}$ for $12 \mathrm{~d}$ for eastern oyster larvae, whereas larval development continued normally at suspended sediment concentrations less than $750 \mathrm{mg} / \mathrm{L}$ for Japanese oysters $C$. gigas. whereas greater concentrations consistently had lethal effects at durations of $12 \mathrm{~d}$. Responses of adult bivalves to suspended sediment concentrations is presented in Figure 104. Different species are included in the figure, among which only M. edulis is relevant for this study. This involved increased growth rates in high algal concentrations after the addition of silt (Wilber \& Clarke 2001). Mortality effects included are for northern quahogs, eastern oysters and softshell clams. 

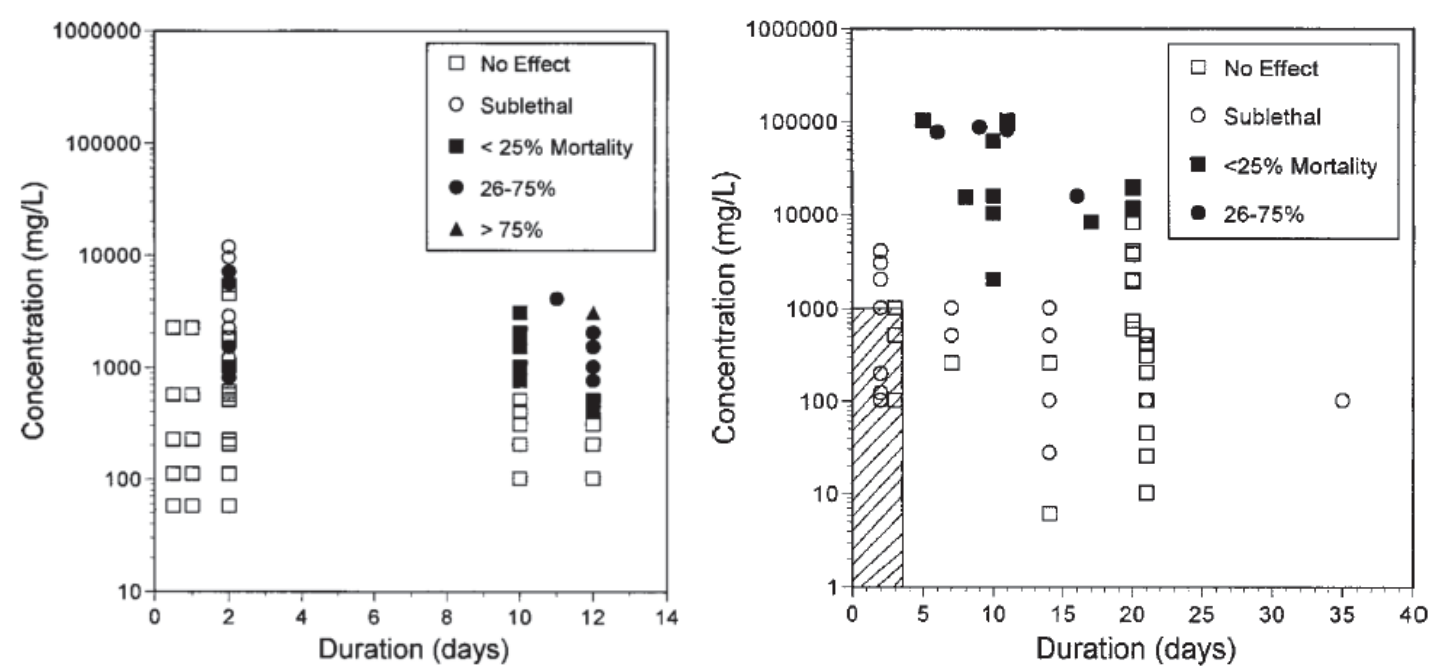

Figure 104 Responses of larval (left) and adult (right) bivalves to suspended sediment concentrations at the given exposure durations (Wilber \& Clarke 2001).

\section{A24.3.4 Oxygen demand and extraction efficiency}

The effects of particle concentration on the rate of oxygen uptake by Mytilus edulis was studied by adding fine surface sediments, collected from mudflats, to seawater (Widdows et al. 1979). There was no effect of particle concentration (seston concentration varying from 3 to $280 \mathrm{mg} / \mathrm{l}$ ) on the rate of oxygen uptake by $M$. edulis.

Oxygen uptake can also be expressed as oxygen extraction efficiency. This can be defined as the oxygen consumed per hour as a percentage of the oxygen made available to the mussel per hour by the ventilation current (Widdows et al. 1979). The relationship between changes in particle concentrations and the oxygen extraction efficiency of $M$. edulis is illustrated in Figure 105. As the ventilation rate declined with increasing particle concentration, there was a decrease in the amount of oxygen delivered to the gills per unit time. In order to maintain a constant metabolic rate independent of seston concentration, the extraction efficiency has to increase exponentially with increasing particle concentration (Widdows et al. 1979). By increasing the oxygen extraction efficiency up to $25 \%, M$. edulis has been shown to maintain a constant metabolic rate up to concentrations of at least $280 \mathrm{mg}$ seston/l (Widdows et al. 1979). Above this seston concentration the oxygen extraction efficiency is expected to increase rapidly. It is unknown whether these high extraction efficiencies can be maintained. If not, the rate of oxygen uptake must decline (Widdows et al. 1979). 


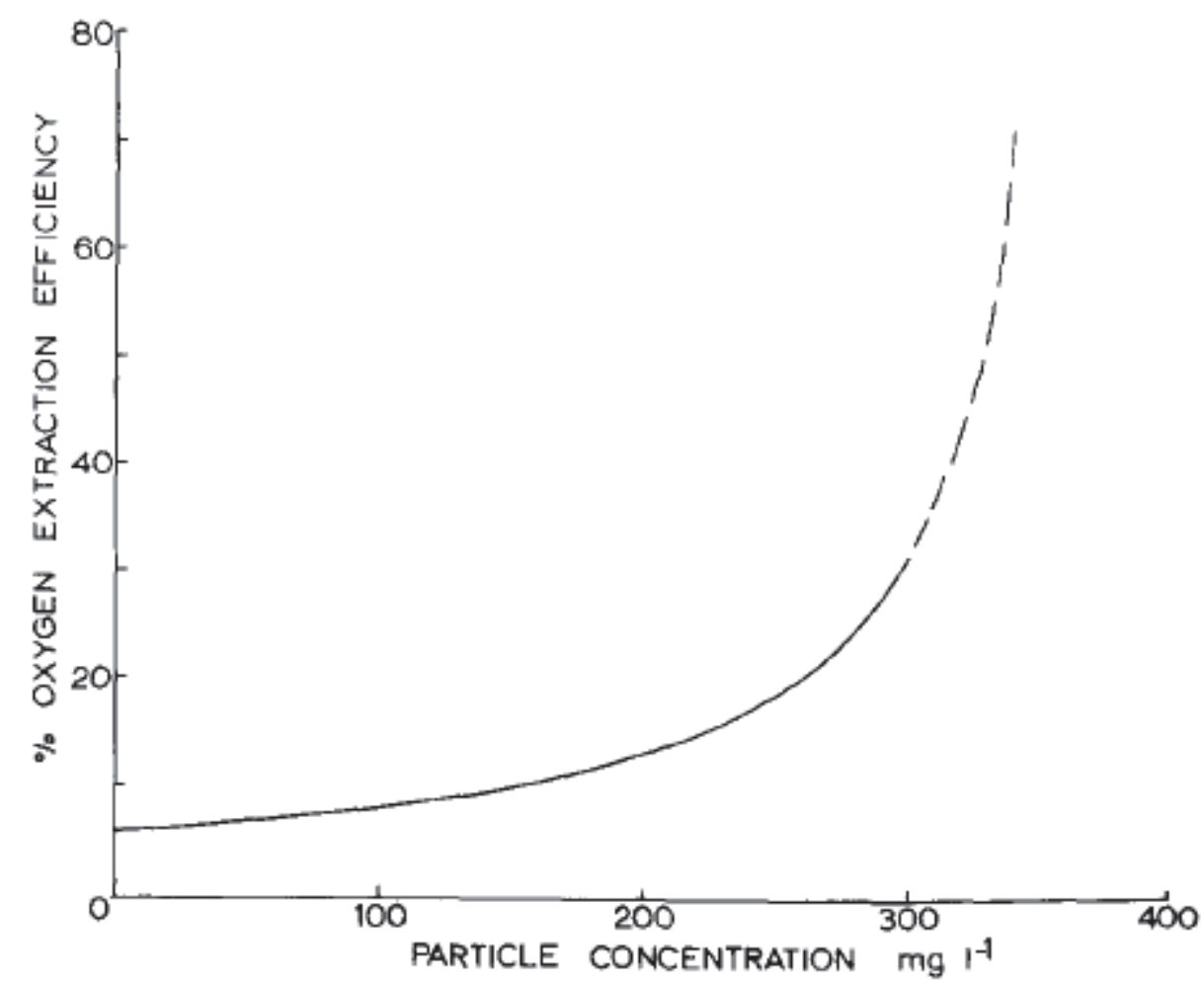

Figure 105 Relationship between oxygen extraction efficiency and particle concentration for a $7 \mathrm{~cm}$ mussel (Mytilus edulis) at $16^{\circ} \mathrm{C}$. Dashed line represents predicted efficiency of oxygen extraction if metabolic rate is maintained constant above $280 \mathrm{mg} / \mathrm{l}$ (Widdows et al. 1979).

\section{A24.3.5 Filtration-, clearance- and pumping rate}

\section{A24.3.5.1 Definitions}

Filtration rate is defined as the mass of particles cleared per unit time (e.g., $\mathrm{mg} \mathrm{h}^{-1}$ ). Clearance rate $\left(C ; \mathrm{L} \mathrm{h}^{-1}\right)$ is used as measure of water processing. The filtration rate can be calculated from the clearance rate and particle concentration $(\mathrm{mg} / \mathrm{l})$. Closely related to clearance rate is the pumping ( = ventilation) rate $\left(P ; L^{-1}\right)$ which is defined as the flow delivered by the ciliary activity of the gill (Cranford et al. 2011). Clearance and pumping rates are equal if all particles in the inhalant current are removed from suspension, i.e. 100\% retention efficiency (Widdows et al. 1979). Small particles ( $<2 \mu \mathrm{m}$ diameter) in natural waters are not effectively retained by most species, but these particles can account for a high proportion of the total suspended particulate matter. Clearance rate, therefore, is measured for a particle size range that is retained by the species with $100 \%$ efficiency (> 4 to $7 \mu \mathrm{m}$ diameter) (Cranford et al. 2011).

\section{A24.3.5.2 Normal clearance and pumping rates}

Feeding rate is influenced by current speed (i.e. reduction in feeding rate with increasing flow velocity), temperature (the viscosity/temperature relationship limits the maximum clearance rate) and salinity (disrupts normal feeding physiology). The maximum capacity for food intake is ultimately limited by the morphological constraint of a limited gut volume and the time required to digest food (Cranford et al. 2011). Feeding rate is thus also related to the size of the species. The clearance and pumping rate as a function of the size of different shellfish species found in literature are presented in Table 21. 
Table 21 Clearance and pumping rate ( $C$ and $P ; L h-1)$ as a function of the size of different shellfish species according to the allometric equation $Y=a X b$, where $Y$ is $C$ or $P$, and $X$ is dry tissue weight $(W ; g)$ or shell length (L; mm) ((Cranford et al. 2011).

\begin{tabular}{|c|c|c|c|c|c|}
\hline Species & Size & $\mathrm{T}\left({ }^{\circ} \mathrm{C}\right)$ & $\mathbf{n}$ & $\begin{array}{l}\text { Weight or length } \\
\text { relationship }\end{array}$ & Reference \\
\hline \multirow{4}{*}{$\begin{array}{l}\text { Cerastoderma } \\
\text { edule }\end{array}$} & - & $9.5-15$ & 53 & $C=2.59 \pm 0.12 W^{0.51 \pm 0.02}$ & Smaal et al. (1986) \\
\hline & $0.03-1.4 \mathrm{~g}$ & $1.4-18.5$ & 134 & $\mathrm{C}=1.44 \mathrm{~W}^{0.69}$ & Smaal et al. (1997) \\
\hline & - & - & - & $C=a W^{0.57}$ & Urrutia et al (1996) \\
\hline & $10-40 \mathrm{~cm}$ & - & 68 & $C=0.60 W^{0.51}$ & Newell and Bayne (1980) \\
\hline \multirow[t]{4}{*}{ Crassostrea gigas } & $0.1-3.0 \mathrm{~g}$ & $5-25$ & 316 & $C=3.92 \pm 0.84 W^{0.50 \pm 0.17}$ & Bougrier et al. (1995) \\
\hline & $0.02-0.4 \mathrm{~g}$ & $10-13$ & 38 & $\mathrm{C}=\mathrm{aL}^{0.57}$ & Ren et al. (2000) \\
\hline & & & & $C=0.016 L^{1.46}$ & \\
\hline & $0.05-0.81 \mathrm{~g}$ & 20 & 10 & $\mathrm{C}=2.28 \mathrm{~L}^{0.73}$ & Gerdes (1983) \\
\hline \multirow[t]{11}{*}{ Mytilus edulis } & $0.01-2.1 \mathrm{~g}$ & $10-12$ & 24 & $P=3.16 W^{0.72}$ & Jones et al. (1992); maximum \\
\hline & & & & $P=0.0037 L^{2.09}$ & \\
\hline & $0.01-2.1 \mathrm{~g}$ & $10-12$ & 24 & $\mathrm{P}=1.78 \mathrm{~W}^{0.70}$ & Jones et al. (1992); mean \\
\hline & & & & $P=0.0024 L^{2.19}$ & \\
\hline & $0.02-3.90 \mathrm{~g}$ & $0.4-19$ & 139 & $C=1.66 \pm 0.55 W^{0.57 \pm 0.17}$ & Smaal et al. (1997) \\
\hline & $45-57 \mathrm{~mm}$ & - & - & $\mathrm{C}=\mathrm{aW} \mathrm{W}^{0.45}$ & Hawkins et al. (1985) \\
\hline & $0.07-4.20 \mathrm{~g}$ & $0-15$ & 128 & $C=1.73 \pm 0.19 W^{0.41 \pm 0.08}$ & Thompson (1984) \\
\hline & $0.01-1.17 \mathrm{~g}$ & $9-15$ & 20 & $C=1.65 \pm 0.79 W^{0.61 \pm 0.20}$ & Smaal et al. (1986) \\
\hline & $0.01-2.4 \mathrm{~g}$ & 15 & 50 & $C=2.65 W^{0.38}$ & Widdows (1978) \\
\hline & $0.15-2.9 \mathrm{~g}$ & $7-21$ & 122 & $C=1.98 \pm 0.49 W^{0.45 \pm 0.24}$ & $\begin{array}{l}\text { Bayne and Widdows (1978) - } \\
\text { Lyner }\end{array}$ \\
\hline & $0.13-2.8 \mathrm{~g}$ & $10-17$ & 105 & $C=1.57 \pm 0.36 W^{0.47 \pm 0.11}$ & $\begin{array}{l}\text { Bayne and Widdows (1978) - } \\
\text { Cattewater }\end{array}$ \\
\hline
\end{tabular}

Cranford et al. (2011) reanalysed the parameters of the allometric relationships reported in eight studies of $C$ max measurements on 13 bivalve species of different sizes, and provided the following average ( \pm standard deviation $[S]$ ) relationships for dry tissue weight $(W)$ and shell length $(L)$ :

$$
\begin{aligned}
& C_{\max }\left(\mathrm{L} \mathrm{h}^{-1}\right)=6.54 \pm 2.41 W^{0.72 \pm 0.09} \\
& C_{\max }\left(\mathrm{L} \mathrm{h}^{-1}\right)=0.0036 \pm 0.10 L^{1.60 \pm 0.45}
\end{aligned}
$$

Pumping rate (filtration) and pressure to maintain flow have been extensively studied for Mytilus edulis so the power delivered by the mussel pump to the water flow is known (1.1\% of total respiratory power), whereas the actual cost based on gill respiration is much higher (19\%) (Riisgård et al. 2015).

There is a large degree of variability in individual pumping rates, as shown for Mytilus edulis in Figure 106, which includes data on the full range of measurements from mussels of different size, including the maximum rate. 


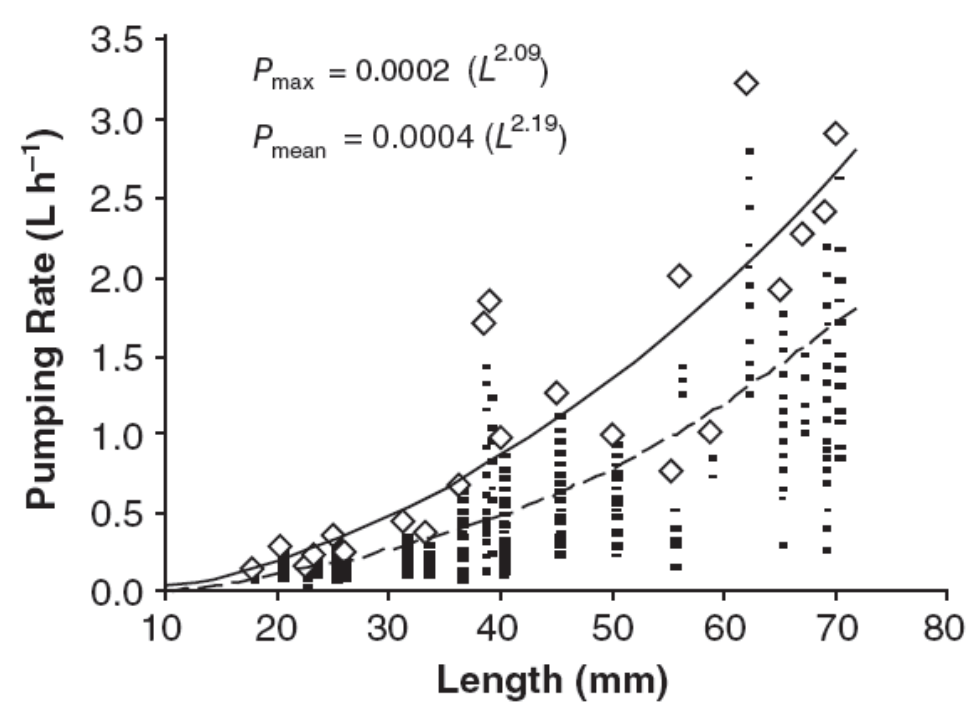

Figure 106 Allometric relationship between pumping rate $(P)$ of the mussel Mytilus edulis and shell length (L). Multiple individual measurements for each mussel are shown, including the maximum ( $P$ max; $\diamond)$. Regression lines and equations are shown for average $P$ ( $P_{\text {mean }}$; broken line) and $P$ max (solid line) values (Cranford et al. 2011).

\section{A24.3.5.3 General aspects of effects of suspended sediments}

Filter feeding bivalves are able to adjust these rates depending on the quantity and quality of the suspended particulate matter (Foster-Smith 1975, Widdows et al. 1979, Navarro et al. 1992, Prins et al. 1994, Cranford et al. 2011, Kamermans et al. 2013, Riisgård et al. 2015). Low concentrations of particulate inorganic matter already affect the food-uptake rate. This is because the filterfeeding shellfish need to invest time and energy in the processing of the inorganic matter (Wijsman et al. 2012).

The dependence of clearance rate with the concentration of total suspended particles has been described differently: (i) a constant clearance rate until a threshold concentration (saturation value), from which there is a decrease and (ii) a continuous decrease of clearance rate (Saraiva et al. 2011). Mostly the first applies: filtration rate increases from a low threshold concentration until a maximum ingestion rate, above which there is a decline in filtration rate, as observed for $M$. edulis (Widdows et al. 1979, Newell et al. 2001), C. gigas (Barillé et al. 1997) and C. edule (Navarro \& Widdows 1997). However, this pattern is not always observed, e.g. a decline in filtration rate for C. gigas with increasing seston concentration has not been found by Ren et al. (2000). For $E$. directus and S. plana there was no information found on the relationship between the clearance rate and SPM. The deposit-feeder Scrobicularia plana can feed in suspensions of Phaeodactylum up to a concentration of $10 \times 10^{10}$ cells $/ \mathrm{ml}$ without any reduction in the rate that water is filtered (Hughes, 1969 in (Foster-Smith 1975)).

For suspension feeding bivalves living in turbid areas, it is important to keep high rates of filtration and rejection to ensure an optimal food supply (Urrutia et al. 1997). It seems that M. edulis and $C$. gigas are more efficient feeders at high seston concentrations than C. edule (Foster-Smith 1975, Hawkins et al. 1998). However, C. edule was also found to effectively regulate feeding mechanisms (Iglesias et al. 1992). Cockles fed organically rich diets were found to regulate rates of food ingestion mainly by adjusting clearance rates to maintain filtration of suspended matter virtually independent from changes in particle concentration. Raising the clearance rate when increased seston concentrations lowers the quality of SPM, has an important adaptive value in helping to 
maximize energy intake (Urrutia et al. 1997). Conversely, with diets consisting of particles low in food value, clearance rates were kept nearly constant and rates of ingestion were regulated by increasing pseudofaeces production (Iglesias et al. 1992). The composition of seston has an important effect on the response of animals to high concentration of particles. When food is composed mainly of particles of high quality, there is no need of rejection to rise the organic content of ingested matter (Urrutia et al. 1997). The ability of sorting particles before ingestion and the capability of adjusting clearance rate are thus key elements in the feeding behaviour that enable bivalves to be well adapted to cope with changes in SPM.

Clearance rate of bivalves related to SPM concentration was modelled by Saraiva et al. (2011), see Figure 107.

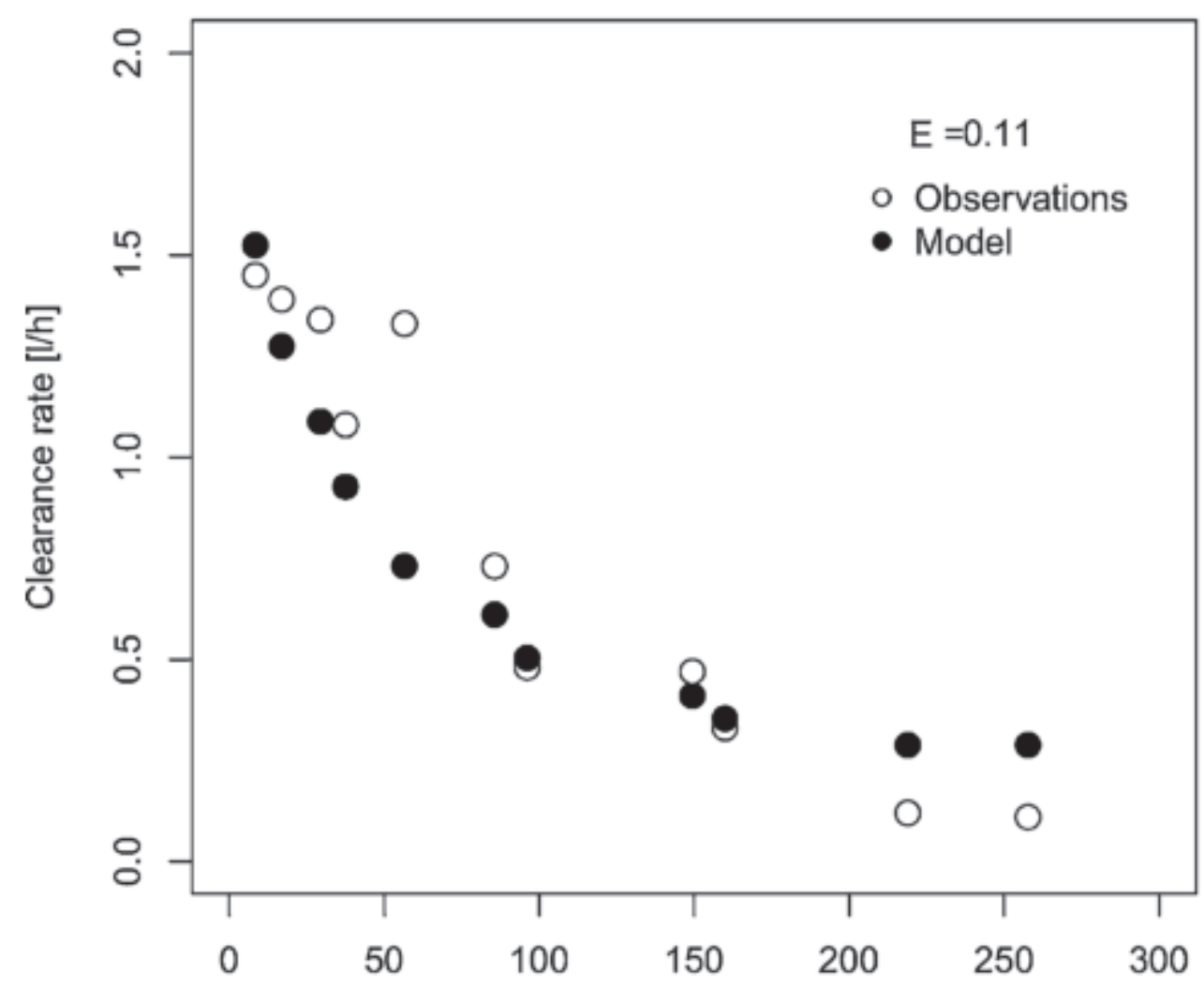

Figure 107 Clearance rate: showing model results (closed symbols) and observations for M. edulis by Widdows et al. (1979) (open symbols). E-value reflects the variance of the error obtained for each data point and TPM represents total particulate matter concentration in the water column (Saraiva et al. 2011).

Some general relations between SPM and filtration rate for different species are shown in Table 22. 
Table 22 Relationships in comparing the feeding physiology of suspension-feeding bivalve molluscs (Hawkins et al. 1998)

Relations describing combined influences of the total particulate mass of natural seston (TPM; $\mathrm{mg}^{-1}$ ), the organic content of that seston ( $\mathrm{OC}$; fraction), filtration rate $\left(\mathrm{FR}\right.$; total $\mathrm{mg} \mathrm{h}^{-1}$ ) and the organic content of ingested matter (OCI; fraction) on component processes of net organic absorption rate (NOAR; $\mathrm{mg} \mathrm{h}^{-1}$ ) in Cerastoderma edule, Crassostrea gigas and Mytilus edulis, recorded whilst feeding upon natural seston in the bay of Marennes-Oléron, France with TPM and OC of up to $90 \mathrm{mg}^{-1}$ and 0.22 , respectively

\begin{tabular}{|c|c|c|c|}
\hline Dependent variable & Equation & $r^{2}$ & Residual df \\
\hline \multicolumn{4}{|l|}{ Filtration rate } \\
\hline Cerastoderma edule & $\begin{aligned} \mathrm{FR} & =0.36( \pm 0.44) \times \mathrm{TPM}^{0.98( \pm 0.29)} \\
& \times \mathrm{OC}^{-0.63( \pm 0.55)}\end{aligned}$ & 0.46 & 125 \\
\hline Crassostrea gigas & $\mathrm{FR}=0.64( \pm 1.25) \times \mathrm{TPM}^{1.22( \pm 0.45)}$ & 0.45 & 75 \\
\hline Mytilus edulis & $\begin{aligned} \mathrm{FR} & =4.13( \pm 9.28) \times \mathrm{TPM}^{1.91( \pm 0.34)} \\
& \times \mathrm{OC}^{2.26( \pm 1.43)}\end{aligned}$ & 0.85 & 134 \\
\hline \multicolumn{4}{|l|}{ Ingestion rate } \\
\hline Cerastoderma edule & $\mathrm{IR}=6.13( \pm 1.19) \times \mathrm{TPM}^{0.19( \pm 0.10)}$ & 0.30 & 126 \\
\hline Crassostrea gigas & $\mathrm{IR}=1.07( \pm 1.37) \times \mathrm{TPM}^{0.69( \pm 0.60)}$ & 0.29 & 75 \\
\hline Mytilus edulis & $\begin{aligned} \mathrm{IR} & =18.8( \pm 39.1) \times \mathrm{TPM}^{1.33( \pm 0.30)} \\
& \times \mathrm{OC}^{3.00( \pm 1.44)}\end{aligned}$ & 0.81 & 134 \\
\hline \multicolumn{4}{|c|}{ Net organic selection efficiency } \\
\hline Cerastoderma edule & $\begin{array}{l}\text { NOSE }=0.86( \pm 0.04)-[0.050( \pm 0.004) \\
\quad \times(1 / \mathrm{OC})]+[0.000031( \pm 0.000018) \\
\quad \times \mathrm{FR} \times(1 / \mathrm{OC})]\end{array}$ & 0.48 & 125 \\
\hline Crassostrea gigas & $\begin{array}{c}\mathrm{NOSE}=-0.04( \pm 0.15)+[0.032( \pm 0.022) \\
\quad \times(1 / \mathrm{OC})]+[0.007( \pm 0.002) \times \mathrm{FR})] \\
\quad-[0.00078( \pm 0.00036) \times \mathrm{FR} \times(1 / \mathrm{OC})]\end{array}$ & 0.46 & 74 \\
\hline Mytilus edulis & $\begin{array}{l}\text { NOSE }=0.77( \pm 0.35)-[0.097( \pm 0.044) \\
\quad \times(1 / \text { OC) }]+[0.0016( \pm 0.0004) \times \text { FR })]\end{array}$ & 0.71 & 134 \\
\hline \multicolumn{4}{|c|}{ Net absorption efficiency from ingested organics } \\
\hline Cerastoderma edule & $\begin{array}{l}\text { NAEIO }=1.01( \pm 0.02)-[0.062( \pm 0.004) \\
\quad \times(1 / \mathrm{OCI})]\end{array}$ & 0.53 & 126 \\
\hline Crassostrea gigas & $\begin{array}{l}\text { NAEIO }=0.74( \pm 0.07)-[0.084( \pm 0.018) \\
\quad \times(1 / \text { OCI })]\end{array}$ & 0.52 & 75 \\
\hline Mytilus edulis & $\begin{array}{l}\text { NAEIO }=1.15( \pm 0.03)-[0.149( \pm 0.004) \\
\quad \times(1 / \text { OCI })]\end{array}$ & 0.98 & 135 \\
\hline
\end{tabular}

Note that relations were derived using different temperatures to determine OC during work for each species, with consequences as are discussed in the text. Refer to Table 1 for the full definition of all acronyms. All data for each species are standardised for individuals of $0.5 \mathrm{~g}$ dry soft tissue. Parameters are presented $\pm 95 \%$ confidence intervals. All equations are significant at $P<0.000001$. 
A meta-analysis was conducted on average clearance rate measurements extracted from 133 published studies on different species (Cranford et al. 2011), see Table 23. The average clearance rates reported in Table 23 for different bivalve species are useful for the first-order approximation of population and community clearance values. However, the authors note that, because of the very wide range of clearance rates and $b$-values reported for individual bivalve populations, it is important to measure actual clearance rates at the study site during the time(s) of year that is (are) pertinent to the specific application of the data. Seston concentration has a strong influence on bivalve clearance rate and explains a large fraction of the variance in clearance rate measurements. In areas where the nutritional quality of the seston is generally high, there is little bioenergetic benefit to regulating particle selection (pseudofaeces production), so the regulation of clearance rate with changing food abundance is the more effective strategy. Bivalves residing in more turbid regions with variable seston conditions benefit from regulating pseudofaeces production, but those species that are best suited to high turbidity also regulate clearance rate to maximize energy intake (Cranford et al. 2011). There is, however, a general lack of correlation between clearance rate and ambient environmental conditions (Cranford et al. 2011).

Table 23 Summary statistics on average ( \pm 2 SE) standardized ( $1 \mathrm{~g}$ dry tissue weight and $60 \mathrm{~mm}$ shell length or height) clearance rate values as reported in 133 papers and after standardization using average $b$-values ( $n=$ number of mean values applicable) (Cranford et al. 2011)

\begin{tabular}{|c|c|c|c|c|}
\hline & Mussel & Scallop & Oyster & Cockle \\
\hline \multicolumn{5}{|c|}{ Dry tissue weight as reported $\left(\mathrm{Lg}^{-1} \mathrm{~h}^{-1}\right)$} \\
\hline$n$ & 401 & 123 & 123 & 111 \\
\hline Median & 2.32 & 2.63 & 3.00 & 3.37 \\
\hline Mean $\pm 2 \mathrm{SE}$ & $2.98 \pm 0.23$ & $3.63 \pm 0.59$ & $3.47 \pm 0.49$ & $3.53 \pm 0.44$ \\
\hline \multicolumn{5}{|c|}{ Shell length (or height) as reported ( ind. $\left.^{-1} \mathrm{~h}^{-1}\right)$} \\
\hline$n$ & 72 & - & - & - \\
\hline Median & 3.18 & - & - & - \\
\hline Mean $\pm 2 \mathrm{SE}$ & $3.23 \pm 0.34$ & - & - & - \\
\hline \multicolumn{5}{|c|}{ Dry tissue weight using $b=0.58\left(\mathrm{Lg}^{-1} \mathrm{~h}^{-1}\right)$} \\
\hline$n$ & 357 & 172 & 68 & 90 \\
\hline Median & 2.46 & 3.21 & 2.05 & 3.57 \\
\hline Mean $\pm 2 \mathrm{SE}$ & $3.24 \pm 0.26$ & $5.10 \pm 0.76$ & $2.54 \pm 0.48$ & $3.58 \pm 0.38$ \\
\hline $\begin{array}{l}\text { Seston-based diet } \\
\text { mean } \pm 2 \text { SE }(n)\end{array}$ & $2.43 \pm 0.28(161)$ & $3.49 \pm 0.86(79)$ & $4.78 \pm 0.56(10)$ & $2.81 \pm 0.66(50)$ \\
\hline $\begin{array}{l}\text { Algae-based diet } \\
\text { mean } \pm 2 \mathrm{SE}(n)\end{array}$ & $4.18 \pm 0.41(178)$ & $6.66 \pm 1.27(79)$ & $2.15 \pm 0.49(58)$ & $4.12 \pm 0.54(61)$ \\
\hline \multicolumn{5}{|c|}{ 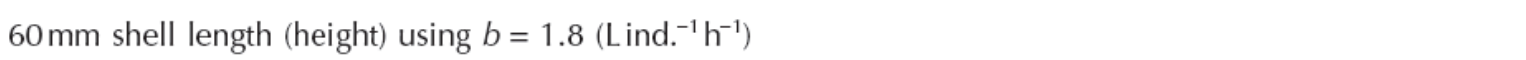 } \\
\hline$n$ & 361 & 130 & 49 & 67 \\
\hline Median & 3.26 & 5.18 & 1.09 & 5.30 \\
\hline Mean $\pm 2 \mathrm{SE}$ & $3.82 \pm 0.28$ & $7.89 \pm 1.37$ & $1.39 \pm 0.28$ & $6.03 \pm 0.81$ \\
\hline
\end{tabular}

Statistical descriptions of clearance rates responses to natural dietary conditions have been developed for several bivalve species, of which the relevant species for the Ems estuary are presented in Table 24. 
Table 24 Significant $(P<0.05)$ regressions between bivalve clearance rate $(C)$ and the total particulate matter concentration (TPM) and organic content (OC; proportion) of TPM. (Cranford et al. 2011)

\begin{tabular}{|c|c|c|c|}
\hline Species and source & Equation & Applicable range & $r^{2}$ \\
\hline \multicolumn{4}{|l|}{ Cerastoderma edule } \\
\hline Iglesias et al. (1996) & $C\left(\operatorname{Lg}^{-1} \mathrm{~h}^{-1}\right)=\mathrm{e}^{(0.3968-0.2118(\mathrm{PP}) \times \mathrm{OC})}$ & $5-22 \mathrm{mgTPML}^{-1}$ & 0.50 \\
\hline Navarro et al. (1992) & $C\left(\mathrm{Lg}^{-1} \mathrm{~h}^{-1}\right)=\mathrm{e}^{(-0.0409 \times \mathrm{Vol}+0.560)}$ & $3-30 \mathrm{mg} \mathrm{TPML}^{-1}$ & 0.66 \\
\hline \multicolumn{4}{|l|}{ Crassostrea gigas } \\
\hline Ren et al. (2000) & $\begin{array}{l}C\left(\mathrm{Lh}^{-1} \mathrm{~cm}^{-1}\right)=0.17 \times(\mathrm{TPM} /(\mathrm{TPM}+0.39)) \\
\times \mathrm{e}^{(-0.00378 \times \mathrm{TPM})}\end{array}$ & $\sim 1-650 \mathrm{mg} \mathrm{TPML}^{-1}$ & 0.79 \\
\hline \multicolumn{4}{|l|}{ Mytilus edulis } \\
\hline Hawkins et al. (1996) & $C\left(\mathrm{Lg}^{-1} \mathrm{~h}^{-1}\right)=0.60+0.039(\mathrm{TPM})$ & $5-120 \mathrm{mg} \mathrm{TPML}^{-1}$ & 0.54 \\
\hline Strohmeier et al. (2009) & $\begin{array}{l}C\left(\mathrm{Lh}^{-1}\right)=5.35-0.67(\mathrm{Chl})+0.56(\operatorname{lnChl}) \\
+0.001 /(\mathrm{Chl})\end{array}$ & $0-3 \mu \mathrm{gChl} \mathrm{L}{ }^{-1}$ & 0.34 \\
\hline
\end{tabular}

\section{A24.3.5.4 Blue mussel}

The uptake and release of material by mussels (Mytilus edulis) in Marennes-Oléron Bay (SW France) were measured in situ by Smaal \& Zurburg (1997). There was a large variation in seston concentrations, owing to high sedimentation/erosion dynamics, with maximum values up to 330 $\mathrm{mg} / \mathrm{l}$. Average clearance rates per tidal cycle ranged from 0.9 to $2.7 \mathrm{l} / \mathrm{g}$-AFDW/h throughout the season (Smaal \& Zurburg 1997).

The effects of particle concentration on filtration by Mytilus edulis was studied by adding fine surface sediments, collected from mudflats, to seawater (Widdows et al. 1979). Filtration was expressed as clearance rate. The authors found a negative correlation between clearance rate and particle concentration (

Figure 108, Table 25). Clearance rate declined with increasing particle concentration, and resulted in a minimal feeding and pumping activity at approximately 220,260 and $330 \mathrm{mg} / 1$ for mussels 3 , 5 and $7 \mathrm{~cm}$ in length, respectively (Widdows et al. 1979). 

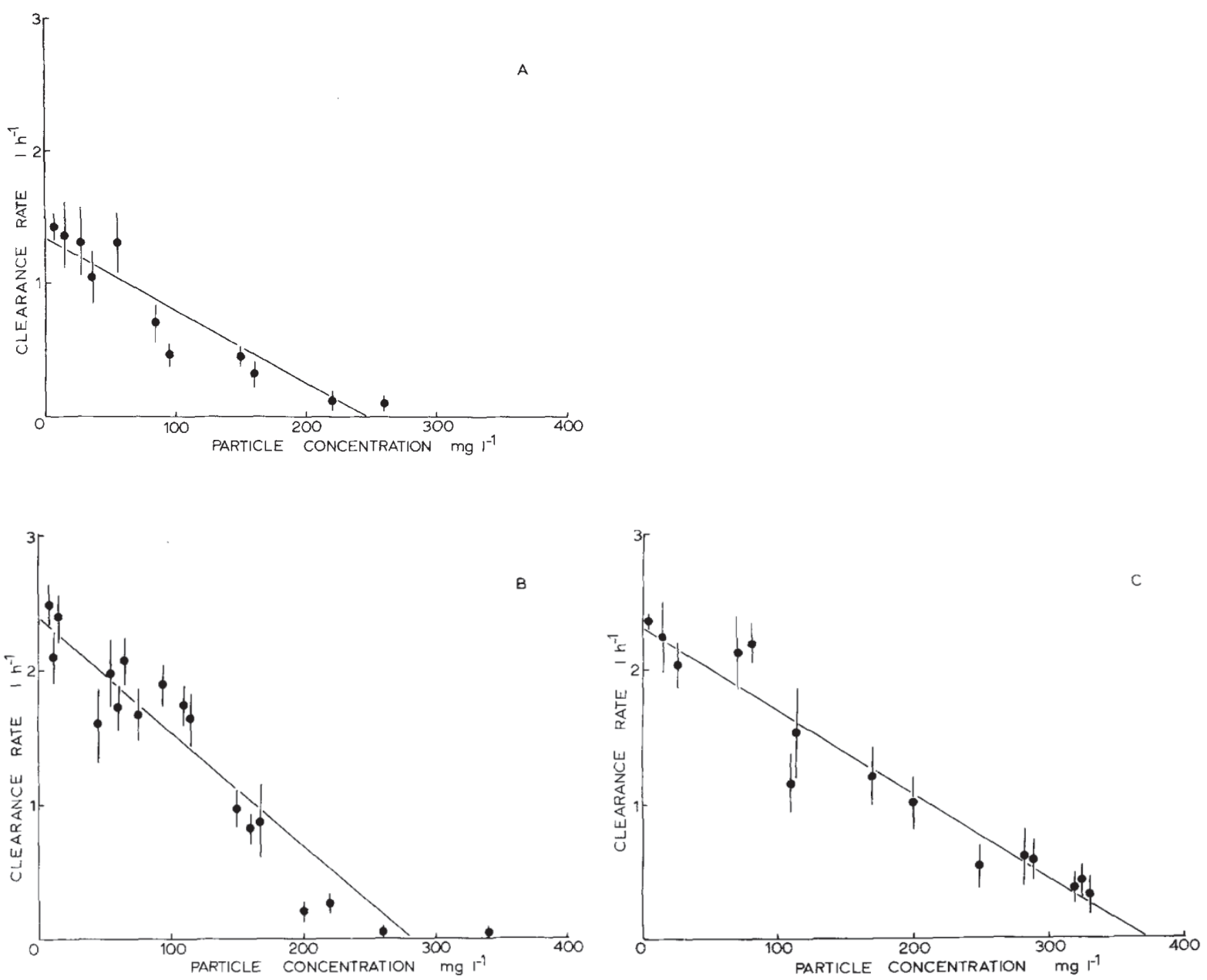

Figure 108 Mytilus edulis. Effect of particle concentration on clearance rate of (A) $3 \mathrm{~cm},(B) 5 \mathrm{~cm}$, and (C) 7 $\mathrm{cm}$ mussel. Values are means \pm standard errors (Widdows et al. 1979). Linear regression functions of these figures are presented in Table 25.

Table 25 Parameters ( $a$ and $b$ ) describing the linear relation between clearance rate and particle concentration $(W$, in $\mathrm{mg} / \mathrm{l})$ in the function clearance rate $=a-b * W$ for different sized mussels (Widdows et al. 1979)

\begin{tabular}{|c|c|c|c|}
\hline Shell length $(\mathbf{c m})$ & $\mathbf{a}(\mathrm{SD})$ & $\mathbf{b}$ & r-value \\
\hline 3 & $1.356( \pm 0.087)$ & $0.00546( \pm 0.0007)$ & -0.73 \\
\hline 5 & $2.416( \pm 0.007)$ & $0.0086( \pm 0.0005)$ & -0.85 \\
\hline 7 & $2.300( \pm 0.099)$ & $0.0061( \pm 0.0005)$ & -0.82 \\
\hline
\end{tabular}

The clearance rate of Mytilus edulis was independent of seasonal temperature (Widdows et al. 1979). The filtration rate increased with higher particle concentrations until a maximum rate was reached, after which the filtration rate declined with further increases in particle concentration, see Figure 109 (Widdows et al. 1979). 


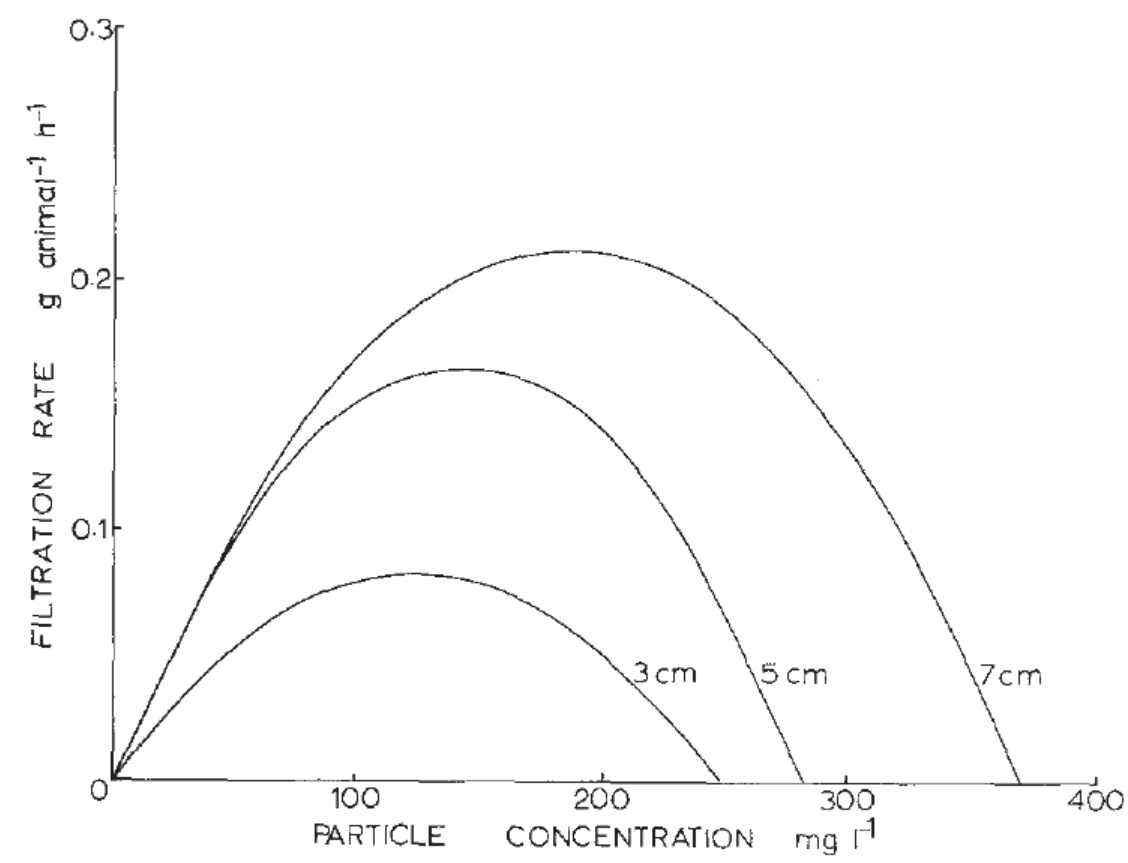

Figure 109. Effect of particle concentration ranging from 0 to $370 \mathrm{mg} / \mathrm{l}$ on filtration rate of Mytilus edulis in three size classes (3, 5 and $7 \mathrm{~cm}$ shell length) (Widdows et al. 1979).

It was observed that the net selection efficiency of $M$. edulis with which filtered organics were selectively retained for ingestion increased rapidly with the rate at which seston was filtered, this increase being faster for seston of higher organic content (Hawkins et al. 1996). The result was that the organic content of ingested matter was enriched by up to 5 times the organic content of filtered particles. Further, net absorption efficiency for ingested organics varied in strong positive relation with the organic content of ingested material. Therefore, rates of organic absorption increased with seston filtration rate, and net energy balance increased despite the decreasing organic content of particles available at higher concentrations (Hawkins et al. 1996).

\section{A24.3.5.5 Cockle}

The suspension-feeding activity of $C$. edule was investigated in response to a wide range of experimental seston concentrations (total particulate matter) which approximated the changes in seston quality and quantity found in the natural environment over tidal cycles of sediment resuspension (Navarro \& Widdows 1997). The different seston concentrations were produced by adding increasing quantities of resuspended fine sediment to a relatively low and constant algal concentration ( $0.59 \mathrm{mg}$ dry wt/l). The organic content of the diets decreased with the increasing seston concentration, from $38.8 \%$ at the lowest concentration to $14 \%$ at the highest concentration. The results suggest that $C$. edule can compensate efficiently for a decrease in seston quality over a wide range of seston concentration (1.6 to $300 \mathrm{mg} / \mathrm{l}$ ) by maintaining an effective pre-ingestive mechanism of selection for organic particulate matter, as well as increasing filtration and rejection rates (Navarro \& Widdows 1997). Consequently, this species is well adapted to living in turbid environments, such as intertidal mudflats, which are characterised by marked fluctuations in seston quality and quantity, caused by resuspension of fine sediments. 
A significant negative relationship was found between clearance rate of $C$. edule and seston concentration, with very low values at the maximum concentration of $570 \mathrm{mg} / \mathrm{l}$ of total seston (Navarro \& Widdows 1997). Filtration rate increased with seston concentration from a minimum value of $2.67 \mathrm{mg} \mathrm{h}-$-' $^{2} 1.6 \mathrm{mg} / \mathrm{l}$ to a maximum of $84.0 \mathrm{mg} \mathrm{h}$-' at a concentration of $300 \mathrm{mg} / \mathrm{l}$. Above $300 \mathrm{mg} / \mathrm{l}$ there was an abrupt decline in filtration rate.

The filtration rate for $C$. edule depending on variable seston conditions at the bay of MarennesOleron (W. France) was simulated by the function: 0.6397 TPM $^{1.197}$ total concentration of particulate matter (TPM; mg/I) (Urrutia et al. 1997). The effect of suspended natural sediment on C. edule was also studied on a population located on a tidal mud-flat in the Mundaca estuary, Spain (Navarro et al. 1992). Rates of filtration and ingestion of total particulate matter (TPM) and particulate organic matter (POM) were fitted as a function of seston concentration (Figure 110 and Figure 111, respectively).

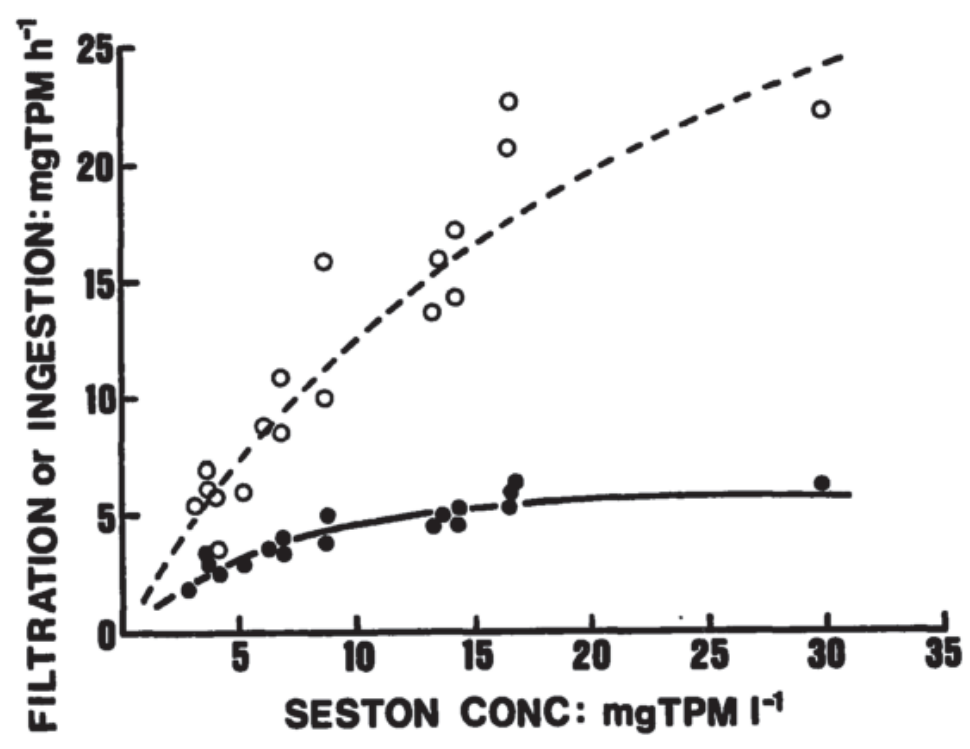

Figure 110 Rates of filtration (o) and ingestion ( $\bullet$ ) of total particulate matter (mg TPM. $h$-1) as a function of seston concentration (mg TPM. L-1) for the cockle C. edule (Navarro et al. 1992). Fitted models were (Navarro et al. 1992):

$$
\begin{aligned}
& F R=28.095 \pm 4.416\left(1-e^{-0.063 \pm 0.016 \mathrm{TPM}}\right)\left(r^{2}=0.879\right) \\
& I R=5.980 \pm 0.341\left(1-e^{-0.157 \pm 0.021 \mathrm{TPM}}\right)\left(r^{2}=0.845\right) .
\end{aligned}
$$




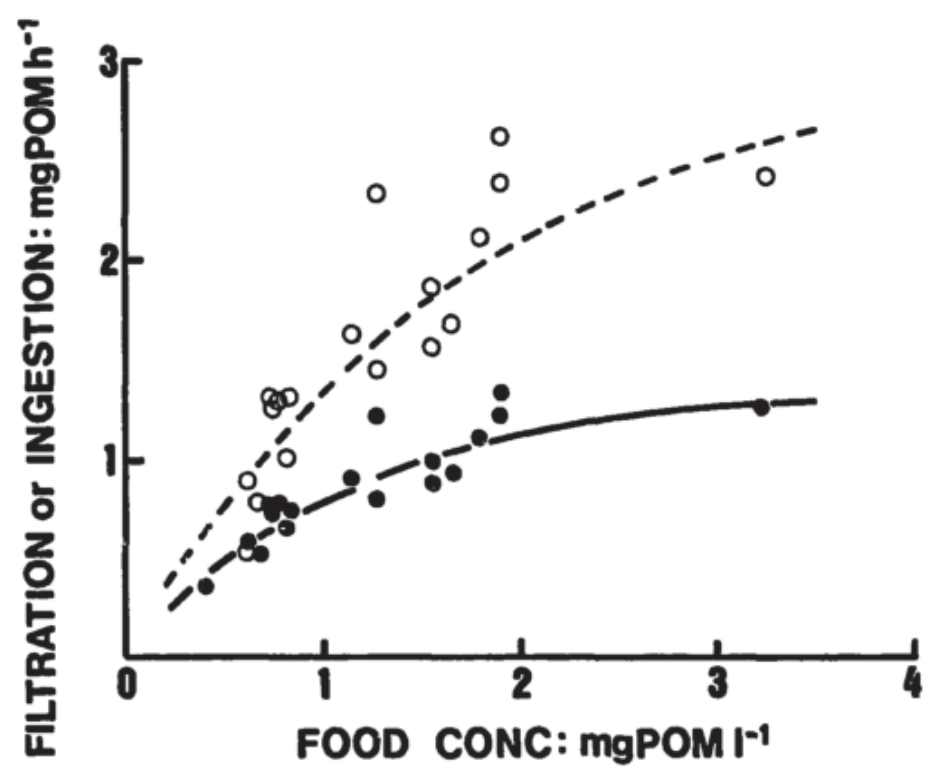

Figure 111 Rates of filtration (o) and ingestion (•) of particulate organic matter (mg POM. $h$ - 1) as a function of seston concentration (mg POM. L-1) for the cockle C. edule (Navarro et al. 1992). Fitted models were (Navarro et al. 1992):

$$
\begin{aligned}
& F R=3.013 \pm 0.523\left(1-e^{-0.639 \pm 0.186 \text { POM }}\right)\left(r^{2}=0.765\right) \\
& I R=1.386 \pm 0.142\left(1-e^{-0.892 \pm 0.180 ~ P O M}\right)\left(r^{2}=0.781\right)
\end{aligned}
$$

\section{A24.3.5.6 Peppery furrow shell}

No information was found for this species.

\section{A24.3.5.7 Japanese oyster}

The uptake and release of material by oysters (Crassostrea gigas) in Marennes-Oléron Bay (SW France) were measured in situ by Smaal \& Zurburg (1997). There was a large variation in seston concentrations, owing to high sedimentation/erosion dynamics, with maximum values up to 330 $\mathrm{mg} / \mathrm{l}$. Average clearance rates per tidal cycle ranged from 4 to $7 \mathrm{l} / \mathrm{g}-A F D W / \mathrm{h}$ throughout the season (Smaal \& Zurburg 1997).

The filtration rate for $C$. gigas was described by Ren et al. (2000) as:

$\mathrm{FR}=\mathrm{FR}_{\max } \times \mathrm{S} /\left(\mathrm{S}+\mathrm{S}_{\text {HHF }}\right)$,

where $F R(\mathrm{mg} / \mathrm{h} / \mathrm{cm})$ is for a standard $1 \mathrm{~cm}$ oyster, $F R_{\max }(\mathrm{mg} / \mathrm{h} / \mathrm{cm})$ is the maximum $F R$ and $S_{\text {HFF }}$ $(\mathrm{mg} / \mathrm{l})$ is the half-saturation $\mathrm{S}$ (half the concentration at which maximum filtration occurs). The best-fit parameters were estimated to be: $\mathrm{FR}_{\max }=16.5(\mathrm{mg} / \mathrm{h} / \mathrm{cm})$ and $\mathrm{S}_{\text {HFF }}=51.5(\mathrm{mg} / \mathrm{h} / \mathrm{cm})(\mathrm{n}=$ $\left.280, R^{2}=0.79\right)($ Ren et al. 2000).

In field experiments at high seston (mixtures of silt and phytoplankton) concentrations (64.4 mg/l) oysters (C. gigas) could only capture particles $>12 \mu \mathrm{m}$ at $100 \%$ efficiency (Barillé et al. 1993). Filtration and rejection rates of $C$. gigas declines above SPM concentration of $90 \mathrm{mg} / \mathrm{l}$ (Barillé et al. 1997), see Figure 112. Note that the ingestion rate plotted with the data slightly decreases with increasing seston concentrations (Barillé et al. 1997). Clearance rates of $C$. gigas in New Zealand, 
where seston concentrations typically range from 5 to $15 \mathrm{mg} / \mathrm{l}$, increased rapidly with increasing seston concentration, peaked at about $10 \mathrm{mg} / \mathrm{l}$, above which it consistently decreased (Ren et al. 2000). Other studies have found the clearance rate of $C$. gigas to be independent of seston concentrations under $50 \mathrm{mg} / \mathrm{l}$, while above this level, an oyster will reduce its pumping rate with increasing particulate concentration (Rena \& Ross 2001). Very high levels of particulate concentration will result in the clogging of oyster's pallial organs. The clogging level was found to be $196 \mathrm{mg} / \mathrm{l}$, after which the clearance declined dramatically (Barille and Prou, 1993 in Rena \& Ross, 2001). For the development of a Dynamic Energy Budget (DEB) model of $C$. gigas, the clearance rate was described as a continuous function of seston concentration (Rena \& Ross 2001):

$\mathrm{CR}=\mathrm{CR} /\left\{1+\exp \left[\left(\mathrm{S}-\mathrm{S}_{\mathrm{CR}}\right) / \mathrm{S}_{\beta}\right]\right\}$,

where $S$ is the seston concentration, $S_{C R}$ is the clogging level (parameterised at $200 \mathrm{mg} / \mathrm{l}$ ) and $S_{\beta}$ is a coefficient determining the rate of decrease in clearance rate (parameterised at $18.6 \mathrm{mg} / \mathrm{l}$ ) (Rena \& Ross 2001).

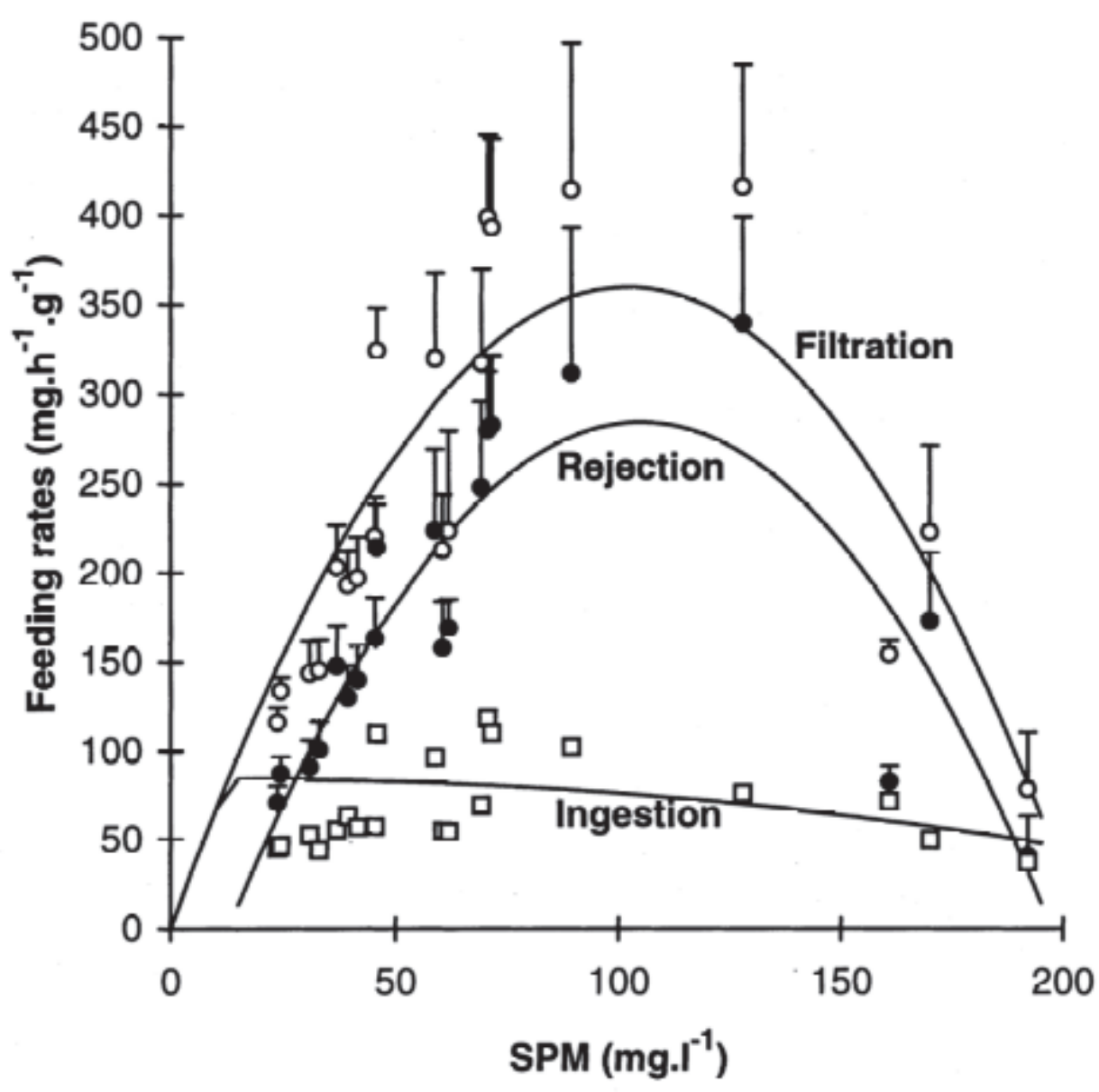

Figure 112 Rates of filtration of C gigas (FR; $\mathrm{mg} / \mathrm{h} / \mathrm{g},(\mathrm{o}))$ rejection $(R R ; \mathrm{mg} / \mathrm{h} / \mathrm{g},(\bullet))$, ingestion $(I R ; \mathrm{mg} / \mathrm{h} / \mathrm{g}$, ()) as a function of suspended particulate matter concentration (SPM; $m g / l)$ for individuals. Data are means +95\% CL (n=6)(Barillé et al. 1997). 


\section{A24.3.5.8 Razor clam}

Laboratory experiments were carried out with Ensis directus to estimate clearance rate as a function of the amount of food and presence of silt (Kamermans et al. 2013). Clearance rates of $E$. directus varied between 0.7 and $5.9 \mathrm{l} / \mathrm{h} / \mathrm{g}$ DW. At a silt concentration of $300 \mathrm{mg} / \mathrm{l}$ clearance rates were significantly lower (16-56\%) than rates at $150 \mathrm{mg} / \mathrm{l}, 50 \mathrm{mg} / \mathrm{l}$ and $0 \mathrm{mg} / \mathrm{l}$ silt. This indicates that only the highest silt concentration induced a reduction in clearance rate. The tested food levels did not influence the clearance rate of E. directus (Kamermans et al. 2013).

\section{A24.4 Synthesis}

Below the quantitative relations between SPM concentrations and filter feeding of Blue mussel (Mytilus edulis), Cockle (Cerastoderma edule), Peppery furrow shell (Scrobicularia plana), Japanese oyster (Crassostrea gigas) and Razor clam (Ensis directus) are summarised.

\section{A24.4.1 Effect of silt concentrations}

Effect values found in literature are presented in Table 26. Effect concentrations for reduced clearance rate of $E$. directus were found in literature: at a silt concentration of $300 \mathrm{mg} / \mathrm{l}$ clearance rates were significantly lower (16-56\%), at lower concentrations (i.e. $\leq 150 \mathrm{mg} / \mathrm{l}$ ) no effects were observed (Kamermans et al. 2013). Clearance rate declined with increasing particle concentration, and resulted in a minimal feeding and pumping activity at approximately 220,260 and $330 \mathrm{mg} / 1$ for mussels (M. edulis) 3, 5 and $7 \mathrm{~cm}$ in length, respectively (Widdows et al. 1979). The threshold seston concentration for $C$. edule above which pseudofaeces production is initiated is $1.54 \mathrm{mg}$ TPM/I (Navarro et al. 1992) and for M. edulis $1 \mathrm{mg} / \mathrm{l}$ (Kiorboe et al. 1980). Above $300 \mathrm{mg} / \mathrm{l}$ there was an abrupt decline in filtration rate of $C$. edule (Navarro \& Widdows 1997). Filtration and rejection rates of $C$. gigas declined above SPM concentration of appr. $110 \mathrm{mg} / \mathrm{l}$ (Barillé et al. 1997). Oxygen uptake of $M$. edulis was not affected at the highest SPM concentration of $280 \mathrm{mg} / \mathrm{l}$ (Widdows et al. 1979). Scope for growth of $C$. gigas decreases at concentrations above $90 \mathrm{mg} / \mathrm{l}$ (Barillé et al. 1997).

\section{A24.4.2 Pseudofaeces production}

A quantified relationship between suspended sediment concentration and pseudofaeces production was described for the Japanese Oyster. The amount of matter retained by the gills of $C$. gigas and rejected as pseudofaeces ( $\mathrm{PF}, \mathrm{mg} \mathrm{h}^{-1} \mathrm{~g} \mathrm{DW}^{-1}$ ) was linearly correlated with the total amount of suspended matter (TSM, mg I-1) in seawater (Lannou et al. 1992)): PF = 1.81 TSM -8.35 $(r=0.94, n=92)$. 
Table 26 Effect values of suspended matter concentrations

\begin{tabular}{|c|c|c|c|c|}
\hline Parameter & Effect & $\begin{array}{l}\text { Conc. } \\
\text { (mg/l) }\end{array}$ & Species & Reference \\
\hline \multirow[t]{5}{*}{ Clearance rate } & $16-56 \%$ reduction & 300 & E. directus & Kamermans et al. (2013) \\
\hline & No effect & 150 & E. directus & Kamermans et al. (2013) \\
\hline & Minimum rate & 220 & M. edulis (3 cm length) & Widdows et al. (1979) \\
\hline & Minimum rate & 260 & M. edulis (5 cm length) & Widdows et al. (1979) \\
\hline & Minimum rate & 330 & M. edulis (7 cm length) & Widdows et al. (1979) \\
\hline \multirow{8}{*}{$\begin{array}{l}\text { Pseudofaeces } \\
\text { production }\end{array}$} & Threshold & 1.54 & C. edule & Navarro et al. (1992) \\
\hline & Threshold & 4.8 & C. edule & Navarro \& Widdows (1997) \\
\hline & Maximum & 300 & C. edule & Navarro \& Widdows (1997) \\
\hline & Threshold & 1 & M. edulis & Kiorboe et al. (1980) \\
\hline & Threshold & $4.5-5.0$ & M. edulis (> $5 \mathrm{~cm}$ length) & Widdows et al. (1979) \\
\hline & Maximum & 200 & M. edulis & Widdows et al. (1979) \\
\hline & Decrease & $>200$ & M. edulis & Widdows et al. (1979) \\
\hline & \multicolumn{2}{|c|}{ Threshold (assumed) >2 } & E. directus & Kamermans et al. (2011) \\
\hline Oxygen uptake & No effect & 280 & M. edulis & Widdows et al. (1979) \\
\hline \multirow[t]{3}{*}{ Filtration rate } & Decrease & $>300$ & C. edule & Navarro \& Widdows (1997) \\
\hline & Decrease & $>90$ & C. gigas & Barillé et al. (1997) \\
\hline & Decrease & $>120$ & M. edulis & Widdows et al. (1979) \\
\hline Scope for growth & Decrease & $>90$ & C. gigas & Barillé et al. (1997) \\
\hline \multirow[t]{2}{*}{ Mortality } & Observed effect & 1220 & M. edulis & $\begin{array}{l}\text { Purchon (1937) in } \\
\text { Mainwarring et al. (2014) }\end{array}$ \\
\hline & No effect & $<750$ & C. gigas (larvae) & Wilber \& Clarke (2001) \\
\hline
\end{tabular}

Table 27 Filtration rate ( $F R$ ) in relation to the total particulate mass of natural seston (TPM; $m g / l)$ and the organic content of that seston (OC; fraction)

\begin{tabular}{|c|c|c|}
\hline Species & Relationship & Reference \\
\hline \multirow[t]{3}{*}{ C. edule } & $F R=0.36( \pm 0.44) \times T^{T P M}{ }^{0.98( \pm 0.29)} \times \mathrm{OC}^{-0.63( \pm 0.55)}$ & Hawkins et al. (1998 \\
\hline & $F R=28.095 \pm 4.416\left(1-e^{-0.063 \pm 0.016 ~ T P M}\right)$ & Navarro et al. (1992) \\
\hline & $F R=0.6397 \times T^{1} M^{1.197}$ & Urrutia et al. (1997) \\
\hline \multirow[t]{3}{*}{ C. gigas } & $\mathrm{FR}=0.64( \pm 1.25) \times \mathrm{TPM}^{1.22( \pm 0.45)}$ & Hawkins et al. (1998) \\
\hline & $F R=16.5 \times \mathrm{TPM} /(\mathrm{TPM}+51.5)$ & Ren et al. (2000) \\
\hline & $F R=0.73( \pm 0.29 S D) S P M^{1.16( \pm 0.08 ~ S D)}$ & Barillé et al. (1997) \\
\hline M. edulis & $\mathrm{FR}=4.13( \pm 9.28) \times \mathrm{TPM}^{1.91( \pm 0.34)} \times \mathrm{OC}^{2.26( \pm 1.43)}$ & Hawkins et al. (1998) \\
\hline
\end{tabular}

\section{A24.4.3 Filtration rates}

Relationships between suspended sediment concentration and filtration rates found in literature are presented in Table 27. 


\section{A24.5 Summary and conclusions}

The available knowledge on the effect of suspended sediments on filter feeding bivalves is mainly focussed on blue mussel (Mytilus edulis), cockle (Cerastoderma edule), Japanese oyster (Crassostrea gigas). Less information is available for razor clam (Ensis directus) and hardly any information is available for peppery furrow shell (Scrobicularia plana), Based on available sensitivity assessments in literature the sensitivity of $S$. plana is not expected to be very different to that of the other species.

\section{A24.5.1 Mechanism of filter feeding of bivalves}

The bivalve species use their gills and palps for feeding and rely on ciliated structures to capture and transport suspended particulate matter for selection and ingestion. A water current is produced which displaces water within the shells and replaces it with water from outside the shells (i.e. the pump). Particles from the water are captured by the gills and edible parts are roughly selected. It is suggested that particles larger than $4 \mu \mathrm{m}$ are withheld. The digestible parts of the SPM such as phytoplankton, bacteria and decomposable detritus are transported to the palps whereas non-edible parts are secreted as pseudofaeces.

\section{A24.5.2 Effect of suspended sediment}

- Bivalves are generally silt-tolerant organisms that survive in naturally silty areas.

- Potential effects of increased suspended matter concentrations in the water column on bivalves are:

- Decreased food intake (clearance rate, filtration rate)

- Increase of pseudo faces

- Decreased ventilation rate

- Higher respiration energy demand (respiration, assimilation)

- Growth inhibition

- Mortality

- Effects of increased SPM mostly follow an optimum curve relationship implying that adverse effects may only occur when exceeding optimum values.

- Many bivalve species can adjust their gills and palps, i.e. their ctenidial morphology, to cope with high levels of suspended matter.

- Quantitative effect values were found for M. edulis, C. edule, C. gigas and E. directus. However, values are not complete for all effects and species. No effect values were found for S. plana.

- Quantitative relationships between SPM and pseudofaeces production was only found for C. gigas. Quantitative relationships between SPM and filtrated rates were found for $M$. edulis, C. edule and C. gigas. No quantitative relationships were found for E. directus and $S$. plana. 


\section{A25 Tuning the model}

\section{A25.1 Chloride distribution, dispersion settings and model results}

\section{A25.1.1 Time series results}

In this appendix, a complete overview of the modelled chloride concentrations is presented. It reflects the success (and sometimes failure) of the underlying transport description of the EmsDollard model. For compartment names and numbers, the reader is referred to Table 2.

First, timeseries are shown, and at the end an averaged system pattern.
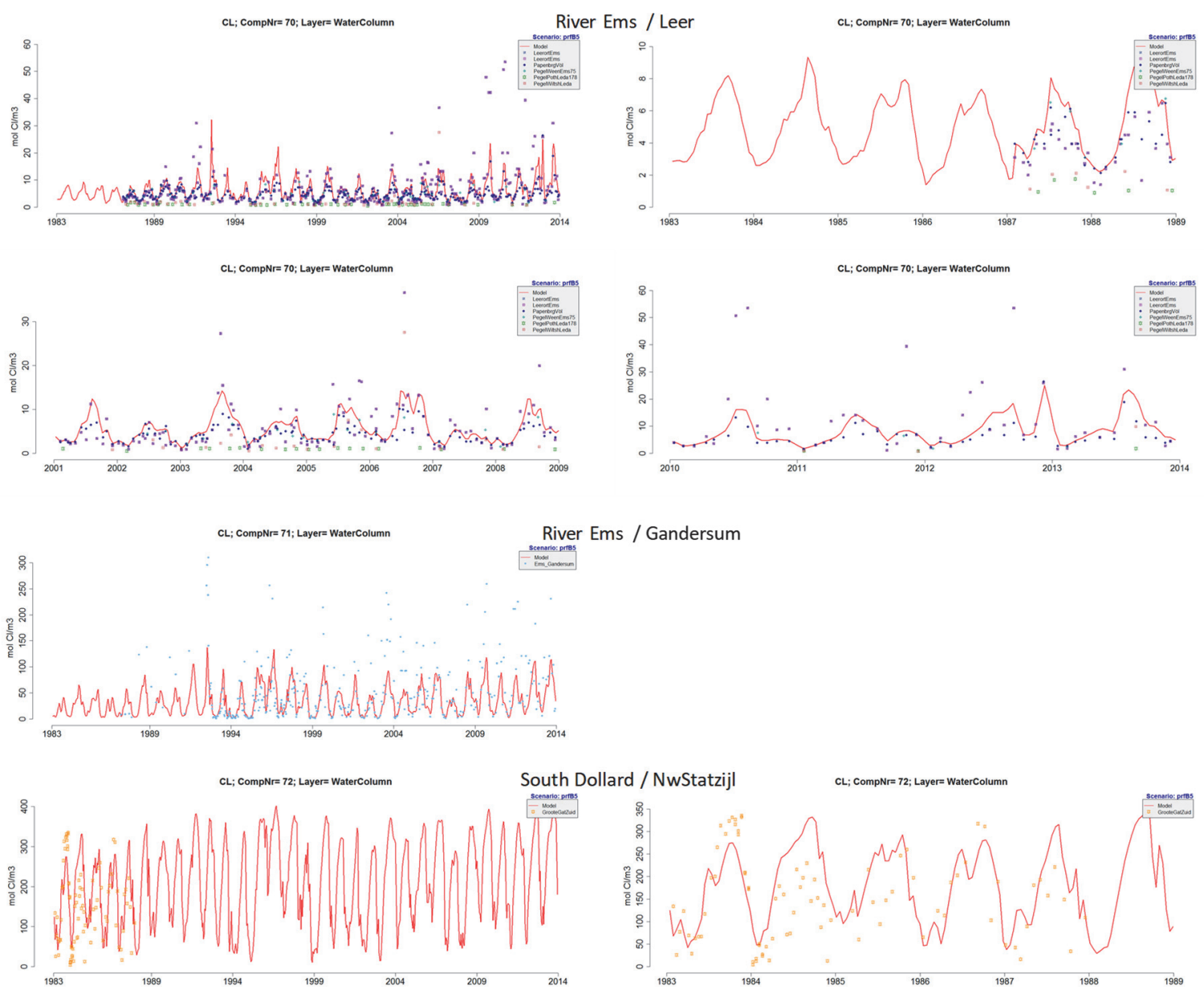

Figure 113 Chloride concentrations (modelled: lines, and data: dots) in the Ems river at Leer (upper four graphs) and Gandersum and (lower graphs) in the Dollard close to Nieuw Statenzijl. For compartment numbers see Figure 38, Table 2. 

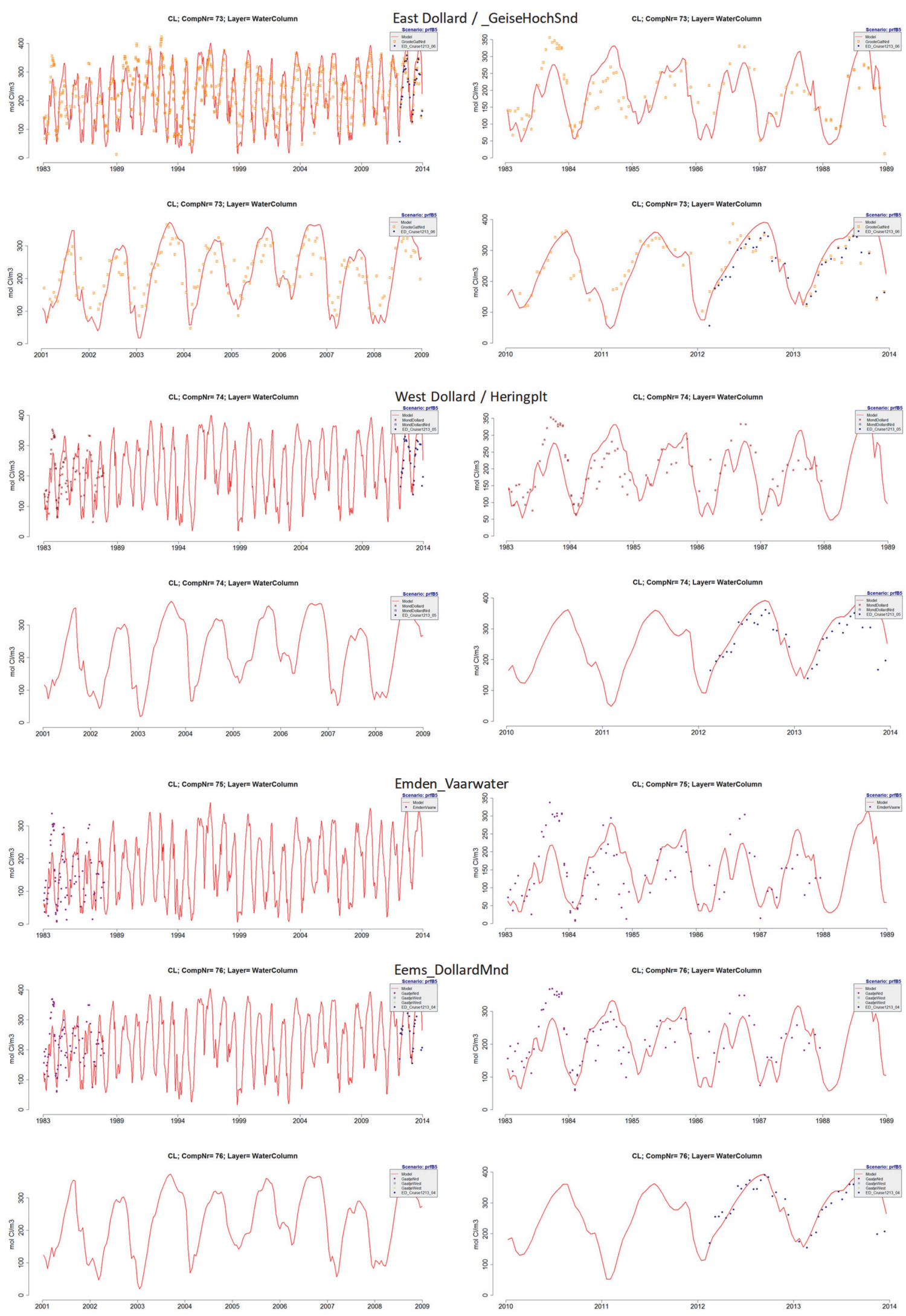

Figure 114 Chloride concentrations (modelled: lines, and data: dots) in the eastern part of the Dollard (upper four graphs), the western Dollard (next four graphs), the last part of the Ems river entering the estuary and the so-called Dollard-mouth (compnr 76, that is main compartment 107). For compartment numbers see, Figure 38, Table 2. 

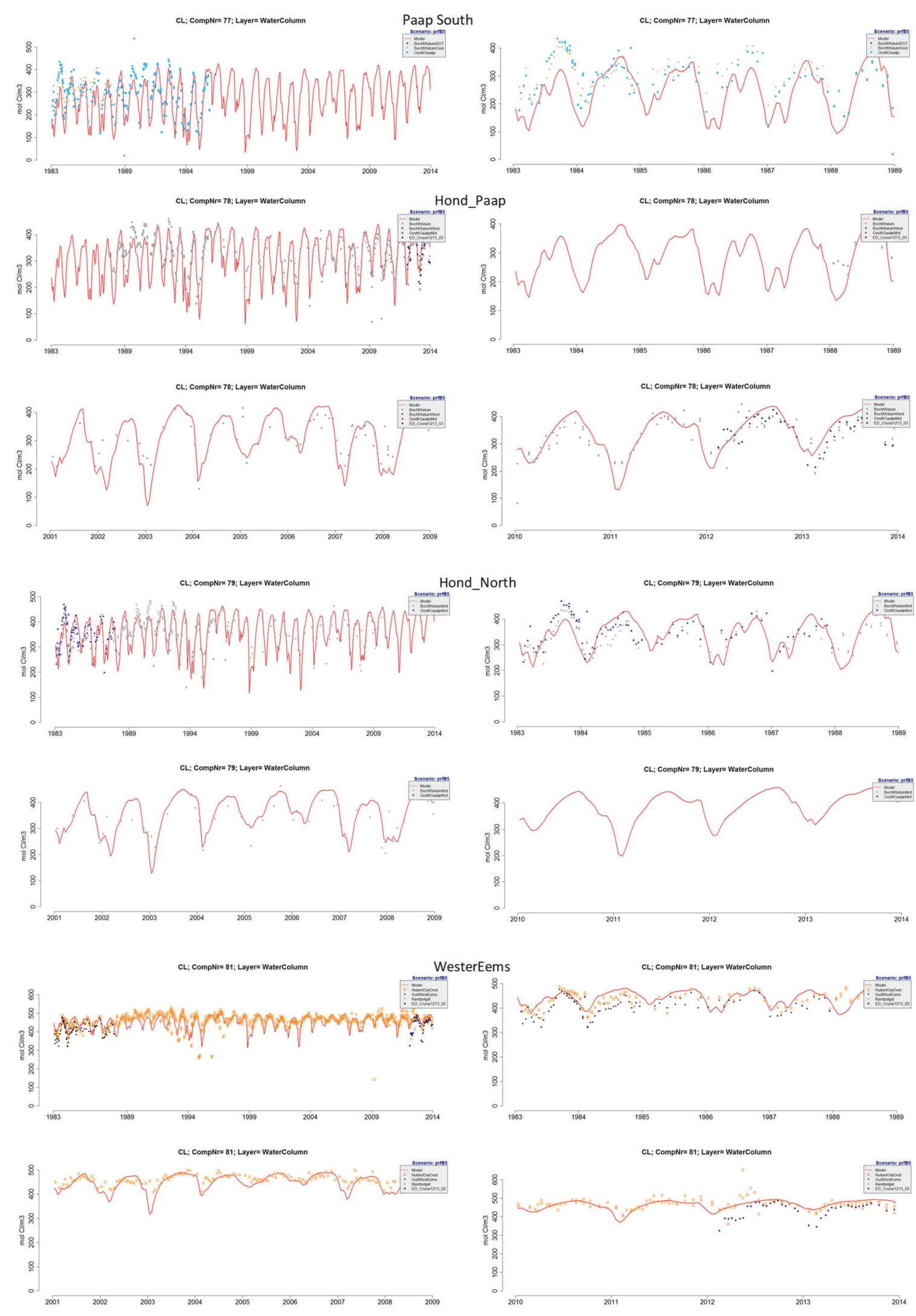

Figure 115 Chloride concentrations (modelled: lines, and data: dots) in the Ems estuary at Paap-south (upper graphs, main comp 108), Hond-Paap-mid (next two graphs, main comp 109), Hond-north (next four graphs, main comp 110) and Westerems (last four graphs, main comp 112). For compartment numbers see Figure 38, Table 2. 

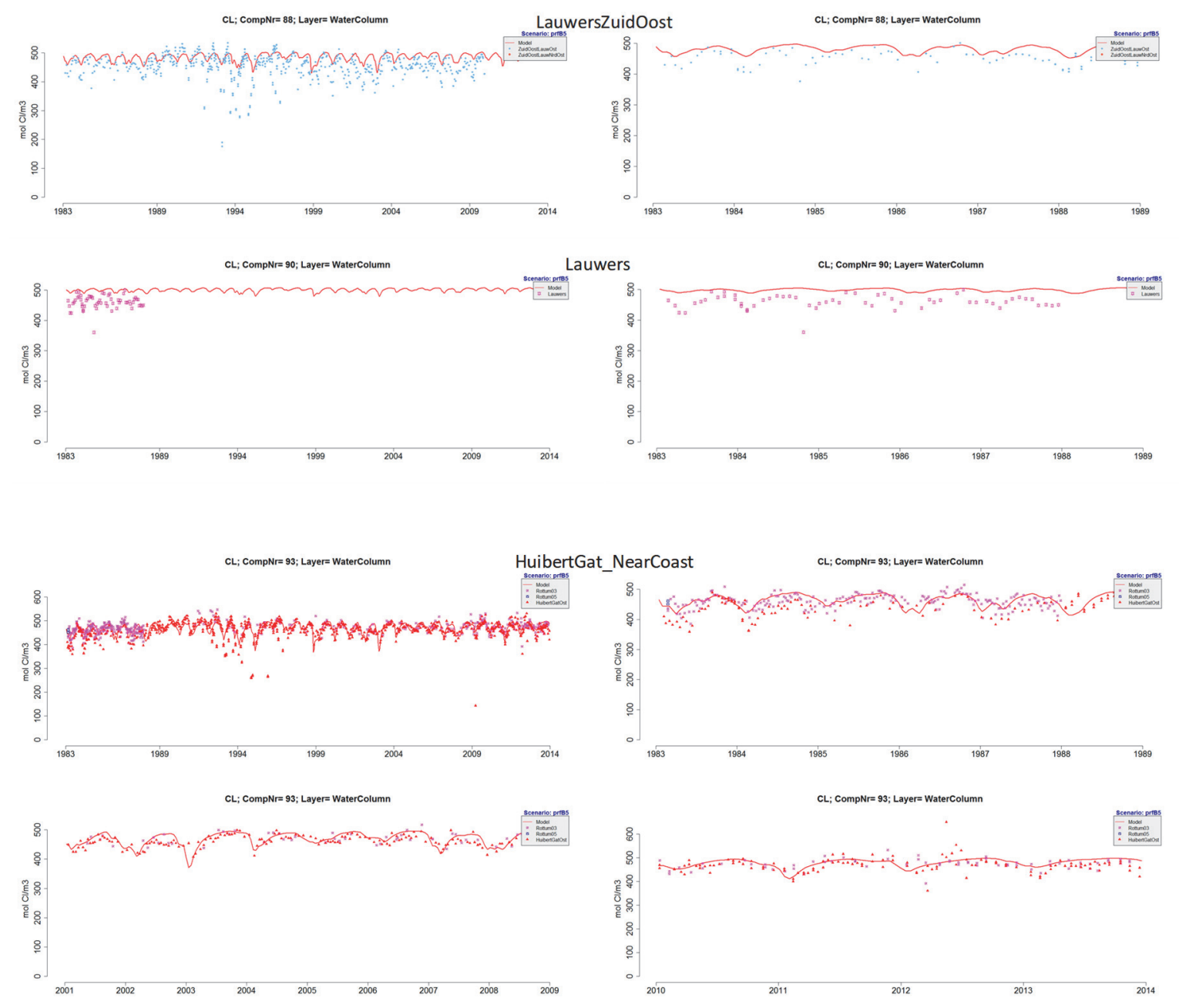

Figure 116 Chloride concentrations (modelled: lines, and data: dots) in the Ems estuary at lauwers-Zuidoost (upper graphs, main comp 119), Lauwers (next two graphs, main comp 121) and Huibert Gat near-coast (next four graphs, main comp 124). For compartment numbers see Figure 38, Table 2. Where most model results fit reasonably, they fail for the Lauwers-area. This must be because inflow of water from the nearby area close to the Lauwersmeer, containing some fresh water, is neglected.

From the figures it can be concluded that chloride contents are simulated rather well, except for the area close(r) to the Lauwersmeer (upper graphs in Figure 116). The fresh water input there was not included in the model and thus main compartments 120-122 (and others close to these) contain too much marine water. This will return in all nutrient results.

\section{A25.1.2 Spatial distribution}

The spatial distribution as measured during the 2012-2013 research by Riegman et al (reported by Brinkman et al, 2014 and 2015), Figure 117Figure 118, is not easily compared to the simulation results Figure 118, but it reflects the timeseries results presented above. 

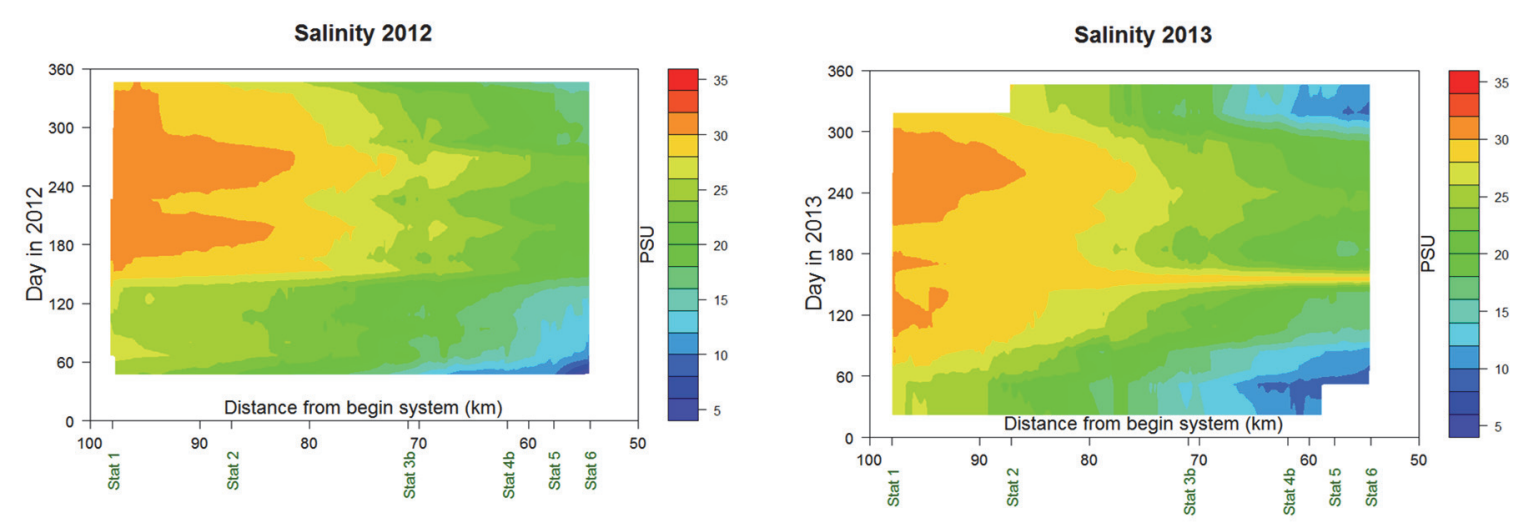

Figure 117 Longitudinal distribution of salinity in the system during 2012 and 2013, as recorded by the PocketBox (Brinkman et al, 2014). Horizontal axis: distance from Herbrum (left=North Sea). Station nrs and km-values are shown. Vertical axis is time (from 1 Jan 2012 (lower) to 31 dec 2013 (upper). Sampling stations 1-6 mentioned, see also Figure 118.

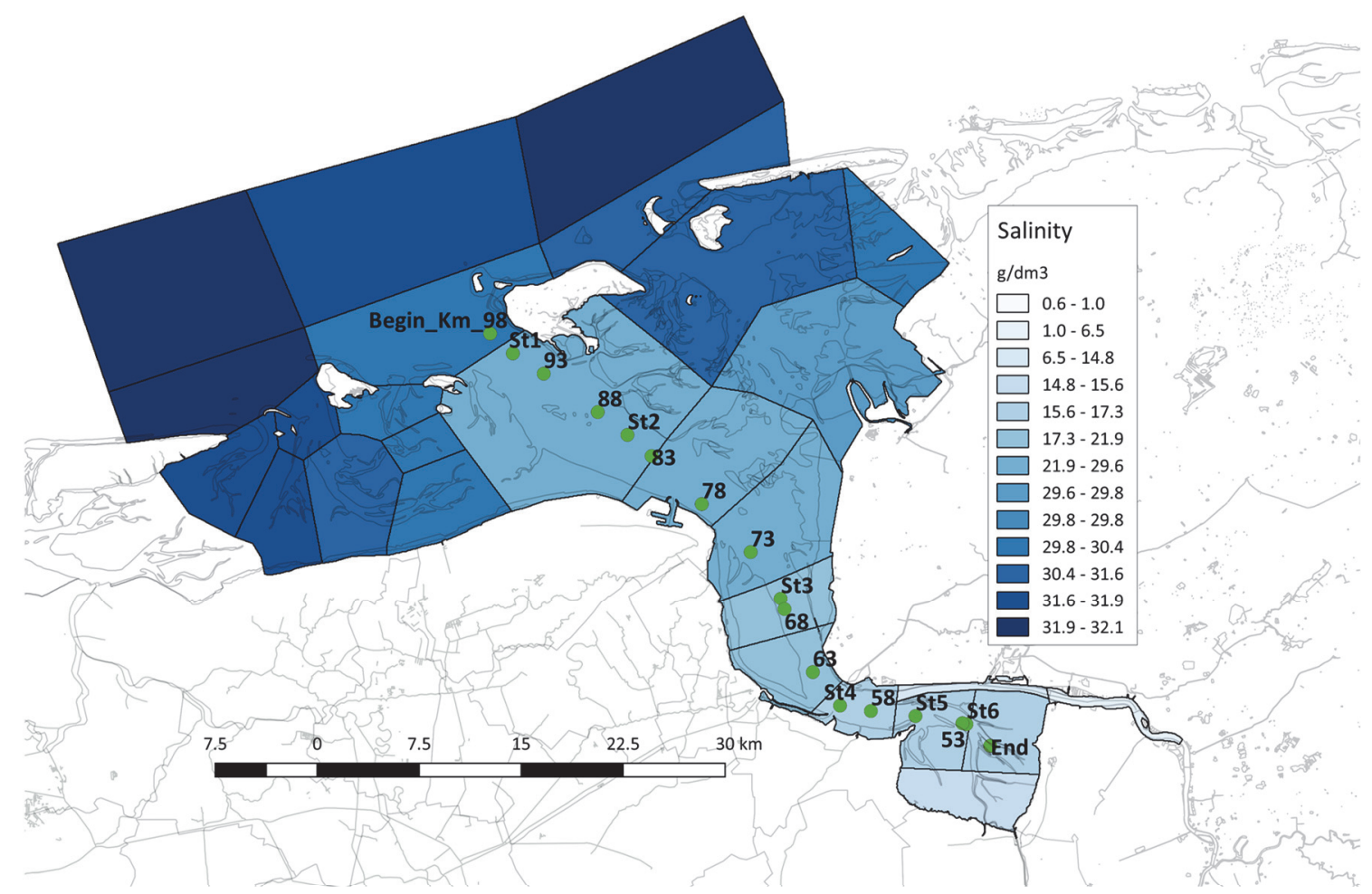

Figure 118 Spatial pattern of all-averaged (years 2010-2013) salinity in the system. Compare with Figure 117. The km-marks are mentioned in Figure 117 as well (starting at Herbrum as km 0)..

\section{A25.1.3 Possible improvements}

Most important improvement is to include the Lauwersmeer-outflow area (called the Zoutkamperlaag), and (thus) the outflow of fresh water from the Lauwersmeer. 


\section{A25.2 Resuspension parameters and model results}

\section{A25.2.1 Introduction}

Next to the chloride distribution, the suspended solids content of the water column must appear as well as possible in the ecosystem simulations. Where a correct chloride distribution stands for a correct mixing of water bodies is a correct representation of the suspended sediment content a must to find correct light climate values for each site, and this will affect the computed primary production.

The parameters $k_{1}$, richt $t_{0}$ (two values), $\zeta$ and $k_{\text {flowfact }}$ from appendix $A 20$ must be found. The last one is estimated based on a zero wind-situation, and lowest values for suspended matter. The others are estimated based on observations and simulations performed. Observations are scarce, and no high frequency data are available, just the Rijkswaterstaat monthly MWTL-monitoring data.

The equations contain more parameters, like the settling velocities of the several components. These are computed first, based on eq. E.12 (appendix A20), assuming a spherical shape and a specific mass of $2.6 \mathrm{~kg} \mathrm{dm}^{-3}$. These are shown in Figure 119. Next, for the several fractions some other values were chosen.

Available RWS-data (not analysed further here) show that the amount of particles $<16 \mu \mathrm{m}$ roughly equals the amount of 16-63 $\mu \mathrm{m}$ particles (in mass unit). Some of the RWS-MWTL samples have been analysed for particle size distribution.

More data come from Postma (1961), who showed that

- Suspended sediment content is much lower at low tide than at high tide. He explained this as a consequence of the longer low velocity period around high tide compared to the shorter low velocity period at low tide time. As a result, more sedimentation can take place around high tide.

- Suspended sediment contents at the water surface are much higher during flood than during ebb.

- Suspended sediment contents at the bottom are much lower during flood than during ebb

- Fine particles $(<8 \mu \mathrm{m})$ constitute about $40-70 \%$ of all suspended sediment. Especially when suspended sediment content is low, the part of fine particles is highest.

- Sum of particles $<16 \mu \mathrm{m}$ is only slightly higher than $<8 \mu \mathrm{m}$.

- Sum of particles $<32 \mu \mathrm{m}$ is $60-80 \%$ of the total.

- Sum of particles $<64 \mu \mathrm{m}$ is $80-95 \%$ of the total.

Particles $>64 \mu \mathrm{m}$ are rare. Postma (1961) writes that the part of sandy components depends a lot on wind. Thus, Lutum02 and Silt0216 are the most important fractions; the MWTL-data support that.

The values used as settling velocities are listed in appendix A28 (containing all parameters used). The values of $r_{3}$ (wind velocity power) is 1.4. See also Aalderink et al (1984). Experience with earlier EcoWasp-computations gave the impression that 2.0 was a too high power-value.

The values for the wind direction were simply estimated: when is an area sheltered from the wind, and when is it exposed? Now, it is nothing more than a rough 'guess'. In future, results from simulations on silt dynamics with an appropriate model (like done for the KRW_Slib-study, see Brinkman, 2015) may result in better estimates. 

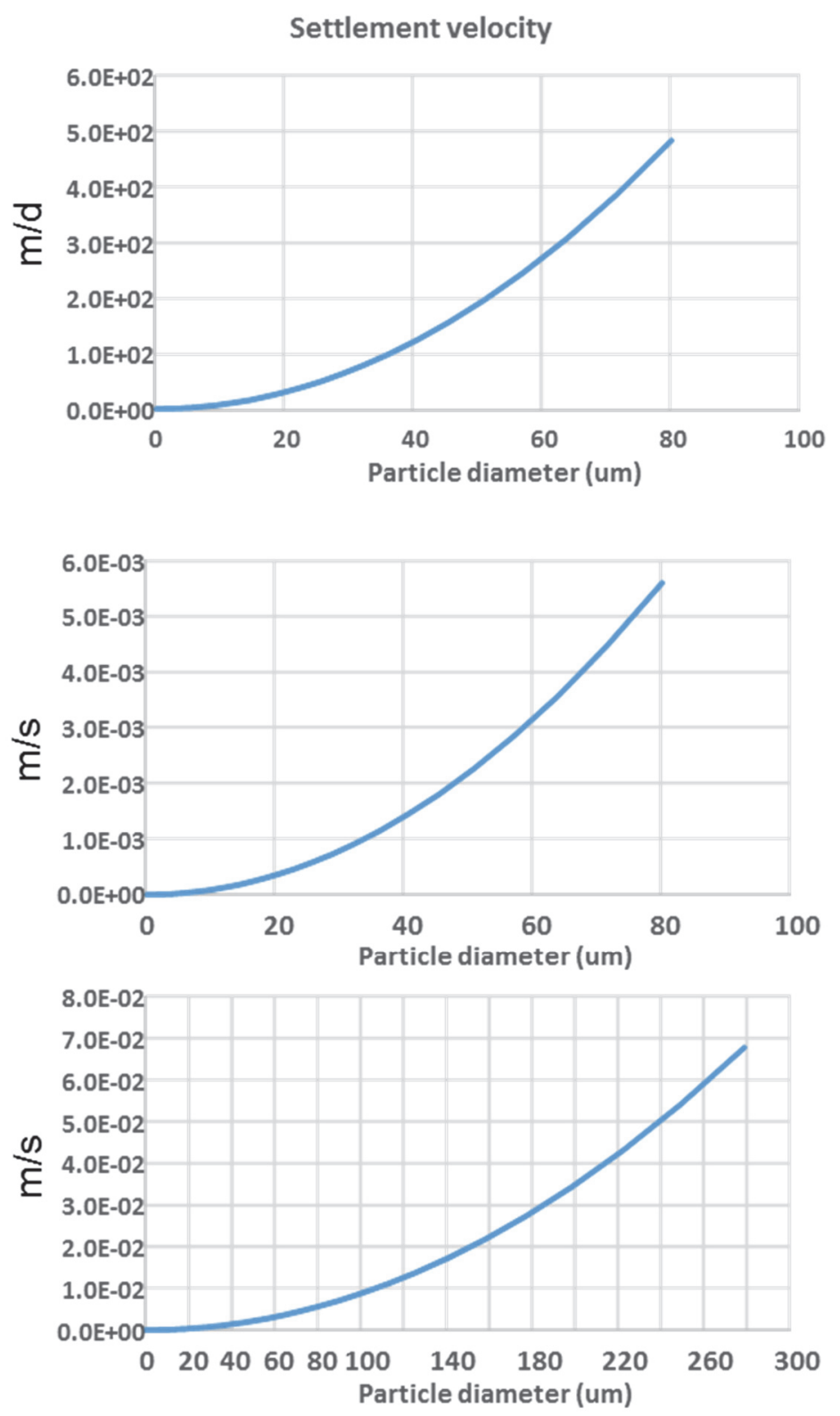

Figure 119 Settling velocities according to volgens Stokes'law; specific mass water $=1 \mathrm{~kg} \mathrm{dm}^{-3}$, of the solids $2.6 \mathrm{~kg} \mathrm{dm}^{-3}$. Kinematic viscosity $=10^{-6} \mathrm{~m}^{2} \mathrm{~s}^{-1}$.

\section{A25.2.2 Time series results}

Data available are the RWS-monitoring data (MWTL) and the IMARES-monitoring data for 2012 and 2013 in the framework of the "Onderzoek slibhuishouding Eems-Dollard" (Research Mud Dynamics Ems-Dollard). The figure is presented below, together with simulation results.

Without going into detail, the following Figure 120 represents the final results found, after the search for the best resuspension parameter settings. Parameter values are listed in appendix A28. 

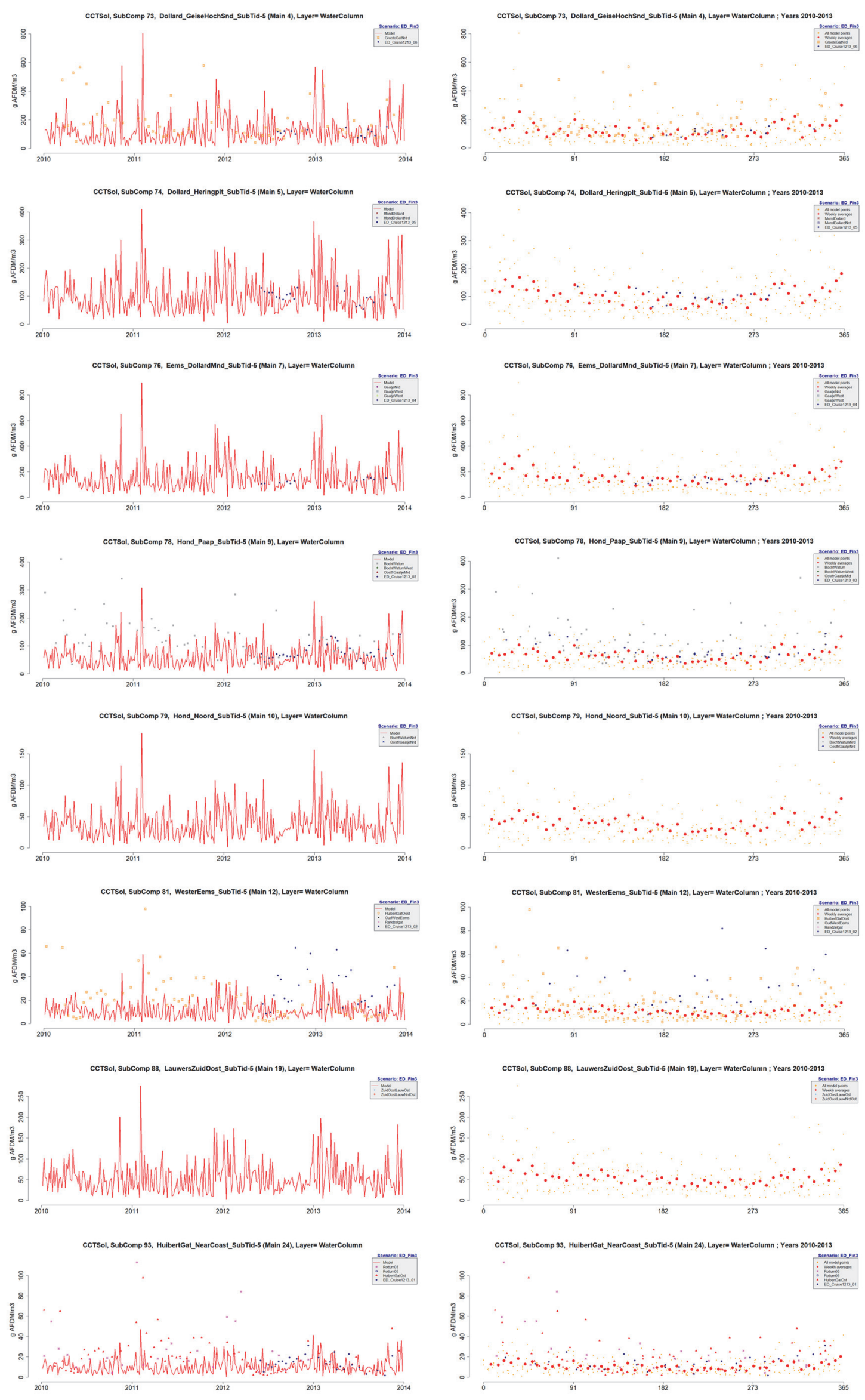

Figure 120 Suspended solids in the Ems-Dollard, period 2010-2013. Left: each 5-days results, right: seasonal pattern. For compartment numbers, see Table 2. Parameter values for this simulation ("ED_Fin3") are all listed in app. A28. 


\section{A25.2.3 Spatial distribution}

A standard situation computed by Deltares is copied from the report by Van Maren et al (2014), and serves as extra information on the suspended solid distribution in the area (Figure 123). Results from the IMARES 2012-2013 research (Brinkman et al, 2014) is shown in Figure 121. The present results (Figure 122) match the distribution rather well, although there are a few discrepancies close to the Groninger sea shore (tidal flats have somewhat lower solid content that parts a bit further away from the shore), and second, the German tidal flats have lower suspended solid values than the Dutch 'counter'-tidal flats.
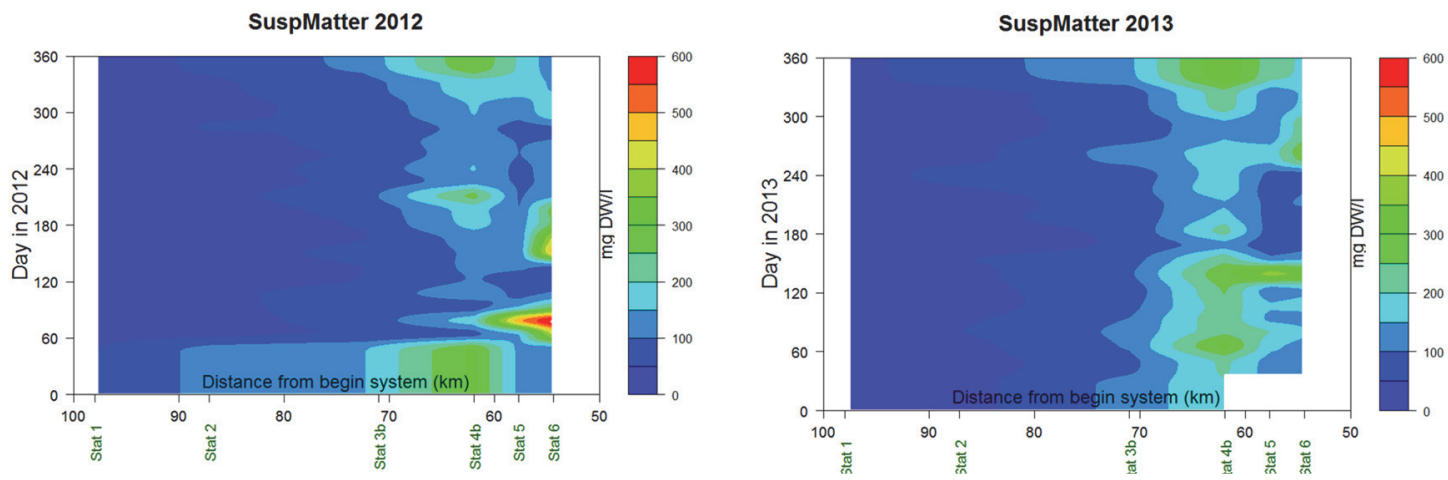

Figure 121 Longitudinal distribution of suspended matter during the 2012-2013 IMARES-research as recorded by the PocketBox (Brinkman et al, 2014). Horizontal axis: distance from Herbrum (left=North Sea). Station nrs and km-values are shown. Vertical axis is time (from 1 Jan 2012 (lower) to 31 dec 2013 (upper). Sampling stations 1-6 mentioned, see also Figure 122.

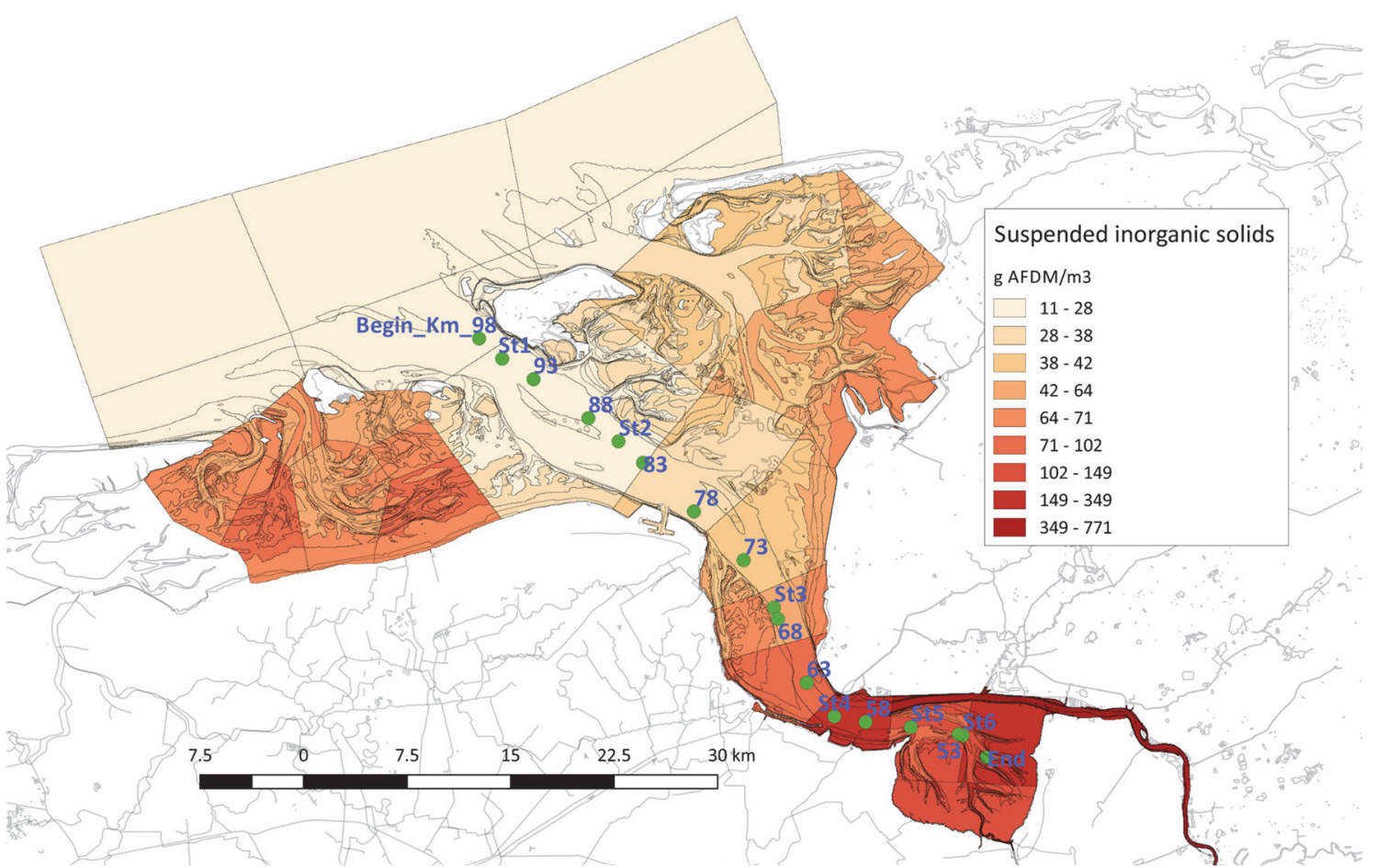

Figure 122 Computed suspended sediment contents of the water column, averaged for the period 2010-2013. The km-marks ate the same as shown in Figure 121. 


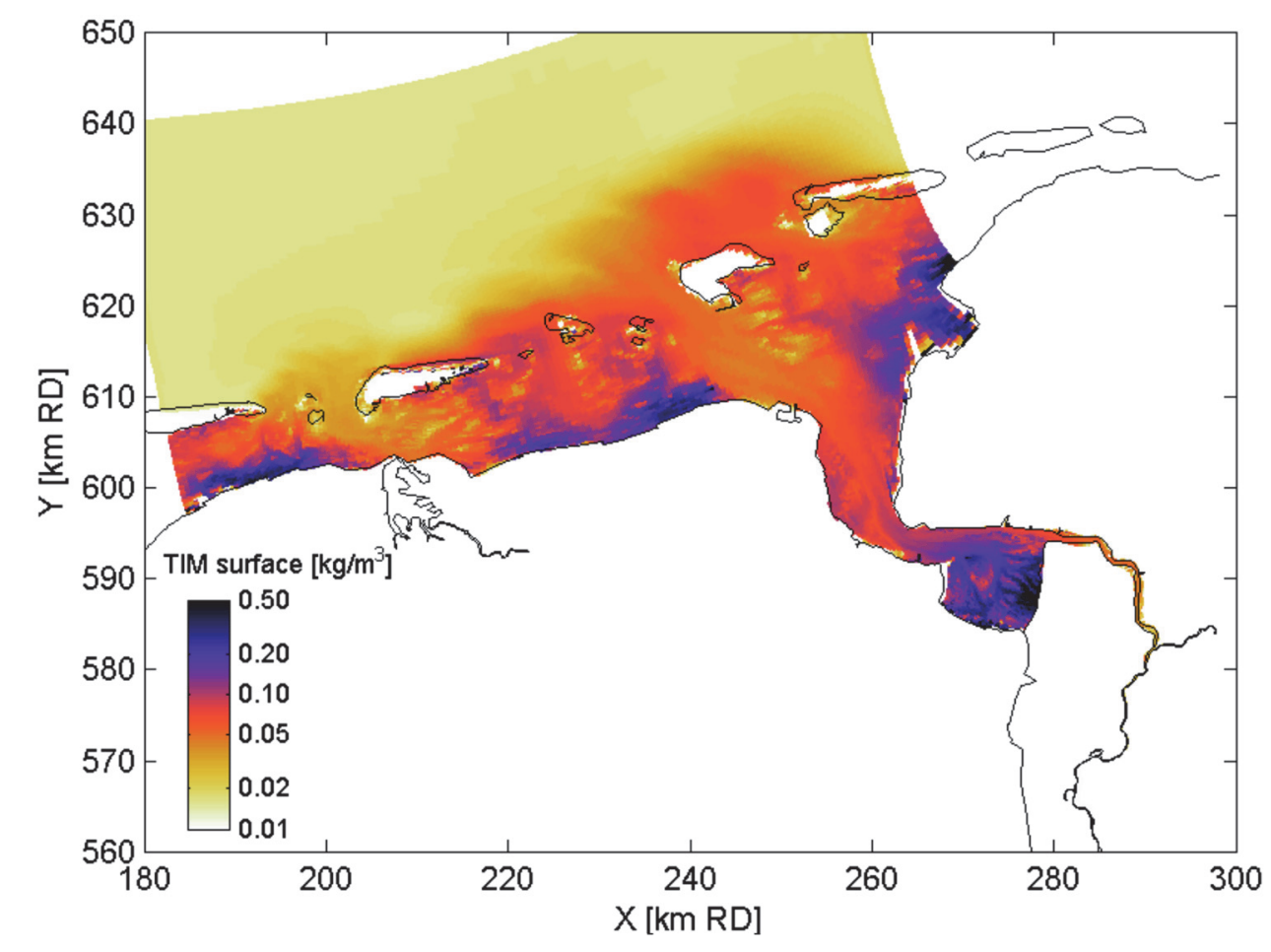

Figure 123 Computed suspended sediment contents of the water column, surface values. Copied from Van Maren etal, 2014: (their Figure 5.9) Yearly averaged surface suspended sediment concentration in the WED model domain (in $\mathrm{kg} / \mathrm{m3}$ ), computed with the reference settings.

\section{A25.2.4 Conclusions}

Although (see the time series in Figure 120) in some compartments the computed values differ from the observations, the spatial pattern matches more or less with the computations performed by Deltares during the 2012-2013 Ems-Dollard research (Maren et al, 2014).

One part that could not be verified now is that the parameter setting is thus that suspended matter content above tidal flats on average exceeds the one in the channels. It is not clear now whether this is reasonable or not. The Deltares results as shown in Figure 123 also do suggest this, but also in that case it is not clear to what extend data support their results. One argument that does support this situation is that, if there is some kind of an equilibrium between transport to the tidal flats and transport fróm the tidal flats, then one may expect higher suspended matter contents above the tidal flats. The reason is that transport to the flats merely takes places deep in the water column because of tidal currents (and thus hardly affects the upper water column suspended solids content of the channels and the subtidal areas), and transport from the tidal flats to the channels is a result of wind influence, and produces a well-mixed water column above the flats.

The pattern shown in Figure 122 differs a bit from the one in Figure 123. Result on the tidal flats close to the Groninger shore (north side) should be a bit higher than the one for the tidal flats between the shore and the islands of Rottumeroog and Schiermonnikoog. Similarly, the German tidal flats might have a bit higher values. So, this is one of the possible improvements.

Together with the light attenuation results (next section 0 ) it can be concluded that suspended solid values are about conform the data. The model presently computes a column averaged value; 
it is assumed that the water column is well-mixed. This can be improved, since there are large vertical differences (Postma, 1961; Brinkman et al, 2014).

\section{A25.2.5 Possible improvements}

Activate the computation of vertical profiles. This option is already present in the model, but not applied yet. Next, the distribution on the tidal flats between the Groninger shore and the islands of Schiermonnikoog and Rottumeroog may be improved. Finally, the German tidal flats now have lower suspended solids simulated than the Dutch 'counter'-tidal flats. This does not seem plausible, thus must be improved also. 


\section{A25.3 Light attenuation and model results}

\section{A25.3.1 Introduction}

The effect of all suspended solids on the water column light climate is visible as the extinction coefficient; although also phytoplankton adds to the light attenuation. In most cases, this contribution is not relevant, except during phytoplankton blooms in those areas where suspension of solids is minor. But this is a rare situation in this area.

\section{A25.3.2 Timeseries}

For those compartments where extinction coefficient values are available, simulation results for the years 2010-2013 are presented (Figure 124). Close examination shows that in all compartments shown, the computed light attenuation coefficients are of the same order and values as the measured ones. There is one exception: for compartment 81 the data assessed during the IMARES-study in 2012 and 2013 are substantially higher than the RWS-values, and the simulated ones. The reason is not clear, yet.

\section{A25.3.3 Spatial distribution}

Figure 126 shows the computed average light attenuation coefficient (years 2010-2013), and Figure 125 the longitudinal observations during the 2012-2013 IMARES-research (Brinkman et al, 2014). In Figure 127 and Figure 128, the differences between the summer and winter period, as computed, is shown. This reflects the characteristic as seen in the measurements that in summer light attenuation is lower than in winter, an effect of weather conditions. Largest difference can be found for station 3 , with values of about $8 \mathrm{~m}^{-1}$ and more in winter and less than $5 \mathrm{~m}^{-1}$ in summer. The simulation values are substantially lower, a difference also already observed in appendix A20 on solid concentrations. It is this site especially where improvements are possible.

\section{A25.3.4 Suggestions and possible improvements}

- Like the remarks given in appendix A25.2.5, the distribution of lower and higher light attenuation coefficients must be improved (although differences certainly are not major): close to the Groningen north shore, and on the German tidal flats.

- Also, the attenuation coefficient, and the concentration of suspended solids, can be adjusted around monitoring site 3 in Figure 126. 

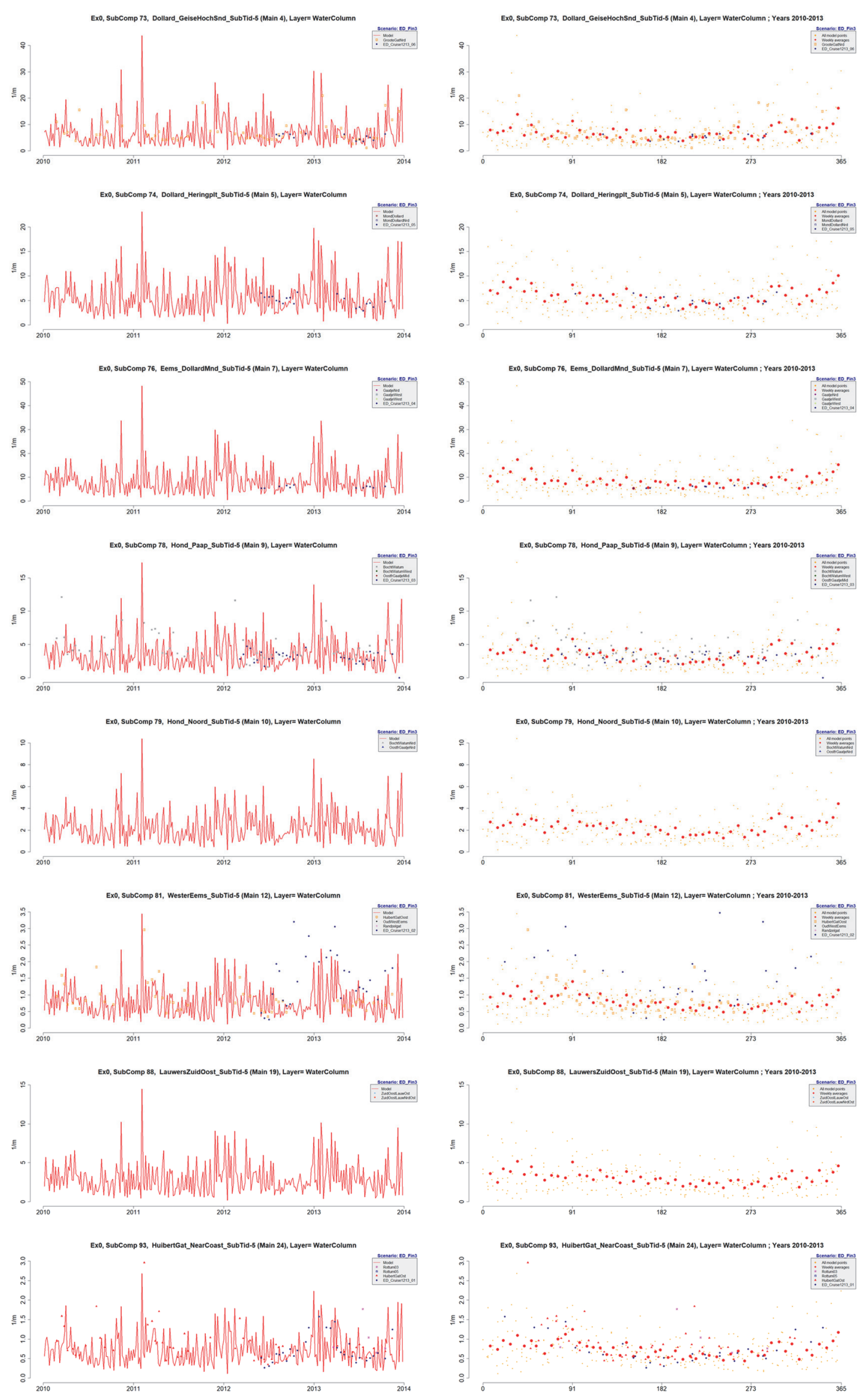

Figure 124 Light attenuation coefficients in the Ems-Dollard, period 2010-2013. Left: each 5-days results, right: seasonal pattern. For compartment numbers, see Table 2. Parameter values for this simulation ("ED_Fin3") are all listed in app. A28. 
Kd.1.m 2012

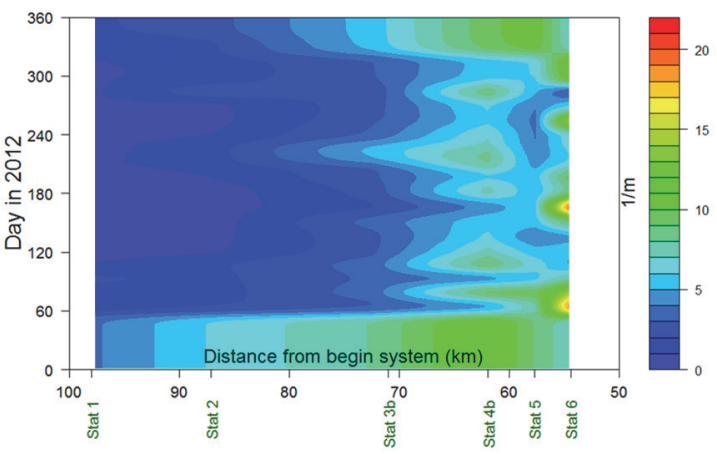

Kd.1.m 2013

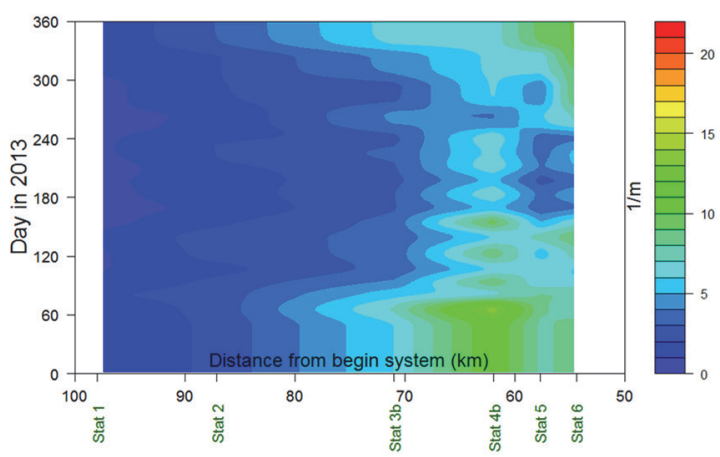

Figure 125 Longitudinal distribution of the light attenuation coefficient during the 2012-2013 IMARESresearch as recorded by the PocketBox (Brinkman et al, 2014). Horizontal axis: distance from Herbrum (left=North Sea). Station nrs and km-values are shown. Vertical axis is time (from 1 Jan 2012 (lower) to 31 dec 2013 (upper). Sampling stations 1-6 mentioned, see also Figure 122.

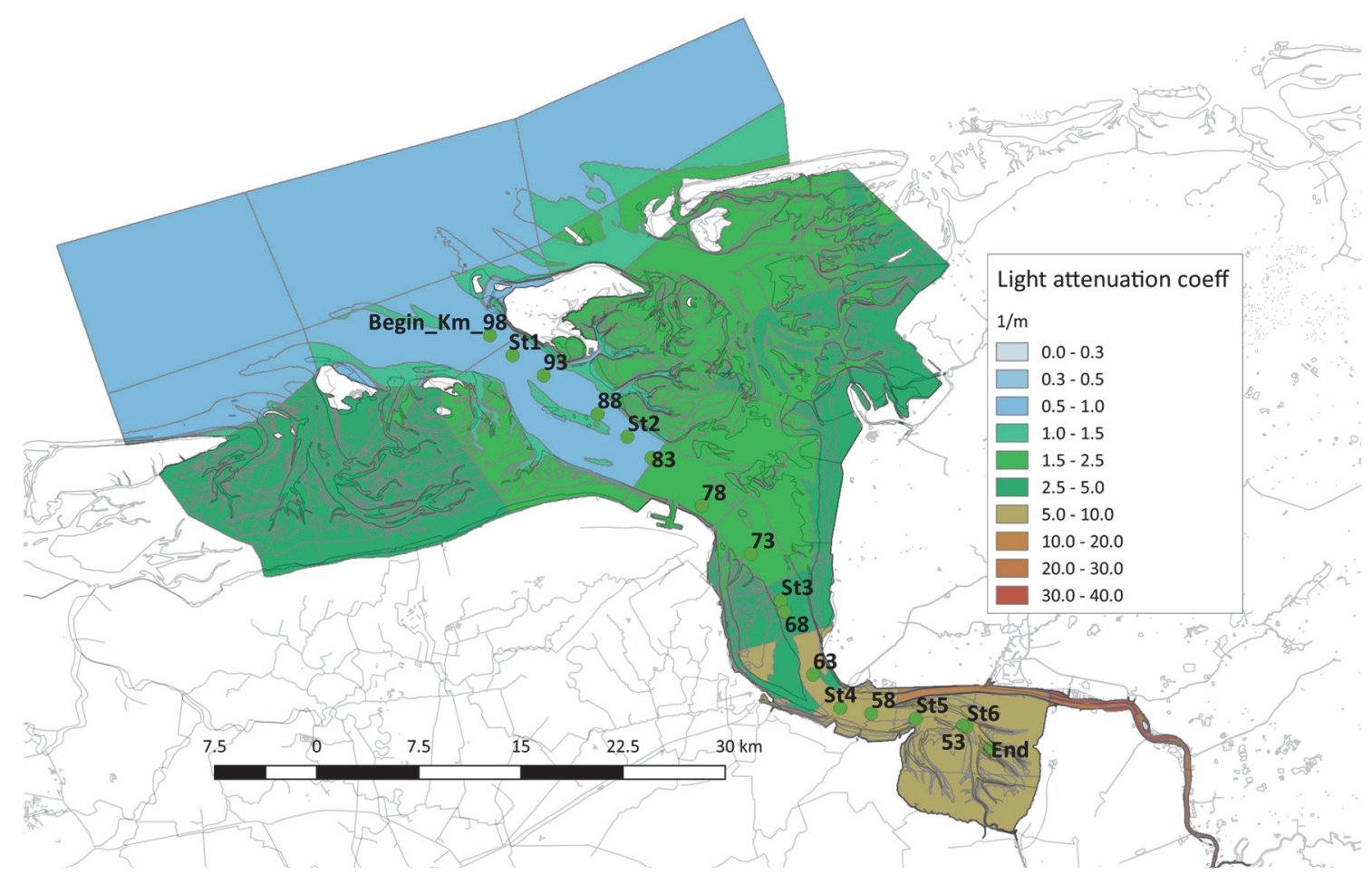

Figure 126 Computed year averaged light attenuation coefficients $(1 / \mathrm{m})$ in the Ems-Dollard (whole period 2010-2013). Simulation ED_Fin3. The km-marks are the same as those in Figure 125 (colors are not the same) 


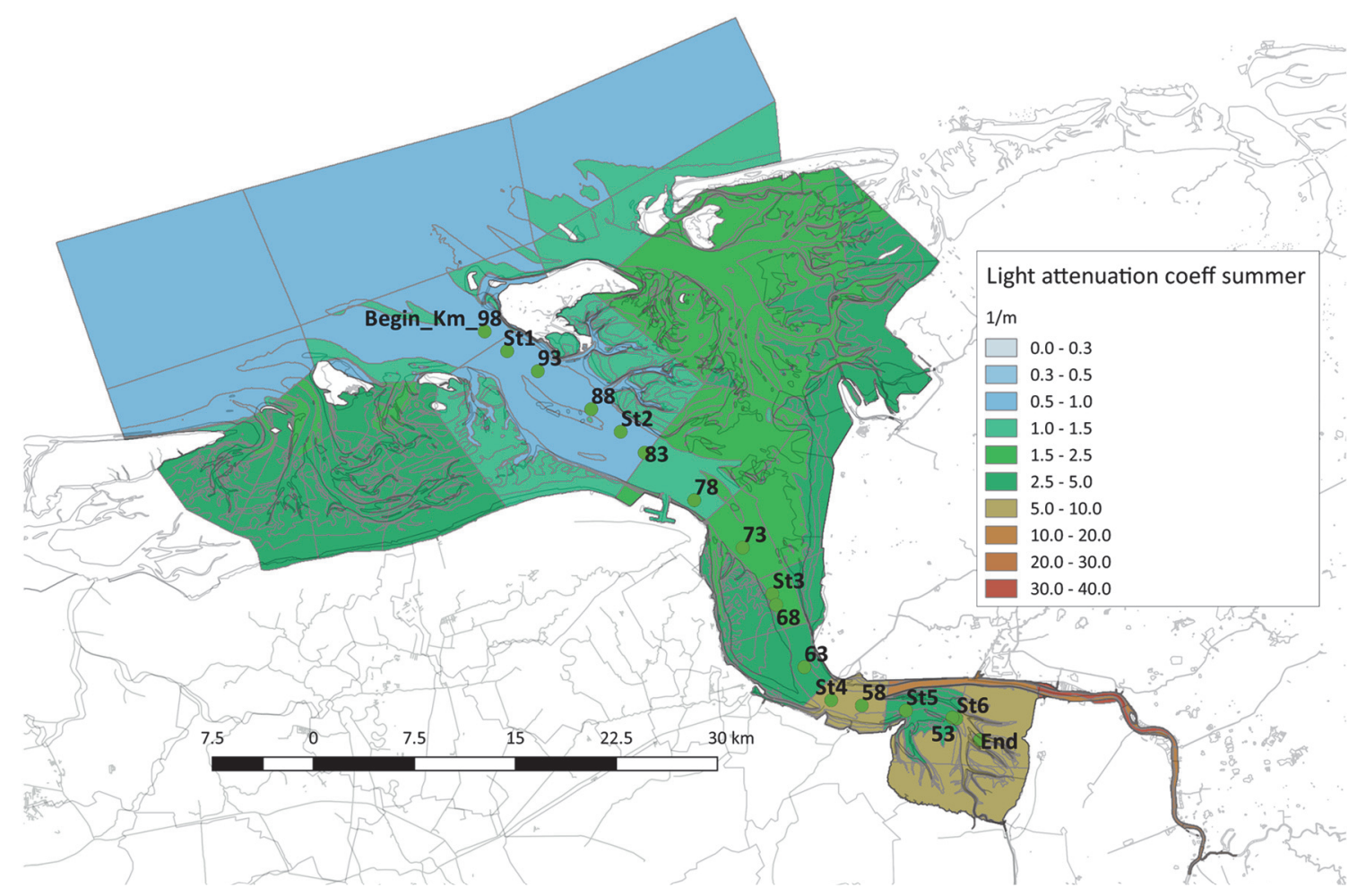

Figure 127 Computed summer (April-September) averaged light attenuation coefficients $(1 / \mathrm{m})$ in the EmsDollard (whole period 2010-2013). Simulation ED_Fin3. The km-marks are the same as those in Figure 125 (colors are not the same)

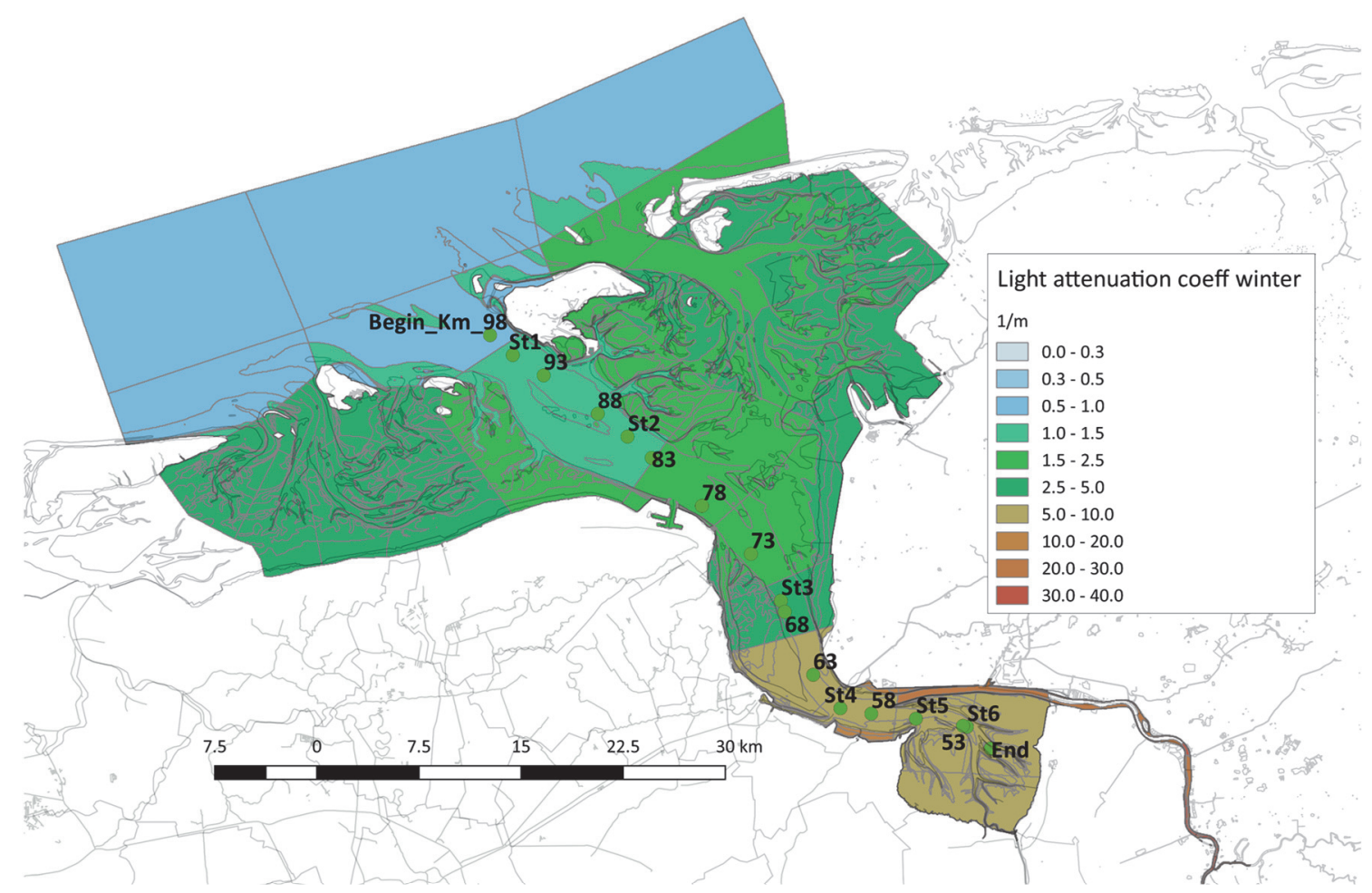

Figure 128 Computed winter (November-February) averaged light attenuation coefficients $(1 / \mathrm{m})$ in the EmsDollard (whole period 2010-2013). Simulation ED_Fin3. The km-marks are the same as those in Figure 125 (colors are not the same) 


\section{A25.3.5 Light at the sediment surface}

In Figure 129 the summer average light intensity at the sediment surface is shown. Even in the deepest offshore parts, some light is still present, even a bit more than in the deepest gully parts inside the estuary. This result is very sensitive to variations in the light attenuation coefficient, so, with some somewhat other values the result will change most in the deepest parts.

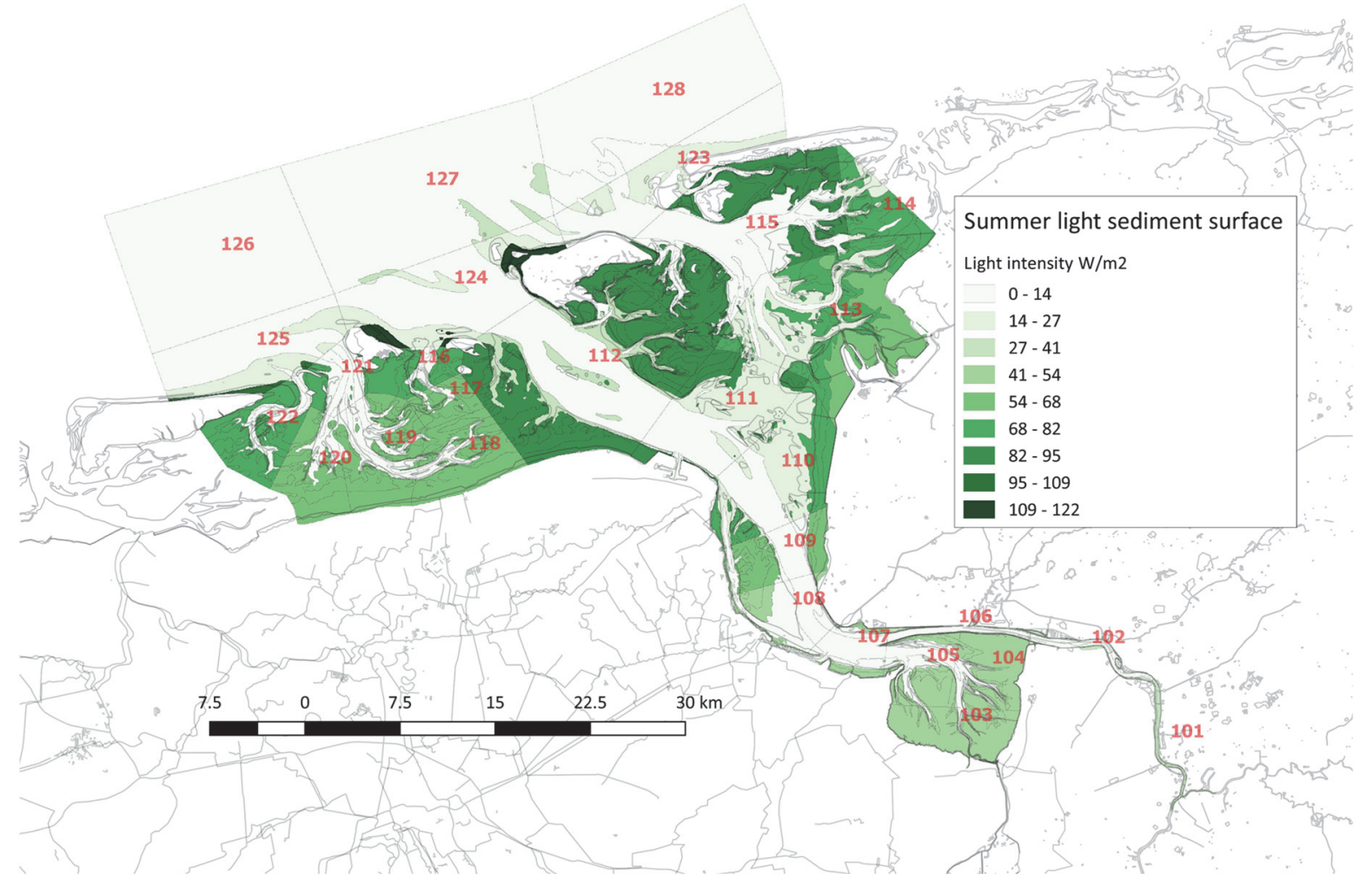

Figure 129 Light intensiy in summer at sediment surface $\left(\mathrm{W} \mathrm{m}^{-2}\right)$ 


\section{A25.4 Biologically active variables}

\section{A25.4.1 Introduction}

Next to the distribution of chloride (appendix A25) and the dynamics of suspended matter and the extinction coefficient (appendix A20), the simulation results for biologically involved dissolved and solids substances are briefly discussed in the next section of this appendix A25.

Suspended matter and salinity could be tuned separately, since there are none (salinity) or few (suspended matter) feedbacks. The result of the rest of the model does not affect salinity or hardly affects the extinction coefficient. For the latter: the amount of algae and detritus is not neglectable, but adds just a bit to the inorganic suspended matter.

It appeared to be very time-consuming to reach a certain level of fit for the other characteristics. Several hundreds of simulations were needed, most of these for the periods 2005-2013, 2005$2008,2010-2013$. The nine-years period was too time consuming (about 1-2 hours computing time) each time, and therefore it was turned into a four-years simulation. The last-mentioned period was preferred at the end since it also covers the IMARES-monitoring period in 2012-2013.

Also, it is our conviction that simulation over more than one year is obligatory. In those cases, the result of the first year is used for the second, and so on, and simulating more years (say, four at least) serves as a proof that the model is stable. Note that in all cases, as number of precomputations (this may be up to over forty runs) have been performed to minimize initialisation effects.

\section{A25.4.2 Introduction (2)}

A (very) complicating factor was (and is) that many of the modelled processes significantly contribute to the computed values ánd that many feedback mechanisms are present. For example, at a certain moment, computed water column ammonium contents were quite close to the data, but for nitrate it was far away. At the same time, benthic chlorophyll-a results were far too low. After changing the benthic primary production drastically (available data from the 2012-2013 IMARES research did not give precise results) it became much better for benthic diatoms, ánd suddenly $\left[\mathrm{NO}_{3}{ }^{-}\right]$came closer to the data. But now, $\left[\mathrm{NH}_{4}{ }^{+}\right]$was out of the range, and much too high. Such things occurred repeatedly.

Another example -especially of feedback mechanisms- is presented in the next section A25.4.3. That short sidestep is relevant in the case of finding appropriate zoobenthos and zooplankton parameters and where classical predator-prey relationships must be solved. For example: tuning phytobenthos parameters not only involves growth and respiration, but also grazing upon benthic algae. At the other side: growth rates and biomasses of benthic algae affect food availability for mudsnails directly and thus affect their own loss term.

Now, not an intelligent parameter estimation routine is included in the scripts that run EcoWasp, although it is very well possible to do this (after some probably time-consuming effort). Thus, it is a matter of trial-and-error, and learning from the results.

This all together supports the idea that an ecosystem model certainly is not a system with which one easily can obtain any wanted result, but that everything computed must fit to the observations. And if yes, then one may expect that the model is also well representing the included system processes. 


\section{A25.4.3 Phytobenthos parameter tuning, Mudsnail and Copepod}

As an example, the search for mudsnail and phytobenthos parameters is illustrated here. Note that final phytobenthos results are presented in appendix A25.11 mudsnail results in A25.12.1 and benthic primary production in A25.15. Thus, results in this example are not the final results.

A first example is in Figure 130, showing that mudsnails reduce benthic diatom development, and consequently also pelagic chlorophyll concentrations.

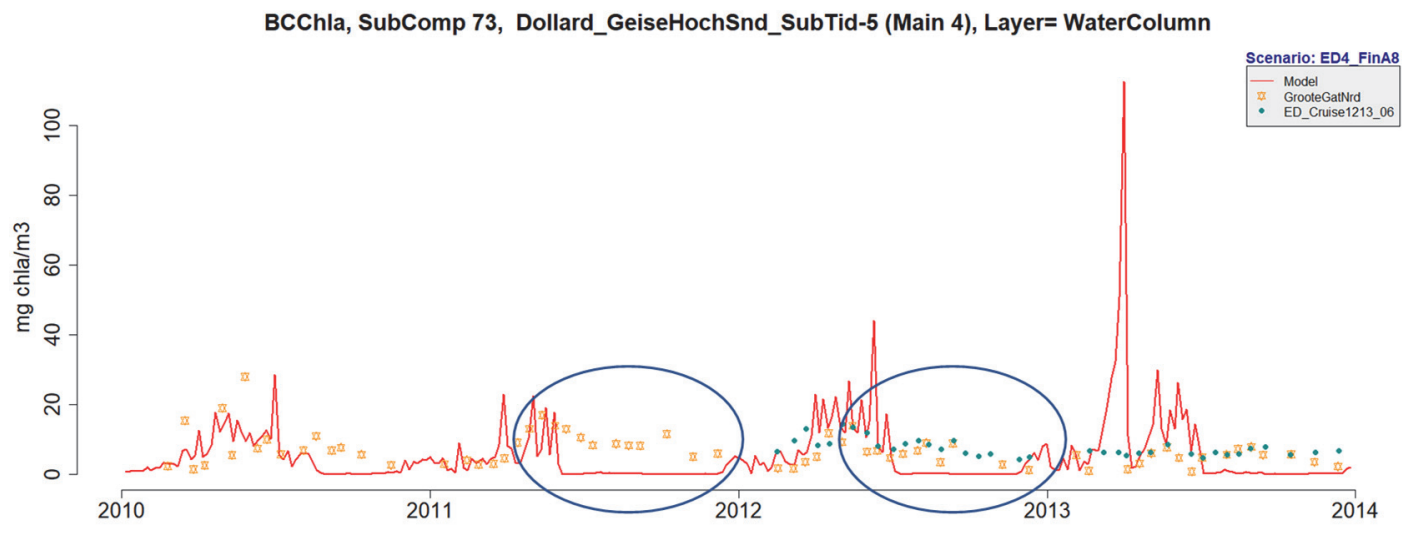

CV_SumFauDens-C_HYDROB, SubComp 49, Dollard_GeiseHochSnd_Tidal_0_20 (Main 4), Layer= WaterColumn

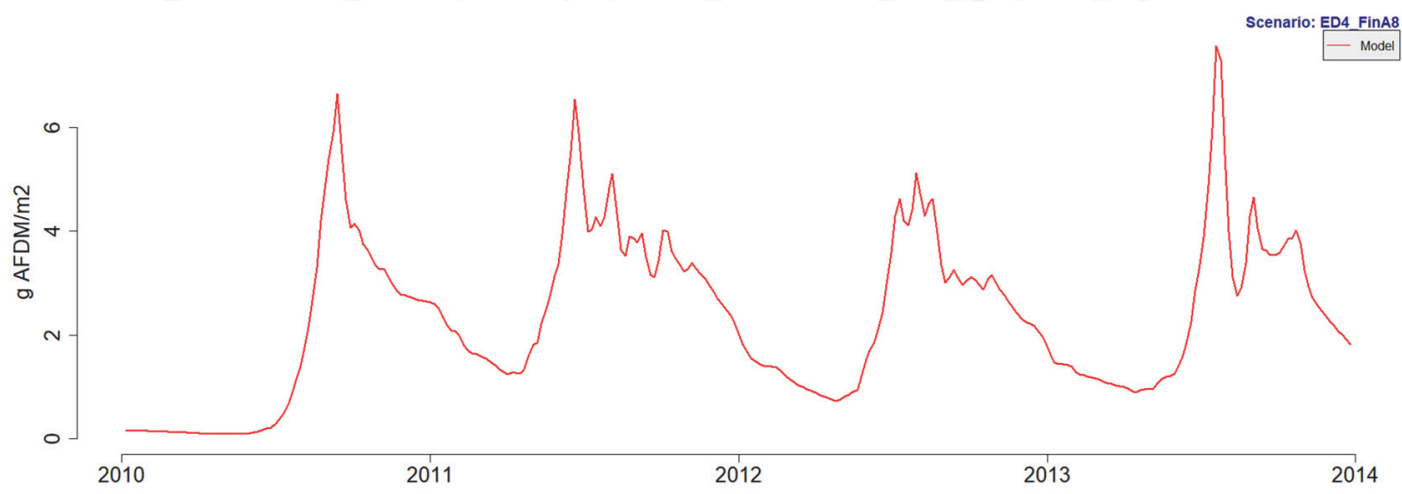

CVV_Chla, SubComp 49, Dollard_GeiseHochSnd_Tidal_0_20 (Main 4), Layer=1

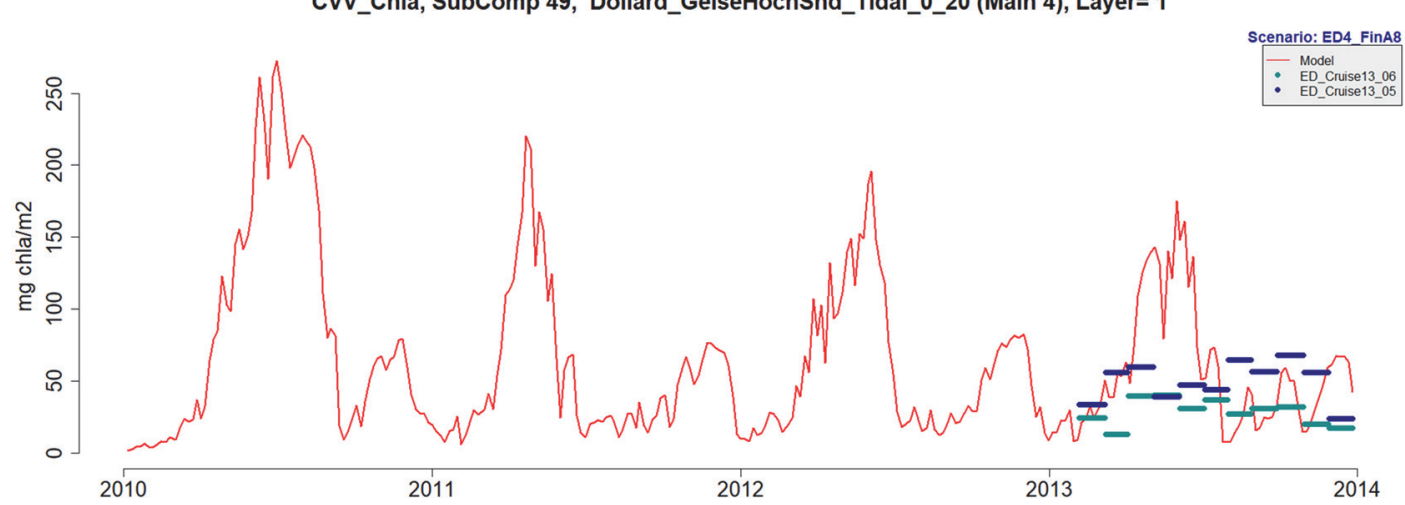

Figure 130 Development of pelagic (upper), benthic (lower) chlorophyll and mudsnail (middle) biomass in the Dollard area.

Next, but this is not show here, it appeared that when copepods were introduced with suspended benthic diatoms as a small part of their food, benthic diatoms disappeared as well as soon as copepods became abundant in the model. Copepods were assumed to feed mainly on detritus- 
associated bacteria, these bacteria were modelled as a fixed part of detritus. Since there is plenty of detritus available, copepods could grow to large densities and then, although benthic diatoms were only a small part of their food uptake, it was large in an absolute sense. And therefor, leading to low water column benthic diatom content. Since there is an intense exchange between benthic diatoms in the water column and those in the sediment top layer, this process affected benthic diatom presence in the sediment as well, resulting in a steep decrease of phytobenthos content and thus of benthic primary production. For bottom dwellers as Hydrobia I would mean a depletion of food, and thus lowering Hydrobia-densities, but Copepods are dependent on other food sources, and thus, a BDIA-depletion hardly affects Copepod-abundance.

\section{A25.5 Dissolved inorganic matter and model results}

\section{A25.5.1 Dissolved oxygen $\left(\mathrm{O}_{2}\right)$, time series}

Oxygen is produced because of primary production, and consumed during oxidation of organic (and inorganic: $\mathrm{NH}_{4}$ ) substances. Both are important regulating the concentrations in the water column together with the atmosphere-water exchange; the latter governed by temperature, salinity dependent solubility and the wind plus current driven air/water-exchange parameter. In the Ems river during summer and autumn, the intensity of the consumption process is far more important than oxygen production and air/water exchange cannot compensate this deficiency.

Generally, the model reproduces oxygen concentrations well, except for the Ems river (not shown) and station 3 (compartment 107), where the Ems river enters the estuary. It must be realized that all data represent surface concentrations and the model result represent the whole water column averaged values. Since $\left[\mathrm{O}_{2}\right]$ always is lower close to the sediment, column averages are always lower (to much lower) than surface values indicate. So, differences between data and model may be smaller than suggested here by the figures. Figure 132 shows the whole period averaged spatial distribution of $\left[\mathrm{O}_{2}\right]$. Note that this includes the salinity effect: saturation values are considerably lower in saline waters.

\section{A25.5.2 Spatial distribution}

During the 2012-2013 IMARES-research, oxygen patterns along the system axis have been recorded, see Figure 133. Model results shown in Figure 132. Lowest values of about 200-250 $\mathrm{mmol} \mathrm{m} \mathrm{m}^{-3}$ thus are a bit above the simulated vales, although differences are not major.

\section{A25.5.3 Suggestions and possible improvements}

Oxygen concentrations seem to be simulated rather well, but

- $\quad$ Check the computed air/water exchange (not mentioned here) with literature values

- Check the computed sediment/water exchange (not mentioned here) with literature values.

For the latter, see also section A25.17. 

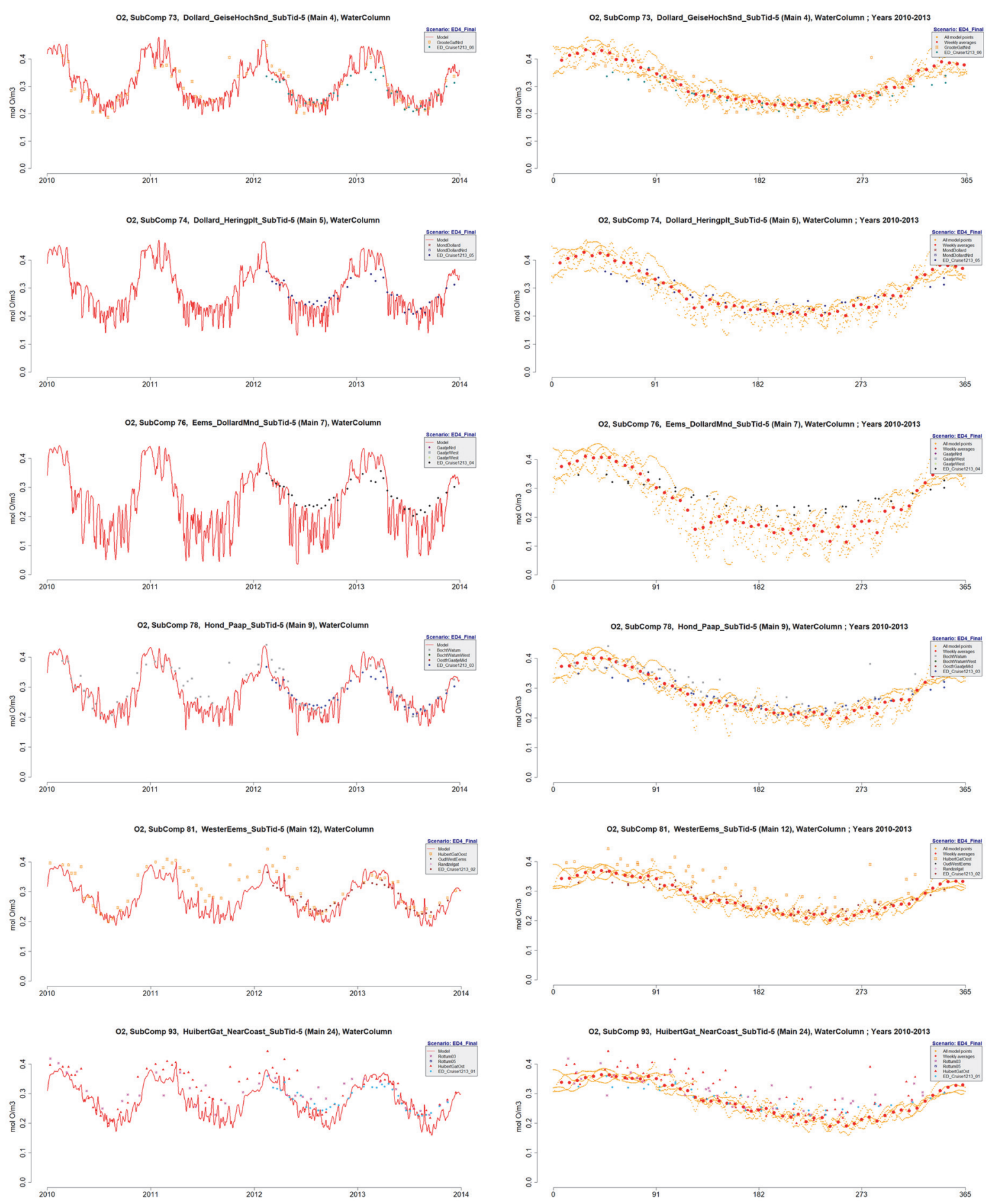

Figure 131 Oxygen concentrations in the system. Upper: Dollard mouth, main compartment 7, $2^{\text {nd }}$ : Hond \&Paap (main compartment 9. $3^{\text {rd }}$ where Ems rivers enters the system. $4^{\text {th }}$ Hond \& Paap. $5^{\text {th }}$ : boundary Westerems and Doekegat. $6^{\text {th }}$ (lowest) boundty of Westerems and Huibert Gat . Left 2010-2013, right: average seasonal pattern. Line=simulation, dots data. Column averaged (completely mixed water column). 


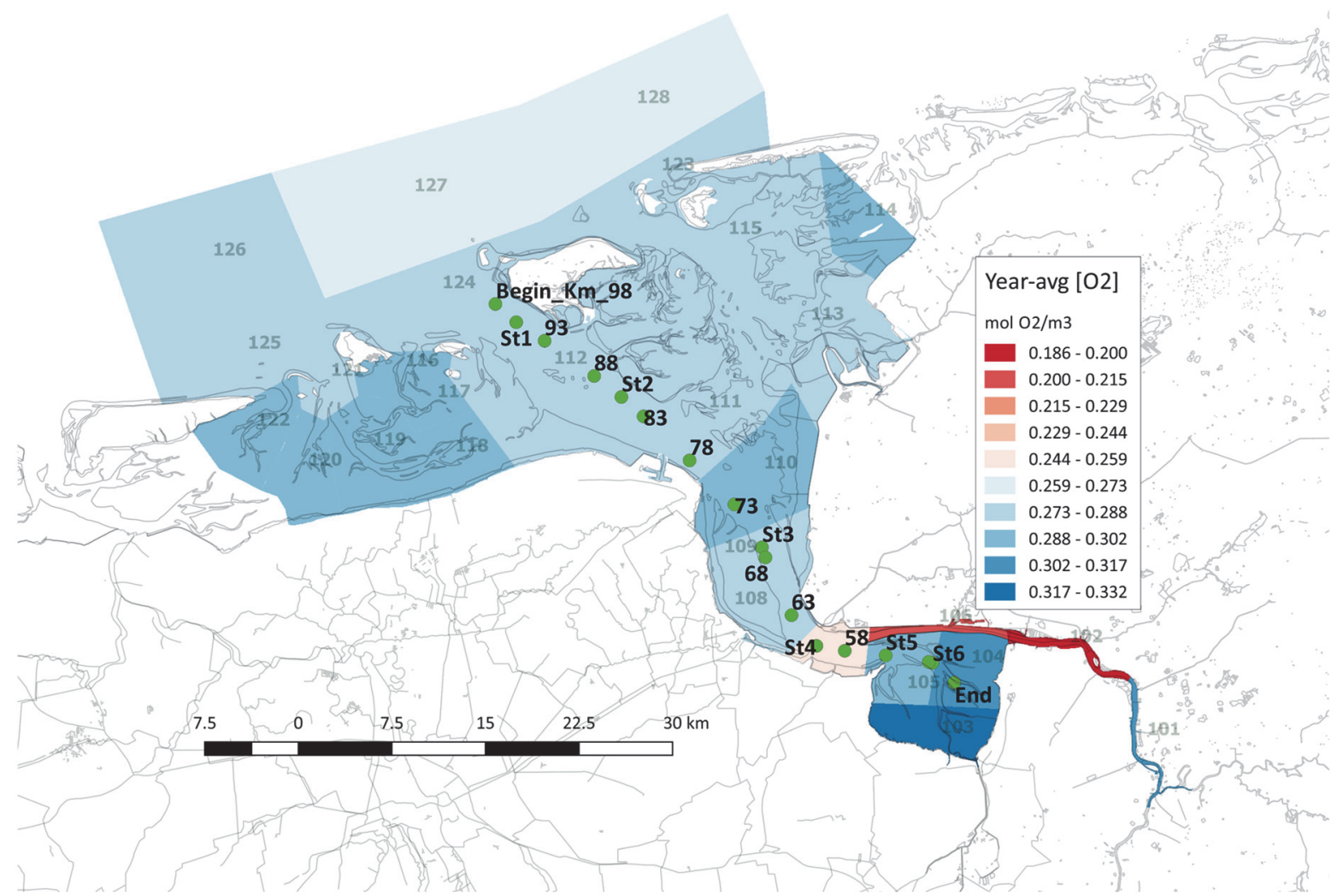

Figure 132 Spatial distribution oxygen concentration $\left(\mathrm{mol}_{2} \mathrm{~m}^{-3}\right)$, averaged over whole 2010-2013 period. Sampling station numbers shown. Station positions (kms from Herbrum) marked, see also Figure 133 (color pattern differs). 


\section{O2_PBox_umol 2013}

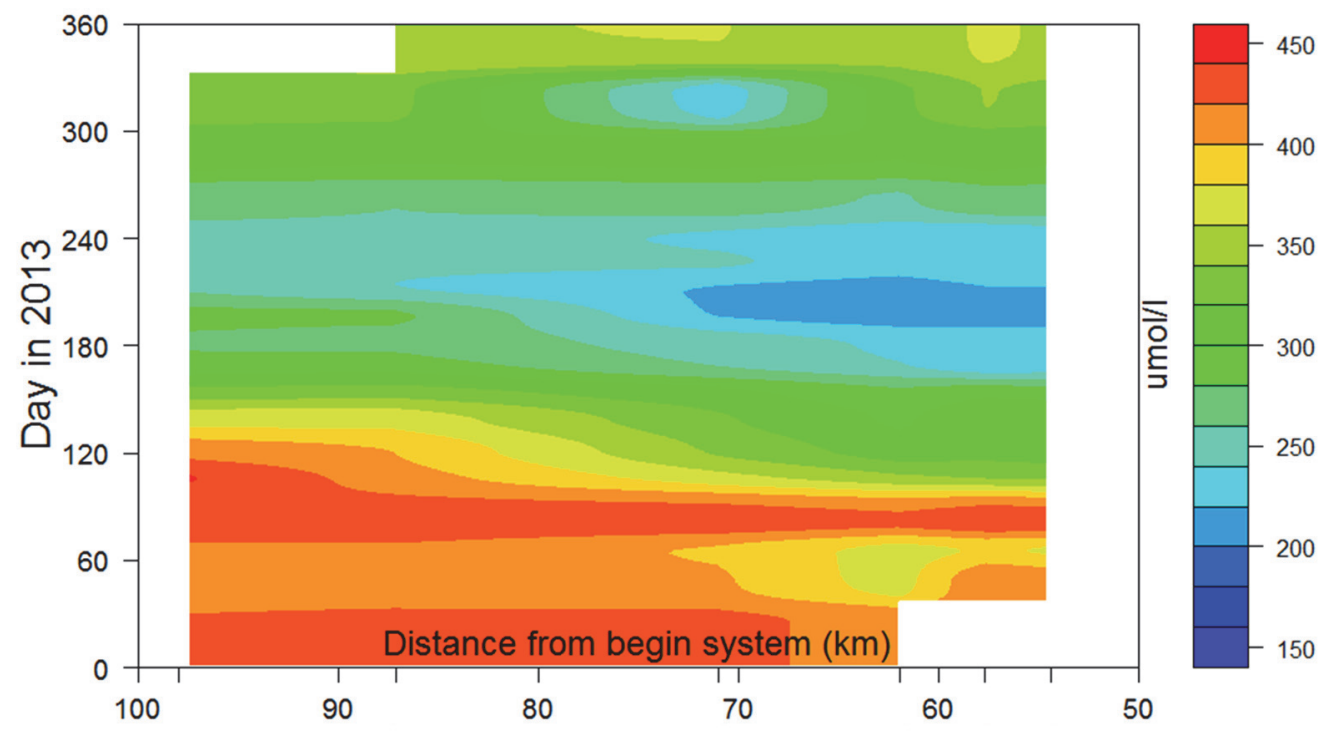

O2_PBox_umol 2012

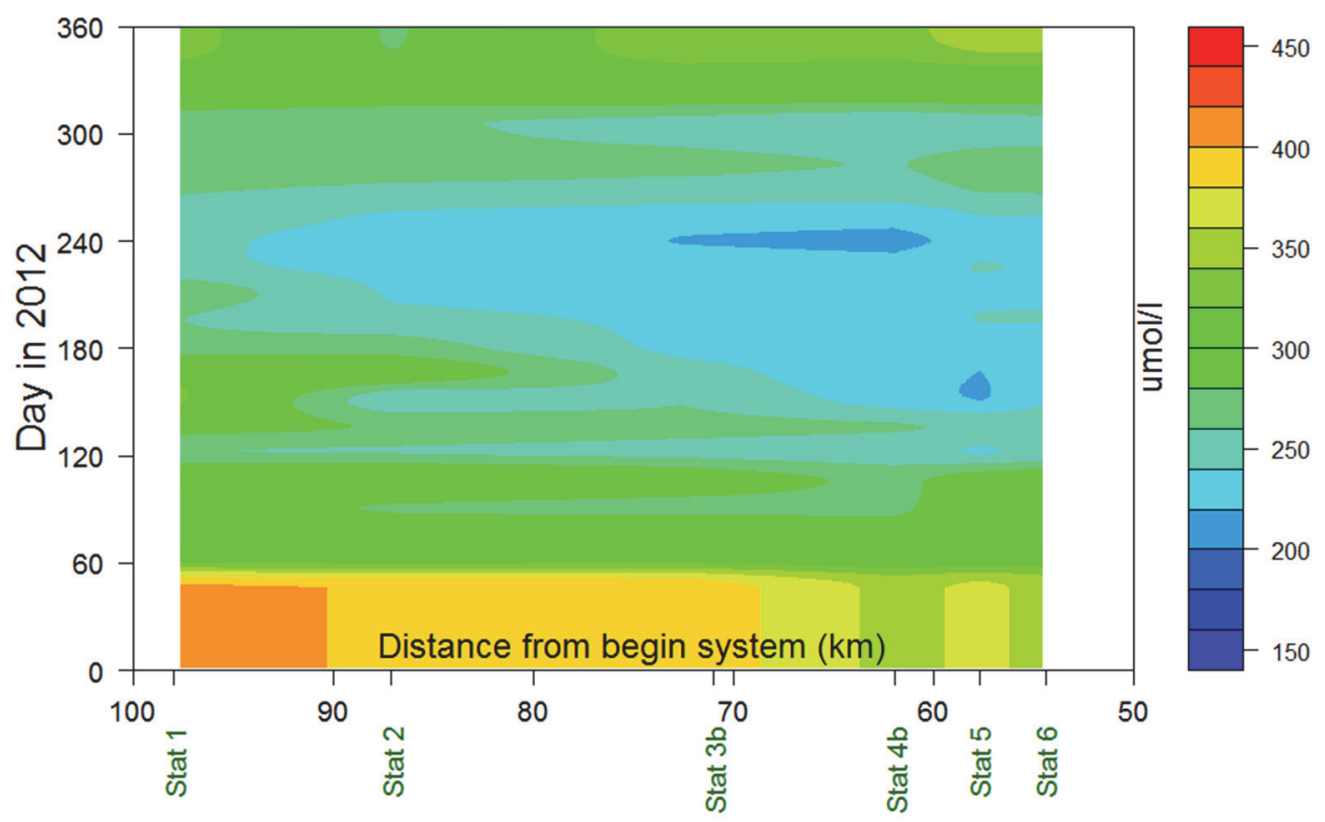

Figure 133 Oxygen observations in 2012-2013 during the IMARES research by Riegman et al, reported by brinkman et al (2015). Horizontal axis: distance from Herbrum (left=North Sea). Station nrs and km-values are shown. Vertical axis is time (from 1 Jan 2012 (lower) to 31 dec 2013 (upper). "PBox" stands for PocketBox, the instrument used. Data in umol/l (== $\left.\mathrm{mmol} \mathrm{m} \mathrm{m}^{-3}\right)$. Color patterns differs from Figure 132. 


\section{A25.6 Dissolved inorganic phosphorus ortho-P}

\section{A25.6.1 Introduction}

Dissolved phosphorus data show that the Ems-Dollard system substantially differs from e.g. the Western Dutch Wadden Sea. Where in most fresh and marine systems highest ortho-P values are found during the winter season, and lowest at the end of the first growing season (mostly around April) one can observe that in the Dollard and its tributaries highest ortho-P values are found in summer.
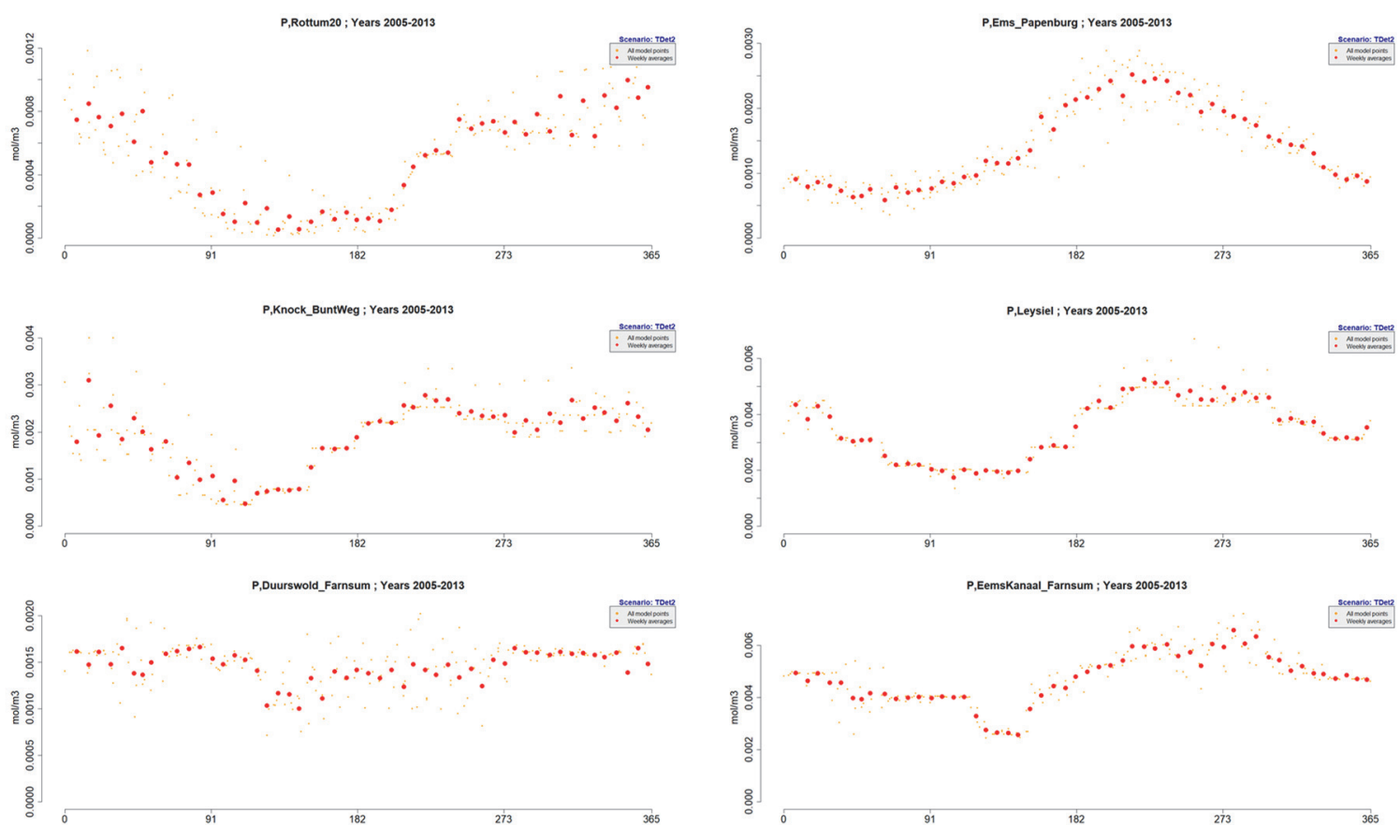

Figure 134 Concentrations of ortho-P in a number of boundaries. Upper left: North Sea coastal zone: showing a 'normal' pattern with highest values in winter, and lowest in April-May. Upper right: the Ems-river at Papenburg, with highest values in summer, and lowest in winter \& early spring. The other examples are a bit in-between.

The reason for this pattern is that [o-P] is not governed by adsorption (in winter) and consumption by primary production plus desorption (in spring), but by mineralization of organic matter. This merely takes place at higher temperatures, and thus in summer.

For some reason, it appears that phosphorus dynamics in this system is difficult to model.

One part of that difficulty obviously is related to the description of adsorption of phosphate onto sediment particles. Two model situations provide extremes: one with adsorption to solids, and one without; both without further tuning, so it concerns early simulations (not the final one discussed in this appendix). In Figure 136, both situations are illustrated, with the one in the Dollard area as extreme regarding the misfit between computed and observed $[\mathrm{P}]$ with adsorption included. Without adsorption, the misfits change into the opposite direction. So, obviously, adsorption is overestimated in the present model. Figure 135 gives a better sight on what is happening: with adsorption switched on, computed autumn-winter concentrations are far below the observations, a result of a too strong adsorption. The opposite occurs when adsorption is absent in the model. Changing the adsorption parameters, however, only slightly affects the [o-P] in the water column. At this moment it is not well understood why this happens. 
The real situation must be somewhere in between, and the target for next research is to find proper adsorption parameters, ór finding better estimates for the amount of adsorbent present (which also is a possible cause).
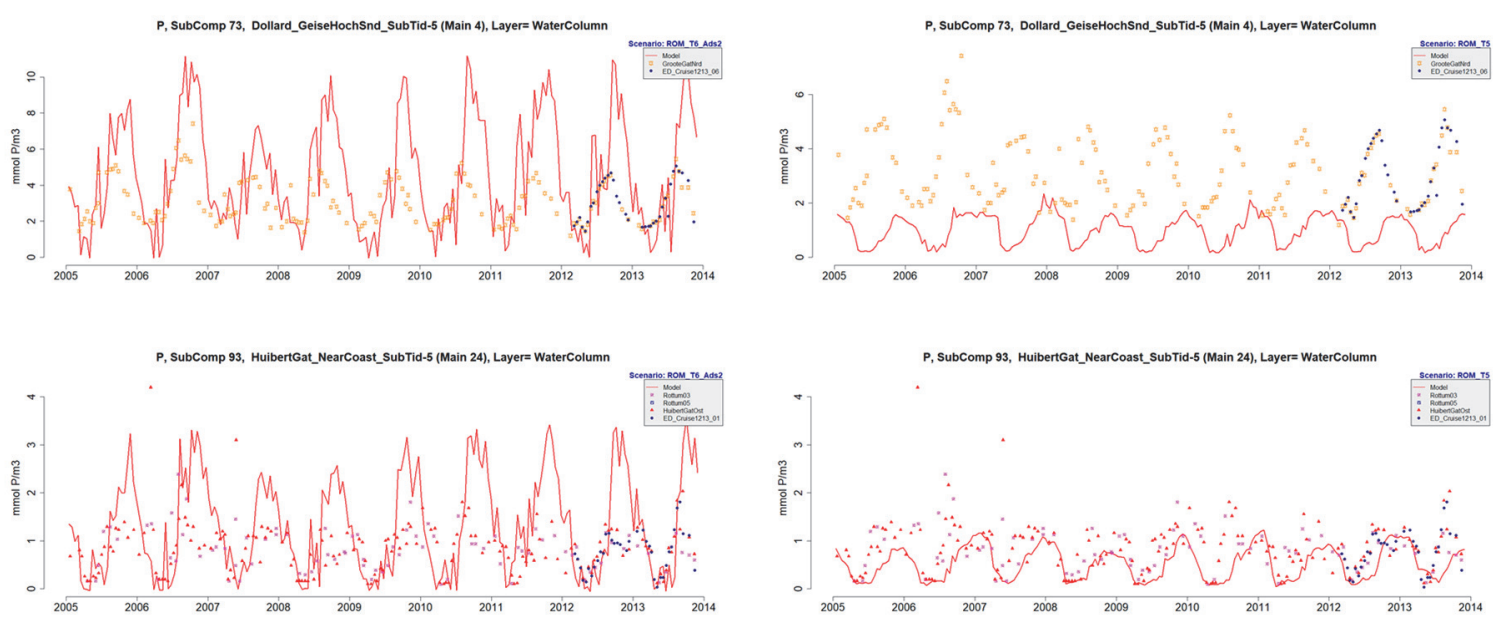

Figure 136 Effect of excluding (left) and including (right) adsorption of phosphate onto sediment particles. $[o-P]$ in the water column of two compartments, see Table 2 and Figure 38 for situation of subcompartments.

P, SubComp 81, WesterEems_SubTid-5 (Main 12), Layer= WaterColumn ; Years 2005-2013

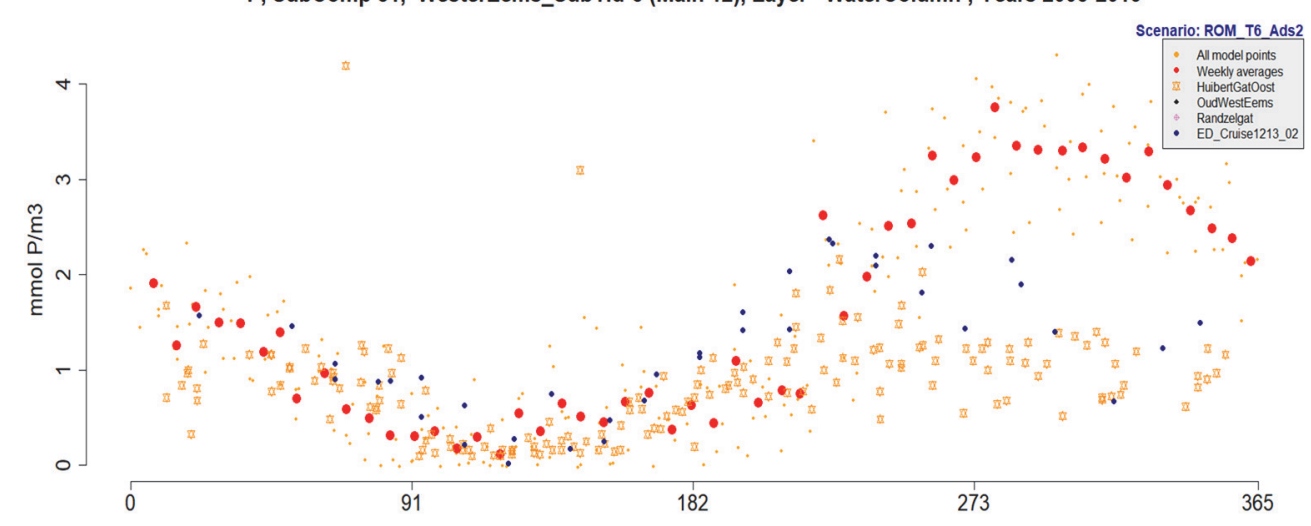

P, SubComp 81, WesterEems_SubTid-5 (Main 12), Layer= WaterColumn ; Years 2005-2013

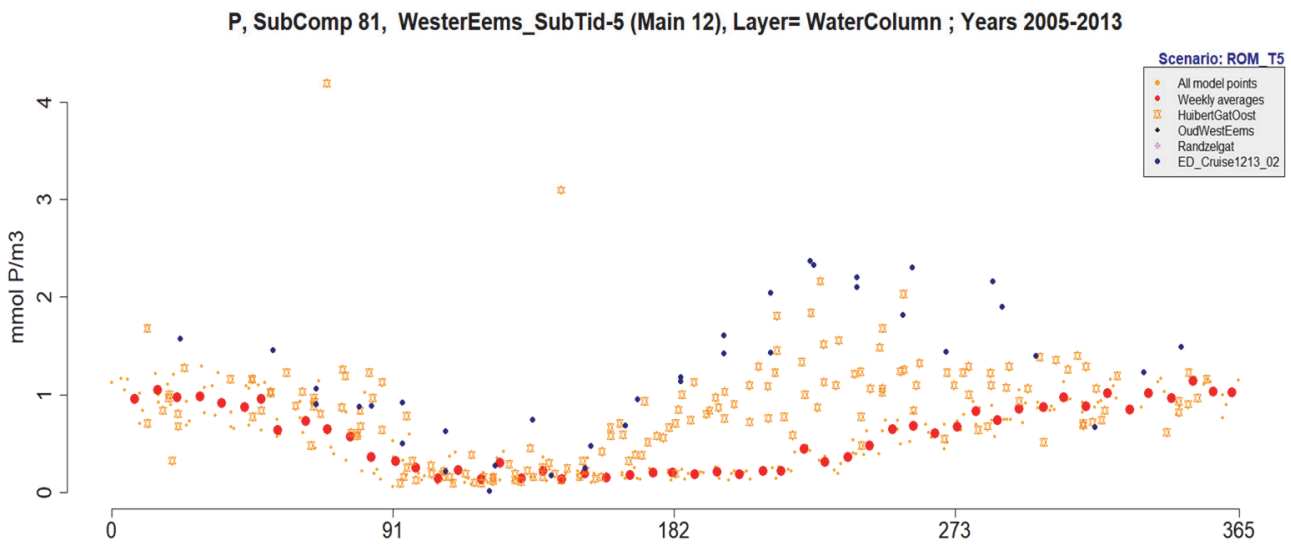

Figure 135 Details for main compartment 112 (Westerems) for the situation without (upper) and with adsorption (lower). In spring and summer, concnetrations are reprocued well, but in autumn the observations are well below the model results when adsorption is omitted, and well above when adsorption is switched on. 


\section{A25.6.2 Time series}

Final simulation results for this report are shown in Figure 137. It must be concluded that the remarks made before still cover the present simulation results: a closer look at the adsorption properties is needed.
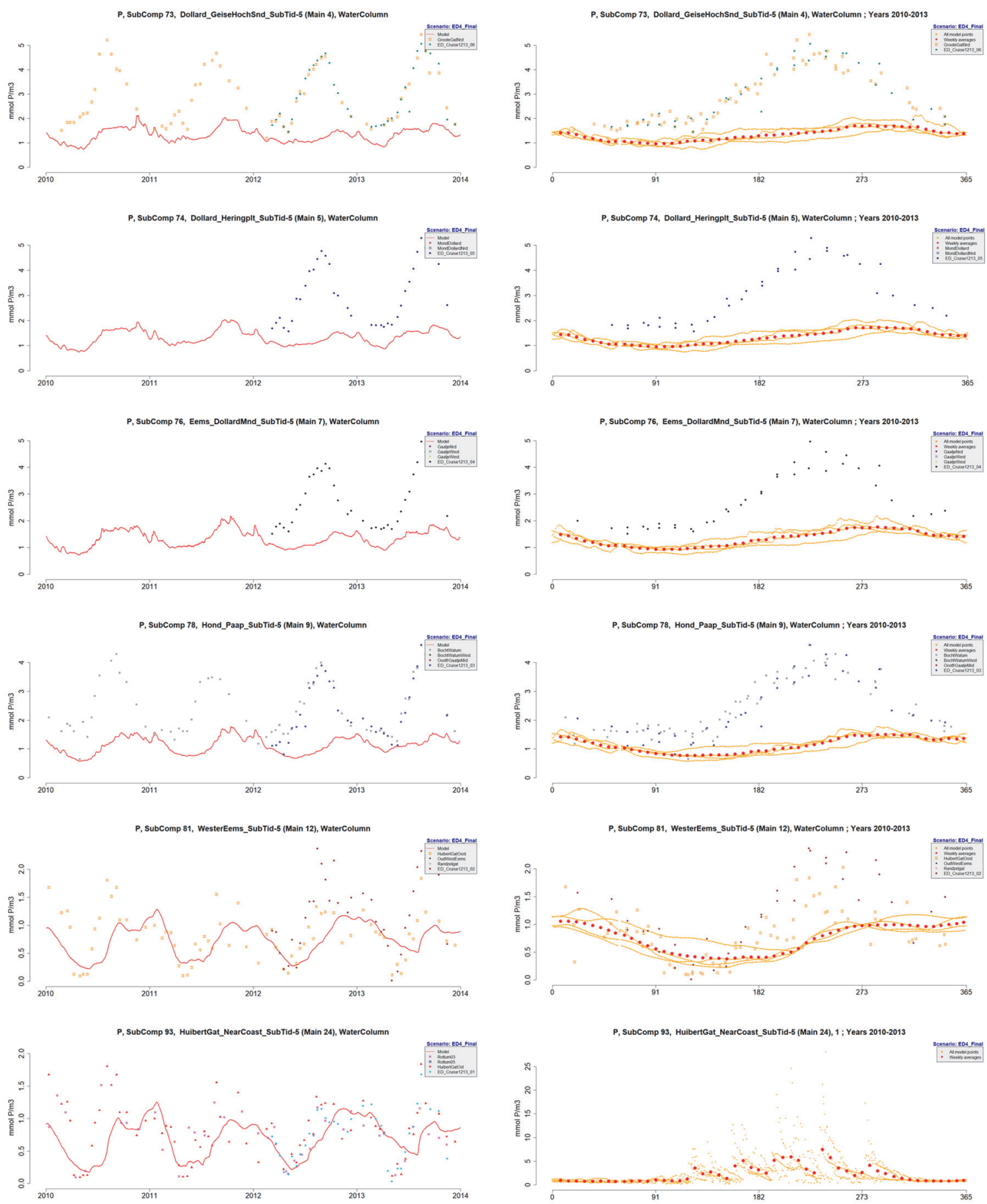

Figure 137 Ortho-phosphate concentrations in the system $\left(\mathrm{mmol} \mathrm{m}^{-3}\right)$. Upper: Dollard mouth, main compartment 7, 2nd: Hond \& Paap (main compartment 9. 3rd where Ems rivers enters the system. 4th Hond \& Paap. 5th: boundary Westerems and Doekegat. 6th (lowest) boundary of Westerems and Huibert Gat. Left 2010-2013, line=simulation, dots data. Right: average seasonal pattern; large red dots give average values; orange dots present all simulated values. Data as left panels. Column averaged (completely mixed water) 


\section{A25.6.3 Spatial distribution}

During the 2012-2013 IMARES-research, phosphate patterns along the system axis have been recorded, see Figure 133. Model results are shown in (with remarks made before on the misfit between observations and model results).

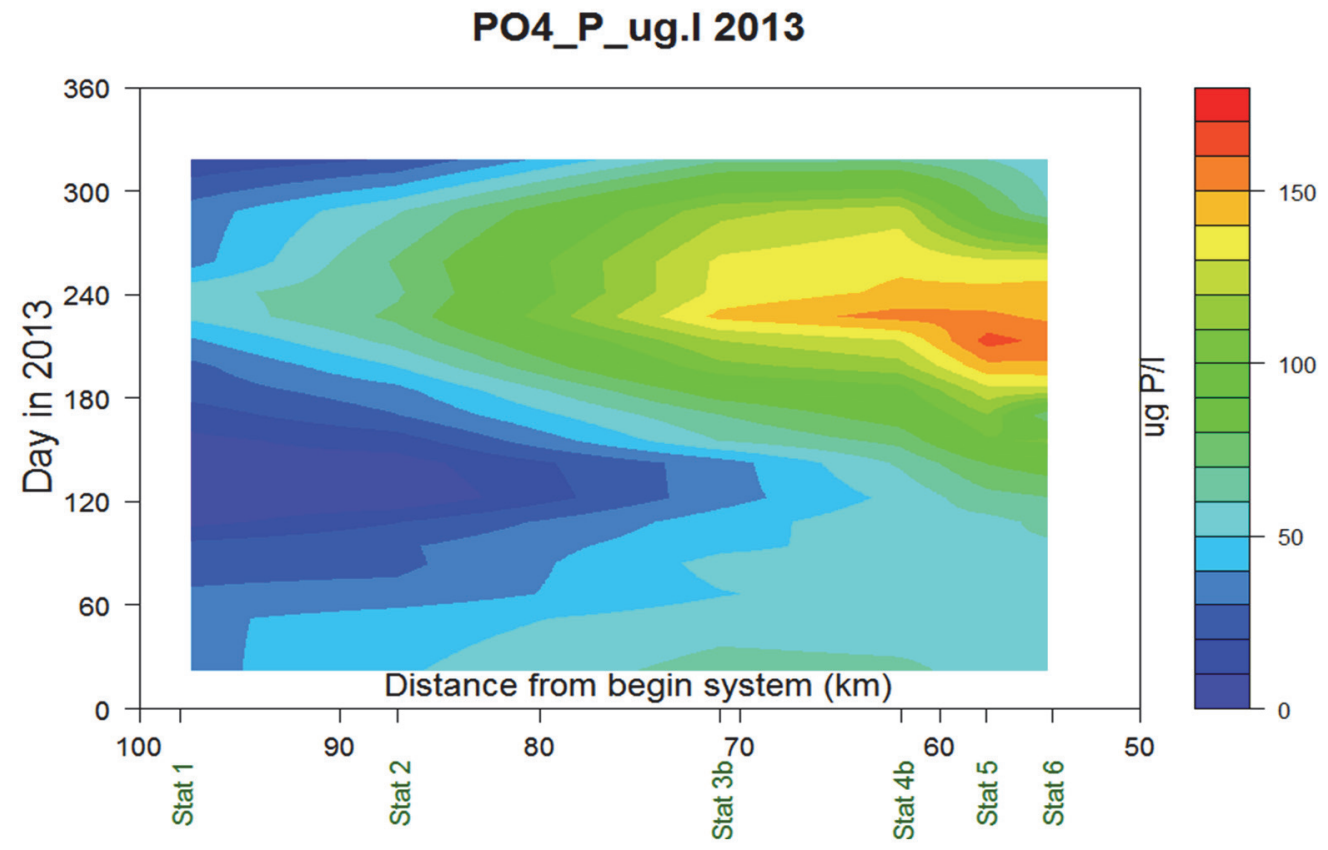

PO4_P_ug.I 2012

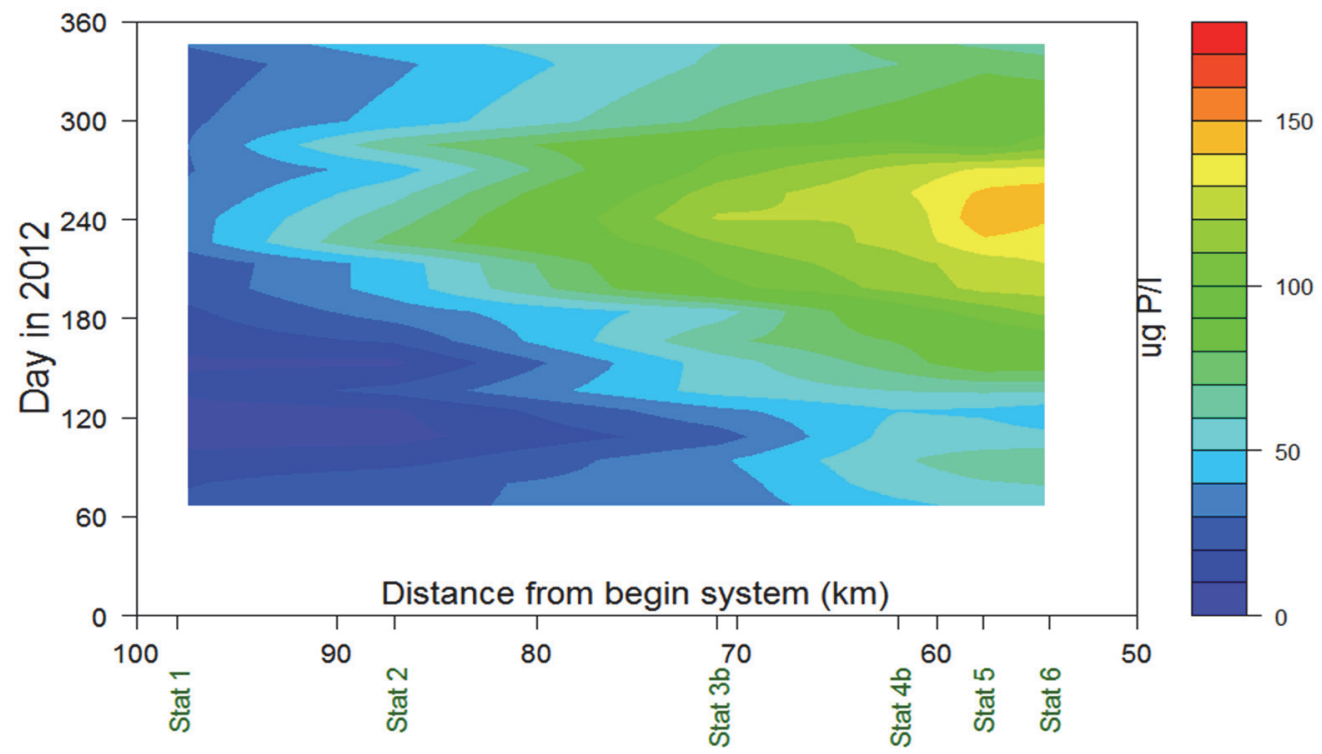

Figure 138 Ortho-phosphate observations in 2012-2013 during the IMARES research by Riegman et al, reported by Brinkman et al (2015). Horizontal axis: distance from Herbrum (left=North Sea). Station nrs and km-values are shown. Vertical axis is time (from 1 Jan 2012 (lower) to 31 dec 2013 (upper). Values in ug P $\mathrm{dm}^{-3}$. Maximum values clearly in late summer. 


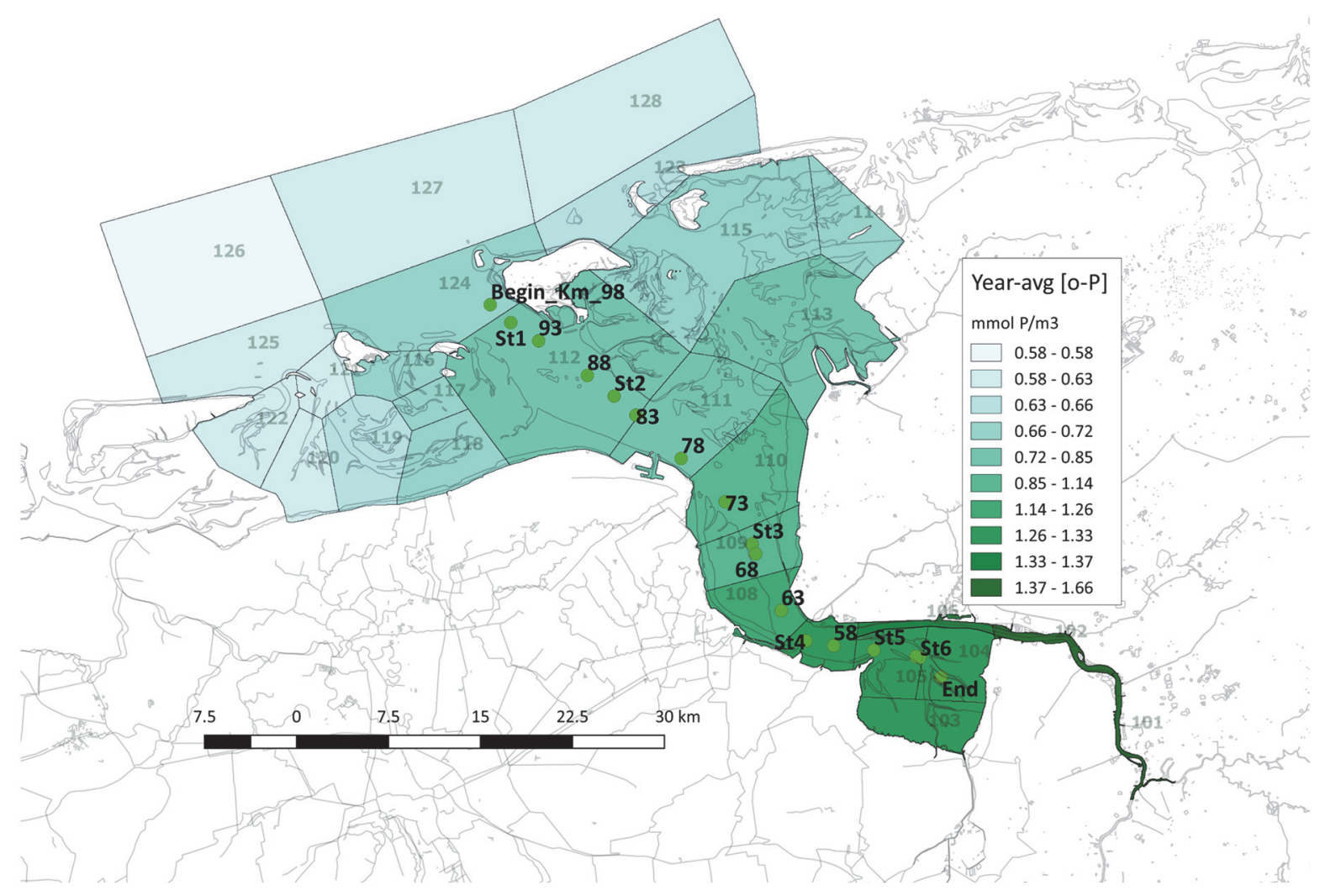

Figure 139 Spatial distribution $P$ concentration $\left(\mathrm{mmol} \mathrm{P}^{-3}\right.$ ), averaged over whole 2010-2013 period. Sampling station numbers shown. Station positions ( $k m s$ from Herbrum) marked, see also Figure 138 (color pattern differs). Transformation of values: $1 \mathrm{mmol} \mathrm{m}^{-3}$ equals $31 \mu \mathrm{g} \mathrm{P{ } ^ { - 1 }}$.

\section{A25.6.4 $P$ in pore water and adsorption in sediment top layer}

In Figure 140, simulated values of $[P]$ in pore water and the amount of adsorbed P. According to the simulations, adsorbed $\mathrm{P}$ is almost two-three orders of magnitude higher than the dissolved part. An idea of the simulated seasonal variation is given in Figure 141. Porewater $P$ follows the adsorbed amount almost exactly, a result of the fast adsorption/desorption process.

Since further data lack, further discussion is omitted here.

\section{A25.6.5 Suggestions and possible improvements}

- Find better adsorption parameters

- Compare computed water sediment fluxes with available observations (from other parts of the Wadden Sea or even the North Sea coastal zone) 

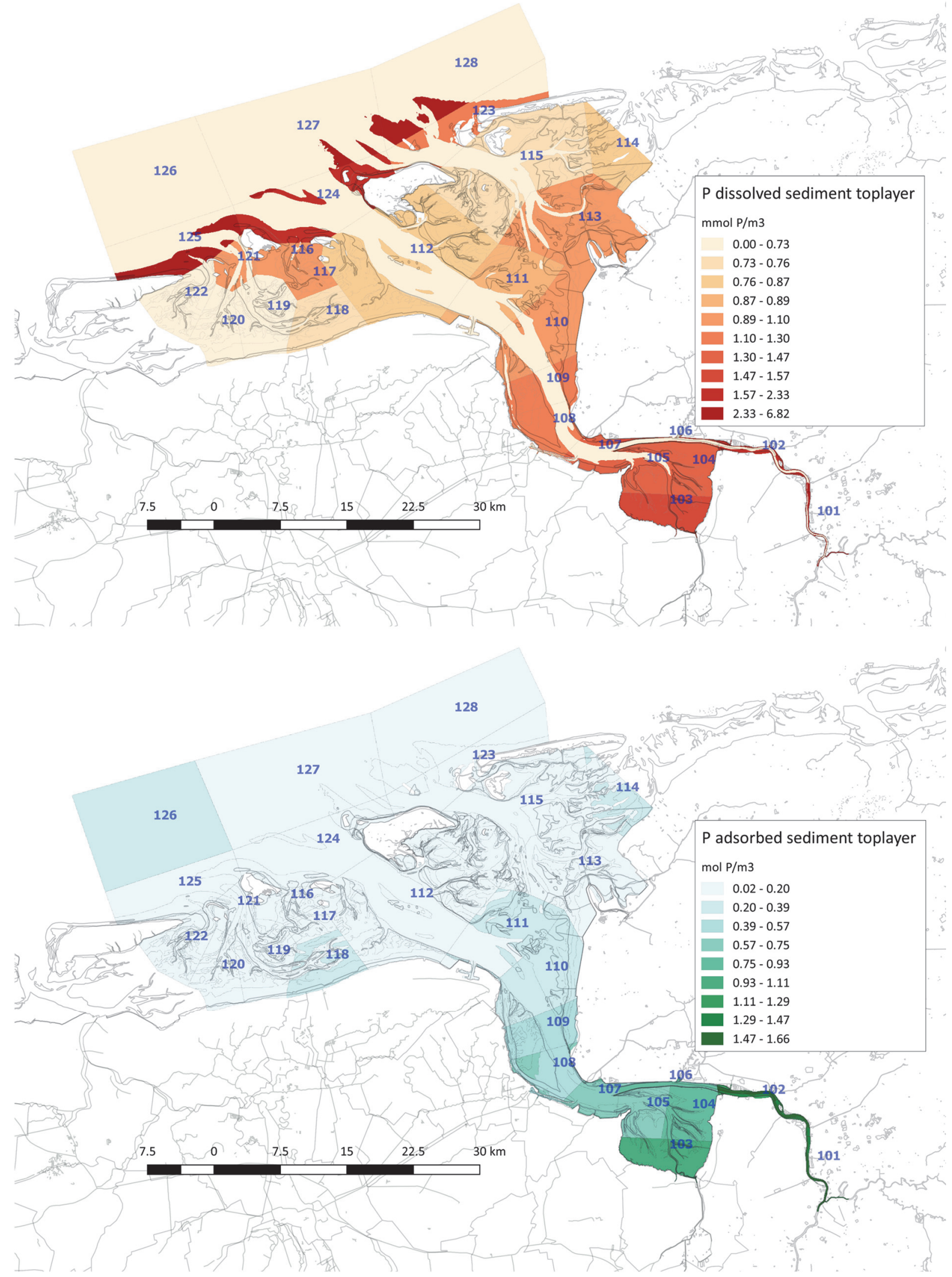

Figure 140 Sediment $P$. Upper: computed $[P]$ in pore water of the sediment top layer (in $\mathrm{mmol}^{\mathrm{P} \mathrm{m}^{-3}}$ ). Lower:

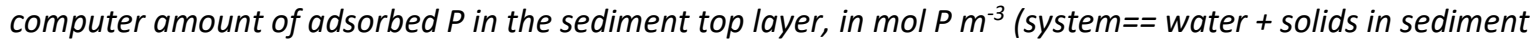
top layer). Thus, adsorbed $P$ in the sediment top layer is 2-3 orders higher than dissolved $P$. 
P, SubComp 78, Hond_Paap_SubTid-5 (Main 9), 1

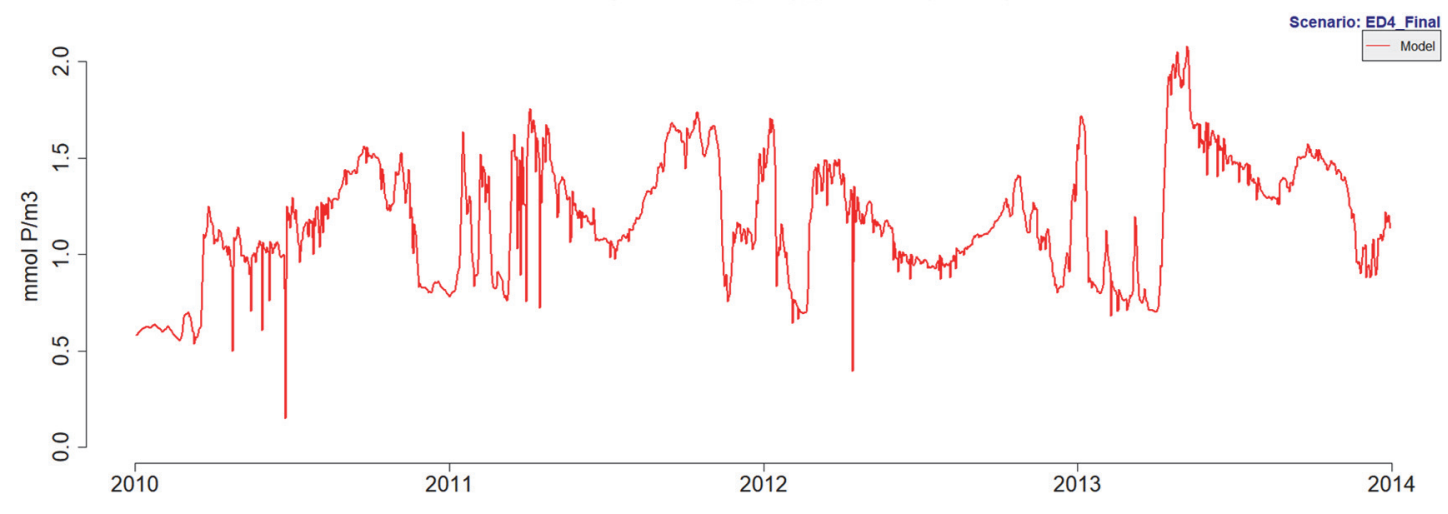

P, SubComp 78, Hond_Paap_SubTid-5 (Main 9), 1

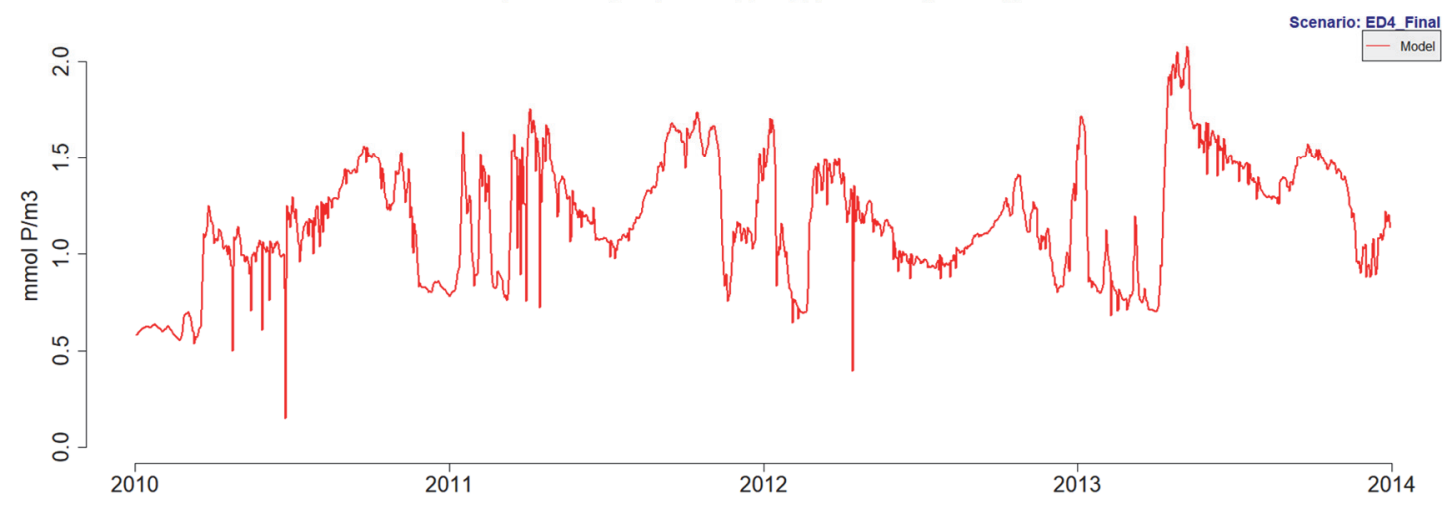

Figure 141 Seasonal variation in simulated pore water dissolved $P$ (upper) and adsorbed $P$ (lower) at subcompartment 78 (main compartment 109). Max-min difference is almost half the maximum values. Data in mmol $P m^{-3}$ (pore water volume) for dissolved $P$ and $P m^{-3}$ (system volume) for adsorbed $P$. 


\section{A25.7 Silicate}

\section{A25.7.1 Introduction}

The difficulties that arose since there were hardly data available for the fresh water sources have already been mentioned in section 3.3.6; consequently, all simulation results depend on the assumption made for this model. Thus, the results partly reflect the choice of the input values. Stolte et al (2015) applied a linear extrapolation of [Si]-observations and salinity towards zerosalinity. It is a possibility as well, although in that case any local process effect is neglected.

Also, like the situation for phosphorus, adsorption plays a role, although silicate adsorption onto solid particles is less strong than that of phosphate. And thus, less effect is to be expected.
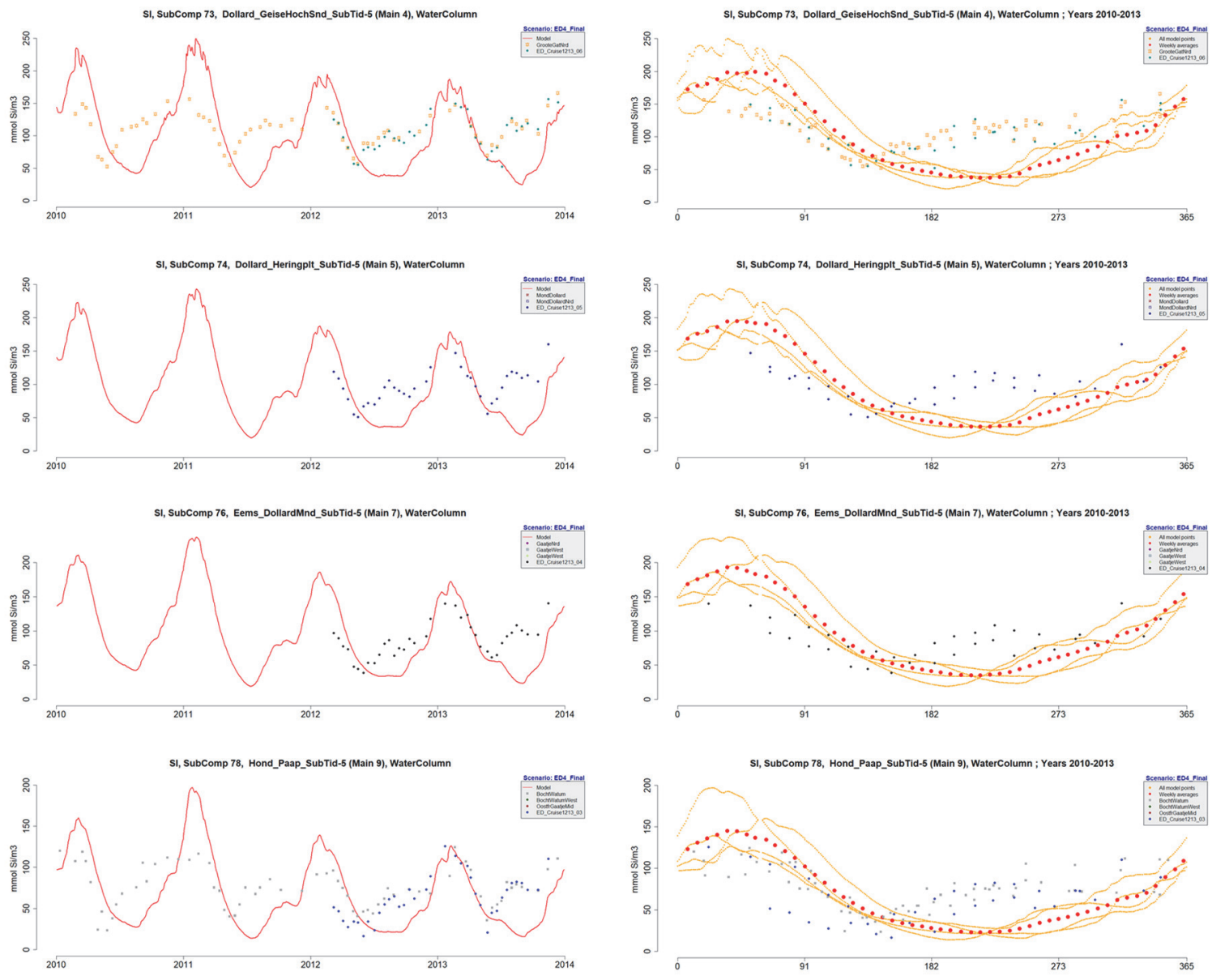

Figure 142 Silicate concentrations in the system $\left(\mathrm{mmol} \mathrm{m}^{-3}\right)$. Upper: Dollard mouth, main compartment 7, 2nd: Hond \&Paap (main compartment 9). 3rd where Ems rivers enters the system. 4th Hond \& Paap. Left 2010-2013, line=simulation, dots data. Right: average seasonal pattern; large red dots give average values; orange dots represent all simulated values. Data as left panels. Column averaged (completely mixed water). Continued in Figure 143

\section{A25.7.2 Time series}

For silicate, a similar response as the one for phosphate might be expected (due to the adsorption behaviour), but here the model computes a too large seasonal variation. Especially (late) summer and autumn values are underestimated. Here, the character of the silicate release process may be 

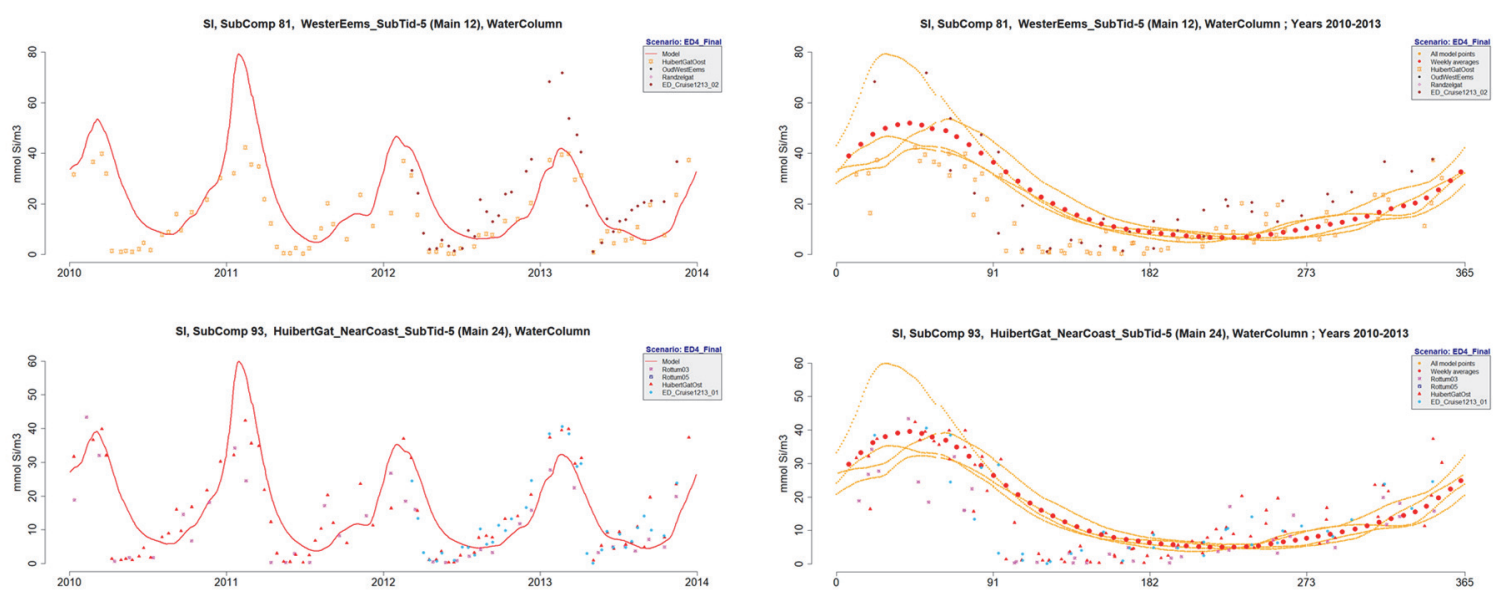

Figure 143 Silicate concentrations in the system $\left(\mathrm{mmol} \mathrm{m}^{-3}\right)$. Upper: boundary Westerems and Doekegat. Lower: boundary of Westerems and Huibert Gat.

modelled not completely correct. This is merely a dissolution of the remaining frustules than a product of microbiological mineralization. May be this process is faster than modelled.

\section{A25.7.3 Spatial distribution}

During the 2012-2013 IMARES-research, dissolved silicate has been monitored as well (Figure 145). Comparing with simulated values (Figure 144) is hard, but at least patterns like a bit alike.

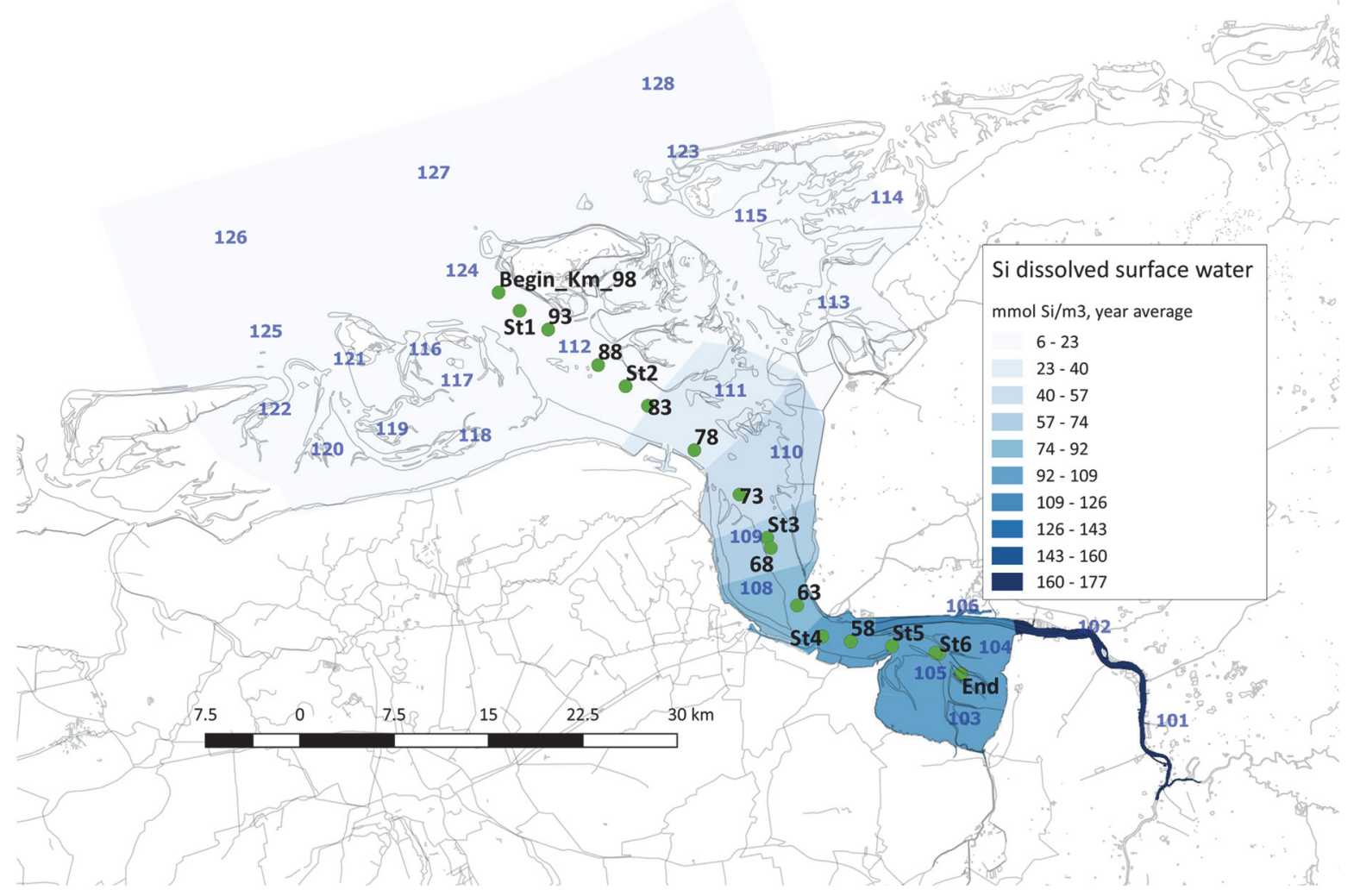

Figure 144 Spatial distribution Si concentration ( $\mathrm{mmol} \mathrm{Si} \mathrm{m}^{-3}$ ), averaged over whole 2010-2013 period. Sampling station numbers shown. Station positions (kms from Herbrum) marked, see also Figure 145 (color pattern differs). Transformation of values: $1 \mathrm{mmol} \mathrm{Si} \mathrm{m}^{-3}$ equals $28.1 \mathrm{mg} \mathrm{Sil}^{-1}$. 


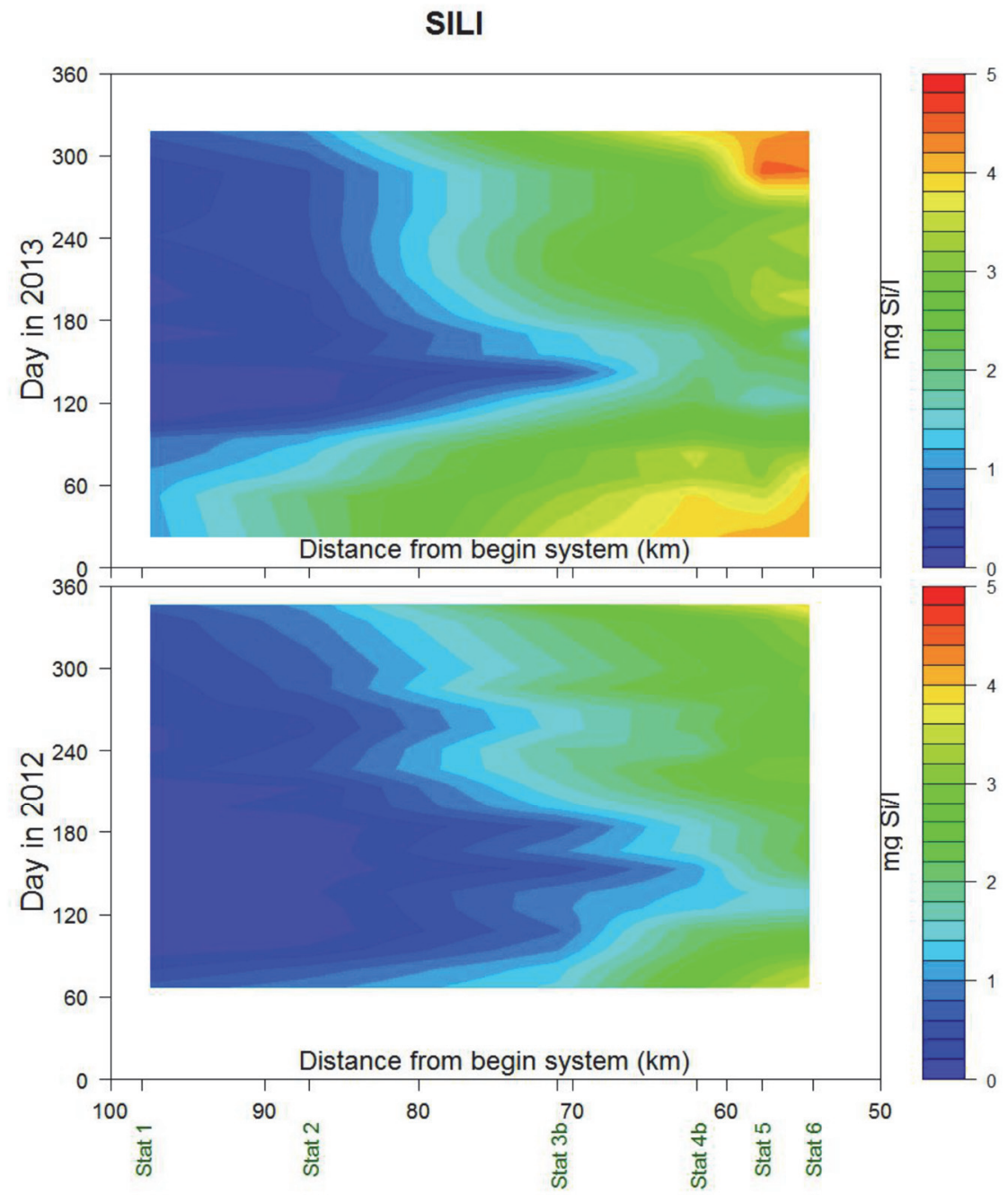

Figure 145 Dissolved silicate observations in 2012-2013 during the IMARES research by Riegman et al, reported by Brinkman et al (2015). Horizontal axis: distance from Herbrum (left=North Sea). Station nrs and km-values are shown. Vertical axis is time (from 1 Jan 2012 (lower) to 31 dec 2013 (upper). Values in $\mathrm{mg} \mathrm{Si} \mathrm{dm}^{-3}$. Maximum values in winter.

\section{A25.7.4 Simulated silicate pore water concentrations and adsorbed silicate}

In Figure 146, pore water concentrations and adsorbed silicate, as simulated, are presented. Ratio of adsorbed and dissolved $\mathrm{Si}$ is about one order (to two) of magnitude, so much smaller than for phosphate. This is a consequence of the lesser adsorption strength of silicate, see e.g. Sigg (1979) and Breeuwsma (1973). As a result, the seasonal variations are larger than for $P$.

\section{A25.7.5 Suggestions and possible improvements}

- $\quad$ Find better parameter values for frustule dissolution 

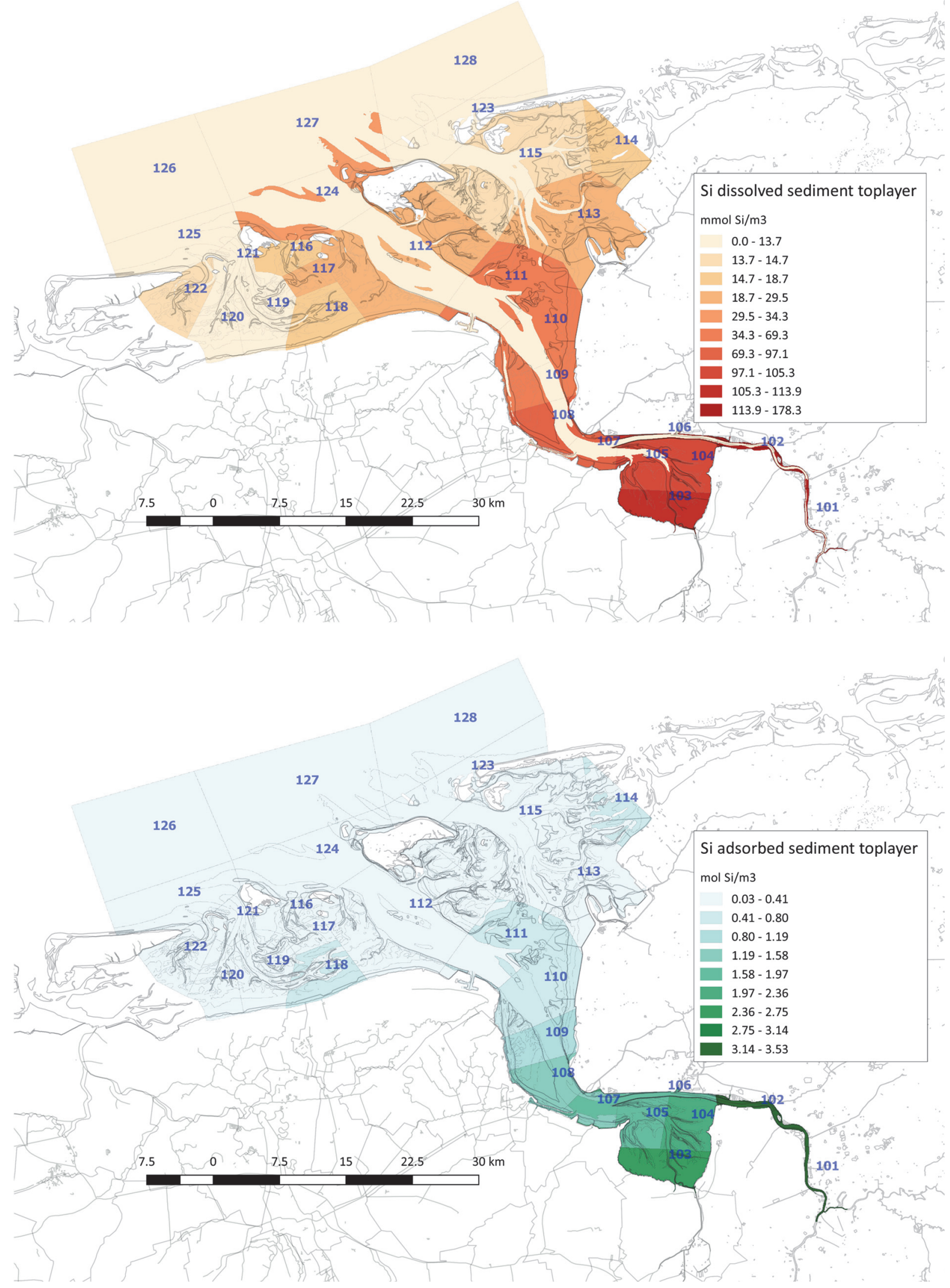

Figure 146 Sediment Si. Upper: computed [Si] in pore water of the sediment top layer (in mmol Si $\mathrm{m}^{-3}$ ). Lower: computer amount of adsorbed Si in the sediment top layer, in mol Si $\mathrm{m}^{-3}$ (system== water + solids in sediment top layer). Thus, adsorbed Si in the sediment top layer is roughly 1 order of magnitude higher than dissolved Si. 


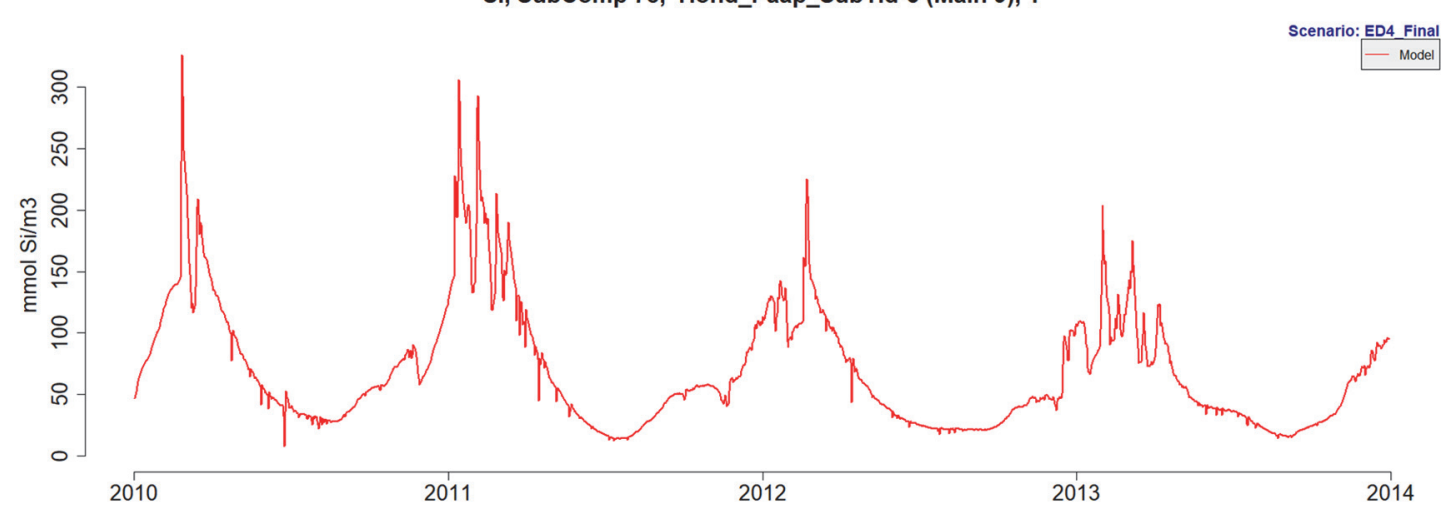

Adsorbed-SI, SubComp 78, Hond_Paap_SubTid-5 (Main 9), 1

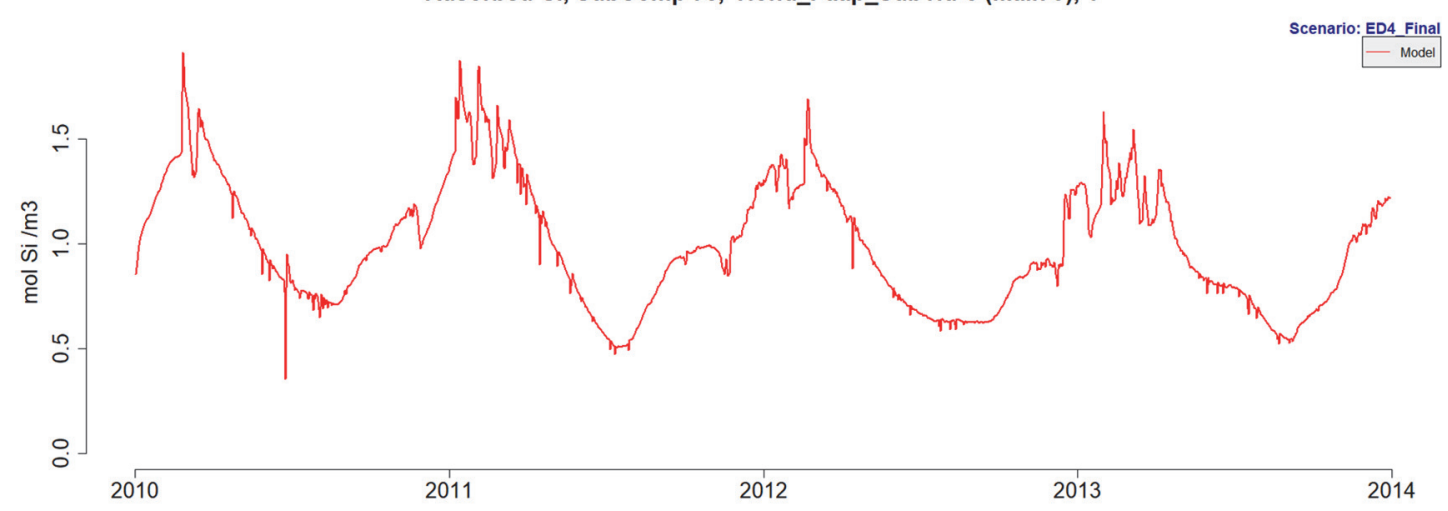

Figure 147 Seasonal variation in simulated adsorbed Si (upper) and pore water dissolved Si (lower) at subcompartment 79 (main compartment 110). Max-min difference is almost 4/5 the maximum values for

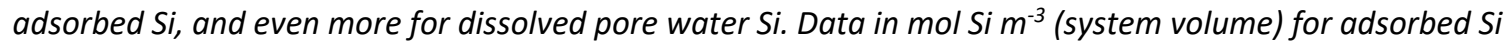
and $\mathrm{mmol} \mathrm{m}^{-3}$ pore water for dissolved Si.

\section{A25.8 Nitrate NO3}

\section{A25.8.1 Introduction}

Nitrate dynamics is related to phosphorus dynamics (as are most of the substances discussed here), since the phytoplankton activity is affected by phosphorus, and thus the use of ammonium and nitrate for production of cells, and, later, of nitrate for the oxidation of dead organic matter. See Figure 148 for an example; both concern preliminary simulations. It would be beyond this report to discuss this in detail here. Here, presentation of results is restricted to observations and related mode results. 

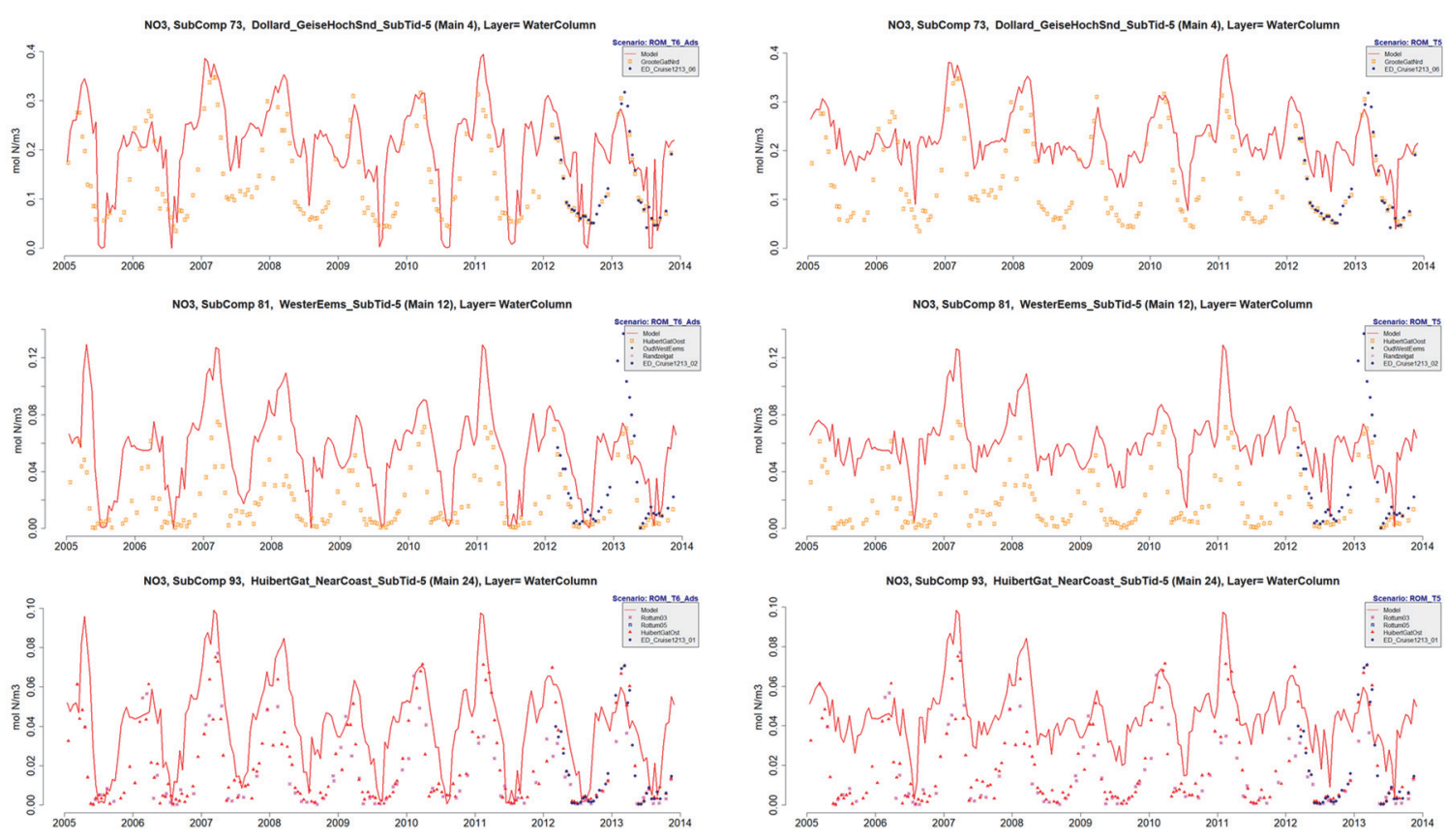

Figure 148 Effect of excluding (left) and including (right) adsorption of phosphate onto sediment particles. [nitrate] in the water column of three compartments, see Table 4 and Figure 20 for situation of subcompartments. One of the preliminary computations shown.

\section{A25.8.2 Time series}

Time series for six compartments are shown in Figure 149. The results indicate that in all compartments shown, simulated values are above observed ones. This is especially the case in the summer. Following Figure 148, a more correct phosphate adsorption description may improve the results a bit, but also, adsorption probably has most effect in the shallow areas, thus the Dollard, and less in the deeper areas. Thus, it is not expected that this will improve results considerably. Here, it must be mentioned that previous simulations showed considerably better results. Better results are needed.

\section{A25.8.3 Spatial distribution}

In Figure 151, observations during the 2012-2013 IMARES-research are shown. Comparing the results with the simulation results (Figure 150) is not easy, but patterns are more or less similar.

\section{A25.8.4 Suggestions and possible improvements}

- Find better nitrification rate parameters (and probably also: descriptions of the process)

- Check why these results differ from the mentioned previous ones where the fit between data and model results were better.

- Check the computed sediment/water exchange (not mentioned here) with literature values.

For the latter, see also section A23.15. 

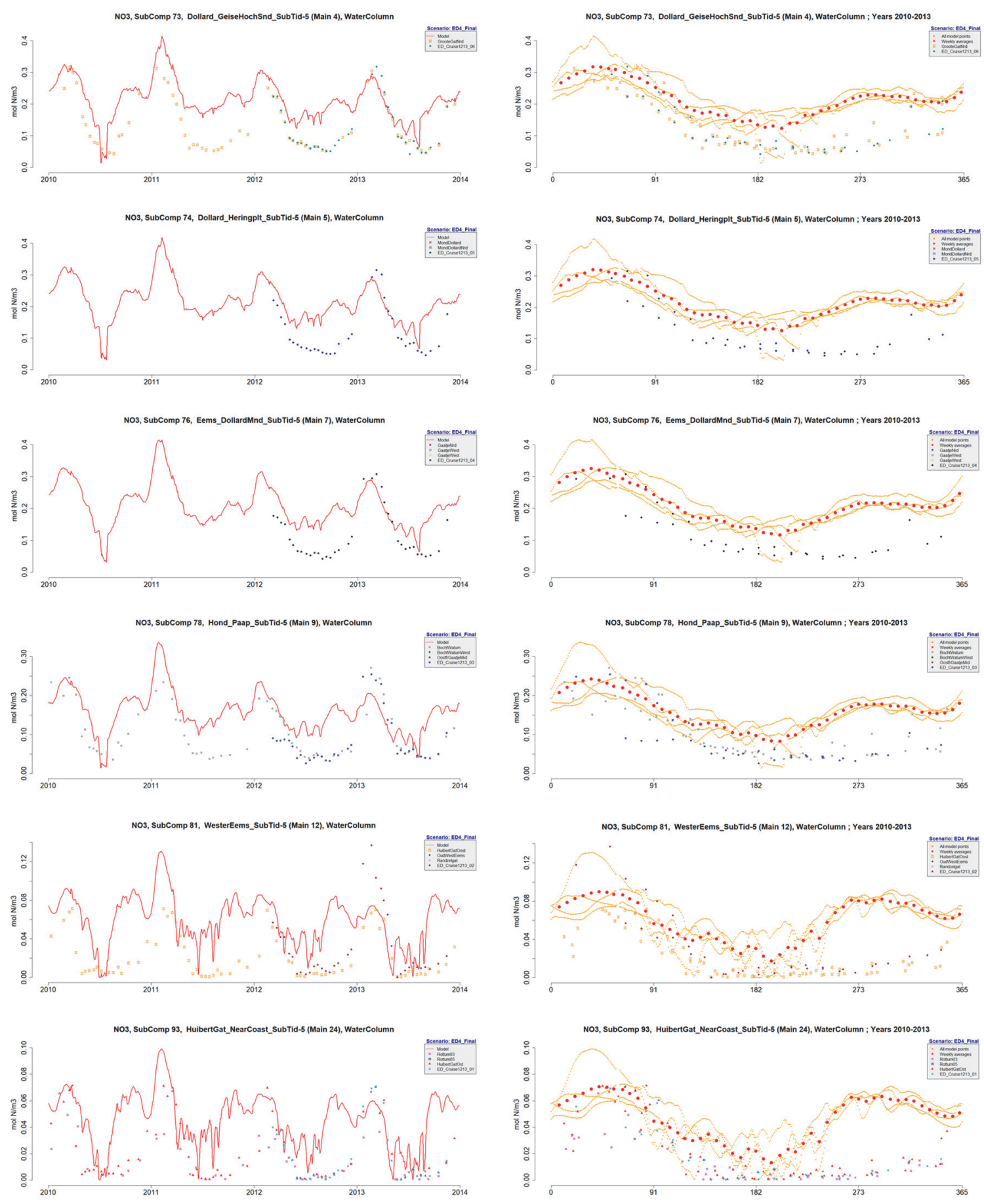

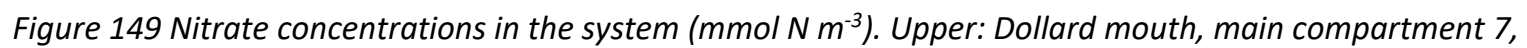
2nd: Hond \&Paap (main compartment 9). 3rd where Ems rivers enters the system. 4th Hond \& Paap. 5th: boundary Westerems and Doekegat. 6th (lowest) boundary of Westerems and Huibert Gat. Left 2010-2013, line=simulation, dots data. Right: average seasonal pattern; large red dots give average values; orange dots represent all simulated values. Data as left panels. Column averaged (completely mixed water). 


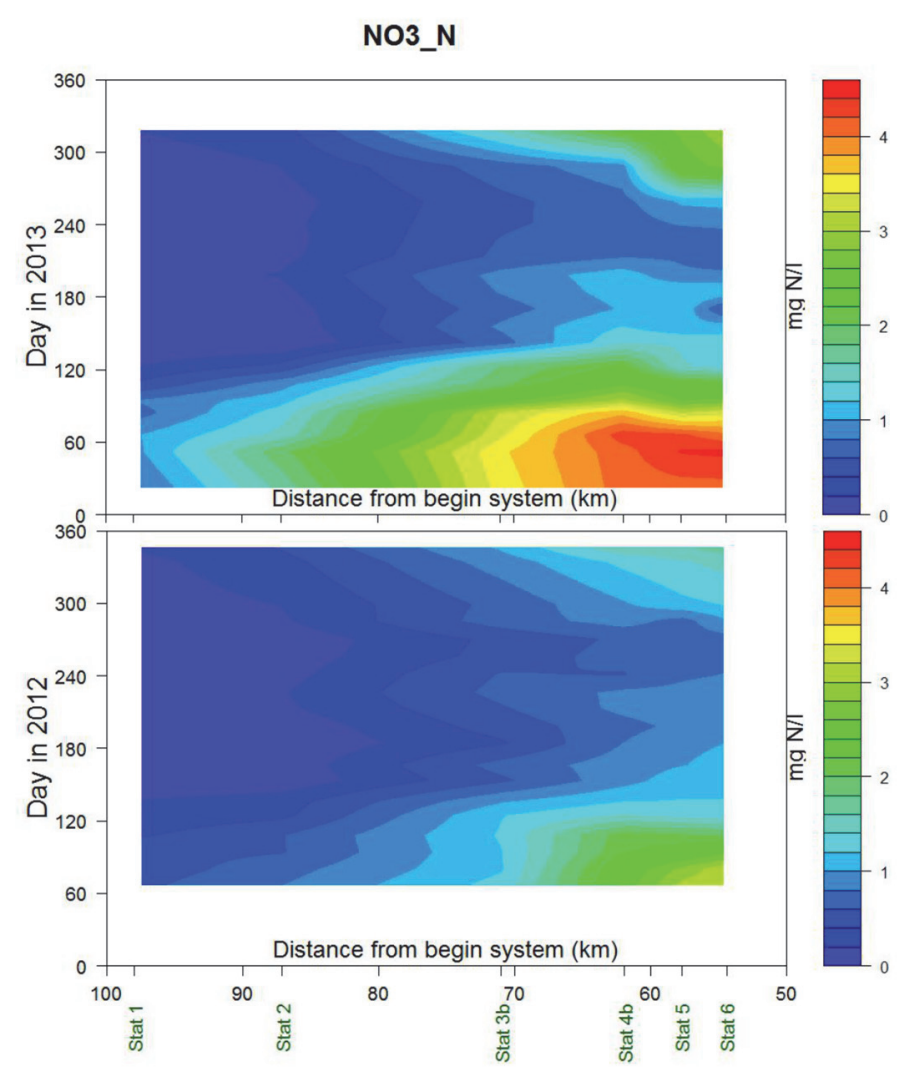

Figure 151 Nitrate observations in 2012-2013 during the IMARES research by Riegman et al, reported by Brinkman et al (2015). Horizontal axis: distance from Herbrum (left=North Sea). Station nrs and km-values are shown. Vertical axis is time (from 1 Jan 2012 (lower) to 31 dec 2013 (upper). Values in mg NO3-N dm ${ }^{-3}$. Maximum values clearly in winter.

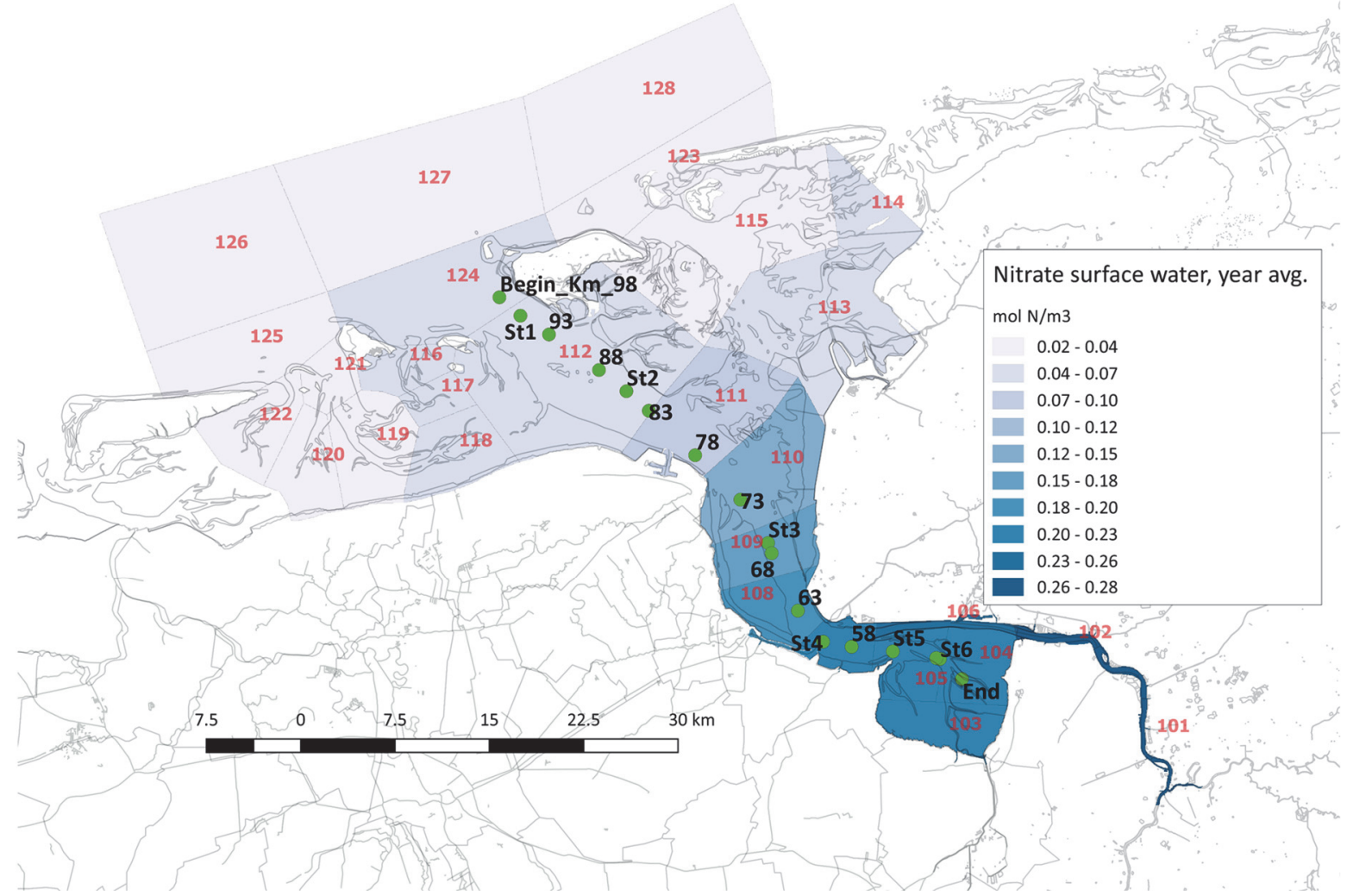

Figure 150 Spatial distribution nitrate concentration $\left(\mathrm{mol} \mathrm{N} \mathrm{m}^{-3}\right)$, averaged over whole 2010-2013 period. Sampling station numbers shown. Station positions (kms from Herbrum) marked, see also Figure 151(color pattern differs). Transformation of values: $1 \mathrm{mmol} \mathrm{N} \mathrm{m}^{-3}$ equals $14 \mathrm{mg}_{\text {Nitrate- }} \mathrm{N} \mathrm{I}^{-1}$. 


\section{A25.9 Ammonium NH4}

\section{A25.9.1 Introduction}

Ammonium usually is a substance hard to model. The reason mostly is not clear, but it is the experience of many modellers. The complexity of processes that contribute to $\left[\mathrm{NH}_{4}{ }^{+}\right]$obviously is not well included in most models. At least one process was not included in the model: adsorption of ammonium onto humus particles. This $\mathrm{NH}_{4}$-adsorption is less strong than that of phosphate or silicate onto iron- and/or aluminium oxides, but since the amount of ROM (being mostly humuslike substances) is large, it might have at least some influence. It, however, is not expected to explain the largest part of the deviations.

\section{A25.9.2 Time series}

Figure 152 shows present simulation results. It is obvious that the more to the inner part of the system, the more model results and observations deviate. In the Dollard area, differences are enormous. This is the reason that further data presentation is omitted. The process of ammonium production is far overestimated in these inner areas, or oxidation/use of ammonium is underestimated. While data show lowest values in summer, model results have an opposite pattern.

To exclude a possible effect of ammonium input from the Westerwoldse $\mathrm{Aa}$, for example, that ammonium time series used is plotted in Figure 153. Maximum values of about $0.2 \mathrm{~mol} \mathrm{~m}^{-3}$ could account for part of the effects, be it not that the maximum input values are in late winter - early spring, and it concerns short during peaks, while maximum simulated values of $0.2 \mathrm{~mol} \mathrm{~m}^{-3}$ are in autumn and cover a much longer during period. Thus, in-system model processes are responsible for the overestimation.

May be the oxidation of ammonia is one of the processes being underestimated. However, although nitrate concentrations certainly are not overestimated in winter, the same is valid here: nitrate content is overestimated in autumn, thus, a larger ammonium oxidation would lead to even more nitrate, and that would not contribute to better nitrate simulation results. As a first guess it would be that denitrification plays a much larger role that computed now (see also appendix A25.18).

\section{A25.9.3 Suggestions and possible improvements}

- Study the effect of ammonium adsorption

- Check further what might cause the overestimation of ammonium.

- Check sediment/water exchange rates with possibly available data 

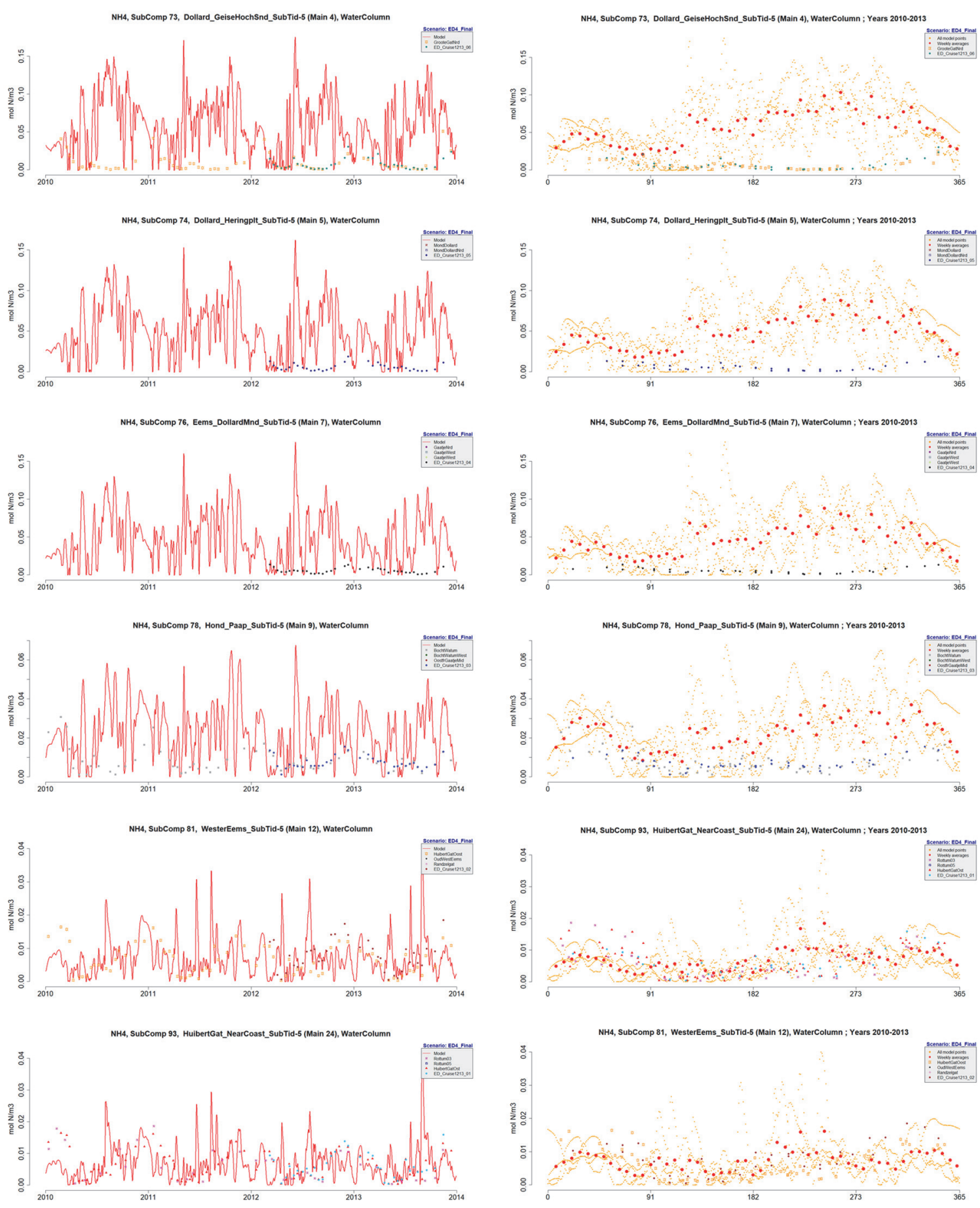

Figure 152 Ammonium concentrations in the system $\left(\mathrm{mmol} \mathrm{N} \mathrm{m}^{-3}\right)$. Upper: Dollard mouth, main compartment 7, 2nd: Hond \& Paap (main compartment 9). 3rd where Ems rivers enters the system. 4th Hond \& Paap. 5th: boundary Westerems and Doekegat. 6th (lowest) boundary of Westerems and Huibert Gat. Left 2010-2013, line=simulation, dots data. Right: average seasonal pattern; large red dots give average values; orange dots represent all simulated values. Data as left panels. Column averaged (completely mixed water). 

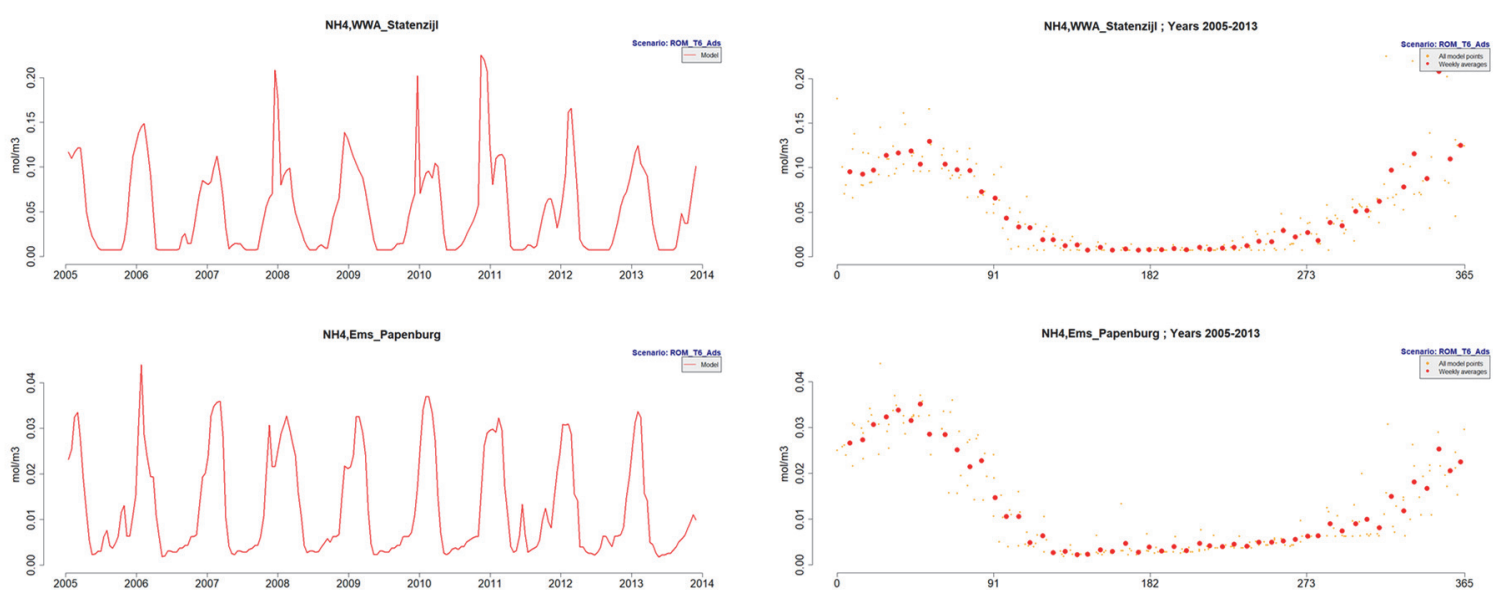

Figure 153 Input values used for $\mathrm{NH}_{4}-\mathrm{N}$ for the Westerwoldse Aa (upper) and Ems river at Papenburg (lower) (right). Maximum values in the Ems river are substantially lower than in the Westerwoldse Aa.

\section{A25.10 Pelagic chlorophyll-a}

\section{A25.10.1 Introduction}

Pelagic chlorophyll-a is not really included in the model, but computed based on an assumed chlorophyll-a/dry mass ratio. This ratio is fixed (no seasonal variation), and specific for each phytoplankton type: for each type the ratio is set a priori as model parameter. See appendix A28 for all values. On storage of the phytoplankton biomass values, chlorophyll simply is computed from these values.

\section{A25.10.2 Time series}

Results in Figure 154 show that most results fit the observations rather well, although some peaks did not appear in most data. Values for main compartments 5 and 7, however, show much higher observations than computed. This is at least partly due to the river Ems: in the river compartments also observed chlorophyll values are above the simulated ones. This demands extra attention in following research. For the Dollard compartment (73, main compartment 4$)$, the computed seasonal pattern is somewhat out of phase: maximum values are reached too early in the year, and computed summer values are too low.

The computation of pelagic chlorophyll is complicated since resuspension of benthic diatoms plays an important role, especially in the shallow Dollard area (De Jonge, 1992; De Jonge \& Van Beusekom, 1992; De Jonge \& Colijn, 1994; De Jonge et al, 2012; Wanink et al, 2014). This is illustrated by Figure 155). In the model, $1 \mathrm{~g}$ of benthic diatoms contain $9 \mathrm{mg}$ chla, thus main compartment 104 (upper figure in Figure 155) maximally contains $4 \mathrm{~g}$ benthic diatoms $\mathrm{m}^{-3}$, or about $36 \mathrm{mg}$ benthic chla $\mathrm{m}^{-3}$, which comes close to all the pelagic values computed. Data (see Wanink et al, 2014 and Brinkman et al, 2014, 2015) indicate that in this area over 50\% is of benthic origin, thus the results overestimate this situation somewhat. It does imply that wind effects and the benthic diatom patterns are partly (to merely) governing the pelagic chlorophyll values (in 

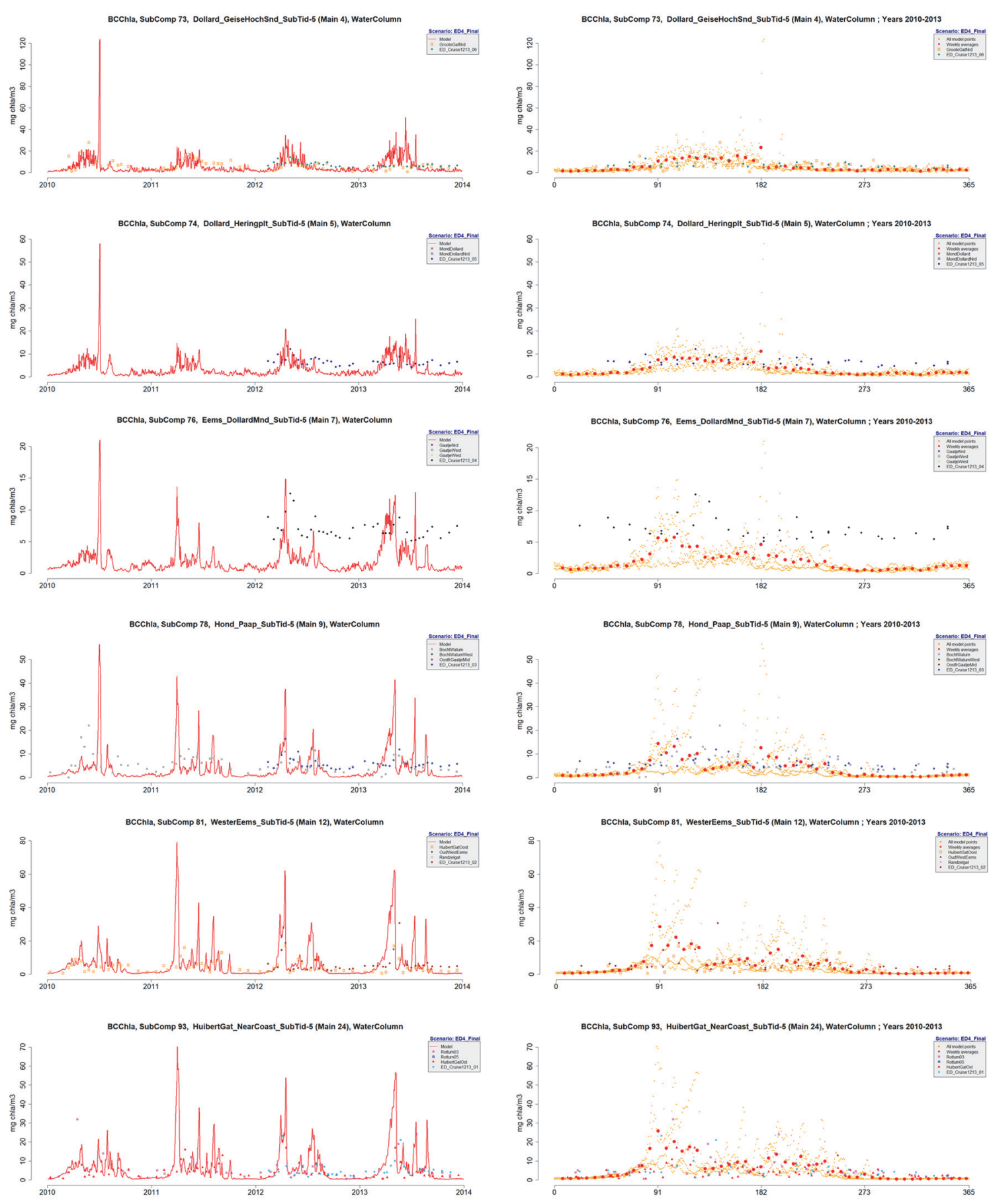

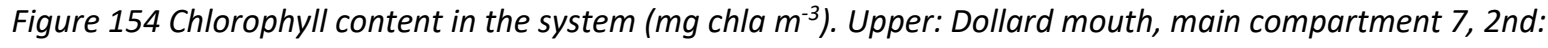
Hond \& Paap (main compartment 9). 3rd where Ems rivers enters the system. 4th Hond \& Paap. 5th: boundary Westerems and Doekegat. 6th (lowest) boundary of Westerems and Huibert Gat. Left 2010-2013, line=simulation, dots data. Right: average seasonal pattern; large red dots give average values; orange dots represent all simulated values. Column averaged (completely mixed water). 

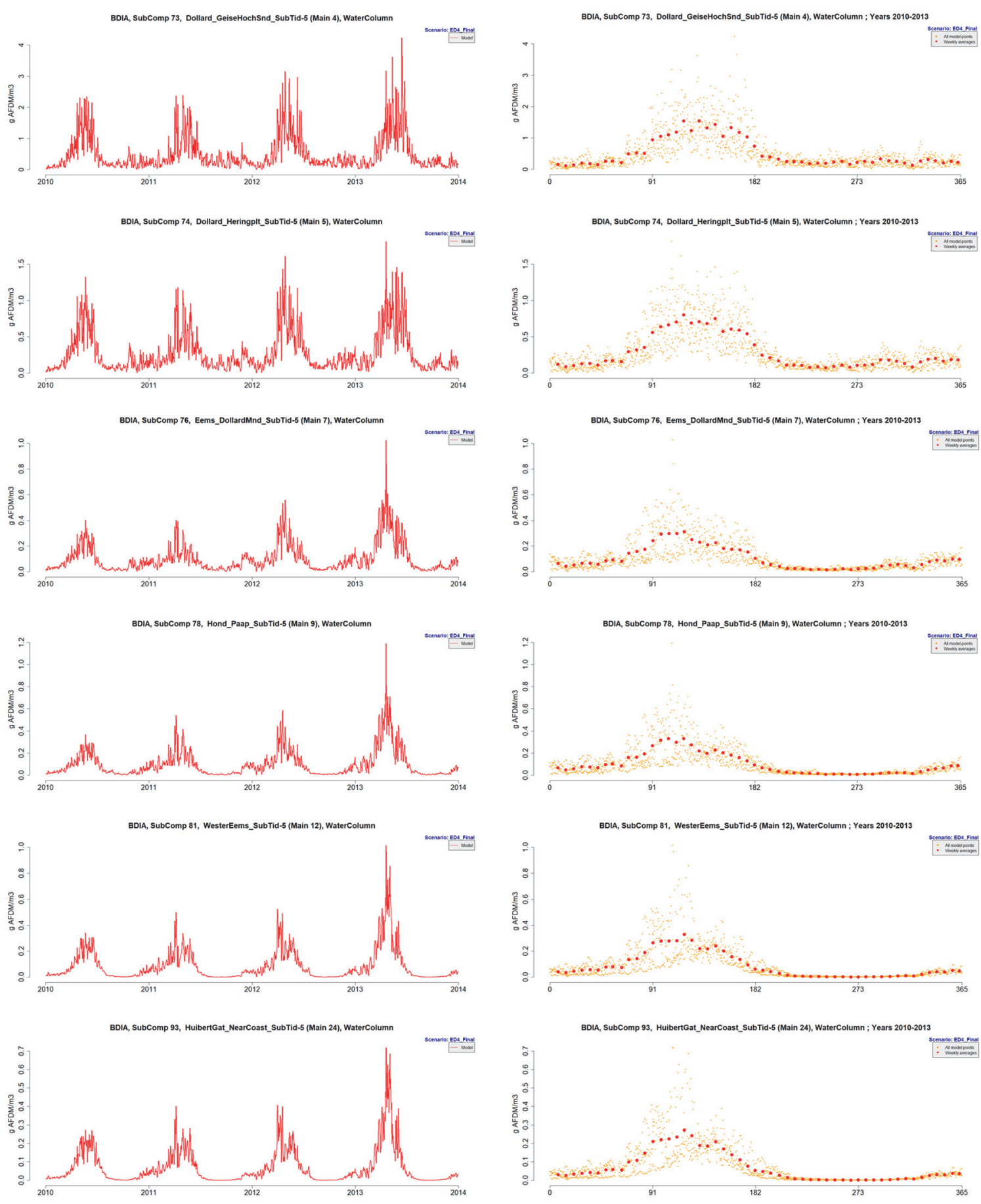

Figure 155 Benthic diatoms resuspended in the water column ( $g$ AFDM $m^{-3}$ ). Upper: Dollard mouth, main compartment 7, 2nd: Hond \&Paap (main compartment 9). 3rd where Ems rivers enters the system. 4th Hond \& Paap. 5th: boundary Westerems and Doekegat. 6th (lowest) boundary of Westerems and Huibert Gat. Left 2010-2013, line=simulation, dots data. Right: average seasonal pattern; large red dots give average values; orange dots represent all simulated values. Column averaged (completely mixed water). In the model, $1 \mathrm{~g}$ of benthic diatoms contain $9 \mathrm{mg}$ chla, thus main compartment 104 (upper figure) contains about $15 \mathrm{mg}$ benthic chla $\mathrm{m}^{-3}$ (with a maximum of about 30) which is over half of (to almost all) the pelagic values computed.

reality, but it also occurs in the model). Also, the relatively flat seasonal pattern also is explained by benthic diatom contribution. 


\section{A25.10.3 Spatial distribution}

In Figure 156, observations during the 2012-2013 IMARES-research are shown. Comparing the results with the simulation results (Figure 157) is not easy, but patterns are more or less similar.

For those compartments that have a large fraction being tidal flat, high year-averaged chlorophylla values are computed. This is caused by the year-through large contribution of benthic diatoms to pelagic chlorophyll-a content.

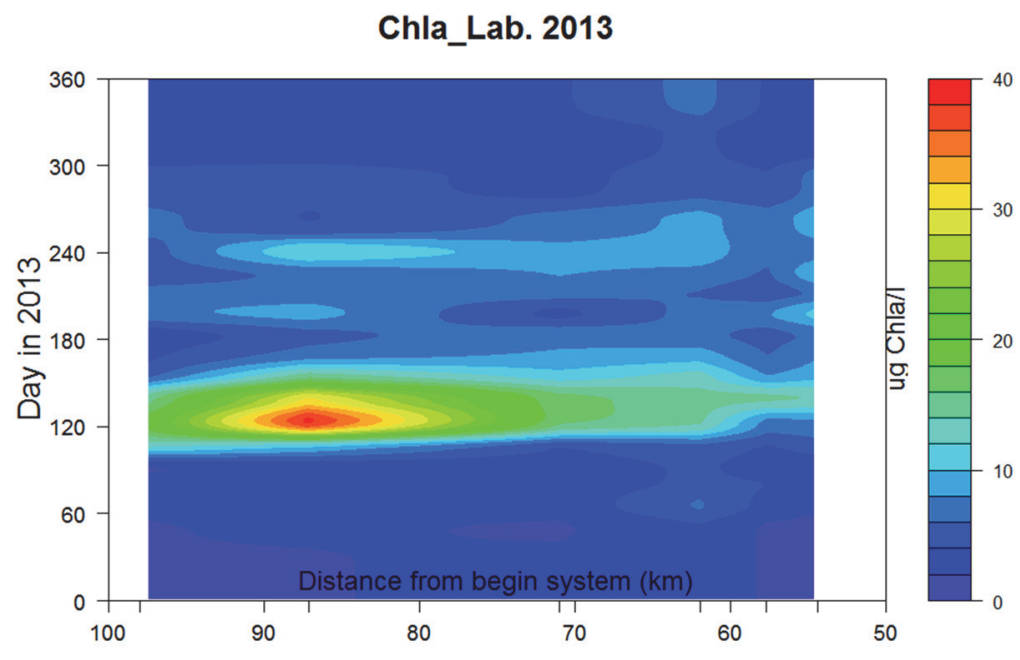

Chla_Lab. 2012

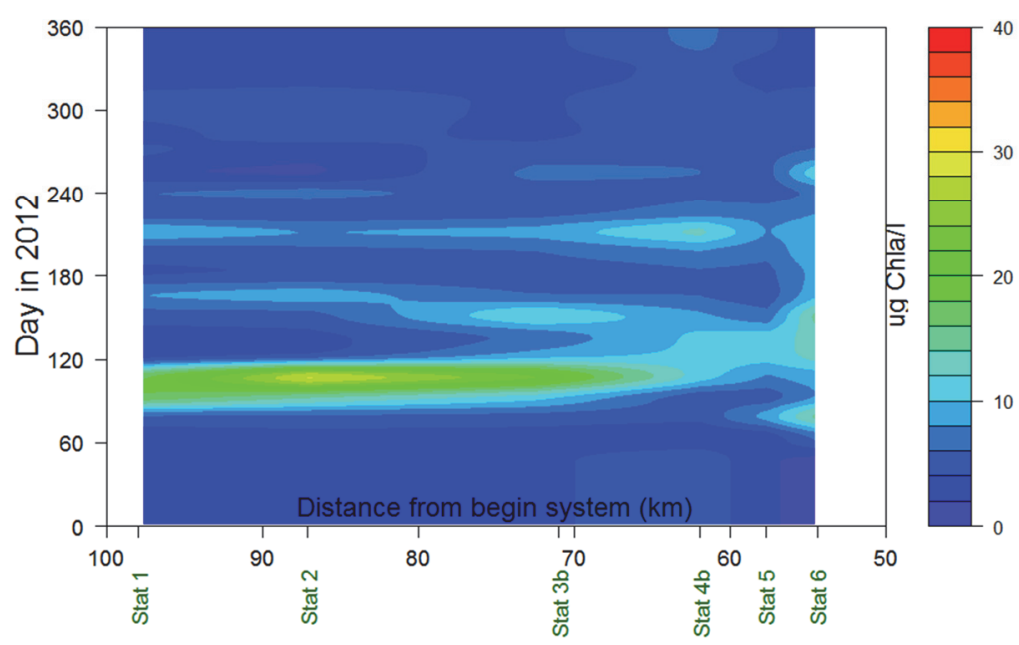

Figure 156 Chlorophyll-a observations in 2012-2013 during the IMARES research by Riegman et al, reported by Brinkman et al (2015). Data obtained after sample analysis in laboratorium. Horizontal axis: distance from Herbrum (left=North Sea). Station nrs and km-values are shown. Vertical axis is time (from 1 Jan 2012 (lower) to $31 \mathrm{dec} 2013$ (upper). Values in $\mathrm{mg} \mu \mathrm{g}$ chla dm ${ }^{-3}$. Maximum values clearly in March-April. 

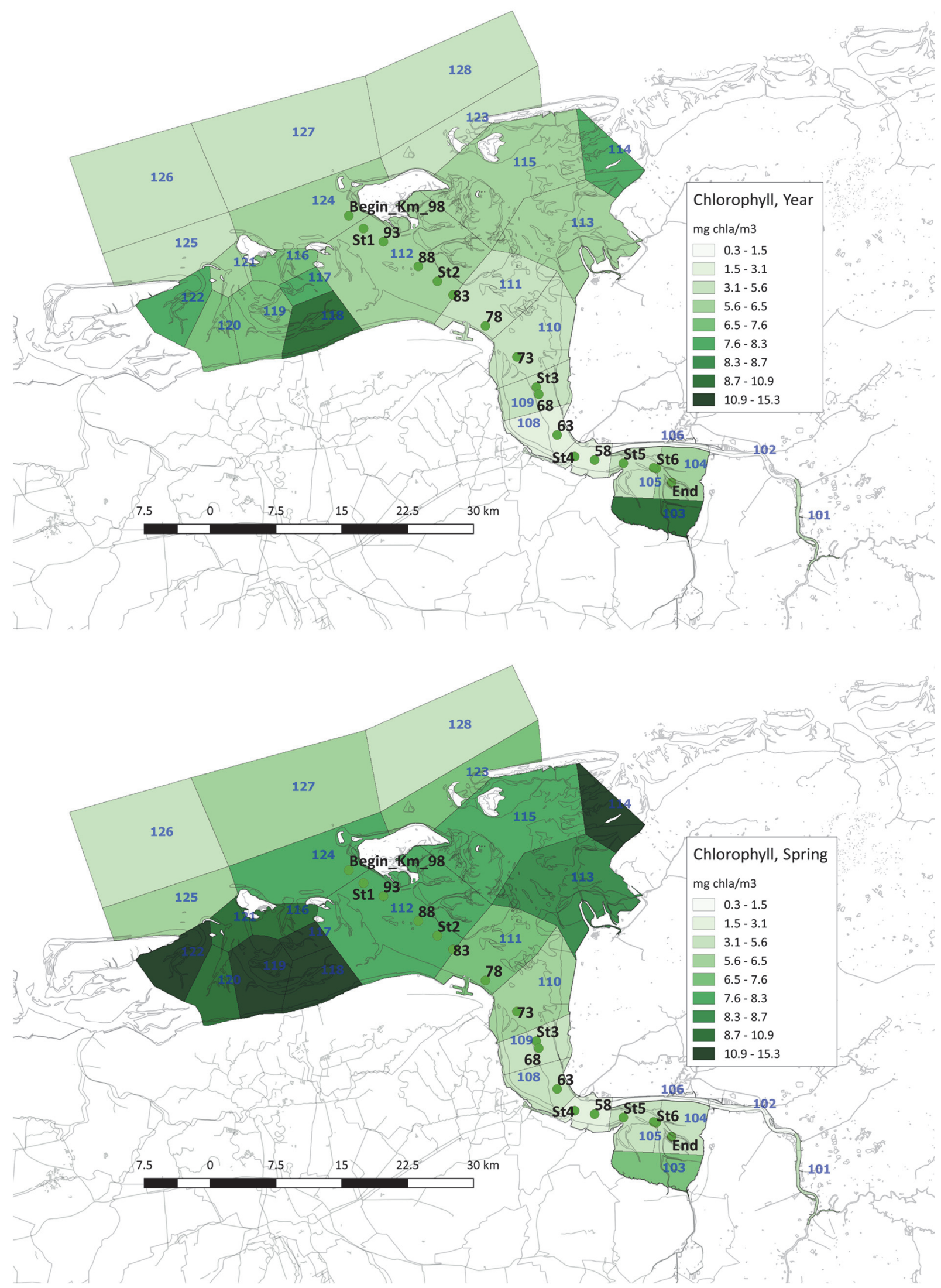

Figure 157 Spatial distribution chlorophyll concentration ( $m g$ chl-a m $\mathrm{m}^{-3}$ ), averaged over whole 2010-2013 period. Sampling station numbers shown. Station positions (kms from Herbrum) marked, see also Figure 156 (color pattern differs). Upper: whole year, lower: spring months only (January-April). Summer in Figure 158. 


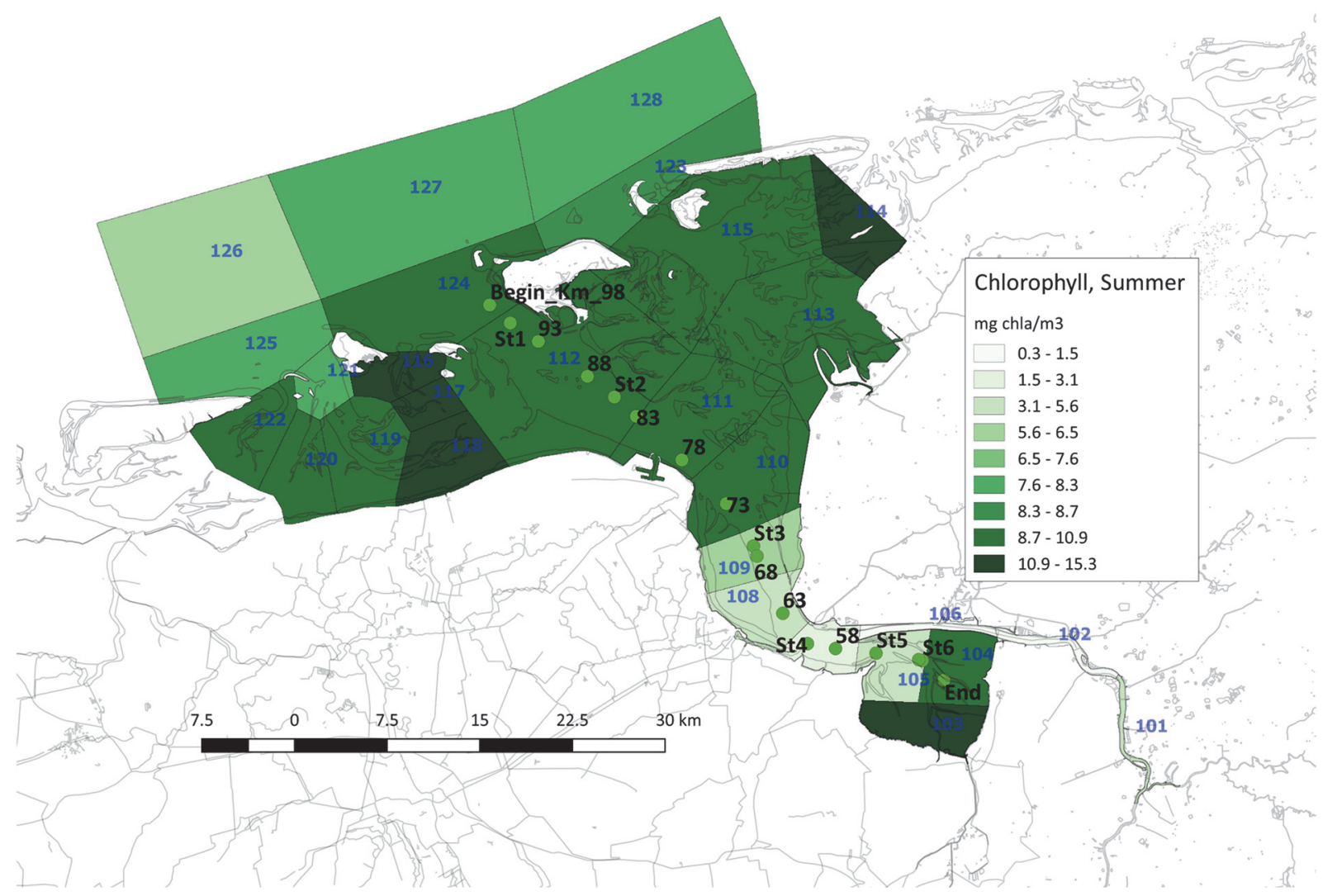

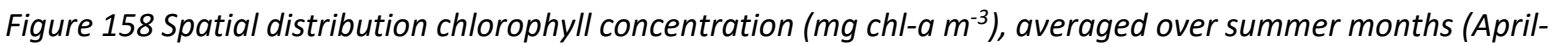
September) whole 2010-2013 period. Sampling station numbers shown. Station positions (kms from Herbrum) marked, see also Figure 156 (color pattern differs). Year and spring in Figure 157.

\section{A25.10.4 Suggestions and possible improvements}

- Chlorophyll in the Ems river needs better tuning

- Better tune the effect of salinity on freshwater and marine phytoplankton

Tuning both parts better probably will also improve the chlorophyll simulation results for main compartments 107-109 (numbers mentioned in Figure 157), now results are far below observations. These improvements are really needed since these are target compartments for all attempts to improve water transparency.

One other possible improvement is

- Model the chlorophyll content of the phytoplankton. Thus, production rates per unit of biomass change during the season and depend on external conditions. This asks for new descriptions in the model. 


\section{A25.11 Benthic chlorophyll-a}

\section{A25.11.1 Introduction}

Benthic chlorophyll, as was the case for pelagic chlorophyll, is not really included in the model, but computed based on an assumed chlorophyll-a/dry mass ratio. This ratio is fixed (no seasonal variation), and specific for each phytoplankton type: for each type the ratio is set a priori as model parameter. See appendix A28 for all values. On storage of the phytoplankton biomass values, chlorophyll simply is computed from these values. In the sediment, only benthic diatoms occur.

Benthic chlorophyll simulation results may differ per tidal part in each main compartment since light availability is less when the part is flooded more often. Grazing by snails causes phytobenthos (and thus chlorophyll) losses in the model. Presently, this is not restricted to tidal areas.

Since it was thought to be useful, not only simulation results for those tidal subcompartments where samples have been taken are used as comparison, but also neighbour sub-compartments. It appeared that differences in lower and mid- tidal levels in each main compartment were too small to make separate presentation necessary.

\section{A25.11.2 Time series}

Results in Figure 159 show that most results fit the observations rather well. Some data for neighbour compartments also differ substantially in some case, and thus, data for more than one monitoring site is included in a sub-compartment graph. Enormous differences between data and simulation results do not occur; results also are in line with previous research (e.g. De Jonge \& Colijn, 1994, see Figure 160 ; De Jonge et al, 2012). One aspect certainly is not correct: results for different tidal areas within the same main compartment hardly differ, and that is not what is expected. Reason probably is that there is a strong exchange between the water phase of all subcompartments within one main compartment, and since resuspension/settlement (of also benthic diatoms) is a major process, also phytobenthos will be mixed. Opposite may be that this is not the case for the subtidal compartment in the same main compartment. Thus, further data analysis is needed.

The model computes maximum chlorophyll values for the early summer months, while in reality, the seasonal distribution is rather flat. Early spring and late autumn values still may be of almost the same order as those in summer. Model results are partly steered by bottom dweller grazing (called Hydrobia in the model).

Comparison with older data as presented in Figure 160, thus for years not covered by the present simulations, must be done by eye. 

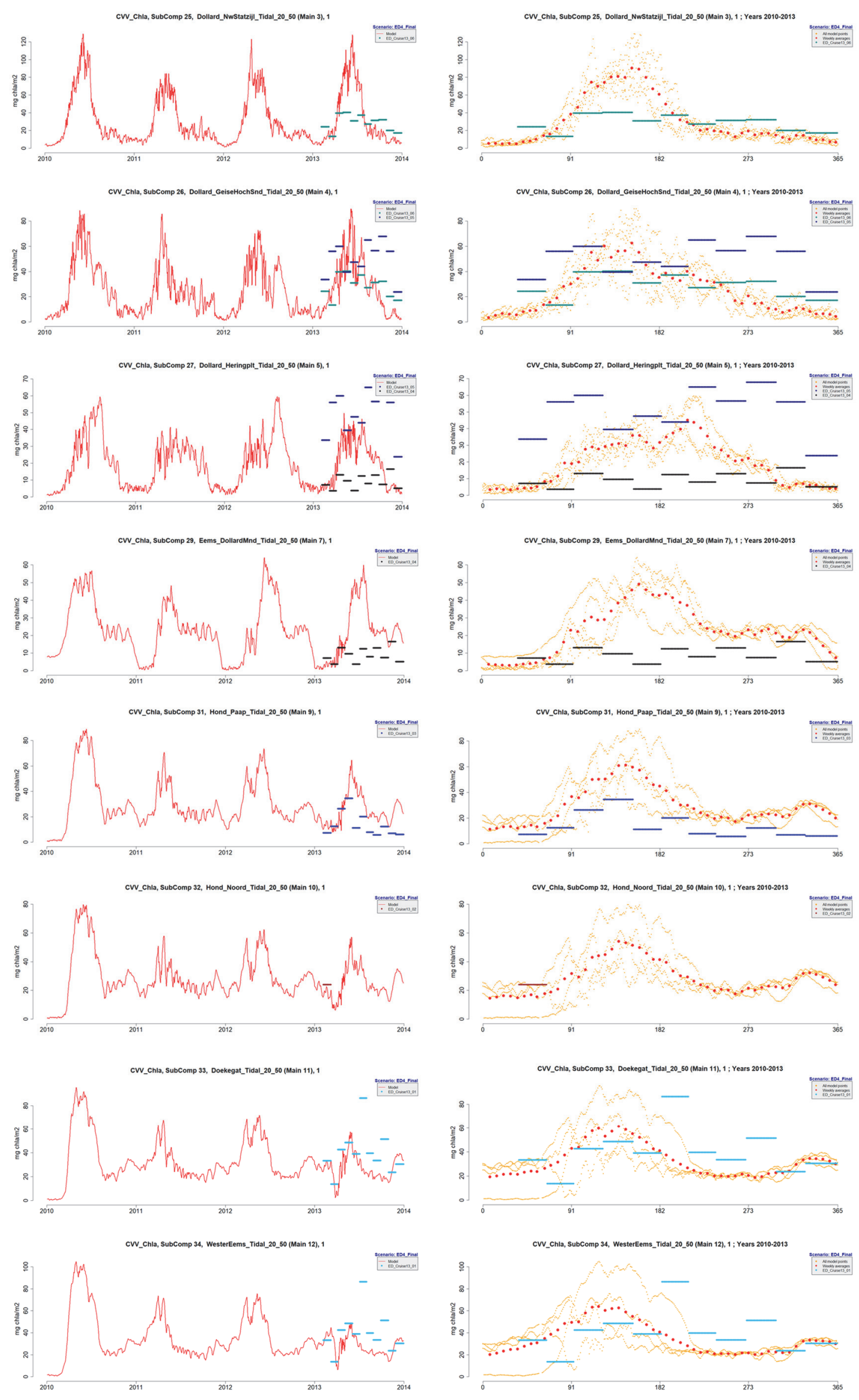

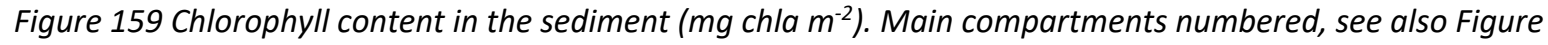
161. Results for tidal sub-compartment that is $20-50 \%$ of the time dry are shown; there is hardly a difference between the three tidal regions. See text for explaination. 

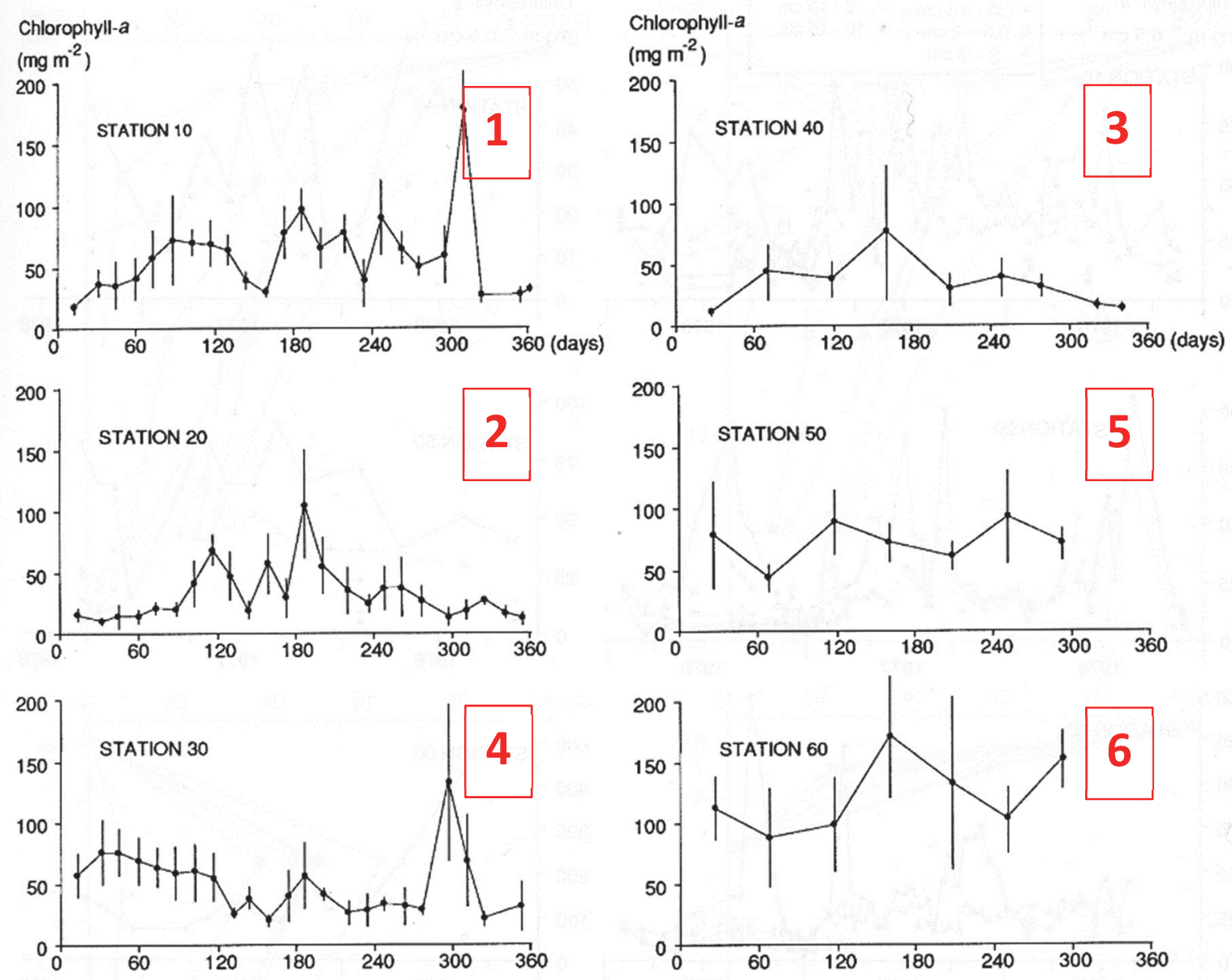

Fig. 4. Plots of the mean chlorophyll-a concentrations in the upper $0.5 \mathrm{~cm}$ sediment layer of the six main stations in 1977 . Bars represent standard deviations.

Figure 160 Chlorophyll-a densities in Eems-Dollard sediments, as determined by De Jonge \& Colijn (1994). Figure is a copy from their publication. Present station numbers (see e.g. Figure 161) are mentioned in bold red.

\section{A25.11.3 Spatial distribution}

The computed spatial distribution is shown in Figure 161. Chlorophyll in the deeper parts is partly a result of sedimentation of suspended benthic diatoms. Also, some growth occurs. See also appendix A25.15. Probably (see also the remark on the almost absent differences between the different parts of the tidal flats) a better description of the suspension/sedimentation process will change this characteristics towards phytobenthos being almost absent in the subtidal areas.

\section{A25.11.4 Suggestions and possible improvements}

- Check the reason of the very small differences between the three tidal parts in each main compartment

- Make early spring and late autumn values more like summer values

- Include other fauna that also grazes upon phytobenthos (e.g. worms and/or shellfish). 


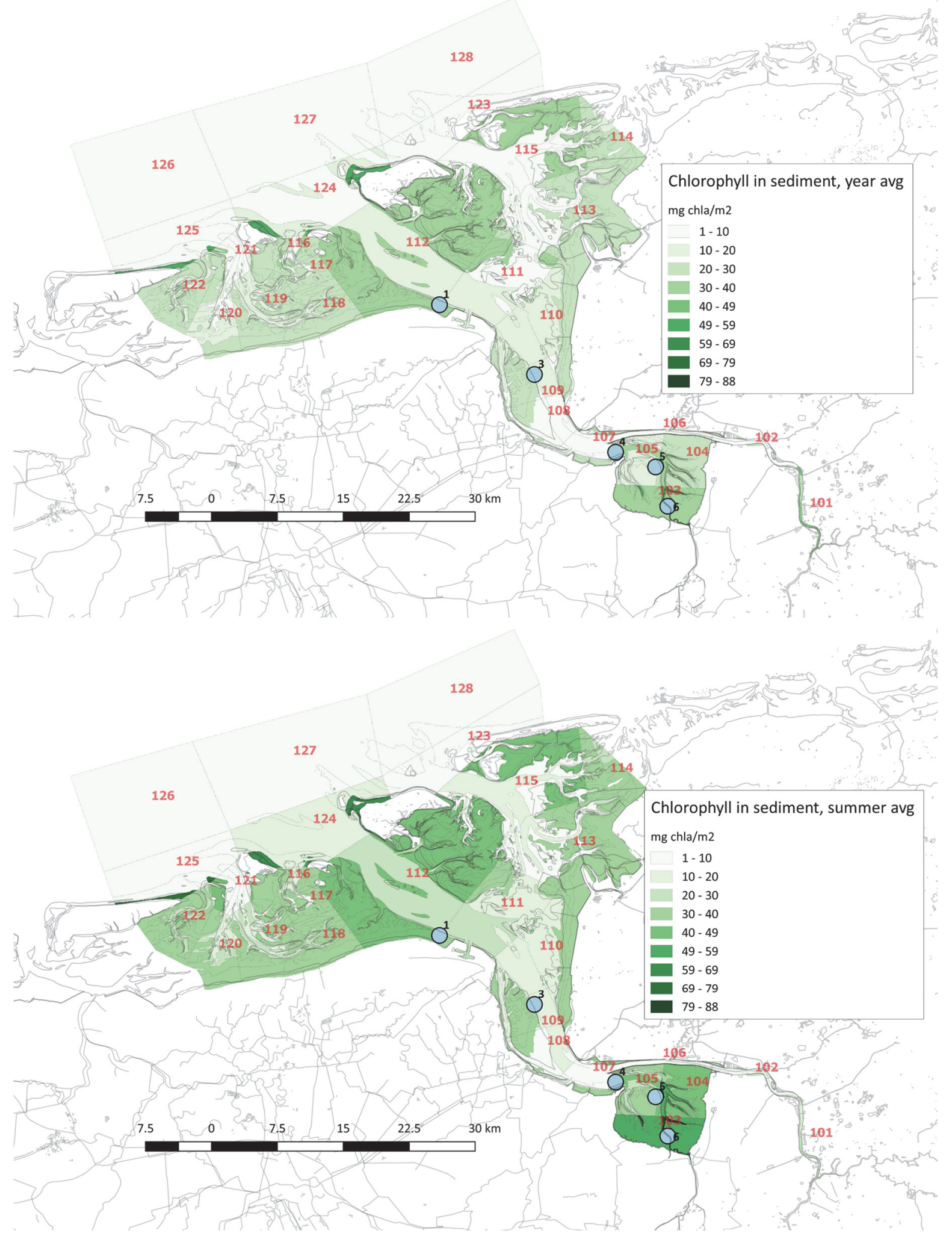

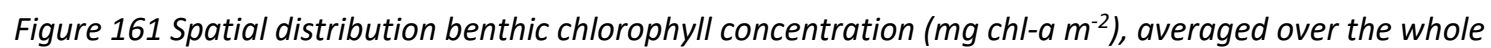
2010-2013 period. Upper: whole year, lower: summer months only (april-september). 


\section{A25.12 Zoobenthos and zooplankton}

\section{A25.12.1 Mudnails}

Observations of sediment browsers (Mudsnail and Periwinkle) are pictured in Figure 162; Figure 164 gives the present model result. The computed distribution is remarkably like the data, the computed values are slightly below these.

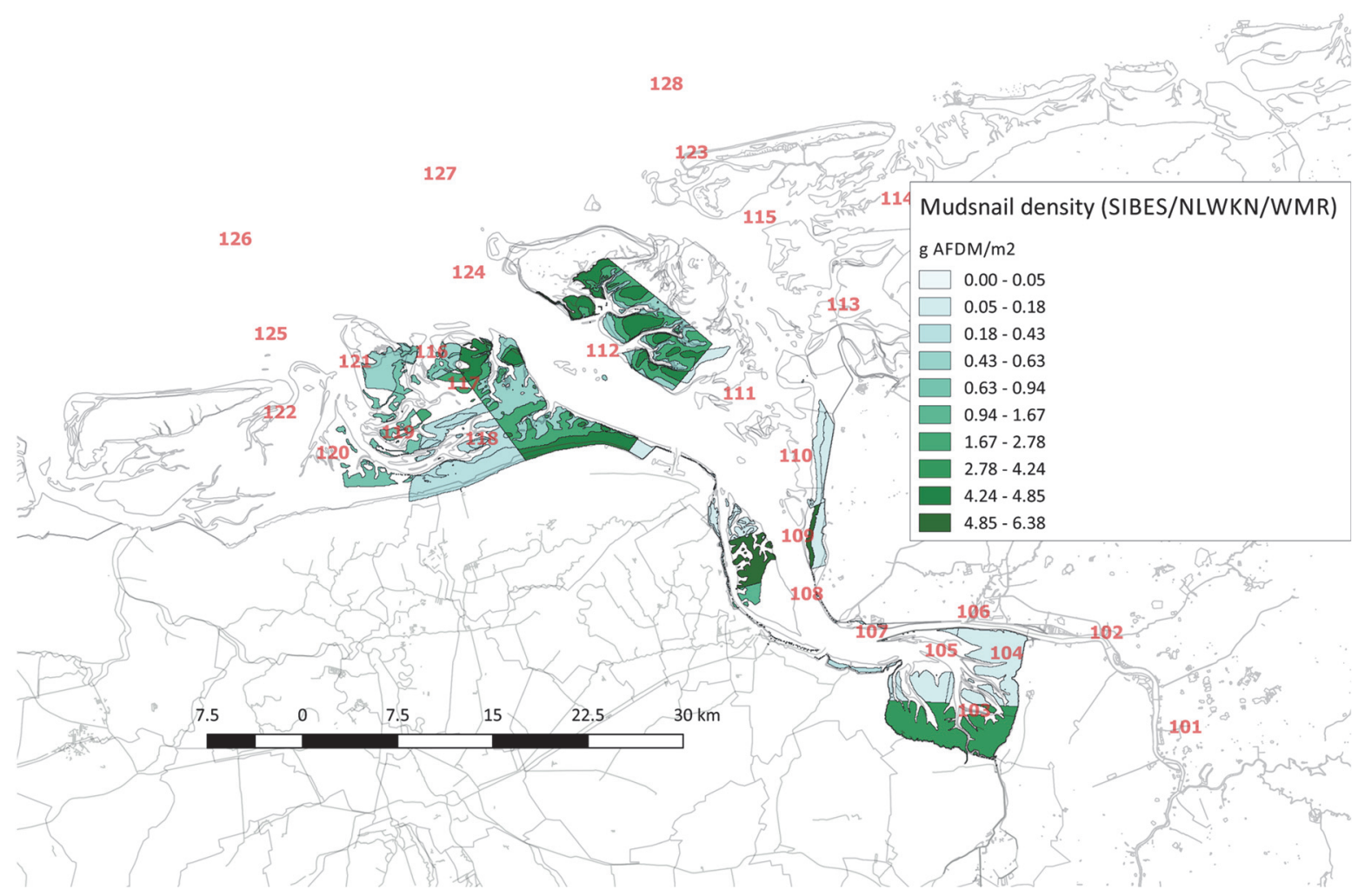

Figure 162 Distribution of Mudsnails (upper) and Periwnkles (lower) in the estuary. Data from NLWKN and SIBES. Both figures same scale. The data are too high, see remarks in the text on these results.

The reason for the differences are not clear yet. One aspect that will be important is the moment of the inventories: the simulations show considerable seasonal differences (see Figure 163 for example). But it is not clear what these variations are in the field.

A second reason may be the way the observation averages have been computed: just the simple average of the observations (including the zero's). here's a difficulty: when examining the data carefully, one can see that some field values are exceptionally high, resulting in high values for the sub-compartment where these values have been found. Thus, a better data analysis is needed to be able to get better averaged field values.

Periwinkles hardly occur in the observations, and are omitted here. 


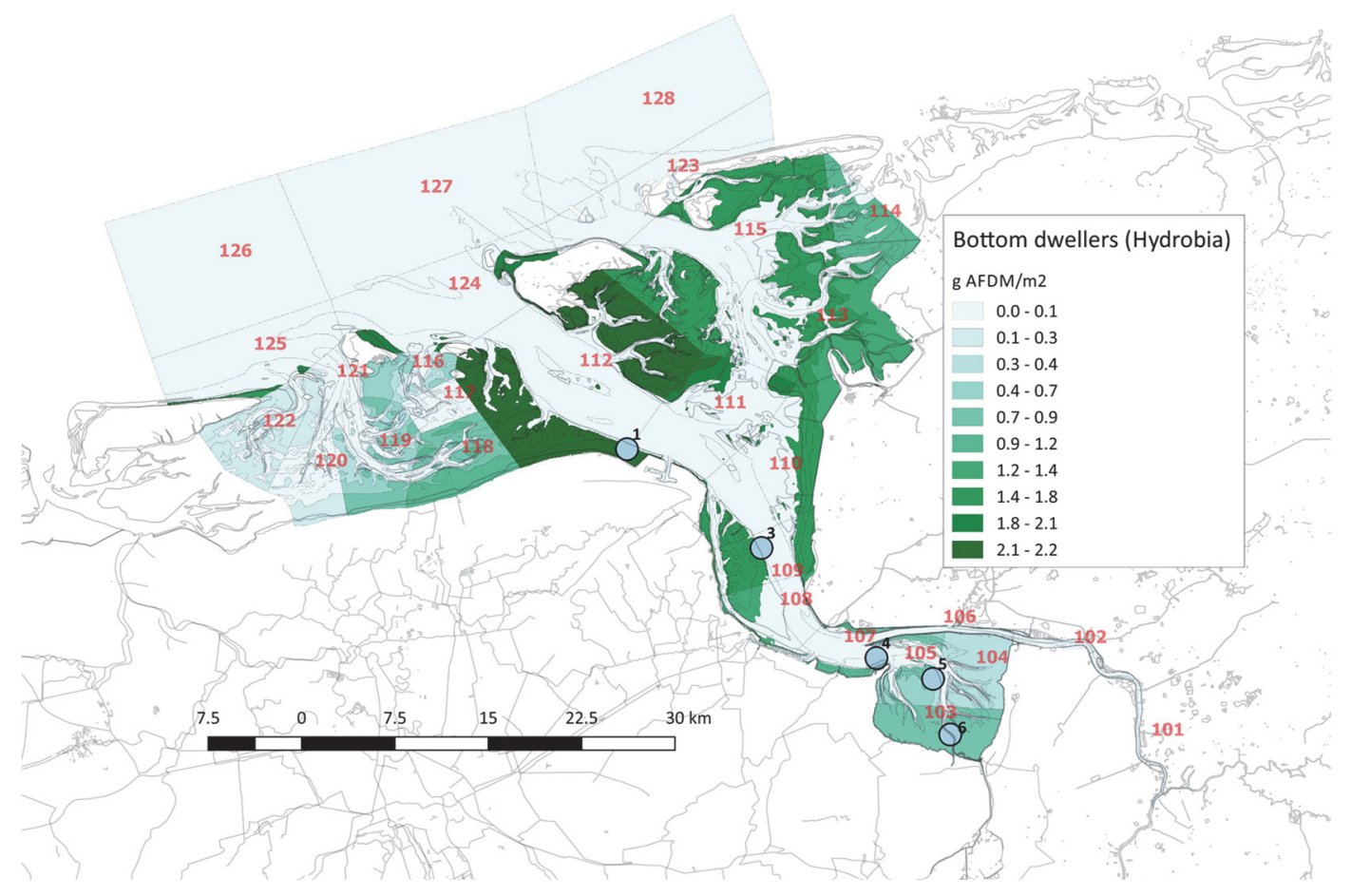

Figure 164 Distribution of bottom dwellers ("Hydrobia") as computed by the model. Yearaveraged values.
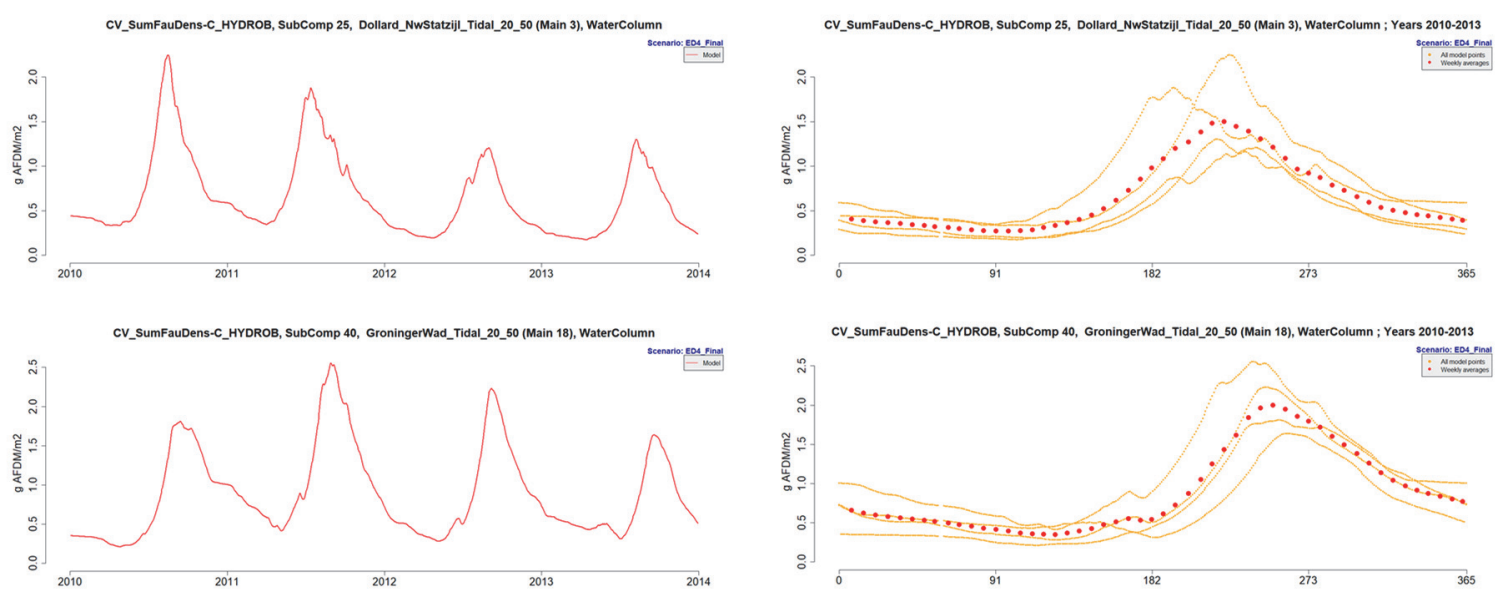

Figure 163 Computed Hydrobia; variations with years and season. Upper: Dollard area, lower Groninger tdal flats.

\section{A25.12.2 Filter feeders}

Observations of filter feeders (as simple averages) are presented in Figure 168. The same remarks as made above for snails are relevant here: in the SIBES and NLWKN-data, there are many zero's in the observations, but these have not been taken into consideration. Just the average values per sub-compartment are computed, as linear averages. So, the distribution type was not considered. Next, the IMARES/WMR-data are strongly biased towards large concentrations of mussels and cockles, since the sampling method of the institute is "stratified". This means that the large concentrations of cockles and mussels are visited, thus avoiding zero observations as much as 


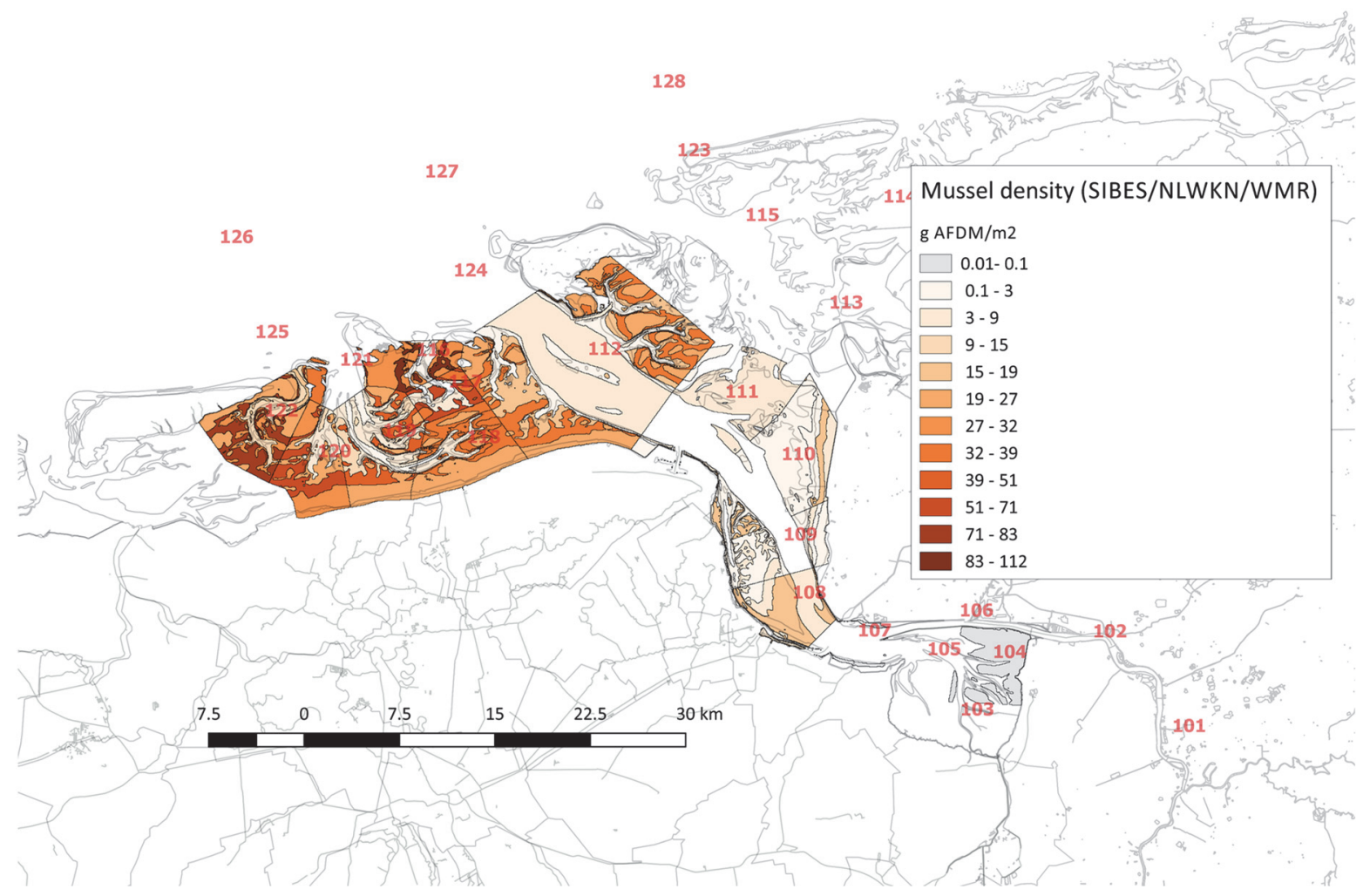

Figure 166 Observed densities of filter feeders (g AFDM/m2) (sum of Blue Mussel, Cockle, Japanese Oyster, Ensis, Scrobularia), thus, small molluscs excluded. Data from NLWKN, SIBES and IMARES/WMR.

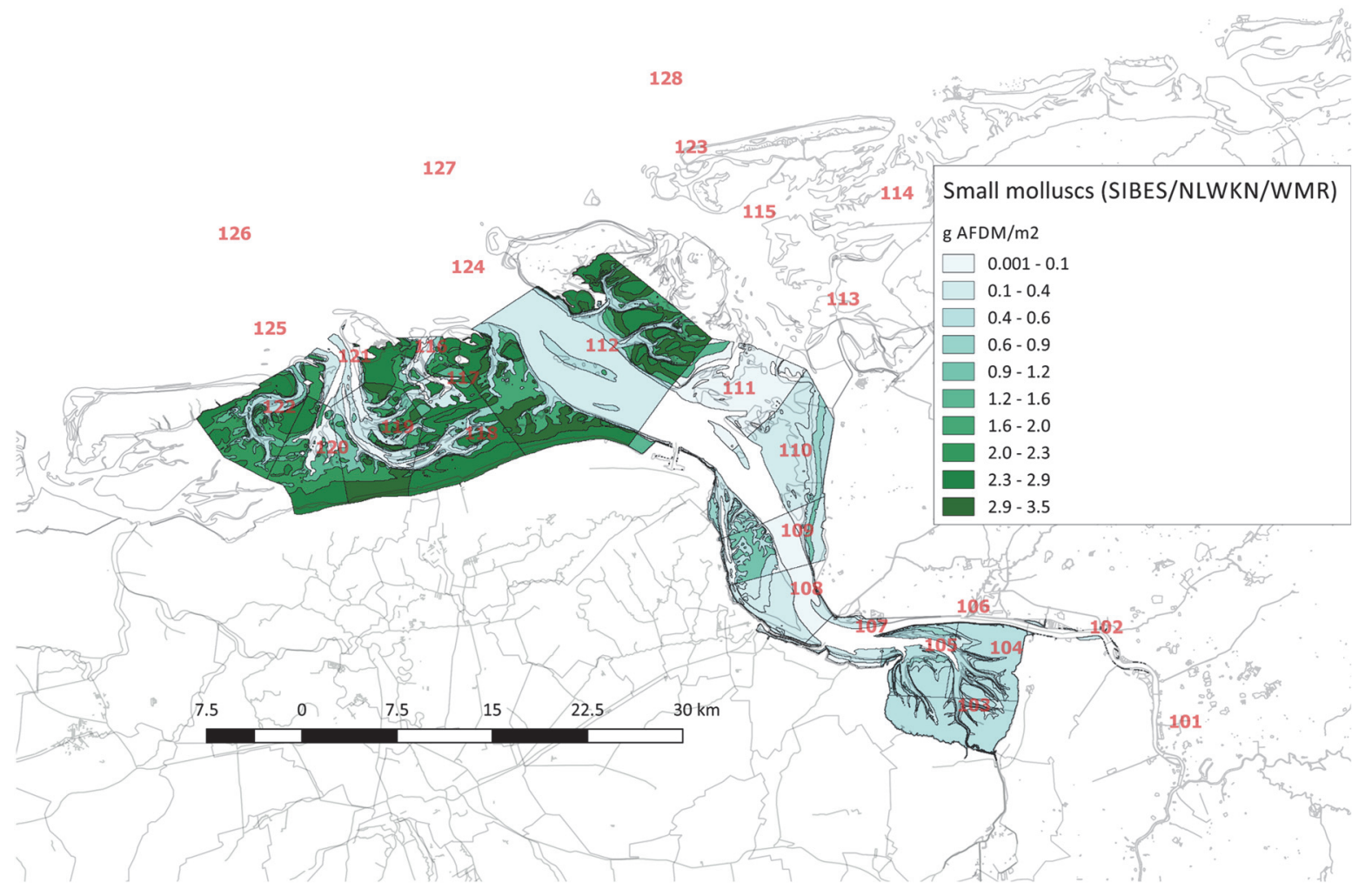

Figure 165 Observed densities of small filter feeders ( $g$ AFDM/m2) (sum of other shell fish, next to the one from Figure 166). Data from NLWKN, SIBES and IMARES/WMR. 
possible. The elaboration of the data occurs in such a way that these zeros are considered, thus resulting in (much) more correct values. Here, a simple straight-forward has been applied: each sampling point was assumed to represent 12.8 ha (see Van den Ende et al, 2012) although it depends on the sampling site.

Then, the computations fail to properly distinguish between tidal flat and gullies. The computed biomasses depend on i) food availability and ii) the effect of silt; not considered was iii) predation by epibenthic animals and iv) the vertical profile of suspended matter in the water column. Thus, in the model, the gullies are a much better place for filter feeders than the tidal flats; the silt conditions are about similar, and the time available for feeding is much longer. In reality, predation by epibenthic animals (starfish, crabs, shrimps) negatively affect the occurrence of mussels and other shellfish below low water level (see e.g Saier, 2000ª,b; Buschbaum 2000,2001; Saier \& Buschbaum, 2001; Saier et al, 2002). Predation by birds affect the occurrence of mussel beds on the tidal flats, but here, they are hindered by the water. Thus, a region on the tidal flats with low emersion times, is preferred by most shell fish species, especially those living on the surface (e.g. the Blue Mussel). This is reflected by the musselbed habitat maps as presented by Brinkman et al (2002). This map has been updated later (Smaal et al, 2014). In Figure 167, an example for adult mussels in autumn is shown ${ }^{1}$.

, an

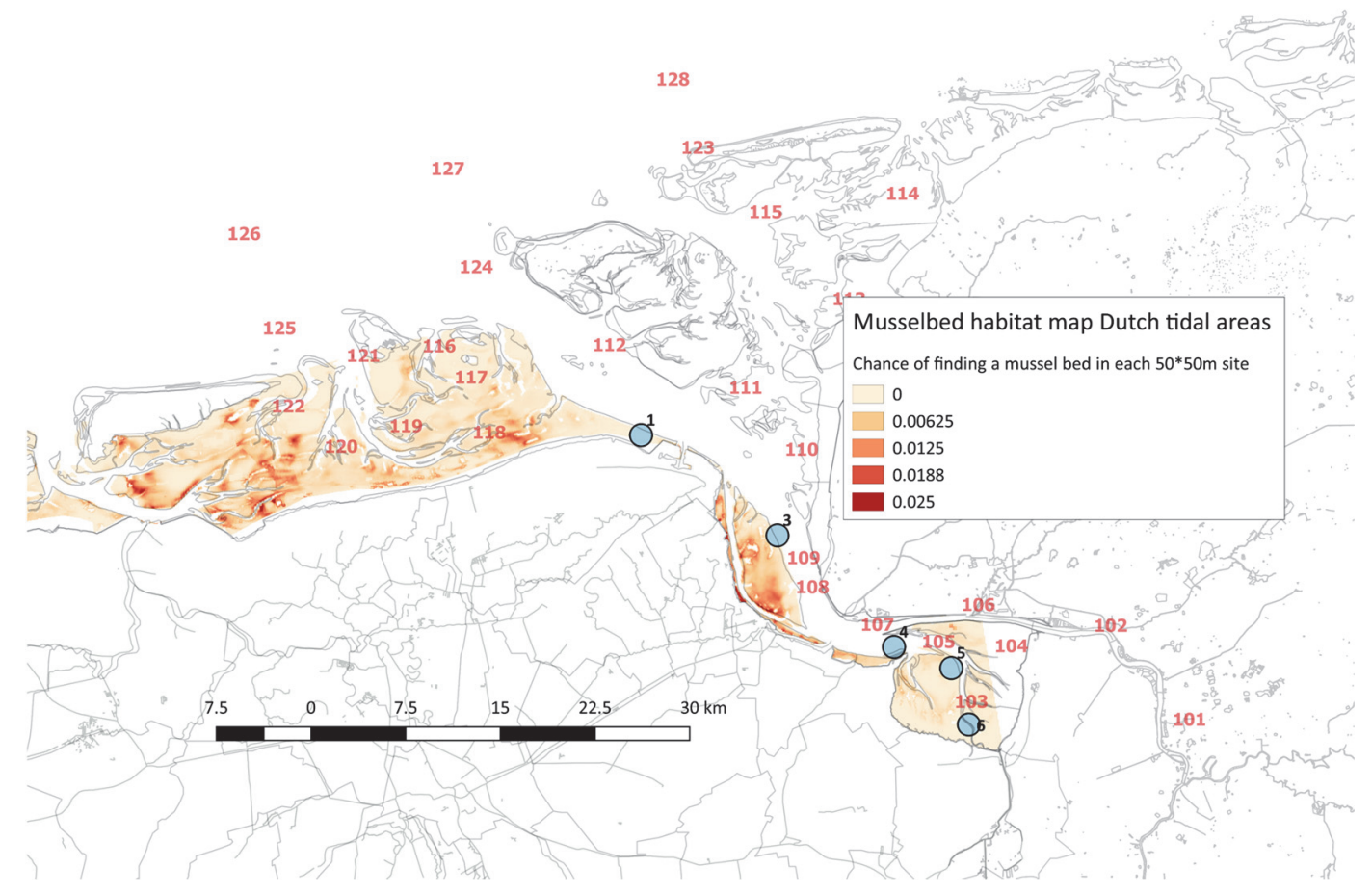

Figure 167 Musselbed habitat map for adult mussels (Mytilus edulis) in autumn. The map shows the chance to find a mussel bed in a 50*50 grid cell. See also appendix A21.

Simulation results for filter feeders ("Mussels", in the simulations) are shown in Figure 168. Best areas are at the North Sea-side of the area, and that is according to the data. Since data for the

\footnotetext{
${ }^{1}$ Musselbed habitat maps were constructed for mussel see, half grown mussels and adult mussels in spring and in autumn.
} 

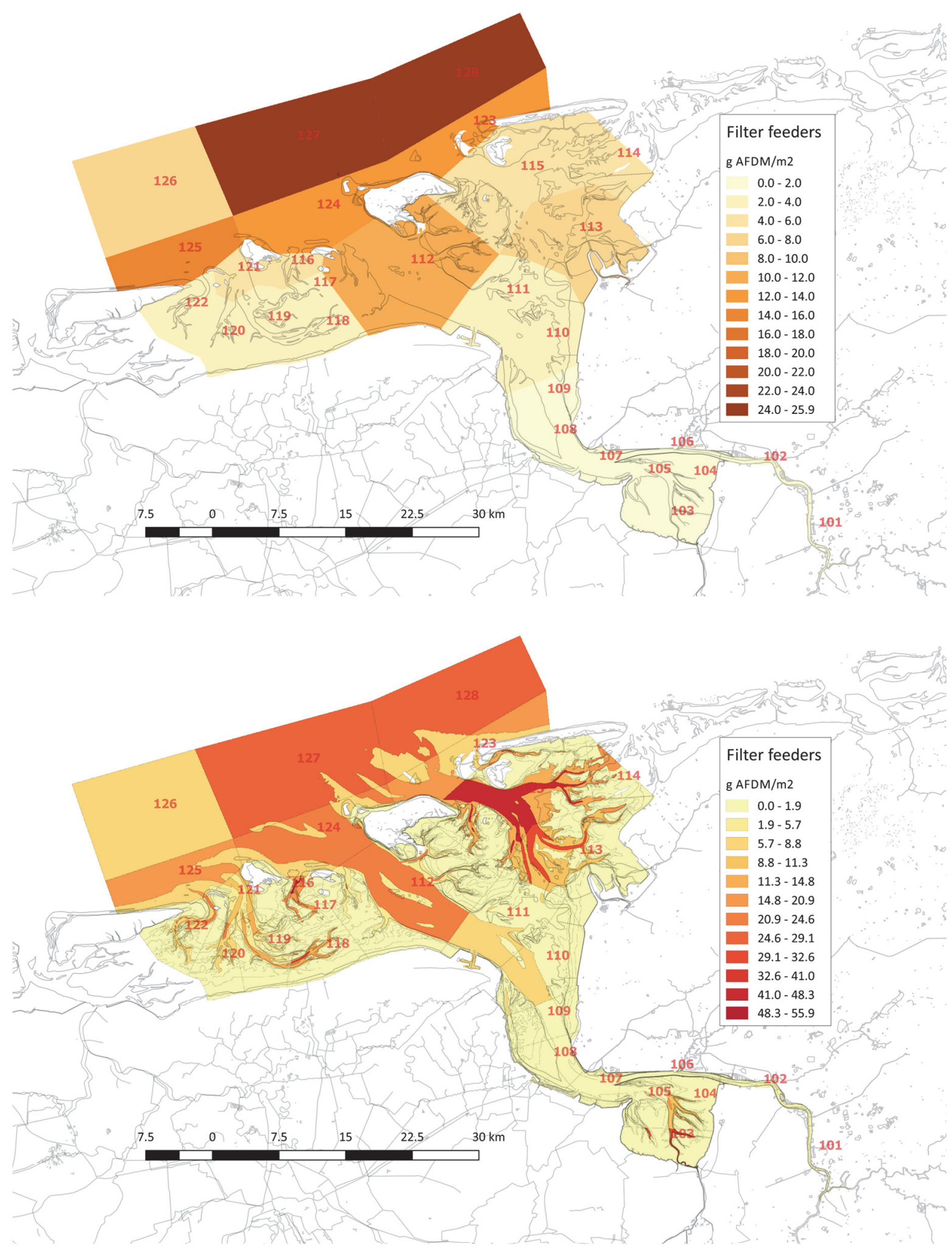

Figure 168 Distribution of filter feeders, as computed (year-average). Upper: filter feeders assigned to main compartments. Lower: same, but assigned to sub-compartments.

more off-shore area are absent, those results cannot be verified. Observations generally are above the simulated ones. Here also, seasonal variations may be important. Striking is the difference between the lower Figure 168 and Figure 166. The latter shows highest densities on the tidal flats, the first in the gullies. This is explained before. The low densities computed for the tidal flats near Schiermonnikoog have to do with the absence of the Lauwersmeer contribution. The high 
densities in the gully near Nieuw-Statenzijl (Dollard) probably is due to a still not correct description of the influence of silt on filtration activities. Also, the too high primary production (see appendix A25.14) may be a cause.

\section{A25.12.3 Microzooplankton}

For microzooplankton, almost any field value is absent. For the western Wadden Sea some data are available (Brussaard et al, 1995, 1996), see. These have been used for comparison.

Microzooplankton has picophytoplankton as main food source, and next to that, it is capable (in the model) to use a (small) part of other algae, and some detritus (see appendix A28.4 for a full description).

A typical pattern is shown in Figure 169. Results for the Dollard area (Figure 170) and closer to the North Sea boundary (main compartments 12 and 24) are presented in Figure 171. Note the different scales. Model results show too sharp and too high peaks, but on the other hand, the order of magnitude on average is not far from the Brussaard-data. The model computes that for the Dollard area microzooplankton hardly occurs.

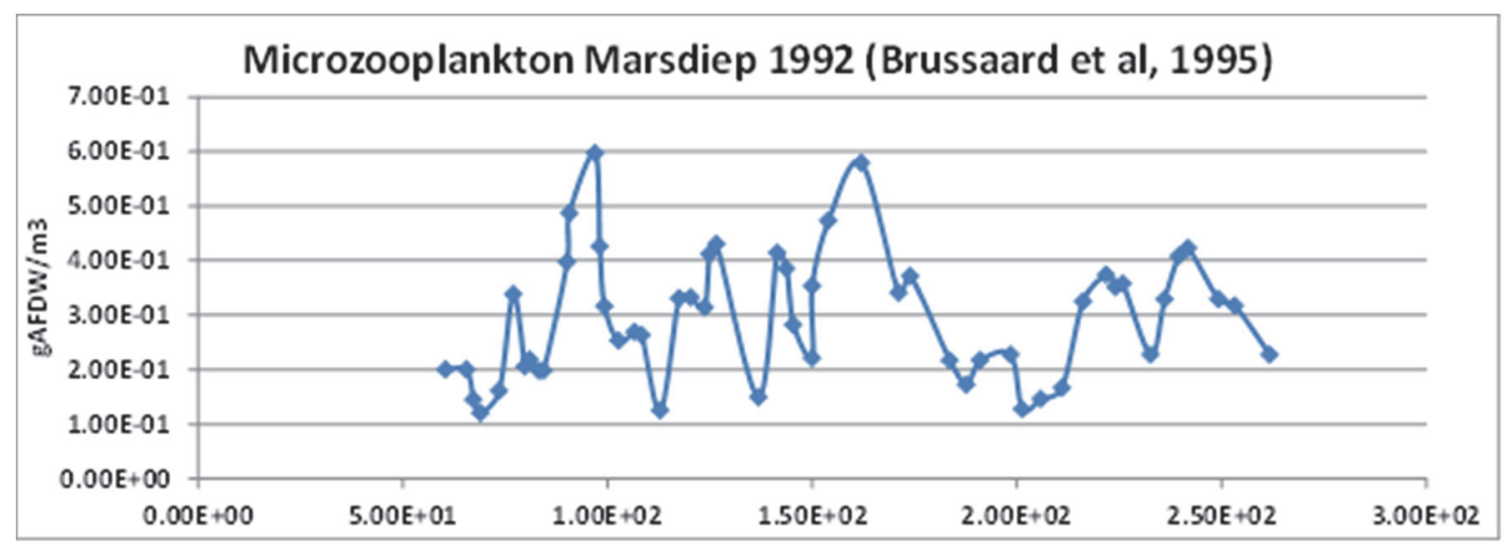

Figure 169 Microzooplankton in the Marsdiep (western Dutch Wadden Sea), 1992, during the season (days 1-300)
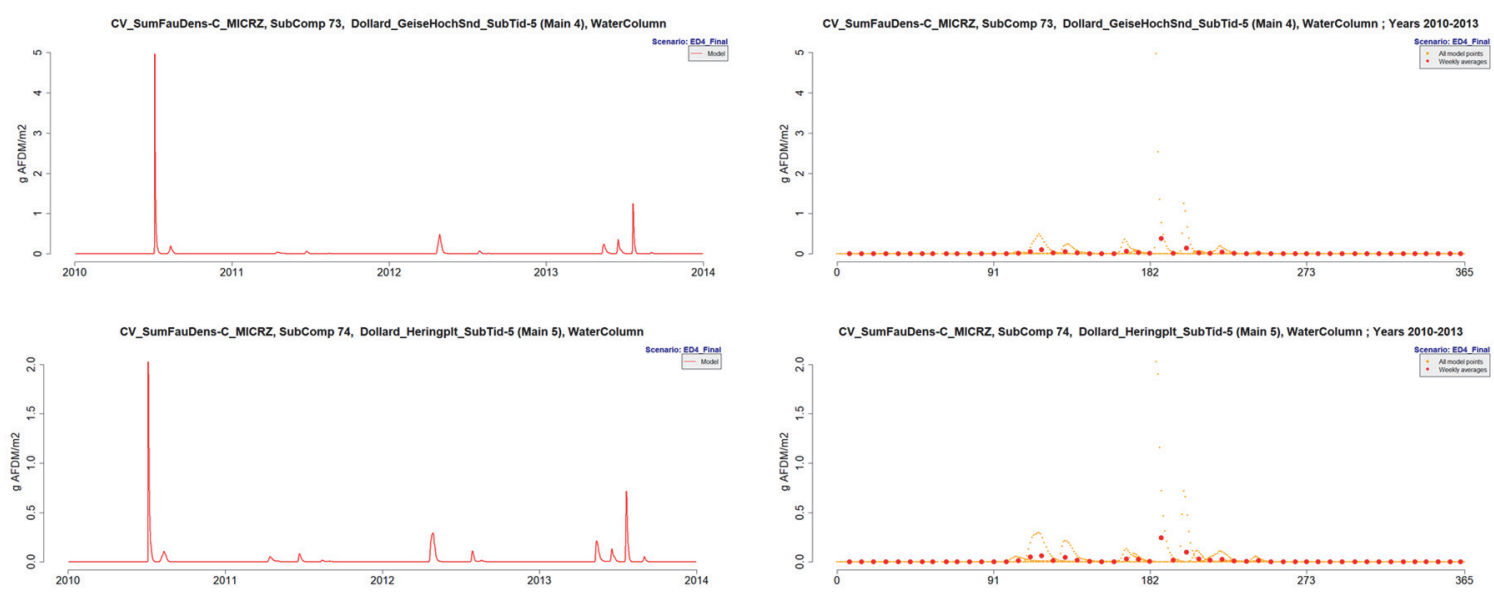

Figure 170 Microzooplankton in the Dollard area (main compartments 4 and 5), model results. Left: 20102013, right: seasonal pattern. 

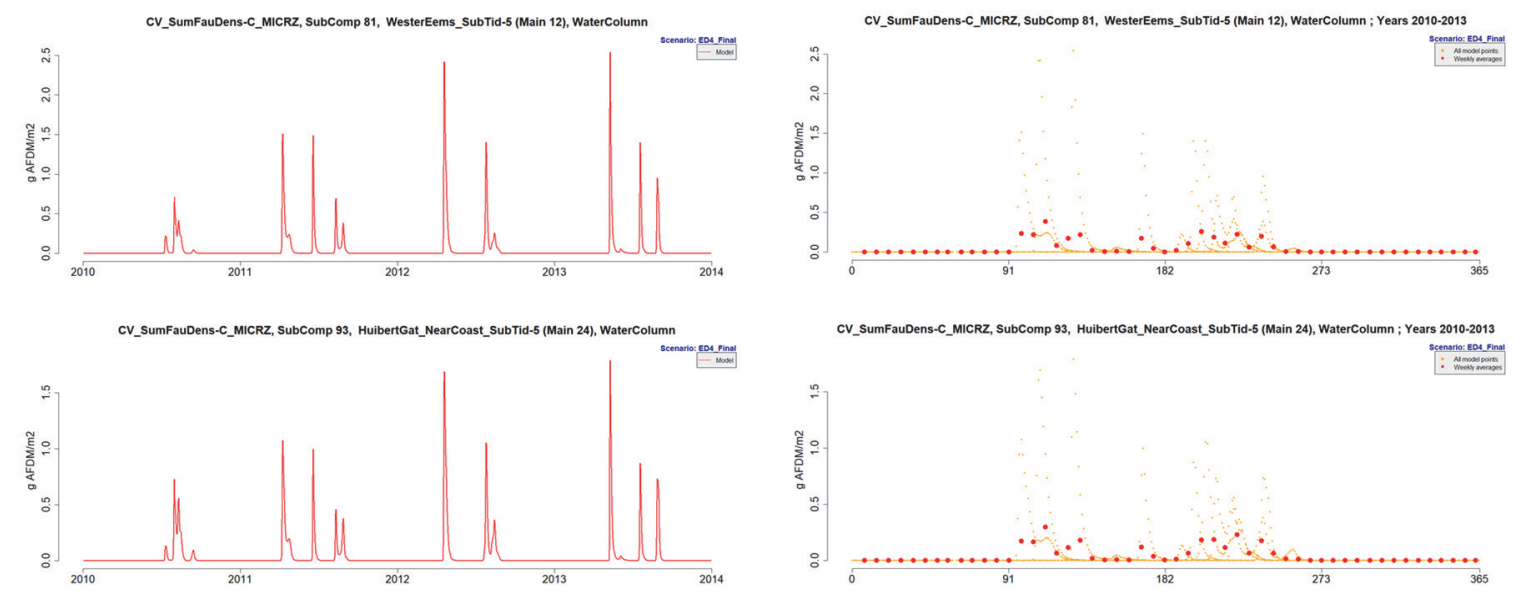

Figure 171 Microzooplankton in main compartments 12 and 24, model results. Left: 2010-2013, right: seasonal pattern.

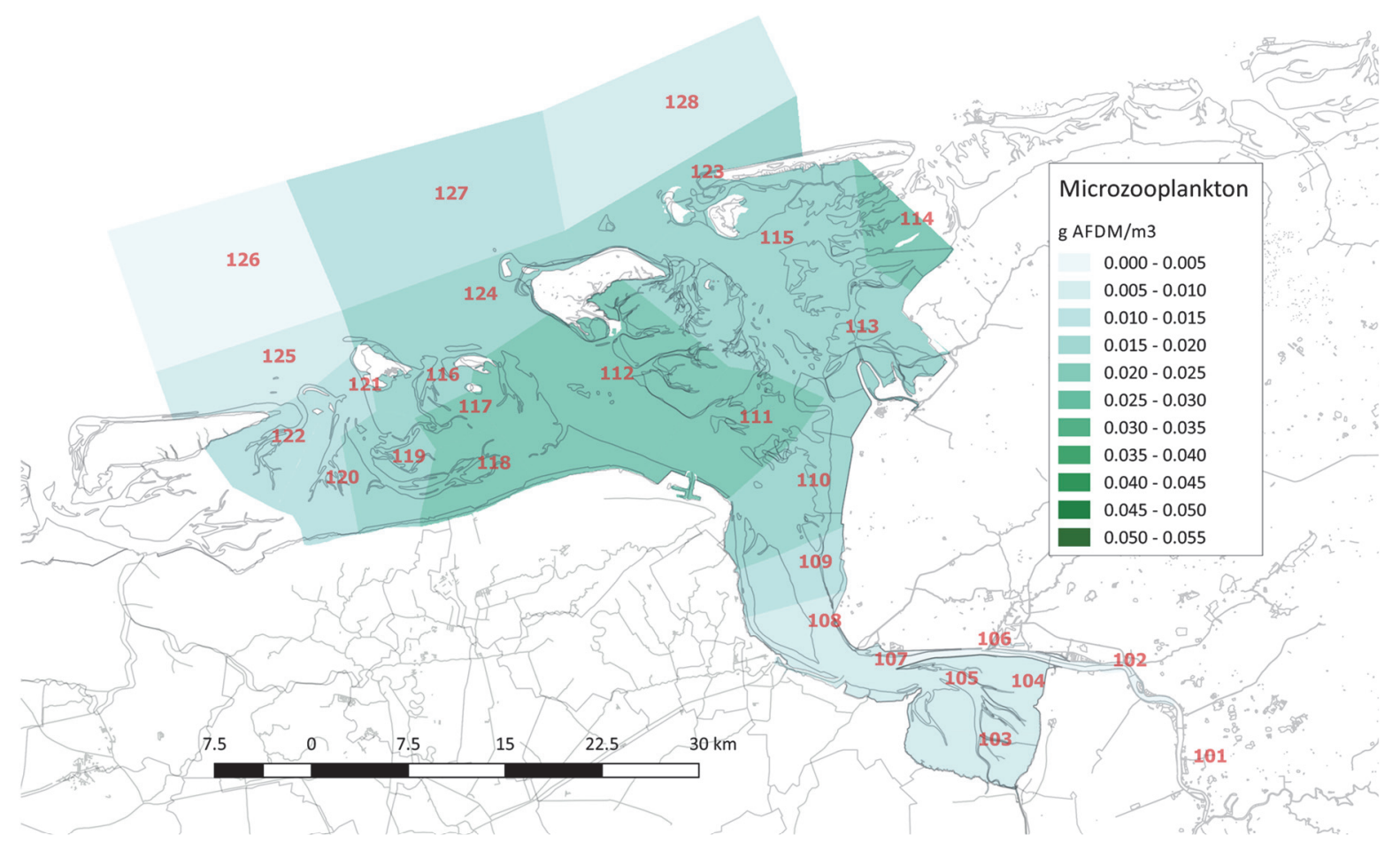

Figure 172 Computed microzooplankton distribution ( $\mathrm{AFDM} \mathrm{m}^{-3}$ )

\section{A25.12.4 Copepods}

The search for a useful parameter setting for copepods had to be stopped. It appeared to take more time to come to an acceptable choice. 


\section{A25.12.5 Suggestions and possible improvements}

Make better overviews of the observations of the benthic fauna (then now has been done by the author): take care of the non-normal distributions of animal numbers in the samples. Possibly a Poisson-like distribution analysis is suited better. Also, the method of stratified sampling by IMARES/WMR should better be taken into consideration, this is to use the several areas that are supposed to be representative for the samples taken instead of one uniform size.

\section{A25.13 Gross pelagic primary production}

\section{A25.13.1 Introduction}

Pelagic primary production has been assessed during the 2012-2013 IMARES research by taking water samples, measure the relationship gross primary production vs light intensity (the so-called PI-curve), and finally compute the gross primary production at each station based on solar light intensity, light attenuation coefficient in the water column and water depth. Thus, data for all the about 40 data and six monitoring stations have been derived. Next, based on available solar light intensities for each day in 2012 and 2013, and assuming that the other primary production variables and parameters (i.e. light attenuation and phytoplankton productivity parameters) stayed constant, primary production at each day in 2012 and 2013 has been estimated. Finally, based on these data, contour plots for [ $\mathrm{x}, \mathrm{time}]$ have been produced (along the estuary axis, and time from 2012-1-1 to 2013-12-31).

Next: depth plays a role. Therefore, some of the next plots are for the subtidal area and for the channel area separately. Especially if the water is relatively clear, this may affect the computed total gross primary production. The effect may be lower than presented here since it is assumed now that the light extinction coefficient is a constant over depth. In reality, the parameter will increase with depth, and thus, a possible contribution of deeper parts to gross primary production will be less than computed here.

Last: gross primary production only concerns the production without respiration losses. With losses, it is called net primary production (next section). Then depth plays a role as well (most times even more) and in deeper areas only respiration occurs. Consequently, the deeper (and the darker) the water column, the larger respiration (oxygen consumption) without production. Net production thus may (or: will) become negative, and affects oxygen concentration in a negative way.

\section{A25.13.2 Time series}

Computed gross primary production values for the water column are shown in Figure 175 - Figure 173. For areas with a low light attenuation coefficient (mostly the outer areas), channel simulation results are a bit above the sub-tidal simulation results. On average, simulation results fit the data rather well, except for the Dollard area where simulation results are too high. Over half of the producing algae in the Dollard area is resuspended phytobenthos, and next to that, a possibly too low attenuation coefficient this might both contribute to this overestimation. However, of these two, Figure 124 (section on light attenuation) does not support this suggestion. 

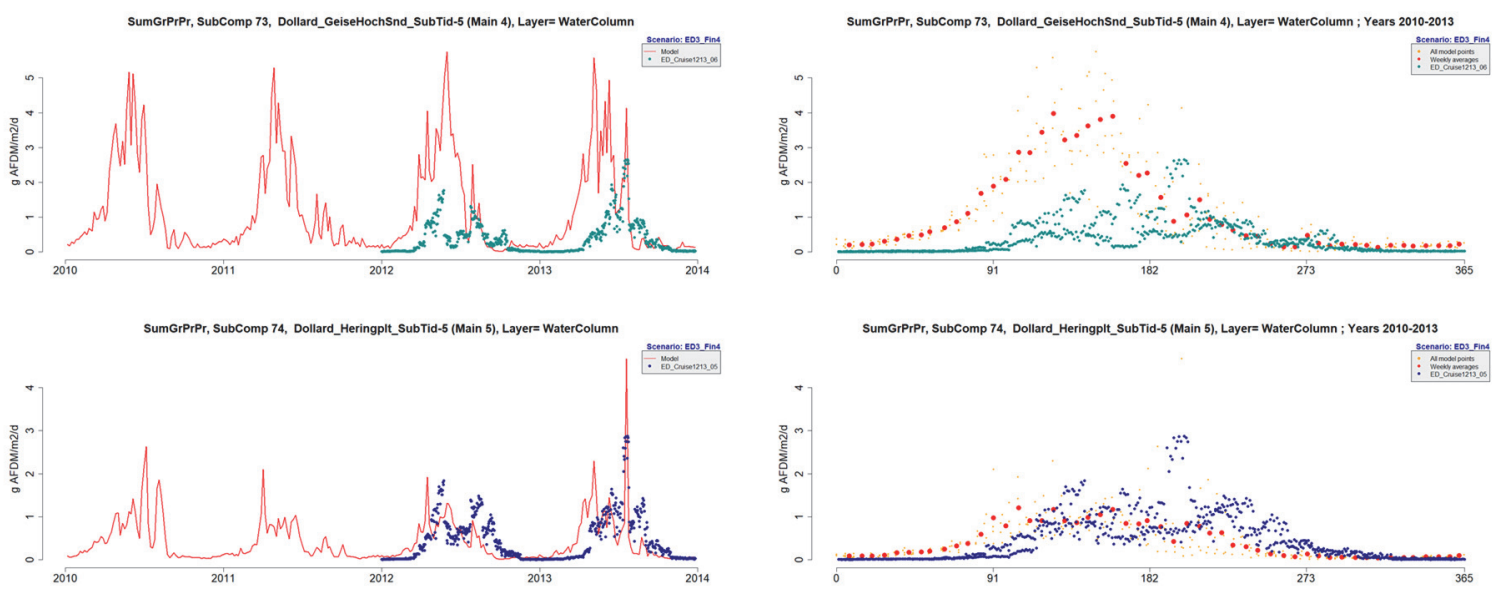

Figure 175 Gross primary production at station 6 and 5. Sub-compartments 73 and 74 are subtidal areas. In $g$ AFDM $m^{-2} d^{-1}$.
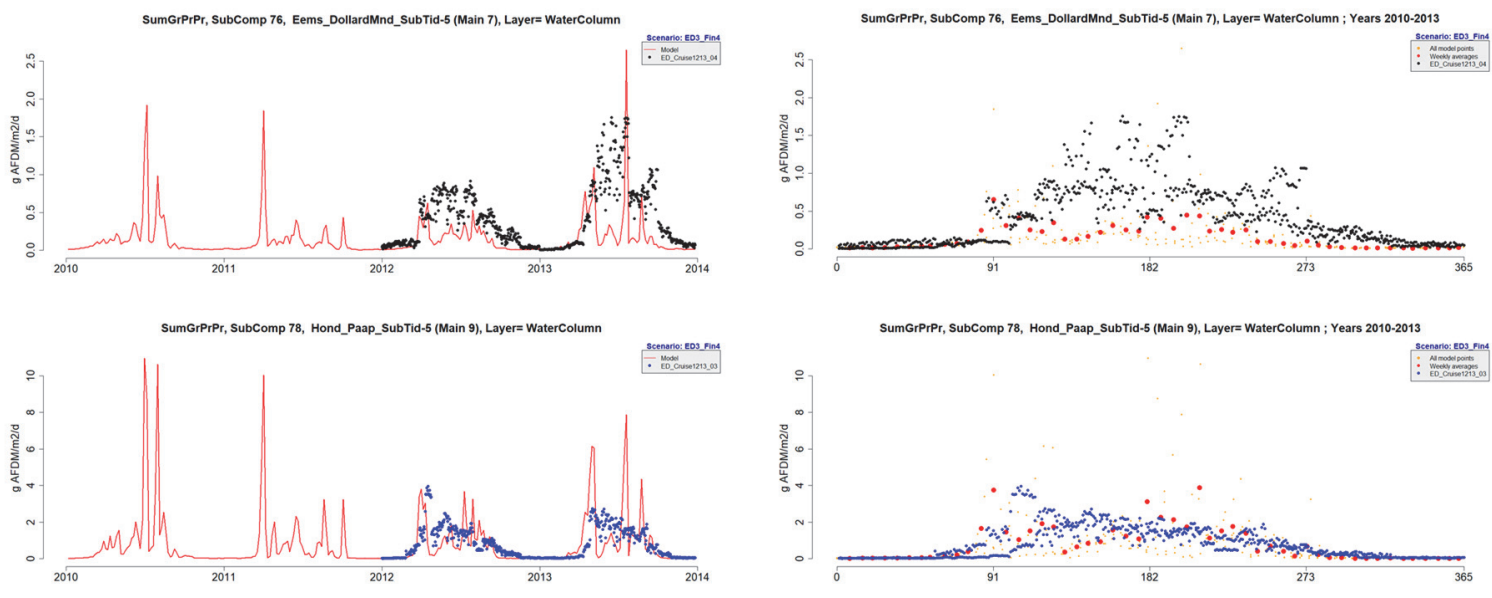

Figure 175 Gross primary production at stations 4 and 3, sub-compartments 76 and 78 , in $g$ AFDM $m^{-2} d^{-1}$
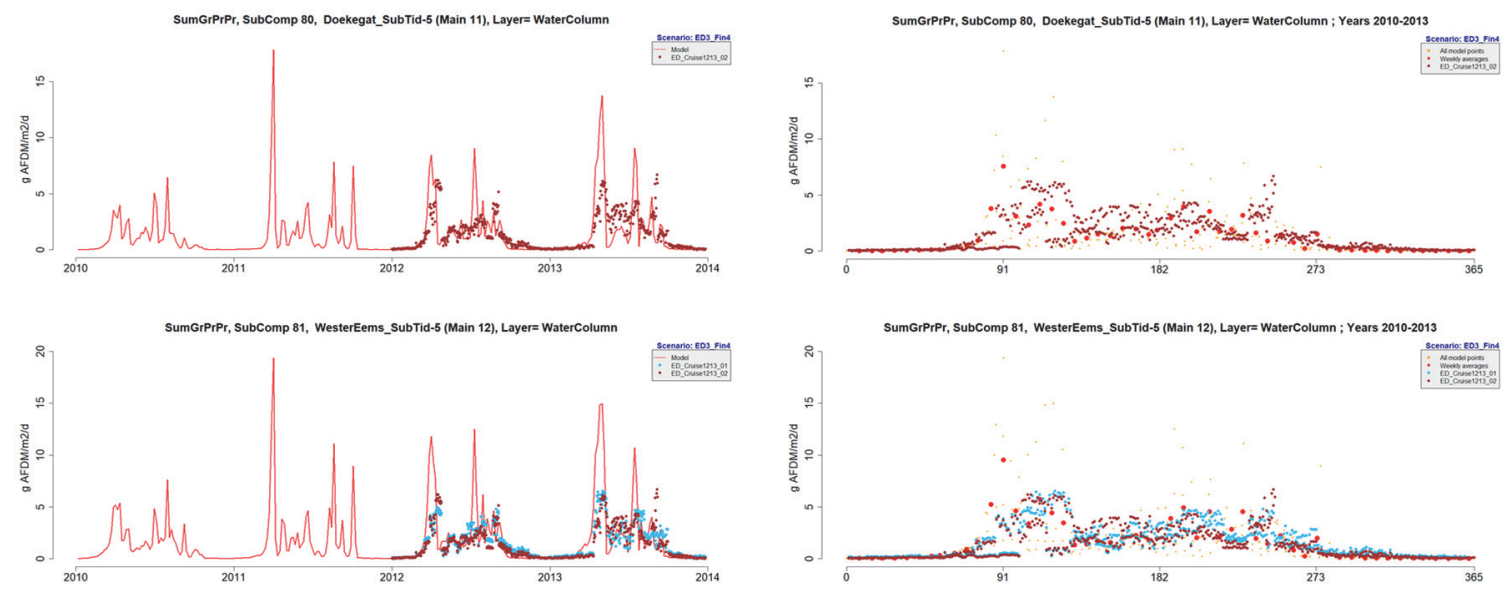

Figure 173 Gross primary production at stations 2 and 1. Sub-compartments 80 and 81 (subtidal area, upper and second row), and 106 and 107 (channel area, $3^{\text {rd }}$ and lowest row), in $g$ AFDM m $\mathrm{m}^{-2} \mathrm{~d}^{-1}$ 


\section{A25.13.3 Spatial distribution}

The computed distribution of the water column gross primary production is presented in Figure 176. The figure clearly illustrates that most of the gross production takes place in the deeper and more near-North Sea parts of the system. Tidal flats and the Dollard area hardly contribute to the overall system production.

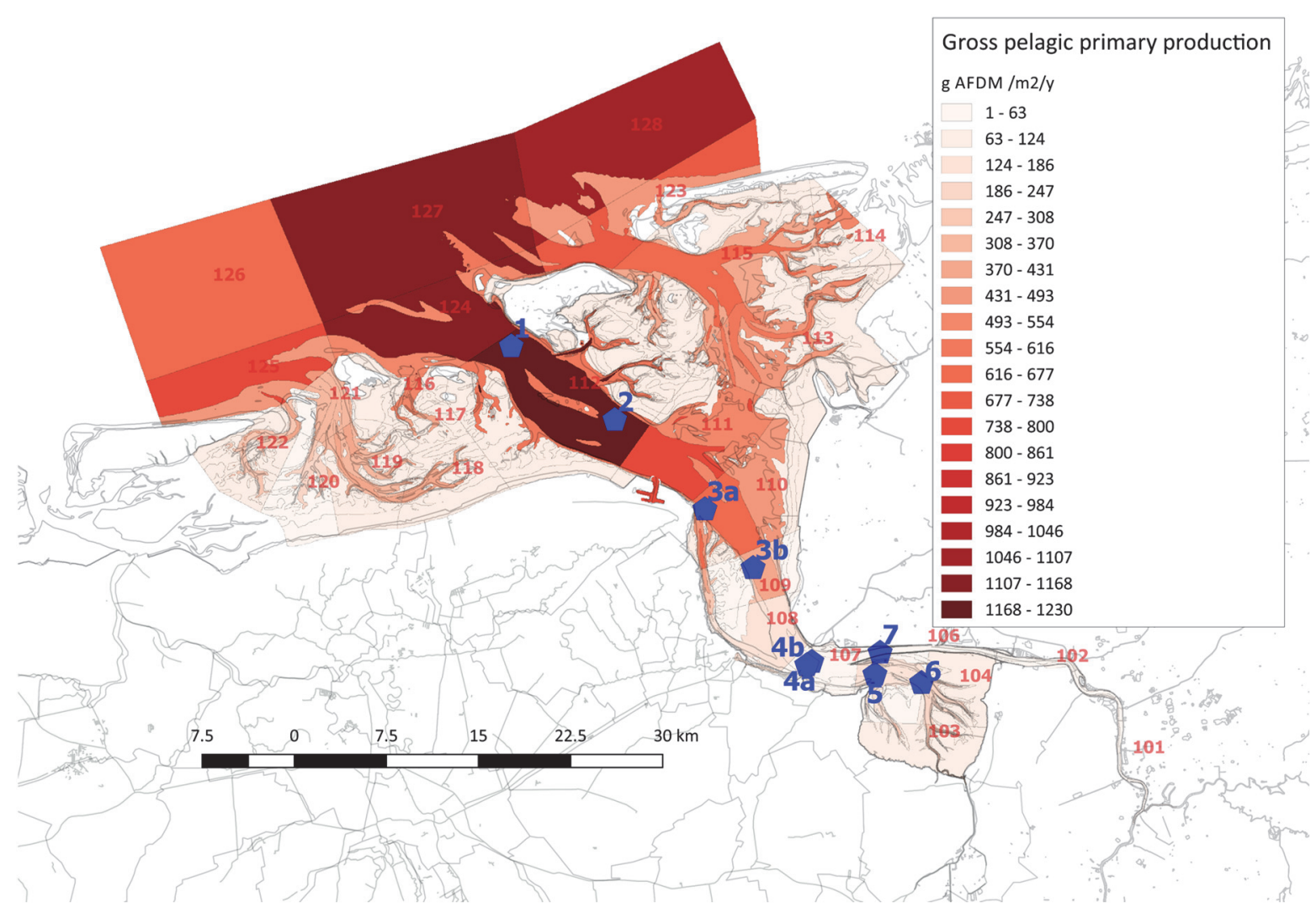

Figure 176 Gross water column primary production as computed by the model. In $g$ AFDM $m^{-2} y^{-1}$. Monitoring station IMARES (2012-2013 research) are added. Note that stations 3a, 4a and 7 have only been visited in the very beginning of the research, and not used to construct Figure 177.

\section{A25.13.4 Comparison with the "Riegman et al" 2012-2013 research results}

The 2012-2013 Ems-Dollard research by Riegman et al (Brinkman et al, 2015) resulted in data for each station, in an estimate for the whole compartments where the sampling points were in, and an estimate for the whole 'pure' Ems-Dollard-system (see Figure 4).

Presently computed gross primary production values for the water column are shown Figure 176; values measured in 2012 (IMARES research, Brinkman et al, 2015) are presented in Figure 177. Presently computed values for stations 1-3 ánd 5 are roughly the same as those computed in $2012 / 2013$. The value for station 4 is lower, those for 6 higher. It was already mentioned before (Appendix A25.10, on pelagic chlorophyll) that for the Dollard mouth (there where the Ems river enters the estuary simulated chlorophyll ) values are too low; and those in the Dollard are too high. Overall are both results not very far from each other, especially when one realizes that the area connected with stations 1 and 2 is much larger than that of station 4 and 6 . 


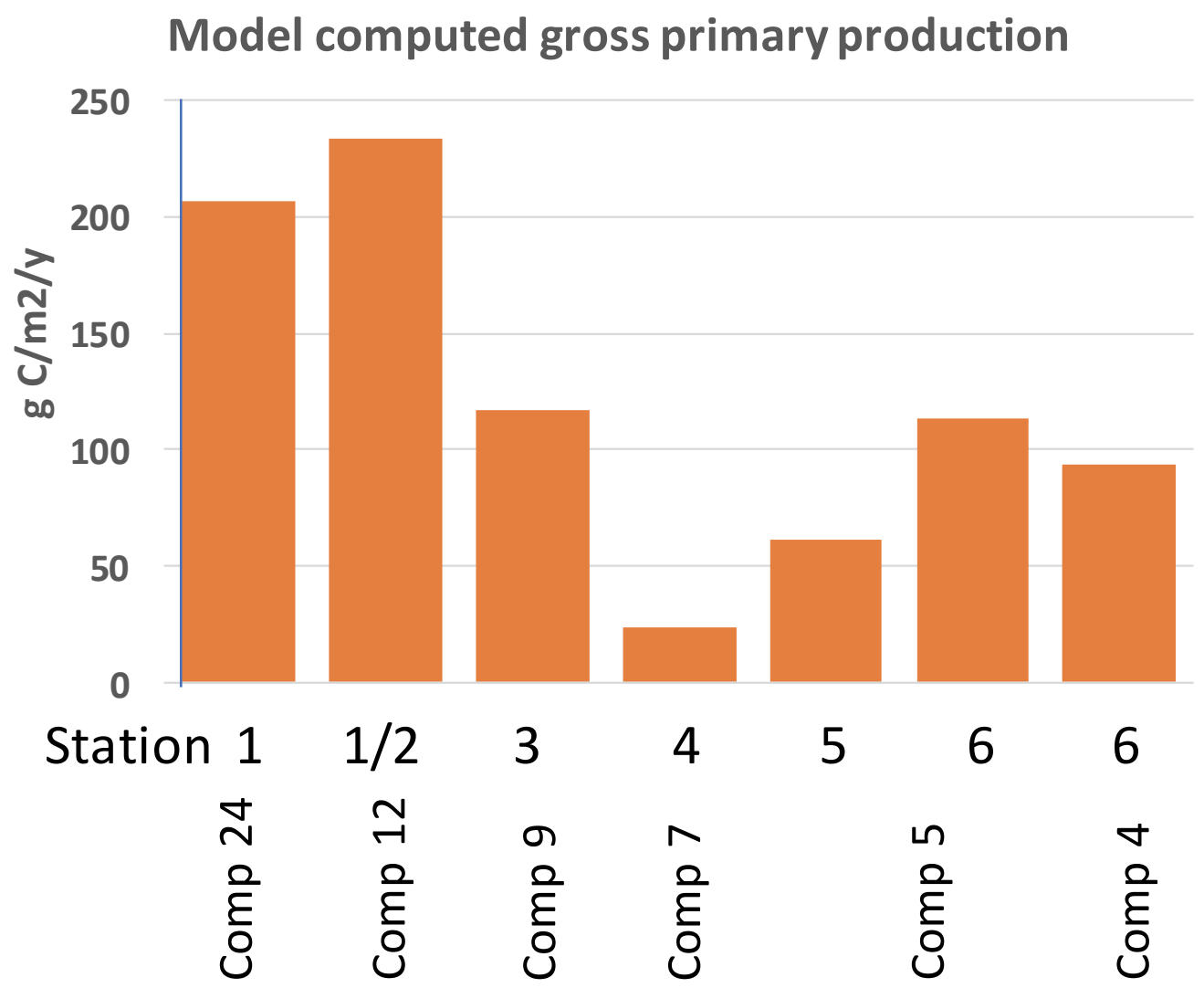

Figure 178 Computed gross primary production for the subtidal areas that have bene subject to the primary production measurements by Riegman et al in 2012/2013 (Brinkman et al, 2015). Note that station is is on the border between compartments 12 and 24;

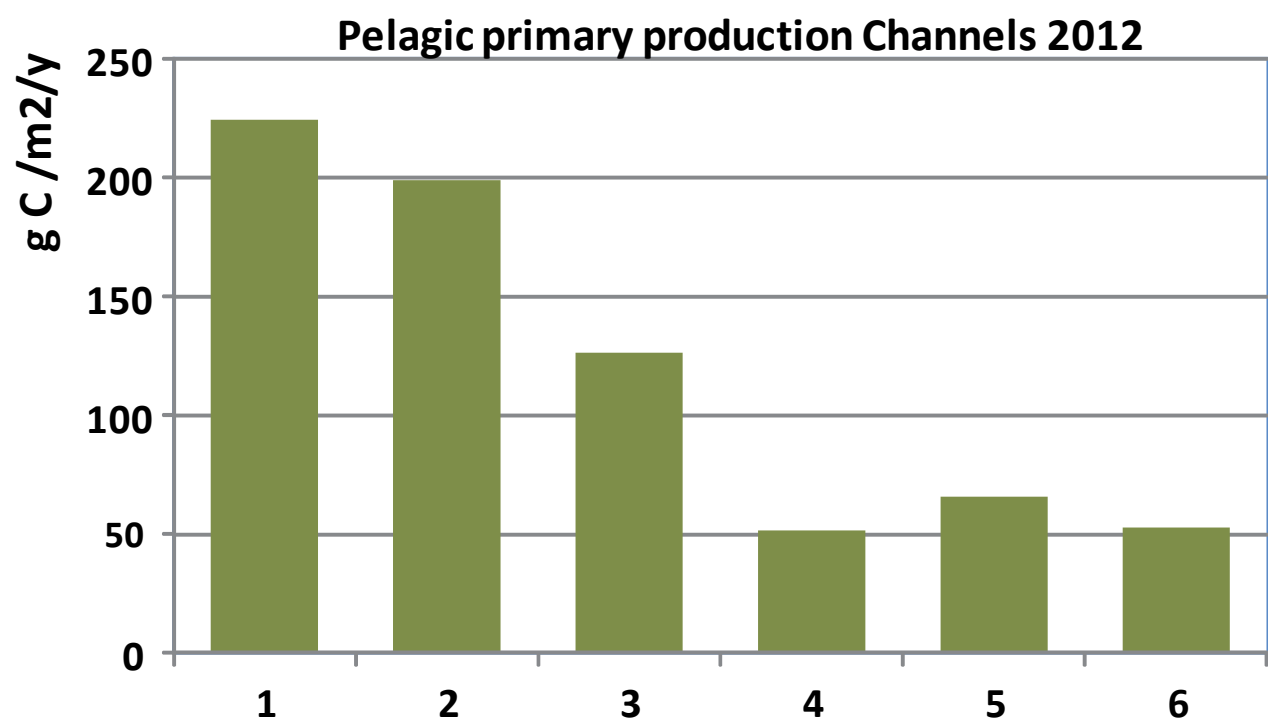

Figure 177 Gross water column primary production in the channels (g C/m2/d) in 2012 (IMARES, Brinkman et al, 2015). Sites are shown in Figure 176 


\section{A25.13.5 Suggestions and possible improvements}

- Better check primary productivity data from the Riegman et al research (Brinkman et al, 2015) with the model results here.

\section{A25.14 Net pelagic primary production}

While field and laboratory data usually concern gross pelagic primary production the nett primary production (the resultant of the gross production and respiration of algae) is most relevant for those animals living from the algae: after all, this is what is left to be eaten. Field values are scarce or even absent. This has to do with the laboratory method applied: when measuring ${ }^{14} \mathrm{C}$-uptake experiments last not too long simply to avoid excretion of (part of) the same ${ }^{14} \mathrm{C}$.

This problem does not exist when oxygen production is used as method, since there always is some uptake (the respiratory part). But this method takes more time to handle, is less accurate and it is harder to discriminate between bacteria (also using oxygen) and algae. Thus, in model computation like the present ones, a respiration rate parameter is used to compute respiration and thus, net production is computed.

Model results are shown in Figure 179; the inner water column parts have a negative contribution to overall net production, and most of the organic matter is produced in the outer areas. This includes the suspended phytobenthos production, but not benthic production. See next section .

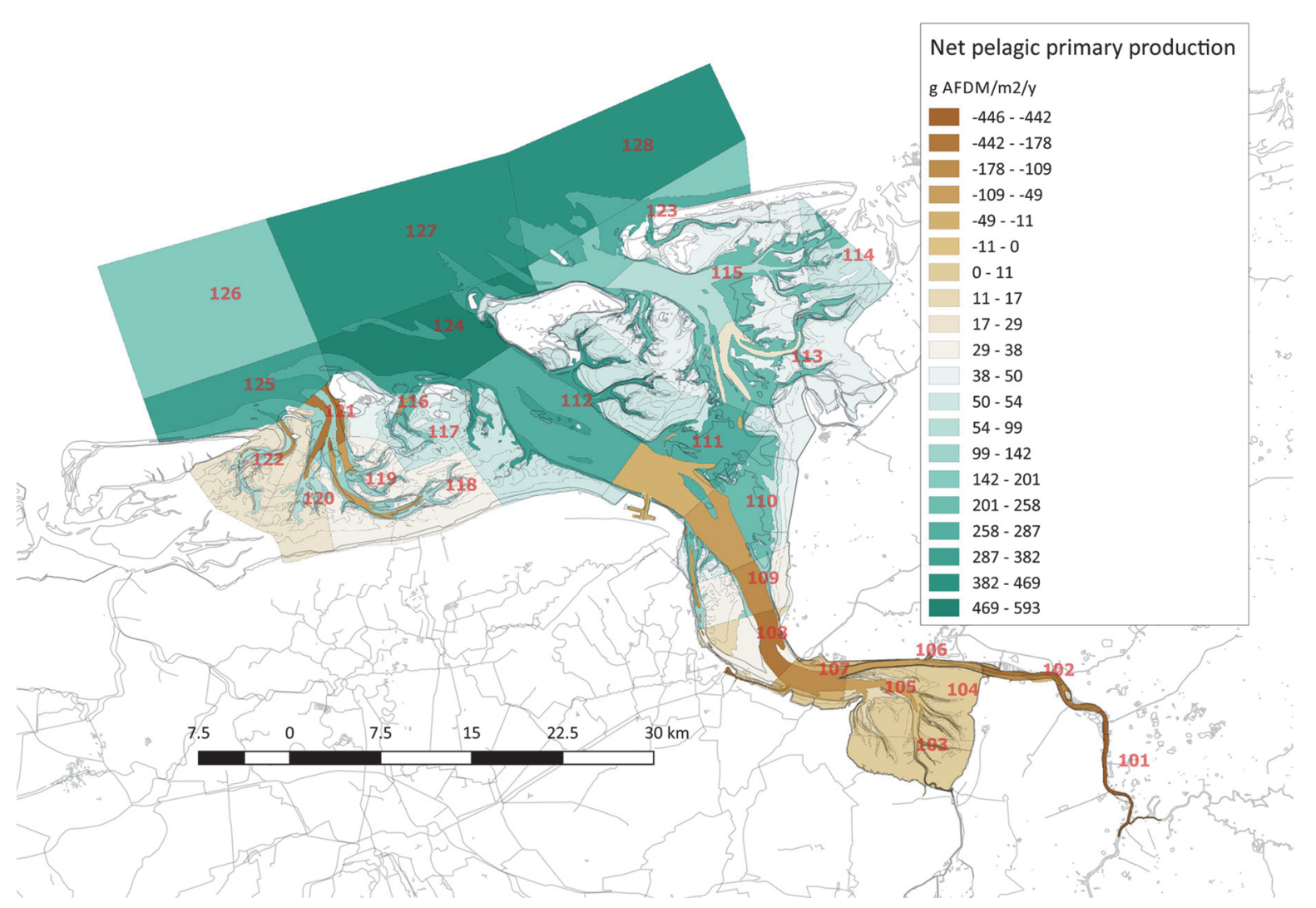

Figure 179 Computed net primary production in the system ( $\mathrm{AFDM} \mathrm{m}^{-2} \mathrm{y}^{-1}$ ) 


\section{A25.15 Benthic primary production}

During the Riegman et al-research, in 2013 also benthic primary production has been subject of the measurements. It was discussed in Brinkman et al (2015), that one of the steps chosen made it very hard to come up with reliable results; possible outcomes varied one order of magnitude. Earlier research (de Jonge, 1992; De Jonge \& Colijn, 1994) came up with values of about 60-230 g C $\mathrm{m}^{-2}$ (sediment surface) $\mathrm{y}^{-1}$; that is, high values in the Dollard area. Note that these values originate from a period with still high organic loads from the Westerwoldse Aa into the Dollard.

Present results are shown in Figure 180. Also in deeper parts, some benthic primary production

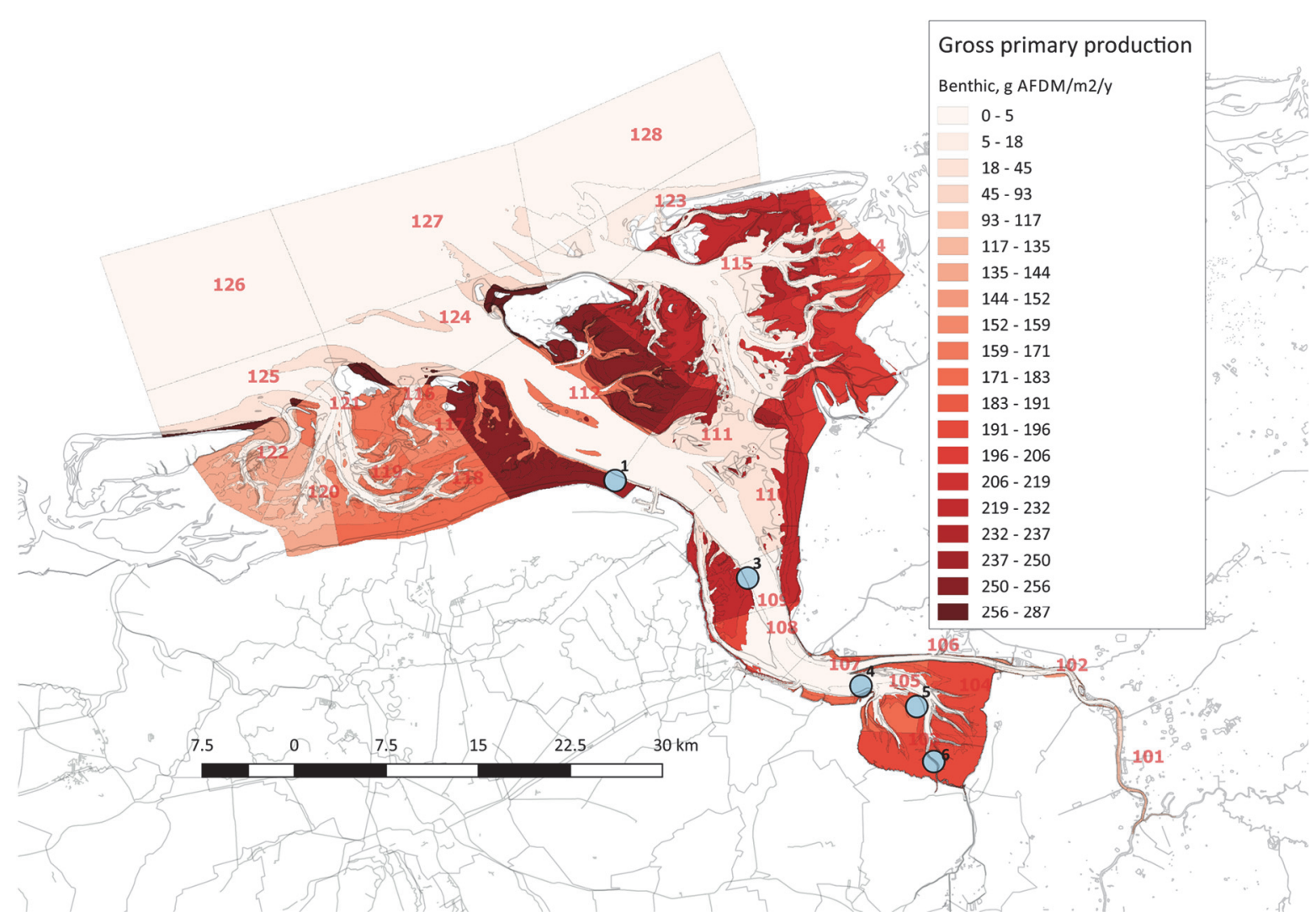

Figure 180 Computed benthic gross primary production, g AFDM $m^{-2} y^{-1}$.

exists. This is in line with the model results on benthic chlorophyll, showing some chlorophyll left in deeper areas. There are three topics that need attention in next research:

- very likely, grazing effects in those areas (e.g. offshore parts) are underestimated.

- there still is some light available in the deepest and off-shore parts of the system (Figure 129), but this is very sensitive to the light attenuation coefficient computed

- part of the benthic algae reaches those areas because of resuspension from tidal flats and subsequent settling in those deeper areas. It is not known how realistic this is.

\section{A25.16 Sum net primary production}

For any system, the resultant of benthic and pelagic production is relevant. The sum of net benthic and pelagic primary production is shown in Figure 182 and Figure 181. Negative contributions are found in the deeper gullies. 


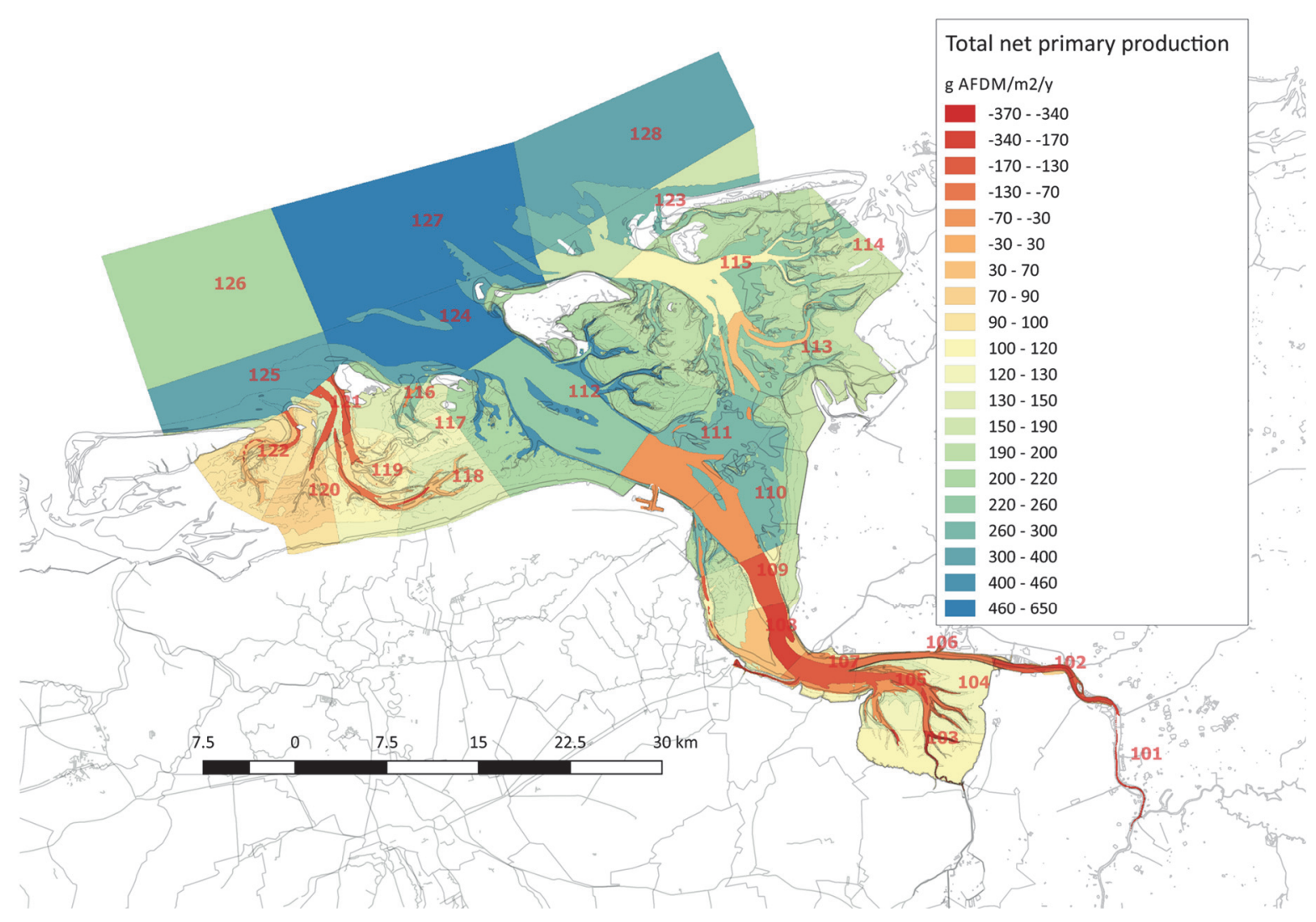

Figure 182 Total net primary production in each sub-compartment, in $g \mathrm{DM} \mathrm{m}^{-2} \mathrm{y}^{-1}$. Multiply with 0.39 to find values in $\mathrm{gC}^{-2} \mathrm{y}^{-1}$.


Figure 181 Same as Figure 182, but positive (upper) and negative (lower) contribution separated. 


\section{A25.17 Oxygen and nitrate sediment penetration depths}

\section{A25.17.1 General}

Mineralization of organic matter in the sediment needs presence of oxygen or nitrate as oxidizing substances. More substances may act as oxidising agents (especially sulphate, present in large amounts), but these are not included in the model. The last process modelled in the model regarding mineralization is the formation of what is called 'reduced substance'. It is chemically defined as methane $\mathrm{CH}_{4}$, but this is only needed to be sure that mass and electron budgets are $\mathrm{OK}$. This 'methane' is nothing else than a result of anaerobic degradation, forming $\mathrm{CO}_{2}$ and $\mathrm{CH}_{4}$ out of organic matter.

Supply of $\mathrm{O}_{2}$ and $\mathrm{NO}_{3}{ }^{-}$to the sediment occurs through diffusion from the water column, and production in the sediment by primary production $\left(\mathrm{O}_{2}\right)$ or oxidation of nitrite $\left(\mathrm{NO}_{3}{ }^{-}\right)$. Diffusion into the sediment depends on the relevant diffusion coefficients (a physical property), sediment porosity (related to the silt content) and bioturbation (accounted for by a simple parameter, and not really accurate in the model). Consumption of both oxidizers depends on the amount of organic matter present (per $\left.\mathrm{m}^{3}\right)$, temperature and the concentration of the oxidizers. The process thus is first order in oxygen and nitrate, but is made zero-order every new time-step. This is a trick to (i) avoid the solution of a first-order differential equation and (ii) to compute a value for the penetration depts. (by its properties, the first-order equation never really reaches zero).

In silty areas (like the Dollard), the content of organic matter per sediment mass is high, but the porosity is low. This i) increases the diffusive supply of oxidizers and ii) decreases the amount of organic matter per unit volume. This has typical effects in the model results.

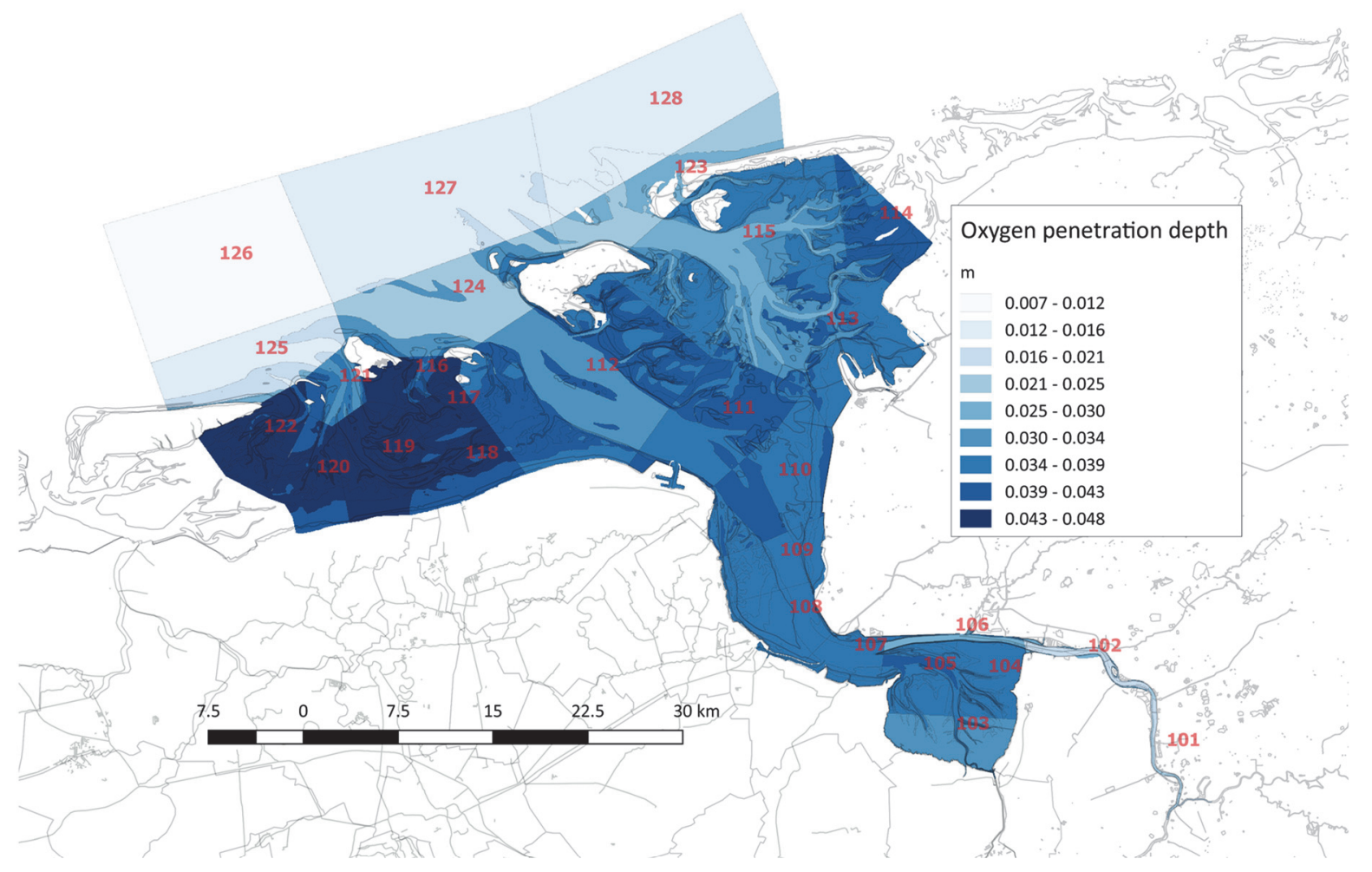

Figure 183 Penetration depth of oxygen in the sediment ( $m$ ) 


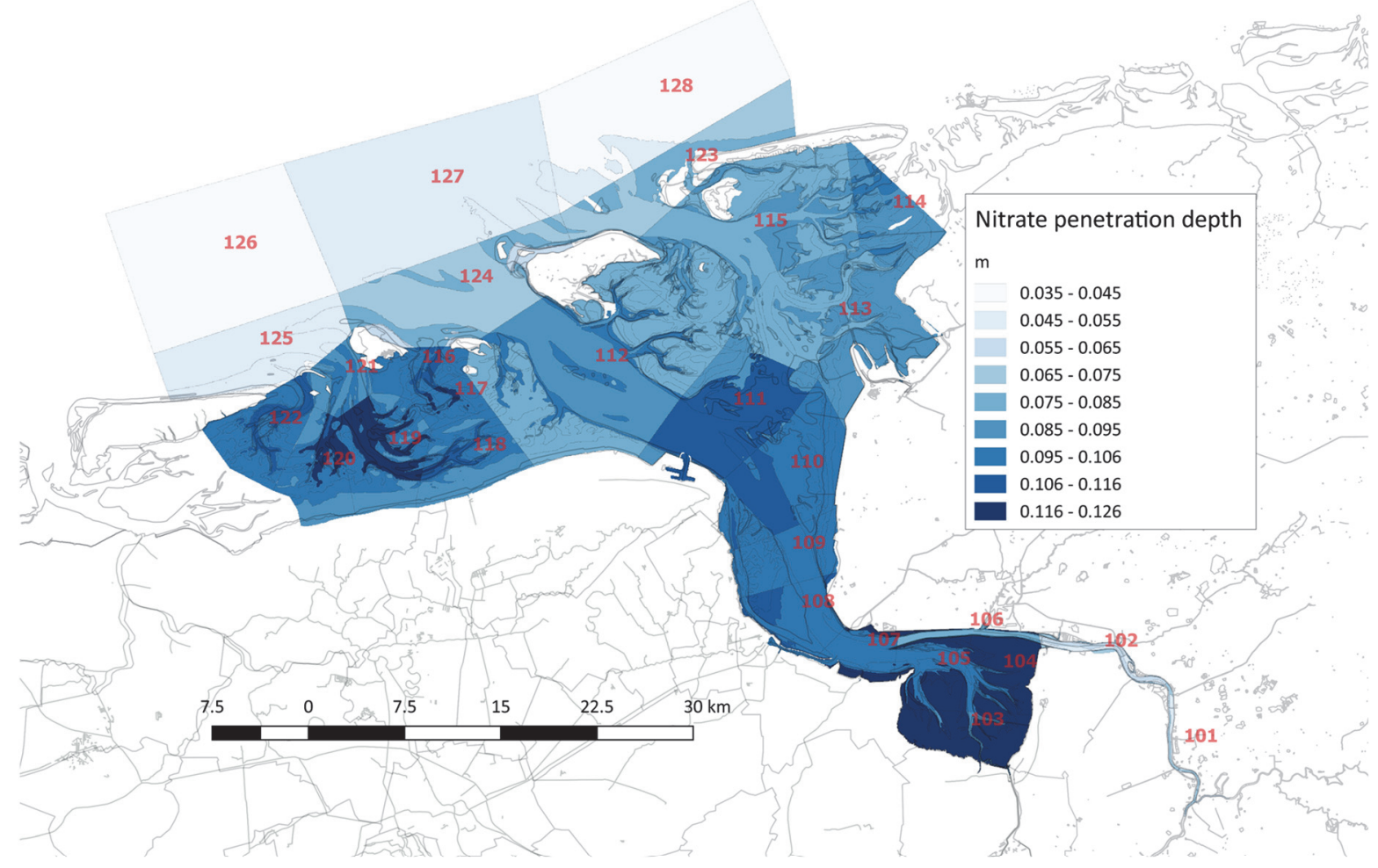

Figure 184 Penetration depth of nitrate in the sediment ( $m$ )

\section{A25.17.2 Spatial distribution}

In Figure 183 and Figure 184, the computed year-average penetration depths of oxygen and nitrate are shown, with highest values on tidal flats. Despite the characteristics mentioned before, the model results are somewhat counter intuitive. Largest values are found for the most active areas, i.e. the tidal flats. It is well-known that these areas sometimes have very thin oxidized sediment top layers. Thus, here's still some work to do.

\section{A25.17.3 Time series}

The computes values for oxygen and nitrate penetration strongly vary with the season, with low values in summer, and highest ones in winter. This of course is because of microbiological activities are (very) low when temperature is low. The highest values are found on tidal flats; it is even more pronounced than shown in Figure 183.

\section{A25.17.4 Suggestions and possible improvements}

- Check computed values with possibly available data (not searched for yet)

- Check results for mineralization rates with possibly available data 

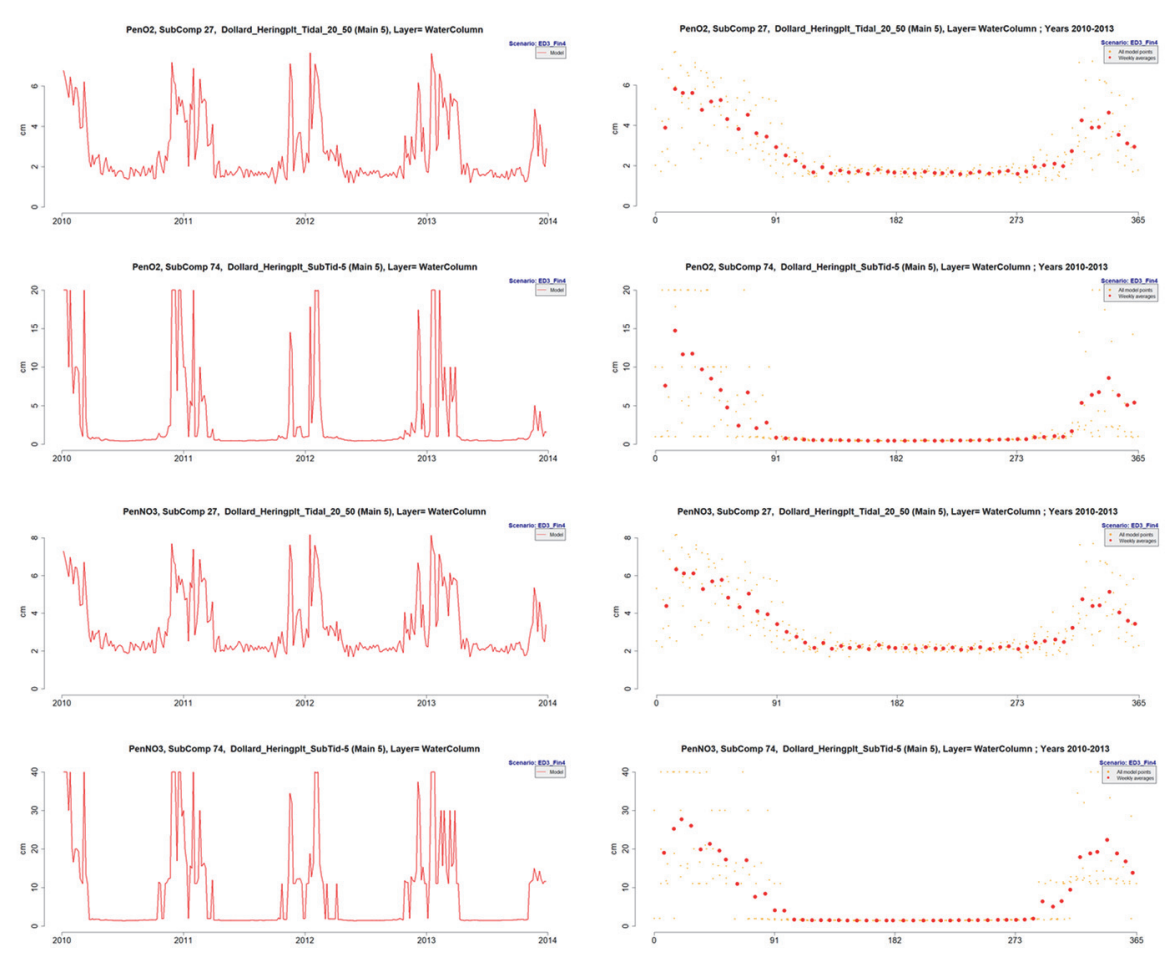

Figure 186 Penetration depths of oxygen and nitrate in tidal flats comp (27) and in gullies (comp 74) in the Dollard (main compartment 6)
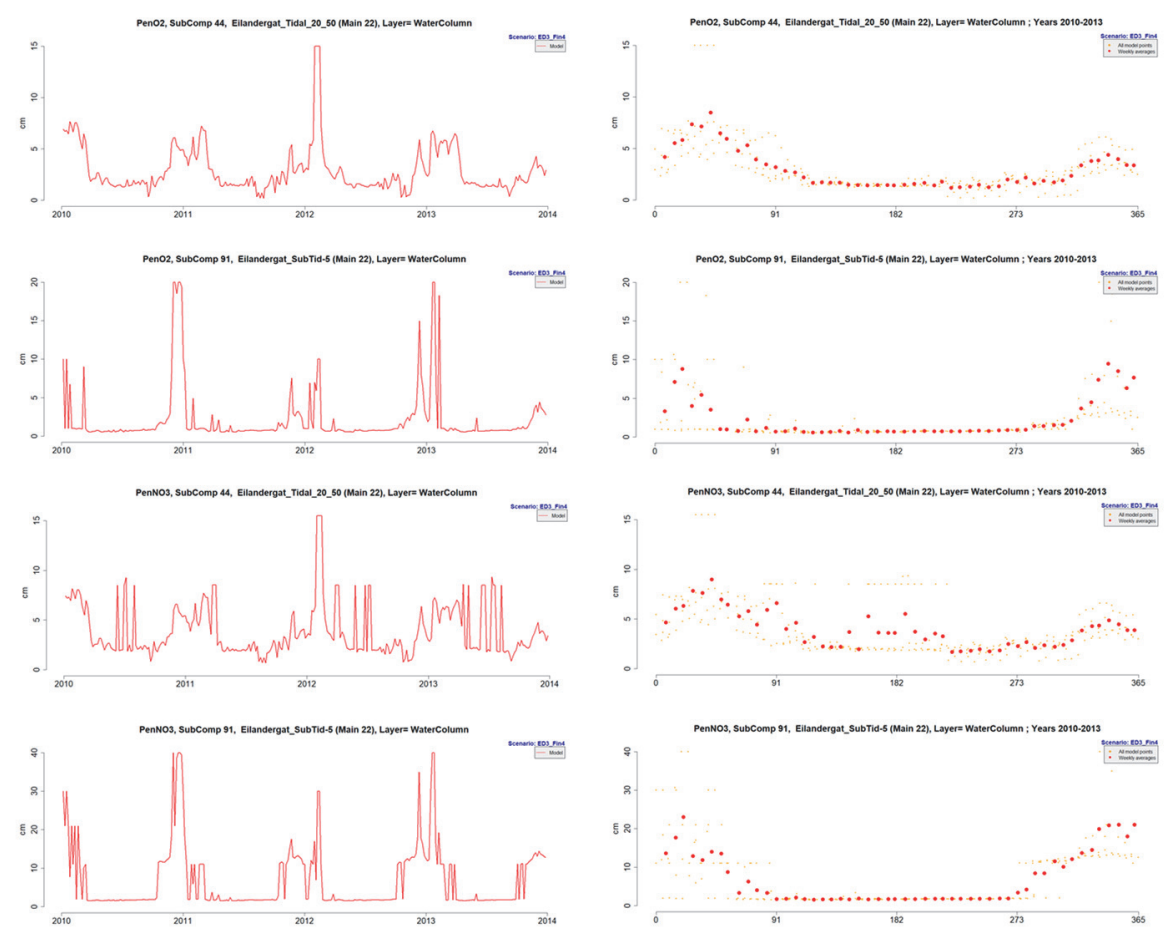

Figure 185 Penetration depths of oxygen and nitrate in tidal flats comp (44) and in gullies (comp 91) in a near North Sea area (Eilandergat) 


\section{A25.18 Nitrification and denitrification}

In the model, nitrification is only computed as the result of nitrite $\left(\mathrm{NO}_{2}^{-}\right)$to nitrate $\left(\mathrm{NO}_{3}{ }^{-}\right)$. If nitrite is not chosen to be present in the model, it concerns the direct oxidation of ammonium $\left(\mathrm{NH}_{4}{ }^{+}\right)$to nitrate. Nitrite is produced previously as oxidation of ammonium $\left(\mathrm{NH}_{4}{ }^{+}\right)$. The process may occur in the sediment and in the water column, and depends on both the oxygen and nitrite (or: ammonium if relevant) concentrations. The process is temperature dependent.

Denitrification is stored as the result of nitrate reduction during the oxidation of organic matter, and only occurs in the sediment, below the $\mathrm{O}_{2}$-containing top layer. Also, the oxidation of " $\mathrm{CH}_{4}$ " by nitrate adds to denitrification. Denitrification is a sink since the product is atmospheric $\mathrm{N}_{2}$, and thus disappears from the system. In Figure 188 and Figure 187Figure 188 both results are shown. Data however, are almost absent. Only a few literature data can be used as comparison.

Kieskamp (1994) mentions maximum denitrification values of $50 \mu \mathrm{mol} \mathrm{N} \mathrm{m} \mathrm{h}^{-1}$, and minimum values of $1 \mu \mathrm{mol} \mathrm{N} \mathrm{m}{ }^{-2} \mathrm{~h}^{-1}$ (winter values, when almost nothing happens) for the western Dutch Wadden Sea tidal flats. The present year-average values of 1.8 to $93 \mathrm{mmol} \mathrm{N} \mathrm{m}^{-2} \mathrm{y}^{-1}$ or 0.005 to 0.3

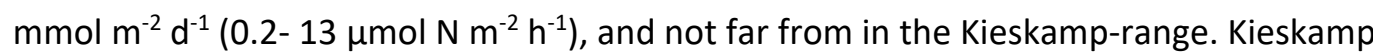
comments that his values are "on the lower edge of the range".

Bruns (1994) found maximum nitrification rates of 0.5-1.5 mmol N m $\mathrm{m}^{-2}$ in silty tidal sediments. The model computes maximum year-average values for tidal flats of about $1 \mathrm{~mol} \mathrm{~N} \mathrm{~m}^{-2} \mathrm{y}^{-1}$, or about $0.3 \mathrm{mmol} \mathrm{N} \mathrm{m}^{-2} \mathrm{~d}^{-1}$; thus, not very far from Bruns' data. A more detailed analysis of the data is beyond the scope of this report.

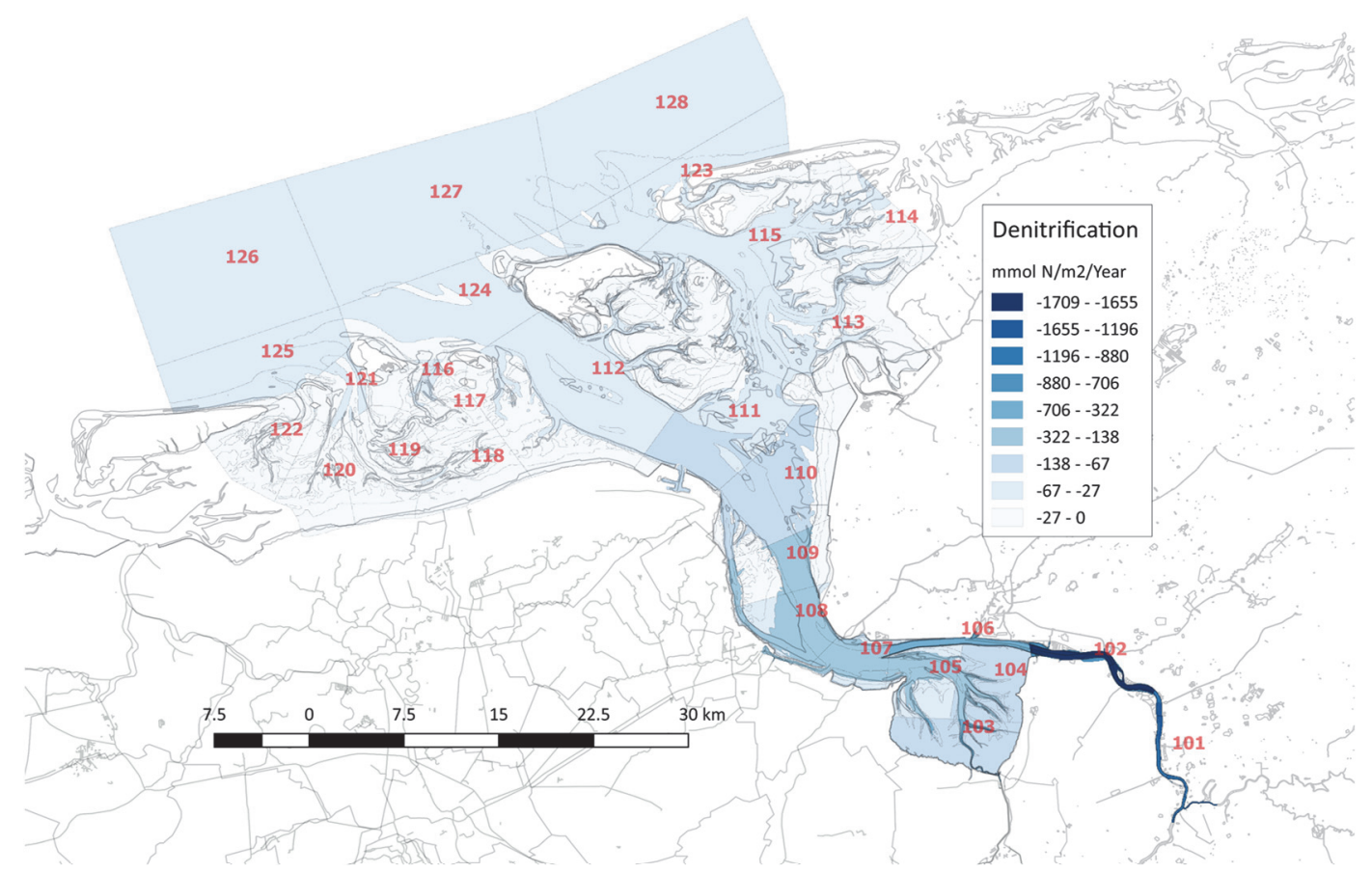

Figure 187 Computed denitrification in the system as mol $\mathrm{N} \mathrm{m}^{-2} \mathrm{y}^{-1}$. 


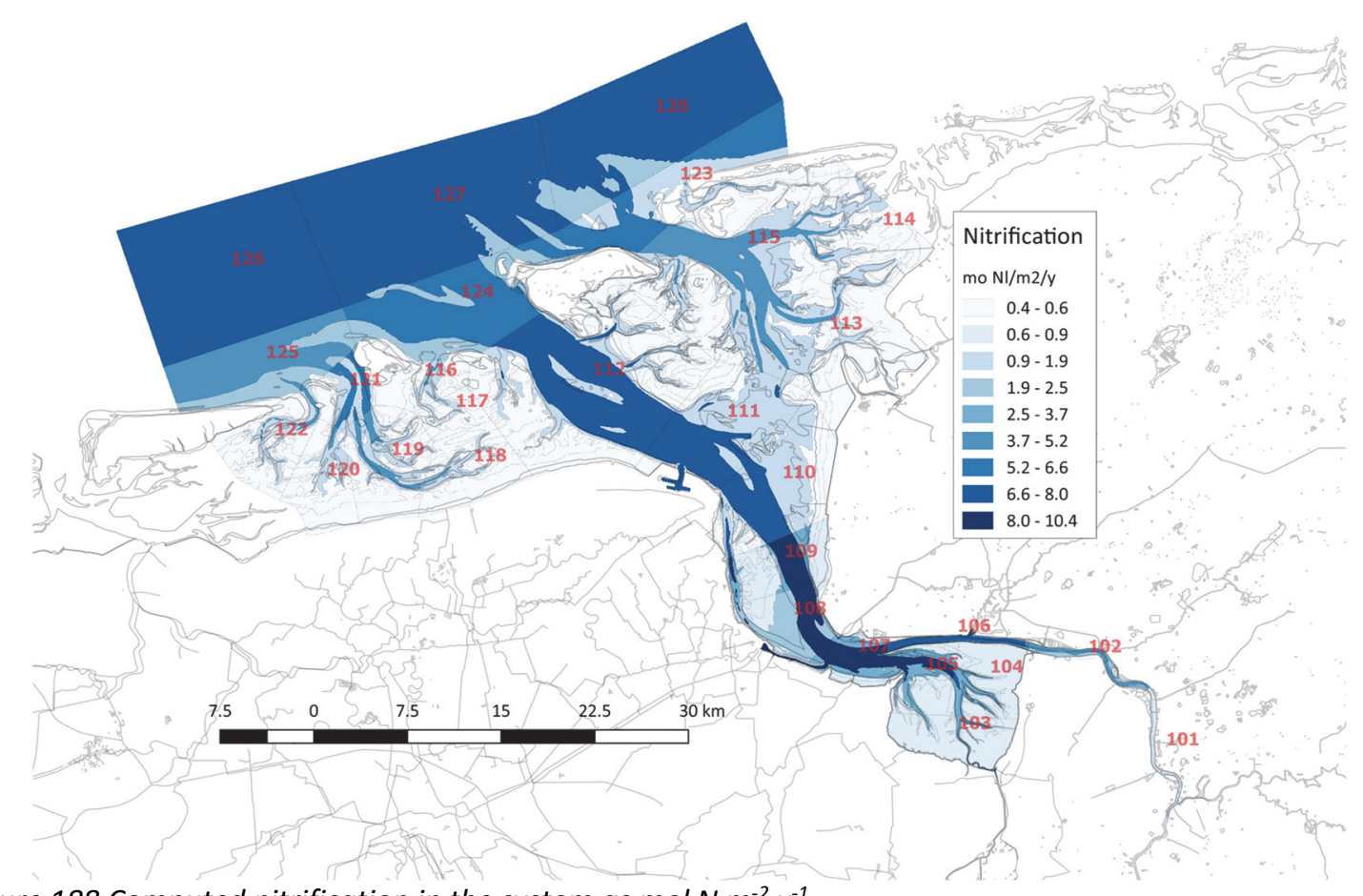

Figure 188 Computed nitrification in the system as mol $\mathrm{N} \mathrm{m}^{-2} y^{-1}$.

\section{A25.18.1 Suggestion and possible improvements}

- Check the parameters chosen for nitrification and denitrification. Better tuning must be possible 


\section{A25.19 Conclusions}

\section{A25.19.1 Data comparison}

This appendix A25 contains a small selection out of the model results, most of these can be compared to available data. However, almost all data available just cover a part of the system, and it appears not always to be easy to find out which model results can be compared to which field or laboratory data. Here certainly is an improvement possible. 


\section{A26 One simulation with changed turbidity}

\section{A26.1 Strategy}

As a final exercise, one simulation was performed with a changed turbidity. The strategy was that the resuspension parameters for solids and detritus were changed with a factor; this is listed in Table 28.

Table 28 Resuspension reduction factor used for the silt-reduction scenario. For compartment numbering see Figure 1. All sub-compartments in each main compartment have the same reduction factor.

\begin{tabular}{|c|c|}
\hline Main compartment & Factor \\
\hline 2 & 0.7 \\
\hline 3 & 0.5 \\
\hline 4 & 0.5 \\
\hline 5 & 0.5 \\
\hline 7 & 0.5 \\
\hline 8 & 0.5 \\
\hline 9 & 0.5 \\
\hline 6 & 0.6 \\
\hline 10 & 0.6 \\
\hline
\end{tabular}

These reductions concern the compartments closest to the site where the Ems river enters the estuary; see Figure 1.

\section{A26.2 Results}

A very brief overview of results is presented here, since in fact all the figures presented in the previous appendix A25 can be repeated here (and more). But we restricted ourselves to a few overview pictures of which we believe primary production (gross and net; Figure 190) and macrofauna densities (bottom dwellers and filter feeders; Figure 189) are the most interesting ones. In both cases, a positive change can be observed, although changes are not very spectacular. According to the present computations, there is hardly a negative effect. One might expect such behaviour, since primary production is fed by nutrients, and if the available nutrients are consumed more inside the system, it might happen that closer to the North Sea it negatively may affect primary production. As far as these first results tell, this not the case. But, care should be taken since a number of model improvements is suggested (see appendix A27), with some of these rather important.

In Figure 192, a time series plot is shown for primary production and total fauna density, averaged for the whole system (including the North Sea coastal zones, and not exclusively for the pure EmsDollard compartments (as shown in Figure 4). Finally, in Figure 191, it is shown how, according to the present simulation, gross primary production changes in the main part of the estuary after a turbidity reduction. The increase shown is higher than is shown in Figure 192, mainly because the other compartments do not change as much as the inner do. 


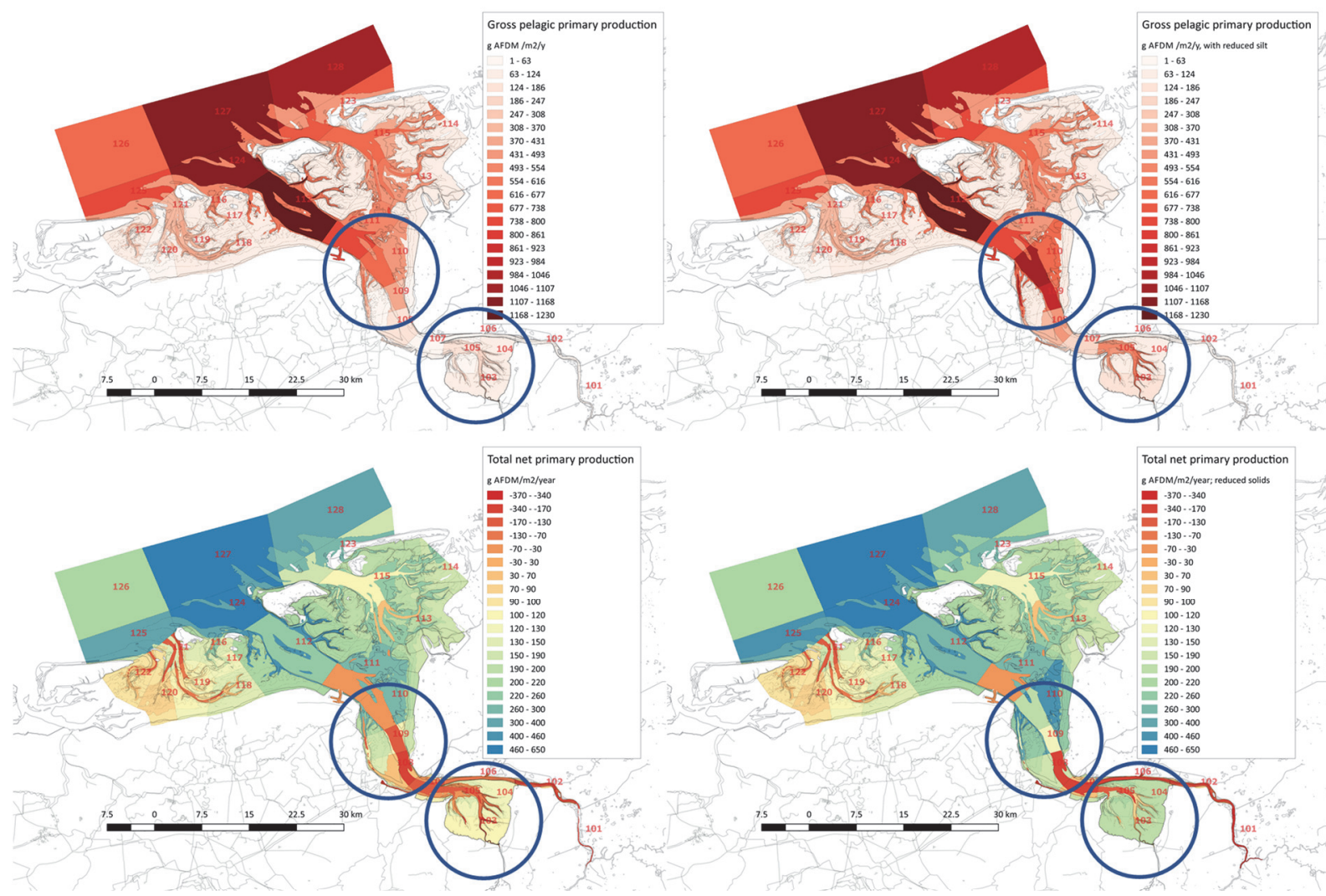

Figure 190 Changes in gross (upper) and net (lower) primary production in the system, standard (left) and after reduction of suspended solids (right, according to Table 28.

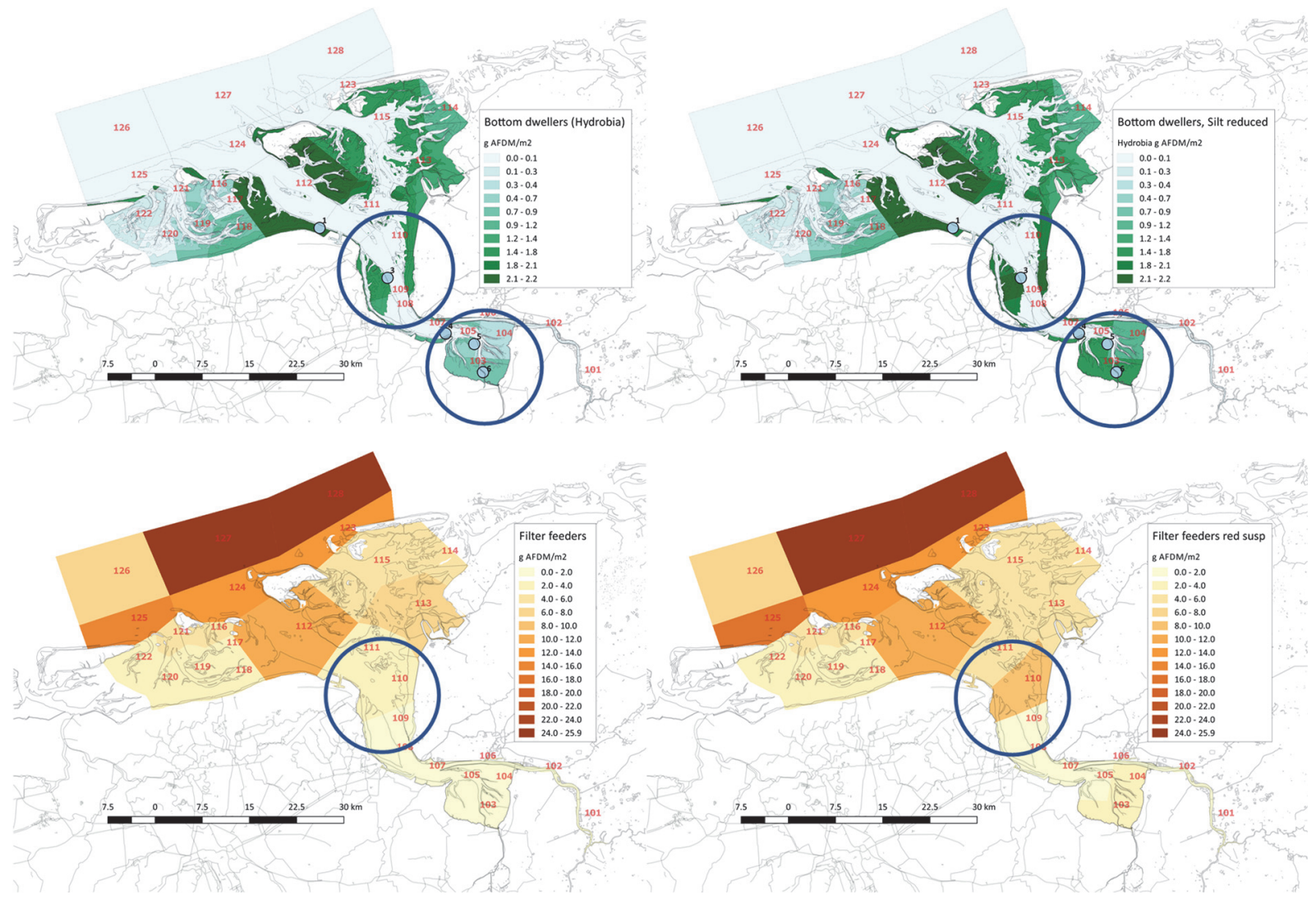

Figure 189 Changes in bottom dwellers (upper) and filter feeders (lower) in the system, standard (left) and after reduction of suspended solids (right, according to Table 26. 

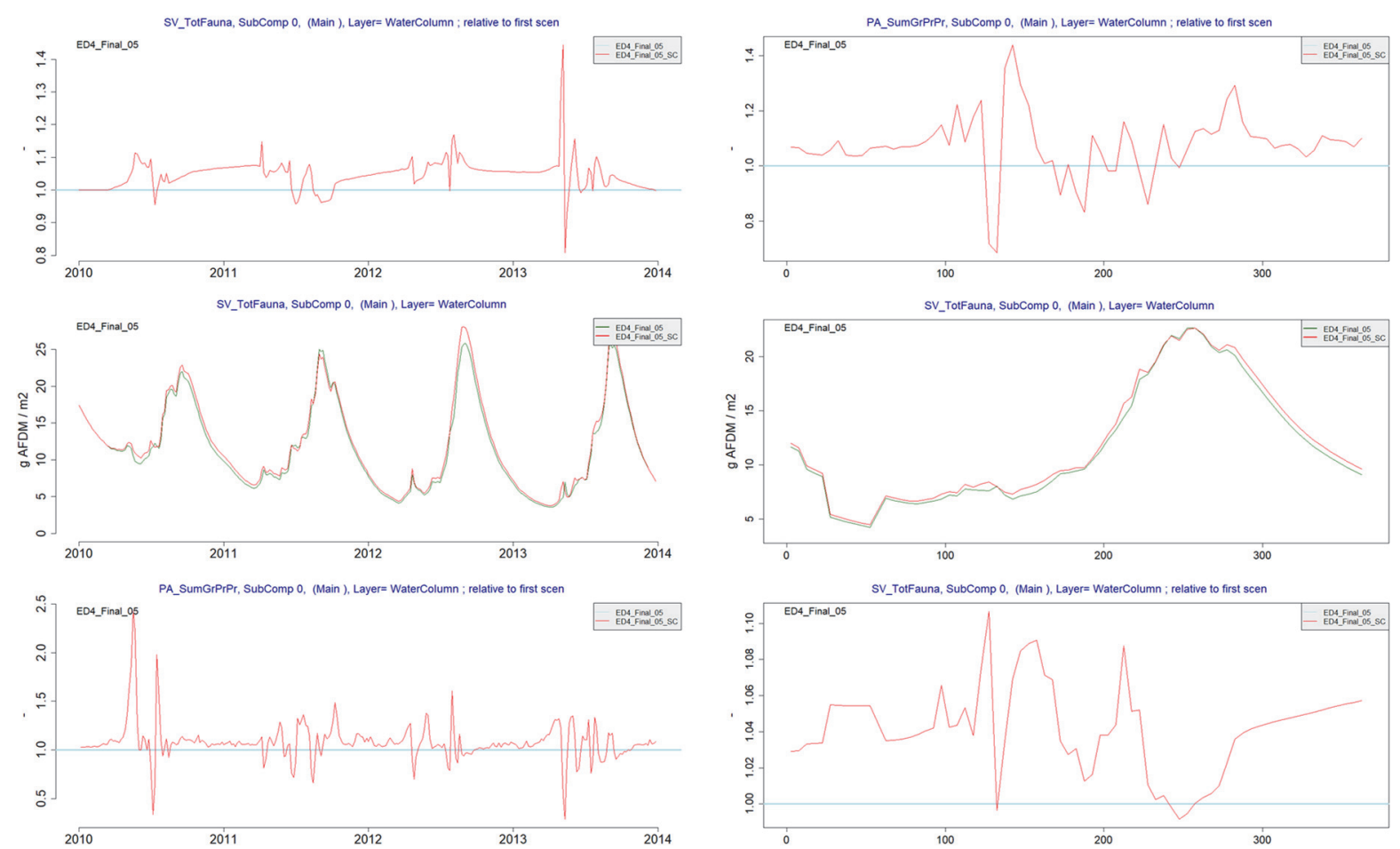

Figure 192 System characteristics changing with a lower turbity in some inner compartments (after Table 28). Upper: relative changes in gross primary production, mid: absolute values computed for sum of all fauna densities, lower: same, but relative changes. The standard situation in the graphs that show relative changes is the basic system (no turbidity changes), this is always the blue horizontal line (upper and lower graphs). Left: situation each year, right: seasonal variation.

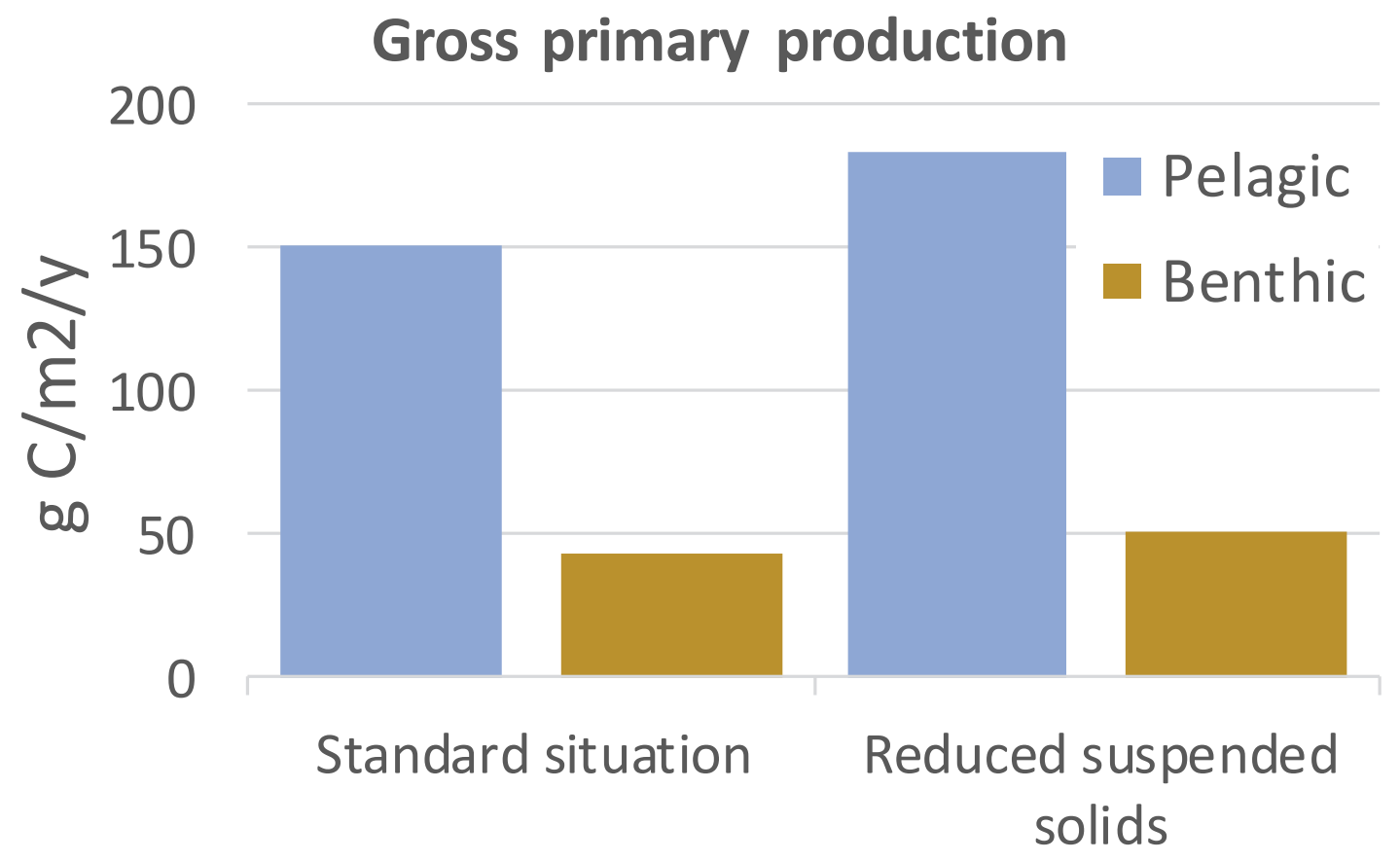

Figure 191 Gross primary production changes in the 'pure' Ems-Dollard estuary (Figure 4) after turbidity reduction (see Table 28) 


\section{A27 Improvements needed/suggested}

\section{A27.1 All suggestions listed}

Most of the sections in appendix A25 (and some mentioned in A26) have one or more suggestions for improvements. These are collected here, just as a list, without further explanation.

On boundaries:

- Most important improvement is to include the Lauwersmeer-outflow area (called the Zoutkamperlaag), and (thus) the outflow of fresh water from the Lauwersmeer.

On silt in the system

- Activate the computation of vertical profiles. This option is already present in the model, but not applied yet. Next, the distribution on the tidal flats between the Groninger shore and the islands of Schiermonnikoog and Rottumeroog may be improved. Finally, the German tidal flats now have lower suspended solids simulated than the Dutch 'counter'tidal flats. This does not seem plausible, thus must be improved also.

On extinction:

- The spatial distribution of lower and higher light attenuation coefficients must be improved (although differences certainly are not major): close to the Groningen north shore, and on the German tidal flats.

- Also, the attenuation coefficient, and the concentration of suspended solids, can be adjusted around monitoring site 3 in Figure 126.

On atmosphere/water exchange:

- Check the computed air/water exchange parameters with literature values

- Check the computed sediment/water exchange (not mentioned here) with literature values.

On adsorption of $\mathrm{P}$ and Si onto sediments

- $\quad$ Find better adsorption parameters

- Compare computed water sediment fluxes with available observations (from other parts of the Wadden Sea or even the North Sea coastal zone)

On phosphorus characteristics:

- Find out what the cause is of the low [P]-values computed (especially in the inner compartments)

For silicate dynamics:

- Find better parameter values for frustule dissolution

- Find better time series for [Si] in tributaries

For nitrification and denitrification:

- Find better nitrification rate parameters (and probably also: descriptions of the process) 
- Check why these results differ from the mentioned previous ones where the fit between data and model results were better.

- Check the computed sediment/water exchange (not mentioned here) with literature values.

For ammonium characteristics:

- Study the effect of ammonium adsorption

- Check further what might cause the overestimation of ammonium.

- Check sediment/water exchange rates with possibly available data

On pelagic chlorophyll:

- Chlorophyll in the Ems river needs better tuning

- Better tune the effect of salinity on freshwater and marine phytoplankton

Tuning both parts better probably will also improve the chlorophyll simulation results for main compartments 107-109 (numbers mentioned in Figure 157), now results are far below observations. These improvements are really needed since these are target compartments for all attempts to improve water transparency.

One other possible improvement is

- Model the chlorophyll content of the phytoplankton. Thus, production rates per unit of biomass change during the season and depend on external conditions. This asks for new descriptions in the model.

On benthic chlorophyll

- Check the reason of the very small differences between the three tidal parts in each main compartment

- Make early spring and late autumn values more like summer values

- Include other fauna that also grazes upon phytobenthos (e.g. worms and/or shellfish).

- check the resuspension and distribution of benthic algae.

On primary production:

- Better check primary productivity data from the Riegman et al research (Brinkman et al, 2015) with the model results here.

- check the activity of suspended benthic algae: is their productivity similar then?

On macrofauna:

- Make better overviews of the observations of the benthic fauna (then now has been done by the author): take care of the non-normal distributions of animal numbers in the samples. Possibly a Poisson-like distribution analysis is suited better. Also, the method of stratified sampling by IMARES/WMR should better be taken into consideration, this is to use the several areas that are supposed to be representative for the samples taken instead of one uniform size.

- Do compute and check better the relationship between suspended matter and filtration characteristics. The material necessary is present: see appendix A24.

On oxygen and nitrate penetration depths: 
- Check computed values with possibly available data (not searched for yet)

- Check results for mineralization rates with possibly available data

On nitrification and denitrification:

- Check the parameters chosen for nitrification and denitrification. Better tuning must be possible.

\section{A27.2 Most relevant improvements}

Some of the suggestions mentioned above are really needed, some others a much less important. The first things to improve concern, according to the authors' opinion:

vii) to include the Lauwersmeer-input (that implies an extension of the model westward),

viii) to implement (==switch on and check) the computation of vertical suspended matter profiles,

ix) to improve the implementation of the effect of suspended solids on fauna,

$x$ check the resuspension and distribution of benthic algae, and their activity when suspended (is their productivity similar then?)

xi) check the reason of the discrepancy between computed and observed [P]

xii) check the reason of the discrepancy between computed and observed $\left[\mathrm{NO}_{3}\right]$ 


\section{A28 Overview of used parameters}

In the next tables, the parameter settings for ions, solids, phytoplankton, detritus and fauna as used for the final simulations are listed. Since these are hard to interpret without the relevant equations, one is referred to the two basic model documents (Brinkman, 1993 and Brinkman \& Smit, 1993).

\section{A28.1 lons/elements}

\begin{tabular}{|c|c|c|c|c|c|c|c|c|c|c|}
\hline \multicolumn{11}{|l|}{ Ion parameters } \\
\hline Ion/Element_Names & $\mathrm{NH} 4$ & $P$ & $\mathrm{CO} 2$ & $\mathrm{O} 2$ & NO3 & $\mathrm{NO} 2$ & Si & $\begin{array}{l}\text { Chlo } \\
\text { ride }\end{array}$ & $\begin{array}{l}\text { Reduced } \\
\text { Comp }\end{array}$ & $\begin{array}{l}\text { RestCh } \\
\text { arge }\end{array}$ \\
\hline Chemical_Formula & $\mathrm{NH} 3$ & H3PO4 & $\mathrm{CO} 2$ & $\mathrm{O} 2$ & $\begin{array}{l}\text { HNO } \\
3\end{array}$ & $\begin{array}{l}\mathrm{HNO} \\
2\end{array}$ & $\mathrm{SiO} 2$ & $\mathrm{Cl}-$ & $\mathrm{CH} 4$ & $\mathrm{H}+$ \\
\hline Transport_tr & 1 & 1 & 1 & 1 & 1 & 1 & 1 & 1 & 1 & 1 \\
\hline Mixing & 1 & 1 & 1 & 1 & 1 & 1 & 1 & 1 & 1 & 1 \\
\hline MolarMass_g & 17 & 97.97 & 30 & 32 & 63 & 47 & 60.09 & $\begin{array}{r}35.4 \\
5\end{array}$ & 16 & 1 \\
\hline ElementMolarMass_g & 14 & 30.97 & 12 & 16 & 14 & 14 & 28.09 & $\begin{array}{r}35.4 \\
5\end{array}$ & 12 & 1 \\
\hline Charge & 0 & 0 & 0 & 0 & 0 & 0 & 0 & -1 & 0 & 1 \\
\hline Elemental Charge & -3 & 5 & 4 & -2 & 5 & 3 & 4 & -1 & -4 & 1 \\
\hline $\begin{array}{l}\text { ElektronAccCap_(mol/m } \\
\text { ol) }\end{array}$ & 0 & 0 & 0 & 4 & 5 & 3 & 0 & 0 & 0 & 0 \\
\hline DiffusionCoeff_m2/s & $\begin{array}{r}1.90 \mathrm{E} \\
-09\end{array}$ & $7.43 \mathrm{E}-10$ & $\begin{array}{r}1.18 \mathrm{E} \\
-09\end{array}$ & $\begin{array}{r}3.47 \mathrm{E} \\
-09\end{array}$ & $\begin{array}{r}1.18 \mathrm{E} \\
-09\end{array}$ & $\begin{array}{r}1.10 \mathrm{E} \\
-09\end{array}$ & $1.00 \mathrm{E}-09$ & $\begin{array}{l}2.00 \\
E-09\end{array}$ & $1.90 \mathrm{E}-09$ & $\begin{array}{r}2.90 \mathrm{E}- \\
09\end{array}$ \\
\hline DiffusionFactor & $\begin{array}{r}1.90 \mathrm{E} \\
+00\end{array}$ & 7.43E-01 & $\begin{array}{r}1.18 \mathrm{E} \\
+00\end{array}$ & $\begin{array}{r}1.00 \mathrm{E} \\
+00\end{array}$ & $\begin{array}{r}1.18 \mathrm{E} \\
+00\end{array}$ & $\begin{array}{r}1.10 \mathrm{E} \\
+00\end{array}$ & $1.00 \mathrm{E}+00$ & $\begin{array}{l}5.76 \\
E-01\end{array}$ & $\begin{array}{r}1.90 \mathrm{E}+0 \\
0\end{array}$ & $\begin{array}{r}2.90 \mathrm{E}+ \\
00\end{array}$ \\
\hline AirExchange_(1/0) & 0 & 0 & 1 & 1 & 0 & 0 & 0 & 0 & 0 & 0 \\
\hline \multicolumn{11}{|c|}{ Adsorption parameters $==============================$ DetrituS } \\
\hline $\begin{array}{l}\text { AdsorptionDetritus- } \\
?(1 / 0)\end{array}$ & ---- & ---- & ---- & ---- & ---- & ---- & ---- & ---- & ---- & ---- \\
\hline DetritusSubstrate & ---- & ---- & ---- & ---- & ---- & ---- & ---- & ---- & ---- & ---- \\
\hline $\begin{array}{l}\text { Rate_parameter_Vads_( } \\
\text { 1/d) aerobe }\end{array}$ & ---- & ---- & ---- & ---- & ---- & ---- & ---- & ---- & ---- & ---- \\
\hline $\begin{array}{l}\text { Rate_parameter_Vads_( } \\
\text { 1/d) anaerobe }\end{array}$ & ---- & ---- & ---- & ---- & ---- & ---- & ---- & ---- & ---- & ---- \\
\hline $\begin{array}{l}\text { MaxAds_Aerobe_(mol/g } \\
\text { ) }\end{array}$ & ---- & ---- & ---- & ---- & ---- & ---- & ---- & ---- & ---- & ---- \\
\hline $\begin{array}{l}\text { MaxAds_Anaerobe_(mo } \\
\text { l/g) }\end{array}$ & ---- & ---- & ---- & ---- & ---- & ---- & ---- & ---- & ---- & ---- \\
\hline $\begin{array}{l}\text { Max_AdsOccupation_Th } \\
\text { eta }\end{array}$ & ---- & ---- & ---- & ---- & ---- & ---- & ---- & ---- & ---- & ---- \\
\hline $\begin{array}{l}\text { Adsorption_parameter_ } \\
\text { a }\end{array}$ & ---- & ---- & ---- & ---- & ---- & ---- & ---- & ---- & ---- & ---- \\
\hline $\begin{array}{l}\text { Adsorption_parameter_ } \\
\text { b }\end{array}$ & ---- & ---- & ---- & ---- & ---- & ---- & ---- & ---- & ---- & ---- \\
\hline $\begin{array}{l}\text { Adsorption_parameter_ } \\
\text { c }\end{array}$ & ---- & ---- & ---- & ---- & ---- & ---- & ---- & ---- & ---- & ---- \\
\hline $\begin{array}{l}\text { Adsorption_parameter__ } \\
\text { d }\end{array}$ & ---- & ---- & ---- & ---- & ---- & ---- & ---- & ---- & ---- & ---- \\
\hline $\begin{array}{l}\text { Adsorption_parameter_ } \\
\text { e }\end{array}$ & ---- & ---- & ---- & ---- & ---- & ---- & ---- & ---- & ---- & ---- \\
\hline $\begin{array}{l}\text { Adsorption_parameter__ } \\
f\end{array}$ & ---- & ---- & ---- & ---- & ---- & ---- & ---- & ---- & ---- & ---- \\
\hline \multicolumn{11}{|c|}{ Adsorption parameters $============================$ Solids } \\
\hline AdsorptionSolids-?(1/0) & ---- & 1 & ---- & ---- & ---- & ---- & 1 & ---- & ---- & ---- \\
\hline SolidSubstrate & ---- & $\begin{array}{l}\text { IronOOH_P } \\
\text { _Comp }\end{array}$ & ---- & ---- & ---- & ---- & $\begin{array}{l}\text { IronOOH_P } \\
\text { _Comp }\end{array}$ & ---- & ---- & ---- \\
\hline $\begin{array}{l}\text { Rate_parameter_Vads_( } \\
\text { 1/d)_aerobe }\end{array}$ & ---- & $2.93 \mathrm{E}-03$ & ---- & ---- & ---- & ---- & $3.00 \mathrm{E}-03$ & ---- & ---- & ---- \\
\hline & & & & & & & & & & \\
\hline
\end{tabular}




\begin{tabular}{|c|c|c|c|c|c|c|c|c|c|c|}
\hline Ion/Element_Names & $\mathrm{NH} 4$ & $\mathrm{P}$ & $\mathrm{CO} 2$ & $\mathrm{O} 2$ & NO3 & NO2 & $\mathrm{Si}$ & $\begin{array}{l}\text { Chlo } \\
\text { ride }\end{array}$ & $\begin{array}{l}\text { Reduced } \\
\text { Comp }\end{array}$ & $\begin{array}{l}\text { RestCh } \\
\text { arge }\end{array}$ \\
\hline $\begin{array}{l}\text { Rate_parameter_Vads_( } \\
\text { 1/d)_anaerobe }\end{array}$ & ---- & $5.00 \mathrm{E}-04$ & ---- & ---- & ---- & ---- & $3.00 \mathrm{E}-04$ & --- & --- & --- \\
\hline MaxAds_Aerobe(mol/g) & ---- & $2.00 \mathrm{E}-03$ & ---- & ---- & ---- & ---- & $1.00 \mathrm{E}-03$ & ---- & ---- & ---- \\
\hline MaxAds_Anaer_(mol/g) & ---- & $8.00 \mathrm{E}-05$ & ---- & ---- & ---- & ---- & 4.00E-05 & ---- & ---- & ---- \\
\hline Max_AdsOccup_Theta & ---- & ---- & ---- & ---- & ---- & ---- & ---- & ---- & ---- & ---- \\
\hline Adsorption_paramet_a & ---- & $4.00 \mathrm{E}-01$ & ---- & ---- & ---- & ---- & $-4.00 E-01$ & ---- & ---- & ---- \\
\hline Adsorption_paramet_b & ---- & $-5.80 \mathrm{E}-01$ & ---- & ---- & ---- & ---- & $-5.80 \mathrm{E}-01$ & ---- & ---- & ---- \\
\hline Adsorption_paramet_c & ---- & $-1.50 \mathrm{E}-02$ & ---- & ---- & ---- & ---- & $-1.50 \mathrm{E}-02$ & ---- & ---- & ---- \\
\hline Adsorption_paramet_d & ---- & ---- & ---- & --- & --- & ---- & ---- & --- & ---- & ---- \\
\hline Adsorption_paramet_e & ---- & $1.09 \mathrm{E}+01$ & ---- & ---- & ---- & ---- & $1.20 \mathrm{E}+01$ & ---- & ---- & ---- \\
\hline Adsorption_paramet_f & ---- & $6.50 \mathrm{E}+00$ & ---- & ---- & ---- & ---- & $8.50 \mathrm{E}+00$ & ---- & ---- & ---- \\
\hline \multicolumn{11}{|c|}{ Reaction parameters $=====================================$} \\
\hline Oxidation_RateParame & $\begin{array}{r}1.00 \mathrm{E} \\
-02 \\
\end{array}$ & --- & ---- & ---- & ---- & $\begin{array}{r}3.00 E \\
-01 \\
\end{array}$ & ---- & ---- & $3.00 \mathrm{E}-01$ & ---- \\
\hline Oxidation_Temp01 & -5 & ---- & ---- & ---- & ---- & 4 & ---- & ---- & -1 & ---- \\
\hline Oxidation_Temp02 & 20 & --- & --- & ---- & ---- & 15 & ---- & ---- & 20 & --- \\
\hline Oxidation_Temp03 & 70 & ---- & ---- & --- & ---- & 20 & ---- & --- & 70 & --- \\
\hline Monod_Conc_own & $\begin{array}{r}1.00 \mathrm{E} \\
-02 \\
\end{array}$ & ---- & ---- & ---- & ---- & $\begin{array}{r}1.00 \mathrm{E} \\
-02 \\
\end{array}$ & ---- & ---- & $3.00 \mathrm{E}-02$ & --- \\
\hline Monod_Conc_O2 & $\begin{array}{r}5.00 \mathrm{E} \\
-02 \\
\end{array}$ & ---- & ---- & --- & ---- & $\begin{array}{r}5.00 \mathrm{E} \\
-02 \\
\end{array}$ & ---- & ---- & $6.00 \mathrm{E}-02$ & --- \\
\hline Monod_Conc_NO3 & ---- & ---- & ---- & ---- & ---- & ---- & ---- & ---- & $2.00 \mathrm{E}-02$ & ---- \\
\hline
\end{tabular}


A28.2 Algae

\begin{tabular}{|c|c|c|c|c|c|c|c|}
\hline Phyto parameters & & & & & & & \\
\hline Phyto_Names & $\begin{array}{l}\text { Benthic- } \\
\text { diatoms }\end{array}$ & Diatoms & $\begin{array}{l}\text { Non- } \\
\text { diatoms }\end{array}$ & $\begin{array}{l}\text { FreshW_ } \\
\text { BlueGree } \\
\text { ns }\end{array}$ & $\begin{array}{l}\text { FreshW_ } \\
\text { Diatoms }\end{array}$ & $\begin{array}{l}\text { FreshW_- } \\
\text { Non_Diat } \\
\text { oms }\end{array}$ & $\begin{array}{l}\text { Pico- } \\
\text { Phyto }\end{array}$ \\
\hline Transport_tr & 1 & 1 & 1 & 1 & 1 & 1 & 1 \\
\hline Mixing & 1 & 1 & 1 & 1 & 1 & 1 & 1 \\
\hline IK Wm-2 & 14 & 9 & 10 & 20 & 8 & 20 & 15 \\
\hline rcgrow d-1 & 3 & 3 & 3.5 & 3 & 4 & 4 & 3 \\
\hline rcresp d-1 & 0.12 & 0.18 & 0.18 & 0.25 & 0.18 & 0.18 & 0.18 \\
\hline rcexcr d-1 & ---- & ---- & ---- & ---- & ---- & ---- & ---- \\
\hline rcmort d-1 & ---- & ---- & ---- & ---- & ---- & ---- & ---- \\
\hline ExtinctionContribution $\mathrm{m}-1 /(\mathrm{g} / \mathrm{m} 3)$ & 0.06 & 0.07 & 0.07 & 0.07 & 0.07 & 0.07 & 0.103 \\
\hline Length $\mathrm{m}$ & $5.00 \mathrm{E}-06$ & $5.00 \mathrm{E}-06$ & $1.20 \mathrm{E}-05$ & $1.20 \mathrm{E}-05$ & $5.00 \mathrm{E}-06$ & $1.20 \mathrm{E}-05$ & $2.50 \mathrm{E}-06$ \\
\hline Chla mg/g & 9 & 9 & 12 & 12 & 9 & 12 & 12 \\
\hline Maximum_conc g DW/m3 & $2.00 \mathrm{E}+02$ & ---- & ---- & ---- & ---- & ---- & ---- \\
\hline MolarMass g & 233.6 & 233.6 & 149.47 & 186.24 & 233.6 & 149.47 & 149.47 \\
\hline sinking_velocity md-1 & $2.00 \mathrm{E}-04$ & $5.00 \mathrm{E}-05$ & $2.00 \mathrm{E}-06$ & $2.00 \mathrm{E}-06$ & $5.00 \mathrm{E}-05$ & $2.00 \mathrm{E}-06$ & 2.31E-06 \\
\hline float_velocity md-1 & ---- & ---- & ---- & ---- & ---- & ---- & ---- \\
\hline Resuspension-r & $3.50 \mathrm{E}-10$ & $1.00 \mathrm{E}-07$ & $9.00 \mathrm{E}-10$ & $9.00 \mathrm{E}-10$ & $5.00 \mathrm{E}-07$ & $9.00 \mathrm{E}-10$ & $9.00 \mathrm{E}-10$ \\
\hline Resusp_power-r3 & 1.5 & 1.5 & 1.5 & 1.5 & 1.5 & 1.5 & 1.5 \\
\hline Resusp_UWI_0 m/s & 1 & 0.5 & 0.5 & 0.5 & 0.5 & 0.5 & 0.5 \\
\hline LiveDepth m & $1.00 \mathrm{E}-03$ & $-1.00 \mathrm{E}-01$ & $-1.00 \mathrm{E}-01$ & $-1.00 \mathrm{E}-01$ & $-1.00 \mathrm{E}-01$ & $-1.00 \mathrm{E}-01$ & $-1.00 \mathrm{E}-03$ \\
\hline Temp01_growth oC & -15 & -10 & -5 & -5 & -15 & -5 & -5 \\
\hline Temp02_growth oC & 20 & 15 & 20 & 30 & 18 & 30 & 25 \\
\hline Temp03_growth oC & 40 & 40 & 40 & 40 & 40 & 40 & 40 \\
\hline Temp01_Respiration oC & -6 & -5 & -5 & -5 & -15 & -5 & -20 \\
\hline Temp02_Respiration oC & 25 & 20 & 25 & 25 & 25 & 25 & 25 \\
\hline Temp03_Respiration oC & 40 & 40 & 40 & 40 & 40 & 40 & 40 \\
\hline Temp01_Mortality oC & ---- & ---- & ---- & ---- & ---- & ---- & ---- \\
\hline Temp02_Mortality oC & ---- & ---- & ---- & ---- & ---- & ---- & ---- \\
\hline Temp03_Mortality oC & ---- & ---- & ---- & ---- & ---- & ---- & ---- \\
\hline Composition mol NH4/g DW & $4.28 \mathrm{E}-03$ & $4.28 \mathrm{E}-03$ & $6.69 \mathrm{E}-03$ & $6.69 \mathrm{E}-03$ & $4.28 \mathrm{E}-03$ & $6.69 \mathrm{E}-03$ & $6.69 \mathrm{E}-03$ \\
\hline Composition mol P/g DW & $1.43 \mathrm{E}-04$ & $1.43 \mathrm{E}-04$ & $2.23 \mathrm{E}-04$ & $2.23 \mathrm{E}-04$ & $1.43 \mathrm{E}-04$ & $2.23 \mathrm{E}-04$ & $2.23 \mathrm{E}-04$ \\
\hline Composition mol CO2/g DW & $2.14 \mathrm{E}-02$ & $2.14 \mathrm{E}-02$ & $3.35 \mathrm{E}-02$ & $3.35 \mathrm{E}-02$ & $2.14 \mathrm{E}-02$ & $3.35 \mathrm{E}-02$ & $3.35 \mathrm{E}-02$ \\
\hline Composition mol O2/g DW & $-1.14 \mathrm{E}-02$ & $-1.14 \mathrm{E}-02$ & $-1.78 \mathrm{E}-02$ & $-1.78 \mathrm{E}-02$ & $-1.14 \mathrm{E}-02$ & $-1.78 \mathrm{E}-02$ & $-1.78 \mathrm{E}-02$ \\
\hline Composition mol Si/g DW & $5.99 \mathrm{E}-03$ & $5.99 \mathrm{E}-03$ & ---- & ---- & $5.99 \mathrm{E}-03$ & ---- & ---- \\
\hline Composition mol RestCharge/g DW & $3.01 \mathrm{E}-02$ & $3.01 \mathrm{E}-02$ & $4.71 \mathrm{E}-02$ & $4.71 \mathrm{E}-02$ & $3.01 \mathrm{E}-02$ & $4.71 \mathrm{E}-02$ & 4.71E-02 \\
\hline C Oxidation number & -1.16 & -1.16 & -0.04 & -0.04 & -1.16 & -0.04 & -0.04 \\
\hline Monod_Factor mol NH4/m3 & $5.00 \mathrm{E}-03$ & $5.00 \mathrm{E}-03$ & $5.00 \mathrm{E}-03$ & $5.00 \mathrm{E}-03$ & $5.00 \mathrm{E}-03$ & $5.00 \mathrm{E}-03$ & $3.00 \mathrm{E}-03$ \\
\hline Monod_Factor mol P/m3 & $1.50 \mathrm{E}-04$ & $1.50 \mathrm{E}-04$ & $2.00 \mathrm{E}-04$ & $2.00 \mathrm{E}-04$ & $1.50 \mathrm{E}-04$ & $2.00 \mathrm{E}-04$ & $1.50 \mathrm{E}-04$ \\
\hline Monod_Factor $\mathrm{mol} \mathrm{CO} 2 / \mathrm{m} 3$ & 8.00E-03 & 8.00E-03 & $8.00 \mathrm{E}-03$ & 8.00E-03 & $8.00 \mathrm{E}-03$ & $8.00 \mathrm{E}-03$ & 8.00E-03 \\
\hline Monod_Factor mol NO3/m3 & $1.50 \mathrm{E}-03$ & $1.50 \mathrm{E}-03$ & $1.50 \mathrm{E}-03$ & $1.50 \mathrm{E}-03$ & $1.50 \mathrm{E}-03$ & $1.50 \mathrm{E}-03$ & $3.00 \mathrm{E}-03$ \\
\hline Monod_Factor mol Si/m3 & $6.00 \mathrm{E}-03$ & $6.00 \mathrm{E}-03$ & ---- & ---- & $6.00 \mathrm{E}-03$ & ---- & $\begin{array}{ll}--- \\
\end{array}$ \\
\hline Sensitivity factor K1 Chloride & ---- & ---- & ---- & $3.00 \mathrm{E}+03$ & $3.00 \mathrm{E}+03$ & $3.00 \mathrm{E}+03$ & $\begin{array}{l}--- \\
\end{array}$ \\
\hline Sensitivity K2 Chloride (mol/m3) & ---- & $\begin{array}{l}--- \\
\end{array}$ & ---- & $2.00 \mathrm{E}+01$ & $2.00 \mathrm{E}+01$ & $2.00 \mathrm{E}+01$ & $\begin{array}{ll}--- \\
\end{array}$ \\
\hline Sensitivity factor K3 Chloride & $\begin{array}{ll}--- \\
-\end{array}$ & $1.00 \mathrm{E}+02$ & $1.00 \mathrm{E}+02$ & ---- & ---- & ---- & $1.00 \mathrm{E}+02$ \\
\hline Sensitivity K4 Chloride (mol/m3) & $\begin{array}{l}--- \\
--\end{array}$ & $1.00 \mathrm{E}+01$ & $1.00 \mathrm{E}+01$ & $\begin{array}{l}--- \\
\end{array}$ & ---- & ---- & $1.00 \mathrm{E}+01$ \\
\hline MortDestination & $\begin{array}{l}\text { Dead_Dia } \\
\text { toms }\end{array}$ & $\begin{array}{l}\text { Dead_Dia } \\
\text { toms }\end{array}$ & $\begin{array}{l}\text { Dead_Su } \\
\text { bstance }\end{array}$ & $\begin{array}{l}\text { Dead_Su } \\
\text { bstance }\end{array}$ & $\begin{array}{l}\text { Dead_Dia } \\
\text { toms }\end{array}$ & $\begin{array}{l}\text { Dead_Su } \\
\text { bstance }\end{array}$ & $\begin{array}{l}\text { Dead_Dia } \\
\text { toms }\end{array}$ \\
\hline ExcretionDestination & $\begin{array}{l}\text { Labile_Or } \\
\text { g_Matter }\end{array}$ & $\begin{array}{l}\text { Labile_Or } \\
\text { g_Matter }\end{array}$ & $\begin{array}{l}\text { Labile_Or } \\
\text { g_Matter }\end{array}$ & $\begin{array}{l}\text { Labile_Or } \\
\text { g_Matter }\end{array}$ & $\begin{array}{l}\text { Labile_Or } \\
\text { g_Matter }\end{array}$ & $\begin{array}{l}\text { Labile_Or } \\
\text { g_Matter }\end{array}$ & $\begin{array}{l}\text { Labile_Or } \\
\text { g_Matter }\end{array}$ \\
\hline
\end{tabular}




\section{A28.3 Detritus}

\begin{tabular}{|c|c|c|c|c|c|c|c|}
\hline \multicolumn{8}{|l|}{ Detritus parameters } \\
\hline Detritus_Names & $\begin{array}{l}\text { Dead_Dia } \\
\text { toms }\end{array}$ & $\begin{array}{l}\text { Dead_Su } \\
\text { bstance }\end{array}$ & Frac2 & $\begin{array}{l}\text { Frac2_Di } \\
\text { at }\end{array}$ & Frustules & $\begin{array}{l}\text { Labile_Org_ } \\
\text { Matter }\end{array}$ & $\begin{array}{l}\text { Refract_Org } \\
\text { Matter }\end{array}$ \\
\hline Transport_tr & 2 & 2 & 2 & 2 & 2 & 2 & 2 \\
\hline Mixing & 1 & 1 & 1 & 1 & 1 & 1 & 1 \\
\hline kmax d-1 & 4.50E-01 & 4.50E-01 & $1.20 \mathrm{E}-01$ & $1.80 \mathrm{E}-01$ & $9.00 \mathrm{E}-02$ & $5.00 \mathrm{E}-02$ & $2.25 \mathrm{E}-03$ \\
\hline totalpha & ---- & ---- & ---- & ---- & ---- & ---- & ---- \\
\hline totminer & ---- & ---- & ---- & ---- & ---- & ---- & ---- \\
\hline ExtinctionContribution $\mathrm{m}-1 /(\mathrm{g} / \mathrm{m} 3)$ & $3.00 \mathrm{E}-02$ & $3.00 \mathrm{E}-02$ & $3.00 \mathrm{E}-02$ & $3.00 \mathrm{E}-02$ & 2.00E-02 & $3.00 \mathrm{E}-02$ & $4.00 \mathrm{E}-02$ \\
\hline Length $\mathrm{m}$ & $1.00 \mathrm{E}-05$ & $1.00 \mathrm{E}-05$ & $1.00 \mathrm{E}-05$ & $1.00 \mathrm{E}-05$ & $1.00 \mathrm{E}-05$ & $1.00 \mathrm{E}-05$ & 1.00E-05 \\
\hline MolarMass g & 220.5 & 142.38 & 108.12 & 1 & 1 & 133.09 & 114.85 \\
\hline sinking_velocity md-1 & 2.20E-05 & $2.20 \mathrm{E}-05$ & $2.20 \mathrm{E}-05$ & $2.20 \mathrm{E}-05$ & $2.20 \mathrm{E}-05$ & $1.00 \mathrm{E}+00$ & 1.00E-04 \\
\hline Resuspension-r & $1.20 \mathrm{E}-10$ & $1.20 \mathrm{E}-10$ & $1.20 \mathrm{E}-10$ & $6.00 \mathrm{E}-11$ & $1.20 \mathrm{E}-10$ & $0.00 \mathrm{E}+00$ & $2.40 \mathrm{E}-11$ \\
\hline Resusp_power-r3 & 1.4 & 1.5 & 1.4 & 1.4 & 1.4 & 1.4 & 1.4 \\
\hline Resusp_UWI_0 m/s & 0.5 & 0 & 0 & 1 & 1 & 1 & 0.5 \\
\hline Temp01_Breakdown oC & 0 & 0 & 0 & 0 & -5 & -2 & 0 \\
\hline Temp02_Breakdown oC & 20 & 20 & 20 & 20 & 25 & 30 & 20 \\
\hline Temp03_Breakdown oC & 80 & 80 & 80 & 80 & 25 & 60 & 80 \\
\hline Composition Mol NH4/gDW & $3.63 \mathrm{E}-03$ & $5.62 \mathrm{E}-03$ & $1.85 \mathrm{E}-03$ & 1.07E-03 & ---- & 4.51E-03 & $2.61 \mathrm{E}-03$ \\
\hline Composition Mol P/gDW & 1.21E-04 & $1.87 \mathrm{E}-04$ & 1.23E-04 & 7.16E-05 & ---- & $1.50 \mathrm{E}-04$ & $4.35 \mathrm{E}-05$ \\
\hline Composition Mol CO2/gDW & 2.27E-02 & $3.51 \mathrm{E}-02$ & 4.63E-02 & $2.69 \mathrm{E}-02$ & ---- & $3.76 \mathrm{E}-02$ & $4.35 \mathrm{E}-02$ \\
\hline Composition Mol O2/gDW & $-1.21 \mathrm{E}-02$ & $-1.88 \mathrm{E}-02$ & $-2.53 \mathrm{E}-02$ & $-1.47 \mathrm{E}-02$ & ---- & $-2.02 \mathrm{E}-02$ & $-2.37 \mathrm{E}-02$ \\
\hline Composition Mol Si/gDW & $5.90 \mathrm{E}-03$ & ---- & ---- & $6.98 \mathrm{E}-03$ & $1.20 \mathrm{E}-02$ & ---- & ---- \\
\hline Composition Mol RestCharge/gDW & 3.10E-02 & $4.80 \mathrm{E}-02$ & $5.75 \mathrm{E}-02$ & $3.34 \mathrm{E}-02$ & ---- & 4.97E-02 & $5.49 \mathrm{E}-02$ \\
\hline C Oxidation number & -1.15 & -0.11 & -0.34 & -1.38 & 0 & -0.18 & -0.29 \\
\hline Destination g/g-Frac2 & 0.1 & 0.2 & ---- & ---- & ---- & 0.05 & ---- \\
\hline Destination g/g-Frac2_Diat & 0.4 & ---- & ---- & ---- & ---- & ---- & ---- \\
\hline Destination g/g-Frustules & ---- & ---- & ---- & 0.35 & ---- & ---- & ---- \\
\hline Destination g/g-Labile_Org_Matter & 0.15 & 0.3 & ---- & ---- & ---- & ---- & ---- \\
\hline $\begin{array}{l}\text { Destination g/g- } \\
\text { Refract_Org_Matter }\end{array}$ & ---- & ---- & 0.15 & 0.05 & ---- & ---- & ---- \\
\hline FaecesDestination & $\begin{array}{l}\text { Frac2_Di } \\
\text { at }\end{array}$ & $\begin{array}{l}\text { Labile_Or } \\
\text { g_Matter }\end{array}$ & $\begin{array}{l}\text { Refract_ } \\
\text { Org_Mat } \\
\text { ter }\end{array}$ & Frustules & Frustules & Frac2 & $\begin{array}{l}\text { Refract_Org } \\
\text { _Matter }\end{array}$ \\
\hline
\end{tabular}




\section{A28.4 Fauna}

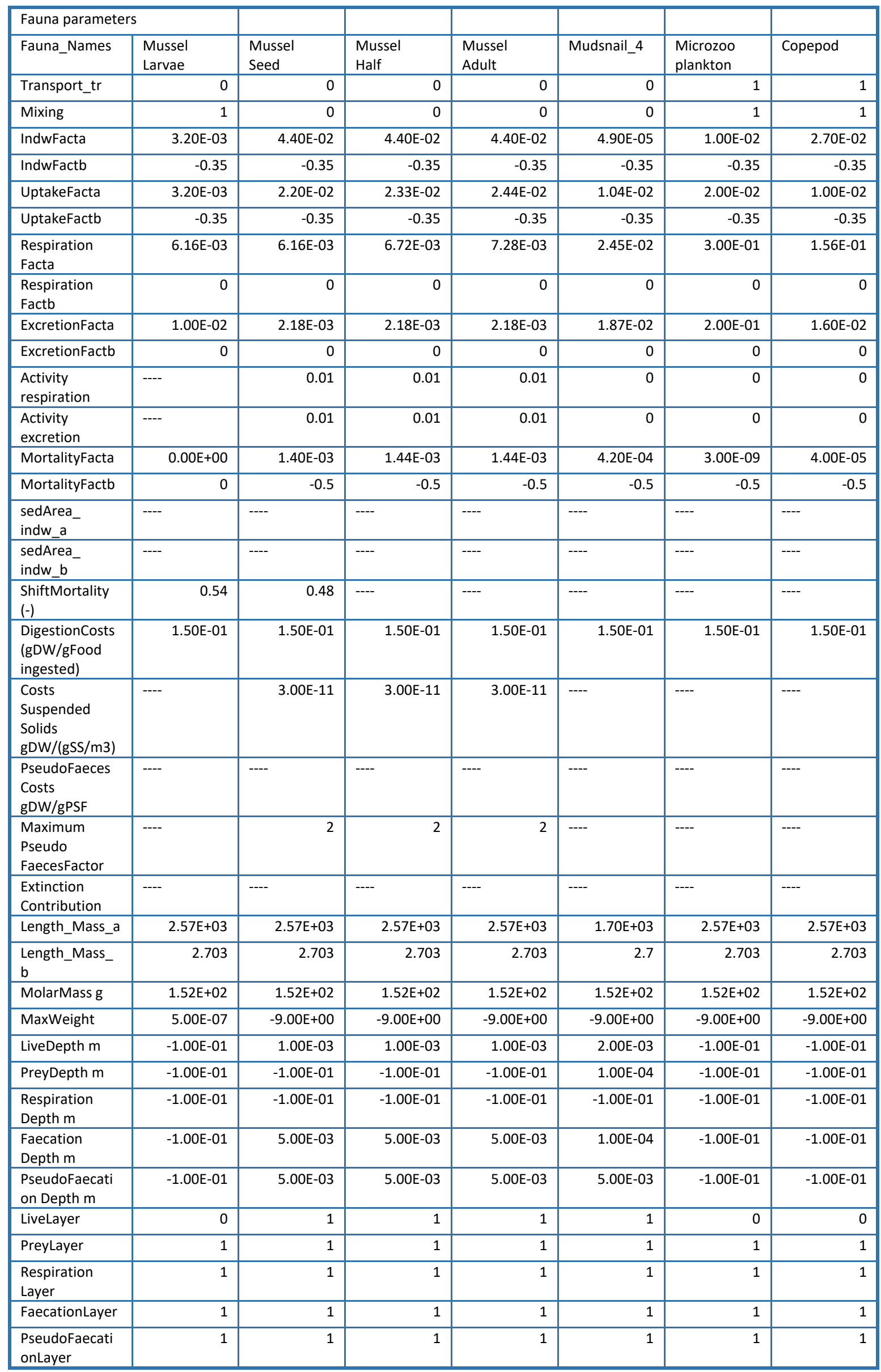




\begin{tabular}{|c|c|c|c|c|c|c|c|}
\hline Fauna_Names & $\begin{array}{l}\text { Mussel } \\
\text { Larvae }\end{array}$ & $\begin{array}{l}\text { Mussel } \\
\text { Seed }\end{array}$ & $\begin{array}{l}\text { Mussel } \\
\text { Half }\end{array}$ & $\begin{array}{l}\text { Mussel } \\
\text { Adult }\end{array}$ & Mudsnail_4 & $\begin{array}{l}\text { Microzoo } \\
\text { plankton }\end{array}$ & Copepod \\
\hline $\begin{array}{l}\text { PreySize } \\
\text { Relative }\end{array}$ & 1 & 1 & 1 & 1 & 1 & 1 & 1 \\
\hline MinRelSize & $1.00 \mathrm{E}-06$ & $1.00 \mathrm{E}-06$ & $1.00 \mathrm{E}-06$ & $1.00 \mathrm{E}-06$ & $1.00 \mathrm{E}-06$ & $1.00 \mathrm{E}-06$ & $1.00 \mathrm{E}-06$ \\
\hline MaxRelSize & $1.00 \mathrm{E}+00$ & $1.00 \mathrm{E}+00$ & $1.00 \mathrm{E}+00$ & $1.00 \mathrm{E}+00$ & $1.00 \mathrm{E}+00$ & $1.00 \mathrm{E}+00$ & $1.00 \mathrm{E}+00$ \\
\hline $\begin{array}{l}\text { MinPreySize } \\
\text { X1 }\end{array}$ & ---- & --- & ---- & ---- & ---- & ---- & ---- \\
\hline $\begin{array}{l}\text { MinPreySize } \\
\text { X2 }\end{array}$ & $1.00 \mathrm{E}-04$ & $1.00 \mathrm{E}-04$ & $1.00 \mathrm{E}-04$ & $1.00 \mathrm{E}-04$ & $1.00 \mathrm{E}-04$ & $1.00 \mathrm{E}-04$ & 1.00E-04 \\
\hline $\begin{array}{l}\text { MinPreySize } \\
\text { X3 }\end{array}$ & $4.00 \mathrm{E}+00$ & 1.00E-01 & $4.00 \mathrm{E}+00$ & $4.00 E+00$ & $4.00 \mathrm{E}+00$ & $4.00 \mathrm{E}+00$ & $4.00 E+00$ \\
\hline $\begin{array}{l}\text { MinPreySize } \\
\text { X4 }\end{array}$ & $4.00 E+01$ & $1.50 \mathrm{E}-01$ & $4.00 \mathrm{E}+01$ & $4.00 E+01$ & $4.00 \mathrm{E}+01$ & $4.00 E+01$ & $4.00 E+01$ \\
\hline $\begin{array}{l}\text { Shift_X1 } \\
\text { withLength }\end{array}$ & ---- & $-5.00 \mathrm{E}-04$ & ---- & ---- & ---- & ---- & ---- \\
\hline $\begin{array}{l}\text { Shift_X2 } \\
\text { withLength }\end{array}$ & ---- & $-4.93 E-04$ & ---- & ---- & --- & ---- & ---- \\
\hline $\begin{array}{l}\text { Shift_X3 } \\
\text { withLength }\end{array}$ & ---- & ---- & ---- & ---- & ---- & ---- & ---- \\
\hline $\begin{array}{l}\text { Shift_X4 } \\
\text { withLength }\end{array}$ & ---- & ---- & ---- & ---- & ---- & ---- & ---- \\
\hline Fixed mass & ---- & ---- & ---- & ---- & $2.00 \mathrm{E}-03$ & $2.00 \mathrm{E}-09$ & 1.99E-05 \\
\hline $\begin{array}{l}\text { Temp01 } \\
\text { Filtration oC }\end{array}$ & -7 & -8 & -10 & -15 & 0 & -10 & 0 \\
\hline $\begin{array}{l}\text { Temp02 } \\
\text { Filtration oC }\end{array}$ & 21.5 & 21.5 & 21.5 & 21.5 & 20.5 & 21.5 & 20.5 \\
\hline $\begin{array}{l}\text { Temp03 } \\
\text { Filtration oC }\end{array}$ & 45 & 45 & 45 & 45 & 45 & 45 & 45 \\
\hline $\begin{array}{l}\text { Temp01 } \\
\text { Respiration oC }\end{array}$ & -20 & -20 & -20 & -20 & -5 & -10 & -5 \\
\hline $\begin{array}{l}\text { Temp02 } \\
\text { Respiration oC }\end{array}$ & 21.5 & 21.5 & 21.5 & 21.5 & 22.5 & 21.5 & 20.5 \\
\hline $\begin{array}{l}\text { Temp03_Respir } \\
\text { ation oC }\end{array}$ & 45 & 45 & 45 & 45 & 45 & 45 & 45 \\
\hline $\begin{array}{l}\text { Temp01 } \\
\text { Mortality oC }\end{array}$ & -20 & -20 & -20 & -20 & -5 & -10 & -5 \\
\hline $\begin{array}{l}\text { Temp02 } \\
\text { Mortality oC }\end{array}$ & 21.5 & 21.5 & 21.5 & 21.5 & 22.5 & 21.5 & 20.5 \\
\hline $\begin{array}{l}\text { Temp03 } \\
\text { Mortality oC }\end{array}$ & 45 & 45 & 45 & 45 & 45 & 45 & 45 \\
\hline $\begin{array}{l}\text { Compostion } \\
\text { Mol NH4 /g } \\
\text { DW }\end{array}$ & $7.23 \mathrm{E}-03$ & $7.23 \mathrm{E}-03$ & $7.23 \mathrm{E}-03$ & $7.23 \mathrm{E}-03$ & 7.23E-03 & $7.23 \mathrm{E}-03$ & $7.23 \mathrm{E}-03$ \\
\hline $\begin{array}{l}\text { Compostion } \\
\text { Mol P /g DW }\end{array}$ & $2.41 \mathrm{E}-04$ & $2.41 \mathrm{E}-04$ & $2.41 \mathrm{E}-04$ & $2.41 \mathrm{E}-04$ & $2.41 \mathrm{E}-04$ & $2.41 \mathrm{E}-04$ & $2.41 \mathrm{E}-04$ \\
\hline $\begin{array}{l}\text { Compostion } \\
\text { Mol CO2 /g DW }\end{array}$ & $3.29 \mathrm{E}-02$ & $3.29 \mathrm{E}-02$ & $3.29 \mathrm{E}-02$ & $3.29 E-02$ & $3.29 \mathrm{E}-02$ & $3.29 \mathrm{E}-02$ & $3.29 \mathrm{E}-02$ \\
\hline $\begin{array}{l}\text { Compostion } \\
\text { Mol O2 /g DW }\end{array}$ & $-1.74 \mathrm{E}-02$ & $-1.74 \mathrm{E}-02$ & $-1.74 \mathrm{E}-02$ & $-1.74 \mathrm{E}-02$ & $-1.74 \mathrm{E}-02$ & $-1.74 \mathrm{E}-02$ & $-1.74 \mathrm{E}-02$ \\
\hline $\begin{array}{l}\text { Compostion } \\
\text { Mol } \\
\text { RestCharge /g } \\
\text { DW }\end{array}$ & 4.69E-02 & 4.69E-02 & $4.69 \mathrm{E}-02$ & 4.69E-02 & 4.69E-02 & 4.69E-02 & 4.69E-02 \\
\hline $\begin{array}{l}\text { C Oxidation } \\
\text { number }\end{array}$ & 0 & 0 & 0 & 0 & 0 & 0 & 0 \\
\hline $\begin{array}{l}\text { CatchPref of } \\
\text { Mussel_Larvae }\end{array}$ & ---- & 1 & 1 & 1 & ---- & ---- & ---- \\
\hline $\begin{array}{l}\text { CatchPref of } \\
\text { Microzooplankt } \\
\text { on }\end{array}$ & ---- & 0.2 & 0.2 & 0.2 & ---- & ---- & ---- \\
\hline $\begin{array}{l}\text { CatchPref of } \\
\text { Benthic } \\
\text { diatoms }\end{array}$ & 1 & 1 & 1 & 1 & 1 & 0.2 & ---- \\
\hline $\begin{array}{l}\text { CatchPref of } \\
\text { Diatoms }\end{array}$ & 1 & 1 & 1 & 1 & 1 & 0.3 & 0.03 \\
\hline $\begin{array}{l}\text { CatchPref of } \\
\text { Non-diatoms }\end{array}$ & 1 & 1 & 1 & 1 & 1 & 0.3 & 0.03 \\
\hline $\begin{array}{l}\text { CatchPref of } \\
\text { FreshW } \\
\text { BlueGreens }\end{array}$ & 1 & 1 & 1 & 1 & 1 & ---- & ---- \\
\hline
\end{tabular}




\begin{tabular}{|c|c|c|c|c|c|c|c|}
\hline Fauna_Names & $\begin{array}{l}\text { Mussel } \\
\text { Larvae }\end{array}$ & $\begin{array}{l}\text { Mussel } \\
\text { Seed }\end{array}$ & $\begin{array}{l}\text { Mussel } \\
\text { Half }\end{array}$ & $\begin{array}{l}\text { Mussel } \\
\text { Adult }\end{array}$ & Mudsnail_4 & $\begin{array}{l}\text { Microzoo } \\
\text { plankton }\end{array}$ & Copepod \\
\hline $\begin{array}{l}\text { CatchPref of } \\
\text { FreshW } \\
\text { Diatoms }\end{array}$ & 1 & 1 & 1 & 1 & 1 & ---- & --- \\
\hline $\begin{array}{l}\text { CatchPref of } \\
\text { FreshW } \\
\text { Non_Diatoms }\end{array}$ & 1 & 1 & 1 & 1 & 1 & --- & --- \\
\hline $\begin{array}{l}\text { CatchPref of } \\
\text { Pico-Phyto }\end{array}$ & 1 & 0.5 & ---- & ---- & ---- & 1 & --- \\
\hline $\begin{array}{l}\text { CatchPref of } \\
\text { Dead_Diatoms }\end{array}$ & --- & 0.2 & 0.2 & 0.2 & 1 & ---- & 0.6 \\
\hline $\begin{array}{l}\text { CatchPref of } \\
\text { Dead } \\
\text { Substance }\end{array}$ & --- & 0.2 & 0.2 & 0.2 & 1 & --- & 0.6 \\
\hline $\begin{array}{l}\text { CatchPref of } \\
\text { Frac2 }\end{array}$ & --- & 0.05 & 0.05 & 0.05 & 1 & --- & 0.3 \\
\hline $\begin{array}{l}\text { CatchPref of } \\
\text { Frac2_Diat }\end{array}$ & ---- & 0.05 & 0.05 & 0.05 & 1 & ---- & 0.3 \\
\hline $\begin{array}{l}\text { CatchPref of } \\
\text { Refract_Org } \\
\text { Matter }\end{array}$ & 0.05 & 0.05 & 0.05 & 0.05 & ---- & 0.07 & 0.2 \\
\hline $\begin{array}{l}\text { Fraction } \\
\text { assimilated of } \\
\text { Mussel_Larvae }\end{array}$ & ---- & 0.8 & 0.8 & 0.8 & --- & ---- & ---- \\
\hline $\begin{array}{l}\text { Fraction } \\
\text { assimilated of } \\
\text { Microzooplankt }\end{array}$ & ---- & 0.8 & 0.8 & 0.8 & ---- & ---- & ---- \\
\hline $\begin{array}{l}\text { Fraction } \\
\text { assimilated of } \\
\text { Benthic } \\
\text { diatoms }\end{array}$ & 0.28 & 0.28 & 0.28 & 0.28 & 0.28 & 0.1 & 0.1 \\
\hline $\begin{array}{l}\text { Fraction } \\
\text { assimilated of } \\
\text { Diatoms }\end{array}$ & 0.28 & 0.28 & 0.28 & 0.28 & 0.28 & 0.28 & 0.1 \\
\hline $\begin{array}{l}\text { Fraction } \\
\text { assimilated of } \\
\text { Non-diatoms }\end{array}$ & 0.56 & 0.56 & 0.56 & 0.56 & 0.56 & 0.3 & 0.1 \\
\hline $\begin{array}{l}\text { Fraction } \\
\text { assimilated of } \\
\text { FreshW } \\
\text { BlueGreens }\end{array}$ & 0.56 & 0.56 & 0.56 & 0.56 & 0.56 & --- & ---- \\
\hline $\begin{array}{l}\text { Fraction } \\
\text { assimilated of } \\
\text { FreshW } \\
\text { Diatoms }\end{array}$ & 0.28 & 0.28 & 0.28 & 0.28 & 0.28 & --- & ---- \\
\hline $\begin{array}{l}\text { Fraction } \\
\text { assimilated of } \\
\text { FreshW } \\
\text { Non_Diatoms }\end{array}$ & 0.56 & 0.56 & 0.56 & 0.56 & 0.56 & ---- & --- \\
\hline $\begin{array}{l}\text { Fraction } \\
\text { assimilated of } \\
\text { Pico-Phyto }\end{array}$ & 0.56 & 0.28 & ---- & ---- & ---- & 0.6 & --- \\
\hline $\begin{array}{l}\text { Fraction } \\
\text { assimilated of } \\
\text { Dead_Diatoms }\end{array}$ & --- & 0.06 & 0.06 & 0.06 & 0.05 & ---- & 0.1 \\
\hline $\begin{array}{l}\text { Fraction } \\
\text { assimilated of } \\
\text { Dead } \\
\text { Substance }\end{array}$ & ---- & 0.1 & 0.1 & 0.1 & 0.1 & ---- & 0.3 \\
\hline $\begin{array}{l}\text { Fraction } \\
\text { assimilated of } \\
\text { Frac2 }\end{array}$ & ---- & 0.05 & 0.05 & 0.05 & ---- & ---- & 0.1 \\
\hline $\begin{array}{l}\text { Fraction } \\
\text { assimilated of } \\
\text { Frac2_Diat }\end{array}$ & ---- & 0.05 & 0.05 & 0.05 & ---- & ---- & 0.1 \\
\hline $\begin{array}{l}\text { Fraction } \\
\text { assimilated of } \\
\text { Refract_Org } \\
\text { Matter }\end{array}$ & 0.02 & 0.02 & 0.02 & 0.02 & ---- & 0.01 & 0.12 \\
\hline $\begin{array}{l}\text { Mort } \\
\text { Destination }\end{array}$ & $\begin{array}{l}\text { Dead_Substa } \\
\text { nce }\end{array}$ & $\begin{array}{l}\text { Dead_Substa } \\
\text { nce }\end{array}$ & $\begin{array}{l}\text { Dead_Substa } \\
\text { nce }\end{array}$ & $\begin{array}{l}\text { Dead_Substa } \\
\text { nce }\end{array}$ & $\begin{array}{l}\text { Dead_Substa } \\
\text { nce }\end{array}$ & $\begin{array}{l}\text { Dead_Substa } \\
\text { nce }\end{array}$ & $\begin{array}{l}\text { Dead_Substa } \\
\text { nce }\end{array}$ \\
\hline
\end{tabular}




\begin{tabular}{|c|c|c|c|c|c|c|c|}
\hline Fauna_Names & $\begin{array}{l}\text { Mussel } \\
\text { Larvae }\end{array}$ & $\begin{array}{l}\text { Mussel } \\
\text { Seed }\end{array}$ & $\begin{array}{l}\text { Mussel } \\
\text { Half }\end{array}$ & $\begin{array}{l}\text { Mussel } \\
\text { Adult }\end{array}$ & Mudsnail_4 & $\begin{array}{l}\text { Microzoo } \\
\text { plankton }\end{array}$ & Copepod \\
\hline $\begin{array}{l}\text { Excretion } \\
\text { Destination }\end{array}$ & $\begin{array}{l}\text { Labile_Org_ } \\
\text { Matter }\end{array}$ & $\begin{array}{l}\text { Labile_Org_ } \\
\text { Matter }\end{array}$ & $\begin{array}{l}\text { Labile_Org_ } \\
\text { Matter }\end{array}$ & $\begin{array}{l}\text { Labile_Org_ } \\
\text { Matter }\end{array}$ & $\begin{array}{l}\text { Labile_Org_ } \\
\text { Matter }\end{array}$ & $\begin{array}{l}\text { Labile_Org_ } \\
\text { Matter }\end{array}$ & $\begin{array}{l}\text { Labile_Org_ } \\
\text { Matter }\end{array}$ \\
\hline
\end{tabular}

\section{A28.5 Spawning}

\begin{tabular}{|l|r|}
\hline Spawning parameters \\
\hline Fauna_Type & \multicolumn{1}{|l|}{ Mussels } \\
\hline Number_spawndays & 130 \\
\hline Spawnday 1 & 0.25 \\
\hline Biomass fraction for spawning day 1 & 210 \\
\hline Minimum age for spawning (days) & 14 \\
\hline Length spawning period (days) & $3.10 \mathrm{E}-08$ \\
\hline Birth mass (g) &
\end{tabular}




\section{A28.6 Solids}

\begin{tabular}{|c|c|c|c|c|c|c|}
\hline \multicolumn{7}{|l|}{ Solid parameters } \\
\hline Solid_Names & $\begin{array}{l}\text { IronOOH_P } \\
\text { Comp }\end{array}$ & Lutum02 & Silt0216 & Silt1663 & SandFine63125 & SandMedium 125250 \\
\hline Transport_tr & 0 & 0 & 0 & 0 & 0 & 0 \\
\hline Mixing & 0 & 0 & 0 & 0 & 0 & 0 \\
\hline $\begin{array}{l}\text { ExtinctionContribution } \\
\mathrm{m}-1 /(\mathrm{g} / \mathrm{m} 3)\end{array}$ & $5.00 \mathrm{E}-02$ & $5.00 \mathrm{E}-02$ & $\begin{array}{r}5.00 \mathrm{E}- \\
02\end{array}$ & $\begin{array}{r}5.00 \mathrm{E}- \\
02\end{array}$ & 3.00E-02 & $1.00 \mathrm{E}-02$ \\
\hline Length m & $1.00 \mathrm{E}-04$ & $1.00 \mathrm{E}-05$ & $\begin{array}{r}1.50 \mathrm{E}- \\
05\end{array}$ & $\begin{array}{r}4.00 \mathrm{E}- \\
05\end{array}$ & $9.00 \mathrm{E}-05$ & 1.70E-04 \\
\hline MolarMass g & 1 & 1 & 1 & 1 & 1 & 1 \\
\hline sinking_velocity md-1 & $8.33 \mathrm{E}-04$ & $8.00 \mathrm{E}-04$ & $\begin{array}{r}8.00 \mathrm{E}- \\
04 \\
\end{array}$ & $\begin{array}{r}2.12 \mathrm{E}- \\
03 \\
\end{array}$ & $1.30 \mathrm{E}-02$ & $3.00 \mathrm{E}-02$ \\
\hline Resuspension $r$ & $5.00 \mathrm{E}-10$ & $5.00 \mathrm{E}-10$ & $\begin{array}{r}5.00 \mathrm{E}- \\
10\end{array}$ & $\begin{array}{r}5.00 \mathrm{E}- \\
10\end{array}$ & $1.00 \mathrm{E}-11$ & 1.00E-11 \\
\hline Resusp_power r3 & 1.5 & 1.5 & 1.5 & 1.5 & 1.5 & 1.5 \\
\hline Resusp UWI_0 m/s & 1 & 0.5 & 0.5 & 0.5 & 5 & 10 \\
\hline
\end{tabular}

